MATHMOD

2015

\title{
MATHMOD 2015 ABSTRACT VOLUME
}

\author{
F. Breitenecker, A. Kugi, I. Troch, Eds.
}

8th Vienna Conference on Mathematical Modelling

February 18-20, 2015

Vienna University of Technolog

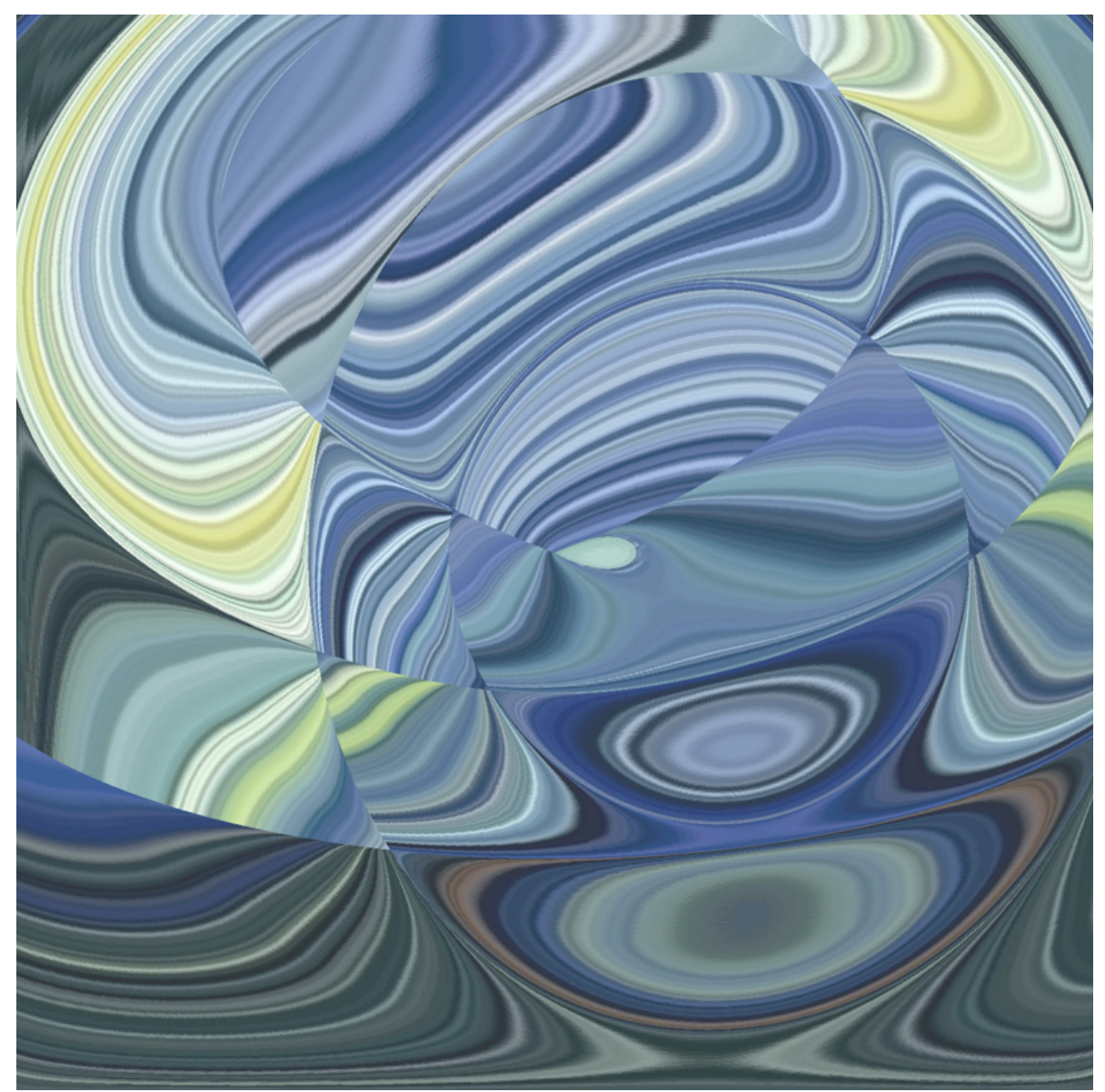




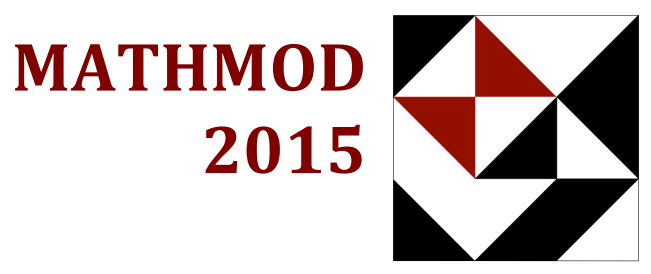



ARGESIM Report No. 44 ASIM Mitteilung AM153

\section{MATHMOD 2015 ABSTRACT VOLUME}

F. Breitenecker, A. Kugi, I. Troch, Eds.

$8^{\text {th }}$ Vienna Conference on Mathematical Modelling

February 18-20, 2015 Vienna University of Technology, Austria 


\title{
ARGESIM Reports
}

Published by ARGESIM and ASIM, German Simulation Society,

Div. of GI - German Society for Informatics / Informatics and Life Sciences

\section{Series Editor:}

Felix Breitenecker, ARGESIM / ASIM, TU Wien

Felix.Breitenecker@tuwien.ac.at

Thorsten Pawletta, ASIM, Univ. Wismar

Thorsten.Pawletta@hs-wismar.de

\section{ARGESIM Report No. 44 \\ ASIM Mitteilung AM153}

Titel: $\quad$ MATHMOD 2015 Vienna - Abstract Volume

Editors: Felix Breitenecker, Andreas Kugi, Inge Troch

Vienna University of Technology, Austria

ISBN: 978-3-901608-46-9 (print)

ISBN: 978-3-901608-40-7 (ebook)

DOI: $\quad 10.11128 /$ arep.44

Pages: $394+x v i$

\author{
ARGE Simulation News (ARGESIM) \\ c/o F. Breitenecker, Div. Mathematical Modelling and Simulation \\ Vienna Univ. of Technology \\ Wiedner Hauptstrasse 8-10, A-1040 Vienna, Austria \\ Tel.: +43-1-58801-10115, Fax: +43-1-58801-10199 \\ Email: info@argesim.org; WWW: http://www.argesim.org
}




\section{MATHMOD 2015 ABSTRACT VOLUME}

Table of Content 
MATHMOD 2015 Abstract Volume 


\section{MATHMOD Plenary Lectures}

Model-Based Approaches for the Future Sustainable Aircraft: The EU ADDSAFE

Project

Goupil, Philippe

Iterative Schemes for Coupled Multiphysical Problems in Electrical Engineering

Schöps, Sebastian

What Has Instrumental Variable Method to Offer for System Identification? Gilson, Marion

Advanced Mechatronics for Precision Engineering and Mechatronic Imaging Systems Schitter, Georg

\section{MATHMOD Full Contributions Biological and Chemical Systems}

Influence of an Asymptotic Pressure Level on the Windkessel Models of the Arterial System

Parragh, Stephanie; Hametner, Bernhard; Wassertheurer, Siegfried

A Compact Mathematical Model of Mandelate Racemase Production and Chaperone Overexpression in E. Coli

Kramer, Bernhard; Tüngler, Ralf; Bettenbrock, Katja; Conradi, Carsten

Efficient DQMOM for Multivariate Population Balance Equations and Application to Virus Replication in Cell Cultures Dürr, Robert; Müller, Thomas; Kienle, Achim

Design of a Molecular Communication Channel by Modelling Enzyme Kinetics Rabenstein, Rudolf

Mathematical Modelling of Molecular Adsorption in Zeolite Coated Frequency

Domain Sensors

Murrieta-Rico, Fabian N.; Mercorelli, Paolo; Sergiyenko, Oleg; Petranovskii, Vitalii; Hernandez-Balbuena, Daniel; Tyrsa, Vera

Modeling Chemical Reaction Networks on the Pontryagin Bundle with the Hamilton-

Pontryagin Approach

Lindhorst, Henning; Waldherr, Steffen

\section{MATHMOD Full Contributions}

\section{Discrete Systems and Manufacturing Applications}

Case Indexing in Case-Based Reasoning by Applying Situation-Operator Model As

Knowledge Representation Model

Sarkheyli, Arezoo; Söffker, Dirk

Integrating Situated Human Interaction Modeling and Stochastic State Automata for

Improved Technical Situation Awareness Muthig, Olga; Wang, Jiao; Deng, Qi; Söffker, Dirk

Adaptive neuro-fuzzy approach to predict tool wear accurately in turning operations for maximum cutting tool utilization Sarhan, Ahmed A.D.

Modeling of Layering Growth Processes Using a Monte Carlo Approach

Rieck, Christian; Bück, Andreas; Tsotsas, Evangelos

Improve Wire EDM Performance at Different Machining Parameters - ANFIS

Modeling

Maher, Ibrahem; Hui Ling, Liew; Sarhan, Ahmed A.D.; Hamdi, M. 


\section{MATHMOD Full Contributions \\ Theoretical Aspects}

Faster Structural Analysis of Differential-Algebraic Equations by Graph Compression Höger, Christoph

Methods for Cellular Automata and Evolution Systems in Modelling and Simulation Schneckenreither, Günter; Popper, Nikolas; Breitenecker, Felix

Lagrangian GSOM Traffic Flow Models on Junctions

Costeseque, Guillaume; Lebacque, Jean-Patrick; Khelifi, Asma

Spatial Effects in Stochastic Microscopic Models - Case Study and Analysis Bicher, Martin; Popper, Nikolas

Alternative Approaches for Groundwater Pollution

Winkler, Stefanie; Bicher, Martin; Breitenecker, Felix

\section{MATHMOD Full Contributions}

\section{Numerical and Computer Modeling}

A Volume Conserving Discrete Formulation of Aggregation Population Balance

Equations on Non-Uniform Meshes Mehakpreet, Singh; Kumar, Jitendra; Bück, Andreas

Alternating Order Algorithm Based on Stages of the Ceschino Method Novikov, Anton; Novikov, Eugeny

Optimal Startup Control of a Steam Power Plant Using the JModelica Platform Fethi, Belkhir; Daniel, Kraus Cabo; Felix, Felgner; Georg, Frey

An Analysis of Performance-Critical Properties of Modelica Models Cafferkey, Neil; Provan, Gregory

\section{MATHMOD Full Contributions Environmental Systems}

Further Developments in Dynamic Modelling of CO2 Capture from Flue Gas Dickinson, Jillian L; Puxty, Graeme; Percy, Andrew; Verheyen, T. Vincent

Pitfalls Associated with Estimation of Radiological Burden of Population Caused by

Radiation Accident

Pecha, Petr

Modelling the Effect of Temperature on Phytoplankton Growth across the Global

Ocean

Grimaud, Ghjuvan Micaelu; Le Guennec, Valérie; Ayata, Sakina-Dorothée; Mairet, Francis; Sciandra, Antoine; Bernard, Olivier

A General Analytical Capture Zone Model: A Tool for Groundwater Remediation

Samani, Nozar; Zarei-Doudeji, Somayeh

\section{MATHMOD Full Contributions Mechanical Systems}

A Closed-Form Approach to Determine the Base Inertial Parameters of Complex

Structured Robotic Systems

Klodmann, Julian; Lakatos, Dominic; Ott, Christian; Albu-Schaeffer, Alin

On the Inertially Decoupled Structure of the Floating Base Robot Dynamics

Garofalo, Gianluca; Henze, Bernd; Englsberger, Johannes; Ott, Christian

Reduced Equations of Motion for a Wheeled Inverted Pendulum

Delgado Londoño, Sergio; gajbhiye, sneha; Banavar, Ravi N. 
Finite Segment Model Complexity of an Euler-Bernoulli Beam 56

Louca, Loucas

Modelling and Simulation of Passive Limited-Slip Differentials 57

Forstinger, Martin; Bauer, Robert; Hofer, Anton

Mathematical Modeling and Analysis of a Hydrostatic Drive Train 58

Zeman, Paul; Kemmetmueller, Wolfgang; Kugi, Andreas

Dynamical Modeling of Constraints with Friction in Mechanical Systems 59

Specker, Thomas; Buchholz, Michael; Dietmayer, Klaus Christian Jürgen

Use of Complex Signals in Modelling of Journal Bearings 60

Wagnerova, Renata; Tůma, Jiří

\section{MATHMOD Full Contributions}

\section{Electrical Systems}

Magnetic Equivalent Circuit Modeling of a Saturated Surface-Mounted Permanent

Magnet Synchronous Machine

Faustner, David; Kemmetmueller, Wolfgang; Kugi, Andreas

Some Model Properties to Control a Permanent Magnet Machine Using a Controlled

Invariant Subspace

Mercorelli, Paolo; Sergiyenko, Oleg; Hernandez-Balbuena, Daniel; Rodriguez-Quiñonez, Julio;

Flores-Fuentes, Wendy; Basaca-Preciado, Luis

Availability Assessment of Electric Power Based on Switch Reliability Modelling with

Dynamic Bayesian Networks: Case Study of Electrical Distribution Network lakehal, abdelaziz; Ghemari, Zine

Modelling, Simulation and Identification of Heat Loss Mechanisms for Parabolic

Trough Receivers Installed in Concentrated Solar Power Plants Caron, Simon; Röger, Marc

Metropolis Monte Carlo Based Relaxation of Atomistic III-V Semiconductor Models Detz, Hermann; Strasser, Gottfried

Correlated Noise Description Using HDLs Haase, Joachim; Lange, André

Pull-in range of the classical PLL with impulse signals Alexandrov, K. D.; Kuznetsov, Nikolay; Leonov, Gennady; Neittaanmaki, Pekka; Seledzhi, Svetlana

Simulation of the Classical Analog Phase-Locked Loop Based Circuits Kuznetsov, Nikolay; Leonov, Gennady; Yuldashev, Marat; Yuldashev, Renat

\section{MATHMOD Full Contributions}

\section{Identification}

Identification of Wiener Time Delay Systems Based on Hierarchical Gradient

Approach Atitallah, Asma; BEDOUI, Saïda; abderrahim, kamel

Towards the Possibility of Applying the Wavelet Analysis to Derive Predicting Models Sakrutina, Ekaterina; Bakhtadze, Natalia

A Nonparametric Measure of Dependence in the Statistical Linearization Chernyshov, Kirill; Sakrutina, Ekaterina

Determination of Leaky Confined Aquifer Parameters by a Multilayer Perceptron

Neural Network Azari, Tahereh; Samani, Nozar; Mansoori, Eghbal 


\section{MATHMOD Full Contributions Control Systems}

Designing Model and Control System Using Evolutionary Algorithms

Corn, Marko; Atanasijevic-Kunc, Maja

Proportional-Integral-Observer: A Brief Survey with Special Attention to the Actual

Methods Using ACC Benchmark

Bakhshande, Fateme; Söffker, Dirk

Bond-Graph-Based Multivariable non linear UIO-Type estimation

Tarasov, Evgeny; Sueur, Christophe; Ould Bouamama, Belkacem; Dauphin-Tanguy,Geneviève

Integrated Optimal Controller for Polyvalent Heating/Cooling Systems with Minimal

Cost and Energy Usage

Muffato, Leonardo Angelo; Bianchi, Mikael; Wemhoener, Carsten; Kunz, Dominique

Multivariable Control of Large Variable-Speed Wind Turbines for Generator Power

Regulation and Load Reduction Njiri, Jackson Githu; Liu, Yan; Söffker, Dirk

Flatness Based Control of a Ball in Tube System Herrmann, Lukas; Broecker, Markus

Ball on Ball: Modeling and Control of a Novel Experiment Set-Up Gahleitner, Reinhard

Multi-Variable Integral Sliding Mode Control of a Two Degrees of Freedom

Helicopter Butt, Saif Siddique; Aschemann, Harald

Flight Path Optimization of a Hang-Glider in a Thermal Updraft Chudej, Kurt; Klingler, Anna-Lena; Britzelmeier, Andreas

Modeling and Control of an Autonomous Hybrid Vehicle for Navigation and Guidance Moriwaki, Katsumi

BEECLUST Used for Exploration Tasks in Autonomous Underwater Vehicles Bodi, Michael; Möslinger, Christoph; Thenius, Ronald; Schmickl, Thomas

\section{MATHMOD Full Contributions \\ Infinite-dimensional Systems}

Boundary Control of a Parallel-Flow Heat Exchange Process with Boundary

Observation Sano, Hideki

Noise and Fluctuations in Nanowire Biosensors Tulzer, Gerhard; Heitzinger, Clemens

Capacity Drop and Traffic Hysteresis As a Consequence of Bounded Acceleration Khoshyaran, Megan; Lebacque, Jean-Patrick

Optimal Design of Elastic Rod Motions Based on a Projection Approach Kostin, Georgy; Saurin, Vasily

\section{MATHMOD Full Contributions Operational Research and Planning}

A Game-Theoretic Calibration Approach for Agent-Based Planning Simulations Buwaya, Julia; Cleophas, Catherine

A Reliability Measure for Time Series Forecasting Predictor George, K.M.; Park, Nohpill; Yang, Zhuxi

Generation of Feasible Petri Net Based Scheduling Problem Solutions Music, Gasper 
Adjacency in Hospital Planning

Lorenz, Wolfgang E.; Bicher, Martin; Wurzer, Gabriel

MODYPLAN: Early-Stage Hospital Simulation Based on Treatment Chains

Wurzer, Gabriel; Lorenz, Wolfgang E.; Rößler, Matthias; Hafner, Irene; Glock, Barbara;

Bruckner, Martin; Popper, Nikolas

\section{MATHMOD Minisymposia}

\section{Biomathematics: What's new?}

Stochastic Model for HIV Dynamics in HIV Specific Helper Cells

Carla, Pinto; Carvalho, Ana

The Effect of Noise Intensity in a Stochastic Model for HIV-Specific Helper Cells Carvalho, Ana; Carla, Pinto

Effect of Drug-Resistance in a Fractional Complex-Order Model for HIV Infection Carla, Pinto; Carvalho, Ana

Modeling HIV Dynamics in the Three Stages of Infection Carvalho, Ana; Carla, Pinto

\section{MATHMOD Minisymposia Model Reduction}

Data-Driven Model Reduction for Weakly Nonlinear Systems: A Summary Antoulas, Athanasios C.

Quadrature-Based IRKA for Optimal H2 Model Reduction Beattie, Christopher A.; Drmac, Zlatko; Gugercin, Serkan

Towards Practical Implementations of Balanced Truncation for LTV Systems Lang, Norman; Saak, Jens; Stykel, Tatjana

A New Error Estimation for Model Order Reduction of Parametrized Evolution

Equations Zhang, Yongjin; Feng, Lihong; Li, Suzhou; Benner, Peter

Automatic Model Reduction of Linear Population Balance Models by Proper

Orthogonal Decomposition Khlopov, Dmytro; Mangold, Michael

A Black-Box Method for Parametric Model Order Reduction Geuss, Matthias; Lohmann, Boris; Peherstorfer, Benjamin; Willcox, Karen Elizabeth

Order-Reduction of Fields-Level Models with Affine and Non-Affine Parameters by

Interpolation of Subspaces Burgard, Stefan; Farle, Ortwin; Klis, Daniel; Dyczij-Edlinger, Romanus

Non-Modal Based Model Reduction for Explicit Crash Codes Fehr, Joerg

Improvement of Krylov-Subspace-Reduced Models by Iterative Mode-Truncation Lein, Claudius; Beitelschmidt, Michael; Bernstein, David

Model Order Reduction for Magneto-Quasistatic Equations Kerler, Johanna; Stykel, Tatjana

Model Order Reduction Approaches for the Optimal Design of Permanent Magnets in

Electro-Magnetic Machines Alessandro, Alla; Hinze, Michael; Lass, Oliver; Ulbrich, Stefan

Fast Simulation of Wireless Power Transfer Systems with Varying Coil Alignment Klis, Daniel; Burgard, Stefan; Farle, Ortwin; Dyczij-Edlinger, Romanus

Interface Reduction for CMS Methods and Alternative Model Order Reduction Holzwarth, Philip; Eberhard, Peter 
A Joint IMEX-MOR Approach for Water Networks

Grundel, Sara; Jansen, Lennart

Optimization Strategy for Parameter Sampling in the Reduced Basis Method

Lapichino, Laura; Volkwein, Stefan

Basis Generation Approaches for a Reduced Basis Linear Quadratic Regulator Schmidt, Andreas; Dihlmann, Markus; Haasdonk, Bernard

A Certified Reduced Basis Approach for Parametrized Linear-Quadratic

OptimalControl Problems with Control Constraints Bader, Eduard; Kaercher, Mark; Grepl, Martin; Veroy-Grepl, Karen

Numerical Investigations of an Error Bound for Reduced Basis Approximations of

Noncoercice Variational Inequalities Glas, Silke; Urban, Karsten

The Empirical Cross Gramian for Parametrized Nonlinear Systems Himpe, Christian; Ohlberger, Mario

Interactive Simulations Using Localized Reduced Basis Methods Buhr, Andreas; Ohlberger, Mario

\section{MATHMOD Minisymposia}

\section{Object-Oriented Modelling, Simulation and Optimization}

Efficient Computation of State Derivatives for Multi-Rate Integration of Object-

Oriented Models Casella, Francesco

Design of Feed Drives with Object-Oriented Behavior Models Özdemir, Denis; Herfs, Werner; Lohse, Wolfram; Brecher, Christian

A Modelica Simulator to Support the Development of the Control System of an

Autonomous All-Terrain Mobile Robot D'Amelio, Enzo Luigi; Bascetta, Luca; Cucci, Davide Antonio; Matteucci, Matteo; Bardaro, Gianluca

Effects of Event-Free Noise Signals on Continuous-Time Simulation Performance Van der Linden, Franciscus L.J.; Klöckner, Andreas; Zimmer, Dirk

Input Design and Parameter Estimation with Open Source Tools Mayr, Simon; Grabmair, Gernot; Reger, Johann

\section{MATHMOD Minisymposia Multiscale Modeling of Transport- and Flow-Problems}

Modelling of Langevin Equations by the Method of Multiple Scales Geiser, Juergen

Reaction-Diffusion Approach to Somite Formation Lemarchand, Annie; Bianca, Carlo

Smoothed Particle Hydrodynamics and Model-Order Reduction for Efficient Modeling of Fluid-Structure Interaction Schörgenhumer, Markus; Humer, Alexander; Gerstmayr, Johannes

Modelling Approach for Mobile and Immobile Transport Problems with Multiple

Time-Scales Geiser, Juergen

Multiscale Analysis of a Retarded Equation: From Kinetic to Macroscopic Scale Bianca, Carlo; Lemarchand, Annie

A Discrete/Continuous Numerical Approach to Multi-Physics Peters, Bernhard; Besseron, Xavier; Estupinan Donoso, Alvaro Antonio; Mahmoudi, Amirhoushang; Mohseni, Mohamad 


\section{MATHMOD Minisymposia Engineering Applications of Fractional Derivatives}

Four Ways to Interpret Temporal Memory Operators in the Wave Equation 173 Holm, Sverre

Recent Results in Fractional-Order Modeling for Multi-Agent Systems and Linear

Friction Welding Goodwine, Bill; Leyden, Kevin

Fractional-Order Modeling of High-Pressure Fluid-Dynamic Flows: An Automotive

Application Lino, Paolo; Maione, Guido; Saponaro, Fabrizio

Optimized Identification Method for Fractional Heating Processes on Heterogeneous 178 Materials Caponetto, Riccardo; Sapuppo, Francesca; Tomasello, Vincenzo

\section{MATHMOD Minisymposia Distributed Parameter Systems}

Infinite Dimensional Port Hamiltonian Representation of Reaction Diffusion Processes ZHOU, Weijun; Hamroun, Boussad; Le Gorrec, Yann; Couenne, Francoise

Distributed and Backstepping Boundary Controls to Achieve IDA-PBC Design

Vu, Trang; Lefevre, Laurent; Nouailletas, Rémy

Asymptotic Stabilisation of Distributed Port-Hamiltonian Systems by Boundary

Energy-Shaping Control

Macchelli, Alessandro; Le Gorrec, Yann; Ramirez, Hector

Heat Transfer with Specular Reflections in an Experimental Annealing Device Jadachowski, Lukas; Steinboeck, Andreas; Kugi, Andreas

How to Choose the State for Distributed-Parameter Systems, a Geometric Point of View Schlacher, Kurt; Schöberl, Markus

Bi-Zone Modelling and Simulation of Twin-Screw Extrusion Process with Variable Screw Speed Lotero, Fernando; Couenne, Francoise; Maschke, Bernhard; Sbarbaro, Daniel G.

Dynamical Models of Axially Moving Rods with Tensile and Bending Stiffness Steinboeck, Andreas; Baumgart, Michael; Stadler, Georg; Saxinger, Martin; Kugi, Andreas

Modeling the Dynamics of a Flexible Belt Drive Using the Equations of a Deformable

String with Discontinuities Vetyukov, Yury; Eliseev, Vladimir; Krommer, Michael

Lagrangian and Port-Hamiltonian Formulation for Distributed-Parameter Systems Schöberl, Markus; Schlacher, Kurt

\section{MATHMOD Minisymposia}

\section{Modelling of Dynamics and Control for Mobile Robots}

Swimming and Crawling Motility at Microscopic Scales: From Biological Templates

to Bio-Inspired Devices DeSimone, Antonio; Cicconofri, Giancarlo

Limbless Locomotion on Solid Surfaces: A Case Study in Soft Bio-Inspired Robotics Cicconofri, Giancarlo; DeSimone, Antonio

Simple Modelling of the Rowing Process 
Modelling of Quasi-Static Motions for Three-Body Mobile Robot

Chernousko, Felix L.; Figurina, Tatiana

Control of a Chain-Like Locomotion System Subject to Undulatory Excitation

Bolotnik, Nikolay; Pivovarov, Mikhail; Zeidis, Igor; Zimmermann, Klaus

An Approach to the Dynamics of Vibration-Driven Robots with Bristles

203 Becker, Felix

Dynamics and Control of a Suction-Type Wall-Climbing Robot

Kuo, Chung-Hsien; Aniroh, Yunafi'atul; Yudha, Andika Pramanta; Chou, Hung-Chyun;

Bolotnik, Nikolay; Valery, Gradetsky; Chernousko, Felix L.

Cascaded Nonlinear Control of a Duocopter with Disturbance Compensation by an

Unscented Kalman Filter Aschemann, Harald; Meinlschmidt, Thomas

Mathematical and Dynamic Model of the Lower Limb Exoskeleton and Synthesis of

Aliseychik, Anton; Orlov, Igor; Pavlovsky, Vladimir E.; Platonov, Alexander; Ptakhin,

Finite Element Approaches for Real-Time Control and Observer Design of Flexible

Rack Feeder Systems

Rauh, Andreas; Warncke, Julia; Kostin, Georgy; Saurin, Vasily; Aschemann, Harald

\section{MATHMOD Minisymposia}

\section{Mathematical Modelling and Control of Bio-Chemical Processes}

Development and Analysis of a Mathematical Model for a Synthetic Biological Cell Schneider, Eugenia; Mangold, Michael

On Stability Analysis of Genetic Regulatory Networks Represented by Delay-

Differential Equations

Haustenne, Laurie; Bastin, Georges; Coron, Jean-michel; Fontaine, Laetitia; Hols, Pascal

Symmetry Principles in Optimization Problems : An Application to Protein Stability

Prediction

Pucci, Fabrizio; Bernaerts, Katrien; Teheux, Fabian; Gilis, Dimitri; Rooman, Marianne

Metabolic Flux Analysis of Hybridoma Cells: Underdetermined Network and

Influence of Batch and Perfusion Operating Modes

Fernandes, Sofia; Bastin, Georges; Vande Wouwer, Alain

Structural Identifiability Analysis of the Anaerobic Digestion Model No. 1 Using a

Local Algebraic Observability Approach

Lauwers, Joost; Nimmegeers, Philippe; Logist, Filip; Van Impe, Jan F.M.

Kriging Based Iterative Parameter Estimation Procedure for Biotechnology

Applications with Nonlinear Trend Functions Freier, Lars; von Lieres, Eric

Set-Membership Identification of an Agro-Ecosystem from a Small Data Set: The Case of Ammonia Volatilisation in a Flooded Rice Field Khairudin, Nurulhuda; Struik, Paul C.; Keesman, Karel J.

Parameter Identification of the Droop Model Using Optimal Experiment Design Benavides, Micaela; Telen, Dries; Lauwers, Joost; Logist, Filip; Van Impe, Jan F.M.; Vande Wouwer, Alain

Macroscopic Modelling of Intracellular Reserve Carbohydrates Production During

Baker's Yeast Cultures Richelle, Anne; Bogaerts, Philippe

Validation of a Simple Fouling Model for a Submerged Membrane Bioreactor Araujo Pimentel, Guilherme; Dalmau, Montse; Vargas, Alejandro; Comas, Joaquim; Rodriguez-Roda, Ignasi; Rapaport, Alain; Vande Wouwer, Alain 
Thermodynamic Behavior-Rules for a Bacterial Individual-Based Model to Study the

Denitrification Process

Araujo Granda, Pablo Alejandro; Gras Moreu, Anna; Ginovart Gisbert, Marta

Modelling Freezing Processes of High Concentrated Systems

Lopez-Quiroga, Estefania; Wang, Rui; Gouseti, Ourania; Fryer, Peter Jonathan; Bakalis, Serafim

\section{MATHMOD Minisymposia Dynamics of Machines and Structures}

Optimal Deployment of a Tethered Satellite Using Tension Control

Steindl, Alois

Bicycle Rider Control Modelling for Path Tracking

Edelmann, Johannes; Haudum, Martin; Plöchl, Manfred

Efficient Simulation of Railway Pantograph/ Catenary Interaction Using Pantograph-

Fixed Coordinates

Ritzberger, Daniel; Talic, Emir; Schirrer, Alexander

Co-Simulation of Matlab and FlightGear for Identification and Control of Aircraft Aschauer, Guilherme; Schirrer, Alexander; Kozek, Martin

Data-based and analytic modelling for model-based control of a flexible beam Dullinger, Christian; Schirrer, Alexander; Kozek, Martin

Analysis of time-varying mechanical systems using the method of impulsive

parametric excitation Pumhössel, Thomas; Hehenberger, Peter

\section{MATHMOD Minisymposia Free Boundary Problems in Applications}

Numerics of Contact Line Motion for Thin Films

Dirk, Peschka

Sharp Interface Limits of an Anisotropic Phase Field Model for Solid-State Dewetting Dziwnik, Marion; Muench, Andreas; Wagner, Barbara

A free boundary problem for interfaces in forward-backward lattice diffusion Helmers, Michael

Sharp Interface Limits of the Cahn-Hilliard Equation with Degenerate Mobility Lee, Alpha Albert; Suli, Endre; Andreas, Muench

A Gradient Flow Approach for Dissipative Equations on a Free Domain Zaal, Martijn

Modelling, Simulation and Stability of Free Surface and Bulk Nanobubbles in

Hydrogen Electrolysis Kimmerle, Sven-Joachim

Towards Topology Optimization in Quasi-Static Elastoplasticity Schleicher, Stephan; Herzog, Roland

A Method to Model Impulsive Multi-Body-Dynamics Using Riemann-StieltjesIntegrals

Michael, Johannes; Gerdts, Matthias 


\section{MATHMOD Minisymposia}

\section{Interdisciplinary Data Based Modelling and Simulation in Health Systems Research}

Data Wrangling: Making Data Useful Again

Endel, Florian; Piringer, Harald

Reproducible Database Queries in Privacy Sensitive Applications

Proell, Stefan; Mayer, Rudolf; Rauber, Andreas

Identifying Structural Changes in Austrian Social Insurance Data

Ortner, Thomas; Filzmoser, Peter; Endel, Gottfried

Visual Analysis of Disease Prevalence Based on Health Accounting Data Piringer, Harald

Simulation of Physiologic Ectopic Beats in Heartbeat Intervals to Validate Algorithms Bachler, Martin; Hörtenhuber, Matthias; Frank, Martin; Wassertheurer, Siegfried; Mayer, Christopher

Modeling Methods Development for Routine Data Based Screening Evaluation:

Decision Making for Organized Abdominal Aortic Aneurysm Screening Zauner, Günther; Urach, Christoph; Wilbacher, Ingrid; Endel, Gottfried

\section{MATHMOD Minisymposia \\ Computational Methods in Modelling and Simulation of Fluid Power Systems}

Multi-Phase and Multi-Component CFD Analysis of a Load - Sensing Proportional

Control Valve

Bigliardi, Elisa; Francia, Marco; Milani, Massimo; Montorsi, Luca; Paltrinieri, Fabrizio;

Stefani, Matteo

A Combined Methodology for Studying the Axial Balancing Mechanism of Orbit

Annular Hydraulic Machines

Bigliardi, Elisa; Francia, Marco; Milani, Massimo; Montorsi, Luca; Paltrinieri, Fabrizio;

Stefani, Matteo

A Study on Mesh Refinement in OpenFOAM for Wave Propagation Problems in Fluid

Power Systems

Fries, Clemens; Manhartsgruber, Bernhard

Low Frequency Correction of a Multi-degrees-of-freedom Model for Hydraulic

Pipeline Systems Mikota, Gudrun

A Reference Model for Modal Approximations of Linear Transmission Line Dynamics Manhartsgruber, Bernhard

\section{MATHMOD Minisymposia}

\section{Systems of Systems Modelling, Simulation and Optimization}

Challenges in the Modelling and Operation of Physically Coupled Systems of Systems Kampert, David; Epple, Ulrich

Mean Field Modeling of Large-Scale Energy Systems

Gentile, Basilio; Grammatico, Sergio; Lygeros, John

A Modelica-Based Modeling and Simulation Framework for Large-Scale Cyber-

Physical Systems of Systems

Nazari, Shaghayegh; Sonntag, Christian; Engell, Sebastian

Levels of Detail and Appropriate Model Types for Virtual Commissioning in 
Equipment Interconnection Models in Discrete Manufacturing

Grüner, Sten; Wagner, Constantin; Weber, Peter; Epple, Ulrich

Modeling and Transformation of Systems of Systems Using Linked Data

Graube, Markus; Urbas, Leon

\section{MATHMOD Minisymposia}

\section{Mathematical Modeling for Nanoelectronic Coupled Problems}

A Fractional Step Method for the Dynamic Electro-Thermal Modelling of Device

Structures

Duque Guerra, David José; Schöps, Sebastian

Model Order Reduction of an Electro-Thermal Package Model

Banagaaya, Nicodemus; Feng, Lihong; Meuris, Peter; Schoenmaker, Wim; Benner, Peter

Measurement Setup for Identifying Parameters of the Encapsulated Bond Wires Petrzela, Jiri; Sotner, Roman; Gotthans, Tomas; Drinovsky, Jiri; Kratochvil, Tomas; Wieers, Aarnout

Uncertainty Quantification in Electro-Thermal Coupled Problems Based on a Power

Transistor Device

Putek, Piotr Adam; Meuris, Peter; Günther, Michael; ter Maten, E. Jan W.; Pulch, Roland; Wieers, Aarnout; Schoenmaker, Wim

Interface Model Integrating Full-Wave Maxwell Simulation Models into Modified

Nodal Equations for Circuit Simulation

Strohm, Christian; Tischendorf, Caren

\section{MATHMOD Minisymposia}

\section{Modeling in Sport and Human Movement Science}

Constants of Movement of Muscular Activation and Force-Velocity Relationship from

Nonlinear Optimization

Penasso, Harald

Normalized and xPower to Generate Pacing Strategies in Road Cycling Dahmen, Thorsten; Saupe, Dietmar

Towards Data-Based Assessment of Individual Tactics Skills in Team Sports Based on

Fuzzy Petri Nets

Serafini, Andrea; Leser, Roland; Hoch, Thomas; Baca, Arnold; Moser, Bernhard Alois

Analysis of Tactical Defensive Behavior in Team Handball by Means of Artificial

Neural Networks

Tilp, Markus; Schrapf, Norbert

Recurrence Analysis of Golfers' Performances on the PGA TOUR

Stöckl, Michael

Modeling Soccer Matches by Recurrence Analyses Lames, Martin; Plück, Denise

\section{MATHMOD Minisymposia}

\section{Modeling and Simulation to Support Sustainable Energy} Systems and Water Treatment

Comparison Case between Modelica and Specialized Tools for Building Modelling

Yebra, Luis J.; Fernandez Gonzalez, Daniel

Environmental Impacts and Benefits of Smart Home Automation: Life Cycle 
The Role and the Impact of Electric Vehicles on the Local Power Grid within the

Northern Finland Climatic Conditions Caló, Antonio; Louis, Jean-Nicolas; Pongrácz, Eva

Evaluation of Fluidized Bed Condition by Image Analysis and Modelling Liukkonen, Mika; Hiltunen, Teri; Hiltunen, Yrjö

Multivariate Analysis Models for Wood Properties Combined with Open Modelica

Model for Process Performance Monitoring Skvaril, Jan; Kyprianidis, Konstantinos; Avelin, Anders; Odlare, Monica; Dahlquist, Erik

Monitoring of Water Processes Using Intelligent Condition Indicators Liukkonen, Mika; Hiltunen, Yrjö; Laakso, Ilkka; Juntunen, Petri

\section{MATHMOD Minisymposia}

\section{Regularization Techniques for DAEs to Improve Model Equations for the Numerical Treatment}

Regularization of Nonlinear DAEs Based on Algebraic Analysis Scholz, Lena; Steinbrecher, Andreas

Regularization of Nonlinear DAEs Based on Structural Analysis McKenzie, Ross; Pryce, John Derwent; Nedialkov, Nedialko; Tan, Guangning

Regularization of Quasi-Linear Differential-Algebraic Equations Steinbrecher, Andreas

Index Reduction and Regularisation Methods for Multibody Systems Pöll, Carina; Hafner, Irene

Regularization of Electrical Circuits Tischendorf, Caren

Comparison of Regularisation Methods Referring to a Multi-Pendulum Case Study Hafner, Irene; Pöll, Carina

\section{MATHMOD Poster Contributions}

Methods of Inverse Simulation for Nonlinear Ship Steering Systems Investigations Murray-Smith, David

Closed form model of manipulators with highly flexible links Scaglioni, Bruno; Ferretti, Gianni

Modeling of Elastic Robotic Arm Using a Soft-Computing Algorithm Tamimi, Hammam; Söffker, Dirk

Modeling, Control, and Powermanagement Optimization for an Emulated Multi-

Source Hybrid Drivetrain Moulik, Bedatri; Söffker, Dirk

Network Based Simulation in Water Construction Brunner, Stefan; Höfinger, Gerhard

A New Tool for Visual Modeling - Rand Model Designer Senichenkov, Yuri Borisovitch; Isakov, Andrey; Kolesov, Yuri Borisovitch

Efficient Modelling Approaches in Control Zupančič, Borut

Numerical Solution of an Optimal Control Problem in Cancer Treatment: Combined

Radio and Anti-Angiogenic Therapy Chudej, Kurt; Wagner, Lisa; Pesch, Hans Josef

Information Management Platform Model for Local Sightseeing and Disaster

Prevention Information Tsuchiya, Takeshi 
Influence of Sample Period Variation to MPC Trajectory Tracking of Mobile Robot

Klancar, Gregor; Skrjanc, Igor

Modeling and Design of High-Speed FM-AFM Driver Electronics Using Cadence

Virtuoso and Simulink Schlecker, Benedikt; Anna, Eichel; Ortmanns, Maurits; Fantner, Georg Ernest; Anders, Jens

Is Driving Assisted Behaviour Infectious? Using Spectral Analysis to Understand Car

Following Behaviour of Naive Drivers Stemmler, Eric; Oeltze, Katharina; Schieß1, Caroline

Modelling of Low Power Electronic Loads in Harmonic Analysis Tokic, Amir; Jukan, Admir; Suljkanovic, Vedran

P(t)mor: Time-Varying Parametric Model Order Reduction and Applications for

Moving Loads Cruz Varona, Maria; Geuss, Matthias; Lohmann, Boris

Advances on the Adaptive Selection of Both Shifts and Reduced Order in H2-PseudoOptimal Model Reduction Castagnotto, Alessandro; Panzer, Heiko K. F.; Wolf, Thomas; Lohmann, Boris

Equations of Motion with Redundant Coordinates for Mechanical Systems on Manifolds Konz, Matthias; Rudolph, Joachim

Combining Discrete Optimization and Simulation to Understand Stochastic Hub Location Problems Dabidian, Peiman; Meier, J. Fabian; Goedicke, Ina; Clausen, Uwe

System Entity Structure Ontology Toolbox for MATLAB/Simulink: Used for Variant Modelling Pawletta, Thorsten; Pascheka, Daniel; Schmidt, Artur

Robotic Control \& Visualization Toolbox for MATLAB Pawletta, Thorsten; Freymann, Birger; Deatcu, Christina; Schmidt, Artur

Two Dimensional Mathematical Model of Heat-Transmission Using MAPLE Perjesi-Hamori, Ildiko

Distributed Parameter Model of the Buck Converter with Constant Inductive Load Huang, Chenzi; Woittennek, Frank; Roebenack, Klaus

Taylor Series Based Solution of Linear ODEs Systems and MATLAB Solvers

Comparison Satek, Vaclav; Kocina, Filip; Kunovsky, Jiri; Schirrer, Alexander

SVD-AORA method for Dynamic Linear Time Invariant Model Order Reduction Kouki, Mohamed; Abbes, Mehdi; Mami, Abdelkader

Modelling "Breaking Bad": An Economic Model of Drugs and Population Dynamics

to Predict How the Series Itself Feeds Back into the Drug Market Rössler, Christiane; Witzmann, Magdalena; Schmickl, Thomas

Physiolibrary 2.3 - An Intuitive Tool for Integrative Physiology Mateják, Marek; Ježek, Filip; Tribula, Martin; Kofranek, Jiri

Model Predictive Control in Small Family House: Extravagance or Future of Energy

Consumption in Households Marušić, Ante; Drazen, Loncar

Comparison between a Non-Linear and Linearized Three-Compartment Model of a

Bioreactor for Hepatocyte Culturing Naghib, Seyed Danial; Di Renzo, Alberto; Curcio, Efrem; De Bartolo, Loredana; Di Maio, Francesco

Taylor Series Based Differential Formulas

Satek, Vaclav; Necasova, Gabriela; Kunovsky, Jiri; Chaloupka, Jan; Veigend, Petr 
MATHMOD 2015 Abstract Volume 


\section{MATHMOD 2015 ABSTRACT VOLUME}

Preface 
MATHMOD 2015 Abstract Volume 


\section{Preface}

MATHMOD 2015 brings together scientists and engineers conducting research in theory and application related to mathematical modelling in the beautiful city of Vienna. This offers the opportunity to present ideas, methods and results, and to discuss experiences or problems with experts from various areas of specialisation. Such discussions often lead to new ideas or innovative solutions.

The scope of the MATHMOD Conference Series intends to cover all aspects of mathematical modelling including modelling theory; first-principles modelling; identification; optimization; model order reduction; automation of modelling and software tools; computer modelling; modelling for/by simulation; co-simulation; modelling standards; qualitative, modular, interdisciplinary modelling; comparison of methods for modelling; alternative modelling methods (CAS, fuzzy, NN, QSS, etc.). Model analysis, calibration and experimental validation of models as well as the elaboration of effects of modelling errors on the overall performance of an engineering system also constitute important topics of this conference. Moreover, contributions with a strong focus on modelling dealing with challenging applications in engineering and natural sciences as well as in environmental systems, biotechnology, operations research, logistics and planning, medicine, physiology and health care are most welcome. The conference also provides the opportunity to discuss aspects of education in/for/with modelling or modelling aspects in the context of scientific computing and realtime applications.

The broad scope also evidences that mathematical modelling serves as a fundamental basis in many scientific disciplines and gains more and more importance in industry.

The plenary lectures of the MATHMOD 2015 conference reflect, at least to some extent, this wide spectrum of important topics in mathematical modelling ranging from applications in aerospace industry over state-of-the-art approaches in system identification and advanced numerical solution concepts for multiphysics problems to challenging examples in mechatronic system integration.

Model-Based Approaches for the Future Sustainable Aircraft: the EU ADDSAFE Project Dr. Philippe Goupil (R\&D Engineer, Airbus Operations S.A.S.)

What has Instrumental Variable Method to Offer for System Identification? Prof. Marion Gilson-Bagrel (Université de Lorraine, Nancy, France)

Iterative Schemes for Coupled Multiphysical Problems in Electrical Engineering Prof. Sebastian Schöps (TU Darmstadt, Germany)

\section{Advanced Mechatronics for Precision Engineering and Mechatronic Imaging Systems Prof. Georg Schitter (TU Vienna, Austria)}

Apart from regular sessions, also a number of minisymposia were organized by experts from different areas: 


\section{Model Reduction}

Martin Grepl (RWTH Aachen, Germany), Boris Lohmann (TU München, Germany)

\& Jens Saak (MPI Magdeburg, Germany)

Object-Oriented Modelling, Simulation, and Optimization: Models, Methods and Tools

Francesco Casella \& Gianni Ferretti (Politecnico di Milano, Italy)

Distributed Parameter Systems - New Methods for Modelling and Control

Markus Schöberl \& Kurt Schlacher (Johannes Kepler University Linz, Austria)

Free Boundary Problems in Applications: Recent Advances in Modelling, Simulation and Optimization

Sven-Joachim Kimmerle (UniBW Munich, Germany) \& Dirk Peschka (WIAS Berlin, Germany)

Engineering Applications of Fractional Derivatives

Mihir Sen \& Bill Goodwine (University of Notre Dame, USA)

Regularization Techniques for DAEs to Improve Model Equations for the Numerical Treatment

Andreas Steinbrecher \& Lena Scholz (TU Berlin, Germany)

Systems of Systems Modelling, Simulation and Optimization

David Kampert (RWTH Aachen, Germany)

Mathematical Modeling for Nanoelectronic Coupled Problems

Caren Tischendorf (Humboldt-Universität Berlin, Germany)

Dynamics of Machines and Structures

Thomas Pumhössel (Johannes Kepler University Linz, Austria) \& Horst Ecker (TU Vienna, Austria)

Modelling of Dynamics and Control for Mobile Robots

Felix L. Chernousko \& Nikolay N. Bolotnik (Russian Academy of Sciences,

Moscow, Russia)

Computational Methods in Modelling and Simulation of Fluid Power Systems

Bernhard Manhartsgruber (Johannes Kepler University, Linz, Austria)

Multiscale-Modelling of Transport- and Flow-Problems

Juergen Geiser (University of Greifswald, Germany)

Modelling and Simulation to Support Sustainable Energy Systems and Water Treatment

Esko Juuso (University of Oulu, Finland)

Mathematical Modelling and Control of Bio-Chemical Processes

Jan van Impe (Katholieke Universiteit Leuven, Belgium) \& Philippe Bogaerts

(Université Libre de Bruxelles, Belgium)

Biomathematics: What's new?

Carla M.A. Pinto (Polytechnic of Porto, Portugal)

Interdisciplinary Data based Modelling and Simulation in Health Systems Research Theoretical Development and Real World Applications

Peter Filzmoser \& Niki Popper (VUT \& DEXHELPP, Vienna, Austria) Modeling in Sport and Human Movement Science

Arnold Baca (University Vienna, Austria)

MATHMOD 2015 provides three basic types of contributions: The first group consists of MATHMOD Full Contributions which were peer reviewed, are presented in a regular session or minisymposium and will appear in the Conference Proceedings published by IFACPapersOnLine (http://www.ifac-papersonline.net/). MATHMOD Discussion Contributions allow the presentation of work in progress either in a minisymposium or in a poster session. 
Finally, MATHMOD Student Contributions are intended to give $\mathrm{PhD}$ students a chance to present their research work in a poster session.

All participants receive a printed Abstract Volume which contains all abstracts of the finally uploaded accepted contributions. Moreover, for convenience of participants, a MATHMOD 2015 Content System on a memory stick contains the electronic preprints of Full Papers and an electronic PDF Abstract Volume as well as further conference information. Both media are included in the conference kit.

As organizers we want to express our sincere thanks to all of you for your help in making the MATHMOD 2015 conference a success. In particular, we appreciate the support of our sponsors and cosponsors. Special thanks go to the members of the International Program Committee who did a great job in organizing the review process and to the organizers of the minisymposia. Moreover, we are very grateful to Andreas Körner from the Institute of Analysis and Scientific Computing and Wolfgang Kemmetmüller from the Automation and Control Institute for their excellent contributions in the organization of the conference. Last but not least, we would like to thank IFAC for the publication arrangement, and the local ARGESIM team for their support in preparing these preprints and the forthcoming proceedings of MATHMOD 2015. 
MATHMOD 2015 Abstract Volume 


\title{
MATHMOD 2015 ABSTRACT VOLUME
}

\author{
MATHMOD Plenary Lectures
}


MATHMOD 2015 Abstract Volume 


\title{
Model-based approaches for the future sustainable aircraft: the EU ADDSAFE project
}

\author{
Philippe Goupil \\ Aircraft Control - EYC, AIRBUS Operations S.A.S. \\ 316 Route de Bayonne, 31060 Toulouse Cedex 09, FRANCE \\ philippe.goupil@airbus.com
}

\begin{abstract}
In this paper a high-fidelity aircraft benchmark, developed by Airbus for advanced flight control related studies, is presented in relation to its use for advanced fault diagnosis within a European $7^{\text {th }}$ Framework Program project termed “Advanced Fault Diagnosis for Sustainable Flight Guidance and Control” (ADDSAFE). This European project was established to investigate and develop advanced model-based fault detection and diagnosis methods advocated by Academia, to assess their viability, performances and robustness as well as to facilitate their transfer to Industry. The importance of the project arose on the one hand, due to the representativeness of the Airbus benchmark, and on the other hand, the industrial benchmarking and validation of the developed designs. This article does not pretend to academic developments but rather to illustrate industrial motivations in such project.
\end{abstract}

Keywords: aircraft, flight control, fault diagnosis, European project, verification and validation. 


\title{
Iterative Schemes for Coupled Multiphysical Problems in Electrical Engineering ${ }^{\star}$
}

\author{
Sebastian Schöps* \\ * Technische Universität Darmstadt, Graduate School for \\ Computational Enginering, Dolivostrasse 15 and Institut für Theorie \\ Elektromagnetischer Felder, Schlossgartenstrasse 8, Darmstadt \\ (e-mail: schoeps@gsc.tu-darmstadt.de).
}

\section{INTRODUCTION}

Multiphysical simulations become increasingly important for engineering applications due to the high accuracy of domain-specific modeling and numerical simulations; effects that were considered previously as negligible cannot be disregarded anymore. In the last decade commercial tools have emerged that can treat many problems but are either too restricted in the coupling, or not efficient enough or do not guarantee convergence. In particular, the monolithic approach to multiphysics, i.e., the solution of all subproblems at once, is often cumbersome or even impossible because incompatible algorithms or software packages are involved. Thus simulation engineers need to couple subproblems in an efficient and stable way, where subdomains are solved separately. This introduces splitting errors (in time or space), which are mitigated by iterative procedures. Well-known examples are splitting schemes for linear systems of equations as Gauss-Seidel or domain decomposition methods in the context of partial differential equations. Typical applications in the domain of electrical engineering require the coupling of electromagnetic fields with thermal, mechanical and electric network problems, see e.g. Clemens et al. (2012).

\section{FIELD-CIRCUIT PROBLEM}

For field-circuit coupled models of electrical energy transducers, two classical approaches are well established. A first approach consists of extracting (constant) lumped parameters or surrogate models from a field model and inserting these as a netlist into a Spice-like circuit simulator. This can be improved by monolithic couplings, where field and circuit models are solved together. We propose a synthesis: the parameter extraction is applied iteratively on time intervals such that nonlinear and transient effects are consistently taken into account; this can be embedded into the framework of waveform relaxation methods Bartel et al. (2013).

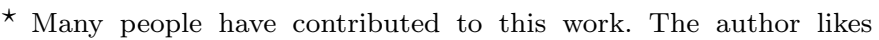
to thank A. Bartel, M. Brunk, M. Clemens, J. Corno, H. De Gersem, C. de Falco, M. Günther, C. Kaufmann and E.J.W. ter Maten. This work is supported by the projects SIMUROM (BMBF 05M2013), nanoCOPS (EU 619166) and the 'Excellence Initiative' of the German Federal and State Governments and the Graduate School of Computational Engineering at TU Darmstadt.
}

\section{FIELD-MECHANICAL PROBLEM}

The Lorentz detuning of an accelerating cavity, which is the change of the resonant frequency due to the mechanical deformation of the cavity wall induced by the electromagnetic pressure is a coupled electromagnetic-mechanical problem. In a first step, Maxwell's eigenproblem is solved with adequate boundary conditions. From this the magnetic field can be obtained. Both fields create a radiation pressure at the boundary of the cavity. The domain is transformed and the computation of the resonant frequency is repeated. This problem fits into the framework of waveform relaxation, however the analysis can be simplified due to the frequency domain assumptions.

\section{FIELD-THERMAL PROBLEM}

In the previous sections we have discussed the mutual coupling of transient and frequency-domain to (quasi-)static problems. The third example revisits the well-known iterative coupling of frequency to time domain problems applied to an electric car charger. Again, the electromagnetic field is given by the Maxwell's equations, however since we are in domains of increased frequency we take the displacement currents into account. This is coupled to the heat equation by the Joule losses. Besides the temperaturedependent electric conductivity, all material parameters are constant. The important modelling step is to relax the coupling of both problems by introducing a averaged heat source btained by converting the electromagnetic fields back to the time domain. Convergence can be assured in view of the works of Kaufmann et al. (2014).

\section{REFERENCES}

Bartel, A., Brunk, M., Günther, M., and Schöps, S. (2013). Dynamic iteration for coupled problems of electric circuits and distributed devices. SIAM J. Sci. Comput., 35(2), B315 - B335. doi:10.1137/120867111.

Clemens, M., Schöps, S., Cimala, C., Gödel, N., Runke, S., and Schmidthäusler, D. (2012). Aspects of coupled problems in computational electromagnetics formulations. ICS Newsletter (International Compumag Society), 3.

Kaufmann, C., Günther, M., Klagges, D., Knorrenschild, M., Richwin, M., Schöps, S., and ter Maten, E.J.W. (2014). Efficient frequency-transient co-simulation of coupled heat-electromagnetic problems. J. Math. Ind., 4(1). doi:10.1186/2190-5983-4-1. 


\title{
What has Instrumental Variable method to offer for system identification?
}

\author{
M. Gilson ${ }^{1,2}$ \\ ${ }^{1}$ University of Lorraine, CRAN, UMR 7039, 2 rue Jean Lamour, F-54519 \\ Vandouvre-les-Nancy, France \\ ${ }^{2}$ CNRS, CRAN, UMR 7039, France, \\ marion.gilson@univ-lorraine.fr
}

\begin{abstract}
Mathematical models of dynamic systems are required in most area of scientific enquiry and take various forms, such as differential equations, difference equations, state-space equations and transfer functions. The most widely used approach to mathematical modeling involves the construction of mathematical equations based on physical laws that are known to govern the behaviour of the system. While the advantage of these methods relies on the deep physical insight of the resulting model, their main drawback is the complexity of the model that makes them difficult to be used in applications such as control system design, prediction or decision making.

An alternative to physically-based mathematical modeling is the so-called data-based system identification, which can be applied to any system where experimental data are available. A large scope of system identification approaches has been developed over the past decades. Amongst these, we can cite the prediction error and maximum-likelihood frameworks (see e.g. Ljung (1999); Söderström and Stoica (1989); Young (2011)), the subspace-based identification (see e.g. Katayama (2005)), the frequency-domain identification (see e.g. Pintelon and Schoukens (2001)), the closed-loop identification case (see e.g. Van den Hof (1998); Forssell and Ljung (1999); Gilson and Van den Hof (2005)).
\end{abstract}

Most physical systems are continuous-time (CT) whereas, mainly due to the advent of digital computers, research on system identification has concentrated on discrete-time (DT) models from underlying CT systems input/output samples. Recently, interest in identification of CT systems from DT data has arisen (see e.g. Garnier and Wang (2008) and references herein) and offer a clever solution in many cases such as irregularly sampled data.

Moreover, systems encountered in practice are often nonlinear or present a time-varying nature. Unlike linearity, non-linearity is a non-property and therefore, non-linearity cannot be defined in a general way. A common framework for the identification of nonlinear models has nevertheless been presented in Sjöberg et al. (1995). Usually, nonlinear models are classified into two classes: non-parametric models and parametric models. However, another type of models has more recently arose the attention of the system identification community and form an intermediate step between Linear Time-Invariant (LTI) systems and nonlinear/time-varying plants: the model class of Linear Parameter-Varying (LPV) systems (Tóth (2010)).

When considering methods that can be used to identify (linear or non linear, CT or DT) models of systems operating in openor closed-loop, instrumental variable (IV) techniques are rather attractive since they are normally simple or iterative modifications of the linear regression algorithm. For instance, when dealing with complex processes, it can be attractive to rely on methods, such as these, that do not require non-convex optimization algorithms. In addition to this computationally attractive property, IV methods also have the potential advantage that they can yield consistent and asymptotically unbiased estimates of the plant model parameters if the noise does not have rational spectral density or if the noise model is mis-specified; or even if the control system is non-linear and/or time-varying, in the closed-loop framework (Gilson and Van den Hof (2005)). Even if several works arise these last ten years, IV methods have not yet really received the attention that it deserves.

This talk is dedicated to the use of IV methods in several cases of system identification. After an introduction of the IV principles, the focus is made on closed-loop system, on LPV models with an application on rainfall-flow modeling and on frequency domain framework.

Further details are provided sin the full paper related to this talk.

\section{REFERENCES}

Forssell, U. and Ljung, L. (1999). Closed-loop identification revisited. Automatica, 35(7), 1215-1241.

Garnier, H. and Wang, L. (eds.) (2008). Identification of continuous-time models from sampled data. Springer Verlag, London.

Gilson, M. and Van den Hof, P. (2005). Instrumental variable methods for closed-loop system identification. Automatica, 41(2), 241-249.

Katayama, T. (2005). Subspace methods for system identification. Springer.

Ljung, L. (1999). System Identification : Theory for the UserSecond Edition. Prentice-Hall.

Pintelon, R. and Schoukens, J. (2001). System identification : a frequency domain approach. IEEE Press, New York.

Sjöberg, J., Zhang, Q., Ljung, L., Benveniste, A., Delyon, B., Glorennec, P.Y., Hjalmarsson, H., and Juditsky, A. (1995). Nonlinear black-box modeling in system identification: a unified overview. Automatica, 31(12), 1691-1724.

Söderström, T. and Stoica, P. (1989). System identification. Prentice-Hall.

Tóth, R. (ed.) (2010). Modeling and identification of linear parameter-varying systems. Lecture Notes in Control and Information Sciences. Springer, Heidelberg.

Van den Hof, P. (1998). Closed-loop issues in system identification. Annual Reviews in Control, 22, 173-186.

Young, P. (2011). Recursive Estimation and Time-Series Analysis. Springer Verlag, London, second edition. 


\title{
Advanced Mechatronics for Precision Engineering and Mechatronic Imaging Systems
}

\author{
G. Schitter \\ *Automation and Control Institute (ACIN), Vienna University of Technology \\ 1040 Wien, Gusshausstraße 27-29, Austria \\ (Tel: +43-1-5880137610; e-mail: schitter@acin.tuwien.ac.at)
}

\begin{abstract}
This paper summarizes how modelling, simulation and model-based design approaches influence the system design and integration of precision mechatronic systems that are used in the hightech industry and in scientific instrumentation for nano-scale imaging. Particular challenges that have to be addressed are positioning with nanometer precision, motion control with high speeds and high precision, and vibration isolation to counteract environmental disturbances. Applications that are discussed and demonstrated are from the fields of atomic force microscopy, confocal scanning laser microscopy, adaptive optics, and precision lithography machines for the production of integrated circuits.
\end{abstract}

Keywords: precision engineering, motion control, mechatronics, scanning probe microscopy, system integration, model-based control.

\section{INTRODUCTION}

Mechatronic systems in the high-tech industry demand a continuous improvement of system performance, such as speed, range, and precision (Munnig Schmidt et al. 2014). For the development, engineering, and control of these systems, modelling and simulation are key enabling technologies that are applied at various steps and levels.

Advanced mechatronic designs and highly sophisticated motion control are prerequisites for the innovation of mechatronic imaging systems, such as atomic force microscopes (AFM), wafer scanners, adaptive optics, and laser scanning microscopes.

This paper discusses an integrated mechatronic design approach, demonstrating the application of modelling and simulation in the various phases of the system design, integration, and control.

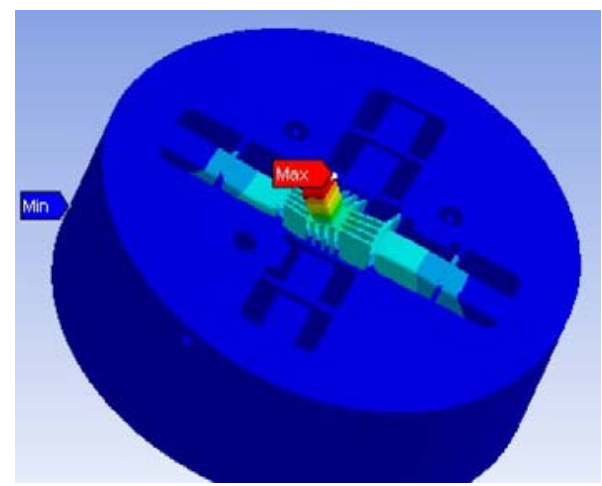

Fig. 1. FEA simulation of a mode-shape of a piezo- and flexure-based scanning unit for high-speed atomic force microscopy.

\section{MECHATRONIC SYSTEM DESIGN}

Already at the system design phase all components involved in the specific application have to be considered. Examples for these components are the mechanical structure of the device, the actuators, the power amplifiers, the sensors, electronics for signal amplification and conditioning, and the real-time control system. The resulting mechatronic system, including all hard- and software components, has to be tailored to and optimized for each specific application. To meet the demanding specifications a well predictable behaviour of the individual system components is required. Utilization of modern engineering tools and software, such as finite element analysis (FEA), is a key factor for modelling and simulation of system dynamics (cf. Fig. 1). At the system level design physics based modelling helps to gain important insights in the structural dynamics, as well as in the interplay

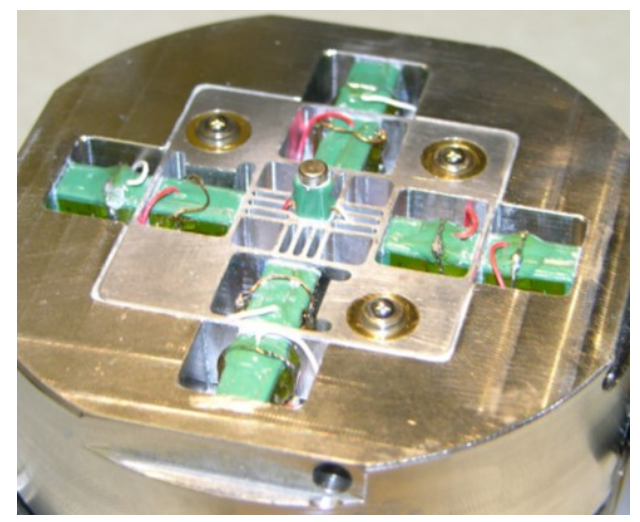

Fig. 2. Flexure-based AFM scanner with piezoelectric actuation for high-speed atomic force microscopy, designed for high stiffness to achieve high resonance frequencies and prevent parasitic motion (Schitter et al. 2007). 
between the individual system components (Schitter et al. 2007). FEA tools enable virtual prototyping of complex mechatronic systems and allow shaping of the mechatronic components for optimizing their performance.

\section{MODEL-BASED CONTROL AND MEASUREMENT}

After building the prototype system (Fig. 2), mathematical models of the actual system dynamics are required as the basis for the control design. These dynamic models of the experimental setup typically are obtained either via system identification, by parameter identification to verify or complete the FEA- or physics based models, or by recording frequency response data and fitting a model to these data (Fig. 3).
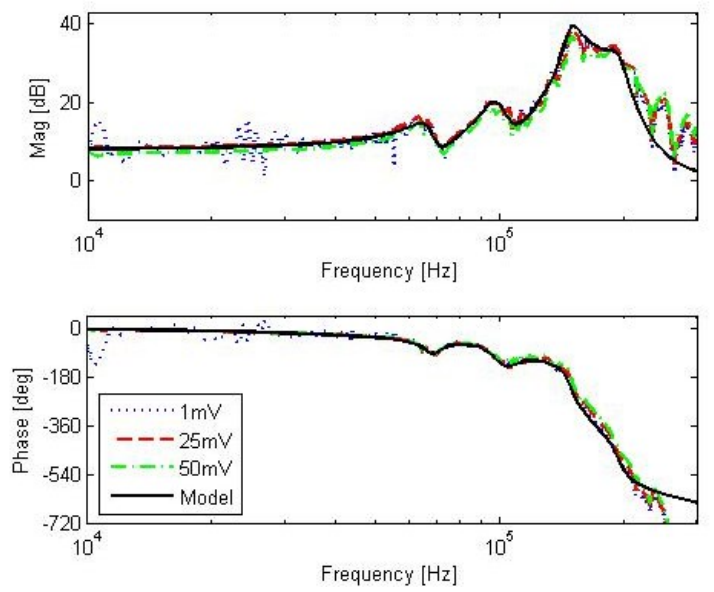

Fig. 3. Measured frequency response data and $10^{\text {th }}$-order fitted model (solid black line) of the vertical axis of a highspeed AFM scanner (Schitter and Phan 2008).

Based on these models, modern control methods are applied, where often feedback and feedforward control are combined in order to achieve a high performance as well as good disturbance rejection and robustness of the controlled mechatronic system (e.g. Schitter et al. 2004).

Furthermore, these models, including the control system, can be used also for model-based measurement (Kuiper et al. 2013). This enables a better and dynamic operation of the metrology equipment, dealing with the design trade-off between measurement speed and accuracy, and for handling of modelling uncertainties.

\section{APPLICATIONS}

These challenges and solutions are illustrated by examples from precision motion control, AFM imaging, confocal laser scanning microscopy (CLSM), and adaptive optics. The examples present the successful implementation of longrange positioning over several $100 \mathrm{~mm}$ with nanometer precision based on zero- and low-stiffness designs. For AFM imaging at high speeds, i.e. up to 1000 times faster than with conventional AFM systems, fast motion control for nanometer precise scanning and topography measurement is presented, as well as the measurement of topography and mechanical sample properties at a high spatial and temporal resolution. For optimizing the spatial resolution of optical imaging systems adaptive optics is demonstrated for the compensation of setup- and sample-induced aberrations, such as for 3D imaging in CLSM (Yoo et al. 2014).

\section{CONCLUSIONS}

This contribution demonstrates the potential to significantly improve the performance of mechatronic imaging systems via an integrated mechatronic design approach, by utilizing the interplay between system design and control design. This allows faster and higher resolution imaging in various applications in research as well as in high-tech industries.

\section{REFERENCES}

Kuiper S., Van den Hof, P.M.J., Schitter, G. (2013). Integrated design of the feedback controller and topography estimator for atomic force microscopy, Control Engineering Practice, volume 21, p. 1110 1120

Munnig Schmidt, R., Schitter G., Rankers, A., Van Eijk, J. (2014). The Design of High Performance Mechatronics: 2nd Edition: High-Tech Functionality by Multidisciplinary System Integration, Delft University Press, ISBN 978-1614993674, Amsterdam

Schitter, G., Stemmer, A., Allgöwer, F. (2004). Robust twodegree-of-freedom control of an atomic force microscope, Asian Journal of Control, volume 6(2), p. $156-163$

Schitter, G., Åström, K.J., DeMartini, B., Thurner, P.J., Turner, K, Hansma, P.K. (2007). Design and modeling of a high-speed AFM-scanner, IEEE Transactions on Control Systems Technology, volume 15(5), p. 906-915

Schitter, G., Phan, N. (2008). Field programmable analog array (FPAA) based control of an atomic force microscope, in Proceedings of 2008 American Control Conference, Seattle, WA, USA, p. 2690-2695

Yoo, H.W., van Royen, M.E., van Cappellen, W.A., Houtsmuller, A.B., Verhaegen, M., Schitter, G. (2014). Automated spherical aberration correction in scanning confocal microscopy, Review of Scientific Instruments, volume 85,123706 
MATHMOD 2015 Abstract Volume 


\title{
MATHMOD 2015 ABSTRACT VOLUME
}

\author{
MATHMOD Full Contributions \\ Biological and Chemical Systems
}


MATHMOD 2015 Abstract Volume 


\title{
Influence of an Asymptotic Pressure Level on the Windkessel Models of the Arterial System
}

\author{
Stephanie Parragh ${ }^{*, * *}$ Bernhard Hametner ${ }^{* *}$ \\ Siegfried Wassertheurer** \\ * Institute for Analysis and Scientific Computing, Vienna University of \\ Technology, Vienna, Austria \\ (e-mail: stephanie.parragh@student.tuwien.ac.at). \\ ** Health $\mathcal{E}$ Environment Department, AIT Austrian Institute of \\ Technology GmbH, Vienna, Austria \\ (e-mail: \{Siegfried.Wassertheurer, Bernhard.Hametner\}@ait.ac.at).
}

\section{INTRODUCTION}

Windkessel models are lumped-parameter models of the arterial system that describe the dynamic relation between blood flow and pressure in the aorta by ordinary differential equations. Despite their simplicity, they are used in many applications including methods for the noninvasive stratification of cardiovascular risk (Hametner et al., 2014). However, even though they have been studied extensively, there is still disunity regarding the question if pressure should be modelled as resulting solely from the ejection of the heart or if an asymptotic pressure level should be included which is independent from cardiac beating (Westerhof et al., 2009). The aim of this work is to mathematically analyse the influence of such a pressure level $P_{\infty}$ on the model behaviour of the four most widely used Windkessel models (two-, three- and four-element Windkessel, the latter in a series as well as a parallel configuration).

\section{METHODS AND RESULTS}

The model equations including the asymptotic pressure level $P_{\infty}$ were formulated and Fourier analysis was performed to study the impact of $P_{\infty}$ on the other model parameters. Therefore, the modelled input impedances $Z_{\text {in }}(f)$ relating pressure to flow in the frequency domain were computed. Furthermore, simulation experiments for $P_{\infty}$ varying from 0 to $75 \mathrm{mmHg}$ using a typical flow curve as input were carried out for parameter values within the physiological range (Kind et al., 2010). The results in fig. 1 show that $P_{\infty}$ mainly affects the diastolic part of the pressure wave, whereas the shape of the systolic upstroke remains almost unchanged. Also, the quantitative differences in the modelled impedances $Z_{\text {in }}$ were overall small and decreasing for increasing frequency. For the most extreme case of $P_{\infty}=75 \mathrm{mmHg}$, differences to $P_{\infty}=0$ $\mathrm{mmHg}$ were, for all four models, in the order of $10^{-3}$ $\mathrm{mmHg} \cdot \mathrm{s} / \mathrm{ml}$ for the modulus and $10^{-1} \mathrm{rad}$ for the phase.

\section{CONCLUSION}

Theoretical as well as numerical results show that including an asymptotic pressure level $P_{\infty}$ mainly affects the
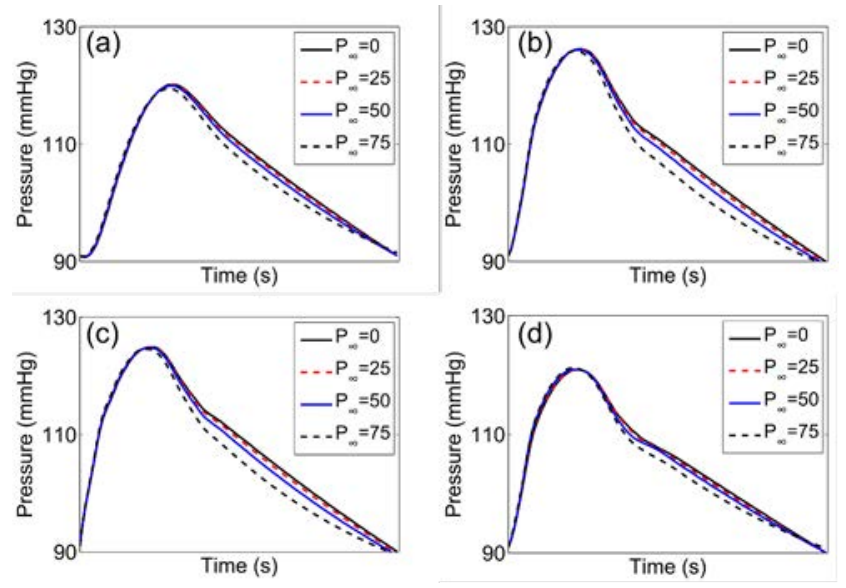

Fig. 1. Pressure waves modelled with the 2-element (a), 3-element (b), serial (c) and parallel (d) 4-element Windkessel model for varying $P_{\infty}$.

low frequency range of the modelled input impedances. In the time domain this results in changes of the diastolic decay of the modelled pressure curves, whereas the systolic upstroke, which is built up from higher frequency components, remains almost unchanged. The additional parameter does neither change the overall qualitative behaviour of the models nor does it affect the model parameters representing physiological characteristics. $P_{\infty}$ might, as an additional degree of freedom, even improve the accuracy of the modelled pressure curves. However, further research is needed to clarify its physiological interpretation as well as its appropriate size.

\section{REFERENCES}

Hametner et al. (2014). Reservoir and excess pressures predict cardiovascular events in high-risk patients. Int. J. Cardiol., 171, 31-6.

Kind et al. (2010). Estimation of three-and four-element windkessel parameters using subspace model identification. IEEE Trans. Biomed. Eng., 57, 1531-8.

Westerhof et al. (2009). The arterial windkessel. Med. Biol. Eng. Comput., 47, 131-41. 


\title{
A compact mathematical model of mandelate racemase production and chaperone overexpression in $\boldsymbol{E}$. coli
}

\author{
Bernhard Kramer* Ralf Tüngler* Katja Bettenbrock* \\ Carsten Conradi* \\ * Max Planck Institute for Dynamics of Complex Technical Systems, \\ Sandtorstr. 1, 39106 Magdeburg, Germany (e-mail: \\ kramer@mpi-magdeburg.mpg.de).
}

$E$. coli is commonly used for recombinant protein synthesis, e.g. in pharmaceutical industry for large-scale production of heterologous proteins like human insulin or other hormones and cytokines. The usually applied high targetprotein (TP) expression rates cause profound changes in the cell metabolism (CM). Besides the high burden for the CM, which results in decreased growth rates and productivity, the cells may also seriously suffer from toxicity of aggregates of the TP, commonly referred to as inclusion bodies (IBs). A common method in industry for TP production is to go for high amounts of IBs which have to be purified after cultivation and cell harvesting. This causes additional challenges and costs after the cultivation process, as solubilization of IBs often requires hazardous chemicals. Moreover the large number of process parameters complicates finding counter strategies for toxic side products like IBs by an empirical trial and error process.

A different approach to increase the amount of soluble recombinant TP is the in-situ concerted overexpression of heat shock proteins (HSPs) like chaperones and proteases. The HSPs influence the correct folding and degradation of the TP and hence decrease the fraction of aggregates and therefore toxic effects. The increased TP production is accompanied by raised stress to the CM due to increased synthesis of HSPs. Therefore it is important to find an optimal balance between TP and HSP production. Due to the complexity and specificity of the interaction between the TP and different chaperones the prediction of suitable overexpression strategies is not straight forward. Moreover various process parameters like growth temperature, media composition, inducer concentration, induction time, and plasmid-related properties like promotor strength and plasmid copy number affect protein biosynthesis. Most promising seems the application of optimal control strategies based on mathematical modelling of TP, HSPs, and CM. However by now no adequate mathematical model (MM) exists that describes the complex dynamic behaviour of recombinant protein synthesis, IB formation, interaction with HSPs, and the influence on the CM.

As a first step we propose a compact MM comprising the key players of recombinant formation, folding, aggregation/disaggregation, and degradation of the TP mandelate racemase (MR) for certain process parameters including the influence of controlled overexpression of selected HSPs. In the MM we consider no substrate limitation and ne-

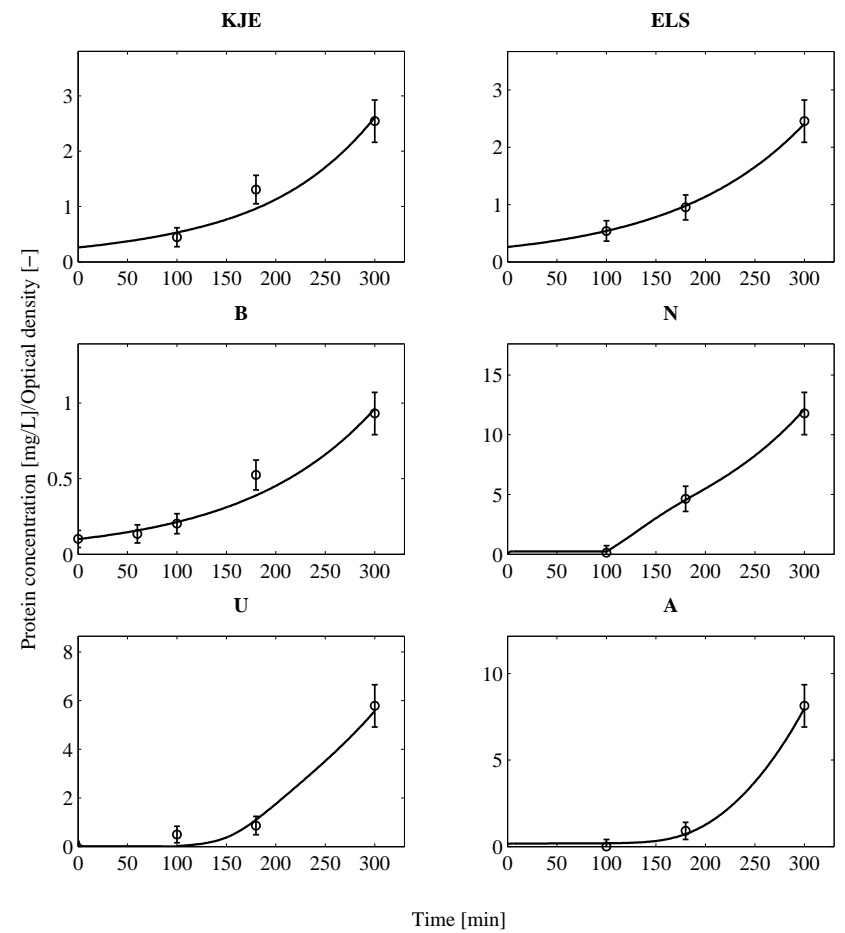

Fig. 1. Simulation results for scenario 1, induction of MR with IPTG at OD 0.2 (100 min). Open circles denote measured values, solid lines simulation results. For details see Fig. 2 of main text.

glect energy species conversion and impact on metabolism. The model comprises six state variables (Biomass B, MR conformations A (aggregates, representing IBs), U (unfolded, dissolved), N (native, dissolved), and chaperone systems KJE and ELS) and ordinary differential equations (ODEs), four input variables (inducer concentrations IPTG, Arabinose, Tetracyclin and growth inhibitor Spectinomycin (Spec)), eleven primary rates, eight secondary rates, and twenty-seven parameters. Resulting ODEs describe B formation, B-dependant formation of MR, MR degradation, chaperone-assisted conversion of MR conformations, and chaperone formation and degradation.

Despite its limited size, the model explains B and chaperone production very well, while MR production is not (yet) fully covered (see Fig. 1 above and Fig. 2 and 3 of main text). 


\title{
Efficient DQMOM for Multivariate Population Balance Equations and Application to Virus Replication in Cell Cultures
}

\author{
R. Dürr ${ }^{*, \star}$ T. Müller* A. Kienle ${ }^{*, * *}$ \\ * Institute for Automation Engineering, Otto-von-Guericke-Universität, \\ Universitätsplatz 2, 39106 Magdeburg, Germany \\ ** Process Synthesis and Process Dynamics Group, \\ Max-Planck-Institut für Dynamik komplexer technischer Systeme, \\ Sandtorsträ̈e 1, 39106 Magdeburg, Germany
}

Heterogeneous processes are found in many fields of applications including process and bioprocess engineering. The dynamics of the corresponding number density distributions can be described conveniently in the framework of Population Balance Modeling. Population Balance Equations represent PDEs which are often multidimensional. The numerical solution of these is quite challenging in particular for high dimensional problems. For this reason the distribution dynamics are usually characterized by the respective moments. Those are closely related to integral measures of the number density distribution like mean and variance. As the corresponding dynamic moment equations can only be computed in a closed form under strict assumptions, approximate moment methods have to be applied. However, existing techniques can not be implemented efficiently for high dimensional problems. In this manuscript an efficient implementation of the Direct Quadrature Method of Moments (DQMOM) is derived using monomial cubature rules. In contrast to general Gaussian cubature formulas where the number of abscissas increases exponentially with dimension, here the numerical effort for the approximation increases only polynomially (see Fig.1). Application is demonstrated for a five dimensional PBE which is based on a single cell model for viral replication in cell cultures, as it can be seen in Fig.2. The algorithm is used to analyze the effects of different model assumptions on the overall dynamics of free virus in the medium (see Fig.3).
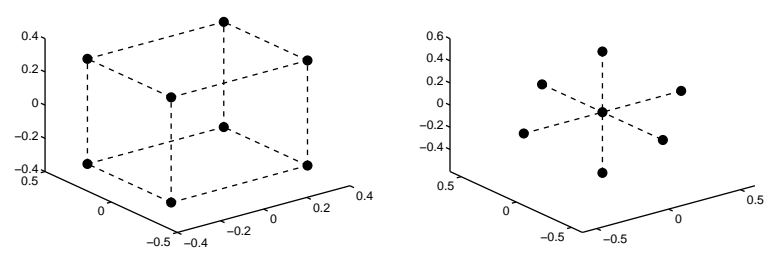

Fig. 1. Abscissas for Gaussian cubature (left) and monomial cubature (right)

\footnotetext{
‡ Correspondence to: R. Dürr, Otto-von-Guericke-Universität, Universitätsplatz 2, 39106 Magdeburg, Germany (e-mail: robert. duerr@ovgu.de)
}

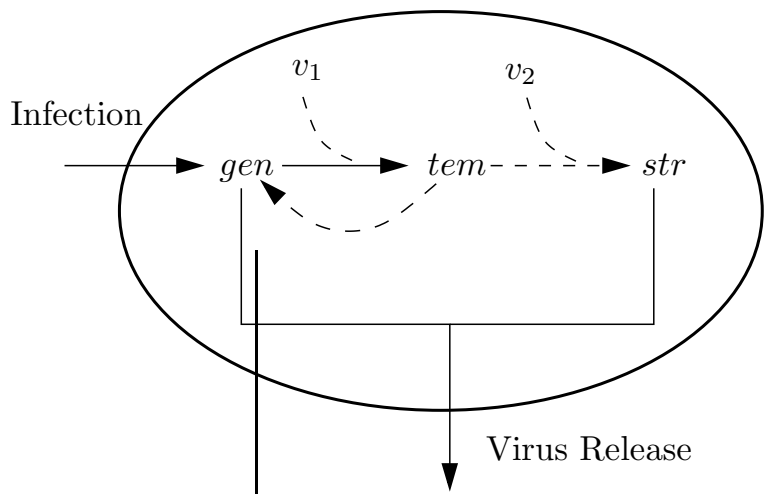

Fig. 2. Single cell scheme for viral replication

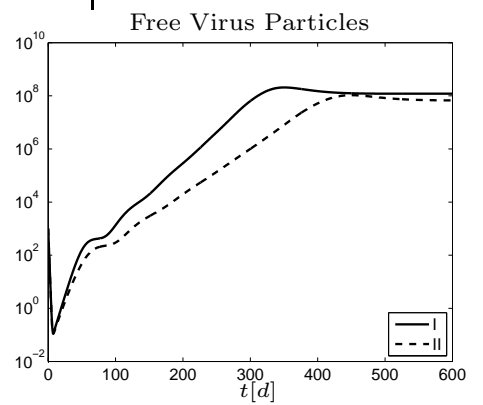

Fig. 3. Comparison of virus dynamics without (solid) and with enzyme degradation (dashed)

\section{REFERENCES}

Dürr, R. and Kienle, A. (2014). The Canadian Journal of Chemical Engineering, 92, 2088-2097.

Haseltine, E.L., Rawlings, J.B., and Yin, J. (2005). Computers $\&$ Chemical Engineering, 29(3), 675 - 686.

Marchisio, D.L. and Fox, R.O. (2005). Journal of Aerosol Science, 36(1), $43-73$.

Müller, T., Dürr, R., Isken, B., Schulze-Horsel, J., Reichl, U., and Kienle, A. (2013). Biotechnology and Bioengineering, 110(8), 2252-2266.

Ramkrishna, D. (2000). Population Balances: Theory and Applications to Particulate Systems in Engineering. Academic Press, San Diego.

Stroud, A. (1971). Approximate Calculation of Multiple Integrals. Prentice-Hall, Englewood Cliffs, N.J., Englewood Cliffs, N.J. 


\title{
Design of a Molecular Communication Channel by Modelling Enzyme Kinetics
}

\author{
Rudolf Rabenstein * \\ * Friedrich-Alexander-Universität Erlangen-Nürnberg (FAU), \\ D-91058Erlangen, Cauerstr.7, Germany (e-mail: rabe@LNT.de).
}

\section{INTRODUCTION}

Classical communication channels are based on the propagation of electromagnetic or acoustic waves. They span ranges from centimeters to astronomical dimensions. Recently, the study of signal transduction networks in biological systems has led to interest in communication concepts that rely on the transfer of molecules instead of wave propagation. Possible applications are on the nano-scale where particle transport is a fast process while senders and receivers for electromagnetic waves are difficult to build. Furthermore, the transport of particles is potentially supported by natural processes like diffusion or advection and does not require the supply of additional energy as in electrical devices.

These concepts have been developed during the last decade and are often refered to as molecular communication. Practical applications are mostly visionary and published results are based on more or less simplified models and idealizing assumptions. The current state is summarized e.g. in Nakano et al. (2013).

Recent studies establish the properties of molecular communication channels from models for particle transfer. Statistical models describe the probability that the arrival of a molecule is detected by a receptor, e.g. Pierobon and Akyildiz (2014); Noel et al. (2014b). Analogies with electrical circuits and coupled differential equations describe the dynamics of molecule concentrations, see Pierobon and Akyildiz (2010); Noel et al. (2014a)

\section{CHANNEL MODEL}

The channel model under investigation has been proposed by Noel et al. (2014a) and is illustrated in Fig. 1. A bitstream $x(n)$ at the input stimulates the emission of substrate molecules at the source. They are emitted at multiples of the bit interval $t_{0}$, i.e. at $t=n t_{0}$. A logical one $(x(n)=1)$ triggers the release of molecules corresponding to an increase of the total substrate concentration $s(t)$ by an amount of $s_{0}$. A logical zero $(x(n)=0)$ does not trigger the release of any molecules.

The environment between source and receptor is characterized by the concentrations of the substrate $s(t)$, the enzyme $e(t)$, and the complex $c(t)$ (see $(1))$. The receptor senses the current substrate concentration $s(t)$ and tries to detect the input bitstream. The balance between substrate, enzyme, and complex is described by the enzyme kinetics (see Noel et al. (2014a))

$$
S+E \underset{k_{-1}}{\stackrel{k_{1}}{\rightleftharpoons}} C \stackrel{k_{2}}{\rightarrow} E+P .
$$

The substrate $\mathrm{S}$ and the enzyme $\mathrm{E}$ form a complex $\mathrm{C}$ at a rate $k_{1}$. In turn the complex decomposes again into $\mathrm{S}$ and $\mathrm{E}$ at a rate $k_{-1}$ and into $\mathrm{E}$ and the product $\mathrm{P}$ at a rate $k_{2}$. The receptor is sensitive to the substrate $\mathrm{S}$ but not to the complex $\mathrm{C}$ or the product $\mathrm{P}$.

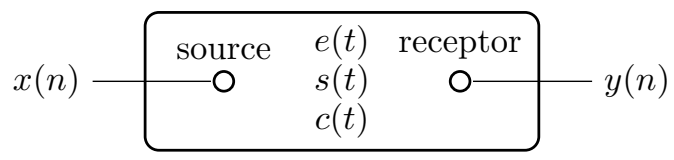

Fig. 1. Channel model for molecular communication.

\section{MAIN RESULTS}

Molecular communication channels based on enzyme kinetics can be analyzed with the mathematical tools of classical Michaelis-Menten models. Care has to be taken to observe the correct relation between substrate and enzyme concentration and between short time and long term behaviour as these are different from the classical MichaelisMenten case. A first order perturbation approach appears to be sufficent for modelling the substrate dynamics. Its range of accuracy for the short term behaviour matches favourably with the lenghts of the bit intervals for a reliable detection.

The final result is a simple expression for the achievable symbol frequency in terms of the chemical parameters of the molecular channel and the detection threshold of the receiver.

\section{REFERENCES}

Nakano, T., Eckford, A.W., and Haraguchi, T. (2013). Molecular communication. Cambridge University Press.

Noel, A., Cheung, K., and Schober, R. (2014a). Improving receiver performance of diffusive molecular communication with enzymes. IEEE Transactions on NanoBioscience, 13(1), 31-43. doi:10.1109/TNB.2013.2295546.

Noel, A., Cheung, K., and Schober, R. (2014b). A unifying model for external noise sources and ISI in diffusive molecular communication. IEEE Journal on Selected Areas in Communications, 32(12), 2330-2343. doi:10.1109/JSAC.2014.2367693.

Pierobon, M. and Akyildiz, I. (2010). A physical end-to-end model for molecular communication in nanonetworks. IEEE Journal on Selected Areas in Communications, 28(4), 602-611. doi: 10.1109/JSAC.2010.100509.

Pierobon, M. and Akyildiz, I. (2014). A statistical-physical model of interference in diffusion-based molecular nanonetworks. IEEE Transactions on Communications, 62(6), 2085-2095. doi: 10.1109/TCOMM.2014.2314650. 


\title{
Mathematical Modelling of molecular adsorption in zeolite coated frequency domain sensors
}

\author{
Fabian N. Murrieta-Rico ${ }^{a}$, Paolo Mercorelli ${ }^{b}$,Oleg Yu. Sergiyenko ${ }^{c}$, Vitalii Petranovskii ${ }^{a d}$, \\ Daniel Hernandez-Balbuena ${ }^{c}$ and Vera Tyrsa ${ }^{c}$ \\ ${ }^{a}$ Universidad Nacional Autónoma de México, Ensenada, BC 22860 Mexico \\ (e-mails:fmurriet@cicese.edu.mx; vitalii@cnyn.unam.mx) \\ ${ }^{b}$ Leuphana University of Lueneburg, Lueneburg, Germany \\ (e-mail: mercorelli@uni.leuphana.de) \\ ${ }^{c}$ Universidad Autónoma de Baja California, 21280 Mexicali, México \\ (e-mails: srgnk@uabc.edu.mx; dhernan@uabc.edu.mx; vera-tyrsa@mail.ru) \\ ${ }^{d}$ On the sabbatical leave at: Departamento de Investigación en Zeolitas \\ Instituto de Ciencias BUAP, Puebla 72570, México
}

\begin{abstract}
Fast and accurate information is important for most monitoring, data acquisition, and monitoring systems; sensors are transducers that allow to accomplish such task. In this work different kinds of resonant sensors known as frequency domain sensors are reviewed; also frequency measurement techniques are explored; finally resolution improvement is proposed and analyzed for using frequency domain sensors by mathematical modelling.
\end{abstract}

Keywords: sensors modeling, zeolite, FDS, microcantilever, frequency measurement, relative error. 


\title{
Modeling chemical reaction networks on the Pontryagin bundle with the Hamilton-Pontryagin approach *
}

\author{
Henning Lindhorst, Steffen Waldherr \\ Institute for Automation Engineering, Otto-von-Guericke-Universität \\ Magdeburg, Magdeburg, Germany (e-mail: henning.lindhorst@ovgu.de, \\ steffen.waldherr@ovgu.de).
}

\section{INTRODUCTION}

In this work, we develop a geometric decription of reversible stoichiometric chemical reaction networks. For this we use a geometric formulation for the system and apply a variational principle. Consider a closed chemical reaction network with $N$ different chemical species and $M$ possible chemical reactions among those species. We assign each species a positive molar concentration $x_{i}$. The stoichiometry of the system is collected in the stoichiometric matrix and we express the temporal change of the concentrations as

$$
\dot{x}=S v, S \in \mathbb{Z}^{N, M}
$$

with the molar fluxes $v$. For this energetic driven approach, we assign each species a chemical potential $\mu_{i}$ given as

$$
\mu_{i}\left(x_{i}\right)=\mu_{i, 0}+R^{*} T \log \left(\frac{x_{i}}{c_{0}}\right),
$$

with a temperature dependent reference potential $\mu_{i, 0}$ the gas constant $R^{*}$, temperature $T$ and a reference concentration $c_{0}$. We define the Lagrangian $\mathcal{L}$ of the system as the negative signed chemical energy

$$
\mathcal{L}(x)=-E_{\text {chem }}=-\sum_{i=1}^{N} \int_{0}^{x_{i}} \mu_{i}(y) \mathrm{d} y .
$$

The stoichiometric matrix is split into a forward $S^{(f)}$ and backward $S^{(b)}$ part, which correspond to the reactants and products of each reaction, as $S=S^{(b)}-S^{(f)}$. With this, we can define the chemical affinities

$$
A^{(f)}=\left(S^{(f)}\right)^{T} \mu, A^{(b)}=\left(S^{(b)}\right)^{T} \mu .
$$

They represent the driving forces of systems and are connected to the fluxes via the resistive relation

$$
\begin{aligned}
& v_{j}^{(f)}=\kappa_{j} \exp \left(\frac{A_{j}^{(f)}}{R^{*} T}\right) \\
& v_{j}^{(b)}=\kappa_{j} \exp \left(\frac{A_{j}^{(b)}}{R^{*} T}\right),
\end{aligned}
$$

with the constants $\kappa_{j}$. The inverse relations define the Lagrangian forces for the system as

$$
\mathfrak{f}_{\mathcal{L}}\left(v^{(f)}, v^{(b)}\right)=\left(\begin{array}{c}
0 \\
-R^{*} T \log \left(K^{-1} v^{(f)}\right) \\
-R^{*} T \log \left(K^{-1} v^{(b)}\right)
\end{array}\right)=\left(\begin{array}{c}
0 \\
A^{(f)} \\
A^{(b)}
\end{array}\right) .
$$

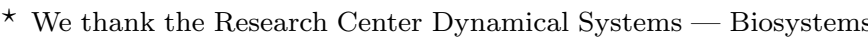
Engineering, Otto-von-Guericke-Universität Magdeburg, for funding.
}

\section{THE LAGRANGE-D'ALEMBERT-PONTRYAGIN PRINCIPLE}

Without going to much into detail, we define a geometric formulation of the system by collecting all admissible concentrations $x$ in a manifold, and show that the fluxes a part of an according tangential space. The affinities and the potentials are collected in a cotangential bundle. This gives us the elements of the Pontryagin bundle $T \mathcal{Z} \oplus T^{*} \mathcal{Z}$ as

$$
\left(z, \dot{z}, z^{*}\right)=\left(x, y^{(f)}, y^{(b)}, \dot{x}, \dot{y}^{(f)}, \dot{y}^{(b)}, \mu, A^{(f)}, A^{(b)}\right) .
$$

We define a Dirac structure $\mathcal{D}=\Delta(z) \oplus \Delta^{\circ}(z)$ on the bundle to express the stoichiometric constraints on the bundle

$$
\begin{aligned}
\Delta(z) & =\left\{\dot{z} \in T_{z} \mathcal{Z} \mid \dot{x}=S\left(\dot{y}^{(f)}-\dot{y}^{(b)}\right)\right\} \\
\Delta^{\circ}(z) & =\left\{z^{*} \in T_{z}^{*} \mathcal{Z} \mid A^{(f)}=\left(S^{(f)}\right)^{T} \mu, A^{(b)}=\left(S^{(b)}\right)^{T} \mu\right\} .
\end{aligned}
$$

Finally, we can apply a variational method, namely the Lagrange-d'Alembert-Pontryagin principle, to derive from the Lagrangian and the Lagrangian forces the equations of motions for the system. The principle reads

$$
\delta \int_{0}^{t_{\text {end }}} \mathcal{L}(z, \tilde{v})-p(\dot{z}-\tilde{v}) \mathrm{d} t+\int_{0}^{t_{\text {end }}} \mathfrak{f}_{\mathcal{L}}(z, \tilde{v}) \delta z \mathrm{~d} t=0,
$$

for constrained variations $\delta$. This leads us to the stoichiometric implicit Euler-Lagrange in the form

$$
\dot{x}=S K \operatorname{Exp}\left(\frac{\left(S^{(f)}\right)^{T} \mu(x)}{R^{*} T}\right)-S K \operatorname{Exp}\left(\frac{\left(S^{(b)}\right)^{T} \mu(x)}{R^{*} T}\right),
$$

with $K=\operatorname{diag}\left(\kappa_{j}\right)$.

\section{CONCLUSION}

We have derived the equations of motion for a reaction network on the Pontryagin bundle. The resulting equations incorporate thermodynamic constraints by construction. The fomulation also allows us to construct variational (and thereby symplectic) integrators of an arbitrary order, as shown in the full contribution. Furthermore, the implicit Euler-Lagrange equations contain the Legendre transformation naturally. This means, our description allows to incorporate a connection between the fluxes and a form of "kinetic energy", without the need for many adjustments. We are working right now on an approach, which includes heat as secondary energy form. 


\title{
MATHMOD 2015 ABSTRACT VOLUME
}

\author{
MATHMOD Full Contributions
}

Discrete Systems and Manufacturing Applications 
MATHMOD 2015 Abstract Volume 


\title{
Case indexing in Case-Based Reasoning by applying Situation Operator Model as knowledge representation model
}

\author{
A. Sarkheyli* D. Söffker* \\ * Chair of Dynamics and Control, University of Duisburg-Essen, \\ Germany \\ (Tel: +49-203-379-1584; e-mail: arezoo.sarkheyli@uni-due.de)
}

\begin{abstract}
:
Case-Based Reasoning (CBR) is an effective technique for solving cognitive problems. It maintains old experiences which are encountered in various problems and presented as cases in a case-base (Bergmann et al. (2005)). Consequently, indexing the cases in memory is an important issue in enhancing retrieval and learning performance in a cognitive system. This paper focuses on this issue and proposes an approach by integrating fuzziness for case indexing in CBR. Generalization and fuzzification of similar cases are the main properties of this approach in improving the performance of retrieval as well as reducing the size of case-base. Also, the connection between signal-based measurement level and problem-oriented behavioral level of the system which is a missing level in actual approaches is realized from a principle point of view in this study. In addition, Situation Operator Model (SOM) proposed by Söffker (2008) as a knowledge representation model is applied to CBR to model the events, related actions, and their effects in term of cases. As an application experiment, COLIBRI developed by DíazAgudo et al. (2007) is utilized as a CBR reference platform. A data set generated using a driving simulator is utilized to learn two different classes of cases (start passing, end passing) generated from maneuvers of drivers. Finally, the performance of retrieval process and similarity error on fuzzy and conventional indexing approaches are measured to reveal the effectiveness of the presented approach.
\end{abstract}

Keywords: Case-Based Reasoning, Situation Operator Model, Retrieval, Case indexing, Fuzzy approach.

\section{REFERENCES}

Bergmann, R., Kolodner, J., and Plaza, E. (2005). Representation in case-based reasoning. The Knowledge Engineering Review, 20(3), 209-213.

Díaz-Agudo, B., González-Calero, P.A., Recio-García, J.A., and Sánchez-Ruiz-Granados, A.A. (2007). Building cbr systems with jcolibri. Science of Computer Programming, 69(1), 68-75.

Söffker, D. (2008). Interaction of intelligent and autonomous systems-part i: qualitative structuring of interaction. Mathematical and Computer modelling of dynamical systems, 14(4), 303-318. 


\title{
Integrating situated human interaction modeling and stochastic state automata for improved technical situation awareness
}

\author{
O. Muthig* J. Wang* Q. Deng* D. Söffker* \\ * Chair of Dynamics and Control, University of Duisburg-Essen, \\ Germany (e-mail: olga.muthig@uni-due.de)
}

\section{MOTIVATION}

An important condition for safe human interaction with the environment is an entire and correct perception of the actual situation. Particularly in complex dynamic situations the human cognitive abilities like planning, making of situated decisions are important for the realization of safe actions. Especially in safety-related activities or working situations with high risk potential the correctness and reliability of situated actions is of great importance. The number of human errors or the related failure rates should be minimized to ensure safe and correct actions and action sequences. Supervising systems should help the human operator dealing with complex management tasks. Those systems have the tasks to increase the human perception to detect possible or upcoming errors in time, and to support human decision processes. Therefore an assistance system must be able to capture relevant information and interaction of the related environment, to structure, and to evaluate them. Suitable aspects and characteristics of a situation must be interpreted with respect to the available knowledge, the appropriate and available actions, as well as with respect to the intended aims and goals. Besides, it is important not only to "understand" the current situation, but also the possible changes, consequently also predictions about the development of the situation and future states. Therefore the understanding of the situation requires abilities like perception, interpretation with respect to the task set or aim, as well as the prediction of future states. These abilities are summarized under the concept "Situation awareness" given in Endsley (1995).

\section{METHODS}

In this contribution, the merging of modeling assumptions is introduced as well as the common application for technical situation recognition in complex scenarios (Fig. 1). By using a driving simulator, the typical behavior of human driver is determined using a help of Hidden-Markov-Model (HMM) approach, introduced in Rabiner (1989). The related Situation-Operator-Modeling methodology, which is introduced in Söffker (2008), allows determining human behavioral errors with respect to actions and decisions using a situated calculated action space. Theoretically this can be done in the complete action space. The combination and fusion of both reliable prediction are to be used for supervision of individual human interaction behavior. The merged supervision and prediction will be presented in this contribution based on the data using a driving simulator.

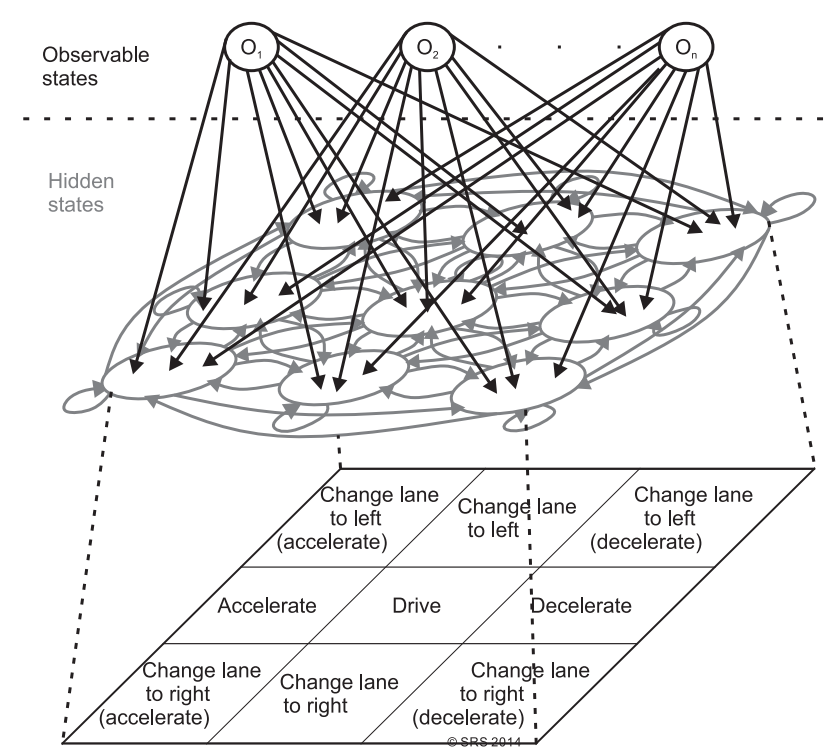

Fig. 1. Example for stochastik state automata

\section{REFERENCES}

Endsley, M.R. (1995). Toward a theory of situation awareness in dynamik systems. Human Factors, 37 (1), 32-64.

Rabiner, L.R. (1989). A tutorial on hidden markov models and selected applications in speech recognition. Proceedings of the IEEE, 77, 257-286.

Söffker, D. (2008). Interaction of intelligent and autonomous systems - part i: Qualitative structuring of interaction. MCMDA-Mathematical and Computer Modelling of Dynamical Systems, 14 (4), 303-318. 


\title{
Adaptive neuro-fuzzy approach to predict tool wear accurately in turning operations for maximum cutting tool utilization
}

\author{
Ahmed A. D. Sarhan \\ Advanced Manufacturing and Material Processing Centre, Department of Mechanical Engineering, Faculty of \\ Engineering, University of Malaya, 50603, Kuala Lumpur \\ (Tel: 0060379674593; e-mail: 1ah_sarhan@um.edu.my)
}

\begin{abstract}
In industry, the capability to predict tool wear is an essential matter as the quality and performance of a cutting tool directly affect the product quality. Developing a model to predict tool wear can help control tool wear and maximize tool utilization. Therefore, this study presents a method of predicting tool flank wear of coated carbide inserts while machining AISI 1050 low carbon steel with a turning operation. The adaptive neuro-fuzzy approach (ANFIS) was implemented in this research. Experiments were conducted based on the Design of Experiments (DOE) technique by developing experiments with four factors at four levels corresponding to the $\mathrm{L}_{16}\left(4^{4}\right)$ experimental array to measure tool flank wear. At the end, a verification test was conducted to illustrate the effectiveness of this approach. Using ANFIS, average prediction accuracy of $92.42 \%$ was obtained and the ANFIS tool wear model developed indicated how the interaction between factors influenced tool wear.
\end{abstract}




\title{
Modeling of layering growth processes using a Monte Carlo approach
}

\author{
Christian Rieck* Andreas Bück* Evangelos Tsotsas* \\ * Chair of Thermal Process Engineering/NaWiTec, Otto-von-Guericke \\ University, Magdeburg, Germany, (e-mail: christian.rieck@ovgu.de)
}

\section{ABSTRACT}

Layering growth processes in fluidized beds are applied in many industries, e.g. the pharmaceutical and food industry, to produce free-flowing and dust free particles. In fluidized bed layering, a liquid containing solid material is sprayed onto particles. The liquid part evaporates and the remaining solid builds a layer around the core particle. This layer can be used to protect an active ingredient (odor or taste masking) or it can contain the active substance itself.

The modeling of particle growth processes in fluidized beds is often done using population balance equations, which require a mathematical formulation of the process kinetics. In many cases, the resulting equations need to be solved numerically. Therefore a discretization is required, which may influence the solution. In this contribution it is presented, how a stochastic modeling approach based on a Monte Carlo method can be used to model a layering growth process by describing micro-scale processes. In case of layering growth in a fluidized bed, the considered microprocesses are droplet deposition on a particle, droplet drying and solidification, see Fig. 1.

In the simulation, droplets are deposited randomly on the particles. After the drying time of a deposited droplet, which is calculated using heat- and mass-transfer correlations, has passed, it is considered to be dry. Then, its remaining solid volume is added to the particle volume. Those micro-processes are executed sequentially in the simulation until a given process time is reached. The presented method allows multiple droplet deposition (a wet droplet can be deposited on a solidified droplet) and accounts for the increase of the particle surface area due to growth in the droplet deposition algorithm.

In order to check the developed algorithm we used a simple population balance model for the dry solid. Both models were fed with identical process parameters and the results were compared. It is shown, that the results obtained with both models are in good agreement with each other. Additionally, the presented Monte Carlo algorithm is tested with experimental data. The experiments were performed in a lab-scale fluidized bed, in which $1 \mathrm{~kg}$ of non-porous glass beads were coated with sodium benzoate solution. The initial particle size distribution and the distribution at the end of the process were measured using a particle size analyzer (Retsch Technology GmbH, type: CAMSIZER). The measured particle size distributions and the particle size distributions obtained with the Monte Carlo algorithm are also in good agreement.

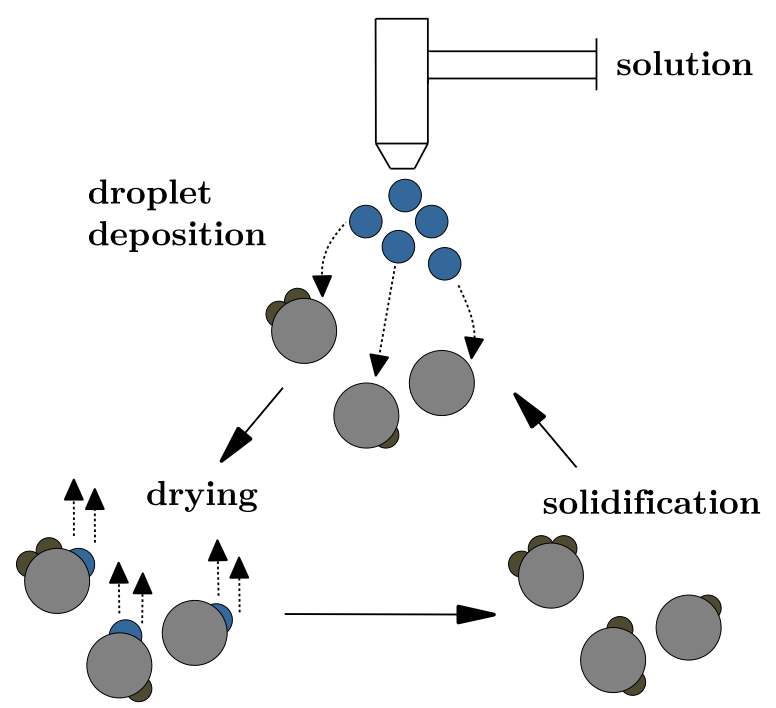

Fig. 1. Scheme of the three micro-processes considered in this study.

The presented algorithm is limited to non-porous particles, which will be solved in the future by including the imbibition of liquid droplets into the porous structure of particles. Another effect which can be included is rewetting of solidified droplets. Due to the deposition of a wet droplet on a dry droplet, solid material may be dissolved leading to changes in the coating layer porosity. Further properties, such as coating uniformity and layer thickness distribution, shall be predicted by this model. 


\title{
Improve wire EDM performance at different machining parameters - ANFIS modeling
}

\author{
Ibrahem Mahera, Liew Hui Ling ${ }^{\text {b }}$ Ahmed A. D. Sarhanc ${ }^{c}$ M. Hamdi ${ }^{d}$ \\ a,b, c, d Centre of Advanced Manufacturing and Material Processing, Department of Mechanical Engineering, Faculty of \\ Engineering, University of Malaya, 50603 Kuala Lumpur, Malaysia \\ ${ }^{a}$ Department of Mechanical Engineering, Faculty of Engineering, Kafrelsheikh University, Kafrelsheikh 33516, Egypt \\ E-mail: ibrahemmaher@eng.kfs.edu.eg \\ ${ }^{c}$ Department of Mechanical Engineering, Faculty of Engineering, Assiut University, Assiut 71516, Egypt
}

\begin{abstract}
This study presents an experimental investigation of wire electric discharge machining (WEDM) for improving the process performance. The effects of the machining parameters were investigated on the machining performance. Adaptive neuro-fuzzy inference system (ANFIS) was applied to determine the effect of significant parameters on WEDM performance. In addition, ANFIS was used to predict the cutting speed (CS), surface roughness (Ra) and heat affected zone (HAZ) in WEDM. The predicted CS, Ra, and HAZ were compared with measured data, and the average prediction error for $\mathrm{CS}, \mathrm{Ra}$, and HAZ were $3.41,3.89$, and 4.1 respectively.
\end{abstract}

Keywords: WEDM, Cutting speed, Surface roughness, Heataffected zone, ANFIS

\section{INTRODUCTION}

WEDM is an electro-thermal machining process for conductive materials. A metal wire electrode with de-ionized water is used to machine metal by the heat produced from electrical sparks (Maher et al., 2014). Several approaches have been used in modelling of WEDM process for improving the productivity and achieving high quality. Fuzzy logic, feed forward neural network, and ANFIS were used to model the process and correlate the input parameters with the performance measures. Because the WEDM involves a lot of machining parameters and multi-performance characteristics, the objective of the present study is to investigate the effects of three machining parameters including peak current (IP), pulse on time (Ton), and wire tension (WT) and develop ANFIS model for improve the performance characteristics namely CS, Ra, and HAZ for AISI 1050 carbon steel.

\section{EXPERIMENTAL WORK}

The levels of machining parameters were chosen according to machining recommendations as shown in Table 1 . The other machining parameters were kept constant as a fixed value during experiments to optimize the process. The experiments were performed using Sodick A500W WEDM machine tool. Hard brass wire with a diameter of $0.2 \mathrm{~mm}$ and tensile strength of $1000 \mathrm{~N} / \mathrm{mm} 2$ was used for machining blocks of AISI 1050 carbon steel. The CS is recorded directly from the WEDM machine tool monitor. The Ra was measured with a stylus-based profilometers. Scanning electron microscope is used to examine the surface characteristics of the machined. Eighteen sets of data were used for training ANFIS.
Table 1. Levels of machining parameters

\begin{tabular}{|c|c|c|c|}
\hline Machining parameter & \multicolumn{3}{|c|}{ Levels } \\
\hline Pulse on time $(\mu \mathrm{s})$ & 0.15 & 0.20 & 0.25 \\
\hline Wire tension $(\mathrm{g})$ & 300 & 350 & 400 \\
\hline Peak current $(\mathrm{A})$ & 16 & 17 & - \\
\hline
\end{tabular}

\section{ANFIS MODELING AND DISCUSSIONS}

Two membership's functions (MF) of peak current and three MFs of the other two parameters were chosen for creating the ANFIS model. The generalized bell membership function (gbellmf) was adopted for the ANFIS training process. The fuzzy rule architecture of ANFIS when gbellmf is adopted consists of 18 fuzzy rules based on the Sugeno fuzzy model. During training, the 18 performance measure values were used to conduct 50 cycles of learning with an average error of $8.37 \times 10-7,2.6 \times 10-6$, and $1.5 \times 10-5$ for $\mathrm{CS}, \mathrm{Ra}$, and HAZ respectively. Four random readings were used as the testing data set to verify the ANFIS model. The obtained average percentage error is $3.41,3.89$, and 4.1 for CS, Ra and HAZ respectively. The low error level signifies that the proposed model can predict $\mathrm{CS}, \mathrm{Ra}$, and HAZ satisfactorily. As conclusion from the ANFIS model results; the CS, Ra, and HAZ thickness increase as Ton and/or IP increase. The WT had a minor effect on CS and HAZ, but it has considerable effect on Ra where, Ra decreases when WT increases.

\section{CONCLUSIONS}

ANFIS was successfully used to develop an empirical model for modelling the relation between the predictor variables and the performance parameters. ANFIS model with gbellmf is accurate and can be used to predict CS, Ra, and HAZ in WEDM operation. Moreover, this study concludes that the IP and Ton are the most significant parameters affecting the CS, $\mathrm{Ra}$ and HAZ. The WT has minor effect on the CS and HAZ but it has great effect on the Ra.

\section{REFERENCES}

MAHER, I., SARHAN, A. A. D. \& HAMDI, M. 2014. Review of improvements in wire electrode properties for longer working time and utilization in wire EDM machining. The International Journal of Advanced Manufacturing Technology, 1-23. 
MATHMOD 2015 Abstract Volume 


\title{
MATHMOD 2015 ABSTRACT VOLUME
}

\author{
MATHMOD Full Contributions \\ Theoretical Aspects
}


MATHMOD 2015 Abstract Volume 


\title{
Faster Structural Analysis of Differential-Algebraic Equations by Graph Compression *
}

\author{
Christoph Höger* \\ * Department of Software Engineering and Theoretical Computer Science, \\ Technische Universität Berlin, Berlin, Germany (e-mail: \\ christoph.hoeger@tu-berlin.de).
}

Keywords: differential equations, structural analysis, performance, continuous systems, linear programming

\section{ABSTRACT}

Modern equation based modeling languages like Modelica offer convenient and safe methods to create large differentialalgebraic equations (DAEs). The composition of those equations from reusable parts routinely yields systems that are not directly applicable to efficient numerical simulation. Implementations like Dymola rely on sophisticated symbolical transformations of the model in question. These symbolical transformations have become absolutely essential when it comes to simulation of non-trivial DAEs, as both users and language rely on them.

This leads to the situation that the symbolic transformations limit the modeling paradigm. One example for this are large, homogeneous systems: A model might contain a large amount of the same sub-components (i.e. to allow for finer granularity in space) and allow for fast (e.g. linearly scaled) simulation. But since index reduction often shows a super-linear asymptotic runtime, the quite bizarre situation might occur that symbolic analysis needs more time then simulation (or fails completely).

Therefore, we consider any optimization in index reduction as highly relevant, especially for systems that might be scaled depending on some structural parameters (i.e. a level-of-detail). As we will show, hierarchic modeling yields the ability to drastically reduce the computational effort necessary for indexreduction by the compression of the input problem.

On a high level, structural analysis by the $\Sigma$-method can be summarized as follows:

(1) Construct a structure-matrix $\Sigma=\left(\sigma_{i j}\right) \in \mathbf{Z}^{n, n}$ of the model, where every $\sigma_{i j}$ indicates the highest derivative of unknown $j$ in equation $i$

(2) Find a maximum-value perfect matching (called a highest value transversal, or HVT in the original work) on the induced weighted bipartite graph

(3) Given that HVT, find the smallest optimal dual solution $c, d \in \mathbf{N}^{n}$

(4) $c_{i}$ is the number of times equation $i$ needs to be differentiated

The static semantics of hierarchical modelig (i.e. the principles of scoping and hiding) allow to reason about the outcome of the

\footnotetext{
* We acknowledge financial support from the German Research Foundation (Deutsche Forschungsgemeinschaft DFG) within the project "Automatische Modellierung und Simulation von technischen Systemen mit Unsicherheiten" (AMSUN).
}

structural analysis: We can enumerate all possible assignments between protected variables and equations and be certain that any assignment found for a larger model must contain one of these sub-assignments. We know that each protected variable can only be matched by an equation in the same instance and we know all those equations. Therefore, given $p$ protected variables and $e>p$ equations, there are at most $\left(\begin{array}{l}e \\ p\end{array}\right)$ possible assignments between them. A component that fulfills $e=p+1$, is called compressible, because exactly one equation must not be assigned to a protected variable (as only equations inside the component can "see" the protected variables).

To validate the optimization we implemented the compressed$\Sigma$-method using a variant of the Jonker-Volgenant algorithm for the LP solution search. We measured the runtime of the structural analysis for different sizes of a simple example circuit that contains many compressible components.

Compressing all sub-components of our example model reduces the size of the structure matrix to only $20 \%$. As table 1 shows, the performance gain is quite impressive. This example is, of course, quite well-posed for this kind of optimization (the Jonkers-Volgenant algorithm required only 1 augmentation in every experiment) and such drastic improvements should not be excepted from every or even the most models.

\begin{tabular}{|c|c|c|}
\hline $\mathrm{n}$ & Compressed Analysis & Analysis w/o compression \\
\hline 500 & $10 \mathrm{~ms}$ & $50 \mathrm{~ms}$ \\
\hline 2500 & $90 \mathrm{~ms}$ & $690 \mathrm{~ms}$ \\
\hline 5000 & $210 \mathrm{~ms}$ & $2960 \mathrm{~ms}$ \\
\hline 12500 & $660 \mathrm{~ms}$ & $17550 \mathrm{~ms}$ \\
\hline 62500 & $10720 \mathrm{~ms}$ & $/$ \\
\hline
\end{tabular}

Table 1.Runtime of model instantiation and compressed $\Sigma$-method

Using this infomation, the size of a model's structure matrix can be reduced significantly, if the model consists of many compressible sub-components. The compressed matrix yields the same highest-value as the original one. Since the time needed to search for a highest-value assignment grows super-linear in the size of the structure matrix, our compression technique significantly reduces the time needed to run a structural analysis of large models. 


\title{
Methods for Cellular Automata and Evolution Systems in Modelling and Simulation
}

\author{
G. Schneckenreither ${ }^{* * *}$ N. Popper ${ }^{*}$ F. Breitenecker ${ }^{* *}$ \\ * dwh simulation services, Neustiftgasse 57-59, 1070 Wien, Austria \\ (e-mail: guenter.schneckenreither@dwh.at). \\ ** Institute for Analysis and Scientific Computing, Vienna University \\ of Technology, Karlsplatz 13, 1040 Wien, Austria
}

\section{INTRODUCTION}

Cellular automata are in many occasions perceived and treated as natural systems consisting of a grid of cells with locally characterised dynamic behaviour. A partially different perception regards cellular automata as a method for modelling and simulation. Of course in both cases the basic ideas and the structure are mostly identical. In the latter case the conception of cellular automata is however used to depict a natural system as an abstract conceptual model and to describe the simplified system in a mathematical fashion. Also the term cellular automaton itself is controversial in this case.

A more abstract approach to cellular automata can be formalised in a functional analytic way as (locally characterised) evolution systems or as strongly continuous semigroups. This is the basis for connecting cellular automata and evolution systems with parabolic partial differential equations or abstract evolution equations (Goldstein (1985); Engel and Nagel (2000); ... ).

\section{EXAMPLE: AGE-STRUCTURED SIR MODEL}

An important demographic parameter in connection with epidemiology is age. An age-structured population was for example investigated by Iannelli and Martcheva (2003) and modelled by the following partial differential equation where $S, I$ and $R$ as well as $P:=S+I+R$ are functions $T \times\left[0, a_{\max }\right] \rightarrow \mathbb{R}_{+}$where $a_{\max }$ is the maximum age and $\mu$ is the force of mortality (or age-dependent natural deathrate).

$$
\begin{aligned}
\partial_{t} S-\partial_{a} S & =-\lambda(t, a) S-\mu(a) S \\
\partial_{t} I-\partial_{a} I & =\lambda(t, a) S-\beta(a) I-\mu(a) I \\
\partial_{t} R-\partial_{a} R & =\beta(a) I-\mu(a) R
\end{aligned}
$$

The parameter

$$
\lambda(t, a):=\int_{0}^{\infty} \kappa(a, s) \frac{I}{P}(t, s) d s
$$

depends on the contact behaviour $(\kappa)$ between different age-groups ( $a$ and $s$ ) of the population.

However for real contact data between individuals with specific age the corresponding evolution system is not locally characterised, because the typical contact behaviour of a population $\kappa(\cdot, \cdot)$ exhibits a shape as illustrated in Fig. 1.

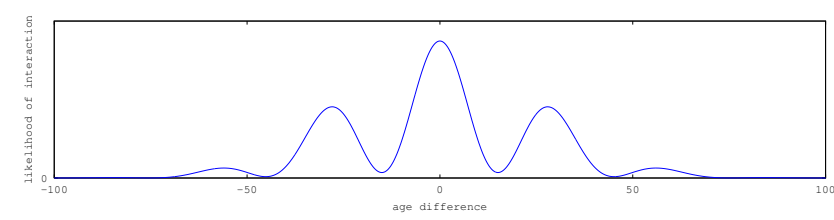

Fig. 1. Typical form of a contact distribution $\kappa(a, a+\cdot)$, describing the likelihood of interaction of a person aged $a$ to other persons with age in $[a-100, a+$ 100]. This figure only shows the general form of a contact distribution. For example ages below 0 are not possible such that this function is not necessarily symmetric. The peaks at -30 respectively +30 indicate the interaction of children with their parents and vice versa (i.e. generations).

\section{CONCLUSION AND OUTLOOK}

Analytic investigations often have to deal with nonlinearities (e.g. Iannelli and Martcheva (2003)). We try to compare experimental data from a cellular automaton implementation with analytic results for our application example especially in context of local characterisation. Also a stochastic cellular automaton model can be used to simulate nonlinear systems. General methods for relating cellular automata and parabolic partial differential equations should be investigated.

\section{REFERENCES}

Engel, K.J. and Nagel, R. (2000). One-Parameter Semigroups for Linear Evolution Equations. Graduate Texts in Mathematics. Springer-Verlag, New York.

Goldstein, J.A. (1985). Semigroups of Linear Operators and Applications. Oxford Mathematical Monographs. Oxford University Press, New York.

Iannelli, M. and Martcheva, M. (2003). Homogeneous dynamical systems and the age-structured SIR model with proportionate mixing incidence. In M. Iannelli and G. Lumer (eds.), Evolution equations: applications to physics, industry, life sciences, and economics, volume 55 of Progress in Nonlinear Differential Equations and Their Application. Birkhuser, Basel. 


\title{
Lagrangian GSOM traffic flow models on junctions
}

\author{
Guillaume Costeseque ${ }^{*, * *}$ Jean-Patrick Lebacque* \\ Asma Khelifi ${ }^{*, * * *}$ \\ * Université Paris-Est, IFSTTAR, GRETTIA, 14-20 Boulevard \\ Newton, Cité Descartes, Champs sur Marne, 77447 Marne la Vallée \\ Cedex 2, France (e-mail: firstname.lastname@ifsttar.fr) \\ ** Université Paris-Est, Ecole des Ponts ParisTech, CERMICS, $6 \& 8$ \\ avenue Blaise Pascal, Cité Descartes, Champs sur Marne, \\ 77455 Marne la Vallée Cedex 2, France. \\ *** Ecole Nationale d'Ingénieurs de Tunis, Rue Béchir Salem Belkhiria \\ Campus universitaire, BP 37, 1002 Tunis Belvédère, Tunisie.
}

\begin{abstract}
This paper is concerned with the macroscopic modeling and simulation of traffic flow on junctions. More precisely, we deal with a generic class of second order models, known in the literature as the GSOM family. While classical approaches focus on the Eulerian point-of-view, here we recast the model using its Lagrangian coordinates and we treat the junction as a specific discontinuity in Lagrangian framework. We propose a complete numerical methodology based on a finite difference scheme for solving such a model and we provide a numerical example.
\end{abstract}

Keywords: Traffic flow; junction; GSOM models; Lagrangian; numerical scheme.

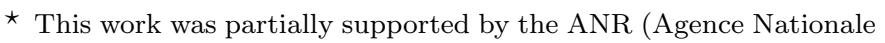
de la Recherche) through HJnet project ANR-12-BS01-0008-01. 


\title{
Spatial Effects in Stochastic Microscopic Models - Case Study and Analysis
}

\author{
Martin Bicher ${ }^{*, * *}$ Niki Popper ${ }^{*, * *}$ \\ * dwh Simulation Services GmbH, Neustiftgasse 57-59, 1070 Vienna, \\ Austria(e-mail: martin.bicher@dwh.at,niki.popper@dwh.at). \\ ** Institute of Analysis and Scientific Computing, Vienna University of \\ Technology, Wiedner Hauptstraße 8-10, 1040 Vienna, Austria (e-mail: \\ martin.bicher@tuwien.ac.at,niki.popper@tuwien.ac.at)
}

\section{INTRODUCTION}

In this paper we will not discuss different motivations and applications of microscopic models, but are presenting a technique to investigate the theoretical background of microscopic models. In context of our work we use the term microscopic model for dynamic (hereby used to indicate temporal dependent) models, consisting of a high number of similar sub-models, which will furthermore be denoted as actors. By this term we want to cover big classes of models like e.g. agent-based models, cellular automata or microsimulation models. As a microscopic model does not necessarily contain a spatial structure we will focus on those kind of models though the presented technique can also be applied on models without spatial relationships.

We furthermore lay special emphasis on the analysis of so called aggregated numbers, typically some kind of sums or statistics. We are analysing the behaviour of those quantities in case of a very large number, respectively in the limit case, an infinite number of individual actors. We especially focus on the influence of spatial relationships between the actors on the aggregated number. Furthermore we apply the results of the theoretical research on three different microscopic models, each of them chosen to particularly point onto an important observation.

\section{METHODS}

We use the diffusion approximation approach derived N.G. Van Kampen (see Kampen, N. G. van (1982)) in order to predict the temporal behaviour of aggregated numbers of the model by differential equations. Diffusion approximation respectively at least its results are, by knowledge of the author, still core of all theorems developed to perform aggregated analysis of microscopic models - so called mean-field theorems (some examples: Boudec et al. (2007). Benoit et al. (2006)).

Applying the method we derive the mean-field theorem (1) to approximate the expectancy value $\vec{\phi}(t)$ of the aggregated microscopic model by the solution of a differential equation and show some convergence results.

$$
\frac{d \vec{\phi}}{d t}=\sum_{i \neq j}\left(\overrightarrow{e_{i}}-\overrightarrow{e_{j}}\right) \omega_{\vec{\phi}, \vec{\phi}+\frac{1}{N}\left(\overrightarrow{e_{i}}-\overrightarrow{e_{j}}\right)}, \vec{\phi}(0)=\vec{\phi}_{0} .
$$
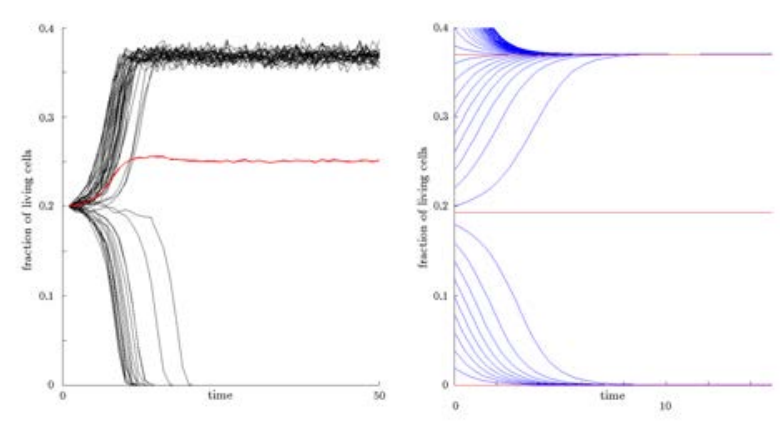

Fig. 1. Left: Sample simulation runs of a modified "Game of Life" model for specific initial condition including mean value (red). Right: Steady state analysis of (1).

\section{TEST CASES}

Furthermore three simple test cases are used to show how the presented technique can be applied to especially calculate means of counting variables for very simplified toy-models. The three cases are chosen properly to draw attention onto three different issues. A simple agent-based model based on a SIR strategy will be used for a direct verification of the mean-equation 1 an how it is applied. A cellular automaton (CA) based on John H. Conways Game of Life furthermore shows that the technique can fail occasionally caused by clustering effects and spatial relationships. A modification of the CA finally helps to understand the value of the theoretical analysis as a steady-state analysis of equation (1) needs to be performed (see Figure 1).

\section{REFERENCES}

Benoit, J., Nunes, A., and Telo da Gama, M. (2006). Pair approximation models for disease spread. The European Physical Journal B, 50(1-2), 177-181. doi: 10.1140/epjb/e2006-00096-x.

Boudec, J.Y.L., McDonald, D., and Mundinger, J. (2007). A generic mean field convergence result for systems of interacting objects. In Proceedings of the 4 th International Conference on the Quantitative Evaluation of Systems, 3-18. IEEE. doi:10.1109/QEST.2007.8.

Kampen, N. G. van (1982). The diffusion approximation for markov processes. In Lamprecht, I and Zotin, A.I. (eds.), Thermodynamics and kinetics of biological processes, 181-195. Walter de Gruyter \& Co. 


\title{
Alternative approaches for groundwater pollution
}

\author{
S. Winkler * M. Bicher * F. Breitenecker * \\ * Vienna University of Technology, Institute for Analysis and Scientific \\ Computing, Wiedner Hauptstrae 8-10, 1040 Vienna (e-mail: \\ stefanie.winkler@tuwien.ac.at,felix.breitenecker@tuwien.ac.at, \\ martin.bicher@tuwien.ac.at).
}

\section{INTRODUCTION}

The pollution of groundwater is an important field of interest regarding the water supply of all countries no matter how poor or rich. The analysis of this problem is based on the study of partial differential equations. In this case it can be restricted to the analysis of the convection diffusion equation. Diffusion equations are not only used to describe distribution of pollution. In biological fields of studies these equations are used to model the development of pattern formation for example in the fur of cats. There are also other fields which are confronted with the analysis for example of reaction-diffusion equation. In the chemistry the mixture of two substances can be simulated using this equation. Also in the finance market another form of the diffusion equation is used to predict the behaviour of stock buyers. The following paper focuses on the different possible approaches for pollution simulation. The first part will deal with the derivation of the regarded equation. Then there are three different types of approaches introduced to analyse the behaviour of this equation. On the one hand there are analytical and numerical methods to solve or approximate the regarded partial differential equations. On the other hand a more stochastic approach will be introduced.

The starting point of this research was a Benchmark of EUROSIM. In this Benchmark a rectangle is given. There is a flux along the $x$-axis which is constant. The given diffusion coefficient is constant as well. Therefore the convection diffusion equation will be analysed in a two dimensional rectangular area with a constant flow along the $x$-axis.

\section{CONVECTION DIFFUSION EQUATION}

The needed convection-diffusion equation can be separated in two parts, each describing a different process. One the one hand there is the oriented movement, called the convection. On the other hand there is a chaotic behaviour which describes the diffusive motion. This movement is characterized by minimal randomized motion of small particles. A transport of particles from regions with high concentration to areas with low concentration can be observed. This behaviour is mathematically formalized in Fick's First Law:

$$
J_{d}: \mathbb{R}^{n} \rightarrow \mathbb{R}^{n} \quad \text { with } \quad J_{d}(\mathbf{x})=-D(\mathbf{x}) \cdot \nabla c(\mathbf{x})
$$

It declares that the flux is proportional to the concentration gradient going from regions with high concentration to regions with low concentration as described in Larsson et al. (2005). The variable $J_{d}$ stands for the diffusive flux. This can be a function of space $x$. The flux is also influenced by the diffusion coefficient $D$ and the concentration $c$.

The oriented movement, the convection, accrues due to a flux. The flux is described with a velocity field $\mathbf{v}$. This vector field contains the flow movement in every possible direction. It can, as well as before, depend on space variables. Due to flux velocity the concentration $c$ of a certain substance at point $\mathbf{x}$ will be transported to the place $\mathbf{x}+t v$ after time step $t$. Therefore the convective flux of mass $J_{c}: R^{n} \rightarrow R^{n}$ can be written as:

$$
J_{c}(\mathbf{x})=v \cdot c(\mathbf{x})
$$

Due to the fact that a closed system is considered the conservation law can be used. In this case it means, that the regarded property does not change. It describes the relation between the time rate of change regarding the concentration of a certain quantity $c$ and the change in space regarding the flux $J$.

$$
\frac{\partial c}{\partial t}+\nabla \cdot J(\mathbf{x})=0
$$

The combination of the equations (1) and (2) results in the replacement of the the flux $J$ in equation (3) with $J=J_{c}+J_{d}$. This leads to the diffusion equation.

$$
\begin{aligned}
\frac{\partial c}{\partial t}+\nabla \cdot J=0 & \Rightarrow \frac{\partial c}{\partial t}+\nabla(-D \cdot \nabla c+v \cdot c)=0 \\
& \Rightarrow \frac{\partial c}{\partial t}=\nabla(D \cdot \nabla c)-\nabla(v \cdot c)
\end{aligned}
$$

\section{DISCUSION}

The main point of this paper is a comparison of different methods simulating convection-diffusion equations. There are three different approaches explained. At first a analytical solution is given. Unfortunately this may not be possible in any given scenario. Therefore the second approach deals with numerical methods solving the partial differential equation. In order to evaluate the results of all approaches properly the analytical solution can be used. The third method covers a stochastic approach, well known as Random Walk. In the paper not only the results but also the advantages and disadvantages of the methods are discussed.

\section{REFERENCES}

S. Larsson and V. Thomee Partielle Differentialgleichungen udn numerische Methoden. Springer, 2005. 
MATHMOD 2015 Abstract Volume 


\title{
MATHMOD 2015 ABSTRACT VOLUME
}

\author{
MATHMOD Full Contributions \\ Numerical and Computer Modeling
}


MATHMOD 2015 Abstract Volume 


\title{
A volume conserving discrete formulation of aggregation population balance equations on non-uniform meshes
}

\author{
Mehakpreet Singh* Jitendra Kumar* Andreas Bück** \\ * Department of Mathematics, Indian Institute of Technology \\ Kharagpur, 721302 Kharagpur, India, (e-mail: \\ jkumar@maths.iitkgp.ernet.in) \\ ** Chair of Thermal Process Engineering/NaWiTec, Otto-von-Guericke \\ University, Magdeburg, Germany,(e-mail: andreas.bueck@ovgu.de)
}

\begin{abstract}
Aggregation is an important size enlargement process in many industries. The modelling and design of the process can be done using the population balance framework, however, in almost every case a numerical solution of the equations must be obtained. In this paper, we present a new numerical scheme (NFVS) for the one-dimensional aggregation population balance equation or Smoluchowski equation on non-uniform grids. We compare the new scheme with a current scheme by Forestier-Coste and Mancini (FVS) considering some classical examples. The simplicity and generality to apply on uniform and non-uniform meshes are main features of the new scheme. Furthermore, the proposed new numerical scheme not only conserves the total volume of individuals in the system and is consistent with the total number of individuals, also higher-order moments are predicted well by the new scheme.
\end{abstract}

Keywords: Aggregation, population balance modelling, finite volume scheme, non-uniform meshes 


\title{
An Alternating Order Algorithm Based on Stages of a Ceschino's Method
}

\author{
Eugeny A. Novikov \\ Institute of Computational Modeling SB RAS ,Krasnoyarsk, Russia \\ e-mail: novikov@icm.krasn.ru \\ Anton E. Novikov \\ Siberian Federal University, SFU, Krasnoyarsk, Russia \\ e-mail: aenovikov@bk.ru
}

The necessity of applying algorithms based on explicit methods appears in numerical large-scale stiff problems solving as shown in. Integration methods on a base of implicit or semi-implicit numerical schemes usually use the decomposition of the Jacobi matrix, that is a separate timeconsuming task. In such a situation, the algorithms based on explicit formulas are more efficient if stiffness of the problem allows to get an approximation to a solution in a reasonable time.

The step control algorithm is usually based on accuracy control of a numerical scheme. It is natural, because the accuracy of the approximation is the main requirement of calculations. However, applying the integration algorithms based on explicit methods to stiff problems leads to the loss of efficiency and reliability. The reason is the contradiction between the accuracy and stability in a settling region which leads to a large amount of the recomputed solutions, whereas the step is much less than the maximum allowable one. The additional stability control of a numerical scheme allows to avoid this problem. At the present time, there are two common approaches to the stability control, based on application of the Dahlquist test equation.

The first is connected with the estimating the maximum eigenvalue of the Jacobi matrix using its norm with the subsequent (in addition to the accuracy control) control of the inequality $h\left\|f_{y}\right\| \leq D$, where $h$ is an integration step, $\left\|f_{y}\right\|$ is a Jacobi matrix norm of an ODE system. Here, $D$ is a positive constant correlated with the size of a stability domain. It is known, that explicit numerical schemes do not involve the Jacobi matrix. Therefore, applying the approach mentioned above leads to the additional calculation of the Jacobi matrix and, consequently, to the increase of computational costs.
The second approach is based on the estimation of the maximum eigenvalue $\lambda_{\max }$ of the Jacobi matrix by the power iterations using the increments of the right-hand part of the system of ordinary differential equations with the subsequent control of the inequality $h\left|\lambda_{\max }\right| \leq D$. This estimation does not lead to the increase of computational costs in all the considered situations.

In this paper, the stability control inequality for the second order Ceschino method is constructed. The first order method based on the stages of the numerical Ceschino formula with a stability interval extended to 32 along the real axis is constructed. A new integration algorithm of alternating order and step formulated. The numerical results of stiff problems solving, confirming the increase of the efficiency due to alternating order and the stability control are given.

This work was partially supported by Project 14-11-00147 of Russian Scientific Foundation.

Keywords - explicit methods, control accuracy and stability, stiff problems. 


\title{
Optimal Startup Control of a Steam Power Plant Using the JModelica Platform
}

\author{
F. Belkhir * D. Kraus Cabo ** F. Felgner * G. Frey * \\ * Chair of Automation, Saarland University, Building A5 1,Germany \\ e-mail:\{fethi.belkhir, felix.felgner, georg.frey\}@aut.uni-saarland.de \\ ** ETSEIB, Polytechnic University of Catalonia, Spain, \\ e-mail:daniel.kraus@estudiant.upc.edu
}

In this contribution, a step toward the immediate usage of Modelica models in the solution of optimal control problems is demonstrated. For this purpose, a model of a steam boiler in a power plant taken from literature was implemented in the high-level modeling language Modelica. The optimal control problem of the steam boiler start-up is formulated and solved using the JModelica framework with the Optimica extension. The results demonstrate the effort saved in solving an optimal control problem when using the JModelica framework in comparison to individual, case- specifically arranged solutions. 


\title{
An Analysis of Performance-critical Properties of Modelica Models
}

\author{
Neil Cafferkey Gregory Provan \\ Insight Centre for Data Analytics, Dept. of Computer Science \\ University College Cork, Cork, Ireland
}

\section{INTRODUCTION}

Equation based object-oriented modelling languages such as Modelica are increasingly used for modelling complex electro-mechanical systems. Although compiling and executing a Modelica model can in some cases take many days, the performance characteristics of Modelica models and compilers are not well understood.

Many simulators have been developed, and in this article we focus on simulation of systems described in the Modelica language (Fritzson, 2010). Simulation of Modelica models is a complex process consisting of numerous distinct phases. At an abstract level, there are two main phases, compilation and execution. Compilation translates the Modelica source code, consisting of a differential algebraic equation (DAE) representation of a model, into a natively executable program for a particular architecture. Once compiled, the program can be run to simulate the model's behaviour.

The compilation phase can be subdivided into several more phases, which are to some extent compiler-specific. This paper focuses on the OpenModelica implementation of Modelica (Fritzson, 2013), and we discuss the phases of that compiler; other compilers (e.g. Dymola and jModelica) follow a similar pattern.

Modelica allows models to be specified by interconnecting components or model sub-systems. Component models may vary significantly in terms of their underlying equations, leading to significant variance in simulation demands. We address two aspects of models, to study their role in simulation complexity: component model specifications, and model structure, based on interconnections of components. In this article, we hence focus on three aspects of Modelica inference:

Compilation Phases By recording timings for individual compilation phases, we highlight the phases that contribute most to compilation time, giving useful information about which sections of code are most worthwhile targeting for future performance improvement. We show that both the Modelica and $\mathrm{C}$ compilation phases are critical, with the interactions being modelspecific; this indicates that one also needs to examine the interactions between the Modelica and $\mathrm{C}$ compilers, and not just the Modelica compilation process.

Model Components Different components may vary in their contribution to simulation performance, so we compare models with different component classes. We

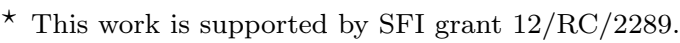

show that different components may have a significant impact on compilation and simulation times.

Model Topology Our experiments analyse differences in performance arising from variation in model structure. We show that different topologies may have a significant impact on compilation and simulation times.

\section{CONCLUSIONS}

To date, most effort has been focused on optimising the Backend phase, e.g. Frenkel et al. (2011, 2012). Our experiments indicate that the Frontend, SimCode and Compile phases can also be critical, with the relative importance of each phase being dependent on the model topology.

Our data show very large differences introduced by variations in network topology, while changes to component types have comparatively little impact. While all model variations considered showed exponential growth in compilation-simulation time (when compilation succeeded), wide differences in their growth rate were observed. The high exponential growth seen in linear circuits of this class can be mitigated through topological inference prior to Modelica simulation. OpenModelica does perform BLT transformations, which is a form of topological inference; however, this inference is done at the equation/variable level, and not at the component-topology level. As a result, BLT transformation does not avoid exponential blowups, and component-topology transformations are needed.

\section{REFERENCES}

Frenkel, J., Kunze, G., and Fritzson, P. (2012). Survey of appropriate matching algorithms for large scale systems of differential algebraic equations. In Modelica'2012, the 9th International Modelica Conference.

Frenkel, J., Schubert, C., Kunze, G., Fritzson, P., Sjölund, M., and Pop, A. (2011). Towards a benchmark suite for Modelica compilers: Large models. In Modelica'2011: The 8th International Modelica Conference.

Fritzson, P. (2010). Principles of object-oriented modeling and simulation with Modelica 2.1. John Wiley \& Sons.

Fritzson, P. (2013). OpenModelica System Documentation. https://openmodelica.org/svn/OpenModelica/tags/ OPENMODELICA_1_9_0_BETA_4/doc/ OpenModelicaSystem.pdf. 


\section{MATHMOD 2015 ABSTRACT VOLUME}

MATHMOD Full Contributions

Environmental Systems 
MATHMOD 2015 Abstract Volume 


\title{
Further Developments in Dynamic Modelling of $\mathrm{CO}_{2}$ Capture from Flue Gas
}

\author{
Jillian Dickinson* Graeme Puxty** \\ Andrew Percy***, T. Vincent Verheyen***
}

*School of Applied Sciences and Engineering, Faculty of Science, Monash University, Australia (Tel: +61 3512262415; e-mail: jillian.dickinson@monash.edu).

**CSIRO Energy Flagship, Newcastle, Australia (e-mail:graeme.puxty@csiro.au)

***School of Applied and Biomedical Sciences, FederationUniversity,

Churchill, Australia (e-mail: vince.verheyen@federation.edu.au,e-mail: andrew.percy@federation.edu.au)

\begin{abstract}
:
Mathematical modelling of $\mathrm{CO}_{2}$ capture from industrial flue gas by absorption into amine solutions such as monoethanolamine (MEA) has been undertaken for decades and steady state, rate-based and dynamic models have been constructed to predict the changes in the process. Recently, dynamic models have been used to predict the effect that physical operational changes have on the absorption process. As more is learnt about the chemistry of MEA and $\mathrm{CO}_{2}$ it becomes evident that the absorption system is losing available MEA, by degradation and by vaporization into the gaseous phase. This paper describes a dynamic model of the absorber column that can be used to predict the reduction of available MEA, the loss of MEA to the atmosphere, and the build-up of heat stable salts. The proposed mathematical model consists of a system of partial differential equations to represent the change of each component with height of the column and with time. It has been validated with data from a pilot capture plant located at the brown coal fired Loy Yang power station in Australia.
\end{abstract}




\title{
Pitfalls Associated with the Estimation of Radiological Burdens on Population Caused by Radiation Accidents
}

\author{
Petr Pecha*, Emilie Pechova** \\ *Institute of Information Theory and Automation, Prague 8, Czech Republic \\ (Tel: +4202 66052009; e-mail: pecha@utia.cas.cz). \\ **Institute of Nuclear Research, Div. EGP, 25068 Rez near Prague, Czech Republic
}

\begin{abstract}
The initial intention of this essay was to compare our environmental code PRIMO with the commonly accepted code COSYMA. All basic output radiological variables are in very good agreement after our careful mutual tuning of all input parameters of both products. However, a certain disproportion was encountered when proceeding to the estimation of radiation doses. It became apparent that due to the hidden variability of the parameters of the dosimetric model, the estimation of the radiological impact on the human body can be generated with a different degree of conservatism. These effects should be accounted for when using the environmental codes for assessment of the radiological impact on a population. An alternative approach of the assessment taking into consideration the variability and subsequently uncertainty of the input parameters can afford more informative answers to the evaluation questions. From this point of view, the PRIMO algorithm follows the research trend of progress from the deterministic calculations towards the probabilistic approach of the consequence estimation.
\end{abstract}

The early stage of a radiation accident has been verified with help of the PRIMO code. This code can be used by the potential authorised contracting authorities planning the construction and operation of a nuclear facility when compliance with the strict obligatory governmental regulations has to be proved. The product was submitted to the Czech Standardisation Board and approved (2013) for its use in the field of nuclear safety. The "worst case" analysis should be performed for a set of prescribed Design Basis Accidents (one of them was the SBLOCA examined here). Before using PRIMO, one must be aware of all pitfalls of the assessment and undertake responsibility for delivery of the relevant input data and correct interpretation of the results. The variations of the inputs should be adjusted with regard to the parameter distributions. In the simplest case the interval of the potential minimum and maximum values based on the expert elicitation procedures can be assumed. If the parameter is assumed to be random, the values should be sampled from its probability density function. We are thus entering the area of the sensitivity and uncertainty studies and up-to-date trends of the probability approach can be followed. The HARP code is designed for either deterministic or probabilistic analysis (HARP, 2013). Simulation of the uncertainty propagation through the model also provides a basis for another main task of the analysis called assimilation of the model predictions with the real measurements coming from the terrain. Data assimilation represents the way to progress from a model to reality and can substantially improve the quality of the model predictions (ASIM, 2012).

The environmental program product HARP (HARP, 2012) includes segmented Gaussian algorithms in the PRIMO and SGPM modifications. The latter version can respect time and spatial weather changes. The complicated scenario of the release dynamics is thus synchronized with the available meteorological forecasts. The SGPM is aimed at detailed modelling in the data assimilation procedures (ASIM, 2013). In the following tests we are using the simpler PRIMO component for description of straightforward radioactivity propagation in the air prospectively proposed for the fast sensitivity analysis and "worst case" studies. The safety studies are also incorporated in the EIA Reports within the licensing process when the operational safety should comply with the obligatory governmental regulations. The detailed comparative study of the PRIMO and COSYMA results is presented here. Possible pitfalls associated with the estimation of radiological burdens on population are demonstrated. 


\title{
Modelling the effect of temperature on phytoplankton growth across the global ocean *
}

\author{
Ghjuvan Micaelu GRIMAUD* , Valérie LE GUENNEC *,** \\ Sakina-Dorothée AYATA ${ }^{* * * * * *}$, Francis MAIRET, ${ }^{*}$ \\ Antoine SCIANDRA ${ }^{* *, * * *}$, Olivier BERNARD* \\ * BIOCORE-INRIA,BP93,06902 Sophia-Antipolis Cedex, France \\ ** UPMC Univ Paris 06, UMR 7093, LOV, Observatoire \\ océanologique, F-06234, Villefranche/mer, France \\ *** CNRS, UMR 7093, LOV, Observatoire océanologique, \\ F-06234, Villefranche/mer, France
}

\begin{abstract}
Phytoplankton are ectotherms and are thus directly influenced by temperature. They experience temporal variation in temperature which results in a selection pressure. Using the Adaptive Dynamics theory and an optimization method, we study phytoplankton thermal adaptation (more particulary the evolution of the optimal growth temperature) to temperature fluctuations. We use this method at the scale of global ocean and compare two existing models. We validate our approach by comparing model predictions with experimental data sets from 57 species. Finally, we show that temperature actually drives evolution and that the optimum temperature for phytoplankton growth is strongly linked to thermal amplitude variations.
\end{abstract}

Keywords: phytoplankton, temperature, evolution, Adaptive Dynamics, microalgae, modelling 


\title{
A General Analytical Capture Zone model: A Tool for Groundwater Remediation
}

\author{
Nozar Samani* $\quad$ Somayeh Zarei-Doudeji*** \\ Department of Earth Sciences, Shiraz University, Shiraz 71454, Iran \\ *(Tel: +98 7132284572 ; e-mail: samani@susc.ac.ir). \\ ** (Tel: +98917 337 1344; e-mail:szarei@shirazu.ac.ir)
}

\begin{abstract}
The capture zone equations of a multi-well system in bounded confined and unconfined aquifers are derived. The aquifer is rectangular shaped in plan view and bounded along all four sides. The boundaries could be in-flow (constant head) or no-flow (barrier) or a combination of both and hence six boundary configurations are formed. Using the image well theory the flow field in bounded aquifers is first transformed to its equivalent in extensive aquifers and then the complex velocity potential theory is applied for the generation of stream function delineating the capture envelope. We show that the derived solution is general and it may be easily reformulated for some existing solutions of capture zone. Our solution is flexible in terms of well number, well location, well type, extraction/injection rate, uniform regional flow rate and direction and number of boundaries. The derived equations are presented in form of dimensionless capture type curves that may be used for the remediation of contaminated groundwater project design, containment of contaminant plumes and the evaluation of surface-subsurface water interaction.
\end{abstract}




\title{
MATHMOD 2015 ABSTRACT VOLUME
}

\author{
MATHMOD Full Contributions \\ Mechanical Systems
}


MATHMOD 2015 Abstract Volume 


\title{
A Closed-Form Approach to Determine the Base Inertial Parameters of Complex Structured Robotic Systems
}

\author{
J. Klodmann, D. Lakatos, C. Ott, A. Albu-Schäffer \\ Institute of Robotics and Mechatronics, German Aerospace Center (DLR), \\ D-82234 Oberpfaffenhofen, Germany (e-mail: julian.klodmann@dlr.de).
}

\section{EXTENDED ABSTRACT}

To increase the dynamic performance and to minimize the steady-state control error, many modern control schemes rely on model knowledge of the plant. Especially in the field of robotics, model based control strategies were often used, including computed torque, adaptive and impedance control (see e.g. Chapter. 9-11 in [1]). These control schemes, where the model parameters have to be a priori known, require the knowledge of the model structure and a parameter identification method [6]

Due to the kinematics of spatial rotations, the inversedynamic equations of multi-body systems are strongly non-linear w.r.t. the state variables. It is a well known fact that, deriving the inverse-dynamic model by the Lagrangian formalism (or by means of the iterative NewtonEuler algorithm), the inertial parameters appear linearly [3]; consequently, the identification model can be formulated linearly w.r.t. the unknown parameters. This property allows the identification of the inertial parameters using linear least-squares methods.

When formulating a robot identification model, a challenging property of multi-body systems has to be considered: the inertia of consecutive bodies is coupled via joints, i.e. in general the set of standard inertial parameters (i.e., mass, mass moment first and second order for each body) consists of dependencies. Furthermore, some parameters do not affect the dynamics. In the case of using the standard inertial parameters, the observation matrix of the identification model is singular and not invertible. Therefore, only the set of identifiable parameters can be estimated, which correspond to non-zero and linearly independent columns of the observation matrix.

To calculate the minimal set of identifiable parameters, which are often referred to as base inertial parameters (BIP), several algorithms have been proposed, i.a. [2-5]. Most of these algorithms use a manual search strategy which can be applied to a special class of robot structures and aim to derive the identifiable parameters in symbolic form. By the mentioned method several algorithms are derived to calculate the BIP of robot structures, where only single degree of freedom joints connect apparent bodies. Two numerical approaches to calculate the BIP have been proposed in [2]. Both methods are applicable for a large class of mechanical systems and straightforward to implement. The set of BIP is obtained in numeric form. The numeric approach given by [2] can be extended to obtain the BIP in symbolic form [4]. Herein also the structure of the dynamic model has to be taken into account.

In this paper, we present a general algorithm to derive the symbolic expressions of a unique set of identifiable param- eters and the corresponding reduced regressor matrix of general linear parameter models. We propose to transform the unknown parameter vector into the row space of the initially rank deficient observation matrix. This leads to a minimum set of identifiable parameters, fully describing the model, and thus to minimum degrees of freedom in the solution of the linear least-squares problem. Regarding the exemplified derivation of the set of BIP of robots, the non-singular linear row space transformation is derived symbolically, without analyzing the structure of the model, in contrast to [3-5]. Furthermore the method applies to complex multi-body structures with multi-degreeof-freedom joints. In addition, as exemplified in the experimental part, the method can be applied to dynamic models with non-collocated placement of the position and torque sensors. Therefore, the proposed closed-form approach is more general than the methods in $[3-5]$. In contrast to the numeric approaches [2], the output of our algorithm is the identification model in symbolic form. This is an advantage, since the resulting model can be further used, for instance, to find optimally exciting observations for the identification procedure, e.g. robot trajectories [6] or to reduce the computational costs to compute the model, e.g. the inverse dynamics of the robot (cf. derivation of BIP).

The paper contains theory of the new method to determine the identifiable parameter model. The procedure of the closed form algorithm is exemplary demonstrated for the double pendulum dynamics. In addition we validate the performance of the method in experiments with a complex structured robotic system. Finally a brief conclusion is given.

\section{REFERENCES}

[1] J. Craig. Introduction to Robotics: Mechanics and Control. 3. Pearson Prentice Hall, 3rd edition, 2005.

[2] M. Gautier. Numerical calculation of the base inertial parameters of robots. In IEEE International Conference on Robotics and Automation, 1990.

[3] M. Gautier and W. Khalil. Direct calculation of minimum set of inertial parameters of serial robots. IEEE Transactions on Robotics and Automation, 6:368-373, 1990.

[4] X. Iriarte, J. Ros, and V. Mata. Symbolic calculation of the base inertial parameters of robots through dimensional analysis. In Multibody Dynamics 2009, 2009.

[5] H. Mayeda, K. Yoshida, and K. Osuka. Base parameters of manipulator dynamic models. IEEE Transactions on Robotics and Automation, 6:312-321, 1990.

[6] J. Swevers, W. Verdonck, and J. De Schutter. Dynamic model identification for industrial robots. Control Systems, IEEE, 27, 2007. 


\title{
On the inertially decoupled structure of the floating base robot dynamics
}

\author{
Gianluca Garofalo* Bernd Henze* Johannes Englsberger* \\ Christian Ott* \\ * Institute of Robotics and Mechatronics, German Aerospace Center \\ (DLR), Wessling, Germany (e-mail: gianluca.garofalo(at)dlr.de).
}

\section{INTRODUCTION}

Insights about the structure of the equations have turned out to be of great usefulness when dealing with floating base robots. In this paper a coordinate transformation is proposed that provides an inertially decoupled structure for the equations of motion of this class of systems. We show that the conservation of momentum directly implies orthogonality relationships between some of the matrices of the dynamic model. These can be used to perform a coordinate transformation that leads to inertially decoupled equations, since in the new coordinates the transformed inertia matrix will be block diagonal. As the center of mass (CoM) has been used both for locomotion and balancing of legged robots because of its decoupled dynamics from the rest of the system, we expect to benefit from our coordinate transformation since it allows to separate the linear and angular centroidal dynamics from the joint dynamics. As an example of application, the proposed transformation is used in the derivation of a humanoid balance controller.

\section{MAIN RESULT}

For floating base robots the state is usually defined by the variables $\boldsymbol{x}$ and $\boldsymbol{v}$, being $\boldsymbol{x}$ the complete configuration coordinates with dimension $n_{v}$ and $\boldsymbol{v} \in \mathbb{R}^{n_{v}}$ the complete velocity coordinates, i.e. including both the floating base and joint coordinates. Indicating with $\tau \in \mathbb{R}^{n_{q}}$ the torques provided by the motors and with $\boldsymbol{w}_{i} \in \mathbb{R}^{6}$ one of the $m$ contact wrenches, then the dynamic model can be written as

$$
\boldsymbol{M}(\boldsymbol{x}) \dot{\boldsymbol{v}}+\boldsymbol{C}(\boldsymbol{x}, \boldsymbol{v}) \boldsymbol{v}+\boldsymbol{g}(\boldsymbol{x})=\boldsymbol{Q}^{T} \boldsymbol{\tau}+\sum_{i=1}^{m} \boldsymbol{J}_{i}^{T}(\boldsymbol{x}) \boldsymbol{w}_{i}
$$

where $\boldsymbol{Q}$ is the matrix that selects the joint velocities $\dot{\boldsymbol{q}} \in \mathbb{R}^{n_{q}}$ out of all the velocity coordinates, i.e. $\dot{\boldsymbol{q}}=\boldsymbol{Q} \boldsymbol{v}$. Moreover, it is always possible to express the generalized momentum $\boldsymbol{h} \in \mathbb{R}^{6}$, i.e. the linear and the angular momentum stacked together, as

$$
\boldsymbol{h}=\boldsymbol{A}(\boldsymbol{x}) \boldsymbol{v} .
$$

From the conservation of the generalized momentum and of the angular momentum, orthogonality relationships between matrices of the dynamic model can be derived. As in Ott et al. (2008), these orthogonality relationships

\footnotetext{
^ This research is partly supported by the Initiative and Networking Fund of the Helmholtz Association through a Helmholtz Young Investigators Group (Grant no. VH-NG-808).
}

are used to derive a velocity coordinate transformation, namely

$$
\begin{array}{ll}
\boldsymbol{\xi}=\left[\begin{array}{c}
\boldsymbol{h} \\
\dot{\boldsymbol{q}}
\end{array}\right]=\boldsymbol{A}_{Q}(\boldsymbol{x}) \boldsymbol{v} & \boldsymbol{A}_{Q}(\boldsymbol{x})=\left[\begin{array}{c}
\boldsymbol{A}(\boldsymbol{x}) \\
\boldsymbol{Q}
\end{array}\right] \\
\boldsymbol{v}=\boldsymbol{A}_{Q}^{-1}(\boldsymbol{x}) \boldsymbol{\xi} . &
\end{array}
$$

In the new coordinates the equations of motion are (omitting the dependencies)

$$
\begin{aligned}
{\left[\begin{array}{ccc}
\boldsymbol{\Lambda}_{p} & \mathbf{0} & \mathbf{0} \\
\mathbf{0} & \boldsymbol{\Lambda}_{l} & \mathbf{0} \\
\mathbf{0} & \mathbf{0} & \boldsymbol{\Lambda}_{q}
\end{array}\right] \dot{\boldsymbol{\xi}}+} & {\left[\begin{array}{ccc}
\boldsymbol{\Gamma}_{p p} & \boldsymbol{\Gamma}_{p l} & \boldsymbol{\Gamma}_{p q} \\
-\boldsymbol{\Gamma}_{p l}^{T} & \boldsymbol{\Gamma}_{l l} & \boldsymbol{\Gamma}_{l q} \\
-\boldsymbol{\Gamma}_{p q}^{T} & -\boldsymbol{\Gamma}_{l q}^{T} & \boldsymbol{\Gamma}_{q q}
\end{array}\right] \boldsymbol{\xi}+\left[\begin{array}{c}
g \boldsymbol{e}_{g} \\
\mathbf{0} \\
\mathbf{0}
\end{array}\right] } \\
= & {\left[\begin{array}{c}
\mathbf{0} \\
\mathbf{0} \\
\boldsymbol{\tau}
\end{array}\right]+\sum_{i} \overline{\boldsymbol{J}}_{i}^{T} \boldsymbol{w}_{i} }
\end{aligned}
$$

where $\overline{\boldsymbol{J}}_{i}(\boldsymbol{x})=\boldsymbol{J}_{i}(\boldsymbol{x}) \boldsymbol{A}_{Q}^{-1}(\boldsymbol{x})$.

\section{APPLICATION}

We propose a balancing controller which exploits the structure of (3). The control law is evaluated in an experiment with the humanoid robot TORO, developed at the German Aerospace Center (DLR) and shown in Fig. 1.

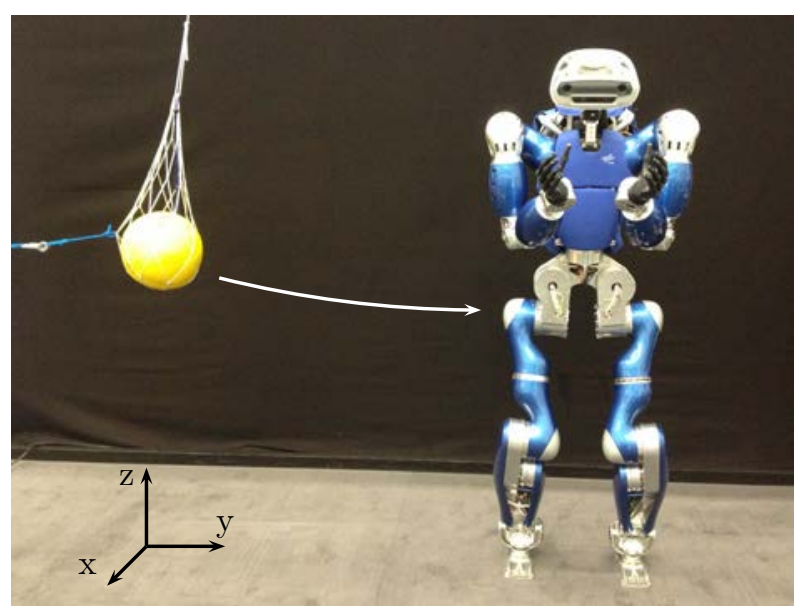

Fig. 1. Setup of the experiment

\section{REFERENCES}

Ott, C., Kugi, A., and Nakamura, Y. (2008). Resolving the problem of non-integrability of nullspace velocities for compliance control of redundant manipulators by using semi-definite Lyapunov functions. In IEEE Int. Conf. on Robotics and Automation (ICRA), 1456-1463. Pasadena, USA. 


\title{
Reduced equations of motion for a wheeled inverted pendulum
}

\author{
Sergio Delgado*, Sneha Gajbhiye ${ }^{* *}$, Ravi N. Banavar ${ }^{* *}$ \\ * Technische Universität München, Boltzmannstr. 15, D-85748, \\ Garching (Tel: +49-89-289 15679; e-mail: s.delgado@tum.de). \\ ** Indian Institute of Technology Bombay, India (e-mail: \\ \{banavar,sneha\}@sc.iitb.ac.in)
}

\begin{abstract}
This paper develops the equations of motion in the reduced space for the wheeled inverted pendulum, which is an underactuated mechanical system subject to nonholonomic constraints. The equations are derived from the Lagrange-d'Alembert principle using variations consistent with the constraints. The equations are first derived in the shape space, and then, a coordinate transformation is performed to get the equations of motion in more suitable coordinates for the purpose of control.
\end{abstract}

Keywords: Underactuated mechanical systems, nonholonomic constraints. 


\title{
Finite Segment Model Complexity of an Euler-Bernoulli Beam
}

\author{
Loucas S. Louca \\ University of Cyprus, Department of Mechanical and Manufacturing Engineering \\ Nicosia, CYPRUS (Tel: +357-2289 2279; e-mail: lslouca@ucy.ac.cy)
}

\section{SUMMARY}

A common approach for modeling the dynamic behavior of distributed parameter systems is the approximation through finite-segment models. These models are able to accurately predict the dynamic behavior of a system given that "adequate" segments are included in the model. Frequencybased methodologies can be used to address the complexity of such models. The purpose of the current work is to address the complexity of distributed parameter using the previously developed activity metric. More specifically the complexity of an Euler-Bernoulli beam model is considered. Bond graph models of this system already exist in the literature and the objective is to identify the necessary complexity (number of segments). A new modeling procedure is proposed for this type of systems where the model starts from simple and the number of segments is increased until an activity based criterion is satisfied. An illustrative example is provided to demonstrate the effectiveness of this methodology.

\section{ACTIVITY METRIC}

The activity metric as shown in (1) has been previously formulated for systems with nonlinearities in both the element constitutive laws and junction structure. In this work, the activity metric is applied to linear systems for which analytical expressions for the activity can be derived, and therefore, avoid the use of numerical time integration that could be cumbersome. The analysis is further simplified if, in addition to the linearity assumption, the system is assumed to have a single sinusoidal excitation, and only the steady state response is examined.

$$
A=\int_{0}^{\tau}\left|P^{\prime}(t)\right| \cdot d t=\int_{0}^{\tau}|e(t) \cdot f(t)| \cdot d t
$$

\section{MODEL COMPLEXITY METHODOLOGY}

In this work the input activity, which provides a measure of the overall system activity level, is used as the metric for identifying the minimum number of segments. The input activity changes as the number of segments varies and the proposition of this new methodology is to increase the number of segments until the input activity reaches a steady state value. This approach is motivated from the principle of the activity metric stating that low activity elements do not contribute to the system behavior and should not be included in the model. In this case the equivalent is that an increase in model complexity producing a "small change" in the input activity is not significant, thus such increase is not necessary for the model accuracy. Again, based on the activity principle, a small change in the input activity is insignificant and thus does not contribute to the overall system behavior. This "small change" is defined through the relative activity $\delta A$ that is mathematically expressed in (2). The input and relative activities vary with the frequency of the excitation, and therefore, the optimum complexity depends on the excitation frequency.

$$
\delta A(\omega, n)=\frac{A^{s s}(\omega, n+1)-A^{s s}(\omega, n)}{A^{s s}(\omega, n)}
$$

\section{RESULTS}

The results for the optimum model complexity of the transverse vibrations of a cantilever beam are shown in Fig. 1. For low frequencies and up to $32 \mathrm{rad} / \mathrm{s}$, 55 segments are necessary to accurately predict the system response. As the input frequency increases the number of segments increases in order to maintain accuracy. It exponentially increases as it approaches the first natural frequency at $262 \mathrm{rad} / \mathrm{s}$ and just before $236 \mathrm{rad} / \mathrm{s}$ the maximum allowable number of segments is reached. More segments need to be added at frequencies around the fundamental natural frequency. At higher frequencies and after $285 \mathrm{rad} / \mathrm{s}$ the number of segments reduces again reaching a minimum of 81 segments at $700 \mathrm{rad} / \mathrm{s}$ and then a similar pattern is repeated for higher input frequencies. For simplicity the optimum complexity is limited to 200 segments.

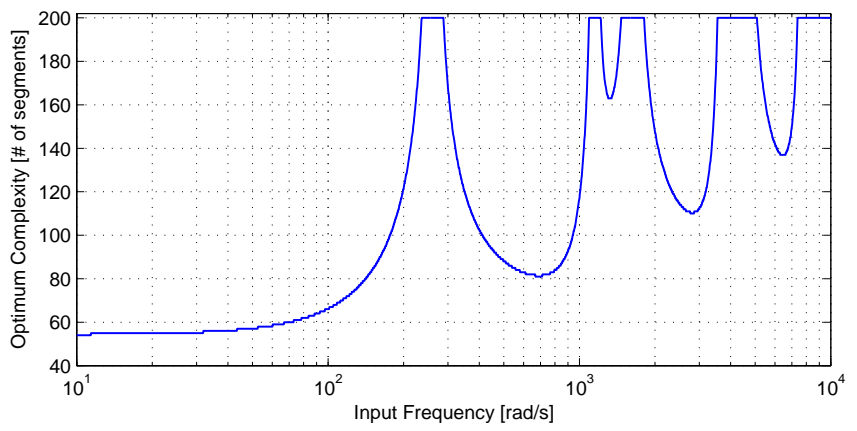

Fig.1: Frequency response of optimum complexity

A new methodology is developed for the systematic modeling of continuous systems that are modeled through the finite segment approach. The previously developed activity metric is used as the basis for determining the optimum number of segments that are needed in order for the model to accurately predict the system dynamic behavior. The activity that flows through the input is used and the optimum complexity model is shown to be accurately predicting the system behavior over a range of input frequencies. 


\title{
Modelling and Simulation of Passive Limited-slip Differentials *
}

\author{
Martin Forstinger* Robert Bauer ** Anton Hofer ${ }^{* * *}$ \\ * Institute of Automation and Control, Graz University of Technology, \\ 8010 Graz, Austria (e-mail: martin.forstinger@tugraz.at) \\ ** Kristl, Seibt $\&$ Co GmbH, 8010 Graz, Austria (e-mail: \\ robert.bauer@ksengineers.at) \\ *** Institute of Automation and Control, Graz University of \\ Technology, 8010 Graz, Austria (e-mail: anton.hofer@tugraz.at)
}

\section{ABSTRACT}

This work starts with the derivation of dynamical models for bevel gear and epicyclic limited-slip differentials. These models include losses and the capability of describing asymmetric torque splitting. It is shown that two different mechanical structures lead to a common system description. Additionally, the stationary torque splitting depending on the system parameters is determined. As Coulomb friction is used to consider both losses and limited-slip functionality simulation is difficult because of a discontinuity at zero angular velocity. A method for overcoming these simulation problems, based on force-balancing, is presented. Simulation studies using the proposed simulation strategy show striking similarities with measurement data from a differential gear test bed.

\section{MODELLING, SIMULATION}

The paper presents two different structures for differential gears, the bevel gear differential and a differential gear based on a planetary gear set. For both types a second order dynamic system model is presented. Both dynamic models include limited-slip functionality, losses and asymmetric nominal torque splitting and can be generalised by the system description

$$
\left[\begin{array}{c}
\dot{x}_{1} \\
\dot{x}_{2}
\end{array}\right]=\mathbf{B u}-\left(\begin{array}{cc}
\alpha_{1} & \alpha_{2} \\
\beta_{1} & \beta_{2}
\end{array}\right)\left[\begin{array}{l}
T_{F 1}\left(x_{1}\right) \\
T_{F 2}\left(x_{2}\right)
\end{array}\right],
$$

where $\mathbf{u}$ is the system input. Because the two friction torques $T_{F 1}$ and $T_{F 2}$ may include static and Coulomb friction this dynamic system belongs to the class of variable dynamic dimension systems (Zanasi et al. (2001)). In addition to modelling special focus of the paper is on the simulation of this particular variable dynamic dimension system. Based on previous work (Morselli et al. (2006)) a simulation strategy for the second order variable dynamic dimension system based on Force-balancing (Karnopp (1985)) is presented. One substantial contribution is a method for determining the friction torques $T_{F 1}$ and $T_{F 2}$ required for simulation depending on the system input $\mathbf{u}$ when both system states are zero.

\footnotetext{
^ This work was supported by Kristl, Seibt \& Co GmbH.
}

\section{RESULTS}

Finally the simulation results are compared to measurement data form a differential gear test bed. Fig. 1 shows the rotational speeds measured at the differential gear's output shafts and additionally the rotational speed of the differential cage. Fig. 2 shows the measured input torque

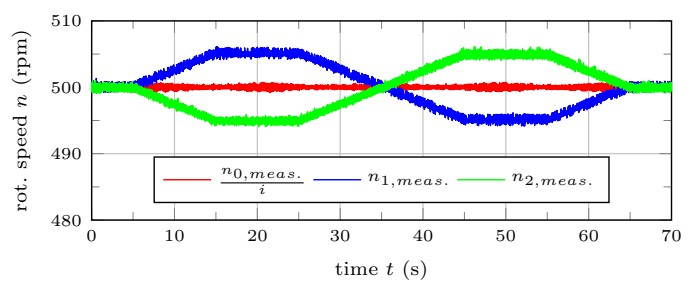

Fig. 1. Measured rotational speeds

$T_{0}$ (grey) scaled by the factor two and the transmission ratio $i$ and a comparison of measured and simulated output torques, showing a very good match.

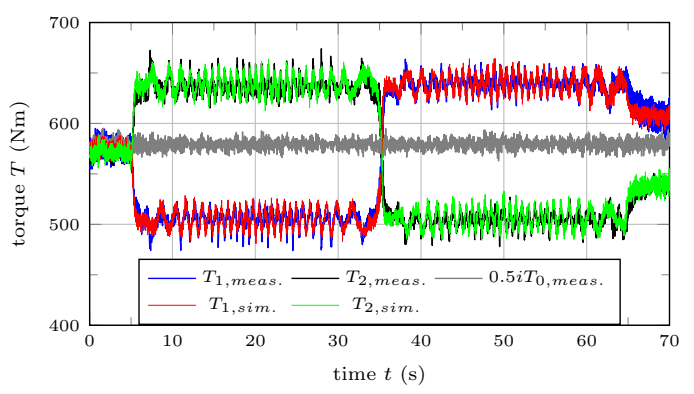

Fig. 2. Measured and simulated torques

\section{REFERENCES}

Karnopp, D. (1985). Computer simulation of stick-slip friction in mechanical dynamic systems. ASME Journal of Dynamic Systems, Measurement, and Control, 107(1), 100-103.

Morselli, R., Zanasi, R., and Sandoni, G. (2006). Detailed and reduced dynamic models of passive and active limited-slip car differentials. Mathematical and Computer Modelling of Dynamical Systems, 12(4), 347-362.

Zanasi, R., Sandoni, G., and Morselli, R. (2001). Simulation of a variable dynamic dimension systems: the clutch example. In European Control Conference (ECC), 31493154 . 


\title{
Mathematical Modeling and Analysis of a Hydrostatic Drive Train
}

\author{
P. Zeman*, W. Kemmetmüller*, A. Kugi* \\ * Automation and Control Institute, Vienna University of Technology, \\ Vienna, Austria (e-mail: zeman@acin.tuwien.ac.at)
}

Hydrostatic drives constitute an advantageous alternative to conventional mechanical gears with a fixed transmission ratio. In a typical setup of a hydrostatic drive train, the energy delivered by an internal combustion engine (ICE) on the primary side of the system is transmitted across a hydraulic circuit to the output on the secondary side. The infinitely variable transmission (IVT) allows to run the ICE in a set point which is optimal from an energetic point of view. Furthermore, recuperation of braking energy can be done in a very efficient way by loading a hydraulic accumulator, see, e.g., Erkkilä et al. (2013). For this reason, hydrostatic drives are a well-established concept, in particular in the field of mobile working machines such as lift trucks or excavators. Current research is concerned with the exploitation of these advantages for passenger vehicles. The hydrostatic drive train represents an alternative concept for automotive manufacturers compared to conventional hybrid drives with electric engines. These serial hydraulic hybrid vehicles (SHHVs) require sophisticated control strategies which translate the torque request of the driver into a suitable actuation of the system by simultaneously fulfilling performance and energy efficiency demands. The basis for the controller design are mathematical models which are tailored to the considered drive train.

Various configurations of SHHVs exist in the literature. Typically, like in Deppen and Alleyne (2012), swash plate controlled variable displacement axial piston pumps/motors are used to control the hydraulic flow between the primary and secondary side of the drive. In most contributions it is assumed that the control of the axial piston units is performed by standard displacement controllers and therefore the underlying dynamics are not accounted for. For the design of highly dynamic torque control strategies, this simplification is not feasible and yields a limited control performance. For this reason, the present contribution deals with the mathematical modeling of an SHHV, where the essential nonlinear dynamics of the axial piston units are taken into account.

The mathematical modeling of variable axial piston pumps is discussed extensively in the literature. In Fuchshumer (2009), a dynamic mathematical model, consisting of the equations of motion of the pump, the swash plate mechanism and the actuating valve, is presented. Because of the different hydraulic and mechanical setting compared to Fuchshumer (2009), a detailed model of the axial piston

\footnotetext{
* The authors from Vienna University of Technology highly appreciate the technical and financial support provided by Robert Bosch $\mathrm{GmbH}$.
}

units of the considered SHHV is derived in the first part of this paper, which captures the essential dynamics of the pump, the displacement system and the actuating valve. Besides, in order to account for the mechanical and hydraulic efficiency of the axial piston units, the torque and volumetric losses are approximated by polynomials which are fitted to stationary measurements. The detailed model thus obtained is suitable to validate different control strategies for the SHHV and to study the influence of model parameters. However, it entails a high computational burden, in particular if it serves as a basis for nonlinear MPC (Model Predictive Control), see, e.g., Grüne and Pannek (2011). For this reason, the present contribution demonstrates how the complexity of the detailed model can be reduced successively by exploiting the singular perturbation theory, see, e.g., Kokotovic et al. (1999). Thereby, the fast dynamics of the detailed model are considered in a quasi-stationary manner, thus leading to a reduced-order model which is suitable for the controller and estimator design. Both the detailed and reduced-order model are validated by measurements on an industrial test bench. It is shown that the reduced-order model almost preserves the accuracy of the detailed model for a quasistationary and a dynamic actuation scenario of the system. Current research is directed to the application of nonlinear MPC to the considered system. Thereby, the reducedorder model serves as a perfect basis for the controller design.

\section{REFERENCES}

Deppen, T.O. and Alleyne, A.G. (2012). Optimal energy use in a light weight hydraulic hybrid passenger vehicle. Journal of Dynamic Systems, Measurement and Control, 134(4).

Erkkilä, M., Bauer, F., and Feld, D. (2013). Universal Energy Storage and Recovery System - A Novel Approach for Hydraulic Hybrid. In Proceedings of the 13th Scandinavian International Conference on Fluid Power, SICFP2013, 92, 45-52. Linköping, Sweden. 3 - 5 June 2013.

Fuchshumer, F. (2009). Modellierung, Analyse und nichtlineare modellbasierte Regelung von verstellbaren Axialkolbenpumpen. Shaker, Aachen, Germany.

Grüne, L. and Pannek, J. (2011). Nonlinear Model Predictive Control: Theory and Algorithms. Communications and Control Engineering. Springer, London.

Kokotovic, P., Khali, H., and O'Reilly, J. (1999). Singular Perturbation Methods in Control: Analysis and Design. Society for Industrial and Applied Mathematics, Philadelphia, USA. 


\title{
Dynamical Modeling of Constraints with Friction in Mechanical Systems
}

\author{
Thomas Specker* Michael Buchholz* Klaus Dietmayer * \\ * Institute of Measurement, Control and Microtechnology, \\ University of Ulm, Albert-Einstein-Allee 41, D-89081 Ulm, Germany \\ (e-mail: thomas.specker@uni-ulm.de,michael.buchholz@uni-ulm.de, \\ klaus.dietmayer@uni-ulm.de)
}

Constraints and friction both exist in almost every mechanical system. However, even on their own, both effects are not easy to describe in a dynamical model of a multibody system without losing numerical stability. Therefore, constraints with friction, i.e. friction only occurring while a constraint is active, is especially challenging. In literature, the combination of constraints and friction is mostly limited to the contact of simple single-body systems. More complex scenarios for multiple contacts with friction of multi-body systems are rather sparse. Approaches in literature that try to deal with those problems are complicated to handle. However, there exist a lot of modeling theories for the single problems of impact or friction.

Regarding dynamic impact models, many approaches can be found in literature. For unilateral contacts, two different kinds of approaches can be distinguished. On the one hand, rigid body impacts are based on the conservation of momentum, being derived from the closely related impact models of Newton or Poisson for single-body systems. This approach approximates the impact by two perfectly smooth and rigid bodies, allowing no deformation or indentation. Extensions are available to introduce dissipation as well as for multi-body systems. On the other hand, continuous force models assume elastic objects and describe the force counteracting the impact in relation to the elastic deformation. These models are mostly based on the Hertzian spring theory and can also be extended by dissipative elements like dampers. Enhancements for multi-body systems are also available.

Both classes of approaches show several problems within the application in multi-body systems and incorporating friction. Due to the lack of a persisting contact within rigid body impact theory, friction can hardly be handled in these approaches. Additionally, the switching behavior of the impact model can cause numerical problems, becoming even worse in multi-contact scenarios of multibody systems. Although the continuous force models are suitable for multiple contacts also including friction in theory, the exponentially growing modeling effort due to underlying piecewise-defined functions and the necessity of considering every contact combination makes the approach very or even too complex in practice.

These disadvantages are avoided by a recently developed modeling approach for unilateral constraints using power-

\footnotetext{
* The authors thank the German Federal Ministry of Economics and Technology for supporting this work through the Central Innovation Program SME within the project KF2648502NT1.
}

based restriction functions. Within this approach, each constraint is approximated by the multiplication of a continuous activation function and a continuous restriction function, whereas each of them can be adapted to the real constraint behavior by two physically interpretable parameters. These restriction functions can easily be integrated in the Lagrangian equations of the second kind, and a calculation of the normal force is also available, which allows the direct integration of additional friction.

Concerning friction, several static and dynamic modeling approaches have been developed since the early works of Coulomb. Static friction models usually comprise Coulomb (or dry) friction, Stribeck friction, and/or viscous friction. Certainly, these static models are not able to describe hysteresis or memory effects, which are dynamically caused by friction. These dynamic effects can be covered by more complex dynamical models, which introduce one or more additional states. Besides other individual disadvantages, these approaches share the usage of stepping or absolute value functions at the zero crossing of the velocity, resulting in unstable numerical behavior in simulations with larger sample times or observer applications.

Especially to overcome this numerical issue, a new dynamic friction model was proposed recently. The model uses continuous functions only to approximate the common static effects. Furthermore, hysteresis and memory effect are embedded by a first order linear parametervarying (LPV) lowpass filter, whose time constant depends on the velocity. This yields high precision for high sample rates, while for low sample rates the numeric stability is guaranteed by rougher approximations.

In this contribution, the combination of this numerically favourable friction model with the restriction function approach for constraint modeling is presented. Inherited from both underlying modeling methods, which are shortly summarized in the contribution, the proposed combination uses continuous functions only to describe or approximate the effects of impact and of friction. It is shown that this approach can be integrated within Lagrangian mechanics, making it easy to use for any kind of mechanical system. The approach is then used to simulate a double inverted pendulum with friction being present if constraints are active. This simulative experiment is described and the results are discussed, showing that very good and reproducible results can be achieved due to the numerical stability of the proposed method. 


\title{
Use of Complex Signals in Modelling of Journal Bearings
}

\author{
Renata Wagnerová* Jiří Tůma** \\ *Vら̌B-Technical University of Ostrava, 17. listopadu 15, 70833 Ostrava \\ Czech Republic, tel: +42059732 4113, renata.wagnerova@vsb.cz. \\ **VŠB-Technical University of Ostrava, 17. listopadu 15, 70833 Ostrava \\ Czech Republic tel: +420 59732 3482, jiri.tuma@vsb.cz
}

\section{INTRODUCTION}

It is known that the journal bearing with an oil film becomes instable if the rotor rotation speed crosses a certain value, which is called the Bently-Muszynska threshold. To prevent the rotor instability, the active control can be employed. The arrangement of proximity probes and piezoactuators in a rotor system is shown in Fig. 1. It is assumed that the carrier ring is a movable part in two perpendicular directions while rotor is rotating. The carrier ring position is controlled by the piezoactuators according to the proximity probe signals, which are a part of the closed loop including a controller, Tůma et al. (2013).

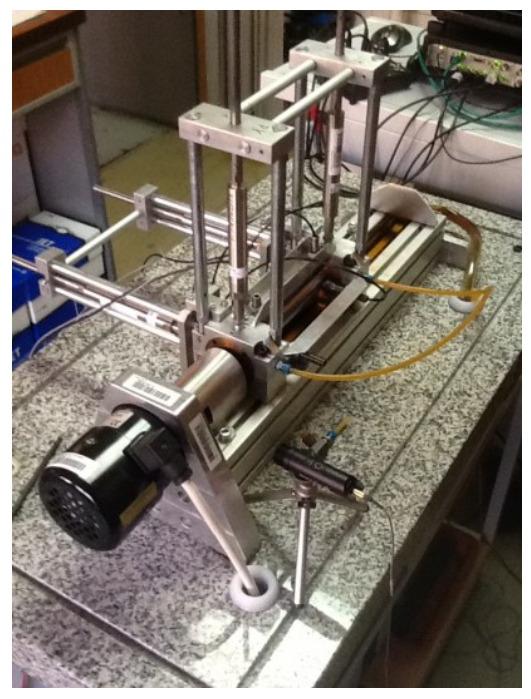

Fig. 1. Test stand for research of active vibration control of journal bearings.

There are many ways how to model a rotor system, but this paper prefers an approach, which is based on the concept developed by Muszynska (1986, 2005) and Bently et al. (1986), who were supported by Bently Rotor Dynamics Research Corporation or on the lubricant flow prediction using a FE method for Reynolds equation solution, Svoboda (2007). The approach based on the Muszynska model enable to describe dynamic behaviour by ordinary differential equation while the other approach uses partial differential equations which are for modelling more complicated. Moreover both approaches achieved same results in behaviour; differences are only for small values of rotor rotation speed but the most interesting values are the high value of rotor rotation speed in which the instability occurs, Mendes et al. (2014).

\section{SIMULATION STUDY OF THE MODEL BEHAVIOUR}

As it was stated before the numeric solution of the journal equation of motion is obtained by using Matlab-Simulink. Thanks to the fact that Matlab-Simulink enables to work with complex signal, it is possible to work with matrix representation of equations describing behaviour of model instead of creating differential equations for each coordinates $\mathbf{x}$ and $\mathbf{y}$. It is only necessary to separate signal to real and imaginary part for operation which are not defined for complex signal, like integration, see Fig. 2.

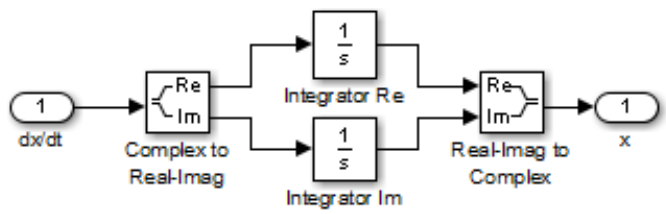

Fig. 2. Integrator calculation for complex signals.

\section{REFERENCES}

Bently, D.E. and Muszynska, A. (1989) Fluid-generated Instabilities of Rotors, Orbit, Volume 10, No. I, April, 1989.

Mendes, R.U. and Cavalca, K. L. (2014) On the Instability Threshold of Journal Bearing Supported Rotors. International Journal of Rotating Machinery. Vol. 2014, Article ID 351261. 17 pp.

Muszynska, A. (1986) Whirl and Whip - Rotor / Bearing Stability Problems. Journal of Sound and Vibration (1986) 110(3), pp 443-462.

Muszynska, A. (2005) Rotordynamics, Taylor \& Francis Group, New York 2005, ISBN 0-8247-2399-6.

Svoboda, R. (2007) Theoretical analysis of influence of bearing bushing external excitation on dynamic properties of the rotor, Technical report TECHLAB No. 07-301 (in Czech).

Tůma, J., Šimek, J. Škuta, J. and Los, J. (2013) Active vibrations control of journal bearings with the use of piezoactuators, In Mechanical Systems and Signal Processing, Vol. 36, 2013, pp. 618-629. 


\title{
MATHMOD 2015 ABSTRACT VOLUME
}

\author{
MATHMOD Full Contributions \\ Electrical Systems
}


MATHMOD 2015 Abstract Volume 


\title{
Magnetic Equivalent Circuit Modeling of a Saturated Surface-Mounted Permanent Magnet Synchronous Machine
}

\author{
David Faustner, Wolfgang Kemmetmüller, and Andreas Kugi \\ Automation and Control Institute, Vienna University of Technology, \\ Gußhausstraße 27-29, 1040 Vienna, Austria (e-mail: \\ \{faustner,kemmetmueller,kugi\}@acin.tuwien.ac.at)
}

Many industrial applications require high performance electrical drive systems. Among three-phase ac machines, permanent magnet synchronous machines (PMSMs) are very popular. The application of rare-earth permanent magnets results in high torque-to-current ratios, whereas the absent rotor windings reduce the overall construction size and improve the energy efficiency. Mathematical modeling of PMSM is still a challenging task and comprises finite element (FE) analysis, magnetic equivalent circuit (MEC) models and analytical models.

Appropriate models have to account for inhomogeneous geometries typically occurring in interior permanent magnet synchronous machines (IPMSMs) and the nonlinear behavior of the iron core material due to saturation. FE models can accurately describe this behavior at expense of high numeric complexity, see, e.g., Tomczuk et al. (2007). The controller design for PMSM is mainly based on analytical models, in particular on fundamental wave models, where homogeneous air gap geometries and a linear material behavior are assumed, cf., e.g., Morel et al. (2008). The MEC modeling approach gives a compromise between model accuracy and model complexity, and is therefore of interest for the development of dynamical simulation models and for the controller design, see, e.g., Ostovic (1989), Bash et al. (2010).

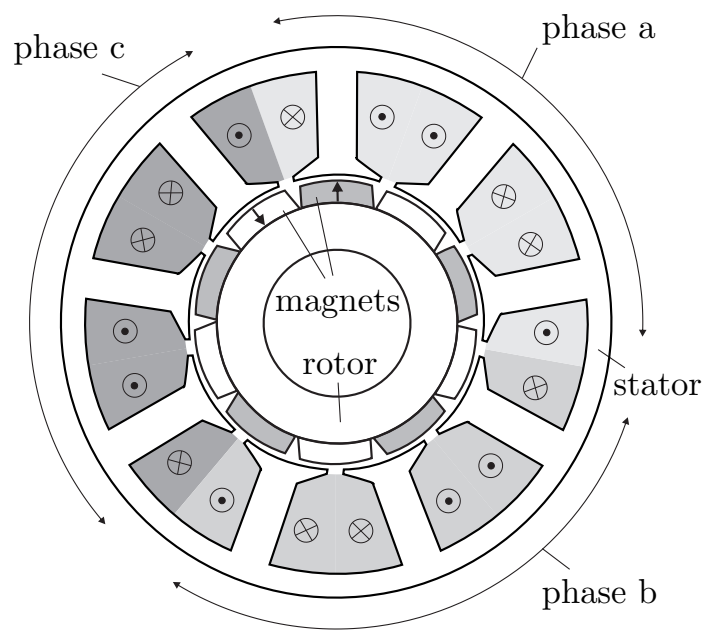

Fig. 1. Cross sectional view of the PMSM.

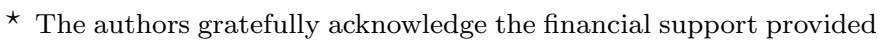
by Bernecker \& Rainer Industrie Elektronik GmbH.
}

This paper presents an MEC model of a surface-mounted permanent magnet synchronous machine (SPMSM) which shows significant magnetic saturation in overload operation, see Fig. 1. Thus, classical linear fundamental wave models are not capable to describe the behavior of the motor in the whole operation range. Starting with a comprehensive permeance network, a minimal set of (nonlinear) equations that describe the static magnetic behavior of the permeance network is derived based on the framework published in Kemmetmüller et al. (2014). This framework is based on network theory which allows a systematical derivation of the corresponding equations. The static magnetic model is extended to account for the flux dynamics corresponding to Faraday's induction law, and by equations which describe the electrical interconnection of the coils of the motor. This results in a state-space model with a minimum number of nonlinear equations and state variables. It is further shown that the model can be calibrated with measurment data, adjusting only the air gap permeances of the permeance network. The accuracy of the proposed model in the whole operation range of the motor is proven by comparisons with measurement results of an industrial test-stand.

\section{REFERENCES}

Bash, M., Williams, J., and Pekarek, S. (2010). Incorporating motion in mesh-based magnetic equivalent circuits. IEEE Trans. Energy Convers., 25(2), 329-338.

Kemmetmüller, W., Faustner, D., and Kugi, A. (2014). Modeling of a permanent magnet synchronous machine with internal magnets using magnetic equivalent circuits. IEEE Trans. Magn., 50(6), 1-14. doi: 10.1109/TMAG.2014.2299238.

Morel, F., Retif, J.M., Lin-Shi, X., and Valentin, C. (2008). Permanent magnet synchronous machine hybrid torque control. IEEE Trans. Ind. Electron., 55(2), 501-511.

Ostovic, V. (1989). Dynamics of Saturated Electric Machines. Springer-Verlag, New York, NY, USA.

Tomczuk, B., Schröder, G., and Waindok, A. (2007). Finite-element analysis of the magnetic field and electromechanical parameters calculation for a slotted permanent-magnet tubular linear motor. IEEE Trans. Magn., 43(7), 3229-3236. 


\title{
Some Model Properties to Control a Permanent Magnet Machine Using a Controlled Invariant Subspace
}

\author{
Paolo Mercorelli* Oleg Sergiyenko** \\ Daniel Hernandez-Balbuena ${ }^{* * *}$ Julio Rodriguez-Quiñonez ${ }^{* * *}$ \\ Wendy Flores-Fuentes ${ }^{* * *}$ Luis Basaca-Preciado** \\ * Institute of Product and Process Innovation \\ Leuphana University of Lueneburg \\ Volgershall 1, D-21339 Lueneburg, Germany \\ Phone: +49-(0)4131-677-5571 \\ Fax: +49-(0)4131-677-5300 \\ Email: mercorelli@uni.leuphana.de \\ ** Applied Physics Department of Engineering Institute \\ Baja California Autonomous University \\ Benito Juárez S/N, 21100 Mexicali, Mexico \\ Phone: +52 6868418200 \\ Email:srgnk@iing.mxl.uabc.mx \\ *** Engineering Faculty of $U A B C$ \\ Baja California Autonomous University \\ Benito Juárez S/N, 21100 Mexicali, Mexico \\ Phone: +52 6868418200 \\ Email:dhernan@uabc.edu.mx,wendy.flores6@uabc.edu.mx \\ julio.rodriguez81@uabc.edu.mx, luis.basaca@cetys.mx
}

\begin{abstract}
In permanent magnetic machines the nonlinearity due to the quadratic terms of the current makes difficulties in the control system. In order to cancel the nonlinearity, a controller based on a controlled invariant subspace for the currents is proposed. Simulation results using real data measurements are reported to validate the proposed technique.
\end{abstract}

Keywords: Modelling, permanent magnets motor, control.

\footnotetext{
* The authors would like to thank Kai Lehmann for the original contribution to this work at the time of the work in IAI (Institute of Automation and Informatics) in Wernigerode, Germany.
} 


\title{
Availability assessment of electric power based on switch reliability modelling with dynamic Bayesian networks: case study of electrical distribution network
}

\author{
A. Lakehal*. Z. Ghemari** \\ * Department of Mechanical Engineering, Mohamed Chérif Messaadia University, P.O. Box 1553, Souk-Ahras, 41000, \\ Algeria (Tel: 213 549020274; e-mail lakehal21@yahoo.fr) \\ ** Electrical Engineering Department, University of M'sila, M'sila, Algeria (e-mail: ghemari-zine@live.fr)
}

\begin{abstract}
As a generalization of the successful hidden Markov models, Dynamic Bayesian Networks (DBNs) are a natural basis for the general temporal action interpretation task. This document provides a conditional probabilistic approach to analyze the energy availability in electrical distribution networks by using Bayesian networks (BN). Firstly a static BN modelling is presented to show the influence of the switch behaviour on the energy availability. Then, the dynamic behaviour of the switch is cared by switch reliability modelling using DBN which permits to predict the energy availability.

The prediction by DBNs discussed in the case study of this paper gives a strong contribution on electrical network supervisory control and it can also be applied to transportation networks.

Keywords: Bayesian network; dynamic Bayesian network, switch reliability; electric power, availability prediction
\end{abstract}




\title{
Modelling, Simulation and Identification of Heat Loss Mechanisms for Parabolic Trough Receivers Installed in Concentrated Solar Power Plants
}

\author{
Simon Caron*, Marc Röger* \\ *German Aerospace Center (DLR), Institute for Solar Research, Plataforma Solar de Almería \\ Tabernas 04200, Spain (Tel: +34 950278 863; e-mail: simon.caron@dlr.de)
}

\section{INTRODUCTION}

Parabolic Trough Receivers (PTRs) represent one of the key components in Concentrated Solar Power (CSP) plants, as their thermal performance significantly influences the solar field operating temperature and thus the power plant overall thermal efficiency. The development of an accurate in-situ receiver heat loss measurement method requires a numerical heat transfer model to separate heat loss mechanisms.

Several receiver heat transfer models have been published in the literature. Two-dimensional heat transfer models based on thermal resistance networks have been implemented elsewhere with Engineering Equation Solver (EES) and validated under steady-state conditions. More detailed threedimensional models combining Finite Element Method (FEM), Computational Fluid Dynamics (CFD), and Monte Carlo Ray-Tracing (MCRT) have also been implemented with ANSYS and these models are more suitable for sun irradiated receivers.

This paper describes a three-dimensional receiver model library which has been extended from two-dimensional models based on thermal resistance networks. This thermodynamical model has been implemented with the object-oriented programming language Modelica, designed for modelling complex physical systems. Steady-state and transient simulations have been performed for single receivers within the Dymola simulation environment.

This receiver model has been validated for steady-state temperature conditions and has been coupled to a derivativefree hybrid optimization routine developed in Matlab in order to identify receiver heat loss mechanisms on the basis of transient measurements. The optimization routine combines a Particle Swarm Optimization (PSO) algorithm and a NelderMead Simplex (NMS) optimization algorithm including search space boundary constraints.

\section{IDENTIFICATION RESULTS}

Parabolic trough receivers with distinct absorber coating and annulus properties have been tested with two different transient excitation profiles, i.e. sinusoidal and ramp-andhold signals.

The identification of PTR key thermal properties was carried out for laboratory sinusoidal measurements and documented for three different receiver categories. Specific heat loss experimental results are shown in Fig. 1 and compared both with steady-state measurements and simulations based on material data.
The observed specific heat loss deviations between steadystate and transient laboratory measurements respectively range from $9 \%$ to $27 \%$ for evacuated receivers with selective coating and from $1 \%$ to $9 \%$ for receivers with black painted absorbers.

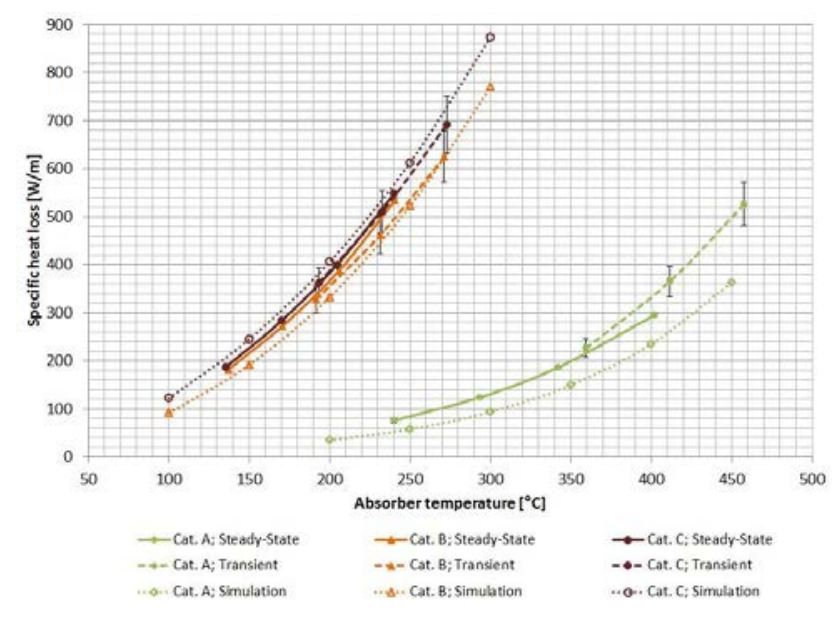

Fig. 1: Comparison between steady-state specific heat loss measurements (Steady-State), transient derived specific heat losses (Transient) and simulates specific heat losses based on material data (Simulation).

\section{CONCLUSION}

The object-oriented model offers great flexibility and heat transfer models can be further refined to capture detailed dynamic heat transfer characteristics.

The optimization criterium and objective function could be simplified further to allow a more direct identification of receiver thermal properties for noisy datasets gained during field measurements. This new procedure is being implemented for noisy datasets where the derivation of transient measurements is less trivial.

\section{REFERENCES}

Caron, S., Röger, M., Pernpeintner, J. (2014), Transient Infrared Thermography Heat Loss Measurements on Parabolic Trough Receivers Under Laboratory Conditions, Paper presented at the SolarPACES 2014 Conference, 16-19 September 2014, Beijing, China.

Röger, M., Potzel, P., Pernpeintner, J., Caron, S. (2014), A Transient Thermography Method to Separate Heat Loss Mechanisms in Parabolic Trough Receivers, ASME Journal of Solar Energy Engineering, Vol. (136), pp.011006-1:011006-9. 


\title{
Metropolis Monte Carlo based Relaxation of Atomistic III-V Semiconductor Models
}

\author{
Hermann Detz* Gottfried Strasser ** \\ * Center for Micro- and Nanostructures, Vienna University of \\ Technology, Vienna, 1040, Austria, (e-mail: \\ hermann.detz@tuwien.ac.at). \\ ** Center for Micro- and Nanostructures, Vienna University of \\ Technology, Vienna, 1040, Austria.
}

We present atomistic simulations of III-V semiconductor materials, which provide an effective way to investigate structural properties at a nanometric scale. Atomistic models cover the range between ab-initio density functional theory methods and continuum-based models. Chemical bonds are described through simplified interaction potentials, while the crystalline structure of the material is preserved. This combination can provide an in-depth description of strain or roughness at heterojunctions, which both allow the optimization of optoelectronic intersubband devices C. Deutsch et al. (2013).

The simulations, described in this work, are performed in initially ideal zincblende lattice cubes with an edge length of six unit cells, as shown in the inset in figure 1. Periodic boundaries in all three dimensions prevent finite size effects. The crystal structure is relaxed using a Metropolis Monte Carlo scheme H. Detz and G. Strasser (2013b). Possible operations are the displacement of a single random atom or a scaling of the whole simulation box. Tersoff potentials are readily available for all binary III-V compounds D. Powell et al. (2007). Although the potentials were optimized for binary compounds, this model was also found to reproduce bulk and shear modulus of ternary alloys over the whole composition range with maximum errors around 5 and $10 \%$, respectively.

Simulations on random alloys led to a compositondependent bimodal bond length distribution, as plotted in figure 1. This is stemming from the constituent binary compounds, which was also observed experimentally. In contrast to this, CuPt-ordered structures, which can form spontaneously, were shown to exhibit a distinct fourfold bond length pattern H. Detz and G. Strasser (2013a). These bond deformations lead to built-in strain up to $1.8 \%$ in the case of $\mathrm{GaAs}_{0.5} \mathrm{Sb}_{0.5}$.

The Metropolis Monte Carlo approach also allows modelling at higher temperatures. The asymmetry of the empirical potential function results in thermal expansion. Calculated thermal expansion coefficients are of the correct order of magnitude, although they are in general overestimated due to the missing Coulomb interaction, which is characteristic of III-V materials.

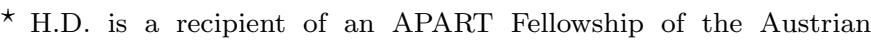
Academy of Sciences. The authors furthermore acknowledge financial support by the Austrian Science Fund (FWF): P26100-N27 (H2N) and F2403-N17 (IRON).
}

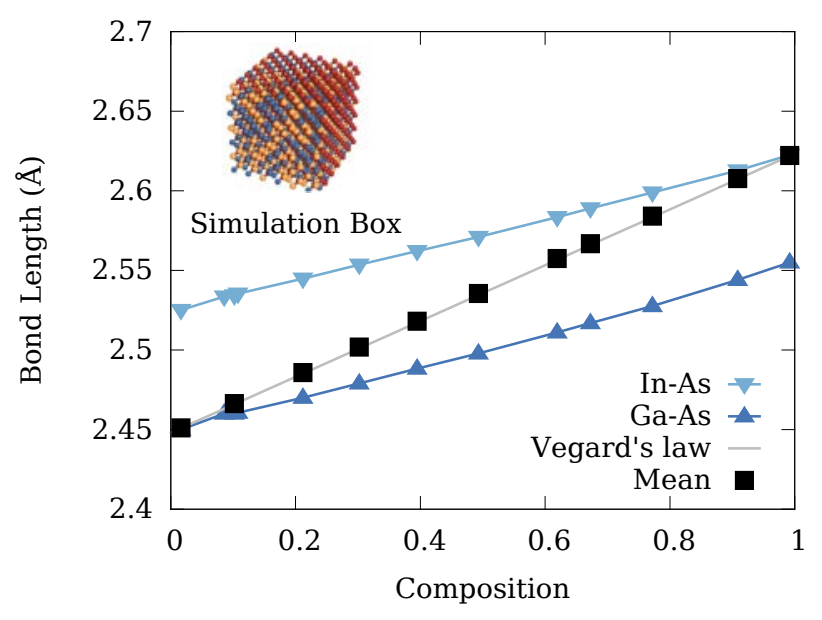

Fig. 1. Bond lengths in $\operatorname{In}_{x} \mathrm{Ga}_{1-\mathrm{x}} \mathrm{As}$ versus In content. The model reproduces the experimentally obtained bi-modal distribution, which on average agrees with Vegard's law. The inset shows the simulation box.

In conclusion, atomistic models based on empirical interaction potentials are shown as aviable way to model structural properties of III-V semiconductor alloys. The elastic behavior of ternary alloys is reproduced with reasonable errors. Furthermore, the thermal expansion coefficients agree by orders of magnitude but will require modifications to the potential functions for better accuracy.

\section{REFERENCES}

C. Deutsch, H. Detz, T. Zederbauer, A.M. Andrews, P. Klang, T. Kubis, G. Klimeck, M.E. Schuster, W. Schrenk, G. Strasser, and K. Unterrainer (2013). Probing scattering mechanisms with symmetric quantum cascade lasers. Optics Express, 21(6), 7209-7215.

D. Powell, M.A. Migliorato, and A.G. Cullis (2007). Optimized tersoff potential parameters for tetrahedrally bonded III-V semiconductors. Phys. Rev. B, 75(11), 115202.

H. Detz and G. Strasser (2013a). Atomistic modeling of bond lengths in random and ordered III-V alloys. J. Appl. Phys., 114(12), 123508.

H. Detz and G. Strasser (2013b). Modeling the elastic properties of the ternary III-V alloys InGaAs, InAlAs and GaAsSb using Tersoff potentials for binary compounds. Semicond. Sci. Technol., 28(8), 085011. 


\title{
Correlated Noise Description Using HDLs
}

\author{
Joachim Haase and André Lange \\ Fraunhofer IIS/EAS, Dresden, Germany \\ e-mail: (joachim.haase| andre.lange)@eas.iis.fraunhofer.de
}

\section{INTRODUCTION}

When a description in the small-signal frequency-domain is available, the standard approach for the analysis of electrical networks that is well-known and widely used in Spice-like simulation engines is to translate the frequencydomain descriptions into linear lumped element circuits or state space equations. This procedure may not be simple and is often inaccurate, in particular when frequencydomain characteristics based on measurements are used.

We describe how an arbitrary frequency-domain behaviour can be described using a system task that makes the current frequency available. This approach is in particular useful when the behaviour at higher frequencies is of interest. Such problems occur when investigating the EMC behaviour. This approach also allows for handling small-signal noise straightforwardly. Furthermore, we can establish a general approach to handle correlated noise sources. This is important in modelling the noise behaviour in systems (Domizioli et al., 2010) and the noise of transistors at higher frequencies (McAndrew et al., 2005).

We show how to make use of an extended small-signal modelling approach to describe any small-signal behaviour, in particular correlated noise sources, using hardware description languages (HDLs) such as the VerilogAMS and VHDL-AMS behavioural modelling languages.

\section{EXTENDED SMALL-SIGNAL FREQUENCY ANALYSIS}

By application of a function that provides access to the frequency $f$, we can apply an approach to extend frequencydomain analysis as described by Haase et al. (2009) for VHDL-AMS. We briefly sketch the main idea. The following relation is given in the frequency-domain

$$
Y(f)=(a(f)+j \cdot b(f)) \cdot X(f),
$$

where $j$ is the imaginary unit, $f$ is the frequency, $X(f)$ and $Y(f)$ are the input and the output resp. in the frequencydomain of an element described by the complex scalar transfer characteristic $a(f)+j \cdot b(f)$. The equation is equivalent to

$$
Y(f)=a(f) \cdot X(f)+b s(f) \cdot j \omega \cdot X(f)
$$

with $\omega=2 \pi f$. The coefficient $b s(f)$ equals $\frac{b(f)}{2 \pi f}$ for frequencies $f>0$. It can be set to zero for the frequency $f=0$. During a DC or transient analysis, $f$ equals zero.

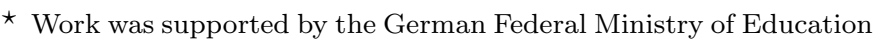
and Research (BMBF, Funding number: 16N12440).
}

When FREQUENCY provides the current frequency in a simultaneous statement, then the following VHDL-AMS description in the frequency-domain is equivalent to equation (2)

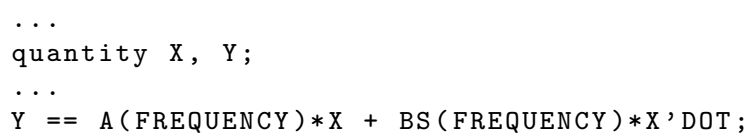

where X'DOT describes the derivative of X w.r.t. time.

\section{HANDLING CORRELATED NOISE SOURCES}

Correlated noise sources are defined by a power spectral density matrix $\mathbf{S}_{\mathbf{x x}}(f)$. Since every correct power spectral density matrix is a complex positive semi-definite Hermitian, a diagonalisation is always possible as follows:

$$
\mathbf{S}_{\mathbf{x x}}(f)=\mathbf{H}(f) \cdot \boldsymbol{\Lambda}(f) \cdot \overline{\mathbf{H}}(f)^{T}
$$

Therefore, correlated noise sources can be described using uncorrelated white noise sources, amplifications, and a linear transfer matrix $\mathbf{H}(f)$ that can be expressed as shown in the previous section. The subsequent figure demonstrates the principle.

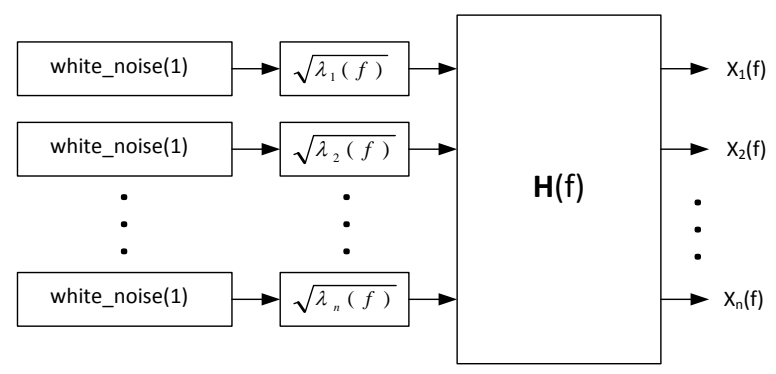

Consequences for the standardisation of behavioural modelling languages are discussed in the full paper.

\section{REFERENCES}

Domizioli, C.P., Hughes, B.L., Gard, K.G., and Lazzi, G. (2010). Noise Correlation in Compact Diversity Receivers. IEEE Trans. Commun., 58(5), 1426-1436.

Haase, J., Hessel, E., and Mammen, H.T. (2009). Proposal to Extend Frequency Domain Analysis in VHDL-AMS. In Proc. Forum on specification Design Languages (FDL 2009), 1-4.

McAndrew, C.C., Coram, G., Blaum, A., and Pilloud, O. (2005). Correlated Noise Modeling and Simulation. In Technical Proceedings of the 2005 NSTI Nanotechnology Conference and Trade Show (Nanotech). 


\title{
Pull-in range of the classical PLL with impulse signals
}

\author{
Konstantin D. Alexandrov ${ }^{\star}$ Nikolay V. Kuznetsov * \\ Gennady A. Leonov ${ }^{\star}$ Pekka Neittaanmäki ${ }^{\star \star}$ \\ Svetlana M. Seledzhi ${ }^{\star}$ \\ Saint-Petersburg State University, Universitetsky pr. 28, \\ Saint-Petersburg, 198504, Russia \\ Dept. of Mathematical Information Technology, University of \\ Jyväskylä, P.O. Box 35 (Agora), FIN-40014, Finland
}

\begin{abstract}
In the present work the pull-in range of the classical PLL with impulse signals is studied. Numerical experiments in MatLab Simulink are presented and two analytical approaches are discussed.
\end{abstract}

Keywords: phase-locked loop, nonlinear analysis, PLL, Best's conjecture, hidden oscillations

\section{INTRODUCTION}

The PLL circuit has been invented by French engineer Henri de Bellescize in 1932. Since then, various modifications of PLL circuits (digital PLL, all-digital PLL, software PLL, neuronal PLL and so on) were developed and new circuits, based on PLL operation principles, were invented (e.g., Costas loop and PLL with squarer). PLLbased circuits are used for carrier recovery, demodulation, frequency synthesis in telecommunications.

Rigorous analysis of PLL-based circuits is a complicated problem, since the consideration of these circuits leads to a system of nonautonomous differential equations. Numerical simulation of PLL-based circuits is also a challenging task since it is necessary to consider simultaneously both high-frequency and low-frequency oscillations.

One important problem in the study of PLL based circuits is to find a pull-in range. There are two effective analytical approaches for the pull-in range estimation are developed. The first one is based on the integration of separatrices and the analysis of their behavior (see pioneering works (Tricomi, 1933; Andronov, 1937; Gubar', 1961)). The second one is based on the frequency methods and Lyapunov function constructions (Lyapunov, 1892; Gelig et al., 1978; Leonov and Kuznetsov, 2014).

In the present paper the problem of the pull-in range estimation is discussed. Simulation and two effective analytical approaches for the pull-in range estimations are discussed. The one of the analytical approaches is based on the integration of separatrices and the analysis of their behavior, while another one is based on the frequency methods and Lyapunov function constructions. One of the problems of the first method is that for any new PD characteristic one has to do a lot of new cumbersome inte-

\footnotetext{
* This work was supported by Saint-Petersburg State University (project 6.39.416.2014, s. 3-4; project 6.38.505.2014, s. 5.)

${ }^{\star \star}$ The author acknowledge the support of Russian Scientific Foundation (project 14-21-00041).
}

grations (as it is demonstrated above). Another problem is that while this approach is useful for the two-dimensional models, its generalization for a multidimensional models is a challenging task. The advantage of this approach is that it allows to estimate also the lock-in range corresponding to a domain of the phase space where the loop achieves a lock without cycle slips. If the aim is to study the pull-in range only then to avoid cumbersome integrations one can use the approach based on Lyapunov function construction for the cylindrical phase space. For the model considered in this paper it is possible to construct Lyapunov function of the type "quadratic form plus the integral of nonlinearity" (see, e.g., (Gelig et al., 1978; ?)). The advantage of this approach is that it allows to consider various PD characteristics at once.

\section{REFERENCES}

Andronov, A.A. (1937). Oscillations.

Gelig, A., Leonov, G., and Yakubovich, V. (1978). Stability of Nonlinear Systems with Nonunique Equilibrium (in Russian). Nauka.

Gubar', N.A. (1961). Investigation of a piecewise linear dynamical system with three parameters. J. Appl. Math. Mech., 25(6), 1011-1023.

Leonov, G.A. and Kuznetsov, N.V. (2014). Nonlinear Mathematical Models Of Phase-Locked Loops. Stability and Oscillations. Cambridge Scientific Press.

Lyapunov, A.M. (1892). The General Problem of the Stability of Motion. Kharkov.

Tricomi, F. (1933). Integrazione di unequazione differenziale presentatasi in elettrotechnica. Annali della $R$. Shcuola Normale Superiore di Pisa, 2(2), 1-20. 


\title{
Simulation of the classical analog phase-locked loop based circuits
}

\author{
N.V. Kuznetsov, G.A. Leonov, M.V. Yuldashev, \\ R.V. Yuldashev \\ Saint-Petersburg State University, Russia \\ Dept. of Mathematical Information Technology, University of \\ Jyväskylä, Finland
}

\begin{abstract}
In this paper a short survey on two approaches to simulation of classical phase-locked loop based circuits (PLLs) is provided. Nonlinear models of PLLs with mixer/multiplier phase detector are considered. Computation of phase detector characteristics for non-harmonic and non-squarewave signals is discussed.
\end{abstract}

Keywords: phase-locked loop, Costas loop, simulation, nonlinear analysis, hidden oscillations

\section{INTRODUCTION}

The Phase-Locked Loop (PLL) circuits were invented at the beginning of twentieth century and widely employed in the radio and television (the demodulation and carrier recovery, the synchronization, and synthesis of frequencies). At present it can be found various types of PLL circuits (analog, analog-digital, digital, software), designed for the operation with different types of signals (sinusoidal, impulse and so on). After PLL realization in form of single chip, PLL started to be widely used in the telecommunication and computer architectures. At present various PLL circuits are also applied to different mechanical devices, location and navigational systems.

For the sake of convenience of the description of PLL operation, it is typically divided into the following blocks: phase detector (PD), low-pass loop filter (LPF), and voltage controllable oscillator (VCO, tunable generator). Such a separation into functional blocks often turns out conditional since in a certain physical realization of PLL it is often impossible to find correct boundaries between these blocks. PLL operation consists in automatic tuning of a phase (frequency) of VCO signal to a phase (frequency) of reference (master) generator signal. The reference generator is usually highly stable and a frequency of its signal is assumed to be constant. The signals from tunable and reference generators enter PD - a nonlinear element, which output includes error signal corresponding to the phase difference of input signals.

To separate high-frequency parasitic component (if such component exists in the loop) from misphasing signal, the signal at the output of PD passes through LPF. A misphasing signal, obtained at the output of filter, is used for the frequency control of tunable generator, the output of which is connected with the control input of the phase detector, providing a negative feedback. After acquisition process the synchronization is achieved, at the output of filter a constant signal is established. This signal corresponds to the frequency deviation of generators Engineers study of stability of the required operating modes, the estimation of attraction domains of these operating modes, and the estimation of the transient time. Various mathematical models of PLL and their numerical-analytical analysis is widely used. To describe operation of PLL it is necessary to consider nonlinear element (PD) operation and the passage from the signal space to signal's phase space and back. Thus, the rigorous mathematical analysis of PLL requires the construction of rigorous nonlinear mathematical models of PLL in the signal space and signal's phase space and justification of the passage between these models. In spite of this, as was remarked by D. Abramovich in his lecture at the American Control Conference 2002, the main direction in modern literature, devoted to the analysis of stability and synthesis of PLL, is the use of simplified linear models, empirical rules, and simulation. However, it is well known that the application of the methods of linearization and linear analysis without rigorous mathematical justification can lead to wrong conclusions.

Precise simulation of the PLL models in the signal space is a challenging task because of the nonlinearity of PD and the high frequencies of the signals: usually a simulation step, which must be sufficiently small to distinctly observe the dynamics of phase detector, makes observation of the dynamics of the whole system a difficult task. Following the classical pioneering books by F.Gardner and A.Viterbi, nowadays to overcame this problem the consideration of the PLL models in signal's phase space is widely used, but it requires (as was remarked above) the construction of the corresponding models of PLL and the rigorous justification of the passage to them and also can lead to wrong conclusion. E.g, in 1961 N. Gubar showed analytically the possibility of the existence of hidden oscillations in twodimensional model of PLL: form a computational point of view in the considered system all the trajectories tend to equilibrium, but, in fact, the domain of attraction of stationary set is bounded. The bifurcations, the study of which requires the development and application of special analytical methods, can also be observed even in the simplest discrete one-dimensional models of PLL. 


\title{
MATHMOD 2015 ABSTRACT VOLUME
}

\author{
MATHMOD Full Contributions \\ Identification
}


MATHMOD 2015 Abstract Volume 


\title{
Identification of Wiener Time Delay Systems Based on Hierarchical Gradient Approach
}

\author{
Asma ATITAlLAH, Saïda Bedoui, Kamel Abderrahim \\ National Engineering school of Gabes \\ University of Gabes, Gabes 6029, Tunisia \\ (e-mail: atitallah.asma@yahoo.fr, saida.bedoui@enig.rnu.tn, \\ kamelabderrahim@yahoo.fr).
}

\begin{abstract}
In this paper, we propose a hierarchical gradient based algorithm to identify simultaneously the parameters of the linear and nonlinear blocks and the time delay of Wiener time delay systems. We separate the nonlinear cost function into two cost functions and present the gradient algorithm for estimating directly the time delay and the parameters. Furthermore, we give an appropriate choice of convergence factor. Numerical simulation results are presented to demonstrate the performance of the proposed method.
\end{abstract}

Keywords: Identification, Nonlinear systems, Wiener systems, Time Delay, Parameter estimation, Hierarchical approach. 


\title{
Towards the Possibility of Applying the Wavelet Analysis to Derive Predicting Models
}

\author{
Ekaterina Sakrutina and Natalia Bakhtadze
}

V.A. Trapeznikov Institute of Control Sciences, 65 Profsoyuznaya, Moscow 117997, Russia (e-mail:bahfone@ipu.ru)

\begin{abstract}
Identification models find out a wide application in modern control systems, decision support systems, play a fundamental role in any activity concerned with the signal processing process. Methods based on the wavelet analysis are characterized with a unique possibility to select «frequency-domain windows». The paper presents methods of identification of non-linear systems, developed by the authors, by using the multi-scale transformation.
\end{abstract}

Keywords: wavelets, system identification, predicting models, associative search. 


\title{
A Nonparametric Measure of Dependence in the Statistical Linearization
}

\author{
Kirill Chernyshov and Ekaterina Sakrutina
}

V.A. Trapeznikov Institute of Control Sciences, 65 Profsoyuznaya, Moscow 117997, Russia (e-mail:myau@ipu.ru)

\begin{abstract}
The paper presents an approach to the statistical linearization of the input/output mapping of non-linear discrete-time stochastic systems driven by a white-noise Gaussian process. The approach is based on applying the contingency coefficient, a nonparametric measure of dependence. Within such an approach, the statistical linearization criterion is the condition of coincidence of the mathematical expectations of the output processes of the system under study and the derived model and the condition of coincidence of the contingency coefficient of the input and output processes of the system and the contingency coefficient of the input and output processes of the model. As a result, explicit analytical expressions to derive coefficients of the weight function of the target linearized model are obtained. The consideration is preceded with an analysis of applying consistent measures of dependence within the system identification.
\end{abstract}

Keywords: consistent measures of dependence, input/output model, contingency coefficient, statistical linearization, system identification. 


\title{
Determination of Leaky Confined Aquifer Parameters by a Multilayer Perceptron Neural Network
}

\author{
Tahereh Azari *. Nozar Samani ** \\ Eghbal Mansoori *** \\ * Department of Earth Sciences, Shiraz University, Shiraz 71454, Iran \\ (e-mail:azari.azadeh@yahoo.com) \\ ** Department of Earth Sciences, Shiraz University, Shiraz 71454, Iran \\ (e-mail: samani@susc.ac.ir) \\ *** School of Engineering, Shiraz University, Shiraz, Iran \\ (e-mail: mansoori@shirazu.ac.ir)
}

\begin{abstract}
In recent years, the artificial neural networks (ANNs) are used as an alternative to the conventional type curve matching techniques for the determination of aquifer parameters. In this paper a multilayer perceptron network (MLPN) is developed for the determination of leaky confined aquifer parameters. Leakage into the aquifer takes place from the storage in the confining aquitard. The network is trained for the well function of leaky confined aquifers. By applying the principal component analysis (PCA) on the adopted training data sets, the topology of the network is reduced and its efficiency increased considerably. The topology of developed network is fixed to $(2 \times 10 \times 2)$ regardless of number of records in the pumping test data. The network generates the match point coordinates for any individual pumping test data set. The match point coordinates are incorporated with Hantush (1960) analytical solution and the aquifer parameter values are determined. The performance of the MLPN is evaluated by real field data and its accuracy is compared with that of the type curve matching technique. The proposed MLPN is recommended as a simple and reliable alternative to the type-curve matching technique.
\end{abstract}




\title{
MATHMOD 2015 ABSTRACT VOLUME
}

\author{
MATHMOD Full Contributions \\ Control Systems
}


MATHMOD 2015 Abstract Volume 


\title{
Designing model and control system using evolutionary algorithms
}

\author{
Marko Corn*. \\ Maja Atanasijević-Kunc** \\ * INEA d.o.o., Ljubljana, Slovenia,(Tel: +386-15138110; e-mail: marko.corn@inea.si). \\ ** Faculty of electrical engineering, University of Ljubljana, Ljubljana, \\ Slovenia (e-mail: maja.atanasijevic-kunc@fe.uni-lj.si)
}

In the paper several types of evolutionary algorithms have been tested regarding the dynamic nonlinear multivariable system model. We have defined three problems regarding the observed system: the first is the so-called grey box identification where we search for the characteristic of the system's valve, the second problem is black box identification where we search the model of the system with the usage of system's measurements and the third one is a system's controller design. We solved these problems with the usage of genetic algorithms, differential evolution, evolutionary strategies, genetic programming and a developed approach called AMEBA algorithm. All methods have been proven to be very useful for solving problems of the grey box identification and design of the controller for the mentioned system but AMEBA algorithm have also been successfully used in black box identification problem where it generated a suitable model.

Atanasijević-Kunc, M. (2005). Multivariable systems, Collection of complex problems (in Slovene) $\left(4^{\text {th }}\right.$ ed., p. 265). Ljubljana, Slovenia: Faculty of Electrical Engineering, University of Ljubljana.

Atanasijević-Kunc, M., Belič, A., \& Karba, R. (2006). Optimal multivariable control design using genetic algorithms. In $5^{\text {th }}$ Vienna Symposium on Mathematical Modeling. Vienna University of Technology.

Beyer, H. G. (2010). The Theory of Evolution Strategies (Natural Computing Series) (p. 400). Springer. Retrieved from http://www.amazon.com/TheoryEvolution-Strategies-NaturalComputing/dp/3642086705

Brownlee, J. (2011). Clever Algorithms: Nature-Inspired Programming Recipes. Search. Swinburne University in Melbourne, Australia.

Corn, M., \& Atanasijević-Kunc, M. (2011). Cell based Genetic Programming Toolbox. In Proceedings of the $20^{\text {th }}$ ERK 2011 Conference (pp. 295-298). Portorož, Slovenia.

Corn, M., \& Černe, G. (2012). A Graph-Based Evolutionary Algorithm : Cell Based Genetic Programming. In Proceedings of the Fifth International Conference on
Bioinspired Optimization Methods and their Applications, BIOMA 2012 (pp. 163-172).

Corn, M., Černe, G., \& Atanasijević-Kunc, M. (2012). Balance Group Model with Smart Grid Elements. In 7th Vienna Conference on Mathematical Modeling: MathMod 2012 (p. 353). Vienna, February 15.18.2012.: Vienna University of Technology.

David Goldberg. (1989). Genetic Algorithms in Search, Optimization and Machine Learning (1st ed.). Massachusetts: Addison Wesley.

Fogel, L. J., Owens, A. J., \& Walsh, M. J. (1966). Artificial Intelligence through Simulated Evolution. John Wiley.

Koza, J. R. (1992). Genetic Programming On the Programming of Computers by Means of Natural Selection (6th ed.). Cambridge, Massachusetts, London, England: MIT Press.

Logar, V., Dovžan, D., Škrjanc, I. (2011). Mathematical Modeling and Experimental Validation of an Electric Arc Furnace. ISIJ International, 51(3), 382-391. doi:10.2355/isijinternational.51.382

Storn, R., \& Price, K. (1997). Differential Evolution - A Simple and Efficient Heuristic for Global Optimization over Continuous Spaces. Journal of Global Optimization, 11, 341-359.

Tan, K. C., \& Li, Y. (2002). Grey-box model identification via evolutionary computing. Control Engineering Practice, 10(7), 673-684. doi:10.1016/S09670661(02)00031-X

Tomažič, S., Logar, V., Kristl, Ž., Krainer, A., Škrjanc, I., \& Košir, M. (2013). Indoor-environment simulator for control design purposes. Building and Environment, 70(0), 60-72. doi:http://dx.doi.org/10.1016/j.buildenv.2013.08.026

Whigham, P. A. (1992). Grammatically-based Genetic Programming. In Workshop on Genetic Programming. From Theory to Real-World Applications (pp. 33-41). Tahoe City, California: Departent of Computer Science, University College, University of New South Vales. 


\title{
Proportional-Integral-Observer: A brief survey with special attention to the actual methods using ACC Benchmark
}

\author{
F. Bakhshande* D. Söffker* \\ * Chair of Dynamics and Control \\ University of Duisburg-Essen, Duisburg, Germany \\ (e-mail: \{fateme.bakhshande,soeffker\}@uni-due.de).
}

\section{ABSTRACT}

This paper gives a brief survey of linear and nonlinear Proportional-Integral-Observer design. This observer has the ability to estimate simultaneously the states and unknown inputs such as disturbances or model uncertainties acting on the system. In literature different system types have been considered for designing PI-Observer which are introduced briefly in this survey. A general formulation of linear and nonlinear systems in the combination with PIObserver is proposed. Several linear and nonlinear design methods have been briefly introduced. Furthermore design methods of Advanced PI-observer and Modified Advanced PI-Observer are presented. Both approaches are based on the PI-Observer but using adaptive gain scheduling approach. Simulation results using American Control Conference (ACC) benchmark are given to evaluate Modified Advanced PI-Observer in comparison to PI-Observer and Advanced PI-Observer. Simulation results illustrate the advantages of Modified API-Observer in the comparison to PI-Observer and Advanced PI-Observer.

\section{INTRODUCTION}

Two classes of observer design methods have been proposed in literature. One is related to state estimation. The well-known Kalman filter (Kalman (1960)) and its extensions are based on a plant model, use or assume knowledge about the measurement noise, are able to work with disturbance descriptions or disturbance model-based observers. Other approaches are designed to estimate states resulting from the underlying and assumed modeling, additionally additional states used to model unknown inputs like disturbances etc. This type can also be used for robust estimation of states in case of modeling uncertainties or additive nonlinear effects. The second type of observer is based on Luenberger observer (Luenberger (1964)) approach and is called Disturbance observer (DO) or later ProportionalIntegral (PI)-Observer. Even if an accurate model of the system is available, in reality the system is affected by unknown inputs, which may be disturbances or unmodeled dynamics. Because of non-efficiency of proportional observers in the presence of unknown input acting to the system, PI-Observer has been proposed to estimate unknown inputs. Some extensions in the structure of PIObserver has been introduced by Lückel and Müller (1979) to improve the observer performance. Increasing the gain of PI-Observer helps to increase the performance of the es- timation, however due to the high gain the performance is influenced by the measurement noise. This result is firstly proposed by Müller (1988) and later including proofs by Söffker et al. (1995). Advanced PI-Observer (APIO) algorithm proposed by Liu and Söffker (2012), is a method used for optimal scheduling of the PI-Observer gain based on a cost function using a bank of PI-Observers. It has the capability to define the relative optimal gain at each step size of the PI-Observer integration routine. Modified Advanced PI-Observer (MAPIO) has been introduced by Bakhshande and Söffker (2014) to achieve better estimation performance during the numerical integration.

\section{REFERENCES}

Bakhshande, F. and Söffker, D. (2014). High-gain scheduling of the proportional-integral-observer. PAMM, Wiley Online Library.

Kalman, R.E. (1960). A new approach to linear filtering and prediction problems. Journal of Fluids Engineering, 82(1), 35-45.

Liu, Y. and Söffker, D. (2012). Variable high-gain disturbance observer design with online adaption of observer gains embedded in numerical integration. Mathematics and Computers in Simulation, 82(5), 847-857.

Lückel, J. and Müller, P. (1979). Verallgemeinerte Störgrößenaufschaltung bei unvollständiger Zustandskompensation am Beispiel einer aktiven Federung. atAutomatisierungstechnik, 27(1-12), 281-288.

Luenberger, D.G. (1964). Observing the state of a linear system. Military Electronics, IEEE Transactions on, $8(2), 74-80$.

Müller, P. (1988). Control of nonlinear systems by applying disturbance rejection control techniques. In Control, 1988, International Conference on, 734-737.

Söffker, D., Yu, T.J., and Müller, P. (1995). State estimation of dynamical systems with nonlinearities by using proportional-integral observer. International Journal of Systems Science, 26(9), 1571-1582. 


\title{
Bond-Graph-Based Multivariable non linear UIO-type estimation
}

\author{
E. Tarasov*, C. Sueur*, B. Ould Bouamama**, G.Dauphin-Tanguy* \\ * Ecole Centrale de Lille, CRIStAL (ex-LAGIS) CNRS UMR 9189, CS 20048, 59651, Villeneuve d'Ascq cedex, France \\ christophe.sueur@ec-lille.fr \\ **Polytech Lille, CRIStAL (ex-LAGIS) CNRS UMR 9189, 59655, Villeneuve d'Ascq cedex, France \\ belkacem.ouldbouamama@polytech-lille.fr
}

This paper studies observers for multivariable systems with nonlinear components and unknown disturbance inputs. In order to apply procedures dedicated to linear systems, the nonlinearity is first modeled as an unknown input variable and an unknown input observer for multivariable linear systems is studied. An integrated approach is proposed from modeling to observer synthesis with the bond graph approach. This result is used to provide a methodology similar to the one applied for the classical input-output decoupling problem in control theory. An application is proposed and simulation results prove the validity of the approach. 


\title{
Integrated Optimal Controller for Polyvalent Heating/Cooling Systems with Minimal Cost and Energy Usage
}

\author{
Leonardo Angelo Muffato* Mikael Bianchi** \\ Carsten Wemhoener ${ }^{* * *}$ Dominique-Stephan Kunz * \\ * Fr. Sauter AG, 4016 Basel, Switzerland (e-mail:leonardo.muffato, \\ dominique.kunz@ch.sauter-bc.com) \\ ** Department of Technology and Innovation, University of Applied \\ Sciences and Arts of Southern Switzerland (SUPSI), 6928 Manno, \\ Switzerland (e-mail: mikael.bianchi@supsi.ch) \\ *** Institute of Energy Technologies, HSR University of Applied \\ Sciences Rapperswil, 8640 Rapperswil, Switzerland (e-mail: \\ carsten.wemhoener@hsr.ch)
}

\begin{abstract}
In this paper the concept of an integrated optimal controller for polyvalent building heating and cooling systems is presented. The controller is named energy optimizer and employs control allocation for minimizing a cost function involving simplified models of several heating/cooling sources while delivering demanded heating/cooling energy for a given building. The cost function may be defined acc. to different objectives, for example to minimize energy usage and energy costs or to maximize the usage of renewable energy sources. Simplified simulation models for several components of a heating/cooling system (i.e. heat sources and sinks, energy diverter and mixer, storage, etc.) were derived and implemented in a simulation environment as well as in an automation station from the company SAUTER ${ }^{1}$. The concept of the energy optimizer was validated using Model-in-the-loop (MIL) and Hardware-in-the-loop (HIL), a detailed plant model was built using CARNOT (2013).
\end{abstract}

Keywords: Building automation, control allocation, optimal building control, control of energy efficient buildings, smart buildings

1 http://www.sauter-controls.com 


\title{
Multivariable Control of Large Variable-Speed Wind Turbines for Generator Power Regulation and Load Reduction
}

\author{
Jackson G. Njiri* Yan Liu* Dirk Söffker* \\ * Chair of Dynamics and Control, University of Duisburg-Essen, \\ Germany (e-mails: \{jackson.njiri, yan.liu, soeffker\}@uni-due.de).
}

In this paper a multivariate control strategy for regulating generator power and minimizing rotor blade loads during high wind speed is proposed. Due to high demand on wind energy, the current trend is to manufacture larger wind turbines with higher ratings. Consequently, structural loads and flexibility increase as the turbines size increases. Notwithstanding these emerging challenges, wind turbines have to cope with seasonal and intermittent wind variability. For this reason, innovative control strategies to limit power generated to the rated value and to mitigate the structural load are required.

In recent years, a number of advanced control schemes have been proposed, but most of them lack the capacity to address the multi-objective problem since they concentrate only on particular objectives while disregarding others. For instance, Xiaodong et al. (2013) and Yao et al. (2009) designed a multivariable LQG optimal controller with torque and common collective pitch angle as the plant control input to realize the goal of regulating power and torque. However, the controller did not take into account the fluctuation of structural loads. Another LQG controller for pitch regulated variable speed wind turbine was introduced by Pintea et al. (2011) to regulate power and reduce structural loads using collective pitch control and torque control. Nevertheless, the performance improvement was not demonstrated since the results were not verified against any of the existing control methods. From the foregoing discussion, it is clear that elaborate control methods are required in order to balance between reliability and quality of the power produced.

In this paper, generator torque is held constant at the rated value, and a fusion of two control algorithms working in tandem is used for pitch control to achieve these objectives. A proportional-integral (PI) baseline controller is used to generate demanded collective pitch angle for regulating generator speed and independent pitch control (IPC) is utilized to reduce structural load. To compute the overall instantaneous turbine control input, collective pitch angle is added to the perturbed IPC pitch angle demand input. The core contribution of this paper is realization of a multi-objective control strategy using IPC. The results indicate improved performance in terms of controlling speed/power as well as mitigating structural loads as illustrated in Fig. 1. Another performance criteria which considers error and control energy as proposed by Liu and Söffker (2014) is also used to compare the two control schemes. Here, the amount of energy exploited for each control scheme is compared with achieved load reduction within a given time window $T$.

It is important to mention that a fictitious $1.5 \mathrm{MW}$ windPACT wind turbine model developed at National Renewable Energy Laboratory (NREL) is used in this study. This model is simulated in aeroelastic Fatigue, Aerodynamics, Structural and Turbulence (FAST) design code.
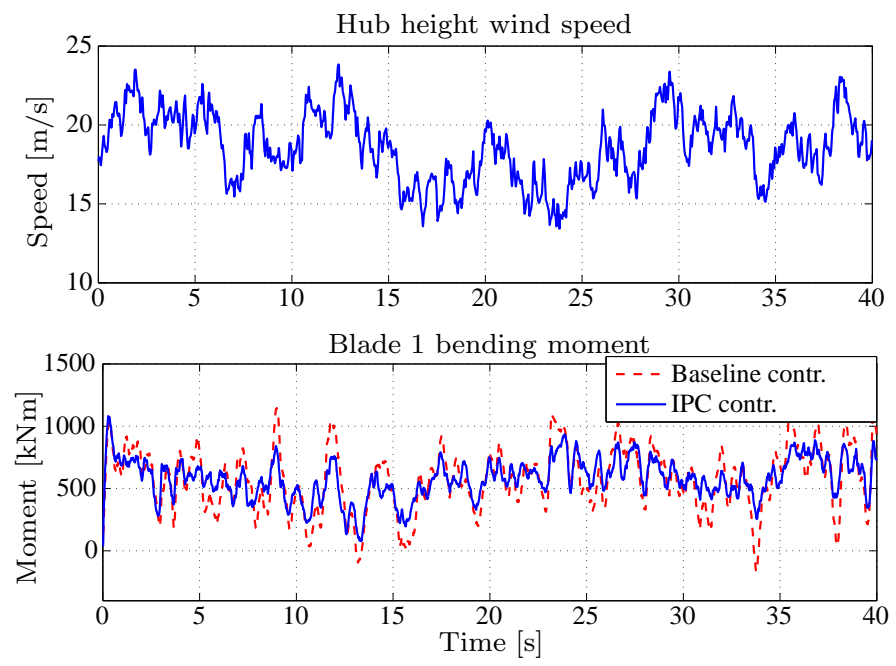

Fig. 1. Variation of blade root moment with wind speed

\section{REFERENCES}

Liu, Y. and Söffker, D. (2014). Robust control approach for input-output linearizable nonlinear systems using high-gain disturbance observer. Int. J. of Robust and Nonlinear Control, 24, 326-339.

Pintea, A., Christov, N., Popescu, D., and Borne, P. (2011). LQG control of horizontal wind turbines for blades and tower loads alleviation. In Preprints of the 18th IFAC World Congress Milano (Italy), 1721-1726.

Xiaodong, W., Shixu, H., Shirong, W., Yingming, L., and Lixia, L. (2013). Multi-objective optimization torque control of wind turbine based on LQG optimal control. In Control and Decision Conference (CCDC), 405 - 408.

Yao, X., Guo, C., Xing, Z., Li, Y., Liu, S., and Wang, X. (2009). Pitch regulated LQG controller design for variable speed wind turbine. In Proceedings ofthe 2009 IEEE International Conference on Mechatronics and Automation, Changchun, China, 845-849. 


\title{
Flatness based control of a ball in tube system
}

\author{
Lukas Herrmann* Markus Broecker ** \\ * University of Heilbronn, 74081 Heilbronn, Germany (e-mail: \\ lukas.herrmann@hs-heilbronn.de). \\ ** University of Heilbronn, 74081 Heilbronn, Germany (e-mail: \\ markus.broecker@hs-heilbronn.de).
}

\section{INTRODUCTION}

Flatness based control, see Fliess et al. (1995) and Lévine (2009), is a method that is often applied to nonlinear systems. In practice, flatness based control achieves precise tracking performance of a flat system output. The desired trajectory for the flat system output is designed with respect to the experience of engineers and the system characteristics. The main advantage of flatness based control is the two-degree-of-freedom structure of the control loop in terms of a feedforward and a feedback control path.

In this contribution flatness based control is applied to a ball in tube system. A ball in tube system is a mechanical, aerodynamical and electrical system that consists of a DC motor that drives a fan for the purpose to lift a table tennis ball in a tube. The ball in tube system is therefore a nonlinear system and the control loop is implemented on a programmable logic controller.

For this purpose the system equations are quoted, considering that the system properties are changing in dependence on a positive or a negative fan acceleration. The proof for the ball position as chosen output shows that the system has the attribute flat. On the basis of this proof a precontrol law is calculated, in order to track the output to a predefined reference trajectory exactly, in case of an ideal model and non-occurring disturbances. Within the whole feedforward control the nonlinear static gain of the DC motor is taken into account by the use of an inverse measured characteristic. The feedforward control is combined with a state feedback and an integral controller.

$$
u=u_{\mathrm{FF}}+r_{\mathrm{w}} \cdot y_{\mathrm{rt}}-\boldsymbol{r}^{\mathrm{T}} \cdot \boldsymbol{x}+k_{\mathrm{I}} \cdot \int\left(y_{\mathrm{rt}}-y_{\mathrm{lin}}\right) \mathrm{d} t .
$$

In this evaluation the performance is compared using the control concept with and without the integral controller. Concluding the disturbance rejection of the ball in tube system is examined.

For the evaluation of the flatness based control the ball in tube system is tested with three different input trajectories. The selected trajectories are chosen as polynomials of the ninth degree, as the relative degree for the ball in tube system is $r=4$. All trajectories have a transition time of $t_{\mathrm{t}}=15 \mathrm{~s}$ and an initial height of $x_{0}=0.15 \mathrm{~m}$. The three input signals solely differ in the final height $x_{\mathrm{e}}$ and their starting point $t_{0}$. The result of one setpoint transition is shown in figure 1.

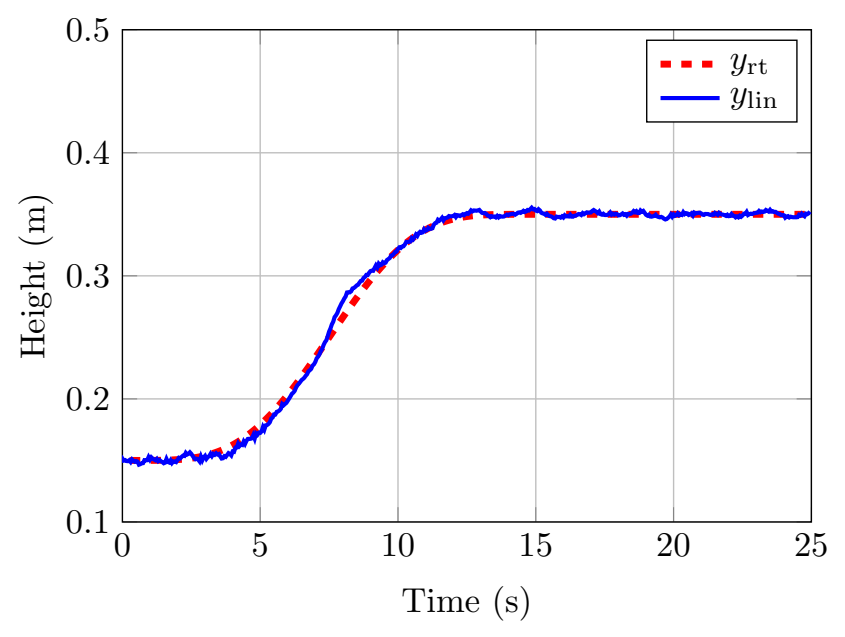

Fig. 1. Comparison of the measured ball position $y_{\text {lin }}$ and the reference trajectory $y_{\mathrm{rt}}$

\section{CONCLUSION}

This paper deals with the derivation and the implementation of a flatness based control for a ball in tube system. The controlled ball in the tube follows a predefined reference trajectory. When the controller has no integral part, the tracking perfomance is not satisfactory. The use of an additional integral controller improves the accuracy of the feedforward and feedback control. Although disturbances can be compensated with the integral controller further investigations may study the implementation of a disturbance attenuation controller on the testbed, see Bröcker (2002). Further investigations of the linearized ball in tube system for the flatness based control can show differences and similarities between the methods for nonlinear and linear systems.

\section{REFERENCES}

Bröcker, M. (2002). On nonlinear disturbance attenuation for hydraulic differential cylinders via tracking control. In 15th IFAC World Congress. Barcelona, Spain.

Fliess, M., Lévine, J., and Rouchon, P. (1995). Flatness and defect of nonlinear systems: Introductory theory and examples. International Journal of Control, 61, 1327-1361.

Lévine, J. (2009). Analysis and Control of Nonlinear Systems: A Flatness-based Approach. Mathematical Engineering. Springer. 


\title{
Ball on Ball: Modeling and control of a novel experiment set-up
}

\author{
Reinhard Gahleitner
}

University of Applied Sciences Upper Austria, School of Engineering and Environmental Sciences (e-mail: reinhard.gahleitner@fh-wels.at)

\section{INTRODUCTION}

This contribution introduces a new control application. The goal is to balance a bowling ball on top of another bowling ball which rotates in three axes (see Fig. 1). The first ball is placed on three so-called omni-wheels in a way that it can rotate in all directions. The second ball moves freely atop the first ball. The balls are ordinary bowling balls without the drill-holes. A model based approach is used for the controller design.

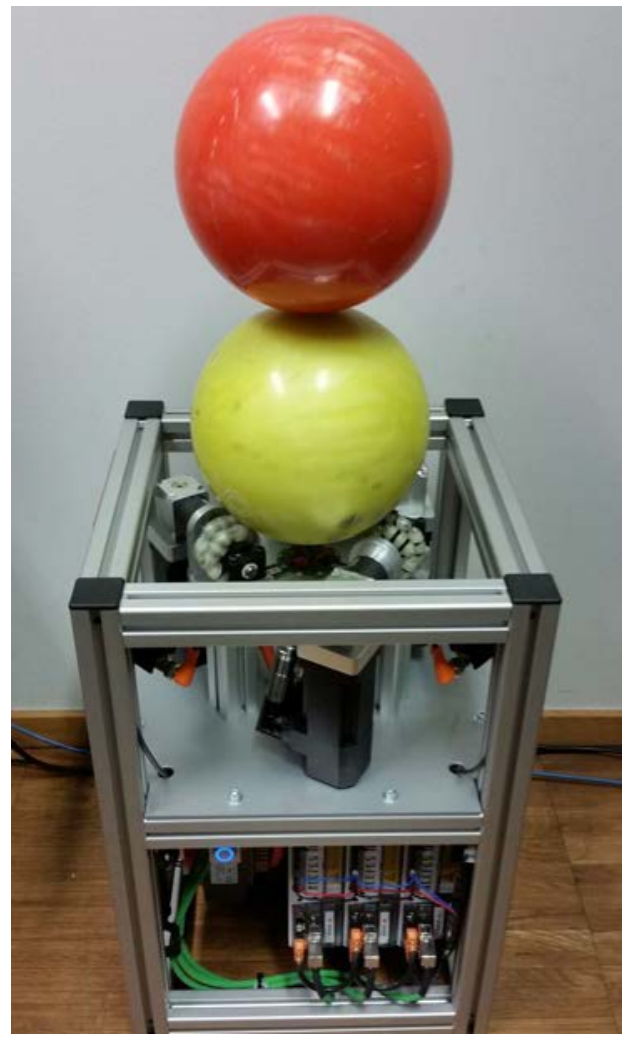

Figure 1. Front view of the Ball on Ball system.

\section{MODELING AND MODEL ANALYSIS}

The mathematical model for the two balls is derived using the Lagrange equations with the torques on the lower ball as inputs. These are generated by a power train, with a servo motor, an angular gear and the omni-wheels. The two balls are modeled as homogenous rigid bodies. There are 8 generalized coordinates, 3 per ball for the rotation and 2 for the position of the upper ball. The assumption that the upper ball is rolling on the lower one, not sliding, gives 2 additional nonholonomic constraints, which reduce the degrees of freedom to 6 .

The mathematical model is simplified with a state transformation and decoupled into subsystems and finally has the form (details are in the full paper)

$$
\begin{aligned}
\dot{\mathbf{q}}_{1} & =\mathbf{T}_{1}^{-1}\left(\mathbf{q}_{1}\right) \boldsymbol{\omega}_{1} \\
\dot{\mathbf{q}}_{\overline{2}} & =\boldsymbol{\omega}_{\overline{2}} \\
\dot{\mathbf{q}}_{2} & =\mathbf{T}_{2}^{-1}\left(\mathbf{q}_{2}\right) \mathbf{T}_{P}\left(\mathbf{q}_{\overline{2}}\right) \boldsymbol{\omega}_{2} \\
\dot{\boldsymbol{\omega}}_{1} & =\mathbf{J}_{1}^{-1}\left(-\overline{\mathbf{Z}}_{1}\left(\mathbf{q}_{\overline{2}}\right) \boldsymbol{\mu}+\boldsymbol{\tau}_{1}\right) \\
\dot{\boldsymbol{\omega}}_{\overline{2}} & =\mathbf{M}_{\overline{2}}^{-1}\left(\mathbf{q}_{\overline{2}}\right)\left(-\mathbf{C}_{\overline{2}}\left(\mathbf{q}_{\overline{2}}, \boldsymbol{\omega}_{\overline{2}}\right) \boldsymbol{\omega}_{\overline{2}}-\mathbf{G}_{\overline{2}}\left(\mathbf{q}_{\overline{2}}\right)-\overline{\mathbf{Z}}_{\overline{2}}\left(\mathbf{q}_{\overline{2}}\right) \boldsymbol{\mu}\right) \\
\dot{\boldsymbol{\omega}}_{2} & =\mathbf{J}_{2}^{-1}\left(-\overline{\mathbf{C}}_{2}\left(\mathbf{q}_{\overline{2}}, \boldsymbol{\omega}_{\overline{2}}\right) \boldsymbol{\omega}_{2}-\overline{\mathbf{Z}}_{2} \boldsymbol{\mu}\right) \\
\mathbf{0} & =\overline{\mathbf{Z}}_{1}^{T}\left(\mathbf{q}_{\overline{2}}\right) \boldsymbol{\omega}_{1}+\overline{\mathbf{Z}}_{\overline{2}}^{T}\left(\mathbf{q}_{\overline{2}}\right) \boldsymbol{\omega}_{\overline{2}}+\overline{\mathbf{Z}}_{2}^{T} \boldsymbol{\omega}_{2}
\end{aligned}
$$

This is a differential algebraic system with 16 differential equations in 16 variables and 2 algebraic equations for the restrictions. The equations for the angular position of the balls are decoupled and can be omitted. This reduces the system by 6 equations and 6 variables. Finally the algebraic restriction are eliminated to get an explicit state space model $\dot{\mathbf{x}}=\mathbf{f}(\mathbf{x}, \mathbf{u})$.

\section{CONTROLLER FOR THE LINEARIZED MODEL}

A linear approximation of the model at the equilibrium point is presented along with the controller design. Implementation on the laboratory model demonstrate the successful application of the model based control concept (see the video [4]).

\section{REFERENCES}

[1] Kammerhofer M., (2008). Modellbildung, Reglerentwurf und Simulation des Modells "Ball on Ball" (german). diploma thesis, University of Applied Sciences Upper Austria, Wels.

[2] Liu, S.Y., Rizal, Y. and Ho, M.T. (2011). Stabilization of a ball and sphere system using feedback linearization and sliding mode control. 8th Asian Control Conference (ASCC), pp. 1334-1339.

[3] Zehetleitner, K., Gahleitner, R. (2014), Modellbasierte Entwicklung in der Industrieautomation am Beispiel eines Laboraufbaus ,Ball on Ball' (german). Matlab EXPO Germany.

[4] YouTube video. ball on ball. http://www.youtube.com/watch?v=NJkYZAxjjT4. 


\title{
Multi-Variable Integral Sliding Mode Control of a Two Degrees of Freedom Helicopter
}

\author{
Saif S. Butt, ${ }^{*}$ Harald Aschemann * \\ * Chair of Mechatronics, University of Rostock, \\ D-18059 Rostock, Germany, \\ (e-mail: \{Saif.Butt, Harald.Aschemann\}@uni-rostock.de)
}

\section{EXTENDED ABSTRACT}

In this paper, a multi-variable nonlinear control-oriented model of a twin rotor aerodynamic system (TRAS) is presented, see Fig. 1. The mathematical description of the multibody system is derived using Lagrange's equations. Here, the former model of the TRAS developed in Butt et al. (2014) is extended to include the moments of inertia of the rotating beam. Based on the system representation, a multi-variable integral sliding mode control is designed to accurately track desired trajectories for both the azimuth angle and the pitch angle. Due to unmeasurable states and uncertainties stemming from simplifications at modelling as well as unknown disturbance torques, a discrete-time extended Kalman filter (EKF) is employed and combined with a discrete-time implementation of the nonlinear control law. In Ahmed et al. (2009), a sliding mode control that deals with couplings of the twin rotor dynamics is considered. An adaptive second order sliding mode control has been proposed in Mondal and Mahanta (2012), however, no experimental validation is presented therein. The proposed control strategy in this paper allows for an excellent tracking behaviour as highlighted by experimental results. The desired and measured trajectories for the azimuth angle and the pitch angle depicted in Fig. 2 are in good agreement during both the transient phase as well as in steady-state. Hence, a precise tracking behaviour is obtained that outperforms the results achieved in Butt et al. (2014).

\section{REFERENCES}

Ahmed, Q., Bhatti, A., and Iqbal, S. (2009). Nonlinear Robust Decoupling Control Design for Twin Rotor System. In Asian Control Conf., 2009. ASCC 2009. Yth, 937-942.

Butt, S.S., Prabel, R., and Aschemann, H. (2014). MultiVariable Flatness-Based Control of a Two Degrees of Freedom Helicopter. In Control, Decision and Information Technologies (CoDIT), 2014 International Conf. on, 321-326.

Mondal, S. and Mahanta, C. (2012). Adaptive Secondorder Sliding Mode Controller for a Twin Rotor MultiInput-Multi-Output System. Control Theory Applications, IET, 6(14), 2157-2167. doi:10.1049/ietcta.2011.0478.

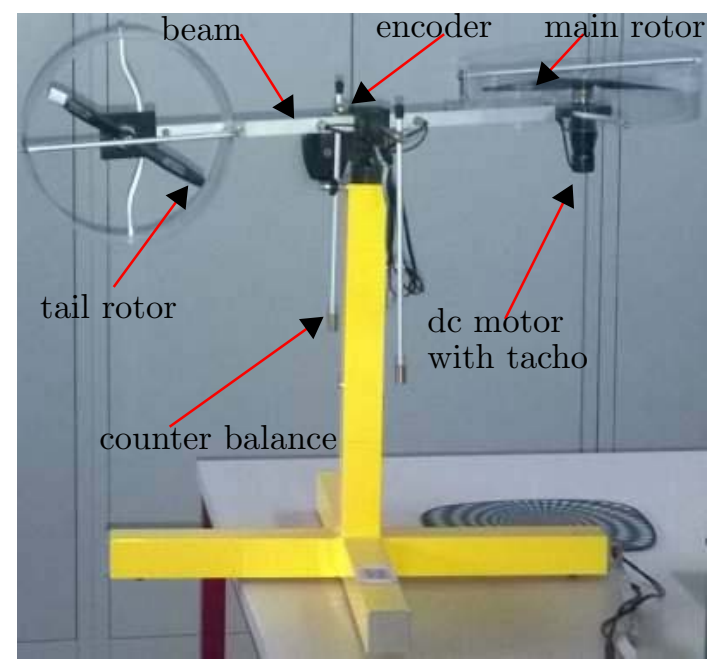

Fig. 1. TRAS set-up at the Chair of Mechatronics, University of Rostock.

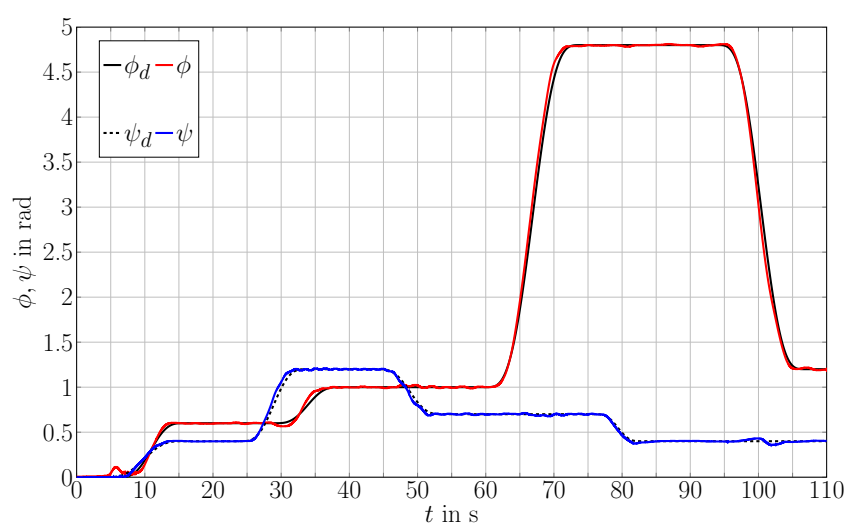

Fig. 2. Desired trajectories and tracking performance w.r.t. the azimuth angle $\phi$ and the pitch angle $\psi$ : experimental results using the proposed integral sliding mode control extended by an EKF. 


\title{
Flight Path Optimization of a Hang-Glider in a Thermal Updraft
}

\author{
Kurt Chudej* Anna-Lena Klingler ${ }^{* *}$ Andreas Britzelmeier *** \\ * Lehrstuhl für Ingenieurmathematik, Universität Bayreuth, 95440 \\ Bayreuth, Germany (e-mail: kurt.chudej@uni-bayreuth.de). \\ ** Fraunhofer-Institut für System- und Innovationsforschung ISI, \\ Karlsruhe, Germany. \\ *** Lehrstuhl für Ingenieurmathematik, Universität Bayreuth, 95440 \\ Bayreuth, Germany.
}

\section{INTRODUCTION}

Hang-gliding is a well-known sport in the Alpes and Franconian Switzerland. Due to favourable thermal upwinds
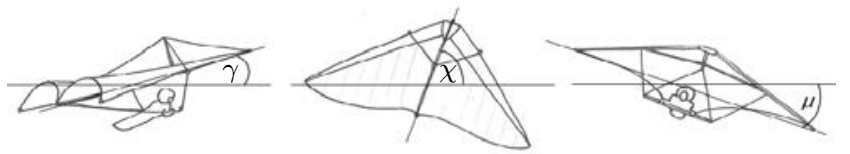

Fig. 1. Path inclination $\gamma$ (state), azimuth angle $\chi$ (state), bank angle $\mu$ (control).

induced through mountains and valleys interesting flights for a long time or over a long range are possible. These flights typically include spiralling trajectories in thermal updrafts. The new enhanced $3 \mathrm{~d}$ model is based on a predecessor model developed by Bulirsch, Nerz, Pesch, von Stryk. This predecessor model was restricted to the flight in a vertical plane and could not capture spiralling trajectories. Numerical solutions for several newly developed thermal updraft models are presented.

\section{OPTIMAL CONTROL MODEL}

We use a point mass modell of the hang-glider with pilot over a flat nonrotating earth. Different scenarios for the thermal updraft are used, e.g. mountain and valley breezes which develop along mountain slopes (Fig. 2). This yields an optimal control problem which is solved
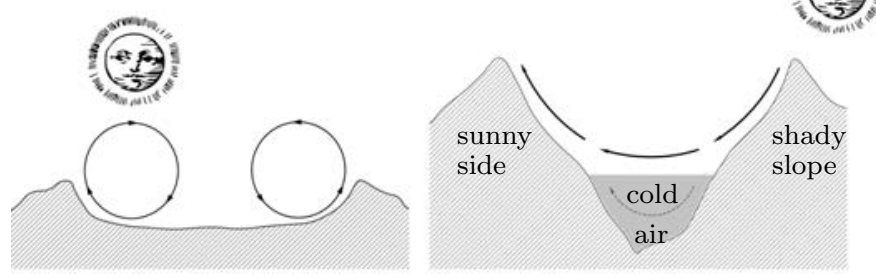

Fig. 2. Mountain und valley breeze.

after a discretization of states and controls by a nonlinear optimization software.
Finally we present some of the numerical results. Fig 3 depicts the numerical solution for a mountain and valley breeze.
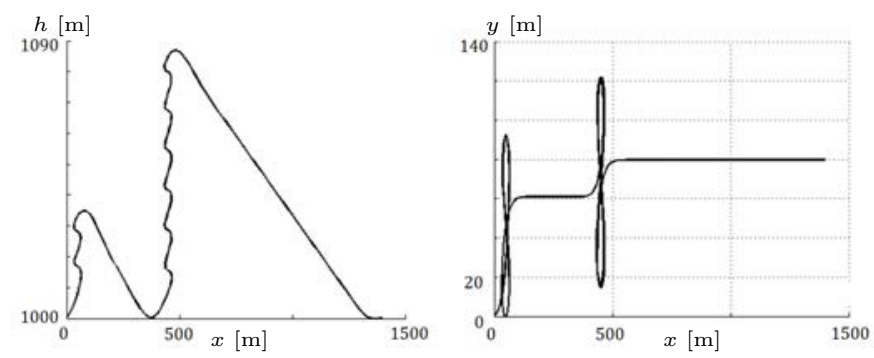

Fig. 3. Flight path in the mountain breeze.

In the first maximum of the mountain breeze, the hangglider gains enough altitude in order to cross the valley with the descending air current without violating the lower altitude constraint. The additional altitude gain in the second maximum of the mountain breeze is used for range maximization. A more detailed view of the trajectory is given in Fig. 4. Please notice that the maximum value of
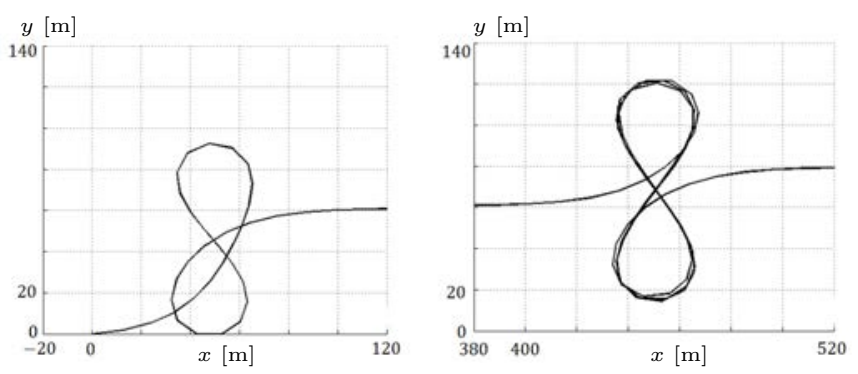

Fig. 4. Detail of Fig 3, left first maximum of mountain breeze, right second maximum of mountain breeze.

the mountain breeze parallel to the $y$ axis is used efficiently in the shape of the loops.

In the presentation we also address the case of a thermal updraft shaped as a flexible hose. 


\title{
Modeling and Control of an Autonomous Hybrid Vehicle for Navigation and Guidance
}

\author{
Katsumi Moriwaki* \\ * Daido University, 10-3, Takiharu-cho, Minami-ku, Nagoya, Aichi, \\ Japan (e-mail:moriwaki@daido-it.ac.jp).
}

\section{INTRODUCTION}

The problems of control of a pure electric vehicle and a hybrid vehicle, however, are easier than a vehicle with an internal combustion engine, because a power train induced by an electric motor has a close affinity with a vehicle control system Ousaka (2001). Steering a car by hand means that the driver plans a path by preview and controls the lateral deviation of the vehicle from the planned path by the steering wheel. In an automatic steering system of autonomous vehicles, this path following is automated. The lateral deviation from the reference path is kept small by feedback control via the steering motors. The reference trajectory may be calculated from the data of a TV camera or a CCD camera. In order to study automation of car steering, the steering model must be extended. The extended model must include not only velocities, but also the vehicle heading and lateral position of the sensor with respect to the reference path. For simplicity this extended model will only be derived using the linearized model of a nonlinear model that is valid for small deviations from a stationary circular path. Introducing the curvature $(=$ the reciprocal of the radius for a circular path) as the input that generates the reference path, the transient rolling motion from straight line to circular path can be controlled continuously. Although the dynamics of an automotive vehicle are designed to be stable in the usual situation, they are described by nonlinear equations. The conventional controller for automatic vehicle steering is obtained via the problem formulation using linearized steering models. The $H_{\infty}$ optimal controller using nonlinear state feedback to the strict nonlinear steering models for automatic vehicle steering system is proposed and the validity of the proposed controller is discussed.

\section{NONLINEAR STATE FEEDBACK $H_{\infty}$ OPTIMAL CONTROLLER OF AN AUTONOMOUS VEHICLE}

The vehicle system can be rewritten as

$$
\begin{gathered}
\dot{x}_{i}(t)=A_{i} x_{i}(t)+B_{i} u_{i}(t)+D_{i} \rho_{\mathrm{ref}}(t) \\
\quad+G_{i}(\Delta) d_{i}(t) \\
y(t)=C_{i} x_{i}(t), \quad(i=1,2)
\end{gathered}
$$

where $x_{1}:=\left[\beta^{T} r^{T} \triangle \psi^{T} y_{\mathrm{S}}^{T} \mid z^{T} \theta^{T} w^{T} q^{T}\right]^{T}$ (: for high velocity) and $x_{2}:=\left[\triangle \psi^{T} y_{\mathrm{S}}^{T} \mid z^{T} \theta^{T} w^{T} q^{T}\right]^{T}$ (: for low velocity).

\footnotetext{
^ This work was not supported by any organization
}

We consider to construct the $H_{\infty}$ optimal controller so that the output $\left[\triangle \psi^{T}, y_{\mathrm{S}}^{T}\right]^{T}$ can be driven to zero as $t \rightarrow \infty$, where $\triangle \psi$ is the deviation of yaw angle and $y_{\mathrm{S}}$ is the deviation from the reference path, respectively (Moriwaki (2005, 2009, 2012)).

[Theorem 1] Consider the nonlinear system with disturbances (1). Let $\gamma>0$. Suppose there exists a $C^{\infty}$ solution $V \geq 0$ to the Hamilton-Jacobi equation

$$
\begin{aligned}
& \frac{\partial V(x)}{\partial x} A x+\frac{1}{2} \frac{\partial V(x)}{\partial x}\left[\frac{1}{\gamma^{2}} G(\Delta) G^{T}(\Delta)-B B^{T}\right] \\
& \times\left(\frac{\partial V(x)}{\partial x}\right)^{T}+\frac{1}{2} x^{T} C^{T} C x=0, V\left(x_{0}\right)=0
\end{aligned}
$$

then the closed-loop system for the feedback

$$
\delta=-B^{T}\left(\frac{\partial V(x)}{\partial x}\right)^{T}
$$

has $L_{2}$ - gain (from $d$ to $\left[y^{T}, \delta\right]^{T}$ ) less than or equal to $\gamma$.

The subscript $i(=1,2)$ in (1) is abbreviated in the Theorem 1.

\section{CONCLUSIONS}

It has been considered the problem of automatic steering of autonomous electric vehicles. The extended model including small nonlinear factors of the steering motion is derived. The optimal regulator for the extended steering system is introduced so that the autonomous vehicle can be driven along the reference path.

\section{REFERENCES}

K. Moriwaki. Autonomous steering control for electric vehicles using nonlinear state feedback $H_{\infty}$ control. Nonlinear Analysis, volume 63, pages e2257-e2268, 2005.

K. Moriwaki and K. Tanaka. Navigation control for electric vehicles using nonlinear state feedback $H_{\infty}$ control. Nonlinear Analysis, volume 71, pages e2920-e2933, 2009.

K. Moriwaki. Drivability Control to Optimize the Operating Freedom in the Hybrid Vehicle Propelling System. Proceedings of the ASME/ISCIE 2012 International Symposium on Flexible Automation, Paper No.ISFA2012-7230, 2012.

T. Ousaka, Y. Daisho, S. Tange, M. Mitani and N. Kurihara and et al. Electric Vehicle Handbook, Maruzen Press, Tokyo, 2001. 


\title{
BEECLUST used for exploration tasks in Autonomous Underwater Vehicles
}

\author{
Michael Bodi ${ }^{*}$ Christoph Möslinger* Ronald Thenius* \\ Thomas Schmickl*
}

* Artificial Life Lab of the Department of Zoology, Karl-Franzens

University,8010 Graz, Austria (e-mail: michael.bodi@uni-graz.at).

\section{INTRODUCTION}

Underwater exploration is still a difficult task these days. In this paper we suggest a swarm of Autonomous Underwater Vehicles (AUVs) for such tasks. These AUVs are controlled by BEECLUST which is a swarm control algorithm inspired by honeybee behaviour.This work discusses the questions whether or not BEECLUST is adaptable for a swarm of AUVs and if this algorithm can be utilized for underwater exploring tasks. The BEECLUST algorithm follows a few simple rules: (I) Robots move around randomly; (II) Whenever a robot meets an obstacle, the robot checks whether the obstacle is another robot or a wall; (III) If the obstacle is a wall, the robot turns and proceeds with step I; (IV) If the obstacle is another robot, the robot stops and calculates a waiting time. The deeper it is, the longer the robot waits; (V) After the waiting time has expired, the robot proceeds with step I.

\section{METHODS}

The following experiments were performed in 'CoCoRoSim', a simulation environment which is specially designed for simulations of AUVs (Read et al., 2013). This simulator was completely written in NetLogo. For these experiments we chose the ground level to be the decisive factor for aggregation. We generated an environment with the dimensions of $100 \cdot 100 \cdot 42$ patches $(\mathrm{l} \cdot \mathrm{w} \cdot \mathrm{h})$. The ground is designed to show a more or less irregular random pattern. As aggregation spots we introduced two depressions in the ground. One depression is located on the front left side of the arena, the other depression is located on the rear right of the arena. Therefore the left depression can be considered to be the optimal aggregation spot whereas the right depression can be considered to be the suboptimal aggregation spot. Within the arena the AUVs moved along the ground terrain using only buoyancy for changing their altitude. At the beginning of each experimental run the AUVs were spawned at random positions. We perfomed experiments varying the size of the AUV swarm and the waiting time.

\section{RESULTS AND DISCUSSION}

Figure 1 shows an example experiment using a waiting time multiplicator of 7 . We show that in groups of one to 30 individuals the fraction of aggregated AUVs correlates to

\footnotetext{
‡ This work was supported by: EU-ICT 'CoCoRo', no. 270382; EUICT 'ASSISIbf', no. 601074
}
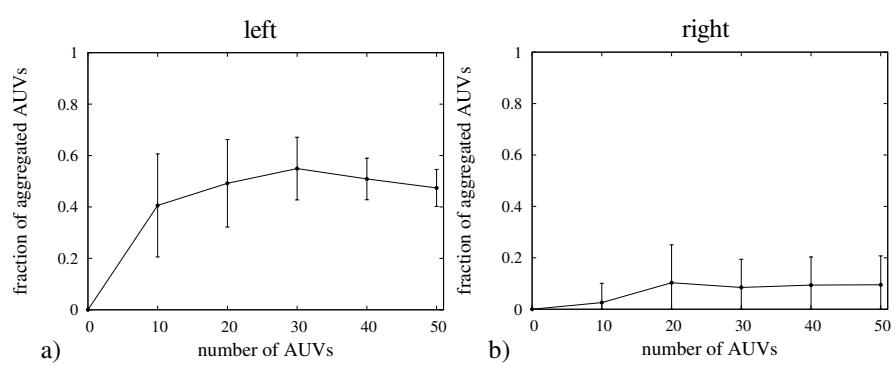

Fig. 1. Fraction and standard deviation of aggregated AUVs. The left target represents the optimal spot for aggregation. $\mathrm{n}=50$ repetitions per experiment.

the total number of AUVs in the swarm: the more individuals the better the aggregation quality. Beyond 30 individuals the aggregation quality decreases. This means that we found an optimal group size of 30 individuals for the given experimental setup. In this case a fraction of 0.549 AUVs $($ stdev $=0.122)$ aggregated at the left target. Figure 1 also clearly shows, that in all cases the swarm aggregated in the left target. The fact that the AUVs 'chose' the optimal aggregation spot does not only show that BEECLUST is suitable for aggregation tasks but proves that AUVs using BEECLUST can also discriminate between different environmental qualities. In our experiments the swarm of AUVs was able to locate the deepest spot of the experimental arena. However BEECLUST works with any given environmental sensor which makes BEECLUST applicable for manifold exploring tasks. Whilst a single AUV could get stuck in a local optimum a swarm of AUVs controlled by BEECLUST would be able to find the global optimum even if the gradient is irregular and complex. The loss of single individuals does not greatly influence the ability to form aggregations (Bodi et al., 2012). Therefore we consider BEECLUST to be a very potent and reliable algorithm for all kinds of swarm robotic applications.

\section{REFERENCES}

Bodi, M., Thenius, R., Szopek, M., Schmickl, T., and Crailsheim, K. (2012). Interaction of robot swarms using the honeybee-inspired control algorithm beeclust. Mathematical and Computer Modelling of Dynamical Systems, 18(1), 87-100. doi:10.1080/13873954.2011.601420.

Read, M., Möslinger, C., Dipper, T., Kengyel, D., Hilder, J., Thenius, R., Tyrrell, A., Timmis, J., and Schmickl, T. (2013). Profiling underwater swarm robotic shoaling performance using simulation. Autonomous Robotic Systems (TAROS). 
MATHMOD 2015 Abstract Volume 


\title{
MATHMOD 2015 ABSTRACT VOLUME
}

\author{
MATHMOD Full Contributions \\ Infinite-dimensional Systems
}


MATHMOD 2015 Abstract Volume 


\title{
Boundary Control of a Parallel-Flow Heat Exchange Process with Boundary Observation
}

\author{
Hideki Sano* \\ * Department of Applied Mathematics, Graduate School of System \\ Informatics, Kobe University, 1-1 Rokkodai, Nada, Kobe 657-8501, \\ Japan (e-mail: sano@crystal.kobe-u.ac.jp).
}

Since the beginning of the 180 's, the design method of finite-dimensional stabilizing controllers for infinitedimensional dynamical systems has been proposed by many researchers. In general, when one constructs a finitedimensional model for an infinite-dimensional system and applies a finite-dimensional controller designed for it to the original infinite-dimensional system, spillover phenomenon may be occured by the influence of unmodeled modes. Sakawa firstly introduced two kinds of finite-dimensional observers for linear diffusion systems to reduce the influence of unmodeled modes for the closed-loop system with the finite-dimensional controller (Sakawa (1 83)). After that, alas called one of them as the residual mode filter (RMF), and clarified that the RMF plays an essential role for the construction of finite-dimensional stabilizing controllers ( alas $\left(\begin{array}{ll}1 & 88\end{array}\right)$ ). On the other hand, Nambu gave the design method of infinite-dimensional stabilizing controllers applicable to a large class of linear parabolic systems, and further accomplished finite-dimensionalization of the controllers (Nambu (1 85, 2005)). Recently, the author showed that the method based on RMF by Sakawa (1 83) was also applicable to a coupled transport-diffusion system related to chemical reaction process (Sano (2012)).

In this paper, we try to extend the result of Sano (2012) to a coupled transport-diffusion system related to parallelflow heat exchange process:

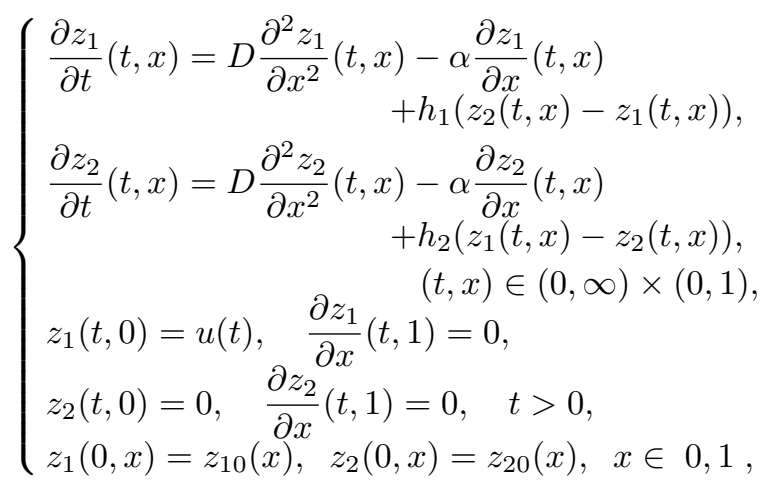

where $z_{1}(t, x), z_{2}(t, x)$ denote the temperatures of fluids at time $t$ and at the point $x \in 0,1$, and $u(t) \in \mathbf{R}$ denotes the control input added to the inlet $x=0$ of tube $1 . D>0$ is the heat diffusion coefficient, $\alpha>0$ the fluid velocity,

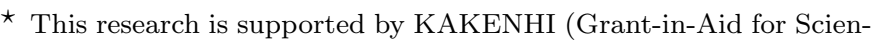
tific Research (C), No. 23540137), Japan Society for the Promotion of Science.
}

$h_{1}, h_{2}>0$ the heat exchange rates between two tubes. For system (1), we consider the output equation $y(t) \in \mathbf{R}^{2}$ :

$$
y(t)=y_{1}(t), y_{2}(t)^{T}=z_{1}(t, 1), z_{2}(t, 1)^{T}, t>0 \text {. }
$$

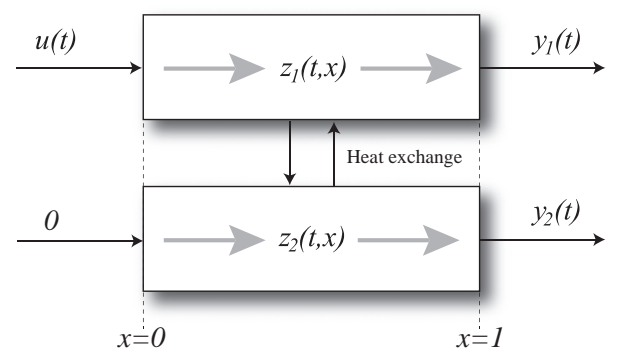

In this paper, we show that the degree of stability of system (1), (2) can be enhanced by using a residual mode filter (RMF)-based controller which is of finite-dimension.

\section{REFERENCES}

M. . alas. Finite-dimensional controllers for linear distributed parameter systems: exponential stability using residual mode filters. J. Math. Anal. Appl., 133: 28326,188 .

R.F. Curtain and H. . Zwart. An Introduction to InfiniteDimensional Linear Systems Theory, Texts in Applied Mathematics, Vol. 21. Springer-Verlag, New York, 15.

T. Nambu. On stabilization of partial differential equations of parabolic type: boundary observation and feedback. Funkcialaj Ekvacioj, Serio Internacia, 28: 26 $28,185$.

T. Nambu. A new algebraic approach to stabilization for boundary control systems of parabolic type. $J$. Differential Equations, 218: 136-158, 2005.

A. Pazy. Semigroups of Linear Operators and Applications to Partial Differential Equations, Applied Mathematical Sciences, Vol. 44. Springer-Verlag, New York, 183.

Y. Sakawa. Feedback stabilization of linear diffusion systems. SIAM J. Control Optim., 21: 66 -6 6, 183.

H. Sano and N. Kunimatsu. An application of inertial manifold theory to boundary stabilization of semilinear diffusion systems. J. Math. Anal. Appl., 1 6: 18-42, 15.

H. Sano. Stability-enhancing control of a coupled transport-diffusion system with Dirichlet actuation and Dirichlet measurement. J. Math. Anal. Appl., 388: 11 41204, 2012.

H. Tanabe. Equations of Evolution. Iwanami, Tokyo, 15 , (in apanese). 


\title{
Noise and Fluctuations in Nanowire Biosensors
}

\author{
Gerhard Tulzer* Clemens Heitzinger*,** \\ * Vienna University of Technology, Wiedner Hauptstrasse 8-10, A-1040 \\ Vienna, Austria. (e-mail: gerhard.tulzer@tuwien.ac.at). \\ ** School of Mathematical and Statistical Sciences, Arizona State \\ University, Tempe, AZ 85287, USA
}

The applicability of nanowire field-effect sonsors for the detection of several types of biomolecules in liquids has been shown experimentally, assuring fast, efficient and label-free detection. However, optimal design is still a main topic of research. Computer simulations are essential at this point, since they enable effective and cheap prediction of the sensor behavior, as has been shown for various types of sensors (Baumgartner and Heitzinger, 2012; Baumgartner et al., 2013; Tulzer et al., 2013).

In this work, we consider hybridization and dissociation processes at a nanowire biosensor. These processes are known to give rise to biological noise (Hassibi et al., 2004) and should therefore be considered as stochastic processes to take into account their random nature.

The stochastic approach for these reactions yields chemical Langevin equations (Gillespie, 2008), which can be solved explicitly under the simplifying assumption of constant amounts of target molecules throughout the liquid. In the more complicated situation of a limited number of target molecules, one has to calculate the moments numerically.

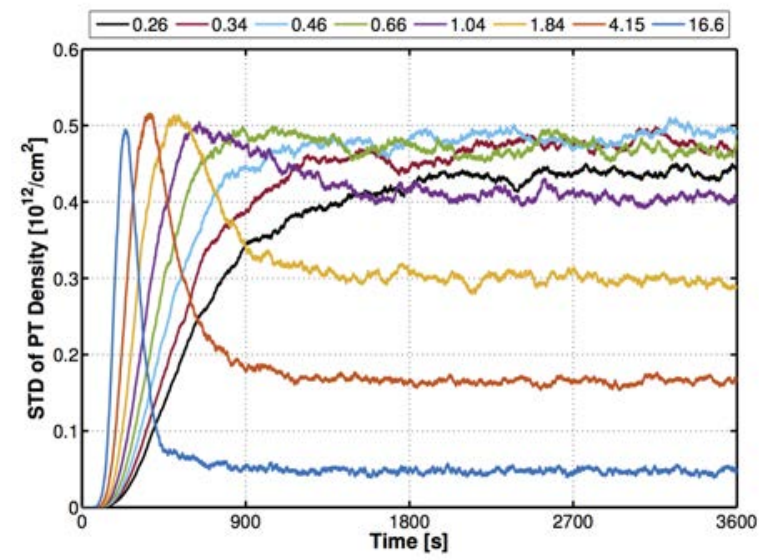

Fig. 1. Evolution of the variance for different target molecule densities, given in $\mu \mathrm{M}$. At higher target molecule densities, the variance is not monotone and shows a maximum after a certain period of time depending on the concentration.

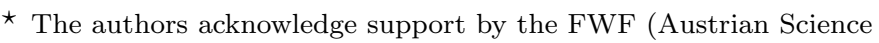
Fund) START project no. Y660 PDE Models for Nanotechnology.
}

The equations describing the surface processes are coupled to a diffusion equation in the bulk liquid phase to model the limited transport of DNA oligomers, which is also considered in a stochastic formulation. After we followed a box-based approach in Tulzer and Heitzinger (2014) to simulate the whole system, we here employ a random-walk based algorithm to calculate the quantities of interest, such as signal-to-noise ratio and response times.

This algorithm turns out to be a lot more efficient than the box-based one. Furthermore, the sampling of realizations can be parallelized very easily, and the implementation of more complicated initial conditions is straightforward, which further increases the applicability of this type of simulations.

The numerical results show that in most cases there is a significant maximum in the variance after a certain period of time, depending on simulation parameters (see Figure 1). The final equilibrium is reached at a lower value, which means that the signal-to-noise ratio is optimal after a certain period of time.

\section{REFERENCES}

Baumgartner, S. and Heitzinger, C. (2012). Existence and local uniqueness for $3 \mathrm{~d}$ self-consistent multiscale models for field-effect sensors. Commun. Math. Sci., 10(2), 693716.

Baumgartner, S., Heitzinger, C., Vacic, A., and Reed, M.A. (2013). Predictive simulations and optimization of nanowire field-effect PSA sensors including screening. Nanotechnology, 24(22), 225503/1-9.

Gillespie, D.T. (2008). Simulation methods in systems biology. In Formal Methods for Computational Systems Biology, 125-167. Springer.

Hassibi, A., Navid, R., Dutton, R.W., and Lee, T.H. (2004). Comprehensive study of noise processes in electrode electrolyte interfaces. Journal of Applied Physics, 96(2), 1074-1082.

Tulzer, G., Baumgartner, S., Brunet, E., Mutinati, G.C., Steinhauer, S., Köck, A., Barbano, P.E., and Heitzinger, C. (2013). Kinetic parameter estimation and fluctuation analysis of $\mathrm{CO}$ at $\mathrm{SnO}_{2}$ single nanowires. Nanotechnology, 24(31), 315501/1-10.

Tulzer, G. and Heitzinger, C. (2014). Fluctuations due to association and dissociation processes at nanowirebiosensor surfaces and their optimal design. Nanotechnology, 26(2), 025502/1-9. 


\title{
Capacity drop and traffic hysteresis as a consequence of bounded acceleration.
}

\author{
M.M. Khoshyaran * J.P. Lebacque ${ }^{* *}$ \\ * ETC Economics Traffic Clinic, 35 avenue des Champs Elysées, F \\ 75008 PARIS, FRANCE (e-mail: etclinic@wanadoo.fr). \\ ** UPE, IFSTTAR-COSYS-GRETTIA, 14-20 Boulevard Newton, Cité \\ Descartes, Champs sur Marne, 77447 Marne-la-Vallée Cedex 2, \\ FRANCE (e-mail: jean-patrick.lebacque@ifsttar.fr)
}

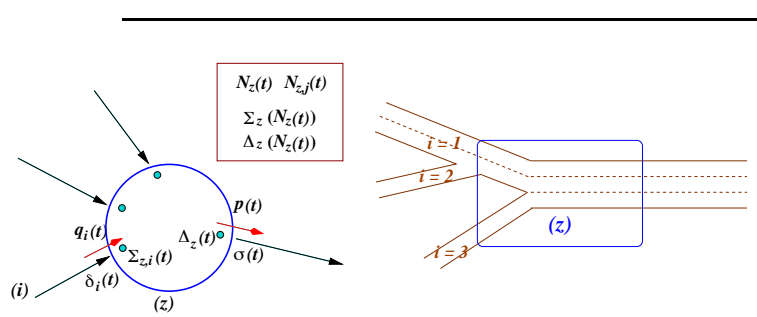

Fig. 1. Pointwise intersection: state variables, example of geometry

The object of this paper is to analyse the impact of merges, lane drops and incidents on capacity drops, a very important aspect for motorway operations.

The idea developed in this paper is that capacity drop results essentially from the impact of conflicts which reduce speed and from bounded acceleration effects which reduce recovery. Traffic on links is assumed to be described by a LWR model. The internal state intersection model introduced and developed in Lebacque and Khoshyaran (2002); Khoshyaran and Lebacque (2009) accounts for conflicts. This model is modified in order to accommodate the impact of the boundedness of traffic acceleration (Lebacque, 2003). The model can be used for capacity drops and merges, and also more complicated settings.

The node is viewed as a buffer which connects the demands of the links entering the intersection with the supplies of the links exiting the intersection. The physical extension of the node is neglected, but it is assumed the node contains vehicles, for instance $N_{z}(t)$ the number of vehicles inside the node $(z), N_{z, i}(t)$ the number of vehicles inside the node originating in link $(i)$. The node is assumed to be endowed with physical properties such as: storage capacity, maximum through-flow, etc... which are expressed by node supply and demand functions

$$
\Sigma_{z}\left(N_{z}(t)\right), \Sigma_{z, i}\left(N_{z}(t), N_{z, i}(t)\right), \Delta_{z}\left(N_{z}(t)\right)
$$

These supply functions are built on the same pattern as the link supply functions. We consider node demand functions which take into account the effects of bounds on acceleration Lebacque and Haj-Salem (2004), i.e. modified demand functions, (refer to figure 2). The model recaptures hysteretic cycles due to variations in upstream demand and recaptures capacity drop (the node output drops when the node becomes congested, as figure 2 shows).

Comparisons are carried out between the model and data collected on the Boulevard Périphérique in Paris for traffic

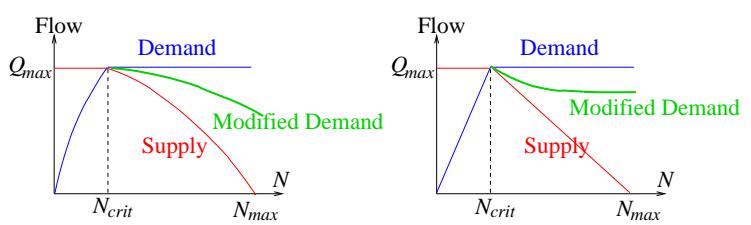

Fig. 2. Node supply and demand functions for different node behaviours

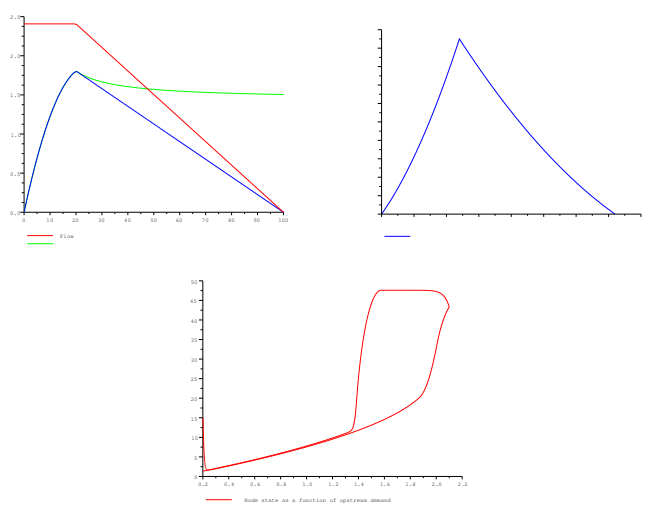

Fig. 3. Hysteresis cycle induced by a peak of demand (top right) on the node outflow: the node state $N$ as a function the upstream demand value $\delta$ (center)Top left in red: node supply

management and driver information and show that the model recaptures capacity drop satisfactorily.

\section{REFERENCES}

Khoshyaran, M. and Lebacque, J.P. (2009). Internal state models for intersections in macroscopic traffic flow models. In Proceedings of TGF'09 (Traffic and Granular Flow 2009).

Lebacque, J.P. (2003). A two phase bounded acceleration traffic flow model: analytical solutions and applications. Transportation Research Record, 1852, 220-230.

Lebacque, J.P. and Haj-Salem, H. (2004). Ramp metering, speed management and braess-like paradoxes. In TRISTAN $V$ : The Fifth Triennial Symposium on Transportation Analysis.

Lebacque, J.P. and Khoshyaran, M. (2002). Transportation planning: the state of the art, chapter First order macroscopic flow models for networks in the context of dynamic assignment, 119-139. Kluwer Academic Press. 


\title{
Optimal Design of Elastic Rod Motions Based on a Projection Approach
}

\author{
Georgy Kostin* Vasily Saurin** \\ * Institute for Problems in Mechanics RAS, Vernadskogo 101-1, \\ 119526, Moscow, Russia (e-mail: kostin@ipmnet.ru). \\ ** Institute for Problems in Mechanics RAS, Vernadskogo 101-1, \\ 119526, Moscow, Russia (e-mail: saurin@ipmnet.ru)
}

\section{INTRODUCTION}

The design of control strategies for dynamic systems with distributed parameters has been actively developed in recent decades. Optimization of elastic structure dynamics is an important part of a large variety of applications in science and engineering. Two different methods to the control design for distributed parameter processes can be emphasized. In the first approach, co-called late lumping, the control is directly designed for distributed parameter models and then converted to a finite approximation. In applications, the second approach, early lumping, is used for numerical control design. Accordingly, the initialboundary value problem is first discretized and reduced to a system of ordinary differential equations.

One of the disadvantages of the early lumping is that it is rather difficult to relate the discretized system with its original distributed model. However, this connection can be estimated by following the method of integrodifferential relations (MIDR) [1]. These estimates allow us to qualify finite-dimensional modelling, refine a coarse solution and make necessary corrections of the control law. The MIDR was extended in [2] to the optimal control design of elastic rod motions. In the paper, this approach is combined with the Ritz method and FEM to minimize the mean energy distributed in an elastic structure during controlled processes.

\section{PROBLEM STATEMENT}

Longitudinal displacements of a thin rectilinear elastic rod are considered. One end of the rod at $x=0$ can move in accordance with some control law $u(t)$ whereas the other end at $x=L$ is free of load (2). In accordance with the MIDR, the controlled motions of the elastic rod can be describe in the variational form

$$
\begin{gathered}
\Phi\left[p^{*}, s^{*}, w^{*}\right]=\min _{p, s, w} \Phi[p, s, w]=0 \\
\Phi[p, s, w]=\int_{\Omega} \varphi d \Omega=0, \varphi=\frac{1}{2}\left(\rho(x) \eta^{2}+\kappa(x) \xi^{2}\right), \\
\eta(t, x)=w_{t}-\rho(x)^{-1} p, \xi(t, x)=w_{x}-\kappa(x)^{-1} s .
\end{gathered}
$$

with Newton's second law, the initial and boundary conditions as the local constraints

$$
\begin{gathered}
p_{t}=s_{x}, t=0: p=p_{0}(x), w=w_{0}(x) ; \\
x=0: w=w_{0}(0)+u(t), u(0)=0 ; x=L: s=0 .
\end{gathered}
$$

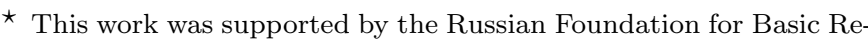
search, project nos. 13-01-00108, 14-01-00282, 15-01-00827 the Leading Scientific Schools Grants NSh-2710.2014.1, NSh-2954.2014.1.
}

Here, $\Omega=(0, T) \times(0, L)$ is the time-space domain with the time interval $T, \rho$ denotes the function of rod linear density, $\kappa$ is its distributed stiffness. The linear momentum density $p(t, x)$, the normal forces in the cross section $s(t, x)$, and the displacements $w(t, x)$ are unknown functions.

The control problem is to find such a function $u^{*}(t) \in \mathcal{U}$ that moves the elastic rod at the fixed time instant $T$ to the finale state

$$
t=T: \quad p=0, \quad w=w_{T}
$$

and minimizes the mean energy $\bar{E}$ of the rod

$$
\begin{gathered}
J\left[u^{*}\right]=\min _{u \in \mathcal{U}} J[u], \\
J=\bar{E}=\frac{1}{2 T} \int_{\Omega}\left(\rho^{-1} p^{2}+\kappa w_{x}^{2}\right) d t .
\end{gathered}
$$

\section{NUMERICAL APPROACH AND RESULTS}

Numerical algorithms for direct and inverse dynamical problems are developed based on the Ritz method and finite element technique with spline approximations of the unknown functions in the space-time domain. The energy error functional $T^{-1} \Phi$ is used to design integral criteria of the solution quality relying on the extremal properties of finite-dimensional variational problems.

The efficiency of the estimates proposed are demonstrated on a example of controlled motions for a homogeneous elastic rod. The piecewise polynomial control of longitudinal displacements is investigated. After FEM discretization, the original optimization problem is reduced to successive solving of two linear algebraic systems. The obtained numerical results are analyzed and discussed.

\section{REFERENCES}

[1] Kostin, G. V., Saurin, V. V. (2012). Integrodifferential Relations in Linear Elasticity. De Gruyter, Berlin.

[2] Kostin, G. V. (2007). The construction of optimal control for the motion of elastic bodies by using the method of integro-differential relations. J. Comput. Syst. Sci. Int., 46(4), 532-542. 


\title{
MATHMOD 2015 ABSTRACT VOLUME
}

\author{
MATHMOD Full Contributions \\ Operational Research and Planning
}


MATHMOD 2015 Abstract Volume 


\title{
A Game-Theoretic Calibration Approach for Agent-Based Planning Simulations
}

\author{
Julia Buwaya* Catherine Cleophas ** \\ * RWTH Aachen University, Research Group Advanced Analytics, \\ 52072 Aachen, Germany (e-mail: julia.buwaya@rwth-aachen.de). \\ ** RWTH Aachen University, Research Group Advanced Analytics, \\ 52072 Aachen, Germany (e-mail: catherine.cleophas@rwth-aachen.de).
}

\begin{abstract}
Simulations are increasingly employed to evaluate alternative planning strategies. With the number of model parameters and their interdependencies grow challenges regarding the validation and calibration of simulation systems. A major challenge lies in calibrating models that include emergent phenomena, as is a frequently stated feature of agent-based simulations. Here heterogeneous groups of agents are directly modeled to enable the consideration of agents' impact on the planning solution and its success. Difficulties in calibration arise as the system's behavior emerges from agents' individual decisions and actions, which cannot be fully observed. In the following we present a novel approach for calibrating agent input parameter values of agent-based simulations for decision support. The approach is based on a game-theoretic model representing an approximation of the dynamic simulation system. Its performance is tested in a meta-simulation framework on the example of a market simulation with a monopolist supplier. The meta-simulation setting allows us to compare calibration results to actual input parameters. The presented work provides an outline for efficient calibration of agent-based simulations in general using a game-theoretic model.
\end{abstract}

Keywords: Agents, Calibration, Decision support systems, Game theory, Integer programming, Simulation. 


\title{
A Reliability Measure for Time Series Forecasting Predictor
}

\author{
K.M. George, N. Park, \& Zhuxi Yang \\ Computer Science Department \\ Oklahoma State University \\ Stillwater, OK 74078 \\ \{kmg,npark, zhuxi\}@cs.okstate.edu
}

Keywords: Time Series, Forecasting, Concordance, Statistical Metric Spaces, Kendall's $\tau$, GINI's mean difference.

A time series is a sequence of observations that are measured at successive times. One of the most common examples of time series is the closing price of stock market indices such as S\&P 500 index, Dow Jones index etc. Time series analysis refers to methods used to develop models for analyzing the data and to extract meaningful statistics and other characteristics of the data. Time series forecasting is the use of a model to forecast future values based on some known past data values before they are measured.

Methods used to study time-series can be broadly classified as parametric methods and non-parametric methods. Several parametric and non-parametric modeling and forecasting methods can be found in the literature. Most methods are based on predictors. In this paper, we propose a method for associating a measure of reliability to a predictor based on the statistical metric space theory. We illustrate it utilizing measures of concordance. In order to accomplish this, we assume a forecasting approach that determines a predictor and constructs forecasting functions that map the predictor to a "current segment" / target and then extend the map to obtain forecast values.

A statistical metric space $S$ is a set of points with a probability distribution function $\pi(x ; p, q)$ of the variable $x$ associated to points $p$ and $q$ in $S$ subject to certain conditions. The function $\pi$ is interpreted as the probability that the distance between $p$ and $q$ is less than $x$. The forecasting approach taken in our research has an algorithmic slant, we interpret a time series $\mathbf{T}$ as a one-directional stream of numbers being printed, one at a time at uniform time intervals. Then, the forecasting problem is to estimate a set of (say $m$ ) numbers to be printed next. From the known stream, we construct a set of n-tuples of the form $p=<p_{1}, p_{2}, \ldots ., p_{n}>$. Each tuple is a continuous segment of the already printed stream and is a subsequence of the stream. Let $S_{n}$ denote the set of all n-tuples constructed from $\mathbf{T}$. Let $t$ be the member of $S_{n}$ consisting of the most recently printed $n$ numbers. We call $t$ the target sequence. We call $S_{n}-\{t\}$ a predictor set. The basic forecasting idea is to identify a member $p$ in $S_{n}-\{t\}$ similar to the target $t$ that is the best predictor candidate (predictor tuple). Then, find a mapping $h: p \rightarrow t$ (prediction/forecasting map) which is extended to calculate the future values of $\mathbf{T}$ as the forecast. We define a SMS structure on $S_{n}$ with a distribution $\pi(x ; p, q)$ where $p, q \in S_{n}$. Then, we interpret $\pi(x ; p, t)$ as a measure of reliability of the forecast.

To implement the approach, a pattern space of dimension $n(n-1) / 2$ is constructed from $S_{n}$. This could be a many-one mapping $M$, from $\mathrm{S}_{\mathrm{n}}$ to $\mathrm{P}_{\mathrm{n}}$. (This essentially partitions $S_{n}$ into clusters.) Choose a predictor $p$ such that the distance between $M(t)$ and $M(p)$ is a minimum (assuming a suitable metric). Once a predictor $p$ is determined, construct a mapping $h: p \rightarrow t$ and a distribution $\pi(x ; p, t)$ which imposes a SMS structure on $S_{n}$. The distribution will be interpreted as a confidence measure or measure of reliability. In short, the methodology consists of four steps namely, predictor set construction, determination of the optimal predictor, construction of prediction map, and construction of a distribution function. Three algorithms are proposed to implement the method. The concordance measures Kendall's $\tau$ and GINI's mean difference are used in the identification of predictor and construction of distribution function. The time complexities of Algorithm 1 and Algorithm 2 are $\mathrm{O}\left(\mathrm{n}^{2}\right)$ where $\mathrm{n}$ is the length of the target sequence. The complexity of Algorithm 3 depends on the method used to construct the mapping function. In the future, we expect to improve the complexities of the algorithms. 


\title{
Generation of Feasible Petri Net Based Scheduling Problem Solutions
}

\author{
Gašper Mušič ${ }^{*}$ \\ * University of Ljubljana, Faculty of Electrical Engineering, Tržaška \\ 25, 1000 Ljubljana, Slovenia (e-mail: gasper.music@fe.uni-lj.si).
}

\section{INTRODUCTION}

The paper focuses on Petri net (PN) models of scheduling problems in manufacturing and explores the possibilities of use of PN models in conjunction with state-of-the-art local search algorithms. The previous works on parameterized conflict resolution strategy and neighbouring solution generation procedures are generalized, so besides standard flow-shop and job-shop problems also less restricted problem classes can be addressed. This way the proposed approach brings PN based scheduling methods closer to the practical needs in manufacturing and services.

\section{PN REPRESENTATIONS OF SCHEDULING PROBLEMS}

Petri nets (Murata, 1989) can be used to effectively model all standard scheduling problem classes (Mušič, 2012). Nevertheless, Petri nets can model a much wider variety of scheduling problems so here some generalizations of the previous work are presented.

PN modelling of manufacturing scheduling problems is based on representation of job orders, workpieces and resource availability by places, and manufacturing actions by transitions. Operation processing times are assigned to transitions and a timing principle described in Napalkova et al. (2014) is used. Depending on the level of details additional places can be used to model activities in progress, intermediate buffers, etc.

\section{A GENERALIZATION OF PN BASED} NEIGHBOUR SOLUTION GENERATION STRATEGY

Local search moves from one candidate solution in the search space $S$ to another as long as necessary. A Petri net extension by predefined sequences (Löscher et al., 2007) enables to parameterize the transition firing sequence and changes in sequence vector can be used to explore various PN-represented schedules by the sequence-supervised simulation. The search for an optimal schedule is then driven by stepwise modifications of feasible firing sequences. A PN based neigbour solution generation strategy was presented in Mušič (2012) where a new solution is obtained by permutation of the jobs sequence for a chosen shared resource. The strategy works well for standard job-shop and flow-shop problems. Unfortunately it fails for more general cases so a generalized solution generation strategy is proposed here.

A process net is derived as a PN model of the scheduling problem without resources. A single sequence vector is used, which is determined by projecting last feasible firing sequence to the set of schedule relevant transitions $T_{j r}$. Given a feasible firing sequence $\sigma$, a neigbour solution is constructed by applying one of the following steps:

Change in resource allocation is made by a choice of transition in the projected firing sequence $\sigma_{r}=P r_{T_{j r}}(\sigma)$ that has an alternative. The alternative transitions are found as conflicting transitions in the process net that are also linked to resources in the full model. The chosen transition is then substituted by one of its alternatives, and the resulting sequence is used for a sequencesupervised simulation run of the original PN.

Change in the processing order. A transition $t_{s 1}$ in the projected firing sequence $\sigma_{r}=\operatorname{Pr}_{T_{j r}}(\sigma)$ is chosen as an initial swap candidate. Then a search for a precedence relation with $t_{s 1}$ within the remaining part of $\sigma_{r}$ is performed. The precedence check is made by the use of the process net and because of inherent parallelism of transition paths that belong to different jobs in such a PN model, only a small part of the PN graph needs to be examined. If a transition $t_{p 1}$ in a precedence relation with $t_{s 1}$ is found the transitions between $t_{s 1}$ and $t_{p 1}$ become swap candidates $T_{s 2}^{\prime}$. An additional precedence relation check is then made for every swap candidate $t_{s 2 c} \in T_{s 2}^{\prime}$ with the section of $\sigma_{r}$ between $t_{s 1}$ and $t_{s 2 c}$. The transitions with no detected precedence relations form a final set of swap candidates $T_{s 2}$. A $t_{s 2} \in T_{s 2}$ is chosen and a new $\sigma$ is obtained by exchange of $t_{s 1}$ and $t_{s 2}$ in $\sigma_{r}$, and a sequence-supervised simulation run.

The frequency of application of each of the two steps and transition choice rules depend on the implementation of the optimization algorithm.

\section{REFERENCES}

Löscher, T., Mušič, G., and Breitenecker, F. (2007). Optimisation of scheduling problems based on timed Petri nets. In 6th EUROSIM Congress on Modelling and Simulation, volume II. Ljubljana, Slovenia.

Murata, T. (1989). Petri nets: Properties, analysis and applications. Proc. IEEE, 77, 541-580.

Mušič, G. (2012). Schedule optimization based on Coloured Petri nets and local search. In 7th Vienna Conference on Mathematical Modelling, full paper preprint volume. ARGESIM, Vienna.

Napalkova, L., Piera, M.A., and Mušič, G. (2014). Performance evaluation of flexible manufacturing systems by coloured timed Petri nets and timed state space generation. In J. Campos, C. Seatzu, and X. Xie (eds.), Formal Methods in Manufacturing, 381-408. CRC Press. 


\title{
Adjacency in hospital planning
}

\author{
Wolfgang E. Lorenz*. Martin Bicher.** \\ Gabriel X. Wurzer *
}

\begin{abstract}
* Digital Architecture and Planning, Institute of Architectural Sciences, Vienna University of Technology, Treitlstraße 3, 1040 Vienna, Austria (e-mail: <firstname.surname>@tuwien.ac.at).

**dwh Simulation Services GmbH, Neustiftgasse 57-59, 1070 Vienna, Austria (e-mail: martin.bicher@dwh.at), Institute of Analysis and Scientific Computing, Vienna University of Technology, Wiedner Hauptstraße 8-10, 1040 Vienna, Austria (email: <firstname.surname>@tuwien.ac.at)
\end{abstract}

\section{INTRODUCTION}

In the preparatory phase of solving an architectural task, the definition of relationships between functional units - usually provided by the client, the staff and/or the architectural planners - plays a central role especially in the case of complex buildings, such as hospitals. Typically recorded in the form of a spreadsheet, information is mapped to relative weights between each pair of units in the range $[-1,1]$ which are put into a (symmetric) adjacency matrix (Fig.1 left). This paper deals with an effective method to automatically translate the matrix into a representation of nodes (corresponding to functional units) and edges (their relations), also called bubble diagram in architectural terms (Fig.1 right). The authors understand their tool as an addition to their proposed early-stage conception rather than for latestage verification and optimization (Wurzer, Lorenz 2012).
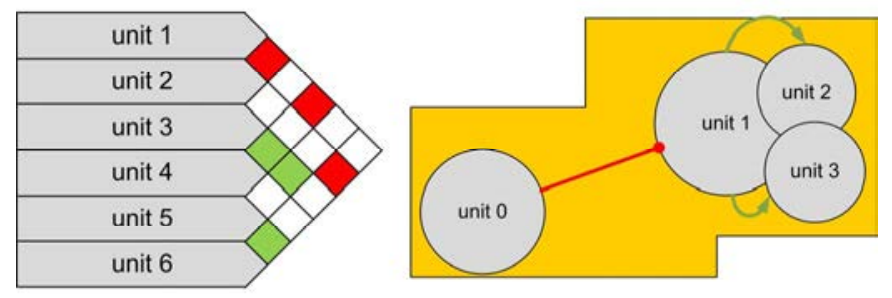

Fig. 1. Simple adjacency matrix (left); Possible spatial solution (right).

\section{THE MODEL}

The authors' method links together three steps of preliminary design: the adjacency matrix, the bubble diagram and to a limited extent, the zoning diagram (White 1986). The goal is to get a preliminary distribution of spaces (bubbles) inside a well-defined area once the adjacency matrix is defined by the client or the planner. As shown in Figure 1 (right) zoning corresponds visually to groups of nodes that stick together.

\subsection{Background}

Spatial relationships are often considered as spatial constraints during automated floor-plan generation for the final design (e.g. Elezkurtaj 2002), also taking into account parameters other than spatial relationships (e.g. noise or proportions). However, key point is that there is no "unique optimal solution for over-constrained problems"
(Balachandran and Gero 1987) and design tasks are overconstrained especially when thinking of hospitals. Thus we seek to establish a planning process where each task is handled only once and in the correct phase of a project (Lohfert 1973).

\subsection{From adjacencies to spatial distribution}

The basic model, implemented in NetLogo already arranges entities spatially and incorporates the requirements directly into the design, allowing an immediate verification (Fig.1 right). The advanced model behaves according to Newton's laws of gravitation and propagates changes to a single relationship immediately to the whole space layout. Considerations, finally, lead to the following second order differential equations:

$\ddot{\bar{x}}_{i}=\sum_{j=1}^{n} A_{i, j} \frac{\vec{x}^{\prime}-\vec{x}}{\left|\vec{x}^{\prime}-\vec{x}\right|^{q}}, \vec{x} \in \Omega^{\circ}$.

In order to avoid standstills a Monte Carlo simulation (rerunning) is proposed, which come up with several results. The final verification is then performed by other (design) criteria.

\section{ACKNOWLEDGEMENTS}

This paper was financed by the ZIT MODYPLAN project, under the call Smart Vienna 2012.

\section{REFERENCES}

Balachandran, M. and Gero, J.S. (1987). Dimensioning of architectural floor plans under conflicting objectives. In Environment and Planning B (14), pp. 29-37.

Elezkurtaj, T, Franck, G. (2002). Algorithmic Support of Creative Architectural Design. In Umbau, 19, Vienna, pp. 1-16.

Lohfert, P. (1973). Zur Methodik der Krankenhausplanung, Werner Verlag, Düsseldorf.

White, E.T. (1986). Space adjacency analysis: Diagramming information for Architectural Design, Architectural Media Tallahassee, USA.

Wurzer, G., Lorenz, W. E. (2012). Pre-Tender Hospital Simulation Using Naive Diagrams As Models. In Proceedings of the International Workshop on Innovative Simulation for Health Care 2012, Dime Università Di Genova, pp. 157-162. 


\title{
MODYPLAN: Early-Stage Hospital Simulation based on Treatment Chains
}

\author{
Gabriel Wurzer*. Wolfgang E. Lorenz.* \\ Matthias Rößler**, Irene Hafner**, Barbara Glock**, Martin Bruckner**, Niki Popper** \\ *Vienna University of Technology, Architectural Sciences (E259), Vienna 1040, Austria \\ (Tel: 01-58801-27201; e-mail: <firstname.surname>@tuwien.ac.at). \\ **DWH Simulation Services, Vienna 1070, Austria (e-mail: <firstname.surname>@dwh.at) and \\ Vienna University of Technology, Analysis and Scientific Computing (E101), Vienna 1040, Austria \\ (Tel: 01-58801-10115; e-mail: <firstname.surname>@tuwien.ac.at).
}

\begin{abstract}
Discrete-event simulation of hospitals typically specifies flow by means of a process graph through which patients are routed. While this is generally fine for models in which processes are clearly defined, e.g. smaller units such as emergency departments, it falls short of capturing the fact that a patient can in principle go from one unit to any other unit if medical procedure requires it. This problem becomes even more evident when modeling a whole hospital, at which level specifying each individual treatment through directed edges and nodes becomes unviably complex. In the past three years, we have been developing a combined hospital simulation/space design tool in which processes are defined individually by patient, as sequence of visited units imported from the Hospital Information System (treatment chains). The major advantage of this approach lies in the fact that we can now design a future space layout in which assumed capacities (staffing, equipment, required space) can be tested against the recorded patient volume, using an Agent-Based Simulation to re-enact each treatment chain. In contrast to other hospital simulations, this approach targets early stages of architectural conception, during which the actual building structure is elaborated. Using the method, we can compare and contrast different layouts during that stage, making simulation a driver for design rather than a tool for late optimization within the final floor plan.
\end{abstract}

\section{INTRODUCTION}

In this paper, we draw together the lessons learned during the development of our combined hospital simulation/space design suite called Modular Dynamic Planning and Simulation for Health Care Facilities, (MODYPLAN) which is based upon the idea of schedule-based simulation (see section 2) using the actual treatment data of a clinic, even though in anonymized form. One of the main realizations leading to the development of the tool was that the hospitals are not conceived from scratch - they are based on preexisting spatial templates (e.g. Neufert 2000) which are adapted to fit the expected patient volume and specialization. There are, nevertheless, a variety of possible options to choose from, leading to the questions of "How to compare two spatial layouts?", which is where simulation comes into play: By letting agents simulate their individual schedule (coined as treatment chain, see section 3), they utilize spaces which have a finite capacity. By recording this utilization, space occupancy and agent histories among different layouts, the program can compare the "performance" of each of them.

From a project view, we have just finished the core implementation and are currently modeling a hospital as a testcase. Our hospital simulation method relies on treatment chains stemming from a Hospital Information System. In contrast to classical process- based simulation, we have no notion of edges; the transitioning between nodes is rather based on an agent's prescribed schedule, making it easy to capture the real flow between different units, departments or even hospitals. The approach has been implemented as our own simulation suite, ((MODYPLAN)), which we will bring to the market in spring of next year.

\section{AUTHORS}

Gabriel Wurzer has done his Ph. D. on Process Visualization and Simulation for Hospital Planning (Vienna UT, 2011). His research in architectural sciences focuses on tool support for early-stage planning of complex buildings.

Wolfgang Lorenz has a $\mathrm{Ph}$. D. degree in Architecture for his thesis on Analysis of Fractal Architecture using the BoxCounting Method (Vienna UT, 2014). He is lecturer on programming for architects and simulation.

Matthias Rößler received his Master's degree on Coupling of Thermodynamical Systems (Vienna UT, 2012) and is currently working on simulation models for DWH Simulation Services as well as on clinical information systems.

Irene Hafner has done her Master's degree on CoSimulation using the Building Controls Virtual Test-Bed. Her current research concerns cooperative and multi-rate simulation, healthcare and production planning.

Barbara Glock Barbara Glock has gotten her Master on $A$ System Dynamics Model of the Prevalance of Obesity in Austria (Vienna UT, 2014). Her current work is in the field of coupling different modelling methods.

Martin Bruckner is a specialist on Agent-Based Modeling currently working for DWH Simulation services. He has completed his $\mathrm{PhD}$ in that same subject and is currently in the way of defending it before Vienna UT.

Niki Popper has a degree in Mathematics and is CEO of DWH Simulation Services, a professional simulation company based in Vienna. He is also lecturer at Vienna 
MATHMOD 2015 Abstract Volume 


\section{MATHMOD 2015 ABSTRACT VOLUMES}

MATHMOD Minisymposium Biomathematics: What's new? 
MATHMOD 2015 Abstract Volume 


\title{
Stochastic model for HIV dynamics in HIV specific helper cells
}

\author{
Carla M.A. Pinto* Ana R.M. Carvalho** \\ * School of Engineering, Polytechnic of Porto, and Centre for \\ Mathematics of the university of Porto, Rua Dr António Bernardino \\ de Almeida, 431, 4200-072 Porto, Portugal (e-mail:cap@isep.ipp.pt). \\ ** Faculty of Sciences, University of Porto, Rua do Campo Alegre s/n, \\ 4440-452 Porto, Portugal (e-mail: up200802541@fc.up.pt)
}

\begin{abstract}
In this paper, we analyse a mathematical model for the dynamics of $\mathrm{CD} 4^{+} \mathrm{T}$ cells in human immunodeficiency virus (HIV) transmission. The model includes antiretroviral therapy (ART), combining reverse transcriptase inhibitors (RTIs) and protease inhibitors (PIs), and drug resistance. We modify a deterministic model proposed in the literature to include stochasticity in the mortality rates of $\mathrm{CD}^{+} \mathrm{T}$ cells and of virus particles. We approximate the probability distributions of the time $t$ to elimination of drug-sensitive infected $\mathrm{CD} 4^{+} \mathrm{T}$ cells and virus particles, using gamma or lognormal functions.
\end{abstract}

Keywords: HIV, mathematical model, deterministic models, stochastic models

\section{INTRODUCTION}

HIV/AIDS epidemics is characterized by a progressive failure of the immune system. AIDS leaves the human body vulnerable to life-threatening opportunistic infections and lymphoma. CD4 ${ }^{+} \mathrm{T}$ cells are important helper cells that organize the immune system's response to bacteria, fungi, and viruses, namely the HIV virus. A $\mathrm{CD} 4^{+} \mathrm{T}$ cells count lower than some value, usually 600 cells $/ \mathrm{mm}^{3}$ indicates the individual is infected with HIV. The treatment for HIV consists of antiretroviral drugs (ART) that reduce HIV transmission and AIDS-related mortality. These drugs work in the body by slowing down the replication of HIV. In the last 2 or 3 decades researchers focused in developing mathematical models for the dynamics of $\mathrm{CD}^{+}{ }^{+} \mathrm{T}$ cells in HIV infection (Wodarz and Hamer [2007]). Wodarz and Hamer [2007] proved that HIV virus ability to infect $\mathrm{CD} 4^{+} \mathrm{T}$ cells influences HIV infection patterns. Namely, the spread of infected $\mathrm{CD} 4{ }^{+} \mathrm{T}$ cells fuels the virus spread, at higher virus loads, and the infected $\mathrm{T}$ cells provide shelter for virus during ART. The framework of the paper is as follows. In Section 2, we describe the deterministic and proposed stochastic models. In Section 3 we present numerical simulations of the model. In Section 4, we conclude our work and provide future research lines.

\section{DESCRIPTION OF THE MODELS}

In this section we describe the proposed stochastic model based on the deterministic model studied in Wang et al. [2014].

\subsection{Deterministic model}

The deterministic model describes the dynamics of the populations of uninfected $\mathrm{CD} 4^{+} \mathrm{T}$ cells, $T$, drug-sensitive infectious virus, $V_{s}$, drug-resistant infectious virus, $V_{r}$, drug-sensitive infected $\mathrm{CD} 4^{+} \mathrm{T}$ cells, $T_{s}$ and drug-resistant infected CD4 ${ }^{+} \mathrm{T}$ cells, $T_{r}$.

The epidemiology of the disease is as follows. The uninfected CD $4^{+}$ $\mathrm{T}$ cells, $T$, are produced at a rate $\lambda$ and die at a rate $d$. These cells, when in contact with HIV, get infected, at a rate $k_{s}$, by drug-sensitive viruses and move, $\tau$ time units later, to the $T_{s}$ class. Moreover, $T$ cells may be infected, at a rate $k_{r}$, by drug-resistant viruses and move to the $T_{r}$ class, after $\tau$ time units later. The term $e^{-m \tau}$ represents the probability of $T_{s}$ and $T_{r}$ cells surviving in the interval $\tau$, where $1 / m$ is the average life time of infected CD $4^{+} \mathrm{T}$ cells, before they become actively productive.

The proportion of $T_{s}$ and $T_{r}$ cells that after contact with the virus is not eliminated by RTIs is $1-n_{r t}^{s}$ and $1-n_{r t}^{r}$, respectively. Parameters $n_{r t}^{s}$ and $n_{r t}^{r}$ Throughout the infection, a proportion $u,(0<u<1)$, of $T_{s}$ cells can become $T_{r}$ cells.

The infected $\mathrm{CD}^{+} \mathrm{T}$ cells and the virus particles die at rates $\delta$ and $c$, respectively. The $V_{s}$ and $V_{r}$ particles are produced by the corresponding infected $\mathrm{CD} 4^{+} \mathrm{T}$ cell populations, with bursting sizes of drug-sensitive strain, $N_{s}$, and of drug-resistant strain, $N_{r}$. The proportion of virus particles that is not eliminated by PIs is $1-n_{p}^{s}$ and $1-n_{p}^{r}$, where $n_{p}^{s}$ is the efficacy of PIs for wild type strain, and $n_{p}^{r}$ is the efficacy of PIs for mutants. 1 The following nonlinear system of ordinary differential equations describes the dynamics of the deterministic model Wang et al. [2014]:

$$
\begin{aligned}
\frac{d T(t)}{d t}= & \lambda-d T-k_{s}\left(1-n_{r t}^{s}\right) V_{s}(t) T(t)-k_{r}\left(1-n_{r t}^{r}\right) V_{r}(t) T(t) \\
\frac{d T_{s}(t)}{d t}= & (1-u) k_{s} e^{-m \tau}\left(1-n_{r t}^{s}\right) V_{s}(t-\tau) T(t-\tau)-\delta T_{s}(t) \\
\frac{d V_{s}(t)}{d t}= & N_{s} \delta\left(1-n_{p}^{s}\right) T_{s}(t)-c V_{s}(t) \\
\frac{d T_{r}(t)}{d t}= & u k_{s} e^{-m \tau}\left(1-n_{r t}^{s}\right) V_{s}(t-\tau) T(t-\tau) \\
& +k_{r} e^{-m \tau}\left(1-n_{r t}^{r}\right) V_{r}(t-\tau) T(t-\tau)-\delta T_{r}(t) \\
\frac{d V_{r}(t)}{d t}= & N_{r} \delta\left(1-n_{p}^{r}\right) T_{r}(t)-c V_{r}(r)
\end{aligned}
$$

\subsection{Stochastic model}

$\mathrm{CD} 4^{+} \mathrm{T}$ cells death and virus's clearance rates are affected by complex biological phenomena (Perelson et al. [1996]). Thus, it is believed that there is randomness involved in these mortality rates. Taking this into account, we include randomness in the previous model (1), replacing parameters $d, \delta$ and $c$ by $d+\sigma_{1} \dot{B_{1}}(t), \delta+\sigma_{1} \dot{B_{1}}(t)$ and $c+\sigma_{2} \dot{B}_{2}(t)$, respectively. Thus, the corresponding system of stochastic differential equations is given by: 


$$
\begin{aligned}
d T(t)= & \left(\lambda-d T-k_{s}\left(1-n_{r t}^{s}\right) V_{s}(t) T(t)-k_{r}\left(1-n_{r t}^{r}\right) V_{r}(t) T(t)\right) d t \\
& -\sigma_{1} T(t) d B_{1}(t) \\
d T_{s}(t)= & \left((1-u) k_{s} e^{-m \tau}\left(1-n_{r t}^{s}\right) V_{s}(t-\tau) T(t-\tau)-\delta T_{s}(t)\right) d t \\
& -\sigma_{1} T(t) d B_{1}(t) \\
d V_{s}(t)= & \left(N_{s} \delta\left(1-n_{p}^{s}\right) T_{s}(t)-c V_{s}(t)\right) d t-\sigma_{2} V_{s}(t) d B_{2}(t) \\
d T_{r}(t)= & \left(u k_{s} e^{-m \tau}\left(1-n_{r t}^{s}\right) V_{s}(t-\tau) T(t-\tau)\right. \\
& \left.+k_{r} e^{-m \tau}\left(1-n_{r t}^{r}\right) V_{r}(t-\tau) T(t-\tau)-\delta T_{r}(t)\right) d t \\
& -\sigma_{1} T_{r}(t) d B_{1}(t) \\
d V_{r}(t)= & \left(N_{r} \delta\left(1-n_{p}^{r}\right) T_{r}(t)-c V_{r}(r)\right) d t-\sigma_{2} V_{r}(t) d B_{2}(t)
\end{aligned}
$$

$B_{1}(t)$ and $B_{2}(t)$ are independent standard Wiener processes. Note that the noise intensity $\sigma$ and the Brownian motion $B(t)$ are the same for uninfected $\mathrm{CD} 4^{+} \mathrm{T}$ cells and infected $\mathrm{CD} 4^{+} \mathrm{T}$ cells, but different for virus particles. It is assumed that biological factors affecting mortality rates of infected $\mathrm{CD} 4^{+} \mathrm{T}$ cells and uninfected $\mathrm{T}$ cells are similar.

\section{NUMERICAL RESULTS}

In this section we present the numerical results for the stochastic model (2). The parameters used in the simulations are in Table (1) and the initial conditions are set to $T(0)=1000, T_{s}(0)=1$ and $V_{s}(0)=T_{r}(0)=V_{r}(0)=0.01$.

\begin{tabular}{ccc}
\hline Parameter & Value & Reference \\
\hline$\lambda$ & 100 & Luo et al. [2012] \\
$m$ & 0.1 & Althaus and De Boer [2011] \\
$k_{s}$ & 0.01 & Wang et al. [2014] \\
$k_{r}$ & $2 \times 10^{-6}$ & Luo et al. [2012] \\
$u$ & $3 \times 10^{-5}$ & Luo et al. [2012] \\
$\delta$ & 1 & Wang et al. [2014] \\
$N_{s}$ & 4800 & Althaus and De Boer [2011] \\
$N_{r}$ & 4000 & Luo et al. [2012] \\
$c$ & 23 & Althaus and De Boer [2011] \\
$\tau$ & 1 & Wang et al. [2014] \\
$n_{r t}^{s}$ & 0.4 & Wang et al. [2014] \\
$n_{r t}^{r}$ & 0.2 & Wang et al. [2014] \\
$n_{p}^{s}$ & 0.1 & Wang et al. [2014] \\
$n_{p}^{r}$ & 0.1 & Wang et al. [2014] \\
$\sigma_{1}$ & 0.1 & Wang et al. [2014] \\
$\sigma_{2}$ & 0.1 & Wang et al. [2014] \\
$\Delta_{t}$ & 0.001 & Wang et al. [2014] \\
\hline
\end{tabular}

Table 1. Parameters used in the numerical simulations of models (1)-(2).

We estimate the probability of the time $t$ to elimination of drugsensitive infected $\mathrm{CD} 4^{+} \mathrm{T}$ cells in the body. From the observation of Figure 1, it is more likely that the body kills all drug-sensitive infected $\mathrm{CD} 4^{+} \mathrm{T}$ cells in 65 days. One can approximate this probability function by a gamma function of the form $\Gamma(20,0.197)$, or by a lognormal function $Y=\log (X)$, where $X \sim N(1.35,0.23)$.

Next, we estimate the probability of the time $t$ to elimination of drugsensitive virus particles in the body (Figure 2). We observe that in $55 \%$ of the trajectories, the body eliminates the drug-sensitive virus particles in less than 70 days. Moreover, in $22 \%$ of the trajectories virus persist 75 days and in $18 \%$ of the trajectories virus is killed at 65 days. This probability of the time $t$ to elimination of drugsensitive virus particles may be approximated by a gamma function $\Gamma(15,0.1905)$, or by a lognormal distribution, $Y=\log (X)$, where $X \sim N(1.1,0.247)$.

\section{CONCLUSIONS}

We proposed a stochastic model for $\mathrm{CD} 4{ }^{+} \mathrm{T}$ cells dynamics in HIV transmission. The model includes ART, combining RTIs and PIs,

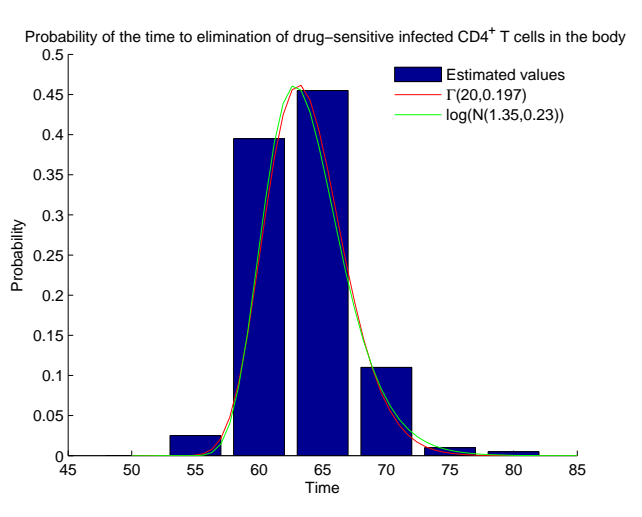

Fig. 1. Probability distribution of the time $t$ to elimination of drugsensitive infected $\mathrm{CD} 4^{+} \mathrm{T}$ cells in the body, for parameter values given in Table 1 and given initial conditions.

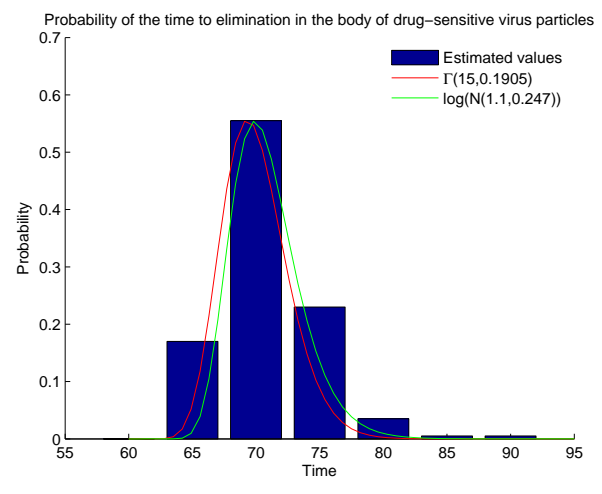

Fig. 2. Probability distribution of the time $t$ to elimination in the body of drug-sensitive virus particles, for parameter values given in Table 1 and given initial conditions.

and drug resistance. We approximate the probability distributions of the time $t$ to elimination of drug-sensitive infected $\mathrm{CD} 4^{+} \mathrm{T}$ cells and virus particles by gamma or lognormal functions. Future work will focus in verifying predictions from the model with real data.

\section{REFERENCES}

Althaus, C.L. and De Boer, R.J. (2011). Implications of ctl-mediated killing of hiv-infected cells during the non-productive stage of infection. PLoS ONE, 6(2), e16468.

Luo, R., Piovoso, M., Martinez-Picado, J., and Zurakowski, R. (2012). Hiv model parameter estimates from interruption trial data including drug efficacy and reservoir dynamics. PLOS ONE, 7(7), e40198.

Perelson, A., Neumann, A., Markowitz, M., Leonard, J., and Ho, D. (1996). Hiv-1 dynamics in vivo: Virion clearance rate, infected cell lifespan, and viral generation time. Science, 271, 1582-1586.

Roy, S. and Wodarz, D. (2012). Infection of hiv specific cd4 t helper cells and the clonal composition of the response. Journal of Theoretical Biology, 304, 143 - 151.

Staprans, S., Barry, A., Silvestri, G., Safrit, J., Kozyr, N., Sumpter, B., Nguyen, H., McClure, H., Montefior, i.D., Cohen, J., and Feinberg, M. (2004). Enhanced siv replication and accelerated progression to aids in macaques primed to mount a cd4 t cell response to the siv envelope protein. Proceedings of the National Academy of Sciences USA, 101(35), 13026-13031.

Wang, Y., Brauer, F., Wu, J., and Heffernan, J. (2014). A delaydependent model with hiv drug resistance during therapy. Journal of Mathematical Analysis and Applications, 414(2), 514 - 531.

Wodarz, D. and Hamer, D. (2007). Hiv treatment as prevention: Natural experiments highlight limits of antiretroviral treatment as hiv prevention. Mathematical Biosciences, 209, 14-29. 


\title{
The effect of noise intensity in a stochastic model for HIV-specific helper cells
}

\author{
Ana R.M. Carvalho* Carla M.A. Pinto** \\ * Faculty of Sciences, University of Porto, Rua do Campo Alegre s/n, \\ 4440-452 Porto, Portugal (e-mail: up200802541@fc.up.pt). \\ ** School of Engineering, Polytechnic of Porto, and Centre for \\ Mathematics of the University of Porto, Rua Dr António Bernardino \\ de Almeida, 431, 4200-072 Porto, Portugal (e-mail:cap@isep.ipp.pt).
}

\begin{abstract}
In this paper, we analyze a stochastic model for the dynamics of $\mathrm{CD} 4^{+} \mathrm{T}$ cells in immunodeficienvy virus (HIV) transmission. The model includes antiretroviral therapy (ART), combining the reverse transcriptase inhibitors (RTIS) and the protease inhibitors (PI), and drug resistance. The stochastic terms are placed in the mortality rates of the $\mathrm{CD} 4^{+} \mathrm{T}$ cells and of the virus particles. The model is simulated for different values of the noise intensity. We observe that as the noise intensity is reduced, the model approaches the patterns of the deterministic model.
\end{abstract}

Keywords: HIV, mathematical model, stochastic models

\section{INTRODUCTION}

The human immunodeficiency virus (HIV) is a retrovirus that causes acquired immunodeficiency syndrome (AIDS) characterized by progressive failure of the immune system, in particular by depleting the $\mathrm{CD}^{+} \mathrm{T}$ cells.

Mortality rates of $\mathrm{CD}^{+} \mathrm{T}$ cells and the clearance rate of HIV involve many complex biological phenomena. For instance, the clearance rate of HIV could be attributed to binding and entry into $\mathrm{CD} 4^{+} \mathrm{T}$ cells Perelson et al. [1996]. Thus, it is fairly accepted that there is randomness involved in these mortality rates. The mathematical modelling of these phenomena involve stochastic terms (that add noise to the system), thus the models are known as stochastic models.

Bearing these ideas in mind, the paper is organized as follows. In Section 2, we briefly describe the stochastic model, a modification of the deterministic model proposed in Wang et al. [2014]. In Section 3, we present the results of the numerical simulations. Finally in Section 4, we conclude our work.

\section{DESCRIPTION OF THE MODEL}

The model describes the dynamics of the populations of uninfected $\mathrm{CD} 4^{+} \mathrm{T}$ cells, $T$, drug-sensitive infectious virus, $V_{s}$, drug-resistant infectious virus, $V_{r}$, drug-sensitive infected $\mathrm{CD}^{+} \mathrm{T}$ cells, $T_{s}$, and drug-resistant infected $\mathrm{CD}^{+} \mathrm{T}$ cells, $T_{r}$. The uninfected $\mathrm{CD} 4^{+} \mathrm{T}$ cells, $T$, are produced at a rate $\lambda$ and die at a rate $d$. These cells, when in contact with HIV, get infected, at a rate $k_{s}$, by drug-sensitive viruses and move, $\tau$ time units later, to the $T_{s}$ class. Moreover, $T$ cells may be infected, at a rate $k_{r}$, by drug-resistant viruses and move to the $T_{r}$ class, after $\tau$ time units later. The term $e^{-m \tau}$ represents the probability of $T_{s}$ and $T_{r}$ cells surviving in the interval $\tau$, where $1 / m$ is the average life time of infected $\mathrm{CD}^{+} \mathrm{T}$ cells, before they become actively productive. The proportion of $T_{s}$ and $T_{r}$ cells that after contact with the virus is not eliminated by RTIs is $1-n_{r t}^{s}$ and $1-n_{r t}^{r}$, respectively. Parameters $n_{r t}^{s}$ and $n_{r t}^{r}$ are the efficacy of RTIS for wild type and mutants, respectivety. Throughout the infection, a proportion $u$, $(0<u<1)$, of $T_{s}$ cells can become $T_{r}$ cells. The infected $\mathrm{CD} 4^{+} \mathrm{T}$ cells and the virus particles die at rates $\delta$ and $c$, respectively. The $V_{s}$ and $V_{r}$ particles are produced by the corresponding infected $\mathrm{CD} 4^{+} \mathrm{T}$ cell populations, with bursting sizes, $N_{s}$ and $N_{r}$, respectively. The proportion of virus particles that is not eliminated by PIs is $1-n_{p}^{s}$ and $1-n_{p}^{r}$, where $n_{p}^{s}$ and $n_{r}^{p}$ are efficacy of PIs for wild type and mutants, respectively. It is considered that there is randomness in mortality rates, replacing the parameters, $d, \delta$ and $c$ for $d+\sigma_{1} \dot{B}_{1}(t), \delta+\sigma_{1} \dot{B}_{1}(t)$ and $c+\sigma_{2} \dot{B}_{2}(t)$, respectively. Functions $B_{1}(t)$ and $B_{2}(t)$ are independent standard Wiener processes. Thus, the system of stochastic differential equations is as follows:

$$
\begin{aligned}
d T(t)= & \left(\lambda-d T-k_{s}\left(1-n_{r t}^{s}\right) V_{s}(t) T(t)-k_{r}\left(1-n_{r t}^{r}\right) V_{r}(t) T(t)\right) d t \\
& -\sigma_{1} T(t) d B_{1}(t) \\
d T_{s}(t)= & \left((1-u) k_{s} e^{-m \tau}\left(1-n_{r t}^{s}\right) V_{s}(t-\tau) T(t-\tau)-\delta T_{s}(t)\right) d t \\
& -\sigma_{1} T(t) d B_{1}(t) \\
d V_{s}(t)= & \left(N_{s} \delta\left(1-n_{p}^{s}\right) T_{s}(t)-c V_{s}(t)\right) d t-\sigma_{2} V_{s}(t) d B_{2}(t) \\
d T_{r}(t)= & \left(u k_{s} e^{-m \tau}\left(1-n_{r t}^{s}\right) V_{s}(t-\tau) T(t-\tau)\right. \\
& \left.+k_{r} e^{-m \tau}\left(1-n_{r t}^{r}\right) V_{r}(t-\tau) T(t-\tau)-\delta T_{r}(t)\right) d t \\
& -\sigma_{1} T_{r}(t) d B_{1}(t) \\
d V_{r}(t)= & \left(N_{r} \delta\left(1-n_{p}^{r}\right) T_{r}(t)-c V_{r}(r)\right) d t-\sigma_{2} V_{r}(t) d B_{2}(t)
\end{aligned}
$$

\section{NUMERICAL RESULTS}

In this subsection we present numerical simulations of the model (1). We apply the Milsthein method Mackevicius [2011]. The parameters used in the simulations are in Table 
(1) and the initial conditions are $T(0)=1000, T_{s}(0)=1$ and $V_{s}(0)=T_{r}(0)=V_{r}(0)=0.01$.

\begin{tabular}{cc}
\hline Parameters & Value \\
\hline$\lambda$ & 100 \\
$d$ & 0.1 \\
$m$ & 0.01 \\
$k_{s}$ & $2.4 \times 10^{-6}$ \\
$k_{r}$ & $2 \times 10^{-6}$ \\
$u$ & $3 \times 10^{-5}$ \\
$\delta$ & 1 \\
$N_{s}$ & 4800 \\
$N_{r}$ & 4000 \\
$c$ & 23 \\
$\tau$ & 1 \\
$n_{r t}^{s}$ & 0.4 \\
$n_{r t}^{r}$ & 0.2 \\
$n_{p}^{s}$ & 0.1 \\
$n_{p}^{r}$ & 0.1 \\
$\Delta_{t}$ & 0.001 \\
\hline
\end{tabular}

Table 1. Parameter values.

We decrease the values of $\sigma_{1}$ and $\sigma_{2}$, the noise intensities. In Figure 1, we observe that the stochastic model approaches asymptotically the disease free equilibrium.

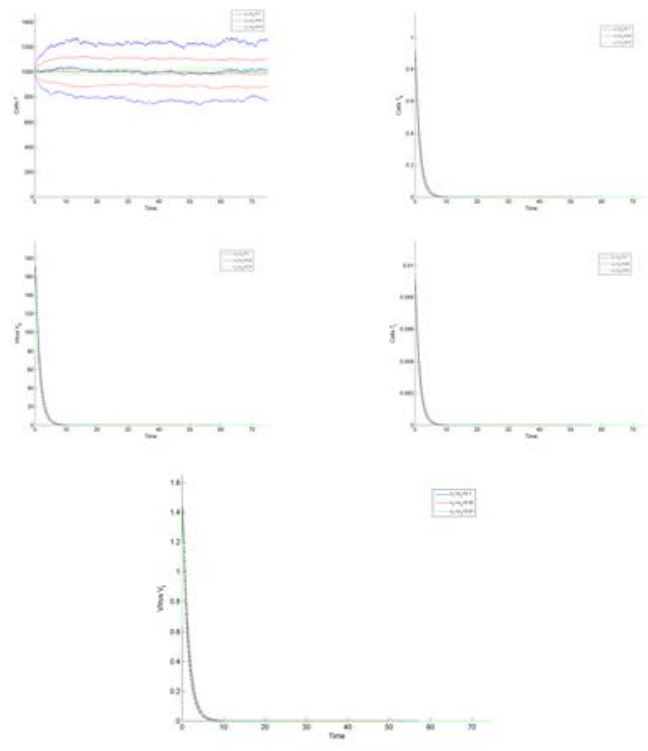

Fig. 1. Dynamics of the variables' averages of the stochastic model (1) using the method of Milsthein, for parameter values of Table 1 and given initial conditions. Blue - noise $\sigma_{1}=\sigma_{2}=0.1$, red - noise $\sigma_{1}=\sigma_{2}=0.05$, green - noise $\sigma_{1}=\sigma_{2}=0.01$.

In Figure 2, we observe that the stochastic model (1) converges to the drug-sensitive endemic equilibrium.

Figure 3 shows the drug-resistance endemic equilibrium of the stochastic model (1).

\section{CONCLUSIONS}

We proposed a stochastic model for $\mathrm{CD} 4^{+} \mathrm{T}$ cells dynamics in HIV transmission. The model includes ART, combining the RTIS and PI, and drug resistance. We simulated the model for different values of the noise intensity. We conclude that as the noise intensity decreases, the variability of the stochastic model decreases and approaches the deterministic model dynamics. This study will continue,

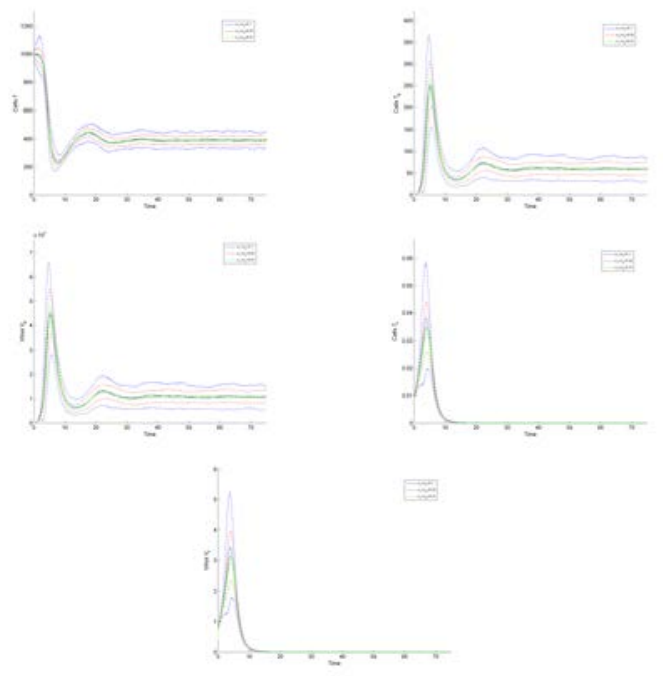

Fig. 2. Dynamics of the variables' averages of the stochastic model (1) using the method of Milsthein, for parameter values of Table 1 , except $k_{s}=2.4 \times 10^{-5}, k_{r}=2 \times 10^{-5}, u=3 \times 10^{-10}$, $N_{s}=4600$ and $N_{r}=2300$, and given initial conditions. Blue noise $\sigma_{1}=\sigma_{2}=0.1$, red - noise $\sigma_{1}=\sigma_{2}=0.05$, green - noise $\sigma_{1}=\sigma_{2}=0.01$

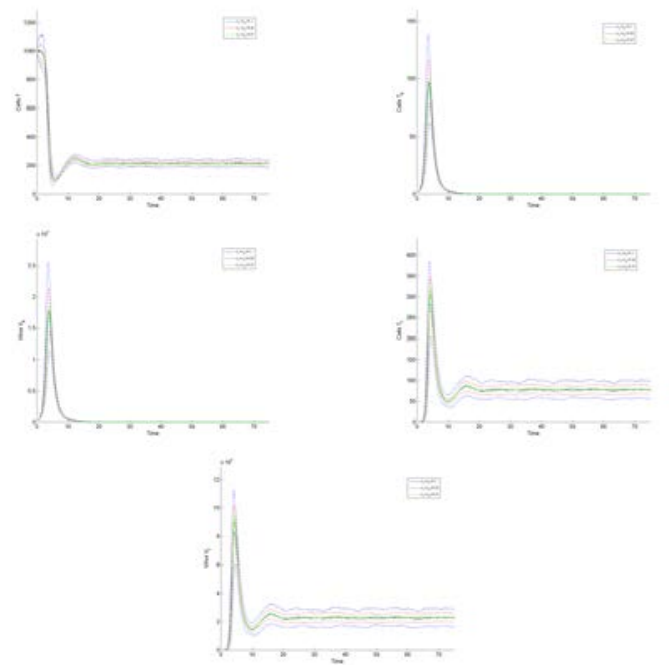

Fig. 3. Dynamics of the variables' averages of the stochastic model (1) using the method of Milsthein, for parameter values of Table 1 , except $k_{s}=2.4 \times 10^{-5}, k_{r}=2 \times 10^{-5}, N_{s}=4700$ and $N_{r}=7500$, and given initial conditions. Blue - noise $\sigma_{1}=\sigma_{2}=0.1$, red - noise $\sigma_{1}=\sigma_{2}=0.05$, green - noise $\sigma_{1}=\sigma_{2}=0.01$.

in a near future, with the comparison of these results with data from real patients.

\section{REFERENCES}

Mackevicius, V. (2011). John Wiley \& Sons, Inc.

Perelson, A.S., Neumann, A.U., Markowitz, M., Leonardy, M.M.J.M., and Ho, D.D. (1996). Hiv-1 dynamics in vivo: Virion clearance rate, infected cell lifespan, and viral generation time. Science, 271, 1582-1586.

Wang, Y., Brauer, F., Wu, J., and Heffernan, J.M. (2014). A delay-dependent model with $\{$ HIV $\}$ drug resistance during therapy. Journal of Mathematical Analysis and Applications, 414(2), $514-531$. 


\title{
Effect of drug-resistance in a fractional complex-order model for HIV infection
}

\author{
Carla M.A. Pinto* Ana R.M. Carvalho** \\ ${ }^{*}$ School of Engineering, Polytechnic of Porto, Porto, Portugal \\ (e-mail:cap@isep.ipp.pt). \\ ** Faculty of Sciences, University of Porto, Porto, Portugal (e-mail: \\ up200802541@fc.up.pt).
}

\begin{abstract}
We study a fractional complex-order (FO) model for drug-resistance in HIV infection during therapy. We simulate the model for different values of the fractional derivative of complex order (FD) $D^{\alpha \pm \jmath \beta}$, where $\alpha, \beta \in \mathbf{R}^{+}$. The FD is a generalization of the integer order derivative where $\alpha=1$ and $\beta=0$. The FO system reveals rich dynamics. The novelty of the paper is attributed to the dynamics of the model promoted by the variation of the complex-order derivative. The obtained interesting dynamics may point in other directions to model the intracellular delay.
\end{abstract}

Keywords: HIV, integer order model, fractional order model, drug-resistance

The human immunodeficiency virus (HIV) is a retrovirus that impairs the immune response system, by targeting the $\mathrm{CD} 4^{+}$helper $\mathrm{T}$ cells. The treatment for HIV/AIDS relies in antiretroviral drugs that suppress HIV viral load below the limit of detection. Some drug classes are the reverse transcriptase inhibitors (RTI) and the protease inhibitors (PI). In the last few years, the mathematical modeling of HIV epidemics and the efficiency of ART regimens has become a reality. Fractional calculus, i.e., non-integer order calculus, has been largely studied by mathematicians and engineers. In our work, we consider the Grünwald-Letnikov formulation. The fractional derivative of complex-order $\alpha \pm \jmath \beta \in \mathbf{C}$, when applied to a system of equations, results in complex valued outcomes. Hartley et al [1999] proposed the association of two complex-order derivatives. One of these associations is given in equation (2).
In this paper, we study a FO model for drug-resistance in HIV infection, during therapy. In Section 2, we describe the model. In Section 3, we simulate the model for distinct values of $\alpha$. In Section 4 , we conclude our work.

\section{DESCRIPTION OF MODEL}

The model describes the dynamics of the populations of the CD4 ${ }^{+}$ $\mathrm{T}$ cells and virus populations. The uninfected $\mathrm{CD} 4^{+} \mathrm{T}$ cells, $T$, are produced at a rate $\lambda$ and die at a rate $d$. When in contact with HIV, they get infected, at a rate $k_{s}$, by drug-sensitive viruses, $V_{s}$, and move to the drug-sensitive infected $\mathrm{CD} 4^{+} \mathrm{T}$ cells class, $T_{s}$. Moreover, $T$ cells may be infected, at a rate $k_{r}$, by drug-resistant viruses, $V_{r}$, and move to the drug-resistant infected $\mathrm{CD} 4^{+} \mathrm{T}$ cells $\left(T_{r}\right)$ class. The proportions of $T_{s}$ and $T_{r}$ cells that, after contact with the virus, are not eliminated by RTI are $1-n_{r t}^{s}$ and $1-n_{r t}^{r}$, respectively. Parameters $n_{r t}^{s}$ and $n_{r t}^{r}$ represent the efficacy rates of RTI for 


$$
Z\left\{\frac{1}{2}\left[D^{\alpha-\jmath \beta} x(t)+D^{\alpha+\jmath \beta} x(t)\right]\right\} \approx \frac{1}{T^{\alpha}}\left\{\sin \left[\beta \ln \left(\frac{1}{T}\right)\right]\left[\beta z^{-1}+\frac{1}{2} \beta(1-2 \alpha) z^{-2}+\cdots\right]+\cos \left[\beta \ln \left(\frac{1}{T}\right)\right]\left[-1+\alpha z^{-1}-\frac{1}{2} \beta\left(\alpha^{2}-\alpha-\beta^{2}+\cdots\right)\right] X(z)\right.
$$

wild type and mutants, respectively. Throughout the infection, a proportion $u,(0<u<1)$, of $T_{s}$ cells can become resistant to drugs and move to the class $T_{r} . T_{s}$ and $T_{r}$ cells die at a rate $\delta$ and viruses are cleared at a rate $c . V_{s}$ and $V_{r}$ particles are produced by the corresponding infected $\mathrm{CD} 4^{+}$populations, with bursting sizes of drug-sensitive strain, $N_{s}$, and of drug-resistant strain, $N_{r}$. The proportion of viruses that is not eliminated by PI is $1-n_{p}^{s}$ and $1-n_{p}^{r}$, where $n_{p}^{s}$ is the efficacy of PI for wild type strain, and $n_{p}^{r}$ is the efficacy of PI for mutants. The nonlinear system describing the dynamics of the fractional model of complex-order is:

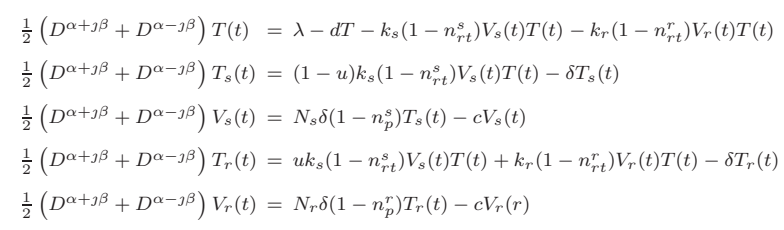

\section{NUMERICAL RESULTS}

In this section we simulate model (3). The parameters are $\lambda=75$, $d=0.1, r=0.03, T_{\max }=1500, k_{s}=2.4 \times 10^{-6}, k_{r}=2 \times 10^{-5}$, $u=3 \times 10^{-5}, \delta=1, N_{s}=4800, N_{r}=4000, c=23, n_{r t}^{s}=0.4$, $n_{r t}^{r}=0.2, n_{p}^{s}=0.1, n_{p}^{r}=0.1$. Initial conditions are $T(0)=1000$, $T_{s}(0)=1$ and $V_{s}(0)=T_{r}(0)=V_{r}(0)=0.01$. We simulate the model (3) for fixed $\beta=0.8$ and vary $\alpha \in\{0.5,0.6,0.7,0.8,0.9,1.0\}$. In Figure 1, we observe that the model (3) approaches asymptotically the disease free equilibrium. Faster transients are seen for decreasing values of $\alpha$. The slowest evolution to equilibrium occurs for $\alpha=1.0$, whereas the fastest transient is for $\alpha=0.5$. This property of the fractional systems is similar to the patterns seen for variation of delays in integer-order models (Wang et al [2014]).

\section{CONCLUSIONS}

We proposed a fractional complex-order model for drug-resistance during HIV therapy. Simulations of the model reveal that the variation of $\alpha$ is similar to the variation of the delay in integer order systems. Future work will focus in the inclusion of the CTL cells in the model.
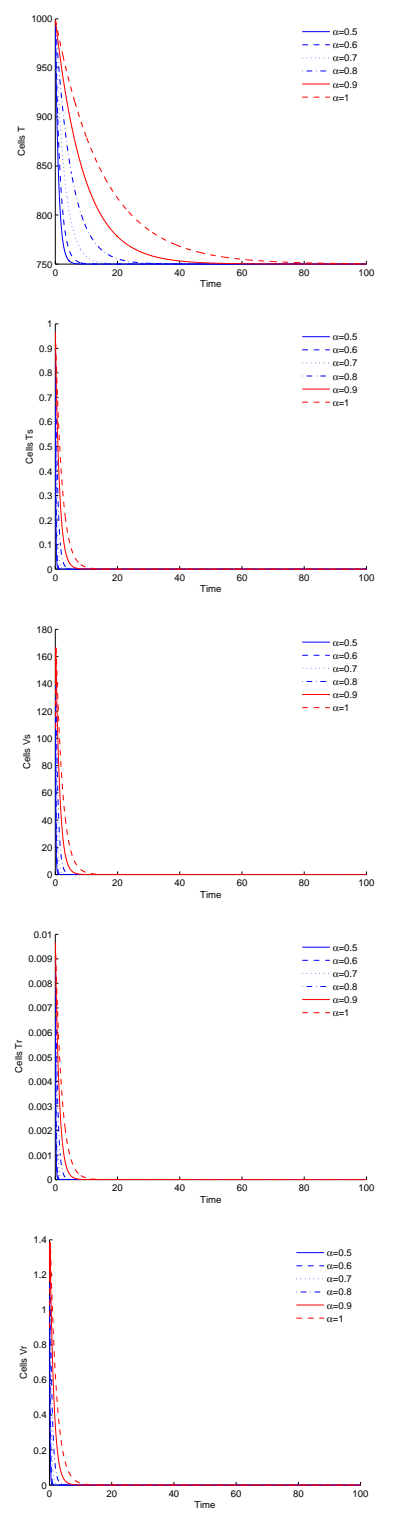

Fig. 1. Disease free equilibrium of the model (3).

\section{REFERENCES}

Hartley TT, Lorenzo CF and Adams JL (2005) Conjugated-order differintegrals. Proceedings of the ASME International Design Engineering Technical Conferences and Computers and Information in Engineering Conference, Long Beach, CA, DETC200584951, on CD-ROM.

Wang Y, Brauer F, Wu J and Heffernan JM (2014) A delaydependent model with HIV drug resistance during therapy. Journal of Mathematical Analysis and Applications, 414: 514-531. 


\title{
Modeling the dynamics of the three stages of HIV infection
}

\author{
Ana R.M. Carvalho** Carla M.A. Pinto* \\ * School of Engineering, Polytechnic of Porto, Porto, Portugal \\ (e-mail:cap@isep.ipp.pt). \\ ** Faculty of Sciences, University of Porto, Porto, Portugal (e-mail: \\ up200802541@fc.up.pt).
}

\begin{abstract}
We study the dynamic of HIV epidemics in the three stages of infection. The proposed mathematical model includes macrophages, CD4 ${ }^{+} \mathrm{T}$ cells and CTLs, and drug-resistance. Numerical simulations of the model show the disease free equilibrium. Future work will focus on the analyses of the equilibria of the model and on the dynamics of the model in the different stages of disease.
\end{abstract}

Keywords: HIV, helper cells, macrophages, CTL, treatment, drug-resistance

The human immunodeficiency virus (HIV) is a deadly disease in the lack of treatment. Time since the initial infection till death in an untreated patient is 9-10 years. HIV infection dilapidates patient's health, since it attacks three extremely important cell populations, the $\mathrm{CD} 4^{+} \mathrm{T}$ cells, macrophages and dendritic cells. The later help to establish the immune system's responses in the presence of viruses and bacteria. The cytotoxic lymphocytes (CTLs) or immune response cells, are the cells set out to eliminate infection by killing infected cells. The treatment for HIV/AIDS relies in antiretroviral drugs that suppress HIV viral load below the limit of detection. The most adopted treatment is a combination of different drugs, such as reverse transcriptase (RTI) and protease inhibitors (PI) that prevent the connversion of HIV RNA to HIV DNA and the production of infectious viral particles, respectively. In the last few years, there has been a major breakthrough in the mathematical modeling of HIV epidemics and the efficiency of ART regimens. Hadjandreou et al Hadjiandreou et al [2009], propose a model for long term HIV dynamics, subjected to continuous and treatment interruptions. In this paper, we study a model HIV epidemics that includes macrophages, CD4 ${ }^{+} \mathrm{T}$ cells, CTLs and drug-resistance. In Section 2, we describe the model. In Section 3, we simulate the model. In Section 4, we conclude our work.

\section{DESCRIPTION OF MODEL}

The model describes the dynamics of the populations of the uninfected $\mathrm{CD}^{+} \mathrm{T}$ cells, $T$, susceptible $\mathrm{T}$ cells, $T_{s}$, resistant $\mathrm{T}$ cells, $T_{r}$, macrophages, $M$, susceptible macrophage, $M_{s}$, resistant macrophages, $M_{r}$, CTLs, $Z$, and susceptible, $V_{s}$, and resistant, $V_{r}$, virus populations. The first two terms in the equations modeling the $T$ and $M$ cells, represent the source of new $T$ and $M$ cells, respectively. CTLs are generated by the first three terms of the corresponding equation. The infection parameters $k_{1}, k_{2}, k_{4}$ denote cells' infection by viruses and infected macrophages. Rates $k_{3}, k_{5}$ denote the killing of the infected helper and macrophage cells by CTLs. Death rates are represented by $\delta_{i}$. Viruses are cleared at a rate $c$. The dynamics concerning drug-resistance are as follows. The 
proportions of $T_{s}$ and $T_{r}$ cells that, after contact with the virus, are not eliminated by RTI are $1-n_{r t}^{s}$ and $1-n_{r t}^{r}$, respectively. Parameters $n_{r t}^{s}$ and $n_{r t}^{r}$ represent the efficacy rates of RTI for wild type and mutants, respectively. Throughout the infection, a proportion $u$, $(0<u<1)$, of $T_{s}$ cells can become resistant to drugs and move to the class $T_{r} . V_{s}$ and $V_{r}$ particles are produced by the corresponding infected $\mathrm{CD}^{+}$and macrophages populations, with bursting sizes of drug-sensitive strain, $N_{s}$, and of drug-resistant strain, $N_{r}$. The proportion of viruses that is not eliminated by PI is $1-n_{p}^{s}$ and $1-n_{p}^{r}$, where $n_{p}^{s}$ is the efficacy of PI for wild type strain, and $n_{p}^{r}$ is the efficacy of PI for mutants. The proposed model introduces some novelty in the equations concerning virus' populations. It is, to our knowledge, the first model combining in this way the dynamics of the several cells. It is a different approach with promising results. The nonlinear system describing the dynamics of the model is:

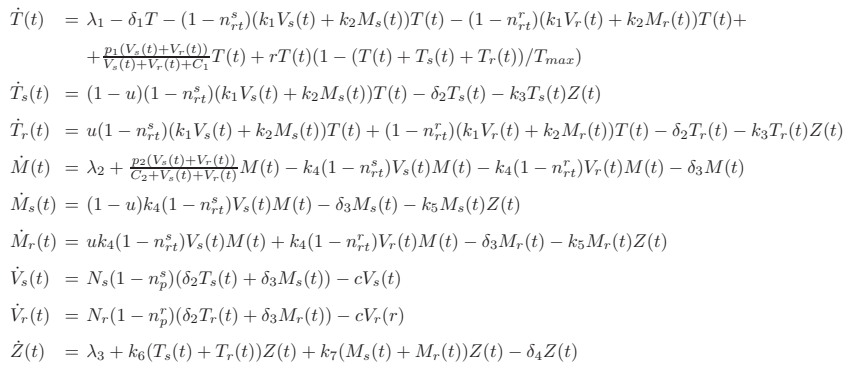

\section{NUMERICAL RESULTS}

We simulate the model (1). The parameters are $\lambda_{1}=10, \delta_{1}=0.02$, $k_{1}=2.4 \times 10^{-5}, k_{2}=1 \times 10^{-6}, n_{r t}^{s}=0.4, n_{r t}^{r}=0.2, p_{1}=38$, $C_{1}=300, r=0.03, T_{\max }=1500, u=3 \times 10^{-5}, \delta_{2}=0.28$, $k_{3}=4.5 \times 10^{-4}, \lambda_{2}=16.3, p_{2}=35, C_{2}=220, k_{4}=4.33 \times 10^{-8}$, $\delta_{3}=0.0285, k_{5}=3 \times 10^{-6} ;, N_{s}=200, n_{p}^{s}=0.1, N_{r}=50, n_{p}^{r}=0.1$, $c=2.39, \lambda_{3}=5, k_{6}=3.3 \times 10^{-4}, k_{7}=6 \times 10^{-9}$ and $\delta_{4}=0.05$. Initial conditions are $T(0)=1000, T_{s}(0)=T_{r}(0)=M_{s}(0)=M_{r}(0)=$ $V_{r}(0)=0, M(0)=30, V_{s}(0)=0.001$ and $Z(0)=333$. Parameters and initial conditions are based in the literature. In Figure 1, we observe that the model (1) approaches asymptotically the disease free equilibrium.
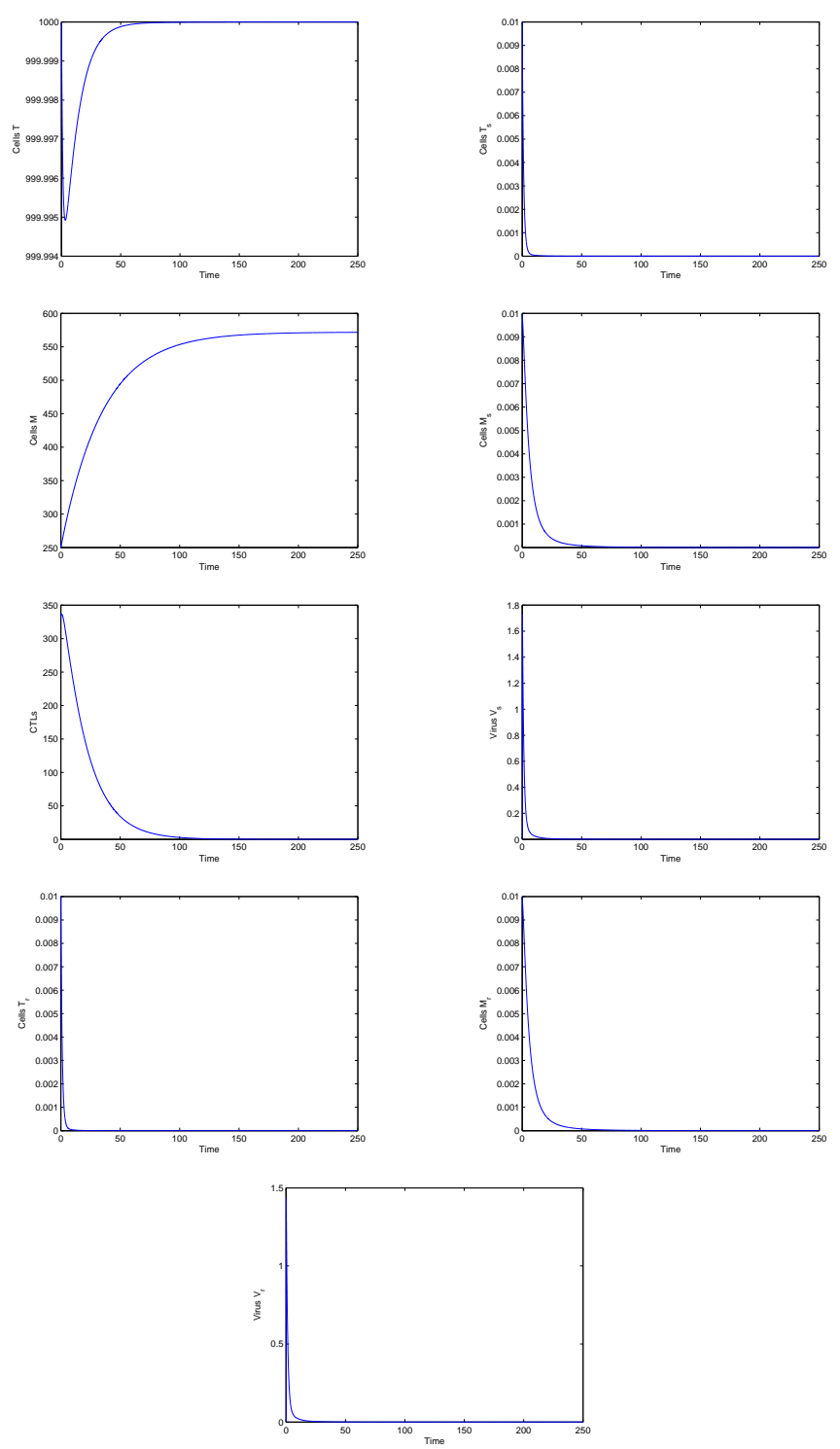

Fig. 1. Disease free equilibrium of the model (1).

3. CONCLUSIONS

We proposed a model for drug-resistance during HIV therapy. Simulations of the model show the disease free equilibrium. Future work wil focus in the analysis of the stability of the distinct equilibria and on the dynamics of the model in the different stages of disease.

\section{REFERENCES}

Hadjiandreou MM, Conejeros R, and Wilson DI (2009) Long-term HIV dynamics subject to continuous therapy and structured treatment interruptions. Chemical Engineering Science, 64:16001617. 


\title{
MATHMOD 2015 ABSTRACT VOLUME
}

\author{
MATHMOD Minisymposium \\ Model Reduction
}


MATHMOD 2015 Abstract Volume 


\title{
Data-driven model reduction for weakly nonlinear systems: A summary*
}

\author{
A.C. Antoulas ${ }^{1,2}$ and I.V. Gosea ${ }^{3}$
}

\begin{abstract}
Model reduction seeks to replace complex dynamical systems with simpler ones, having similar characteristics. One approach is data-driven reduction based on system data which are either measured or computed. In this regard the Loewner framework is a powerful tool for dealing with the reduction of both linear and nonlinear systems.
\end{abstract}

\section{OVERVIEW}

In broad terms model reduction seeks to transform large, complicated models of time dependent processes into smaller, simpler models that are nonetheless capable of representing accurately the behavior of the original process under a variety of operating conditions. The goal is an efficient, methodical strategy that yields dynamical systems evolving in substantially lower dimension spaces (hence requiring far less computational resources for realization) yet retaining response characteristics close to those of the original system.

Interpolatory MOR methods have matured quickly in the last decade. They have emerged as one of the leading choices for truly large-scale problems. These methods have their roots in numerical analysis and linear algebra and are related to rational interpolation. In the case of linear dynamical systems, the main idea behind these methods is to generate a reduced model whose transfer function interpolates that of the original system at select interpolation points. These methods typically require only the solution of large sparse linear systems and are capable of producing locally optimal, high-fidelity, reduced models. Recently, major advances showed how to apply interpolation methods to classes of nonlinear systems.

\section{SOME DETAILS}

We begin by listing the classes of systems which can be treated in our approach. The fist class is composed of linear systems whose dynamics are described in generalized state form as

$$
E \dot{x}(t)=A x(t)+B u(t)
$$

*This work was supported by NSF Grants CCF-1017401 and CCF1320866, as well as DFG Grant AN-693/1-1.

${ }^{1}$ Department of Electrical and Computer Engineering, Rice University, Houston (acalrice.edu).

${ }^{2}$ School of Engineering and Science, Jacobs University, D-28759 Bremen.

${ }^{3}$ School of Engineering and Science, Jacobs University, D-28759 Bremen. (i.gosealjacobs-university.de). where $x(t), u(t)$ are the generalized state, input or excitation, of dimension $n, m$, respectively. Consequently $E, A, B$ are matrices of size $n \times n, n \times n, n \times m$. Recall that if $E$ is singular we are dealing with a descriptor or differential-algebraic representation. The next class is that of bilinear systems, described by

$$
E \dot{x}(t)=A x(t)+N x(t) u(t)+B u(t),
$$

where $N$ is square of size $n$, and for simplicity we have assumed that $m=1$. Finally we consider quadratic bilinear systems $(\mathrm{QB})$, described (for $m=1$ ) by

$$
E \dot{x}(t)=A x(t)+F x(t) \otimes x(t)+N x(t) u(t)+B u(t),
$$

where $F$ has size $n \times n^{2}$ and ' $\otimes$ ' denotes the Kronecker product. In all these cases the output or observation equations are assumed linear in the (generalized) state:

$$
y(t)=C x(t), \quad C \in \mathbb{R}^{p \times n} .
$$

An important recent development is the observation that all nonlinear systems containing analytic nonlinearities can be expressed as quadratic bilinear systems, at the expense of increasing the dimension of the generalized state of the system. Hence QB systems form a very general class of dynamical systems [7].

Model reduction involves approximating the above systems by systems with generalized state $\hat{x}(t)$ of dimension $k \ll n$. For instance, in the linear case we seek matrices $\hat{A}, \hat{E}, \hat{B}, \hat{C}$, of dimension $k \times k, k \times k, k \times m, p \times k$, respectively, such that for the same input $u$, the output of the original system $y$ and that of the reduced system $\hat{y}$ are 'close' (i.e. an appropriate norm of the error $\|y-\hat{y}\|$ is small). All the above categories of systems can be reduced using so-called rational Krylov methods. For details concerning linear systems see [1]. For details concerning bilinear and analytic nonlinear systems, see [2], [3], [4], [5], [6], [7].

One shortcoming of these methods is that it is not a priori clear how the dimension of the reduced system should be chosen, so that certain accuracy requirements are met. In model reduction there are two classes of methods which achieve this a priori objective. The first is balanced truncation and is applicable mostly to linear systems (see [1] for details). The second approach is more recent and is a data-driven approach. This is the Loewner framework 
introduced and elaborated in [9], [10], [12], [13], [14], [15], [16], [11].

The purpose of this presentation is to illustrate some of the features of this approach by applying the Loewner framework to weakly nonlinear systems, that is, nonlinear systems with analytic nonlinearities.

\section{REFERENCES}

[1] A.C. Antoulas, Approximation of large-scale dynamical systems, Book Series Advances in Design and Control volume 6, SIAM Philadelphia (2005), reprinted (2008).

[2] T. Breiten, T. Damm, Krylov subspace methods for model reduction of bilinear systems, Systems and Control Letters, 59: 443-450 (2010).

[3] P. Benner, T. Breiten: Interpolation-Based Model Reduction of Bilinear Control Systems, SIAM Journal on Matrix Analysis and Applications, vol. 33, 2012.

[4] T. Breiten, Interpolatory methods for model reduction of large-scale dynamical systems, PhD Dissertation, Magdeburg, March 2013.

[5] G. Flagg, Interpolatory methods for model reduction of bilinear systems, PhD Dissertation, Virginia Tech, May 2012.

[6] G. Flagg and S. Gugercin, Multipoint Volterra Series Interpolation and $H_{2}$ optimsl model reduction of bilinear systems, Technical Report, Virginia Tech, December 2013.

[7] C. Gu, QLMOR: A Projection-Based Nonlinear Model Order Reduction Approach Using Quadratic-Linear Representation of Nonlinear Systems, IEEE-CAD, vol 30, Sept 2011.

[8] K. Löwner, Über monotone Matrixfunctionen, Mathematische Zeitschrift, 38: 177-216 (1934).

[9] A.J. Mayo and A.C. Antoulas, A framework for the solution of the generalized realization problem, Linear Algebra and Its Applications, vol. 425, pages 634-662 (2007).

[10] S. Lefteriu, A.C. Antoulas: A New Approach to Modeling Multiport Systems from Frequency-Domain Data, IEEE Trans. CAD, vol. 29, pages 14-27 (2010).

[11] A.C. Ionita, Lagrange rational interpolation and its application to approximation of large-scale dynamical systems, $\mathrm{PhD}$ Dissertation, ECE Dept., Rice University, August 2013.

[12] A.C. Antoulas, C.A. Beattie, and S. Gugercin, Interpolatory model reduction of large-scale systems, in Efficient modeling and control of large-scale systems, K. Grigoriadis and J. Mohammadpour Eds, Springer Verlag, pages 3-58 (2010).

[13] S. Gugercin, C.A. Beattie and A.C. Antoulas, Data-driven and interpolatory model reduction, book in preparation, SIAM (2014).

[14] A.C. Antoulas, A.C. Ionita, and S. Lefteriu, On two-variable rational interpolation, Linear Algebra and Its Applications, 436 : 2889-2915 (2012)

[15] A.C. Ionita and A.C. Antoulas, Parametrized model order reduction from transfer function measurements, in Reduced Order Methods for modeling and computational reduction, Book Series: Modeling, Comutations and Applications, A. Quarteroni, G. Rozza (Editors), Springer Verlag (2013).

[16] A.C. Ionita and A.C. Antoulas, Data-driven parametrized model reduction in the Loewner framework, Technical Report, Rice University, March 2013. 


\title{
Quadrature-based IRKA for optimal $\mathcal{H}_{2}$ model reduction
}

\author{
Christopher A. Beattie* Zlatko Drmač ${ }^{*}$ Serkan Gugercin *** \\ * Dept. of Mathematics, Virginia Tech., Blacksburg, VA, 24061-0123 \\ USA (Tel: 540-231-8279; e-mail: beattie@vt.edu). \\ ** Faculty of Science, Department of Mathematics, University of \\ Zagreb, Bijenička 30,10000 Zagreb, Croatia (e-mail: drmac@math.hr) \\ *** Dept. of Mathematics, Virginia Tech., Blacksburg, VA, 24061-0123 \\ USA(e-mail: gugercin@math.vt.edu)
}

\begin{abstract}
Iterative Rational Krylov Algorithm (IRKA) of Gugercin et al. [2008] is an effective tool for optimal $\mathcal{H}_{2}$ rational approximation. Beattie and Gugercin [2012] has recently developed a new formulation of IRKA that only uses transfer function evaluations, without requiring any particular realization; thus extending IRKA to $\mathcal{H}_{2}$ approximation of irrational, infinitedimensional dynamical systems. The main computational cost of this new framework is the need for repeated transfer function evaluations. In this paper, based on the reproducing kernel formulation of the underlying Hilbert space, we introduce a quadrature-based formulation of IRKA where a number of transfer function evaluation are computed only at the beginning (the offline phase) and the IRKA steps (the online phase) never revisit the original transfer function performing all the computations in the reduced order dimension.
\end{abstract}

\section{INTRODUCTION}

Let $H(s)$ be a $\mathcal{H}_{2}$ function where $\mathcal{H}_{2}$ denotes the set of scalar valued functions, $H(s)$, that are analytic for $s$ in the open right half plane, $\operatorname{Re}(s)>0$, and such that for each fixed $\operatorname{Re}(s)=x>0, \sup _{x>0} \int_{-\infty}^{\infty}|h(x+\imath y)|^{2} d y<\infty . \mathcal{H}_{2}$ is a Hilbert space with the $\mathcal{H}_{2}$-inner product

$$
\langle G, H\rangle_{\mathcal{H}_{2}} \stackrel{\text { def }}{=} \frac{1}{2 \pi} \int_{-\infty}^{\infty} \overline{G(\imath \omega)} H(\imath \omega) d \omega
$$

and the $\mathcal{H}_{2}$ norm $\|H\|_{\mathcal{H}_{2}} \stackrel{\text { def }}{=}\left(\frac{1}{2 \pi} \int_{-\infty}^{+\infty}|H(\imath \omega)|^{2} d \omega\right)^{1 / 2}$. Given an order $r$, our goal is to find a reduced model

$$
H_{r}(s):=\mathbf{C}_{r}\left(s \mathbf{E}_{r}-\mathbf{A}_{r}\right)^{-1} \mathbf{B}_{r}
$$

with $\mathbf{A}_{r}, \mathbf{E}_{r} \in \mathbb{R}^{r \times r}, \mathbf{B}_{r} \in \mathbb{R}^{r \times 1}$, and $\mathbf{C}_{r} \in \mathbb{R}^{1 \times r}$, such that $H_{r}(s)$ is an optimal $\mathcal{H}_{2}$ approximation to $H(s)$ :

$$
\left\|H-H_{r}\right\|_{\mathcal{H}_{2}}=\min _{G_{r}: \text { stable }}\left\|H-G_{r}\right\|_{\mathcal{H}_{2}} .
$$

Let $\lambda_{i}$ denote the poles of $H_{r}(s)$. Then, $H_{r}(s)$ satisfies

$$
H_{r}\left(-\lambda_{i}\right)=H\left(-\lambda_{i}\right) \text { and } H_{r}^{\prime}\left(-\lambda_{i}\right)=H^{\prime}\left(-\lambda_{i}\right)
$$

for $i=1, \ldots, r$. For details on the optimal $\mathcal{H}_{2}$ approximation, see Gugercin et al. [2008], Antoulas et al. [2010].

\subsection{Realization-independent IRKA}

Given the interpolation points $s_{1}, \ldots, s_{r}$, construct $H_{r}(s)=$ $\mathbf{C}_{r}\left(s \mathbf{E}_{r}-\mathbf{A}_{r}\right)^{-1} \mathbf{B}_{r}$ using

$$
\left(\mathbf{E}_{r}\right)_{i, j}=-\frac{H\left(s_{i}\right)-H\left(s_{j}\right)}{s_{i}-s_{j}} \text { if } i \neq j, \quad\left(\mathbf{E}_{r}\right)_{i, i}=-H^{\prime}\left(s_{i}\right)
$$

$\left(\mathbf{A}_{r}\right)_{i, j}=-\frac{s_{i} H\left(s_{i}\right)-H\left(s_{j}\right) s_{j}}{s_{i}-s_{j}}$ if $i \neq j,\left(\mathbf{A}_{r}\right)_{i, i}=-\left.[s H(s)]^{\prime}\right|_{s=s_{i}}$

$$
\mathbf{C}_{r}=\mathbf{B}_{r}^{T}=\left[H\left(s_{1}\right), \ldots, H\left(s_{r}\right)\right]
$$

$\star$ This work was supported in part by the National Science Foundation through Grant DMS-1217156.
Then, $H_{r}\left(s_{i}\right)=H\left(s_{i}\right)$ and $H_{r}^{\prime}\left(s_{i}\right)=H^{\prime}\left(s_{i}\right)$ for $i=$ $1, \ldots, r$. This is the Loewner framework Mayo and Antoulas [2007], Antoulas et al. [2010] for interpolation, only requiring $H(s)$ and $H^{\prime}(s)$ evaluations. Then, by incorporating this framework in the implementation of IRKA, Beattie and Gugercin [2012] developed a realization independent formulation of IRKA to tackle the optimization problem (3) by only requiring transfer function evaluations $H(s)$ and $H^{\prime}(s)$ as outlined in Algorithm TF-IRKA below.

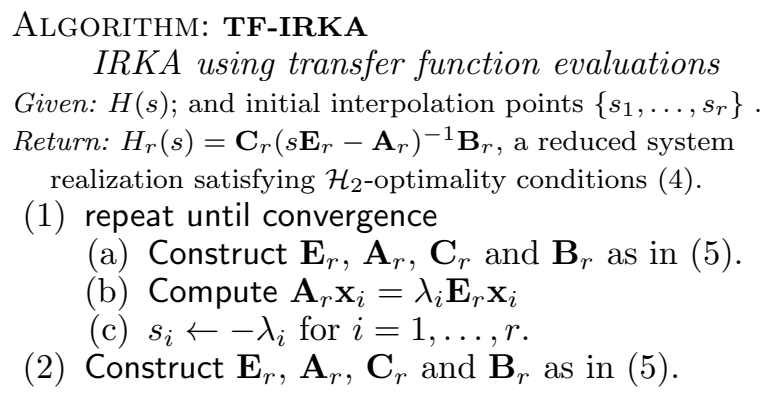

(2) Construct $\mathbf{E}_{r}, \mathbf{A}_{r}, \mathbf{C}_{r}$ and $\mathbf{B}_{r}$ as in (5).

Upon convergence, the reduced model $H_{r}(s)$ satisfies the $\mathcal{H}_{2}$ optimality conditions (4). There is no structural restriction on $H(s)$; it can correspond to, for example, irrational, infinite-dimensional dynamical systems such as those with delays.

\section{QUADRATURE-BASED IRKA}

The main computational cost of Algorithm TF-IRKA is the repeated evaluations of $H\left(s_{i}\right)$ and $H^{\prime}\left(s_{i}\right)$ throughout the iteration. Our goal in this paper is to reduce this cost drastically. The reproducing kernel structure of the underlying $\mathcal{H}_{2}$ space is the first step in achieving this goal 
by replacing function evaluations by a linear functional. Using the $\mathcal{H}_{2}$ inner product definition in (1), it readily follows that for $s_{k} \in \mathbb{C}_{+}$,

$$
H\left(s_{k}\right)=\left\langle\frac{1}{s+s_{k}}, H\right\rangle \text { and } H^{\prime}\left(s_{k}\right)=\left\langle\frac{-1}{\left(s+s_{k}\right)^{2}}, H\right\rangle \text {. }
$$

In other words, the transfer function evaluations can be considered as an inner product evaluations. We note that other kernel choices are possible, such as the Poisson kernel; see e.g. Hoffman [1962]. We leave these details to the full paper.

Next, we approximate these inner-products by a numerical quadrature. For simplicity, consider only $H\left(s_{k}\right)$ computation.

$$
\begin{aligned}
H\left(s_{k}\right) & =\left\langle\frac{1}{s+s_{k}}, H\right\rangle=\frac{1}{2 \pi} \int_{-\infty}^{\infty} \frac{1}{\overline{\imath \omega+s_{k}}} H(\imath \omega) d \omega \\
& \approx \sum_{j=1}^{M} \rho_{j} H\left(\xi_{j}\right) \frac{1}{\overline{\xi_{j}+s_{k}}}=: \tilde{H}\left(s_{k}\right)
\end{aligned}
$$

where, $\rho_{j}$ and $\xi_{j}$ denote, respectively, the quadrature weights and nodes, and $M$ is the number of quadrature nodes. We denote the quadrature approximation to $H\left(s_{k}\right)$ by $\widetilde{H}\left(s_{k}\right)$. A similar approximation is used to obtain the quadrature approximation $\widetilde{H}^{\prime}\left(s_{k}\right)$ to $H^{\prime}\left(s_{k}\right)$. Various options are available for the quadrature selection such as Clenshaw-Curtis, Gauss-Legendre, or Gauss-Hermite quadrature rules. Due to page limitations, details are left to the full paper. Now, given the interpolation points $\left\{s_{1}, s_{2}, \ldots, s_{r}\right\}$, construct the quadrature-based reduced model $\widetilde{H}_{r}(s)=\widetilde{\mathbf{C}}_{r}\left(s \widetilde{\mathbf{E}}_{r}-\widetilde{\mathbf{A}}_{r}\right)^{-1} \widetilde{\mathbf{B}}_{r}$ using

$$
\begin{gathered}
\left(\widetilde{\mathbf{E}}_{r}\right)_{i, j}=-\frac{\widetilde{H}\left(s_{i}\right)-\widetilde{H}\left(s_{j}\right)}{s_{i}-s_{j}} \text { if } i \neq j, \quad\left(\widetilde{\mathbf{E}}_{r}\right)_{i, i}=-\widetilde{H}^{\prime}\left(s_{i}\right) \\
\left(\widetilde{\mathbf{A}}_{r}\right)_{i, j}=-\frac{s_{i} \widetilde{H}\left(s_{i}\right)-\widetilde{H}\left(s_{j}\right) s_{j}}{s_{i}-s_{j}} \text { if } i \neq j,\left(\widetilde{\mathbf{A}}_{r}\right)_{i, i}=-\left.[s \widetilde{H}(s)]^{\prime}\right|_{s=s_{i}} \\
\widetilde{\mathbf{C}}_{r}=\widetilde{\mathbf{B}}_{r}^{T}=\left[\widetilde{H}\left(s_{1}\right), \ldots, \widetilde{H}\left(s_{r}\right)\right]
\end{gathered}
$$

This construction directly leads to a quadrature-based formulation of IRKA for $\mathcal{H}_{2}$ model reduction problem as outlined below in Algorithm Q-IRKA.

\footnotetext{
Algorithm: Q-IRKA

IRKA using quadrature-based function evaluations Given: $\mathbf{H}(s)$; and initial interpolation points $\left\{s_{1}, \ldots, s_{r}\right\}$. Return: $\widetilde{H}_{r}(s)=\widetilde{\mathbf{C}}_{r}\left(s \widetilde{\mathbf{E}}_{r}-\widetilde{b f A}_{r}\right)^{-1} \widetilde{\mathbf{B}}_{r}$, a reduced system satisfying $\mathcal{H}_{2}$-optimality conditions (4) within quadrature error

(1) Evaluate $H(s)$ at the quadrature nodes $\xi_{k}$, for $k=1, \ldots, M$.

(2) repeat until convergence
(a) Construct $\widetilde{\mathbf{E}}_{r}, \widetilde{\mathbf{A}}_{r}, \widetilde{\mathbf{C}}_{r}$ and $\widetilde{\mathbf{B}}_{r}$ as in (8).
(b) Compute $\widetilde{\mathbf{A}}_{r} \mathbf{x}_{i}=\widetilde{\lambda}_{i} \widetilde{\mathbf{E}}_{r} \mathbf{x}_{i}$
(c) $\widetilde{s}_{i} \leftarrow-\widetilde{\lambda}_{i}$ for $i=1, \ldots, r$.

(3) Construct $\widetilde{\mathbf{E}}_{r}, \widetilde{\mathbf{A}}_{r}, \widetilde{\mathbf{C}}_{r}$ and $\widetilde{\mathbf{B}}_{r}$ as in (8).
}

Note that $H(s)$ is computed only initially (the online phase) at the quadrature nodes; the rest of the iteration (the offline phase) never evaluates $H(s)$; only uses the precomputed values. Upon convergence, the reduced-order model satisfies the $\mathcal{H}_{2}$ optimality conditions to the accuracy of the underlying numerical quadrature.

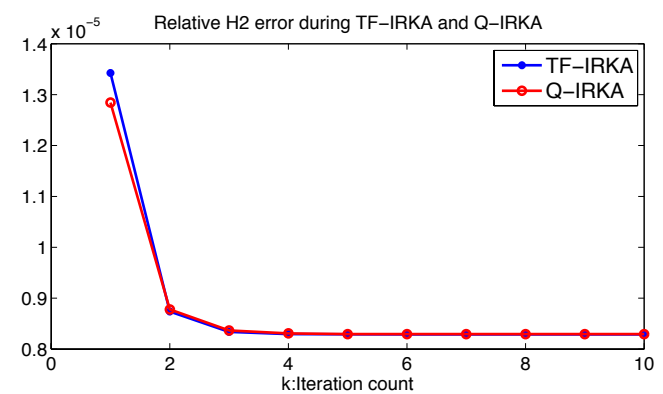

Fig. 1. Evolution of the relative $\mathcal{H}_{2}$ error

Numerical Example: We apply Algorithm Q-IRKA to reduce the Heat Model with $n=197$ from the NICONET Benchmark collection Chahlaoui and Dooren [202]. We use $M=64$ nodes; but due to the conjugacy symmetry, only $32 H(s)$ evaluations are needed. We reduce the order to $r=5$ using both Algorithm Q-IRKA and the exact IRKA implementation Algorithm TF-IRKA. The resulting relative $\mathcal{H}_{2}$ errors due to Q-IRKA and TF-IRKA are, respectively, $7.3592 \times 10^{-4}$ and $7.3637 \times 10^{-4}$; thus Q-IRKA almost exactly replicates the accuracy of the true optimal reduction. The evolution of the $\mathcal{H}_{2}$ error depicted in Figure 1 also shows the effectiveness of Q-IRKA.

\section{CONCLUSIONS AND FUTURE WORK}

In this note, we have introduced a quadrature-based implementation of IRKA. The proposed approach, Q-IRKA, removes the need for repeated function evaluations during the iteration and only uses a set of precomputed values. A numerical examples is used to illustrate the effectiveness of the proposed approach. Due to the page limitation, several details are omitted. These details will be included in the upcoming full paper.

\section{REFERENCES}

A.C. Antoulas, C.A. Beattie, and S. Gugercin. Interpolatory model reduction of large-scale dynamical systems. In J. Mohammadpour and K. Grigoriadis, editors, Efficient Modeling and Control of Large-Scale Systems, pages 2-58. Springer-Verlag, 2010.

C. Beattie and S. Gugercin. Realization-independent $\mathcal{H}_{2}$ approximation. In Proceedings of the 51st IEEE Conference on Decision \& Control, pages 4953-4958. IEEE, 2012.

Y. Chahlaoui and P. Van Dooren. A collection of benchmark examples for model reduction of linear time invariant dynamical systems. Technical report, SLICOT Working Note 2002-2, 202.

S. Gugercin, A. C. Antoulas, and C. A. Beattie. $\mathcal{H}_{2}$ model reduction for large-scale linear dynamical systems. SIAM J. Matrix Anal. Appl., 30(2):609-638, 2008.

K. Hoffman. Banach Spaces of Analytic Functions. Prentice-Hall Inc., 1962.

A.J. Mayo and A.C. Antoulas. A framework for the solution of the generalized realization problem. Linear Algebra and Its Applications, 425(2-3):634-662, 2007. 


\title{
Towards Practical Implementations of Balanced Truncation for LTV Systems
}

\author{
Norman Lang ${ }^{*}$ Jens Saak ${ }^{* * *}$ Tatjana Stykel ${ }^{* * *}$ \\ * Technische Universität Chemnitz, Reichenhainerstraße 39/41, \\ D-09126 Chemnitz (e-mail: \\ norman.lang@mathematik.tu-chemnitz.de). \\ ** Max Planck Institute for Dynamics of Complex Technical Systems, \\ Sandtorstraße 1, D-39106 Magdeburg (e-mail: \\ saak@mpi-magdeburg.mpg.de) \\ *** Universität Augsburg, Universitätsstr. 14, 86159 Augsburg, (e-mail: \\ stykel@math.uni-augsburg.de)
}

\begin{abstract}
We present the application of Balanced Truncation (BT) for linear time-varying (LTV) systems. This directly leads to the solution of the controllability and observability differential Lyapunov equations associated to the LTV system. For large-scale dynamical systems the main task is to efficiently solve these equations with respect to computational cost and memory requirements. Thus, efficient strategies exploiting the low-rank structure of the systems are applied in the context of the matrix-valued time integration schemes. In particular, an $L D L^{T}$-type low-rank splitting is considered in order to avoid the problems arising from the indefinite right hand sides of the algebraic Lyapunov equations that have to be solved inside the time integration schemes.
\end{abstract}

Keywords: Balanced Truncation, model order reduction, time-varying systems

\section{INTRODUCTION}

Many physical phenomena are naturally modeled in terms of linear time-varying (LTV) systems of the form

$$
\begin{aligned}
E(t) \dot{x}(t) & =A(t) x(t)+B(t) u(t), x\left(t_{0}\right)=x_{0}, \\
y(t) & =C(t) x(t),
\end{aligned}
$$

where $E(t), A(t) \in \mathbb{R}^{n \times n}, B(t) \in \mathbb{R}^{n \times m}$ and $C(t) \in \mathbb{R}^{q \times n}$ $x(t) \in \mathbb{R}^{n}$ defines the state vector, $u(t) \in \mathbb{R}^{m}$ the inputs, and $y(t) \in \mathbb{R}^{q}$ are the outputs of the system. The system matrices $E(t), A(t), B(t), C(t)$ are assumed to be continuous and bounded, and $E(t)$ is non-singular for all $t \in\left[t_{0}, t_{f}\right]$. Models of dynamical systems of the form (1) originating from complex physical and technical processes such as mechanical systems, fluid flow or chip design simulation are often of very large dimension $n$. In order to perform real time simulations or controller design, model order reduction (MOR) becomes very important. The goal of any model order reduction procedure is to find a reduced order approximant

$$
\begin{aligned}
\hat{E}(t) \dot{\hat{x}}(t) & =\hat{A}(t) x(t)+\hat{B}(t) u(t), \hat{x}\left(t_{0}\right)=\hat{x}_{0}, \\
\hat{y}(t) & =\hat{C}(t) \hat{x}(t),
\end{aligned}
$$

of (1) with

$$
\begin{aligned}
& \hat{E}(t)=W(t)^{T} E V(t) \in \mathbb{R}^{k \times k}, \\
& \hat{A}(t)=W(t)^{T} A V(t)-W(t)^{T} E \dot{V}(t) \in \mathbb{R}^{k \times k}, \\
& \hat{B}(t)=W(t)^{T} B \in \mathbb{R}^{k \times m}, \hat{C}(t)=C V(t) \in \mathbb{R}^{q \times k},
\end{aligned}
$$

and projection matrices $W(t), V(t) \in \mathbb{R}^{n \times k}$, such that $k \ll n$ and the output $\hat{y}$ of the reduced order model (ROM) yields a significantly small approximation error $\|\hat{y}-y\|$ in a suitable norm. In the remainder, we consider the Balanced Truncation MOR method applied to LTV systems.

\section{BALANCED TRUNCATION FOR LTV SYSTEMS}

The theoretical application of Balanced Truncation (BT) model order reduction for standard LTV systems with $E(t) \equiv I$ is deeply studied in the literature, see e.g., Sandberg (2002); Shokoohi et al. (1983) and the references therein. Its main ingredients are the controllability and observability Gramians $P(t)$ and $Q(t)$ given as the solutions of the differential Lyapunov equations (DLEs)

$$
\begin{aligned}
A(t) P(t)+P(t) A(t)^{T} & -B(t) B(t)^{T}=\dot{P}(t), \\
P\left(t_{0}\right) & =0, \\
A(t)^{T} Q(t)+Q(t) A(t) & -C(t)^{T} C(t)=-\dot{Q}(t), \\
Q\left(t_{f}\right) & =0,
\end{aligned}
$$

associated to (1). Note that the observability Lyapunov equation (4) has to be solved backwards in time.

Given the solutions of (3) and (4), a ROM of the form (2) can be computed via a generalization of the Square Root Balanced Truncation method developed, e.g., Laub et al. (1987) for linear time-invariant (LTI) systems based on a factorization of the Gramians $P(t)=R(t) R(t)^{T}$ and $Q(t)=L(t) L(t)^{T}$. In practice we often observe that $P(t), Q(t)$ are of low numerical rank. Therefore, efficient algorithms exploiting the low-rank property can be used in order to obtain $R(t) \in \mathbb{R}^{n \times r}$ and $L(t) \in \mathbb{R}^{n \times \ell}$ with $r, \ell \ll n$. Furthermore, the DLE can be considered as a special case of the differential Riccati equation (DRE). That is, any integration method for DREs, e.g., discussed 
in Benner and Mena (2013); Lang et al. (2014) can also be employed in the DLE setting.

\section{SOLVING DIFFERENTIAL LYAPUNOV EQUATIONS}

In this contribution, we consider the backward differentiation formulas (BDF) and the Rosenbrock methods applied to the generalized DLE

$$
E(t) \dot{X}(t) E^{T}(t)=F(t, X(t)), \quad X(0)=0,
$$

with

$$
F(t, X(t))=A(t) X(t) E^{T}(t)+E(t) X(t) A^{T}(t)+N(t) N^{T}(t) .
$$

Further, let $\tau_{k}=t_{k}-t_{k-1}$ be the time step size, where $0=t_{0}<t_{1}<\cdots<t_{k_{\max }}=t_{f}$ denote the discrete time instances. These may be determined adaptively. We abbreviate $E_{k}=E\left(t_{k}\right), A_{k}=A\left(t_{k}\right)$ and $N_{k}=N\left(t_{k}\right)$ in the remainder for easier reading.

Backward Differentiation Formulas: The $p$-step BDF method applied to equation (5) has the form

$$
E_{k}\left(\sum_{j=0}^{p} \alpha_{j} X_{k-j}\right) E_{k}^{T}=\tau_{k} \beta F\left(t_{k}, X_{k}\right),
$$

where $X_{k}$ is an approximation to $X\left(t_{k}\right)$. The coefficients $\alpha_{j}$ and $\beta$ are chosen such that the $p$-step BDF method has the maximum possible order $p$, see Hairer and Wanner (2002). Assuming that $X_{0}, \ldots, X_{k-1}$ are already known, the matrix $X_{k}$ can then be determined from the algebraic Lyapunov equation (ALE)

$$
\tilde{A}_{k} X_{k} E_{k}^{T}+E_{k} X_{k} \tilde{A}_{k}^{T}=-N_{k} N_{k}^{T}+E_{k}\left(\sum_{j=1}^{p} \alpha_{j} X_{k-j}\right) E_{k}^{T}
$$

with $\tilde{A}_{k}=\tau_{k} \beta A_{k}-\frac{1}{2} \alpha_{0} E_{k}$. Note that, since for $p \geq 2$ some of the coefficients $\alpha_{j}, j=1, \ldots, p$, are positive, the right-hand side of (6) may be indefinite. Assume that the matrices $X_{j}, j=0, \ldots, k-1$, admit a low-rank $L D L^{T}$ decomposition $X_{j} \approx L_{j} D_{j} L_{j}^{T}$ with $L_{j} \in \mathbb{R}^{n \times \ell_{j}}$, $D_{j} \in \mathbb{R}^{\ell_{j} \times \ell_{j}}$ and $\ell_{j} \ll n$. Then the right-hand side of the ALE (6) takes the form

$$
-N_{k} N_{k}^{T}+E_{k}\left(\sum_{j=1}^{p} \alpha_{j} X_{k-j}\right) E_{k}^{T}=-G_{k} S_{k} G_{k}^{T}
$$

with $G_{k}=\left[N_{k}, E_{k} L_{k-1}, \ldots, E_{k} L_{k-p}\right]$ and

$$
S_{k}=\left[\begin{array}{llll}
I & & & \\
& -\alpha_{1} D_{k-1} & & \\
& & \ddots & \\
& & & -\alpha_{p} D_{k-p}
\end{array}\right] \text {. }
$$

In this case, an approximate solution of the ALE (6) can be determined in the factorized form $X_{k} \approx L_{k} D_{k} L_{k}^{T}$ $L_{k} \in \mathbb{R}^{n \times \ell_{k}}, D_{k} \in \mathbb{R}^{\ell_{k} \times \ell_{k}}$ using the $L D L^{T}$-type ADI or Krylov method presented in Lang et al. (2014) based on earlier ideas in Benner et al. (2009).

Rosenbrock methods: Following the statements in Benner and Mena (2013) for DREs, the general p-stage Rosenbrock method applied to the DLE (5) reads

$$
\begin{gathered}
X_{k+1}=X_{k}+\sum_{j=1}^{p} m_{j} K_{j} \\
\tilde{A}_{k} K_{i} E_{k}^{T}+E_{k} K_{i} \tilde{A}_{k}^{T}=-F\left(t_{k, i}, X_{k}+\sum_{j=1}^{i-1} a_{i j} K_{j}\right) \\
\\
-\sum_{j=1}^{i-1} \frac{c_{i j}}{\tau_{k}} K_{j}-\gamma_{i} \tau_{k} F_{t_{k}},
\end{gathered}
$$

where $\tilde{A}_{k}=A_{k}-\frac{1}{2 \gamma_{i i} \tau_{k}} E_{k}, t_{k, i}=t_{k}+\alpha_{i} \tau_{k}, i=1, \ldots, p$, and $\gamma_{i i}, a_{i j}, c_{i j}, \gamma_{i}, m_{j}, \alpha_{i}$ are the method coefficients, that are available in text books as, e.g. Hairer and Wanner (2002). We denote by $K_{i}$ the $n \times n$ matrix representing the solution of the $i$-th-stage of the method and abbreviate $F_{t_{k}}=\frac{\partial F}{\partial t}\left(t_{k}, X\left(t_{k}\right)\right)$. Again considering a right hand side factorization $-G_{k} S_{k} G_{k}^{T}$ in (7) yields an approximate solution $X_{k} \approx L_{k} D_{k} L_{k}^{T}$ to (5) at time step $t_{k}$. The particular representations of the several Rosenbrock methods and the corresponding low-rank factors $G_{k}, S_{k}$ in the stage equations for $K_{i}, i=1 \ldots, p$ depend on the order $p$ and the choice of the coefficients $\gamma_{i i}, a_{i j}, c_{i j}, \gamma_{i}, m_{j}$ and $\alpha_{i}$. Details on the particular formulations will be presented elsewhere for reasons of space.

\section{CONCLUSION}

The BT MOR method for LTV systems is deeply studied in the literature. Still, as far as the authors know, there is no suitable procedure that is applicable for large-scale LTV systems. Therefore, the authors investigate the application of low-rank based solvers for matrix differential equations previously mentioned for solving large-scale DREs. It is briefly shown that the ideas for DREs also apply to the DLE case. Also the origin of the initial and final conditions for the controllability and observability DLEs will be addressed.

\section{REFERENCES}

Benner, P. and Mena, H. (2013). Rosenbrock methods for solving differential Riccati equations. IEEE Trans. Automat. Control, 58, 2950-2957.

Benner, P., Li, R.C., and Truhar, N. (2009). On the ADI method for Sylvester equations. J. Comput. Appl. Math., 233(4), 1035-1045.

Hairer, E. and Wanner, G. (2002). Solving Ordinary Differential Equations II - Stiff and Algebraic Problems. Springer Series in Computational Mathematics. Springer, second edition.

Lang, N., Mena, H., and Saak, J. (2014). On the benefits of the $L D L^{T}$ factorization for large-scale differential matrix equation solvers. Preprint MPIMD/14-14, Max Planck Institute Magdeburg.

Laub, A., Heath, M., Paige, C., and Ward, R. (1987). Computation of system balancing transformations and other applications of simultaneous diagonalization algorithms. IEEE Trans. Automat. Control, 32(2), 115-122.

Sandberg, H. (2002). Linear Time-Varying Systems: Modeling and Reduction. Ph.d. thesis, Department of Automatic Control, Lund Institute of Technology, Lund (Sweden).

Shokoohi, S., Silverman, L., and van Dooren, P. (1983). Linear time-variable systems: balancing and model reduction. IEEE Trans. Automat. Control, 28, 810-822. 


\title{
An Efficient Output Error Bound for Model Order Reduction of Parametrized Evolution Equations
}

\author{
Yongjin Zhang, Lihong Feng, Suzhou Li and Peter Benner \\ Max Planck Institute for Dynamics of Complex Technical Systems, \\ D-39106 Magdeburg, Germany \\ Corresponding author: Yongjin Zhang, Max Planck Institute for Dynamics of Complex \\ Technical Systems, D-39106 Magdeburg, Germany, zhangy@mpi-magdeburg.mpg.de
}

Numerical simulation of large-scale complex systems is challenging, especially when such a task needs to be done many times under parameter variations, e.g., in the context of optimization, control, and parameter estimation. Model order reduction (MOR) is a useful technique for constructing a low-cost surrogate, i.e., a reduced order model (ROM), which can reproduce the input-output response of the original large-scale system, with compromise on the accuracy to an acceptable extent. To compute a ROM, an efficient a posteriori error estimation is crucial because it enables the computation to be reliable and automatic.

In this work we propose an efficient output error estimation for projection based MOR methods applied to parametrized nonlinear evolution problems. For (nonlinear) evolution equations, time-stepping schemes are often used to solve them, and error estimations for reduced basis methods have been studied in recent years $[1,3]$. The error estimator may loose sharpness when a large number of time steps are needed, because the error estimator is actually a summation of the error over the previous time steps. To circumvent this problem, we introduce a dual system at each time instance in the evolution process. With the help of the dual system and under suitable assumptions, the output error of the reduced model can be estimated more sharply. The proposed output error bound is independent of the choice of the projection matrix, and hence is independent of the MOR methods.

The proposed error estimation is applied to two kinds of evolution problems arising from chromatographic separation processes. One is batch chromatography with bi-Langmuir isotherm equations, which is a nonlinear parametric evolution problem. The other is a linear parametric periodic switching system, the model of simulated moving bed chromatography with linear isotherm equations. Reduced basis methods are used to construct the reduced order model. More precisely, the POD-Greedy algorithm [2] is employed to generate the reduced basis. Figure 1 illustrates the decay of the error bounds and the corresponding true error during the reduced basis extension process for the batch chromatographic model. It is seen that the proposed output error bound (ErrorBound-2) is much sharper than ErrorBound-1 [1, 3]. The resulting reduced models are employed to solve the underlying optimization problems. Employing the reliable compact reduced models, the optimization problems are solved much faster with sufficient accuracy. The results of the optimization of batch chromatography are summarized in Table 1. It is seen that the optimal solution of the ROM based optimization converges to the full order model (FOM) based optimal solution, and the runtime is significantly reduced. The speedup factor ( $\mathrm{SpF}$ ) is 58 .

Table 1: Results of the optimization based on the FOM $(\mathcal{N}=1500)$ and the $\mathrm{ROM}(N=45)$.

\begin{tabular}{lllcc}
\hline Models & Objective & Optimal solution & \#Iterations & Runtime $[\mathrm{h}] / \mathrm{SpF}$ \\
\hline FOM-Opt. & 0.020264 & $(0.07964,1.05445)$ & 202 & $33.88 /-$ \\
ROM-Opt. & 0.020266 & $(0.07964,1.05445)$ & 202 & $0.58 / 58$ \\
\hline
\end{tabular}




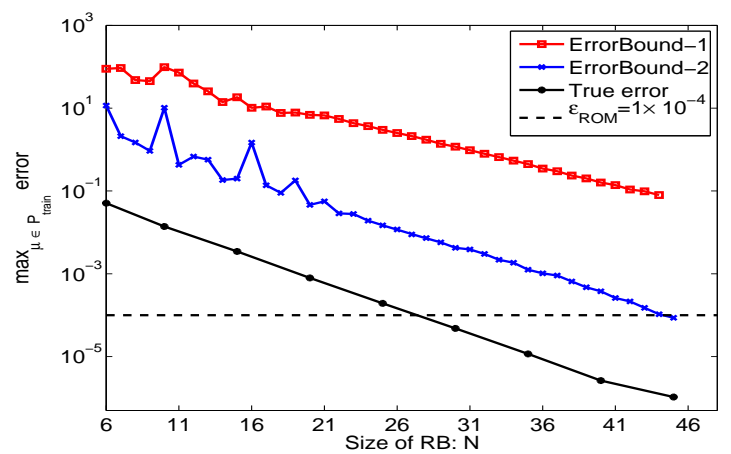

Figure 1: Illustration of the decay of the error bounds and the corresponding true error during the reduced basis extension process for the batch chromatographic model. ErrorBound-1 refers to the output error bound used in [3], while ErrorBound-2 is the proposed output error bound.

\section{References}

[1] M. Drohmann, B. HaAsdonk, And M. Ohlberger, Reduced basis approximation for nonlinear parametrized evolution equations based on empirical operator interpolation, SIAM Journal on Scientific Computing, 34 (2012), pp. 937-969.

[2] B. HAASDONK AND M. OHLBERGER, Reduced basis method for finite volume approximations of parametrized linear evolution equations, M2AN Math. Model. Numer. Anal., 42 (2008), pp. 277-302.

[3] Y. Zhang, L. Feng, S. Li, And P. Benner, Accelerating PDE constrained optimization by the reduced basis method: application to batch chromatography, MPI Magdeburg Preprint MPIMD/14-09, May 2014. Available from http://www.mpi-magdeburg.mpg.de/preprints/. 


\title{
Automatic Model Reduction of Linear Population Balance Models by Proper Orthogonal Decomposition
}

\author{
Dmytro Khlopov* Michael Mangold* \\ * Max Planck Institute for Dynamics of Complex Technical Systems, \\ 39106 Magdeburg, Germany (e-mail: khlopov@mpi-magdeburg.mpg.de, \\ mangold@mpi-magdeburg.mpg.de)
}

\section{INTRODUCTION}

Particle processes are very important for chemical and pharmaceutical industries. The vast majority of products in these industries is produced in particulate form (Wintermantel (1999)). Main examples of such processes are crystallization, granulation, and polymerization.

Particle processes typically consist of the actual dispersed phase and a fluid phase, which stands in exchange with the dispersed phase. Essential processes within the dispersed phase are formation of particles (nucleation), their division into smaller particles (breakage) and union of particles into larger units (aggregation). The dispersed phase with a large number of particles can be described by population balance equations (Ramkrishna (2000)). The equation for the description of behavior of the dispersed phase reads:

$$
\begin{aligned}
& \frac{\partial n}{\partial t}+\underbrace{\frac{\partial}{\partial L_{j}}\left(G_{j} n\right)}_{\text {growth }}+\underbrace{\frac{\partial}{\partial x_{k}}\left(\nu_{k} n+j_{k}^{n}\right)}_{\text {convection/diffusion }}= \\
& \underbrace{\int_{\Omega_{L}} h_{\text {br }} d V_{L}}_{\text {breakage }}+\underbrace{\int_{\Omega_{L}} h_{\text {agg }} d V_{L}}_{\text {agglomeration }}
\end{aligned}
$$

In Eq. (1) $n$ is the number density function of the particle population. This function depends on time, and in addition on external $x_{k}$ and internal $L_{j}$ coordinates which usually describe a geometry configuration and some properties of a system, respectively. The function $G_{k}$ denotes the growth rate of particles in the direction of internal coordinate $L_{j} . \nu_{k}$ is the fluid velocity in the direction of internal coordinate $L_{j}$ and $\mathrm{j}_{k}^{f}$ is the diffusion flow in the direction of external coordinate $x_{k}$. The nonlinear functions $h_{b r}$ and $h_{\text {agg }}$ denote breakage and agglomeration of particles respectively.

The numerical solution of such systems with several internal and external coordinates is hardly possible in real time. To enable model-based process design and process control of systems with particle population in fluid flow, there is a need for reduced models. The reduced models should have much lower system order than the reference models. In this paper Proper Orthogonal Decomposition (Kunisch and Volkwein (2003)) is used for the development of an automatic procedure for model reduction.

\section{MODEL REDUCTION}

Considering only linear models as the simplest case, a linear variant of the population balance equation (1) can be approximated by a set of ordinary differential equations

$$
\frac{d n(t)}{d t}=L_{n} n(t)
$$

where $n(t)$ is the discretized state vector, and $L_{n}$ denotes a system matrix of the reference model.

As a starting point, the detailed reference model has to be solved numerically. Snapshots for each state of the model $n\left(t_{1}\right), n\left(t_{2}\right), \ldots$ are collected and arranged as a matrix $N=\left(n\left(t_{1}\right), n\left(t_{2}\right), \ldots\right)$.

A reduced basis for the snapshot vectors is constructed from the singular value decomposition

$$
N=U \Sigma V^{T}
$$

The new basis vectors are taken as

$$
\psi_{i}^{n}=U_{i}, \quad i=1, \ldots, N^{n},
$$

where $U_{i}$ denotes the $i$ th column of $U$, and $N^{n}$ is the dimension of the reduced basis and correspondingly the order of the resulting reduced model.

The state vector $n(t)$ can be approximated by the following expression:

$$
n(t) \approx \psi^{n} \phi^{n}(t)
$$

where $\psi^{n}=\left(\psi_{1}^{n}, \ldots, \psi_{N^{n}}^{n}\right)$, and $\phi^{n}(t)$ is the coefficient vector of the reduced basis; $\phi^{n}(t)$ will become the state vector of the reduced model.

In order to obtain equations for $\phi^{n}(t)$, the approximation for the state vector $(5)$ is inserted into the discretized population balance equation (2). To make the projection of the residuals on the reduced basis vanish, Galerkin's method of weighted residuals is applied, which leads to

$$
\frac{\partial \phi^{n}(t)}{\partial t}=\underbrace{\psi^{n T} L_{n} \psi^{n}}_{=: L^{\prime}} \phi^{n}(t)
$$

The matrix $L^{\prime}$ on the right-hand side of (6) is a constant matrix and has to be evaluated only once. 


\section{AUTOMATIC PROCEDURE FOR MODEL REDUCTION}

An automatic procedure for the reduction of linear models is implemented in the modeling and simulation environment ProMoT/Diana. ProMoT is a modeling tool written in Common Lisp (Ginkel et al. (2003)). ProMoT provides a general modeling language and outputs to different numerical solution tools. Diana (Krasnyk (2009)) is a simulation tool for the solution and nonlinear analysis of differential algebraic systems.

The developed automatic tool for model reduction is a part of the ProMoT project and hence is written in Common Lisp. One uses Diana simulation tool only as an intermediate step for the numerical solution of the reference model. The main parts of the tool so far are the snapshots reader, the generator of basis functions, the extractor of the system matrix of the reference model and the generator of reduced model.

Via a Python script the user has to define model parameter values, a time range, and an output time interval for collecting snapshots to which the POD method is to be applied. After reading of all necessary information, the snapshots reader runs the $\mathrm{C}++$ code generator for Diana and translates the reference model for which we want to produce a reduced model. Then the snapshots reader runs Diana to generate snapshots and writes them into an output file. The last step of this part is to read the snapshots from the output file into the ProMoT environment and to arrange them into a matrix $N=$ $\left(n\left(t_{1}\right), n\left(t_{2}\right), \ldots\right)$.

When the work of the snapshots reader is finished, the automatic tool starts building the system matrix of the detailed reference model. The main idea in extracting the system matrix is a search of coefficients in the ordinary differential equations which correspond to the desired state variables of the reference model. The general form of an element of the system matrix of a linear autonomous system can be expressed by the following equation:

$$
L_{n i, j}=\frac{\partial O D E_{i}}{\partial s_{j}}
$$

In Eq. (7) $L_{n i, j}$ is an element of the system matrix at $i$ th row and at $j$ th column. $O D E_{i}$ is the right-hand side of the ordinary differential equation corresponding to $i$ th state variable, $s_{j}$ is an symbolic name of $j$ th state variable.

In ProMoT all modeling data is stored in symbolic form. To preserve generality and to preserve the dependency of the reduced model on the model parameters, the system matrix has to keep the elements in a symbolic form. For this purpose the computer algebra system Maxima is used. Since it is written in Common Lisp and can be called directly from Lisp code, Maxima is embedded into the ProMoT core. For convenience of use of the computer algebra system a program interface between ProMoT and Maxima has been developed. The interface allows to convert internal data structures of ProMoT into corresponding Maxima representation and vice versa.
In order to generate the equations of reduced model, it is necessary to calculate the reduced basis of model. A complicated mathematical apparatus is needed to accomplish this, in particular to calculate singular value decomposition of snapshots matrix. For these purposes it was decided to use a specialized software as an external tool. GNU Octave is a high-level interpreted language, primarily intended for numerical computations. To use this numerical tool externally a program interface between ProMoT and Octave has been developed. The interface allows to run Octave in interactive mode, send and receive data, and to apply all the built-in mathematical functions of Octave.

After completion of the above parts it is possible to calculate the system matrix $L^{\prime}$ of the reduced model from Eq. (6). The generator of the reduced model creates a new modeling file into which it writes the set of ordinary differential equations in the following form:

$$
\frac{d \phi_{i}^{n}(t)}{d t}=\sum_{j=1}^{N^{n}} L_{i, j}^{\prime} \phi_{j}^{n}(t)
$$

In Eq. (8) $\phi_{j}^{n}$ is a coefficient of the reduced basis and $N^{n}$ denotes the number of basis functions.

For reconstruction of actual states of the given model $n\left(t_{1}\right), n\left(t_{2}\right), \ldots$ one has to evaluate Eq. (5).

\section{OUTLOOK}

The automatic tool for reduction of linear models has been developed by using proper orthogonal decomposition (POD). The implemented approach has to be extended for nonlinear systems. For these purposes, automatic splitting of the ride-hand sides of differential equations into linear and nonlinear terms has to be done.

\section{REFERENCES}

Ginkel, M., Kremling, A., Nutsch, T., Rehner, R., and Gilles, E.D. (2003). Modular modeling of cellular systems with ProMoT/diva. Bioinformatics, 19(9), 11691176. doi:10.1093/bioinformatics/btg128.

Krasnyk, M. (2009). Ph.D. Thesis. Otto-von-GuerickeUniversity Magdeburg.

Kunisch, K. and Volkwein, S. (2003). Galerkin proper orthogonal decomposition methods for a general equation in fluid dynamics. SIAM Journal on Numerical Analysis, 40(2), 492-515.

Ramkrishna, D. (2000). Population balances: theory and application to particulate systems in engineering. Academic Press.

Wintermantel, K. (1999). Process and product engineeringachievements, present and future challenges. Chemical Engineering Science, 54, 1601-1620. doi: 10.1016/S0009-2509(98)00411-4. 


\title{
A Black-Box Method for Parametric Model Order Reduction
}

\author{
M. Geuss ${ }^{*}$ B. Lohmann ${ }^{*}$ B. Peherstorfer ${ }^{* *}$ K. Willcox ${ }^{* *}$ \\ * Institute of Automatic Control, Technische Universität München, \\ 85748 Garching, Germany (e-mail: matthias.geuss@tum.de). \\ ** Department of Aeronautics and Astronautics, MIT, Cambridge, MA \\ 02139, USA
}

\begin{abstract}
A black-box method for parametric model order reduction is presented that includes method selection, model refinement and error prediction using a cross-validation-based error indicator. The method is demonstrated for the interpolation of reduced system matrices.
\end{abstract}

Keywords: Parametric model order reduction; matrix interpolation; cross-validation; surrogate model; method selection; model refinement; error prediction

\section{INTRODUCTION}

With increasing demands on accuracy for optimization, simulation or control, the modeling of complex systems results in large-scale systems of ordinary differential equations. For many engineering applications the large-scale systems additionally depend on parameters, for example geometry or material parameters, which results in linear time-invariant (LTI), parameter-dependent systems

$$
G(\mathbf{p}):\left\{\begin{aligned}
\mathbf{E}(\mathbf{p}) \dot{\mathbf{x}}(t, \mathbf{p}) & =\mathbf{A}(\mathbf{p}) \mathbf{x}(t, \mathbf{p})+\mathbf{B}(\mathbf{p}) \mathbf{u}(t) \\
\mathbf{y}(t, \mathbf{p}) & =\mathbf{C}(\mathbf{p}) \mathbf{x}(t, \mathbf{p})
\end{aligned}\right.
$$

where the system matrices depend on the vector of parameters $\mathbf{p} \in \mathcal{D}$ with domain $\mathcal{D} \subset \mathbb{R}^{d}$. The vectors $\mathbf{u}(t) \in \mathbb{R}^{m}, \mathbf{y}(t, \mathbf{p}) \in \mathbb{R}^{p}$ and $\mathbf{x}(t, \mathbf{p}) \in \mathbb{R}^{n}$ denote the inputs, outputs and states of the system, respectively. Methods of parametric model order reduction (pMOR) have been developed to construct a parameter-dependent, low-order system which approximates the large-scale system $G(\mathbf{p})$. One can distinguish between global basis approaches such as global POD and local basis approaches such as interpolation of bases, transfer functions or system matrices. Greedy adaptive parameter sampling based on error indicators such as the residual can be applied to the global basis approaches. However, efficient adaptive selection of grid points (model refinement) has not been explored in the literature for local basis approaches due to missing error indicators. Additionally, it is an open question how the optimal interpolation method can be chosen in advance (method selection). In this contribution we propose a cross-validation-based error indicator for efficient method selection and model refinement for local basis approaches and demonstrate the method for the case of the interpolation of local reduced system matrices.

\section{PMOR BY MATRIX INTERPOLATION}

The method of interest interpolates local reduced system matrices to construct a parameter-dependent, reduced system, see Degroote et al. (2010); Panzer et al. (2010); Amsallem and Farhat (2011); Geuss et al. (2013). In the offline phase of the method the parameter domain $\mathcal{D}$ is sampled with a uniform distribution for a set of vectors $\mathcal{P}=\left\{\mathbf{p}_{1}, \ldots, \mathbf{p}_{N}\right\}$ and system $G(\mathbf{p})$ is computed for parameters in the set $\mathcal{P}$. The resulting local systems are reduced individually to order $q \ll n$. After adjustment of the bases of the local subspaces using transformation matrices $\mathbf{T}\left(\mathbf{p}_{i}\right)$ and $\mathbf{M}\left(\mathbf{p}_{i}\right)$, one finally obtains $N$ reduced, nonparametric systems $\hat{G}\left(\mathbf{p}_{i}\right)$ with $i=1, \ldots, N$ :

$$
\hat{G}\left(\mathbf{p}_{i}\right):\left\{\begin{aligned}
\hat{\mathbf{E}}\left(\mathbf{p}_{i}\right) \dot{\hat{\mathbf{x}}}\left(t, \mathbf{p}_{i}\right) & =\hat{\mathbf{A}}\left(\mathbf{p}_{i}\right) \hat{\mathbf{x}}\left(t, \mathbf{p}_{i}\right)+\hat{\mathbf{B}}\left(\mathbf{p}_{i}\right) \mathbf{u}(t) \\
\mathbf{y}\left(t, \mathbf{p}_{i}\right) & =\hat{\mathbf{C}}\left(\mathbf{p}_{i}\right) \hat{\mathbf{x}}\left(t, \mathbf{p}_{i}\right),
\end{aligned}\right.
$$

where $\hat{\mathbf{x}}\left(t, \mathbf{p}_{i}\right) \in \mathbb{R}^{q}$ is the reduced state vector and

$$
\begin{aligned}
& \hat{\mathbf{E}}\left(\mathbf{p}_{i}\right)=\mathbf{M}\left(\mathbf{p}_{i}\right)^{T} \mathbf{E}_{\text {red }}\left(\mathbf{p}_{i}\right) \mathbf{T}\left(\mathbf{p}_{i}\right), \\
& \hat{\mathbf{A}}\left(\mathbf{p}_{i}\right)=\mathbf{M}\left(\mathbf{p}_{i}\right)^{T} \mathbf{A}_{\text {red }}\left(\mathbf{p}_{i}\right) \mathbf{T}\left(\mathbf{p}_{i}\right), \\
& \hat{\mathbf{B}}\left(\mathbf{p}_{i}\right)=\mathbf{M}\left(\mathbf{p}_{i}\right)^{T} \mathbf{B}_{\text {red }}\left(\mathbf{p}_{i}\right), \\
& \hat{\mathbf{C}}\left(\mathbf{p}_{i}\right)=\mathbf{C}_{\text {red }}\left(\mathbf{p}_{i}\right) \mathbf{T}\left(\mathbf{p}_{i}\right) .
\end{aligned}
$$

Then, an interpolant is constructed for every matrix element of parameter-dependent, reduced system matrices $\tilde{\mathbf{E}}(\mathbf{p}), \tilde{\mathbf{A}}(\mathbf{p}), \tilde{\mathbf{B}}(\mathbf{p}), \tilde{\mathbf{C}}(\mathbf{p})$. For example, the interpolant of the $j k$-th coefficient of $\tilde{\mathbf{A}}(\mathbf{p})$ is $\tilde{a}^{j k}(\mathbf{p})=\sum_{i=1}^{N} \omega_{i}^{j k}(\mathbf{p}) \alpha_{i}^{j k}$ with basis functions $\omega_{i}^{j k}(\mathbf{p}): \mathcal{D} \rightarrow \mathbb{R}$ and coefficients $\alpha_{i}^{j k}$. For a cardinal function basis the procedure can be simplified to one interpolant $\tilde{\mathbf{A}}(\mathbf{p})=\sum_{i=1}^{N} \omega_{i}(\mathbf{p}) \hat{\mathbf{A}}\left(\mathbf{p}_{i}\right)$. In the online phase, to obtain a reduced system $\tilde{G}(\mathbf{p})$, the interpolants are evaluated at the parameter vector $\mathbf{p} \in \mathcal{D}$ and their outputs are assembled again into system matrices $\tilde{\mathbf{E}}(\mathbf{p}), \tilde{\mathbf{A}}(\mathbf{p}), \tilde{\mathbf{B}}(\mathbf{p}), \tilde{\mathbf{C}}(\mathbf{p})$. Alternatively, interpolation can take place in the tangent space of a matrix manifold.

\section{BLACK-BOX METHOD}

The black-box method automatically selects the interpolation method, performs the refinement in the parameter space and stops when the maximum predicted error of the interpolated system falls below a given error tolerance. The method is based on a new error indicator. 


\subsection{Cross-validation-based error indicator}

We introduce an error indicator which is based on available information at grid points in $\mathcal{P}$. For that we assign a leave-one-out cross-validation error to each grid point, see e.g. Bishop (2006). For example, if this error indicator is supposed to be calculated for grid point $\mathbf{p}_{i}$, one leaves out this point from set $\mathcal{P}$, builds new interpolants from the matrices at the remaining grid points in $\mathcal{P} \backslash\left\{\mathbf{p}_{i}\right\}$, evaluates them at $\mathbf{p}_{i}$ and computes the error of the interpolated system with respect to the reference system at $\mathbf{p}_{i}$. For error indicators at grid points from $\mathcal{P}$ we build in the following an aggregated error measure or construct a surrogate model to obtain the error indicator for parameters in $\mathcal{D}$.

\subsection{Method selection}

Interpolation methods for pMOR offer a variety of properties. Firstly, there are different basis functions. Secondly, the basis functions can additionally depend on a parameter. Thirdly, the interpolation can take place in different manifolds. This results in a large number of candidate interpolation methods. In order to select the method with the smallest error between the interpolated and directly reduced system for parameters in $\mathcal{D}$, we first assign a crossvalidation error to each grid point in $\mathcal{P}$. The interpolated system is compared to the directly reduced one. Finally, an aggregated error measure such as the root-mean-square error is calculated for the error indicators at grid points in $\mathcal{P}$ for all candidate interpolation methods. The method of interest is then the one with the minimum error measure.

\subsection{Model refinement}

Methods of pMOR based on interpolation require a regular or irregular grid of the parameter domain $\mathcal{D}$. In order to decrease the error of the interpolated model, new grid points are inserted. An refinement scheme identifies regions with high error between the interpolated system and the original system and inserts new grid points there. We again use the cross-validation-based error indicator and assign a cross-validation error to each grid point from $\mathcal{P}$. In contrast to Section 3.2 the interpolated system is compared to the high-order one as we are interested in the overall error and not only in the error due to interpolation. Afterwards, we build a surrogate of the error for parameters in $\mathcal{D}$. This surrogate model is then used to identify regions in $\mathcal{D}$ with high error indicator. Depending on the grid type we distinguish between two procedures. For a regular grid we build a surrogate model by linearly interpolating the error indicators at points from $\mathcal{P}$ and use a gradient-based approach to find the parameters for which the model predicts the maximum error. As a constraint we have to consider that the inserted grid points preserve the structure of the regular grid. For an irregular grid we construct a Kriging surrogate model and use Efficient Global Optimization (EGO) from Jones et al. (1998), where we maximize the Expected Improvement using a gradient-based multistart solver. Additionally, we avoid clustering by introducing constraints into the optimization. For parameters corresponding to the newly added points we perform local model order reduction and update the parameter-dependent, reduced model with the new local reduced systems. As the procedure is iterative, these steps are repeated until the maximum predicted error falls below a given tolerance, which will be explained in Section 3.4. For every grid point high-dimensional operations have to be performed only once and corresponding quantities can be stored and reused in every iteration for the calculation of the cross-validation errors.

\subsection{Model error prediction}

So far we used the cross-validation error in the surrogate model just as an indicator for the (unknown) error. Now we want to predict the maximum error so that we can assess the accuracy of the interpolated system and can stop the refinement procedure in Section 3.3 when the maximum predicted error falls below a given tolerance. The crossvalidation error indicator is in most cases larger than the true error because the cross-validation error was computed with one grid point less. Furthermore, we observed in numerical examples an approximately linear dependence between the cross-validation-based error indicator and the true interpolation error. Therefore, we propose a linear error model like the one suggested by Paul-DuboisTaine and Amsallem (2014) which is obtained via linear regression of an error pool. We save in every iteration the cross-validation-based error indicator and the true interpolation error which are calculated at the new grid points. This gives an error pool which grows with an increasing number of iterations. The maximum predicted error can then be calculated by inserting the maximum cross-validation error into the linear error model which delivers an increasingly accurate prediction with growing error pool.

\section{REFERENCES}

Amsallem, D. and Farhat, C. (2011). An online method for interpolating linear parametric reduced-order models. SIAM J. Sci. Comput., 33(5), 2169-2198.

Bishop, C.M. (2006). Pattern Recognition and Machine Learning. Springer, Berlin.

Degroote, J., Vierendeels, J., and Willcox, K. (2010). Interpolation among reduced-order matrices to obtain parameterized models for design, optimization and probabilistic analysis. Int. J. Numer. Methods Fluids, 63(2), 207-230.

Geuss, M., Panzer, H., and Lohmann, B. (2013). On parametric model order reduction by matrix interpolation. Proceedings of the European Control Conference, 34333438 .

Jones, D.R., Schonlau, M., and Welch, W.J. (1998). Efficient global optimization of expensive black-box functions. J. Global Optim., 13(4), 455-492.

Panzer, H., Mohring, J., Eid, R., and Lohmann, B. (2010). Parametric model order reduction by matrix interpolation. at-Automatisierungstechnik, 58(8), 475-484.

Paul-Dubois-Taine, A. and Amsallem, D. (2014). An adaptive and efficient greedy procedure for the optimal training of parametric reduced-order models. Int. J. Numer. Methods Engrg. Published online. 


\title{
Order-Reduction of Fields-Level Models with Affine and Non-Affine Parameters by Interpolation of Subspaces
}

\author{
S. Burgard * O. Farle* D. Klis* R. Dyczij-Edlinger* \\ * Chair for Electromagnetic Theory, Saarland University, \\ Campus C63, D-66123 Saarbrücken, Germany \\ (e-mail: edlinger@lte.uni-saarland.de)
}

\begin{abstract}
Keywords: Computer aided engineering, numerical analysis, parametric models, parametric
\end{abstract} order reduction, reduced order system.

\section{SUMMARY}

Model-order reduction provides an appealing approach to solving parametric large-scale models stemming from spatial discretization methods. The high-dimensional system at the fields-level is replaced by a surrogate model that is fast to evaluate, at a controllable level of error. This paper presents an interpolation-based order-reduction method for systems with non-affine parameters. The main novelty is the construction of the parameter-dependent projection matrix. For a given error level, the suggested approach reduces the number of instantiations of the fields-level model compared to state-of-the-art methods.

The behavior of physical structures generally depends on a number of parameters, such as materials or shape. Spatial discretization methods, like finite elements (FE), are proven numerical techniques for analyzing physical structures at the fields-level. The resulting models are high-dimensional systems of equations which have to be assembled and solved separately for each considered configuration. Although one single evaluation may not be particularly demanding on modern computers, large evaluation counts, as encountered in parameter studies or numerical optimization, may rapidly exhaust computational resources.

Methods of parametric model-order reduction (PMOR) provide a possible remedy. They approximate the original large-scale model by a cheap reduced-order model (ROM) of low dimension but very similar response. Most PMOR methods are of projection type, i.e., the fields-level model is projected onto a carefully chosen low-dimensional subspace. A necessary condition for the efficient application of this approach is the presence of affine parameter depencence: The parameters must appear explicitly in the fieldslevel model. Typical affine parameters are the frequency and material properties. Geometrical parameters usually enter the fields-level model in a non-affine way. In recent times, projection-based PMOR methods have been extended to systems with non-affine parameters: The PMOR method proposed in this paper constructs a parameterdependent projection matrix by interpolating projection spaces with respect to the non-affine parameters. This approach offers the possibility to make the dimension of the
ROM independent of the number of non-affine parameters. To render the local projection bases compatible for interpolation, a novel projection-and-scaling step is introduced. The present method improves subspace interpolation compared to prior works and achieves higher convergence rates than methods that interpolate ROMs.

Fields-Level System: We consider a linear and timeinvariant numerical model $\Sigma$ with $q$ inputs and outputs, which depend on vectors $\mathbf{s} \in \mathcal{Q} \subset \mathbb{R}^{Q}$ and $\mathbf{p} \in \mathcal{P} \subset \mathbb{R}^{P}$, of affine and non-affine parameters, respectively. To simplify presentation, let the input matrix $\mathbf{B} \in \mathbb{C}^{N \times q}$ depend on $\mathbf{s}$ only, $\mathbf{B}=\mathbf{B}(\mathbf{s})$, and the system matrix $\mathbf{A} \in \mathbb{C}^{N \times N}$ be complex symmetric, $\mathbf{A}=\mathbf{A}^{T}$. Moreover, let $\Sigma(\mathbf{s}, \mathbf{p})$ possess the symmetric structure

$$
\begin{aligned}
\mathbf{A}(\mathbf{s}, \mathbf{p}) \mathbf{x}(\mathbf{s}, \mathbf{p}) & =\mathbf{B}(\mathbf{s}) \mathbf{u}, \\
\mathbf{y}(\mathbf{s}, \mathbf{p}) & =\mathbf{B}(\mathbf{s})^{T} \mathbf{x}(\mathbf{s}, \mathbf{p}),
\end{aligned}
$$

where $\mathbf{x} \in \mathbb{C}^{N}, \mathbf{y} \in \mathbb{C}^{q}, \mathbf{u} \in \mathbb{C}^{q}$ are the solution vector, the output vector, and the input vector, respectively. Let $\mathbf{B}_{m} \in \mathbb{C}^{N \times q}$ denote coefficient matrices, $\Theta_{k}, \hat{\Theta}_{m}$ : $\mathcal{Q} \rightarrow \mathbb{C}$ scalar functions of the affine parameters, and $\mathbf{A}_{k}(\mathbf{p}) \in \mathbb{C}^{N \times N}$ matrix-valued functions of the non-affine parameters. Then $\mathbf{A}(\mathbf{s}, \mathbf{p})$ and $\mathbf{B}(\mathbf{s})$ may be written as

$$
\begin{aligned}
\mathbf{A}(\mathbf{s}, \mathbf{p}) & =\sum_{k} \Theta_{k}(\mathbf{s}) \mathbf{A}_{k}(\mathbf{p}), & \mathbf{s} \in \mathcal{Q}, \mathbf{p} \in \mathcal{P} \\
\mathbf{B}(\mathbf{s}) & =\sum_{m} \hat{\Theta}_{m}(\mathbf{s}) \mathbf{B}_{m}, & \mathbf{s} \in \mathcal{Q} .
\end{aligned}
$$

Reconstruction of affine parametrization: It was proposed in Grepl et al. (2007) and Dyczij-Edlinger and Farle (2009) to replace the coefficients $\mathbf{A}_{k}(\mathbf{p})$ in (2) by affine interpolations $\mathbf{A}_{k}^{\text {in }}(\mathbf{p}) \approx \mathbf{A}_{k}(\mathbf{p})$ with

$$
\mathbf{A}_{k}^{\mathrm{in}}(\mathbf{p})=\sum_{j} \Gamma_{j}(\mathbf{p}) \mathbf{A}_{j, k}
$$

where $\Gamma_{j}: \mathcal{P} \rightarrow \mathbb{R}$ are multivariate interpolation functions and $\mathbf{A}_{j, k} \in \mathbb{C}^{N \times N}$ matrix-valued coefficients. The resulting interpolated model $\Sigma^{\text {in }}(\mathbf{s}, \mathbf{p})$ exhibits affine parametrization only. It reads

$$
\begin{aligned}
\mathbf{A}^{\text {in }}(\mathbf{s}, \mathbf{p}) \mathbf{x}^{\prime}(\mathbf{s}, \mathbf{p}) & =\mathbf{B}(\mathbf{s}) \mathbf{u} \\
\mathbf{y}^{\prime}(\mathbf{s}, \mathbf{p}) & =\mathbf{B}(\mathbf{s})^{T} \mathbf{x}^{\prime}(\mathbf{s}, \mathbf{p})
\end{aligned}
$$

with solution $\mathbf{x}^{\prime}(\mathbf{s}, \mathbf{p}) \in \mathbb{C}^{N}$, output $\mathbf{y}^{\prime}(\mathbf{s}, \mathbf{p}) \in \mathbb{C}^{q}$, and

$$
\mathbf{A}^{\text {in }}(\mathbf{s}, \mathbf{p})=\sum_{k} \Theta_{k}(\mathbf{s}) \mathbf{A}_{k}^{\text {in }}(\mathbf{p})
$$


Order reduction by projection: Let us restrict the domain of the solution $\mathbf{x}^{\prime}(\mathbf{s}, \mathbf{p})$ to a parameter-dependent subspace $\mathcal{S}(\mathbf{p}) \in \mathbf{G r}(N, n)$ of dimension $n$ of the Grassmann manifold $\operatorname{Gr}(N, n)$; see Edelman et al. (1998). Given a basis $\mathbf{V}(\mathbf{p}) \in \mathbb{R}^{N \times n}$ for $\mathcal{S}(\mathbf{p})$, i.e., $\mathcal{S}(\mathbf{p})=\operatorname{range}\{\mathbf{V}(\mathbf{p})\}$, Galerkin testing by $\mathbf{V}(\mathbf{p})$ leads to the $\operatorname{ROM} \widetilde{\Sigma}(\mathbf{s}, \mathbf{p})$

$$
\begin{aligned}
\widetilde{\mathbf{A}}^{\text {in }}(\mathbf{s}, \mathbf{p}) \widetilde{\mathbf{x}}(\mathbf{s}, \mathbf{p}) & =\widetilde{\mathbf{B}}(\mathbf{s}) \mathbf{u}, \\
\widetilde{\mathbf{y}}(\mathbf{s}, \mathbf{p}) & =\widetilde{\mathbf{B}}(\mathbf{s})^{T} \widetilde{\mathbf{x}}(\mathbf{s}, \mathbf{p}),
\end{aligned}
$$

with $\widetilde{\mathbf{x}}(\mathbf{s}, \mathbf{p}) \in \mathbb{C}^{n}, \widetilde{\mathbf{y}}(\mathbf{s}, \mathbf{p}) \in \mathbb{C}^{q}$, and the reduced matrices

$$
\begin{aligned}
\widetilde{\mathbf{A}}^{\mathrm{in}}(\mathbf{s}, \mathbf{p}) & =\mathbf{V}^{T}(\mathbf{p}) \mathbf{A}^{\mathrm{in}}(\mathbf{s}, \mathbf{p}) \mathbf{V}(\mathbf{p}), \\
\widetilde{\mathbf{B}}(\mathbf{s}, \mathbf{p}) & =\mathbf{V}^{T}(\mathbf{p}) \mathbf{B}(\mathbf{s}) .
\end{aligned}
$$

Construction of parameter-dependent subspaces: The domain of non-affine parameters $\mathcal{P}$ is equipped with a grid $\mathcal{G}$ of interpolation points $\mathbf{p}_{i}$, at which the fields-level model $\Sigma(\mathbf{s}, \mathbf{p})$ is instantiated. Since $\Sigma\left(\mathbf{s}, \mathbf{p}_{i}\right)$ depends on affine parameters only, standard PMOR methods can be used to generate orthogonal projection matrices $\mathbf{V}_{i} \in \mathbb{R}^{N \times n}$, with $\operatorname{rank}\left(\mathbf{V}_{i}\right)=n$, at the grid points $\mathbf{p}_{i}$. The next step is to choose a reference basis $\mathbf{V}_{c} \in \mathbb{R}^{N \times n}$ of rank $n$ for each $P$ cell of the grid. Let $\xi_{\mathcal{S}_{i}}\left(\mathbf{V}_{c}\right) \in \mathbb{R}^{N \times n}$ denote the orthogonal projection of $\mathbf{V}_{c}$ to the subspace $\mathcal{S}_{i}=\operatorname{range}\left\{\mathbf{V}_{i}\right\}$. Then we require of $\mathbf{V}_{c}$ that

$$
\operatorname{rank}\left(\xi_{\mathcal{S}_{i}}\left(\mathbf{V}_{c}\right)\right)=n \quad \forall i
$$

holds. With interpolation functions $\Omega_{i}: \mathcal{P} \rightarrow \mathbb{R}$ such that

$$
\Omega_{i}\left(\mathbf{p}_{j}\right)=\delta_{i j}
$$

a parameter-dependent basis $\mathbf{V}(\mathbf{p})$ is given by

$$
\mathbf{V}(\mathbf{p})=\sum_{i} \Omega_{i}(\mathbf{p}) \theta_{i, c} \circ \xi_{\mathcal{S}_{i}}\left(\mathbf{V}_{c}\right) .
$$

Herein, the scalings $\theta_{i, c}: \mathbb{R}^{N \times n} \rightarrow \mathbb{R}^{N \times n}$ are provided to normalize the column vectors after the projection step. Hence the interpolated matrices of (11) take the form

$$
\theta_{i, c} \circ \xi_{\mathcal{S}_{i}}=\mathbf{V}_{i} \mathbf{V}_{i}^{T} \mathbf{V}_{c} \mathbf{D}_{i, c}, \quad \text { with } \mathbf{D}_{i, c}=\operatorname{diag} \theta_{i, c},
$$

and the resulting ROM is given by

$$
\begin{aligned}
& \sum_{i, j, k, l} \Omega_{i}(\mathbf{p}) \Gamma_{j}(\mathbf{p}) \Theta_{k}(\mathbf{s}) \Omega_{l}(\mathbf{p}) \widetilde{\mathbf{A}}_{i, j, k, l} \widetilde{\mathbf{x}}(\mathbf{s}, \mathbf{p})= \\
& \quad \sum_{i, m} \Omega_{i}(\mathbf{p}) \hat{\Theta}_{m}(\mathbf{s}) \widetilde{\mathbf{B}}_{i, m} \mathbf{u}, \\
& \widetilde{y}=\sum_{i, m} \Omega_{i}(\mathbf{p}) \hat{\Theta}_{m}(\mathbf{s}) \widetilde{\mathbf{B}}_{i, m}^{T} \widetilde{\mathbf{x}}(\mathbf{s}, \mathbf{p}),
\end{aligned}
$$

where

$$
\begin{aligned}
\widetilde{\mathbf{A}}_{i, j, k, l} & =\left(\mathbf{V}_{i} \mathbf{V}_{i}^{T} \mathbf{V}_{c} \mathbf{D}_{i, c}\right)^{T} \mathbf{A}_{j, k}\left(\mathbf{V}_{l} \mathbf{V}_{l}^{T} \mathbf{V}_{c} \mathbf{D}_{l, c}\right), \\
\widetilde{\mathbf{B}}_{i, m} & =\left(\mathbf{V}_{i} \mathbf{V}_{i}^{T} \mathbf{V}_{c} \mathbf{D}_{i, c}\right)^{T} \mathbf{B}_{m} .
\end{aligned}
$$

Since the coefficients $\widetilde{\mathbf{A}}_{i, j, k, l} \in \mathbb{C}^{n \times n}$ and $\widetilde{\mathbf{B}}_{i, m} \in \mathbb{C}^{n \times q}$ do not depend on $\mathbf{s}$ and $\mathbf{p}$, they can be computed in an offline step, and the evaluation of (13) becomes independent of the dimension of the fields-level model.

Implementation details: The interpolation grid $\mathcal{G}$ is taken to be a tensor grid, and the interpolation functions for the non-affine parameters, $\Gamma_{j}(\mathbf{p})$ of $(4)$, are products of Lagrange polynomials. Thanks to $\Gamma_{j}\left(\mathbf{p}_{k}\right)=\delta_{i k}$, we have

$$
\mathbf{A}_{j, k}=\mathbf{A}_{k}\left(\mathbf{p}_{\alpha^{j}}\right) \text {. }
$$

For subspace interpolation, we employ piecewise multilinear interpolation functions whose support is given by the hypercubes adjacent to the interpolation point. They are built from univariate hat functions.

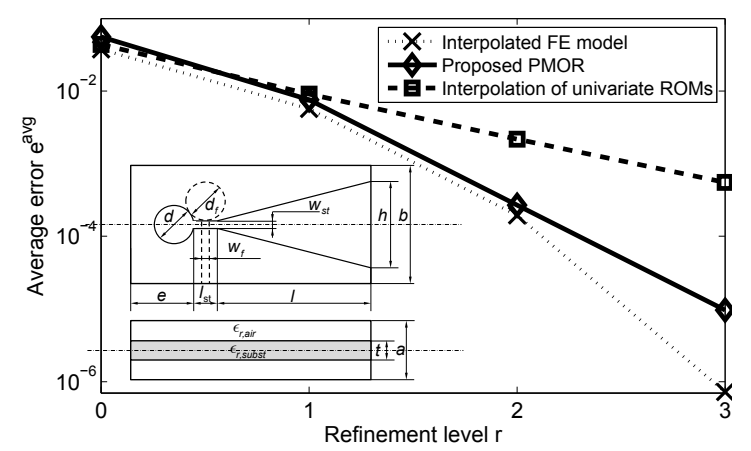

Fig. 1. Convergence of interpolated FE model, present approach, and interplation of univariate ROMs. Dimensions in $\mathrm{cm}: e=1.5, l_{s t}=0.5, w_{s t}=0.1, h=2.2$, $d=1, b=3.2, t=0.288, a=3.45, w_{f}=0.2, d_{f}=0.9$. Material: $\epsilon_{r, \text { air }}=1, \epsilon_{r, \text { subst }}=2.2, \sigma=0, \mu_{r}=1$.

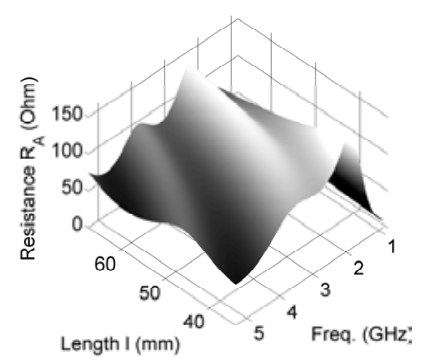

(a) Resistance $R$.

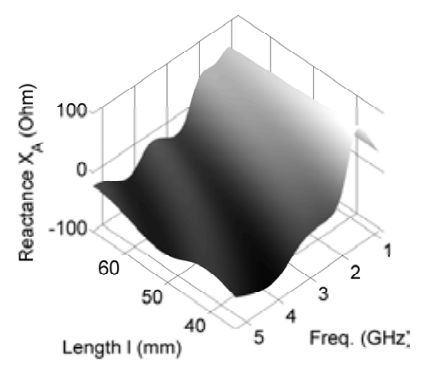

(b) Reactance $X$.
Fig. 2. Input impedance versus length $l$ and frequency $f$.

\section{NUMERICAL EXAMPLE}

The inset of Fig. 1 shows the Vivaldi notch antenna of Shin and Schaubert (1999). We consider the taper length $l \in$ $[3.5,6.5] \mathrm{cm}$ as a non-affine parameter and the frequency $f \in[0.5,5.5] \mathrm{GHz}$ as an affine one. FE discretization leads to a model $\Sigma(f, l)$ of $N=1,045,577$ unknowns. Fig. 1 presents the average error $e^{\text {avg }}$ versus the number of hierarchical refinement steps of $\mathcal{G}$, the refinement level $r$ : The rate of convergence of the present technique is higher than with a competing method and, up to $r=2$, FE matrix interpolation is the dominant source of error. The following data are for $r=2: e^{\text {avg }}=4.25 \cdot 10^{-4}$, which is below typical FE discretization error; off-line time for PROM generation is $50.02 \mathrm{~min}$, and PROM evaluation takes just $525 \mu \mathrm{s}$. Thus, computing the impedance surfaces of Fig. 2 from $318^{2}$ points is accomplished within $53.04 \mathrm{~s}$.

\section{REFERENCES}

R. Dyczij-Edlinger and O. Farle. Finite element analysis of linear boundary value problems with geometrical parameters. COMPEL, 28(4): 779-794, 2009.

A. Edelman, T.A. Arias, and S.T. Smith. The geometry of algorithms with orthogonality constraints. SIAM J. Matrix Anal. Appl., 20(2): 303-353, 1998.

M.A. Grepl, Y. Maday, N.C. Nguyen, and A.T. Patera. Efficient reduced-basis treatment of nonaffine and nonlinear partial differential equations. Math. Model. Numer. Anal., 41(3): 575-605, 2007.

J. Shin and D.H. Schaubert. A parameter study of stripline-fed Vivaldi notch-antenna arrays. IEEE Trans. Antennas Propag., 47(5): 879-886, 1999. 


\title{
Non-Modal Based Model Reduction for Explicit Crash Codes *
}

\author{
Joerg Fehr* \\ * Institute of Engineering and Computational Mechanics, University of \\ Stuttgart, Pfaffenwaldring 9, 70569 Stuttgart (e-mail: \\ joerg.fehr@itm.uni-stuttgart.de).
}

\begin{abstract}
Model order reduction is scarcely used for crash simulations and, if at all, only in its traditional modal forms, e.g. the Craig-Brampton substructure reduction method. In order to use other MOR techniques, based on moment matching or singular value decomposition (SVD), it is necessary to couple LS-Dyna and Radioss with an external model reduction program. Here, the model reduction program Morembs is used for reduction. The purpose of this discussion is to evaluate and compare the implementation of coupling LS-Dyna and Radioss with Morembs. As an example, the flexible degrees of freedom of an anti-roll bar are reduced.
\end{abstract}

Keywords: Model Reduction, Explicit Crash Simulations, Krylov-subspaces, Gramian matrices, Component Mode Synthesis.

\section{INTRODUCTION}

Crash simulations are one of the most computer timeconsuming tasks in car design. The use of model order reduction (MOR) for speedup and data reduction is a logical consequence. For crash simulations however, MOR is predominantly used in its traditional modal forms, e.g. the Craig-Brampton substructure reduction method, if at all. In this research, MOR methods from system dynamics and mathematics are tested with regard to their use in LS-Dyna or Radioss. Contrary to modal reduction methods, MOR techniques, based on moment matching or singular value decomposition (SVD), have error bounds and can be tuned in a certain frequency range. For the application of non-modal MOR techniques it is necessary to couple LS-Dyna and Radioss with an external MOR program. Here, Morembs is used, see Fehr (2011). The focus of this discussion is the implementation and validation of the workflow, excluding the development of a new MOR technique. Therefore, we refer to e.g. Fehr (2011), Holzwarth and Eberhard (2014) for theoretical aspects.

\section{IMPLEMENTATION AND VALIDATION OF MOR WITH MOREMBS}

Our objective is the use of Morembs for MOR of crash models in explicit integrators. In this context, the toolbox is extended with a feature to import FE data from the explicit FE code LS-Dyna. For linear MOR it is necessary to import the system matrices of the linear FE equation

$$
\boldsymbol{M}_{e} \cdot \ddot{\boldsymbol{q}}+\boldsymbol{D}_{e} \cdot \dot{\boldsymbol{q}}+\boldsymbol{K}_{e L} \cdot \boldsymbol{q}=\boldsymbol{f}
$$

the symmetric mass matrix $\boldsymbol{M}_{e}$, the at least positive semidefinite linear stiffness matrix $\boldsymbol{K}_{e L}$, a velocity de-

* The authors would like to thank the German Research Foundation (DFG) for financial support of the project within the Cluster of Excellence in Simulation Technology (EXC 310/1) at the University of Stuttgart. pendent damping term $\boldsymbol{D}_{e} \cdot \dot{\boldsymbol{q}}$, the applied forces $\boldsymbol{f}$ and the nodal information, into the external MOR program. Initially, the full FE model is modeled in LS-Dyna. Subsequently, the system matrices of the FE body are dumped onto the disk in the Harwell-Boeing format. This is achieved by using the implicit solver options of LS-Dyna. Following the dump of the system matrices, all the nodal information plus the system matrices are imported into Morembs. Unfortunately, in Radioss it is not possible to gain access to the assembled system matrices. Therefore, the full FE body needs to be modeled with another FE program, e.g. Abaqus, as used in this discussion. Afterwards, all the MOR features of Morembs can be used to reduce the full FE body. The final step is the simulation of the reduced elastic body with an explicit FE solver used for crash simulations. Therefore, the reduced FE system,

$$
\begin{aligned}
\underbrace{\boldsymbol{V}^{T} \cdot \boldsymbol{M}_{e} \cdot \boldsymbol{V}}_{\overline{\boldsymbol{M}}_{e}} \cdot \ddot{\overline{\boldsymbol{q}}}(t)+\underbrace{\boldsymbol{V}^{T} \cdot \boldsymbol{D}_{e} \cdot \boldsymbol{V}}_{\overline{\boldsymbol{D}}_{e}} \cdot \dot{\overline{\boldsymbol{q}}}(t)+ \\
\underbrace{\boldsymbol{V}^{T} \cdot \boldsymbol{K}_{e} \cdot \boldsymbol{V}}_{\overline{\boldsymbol{K}}_{e}} \cdot \overline{\boldsymbol{q}}(t)=\underbrace{\boldsymbol{V}^{T} \cdot \boldsymbol{f}}_{\overline{\boldsymbol{f}}},
\end{aligned}
$$

where $\boldsymbol{V}$ is the Galerkin projection matrix calculated by the various reduction approaches, is exported into Ls-Dyna and Radioss and simulated thereafter.

In Ls-Dyna, the reduced elastic body is implemented as a superelement based on the Component Mode Synthesis (CMS) approach, see Craig (2000). The CMSsuperelement is represented in the Nastran DMIG-format. For this reason, a new converter is written which exports the reduced elastic body (reduced system matrices) into the ASCII Nastran DMIG-format and enables the reduced elastic body to be simulated in LS-Dyna. The use of standard second order Krylov or Gramian MOR procedures as explained in Fehr (2011) is not feasible. The lack of feasibility stems from the fact that LS-Dyna requires the reduced model given in a CMS-based format. In order to use CMS-based reduction with input- 
output-based MOR methods, like SVD- and Krylov-based reduction, the method of Holzwarth and Eberhard (2014) is facilitated. This allows using SVD- and Krylov-based reduction methods in combination with the CMS approach see Holzwarth and Eberhard (2014).

The implementation of the reduced elastic body in Radioss is not based on a CMS but on a floating frame of reference approach, see e.g. Flidrova et al. (2010). Such an approach allows a correct consideration of the large nonlinear rotations of an elastic body. In Radioss the procedure is based on the four masses approach, see e.g. Flidrova et al. (2010). It implies that the nonlinear rigid body movement of the elastic body is represented by the movement of four masses, described by 15 ansatz functions. An explanation how these ansatz functions are calculated can be found e.g. in Flidrova et al. (2010). Finally, a .fxb file which contains all the necessary information needed to simulate the reduced elastic body in Radioss is obtained.

With the converters implemented, it is examined if the reduction with Morembs yields to the same results as the reduction with traditional workflow in LS-Dyna and Radioss. The application example is the model of a stabilization linkage of a car front suspension. In this example, the displacement of node 20 in $z$-direction is chosen as output of the system. The linkage is excited at node 20 by a sinusoidal force $F$ with a frequency of $1 \mathrm{~Hz}$ and an amplitude of $100 \mathrm{~N}$ in $z$-direction. In Fig. 1, a comparison between the full and the reduced model is drawn. The models are reduced either in Radioss, LS-Dyna or Morembs. Subsequently, the reduced models are simulated with either Radioss or LS-Dyna. Both implementations lead to the correct results.

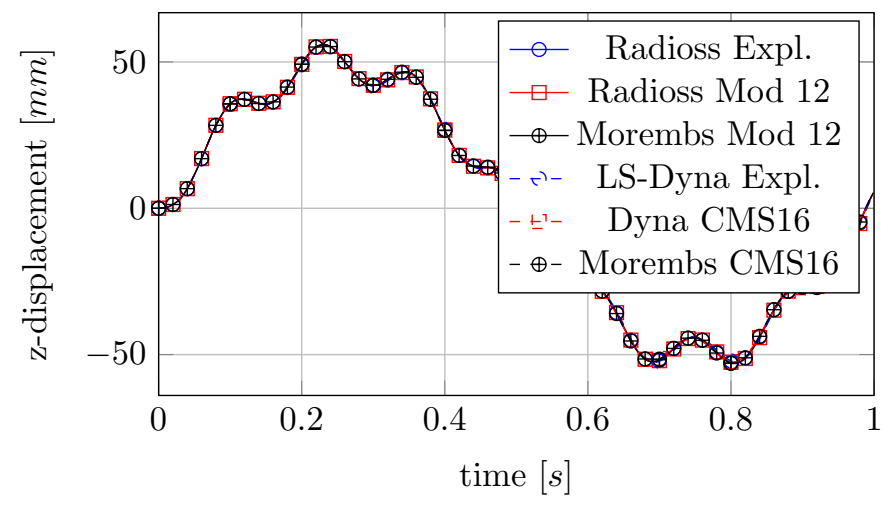

Fig. 1. The displacement of node 20 in $\mathrm{z}$ direction is plotted. The original unreduced Radioss -0 and LS-Dyna- ${ }^{-}-$models are compared with the reduced models. The reduced model is calculated once with the traditional workflow $\square /-\square^{-}$and with the new workflow in Morembs $\rightarrow-/-\oplus-$.

\section{CONCLUSION}

After the successful coupling of LS-Dyna and Radioss to Morembs it is now possible to use non-modal MOR techniques for the simulation of linear reduced elastic bodies within Radioss and LS-Dyna. That is why a sensitivity analysis of the simulation results based on different reduced models is conducted. The influence of the coupling of the flexible body on the environment (a free vs. a fixed

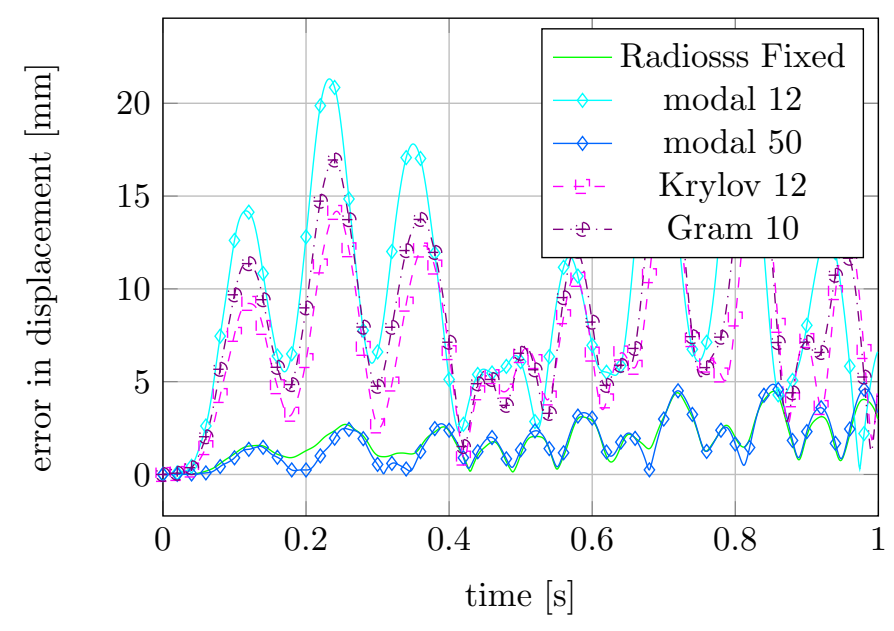

Fig. 2. Displacement error of node 20 in Radioss for different reduced models

approach) and the influence of the different reduced models on the runtime of the reduced models are examined. As an example of the conclusions/results in Fig. 2 the absolute error in the displacement is plotted. In a free system, 50 modal ansatz functions ( line $\rightarrow$ ) are necessary to achieve the same approximation quality as achieved with 10 ansatz functions in a fixed system (line - - ). In addition for the free system, it is revealed that input/output-based reduction methods, like Krylov (line - $\left\llcorner^{-}\right.$) and Gramian based reduction methods (line - - ) reach a better approximation compared to modal reduction methods. With 10 are achieved than with 12 modal ansatz functions $\rightarrow$. Regarding the input/output-based reduction methods the $\mathrm{x}-, \mathrm{y}-, \mathrm{z}-$ degree of freedom at node 1 and node 20 are considered as input and output. Concerning the Krylov reduction method the first two moments at expansion point $s_{k}=i 2 p i 1 \mathrm{~Hz}$ are matched. The Gramian-based reduction is based on a POD-based approximation of the frequency weighted second order position Gramian matrix $\boldsymbol{P}_{p}^{\omega}$ where the interesting frequency range is between $\left[f_{\min }, f_{\max }\right]=[0,20 \mathrm{~Hz}]$.

\section{REFERENCES}

Craig, R. (2000). Coupling of substructures for dynamic analyses: An overview. In Proceedings of the AIAA Dynamics Specialists Conference, Paper-ID 2000-1573, Atlanta, April 5.

Fehr, J. (2011). Automated and error-controlled model reduction in elastic multibody systems, volume 21 of Dissertation, Schriften aus dem Institut für Technische und Numerische Mechanik der Universität Stuttgart. Shaker Verlag, Aachen.

Flidrova, K., Lenoir, D., Vasseur, N., and Jzquel, L. (2010). Modelization by superelements with contact management in explicit car crash simulations. In A. Mikkola (ed.), 1st Joint Int. Conf. on Multibody System Dynamics (IMSD10, Lappeenranta, Finland, 25-27 May 2010).

Holzwarth, P. and Eberhard, P. (2014). Svd-based improvements for component mode synthesis in elastic multibody systems. European Journal of Mechanics A/Solids, 49, 408-418. 


\title{
Improvement of Krylov-Subspace-Reduced Models by Iterative Mode-Truncation
}

\author{
Claudius Lein ${ }^{*}$ Michael Beitelschmidt ${ }^{* *}$ David Bernstein \\ * Technische Universität Dresden, Faculty of Mechanical Science and \\ Engineering, Department of Solid Mechanics, Chair of Dynamics and \\ Mechanism Design, 01062 Dresden, Germany \\ (e-mail: Claudius.Lein@tu-dresden.de) \\ ** (e-mail: Michael.Beitelschmidt@tu-dresden.de)
}

\section{INTRODUCTION}

Model order reduction (MOR) techniques are state of the art and well established in control theory and likewise in structural mechanics, whereof this article focuses the latter. Applications are found in Elastic Multi-Body-Systems (EMBS), where flexible bodies are represented by reduced Finite-Element (FE) models.

Since years, Krylov-subspace-based reduction techniques are common practise. Especially Krylov-subspace-methods (KSM) based on the second-order Arnoldi-algorithm (SOAR) stand out of the broad range of reduction methods available. Besides the classic KSM formulation, a Rational KSM (RKSM) with variable expansion points gives promising results.

But no matter which reduction technique is utilized, the main objective lies in finding a minimal reliable model dimension with desired quality within a predefined frequency range. Current research concerning optimal MOR, usually based on the $\mathcal{H}_{2}$-error, can be found in Bunse-Gerstner et al. (2007) and Gugercin et al. (2008). The works of Eid et al. (2009) and Fehr et al. (2013) deal with generating an optimal reduced model based on RKSM by iteratively choosing the expansion points. In all works, the dimension of the reduced model is fixed by keeping the number of expansion points and the order of the series expansion constant. The drawbacks are the expensive iteration process, since the Krylov-modes need to be recalculated, and the fixed dimension, which is difficult to choose in advance.

The novel approach presented consists of a two-stage strategy and is based on a RKSM reduction with fixed expansion points. Firstly, the reduction is only performed once for an extended order of the series expansion resulting in a fairly large reduced model, called reference model. Secondly, this reference model is improved by iteratively truncating unnecessary Krylov-modes until a minimal reduced model with desired quality is reached. Due to a convergence observation, a reliable model dimension can confidently be determined.

In contrast, two-step reduction methods use two sequential reduction schemes, e.g. KSM followed by Balanced Truncation (BT). The novel approach does not belong to this category, since the second step exhibits no projection matrix. Its benefit is the generation of a minimal possible dimension at a physical error value.
In this article, KSM techniques based on a SOAR-algorithm according to Lehner (2007) pp. 144-146 are considered. The effort of the new method lies in calculating the Krylov-modes onetime, which is potentially faster compared to an iterative calculation of Krylov-modes.

\section{NOVEL APPROACH}

\subsection{Iterative Mode-Truncation}

The distribution of expansion points represented by frequencies $f_{e, i}$ affects the quality of the reduced model. The novel approach constitutes of successively reducing the order $j$ by keeping number and distribution of expansion points $s_{e, i}=\left(2 \pi f_{e, i}\right)^{2}$ constant. Within the desired frequency range $\left[f_{0}, f_{e n d}\right]$ the frequencies $f_{e, i}$ are distributed equidistantly according to the following formula:

$$
\Delta f=\frac{f_{\text {end }}-f_{0}}{n_{e}} \quad, \quad f_{e, i}=f_{0}+(i-0.5) \cdot \Delta f
$$

For quantifying the differences in natural frequencies the Natural Relative Frequency Difference (NRFD) is applied, which compares the natural frequencies of the original and of the reduced model $f_{0, i}$ and $\bar{f}_{0, i}$ for the $p$ first modes within a desired frequency range:

$$
\mathrm{NRFD}_{i}=\left|1-\frac{\bar{f}_{0, i}}{f_{0, i}}\right| \quad, \quad i=1 \ldots p .
$$

The order of series is truncated iteratively at all expansion points until the predefined NRFD-limit is reached, see Fig. 1. The quadratic mean value of the NRFD should be below $0.001 \%$. Alternatively, a maximal NRFD-error below $0.1 \%$ can be used, giving more conservative results.

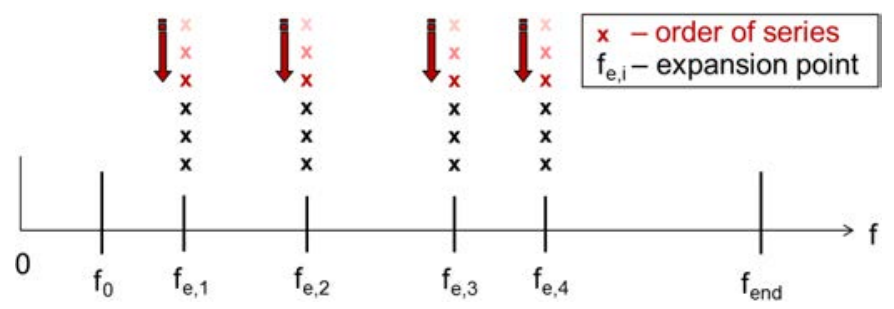

Fig. 1. Schematic diagram of iterative mode-truncation after KSM-reduction, 3 iterations $\left(n_{e}=4, j=6 \rightarrow 3, n=24 \rightarrow 12\right)$ 
The calculation of the reference model is the main cost, which only needs to be done once in advance. The iterative truncation means no additional cost, if the reordering scheme is applied. The kernel for this fast approach is the orthogonalization sequence. Simply neglecting Krylovmodes in $\mathbf{V}$ requires the reordering of the projection matrix according to the order of series expansion. For that reason, the SOAR-algorithm is performed for each expansion point separately.

Finally, the reordered matrix is orthogonalized under application of an overall deflation strategy. Since the orthogonalization does not influence the subspace spanned and only partitions the basis vectors, the following theorem holds: The sequence of vectors before the orthogonalization does not affect the spanned subspace. That way, only the last Krylov-modes are dropped and a recalculation of the whole projection matrix can be avoided. Note that dropping any Krylov-modes departing from the prescribed order would require an expensive and additional orthogonalization.

The principle of reordering after the KSM-reduction is shown in Fig. 2. The Krylov-modes in the projection matrix are sorted in ascending order for each order of the series expansion. Three steps are required:

(1) Calculation of Krylov-modes by SOAR including a block-orthogonalization,

(2) Reordering of Krylov-modes (Fig. 2),

(3) Orthogonalization of all Krylov-modes including an overall deflation strategy.

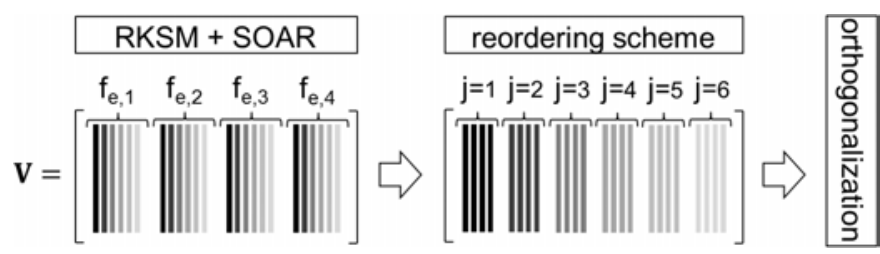

Fig. 2. Reordering scheme for projection matrix

$$
\left(n_{e}=4, j=6, n=24\right)
$$

The procedure of the novel approach is illustrated in Fig. 3 and can be summarized as follows:

(1) Definition of frequency range of interest $\left[f_{0}, f_{\text {end }}\right]$ and number of expansion points $n_{e}$,

(2) Generation of fairly large reduced reference model of dimension $n$ by RKSM,

(3) Iterative procedure:

(a) Truncation of Krylov-modes based on reduced reference model,

(b) Eigenanalysis of truncated model,

(c) Correlation of truncated model and reference model via NRFD, according to (2),

(d) Break if error limit is reached and at least three results are below error limit (to ensure convergence).

\subsection{Convergence}

The important issue for the success of the novel approach is the existence of a distinct convergence behaviour of the truncated results against the reference model. Convergence is reached, if the NRFD-error is below an error limit and

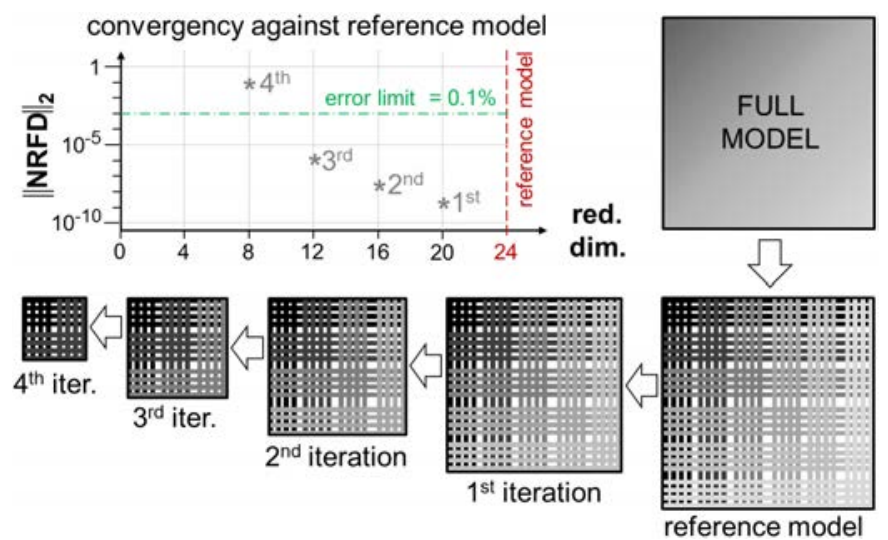

Fig. 3. Schematic diagram of novel approach by convergence observation

monotonically decreasing towards the reference dimension. Otherwise no minimal reliable dimension of the reduced model is ensured. Therefore, the rough size of the reduced reference model must be estimated in advance; If too small, no convergence is achieved, if too large the calculation effort is vast.

\section{CONCLUSION}

Numerical experiments at a framework and a gear box housing show, that minimal models with a reliable dimension are generated. The calculation overhead is adequate and can be compensated by the time saving when further using the smaller reduced models. Due to a reliable model dimension, an expensive eigenanalysis of the original model is avoided.

Extensions could be made by truncating single Krylovmodes instead of dropping the complete order of the series expansion. In principle, the novel two-stage strategy could be applied to nearly any reduction technique, even presumably for a non-orthogonal projection, as long as an iterative truncation of modes is admissible, e.g. CMS. Apart from this, RKSM followed an iterative BT-step in combination with the proposed convergence criterion could lead to sophisticated minimal models.

\section{REFERENCES}

Bunse-Gerstner, A.; Kubalinska, D.; Vossen, G.; Wilczek, D. H2norm optimal model reduction for large-scale discrete dynamical MIMO systems. Journal of Computational and Applied Mathematics, Vol. 233, No. 2010, pp. 1202-1216, 2007.

Eid, R.; Panzer, H. K.; Lohmann, B. How to choose a single expansion point in Krylov-based model reduction? Technical Reports on Automatic Control (TRAC), Vol. 4, Lehrstuhl für Regelungstechnik, Technische Universität München, Germany, 2009.

Fehr, J.; Fischer, M.; Haasdonk, B.; Eberhard, P. Greedy-based Approximation of Frequency-weighted Gramian Matrices for Model Reduction in Multibody Dynamics. Journal of Applied Mathematics and Mechanics, Vol. 93, No. 8, pp. 508-519, 2013.

Gugercin, S.; Antoulas, A. C.; Beattie, C. A. H2 model reduction for large-scale linear dynamical systems. Journal on Matrix Analysis and Applications, Vol. 30, No. 2, pp. 609-638, 2008.

Lehner, M. Modellreduktion in elastischen Mehrkörpersystemen. PhD thesis, Institut für Technische und Numerische Mechanik, Universität Stuttgart, Germany, 2007. 


\title{
Model Order Reduction for Magneto-Quasistatic Equations
}

\author{
Johanna Kerler* Tatjana Stykel ${ }^{*}$ \\ * Universität Augsburg, Universitätsstraße 14, 86159 Augsburg, \\ Germany (e-mail: johanna.kerler@math.uni-augsburg.de, \\ stykel@math.uni-augsburg.de).
}

\begin{abstract}
We consider model reduction of Maxwell's equations arising in magneto-quasistatic problems. A finite element discretization of such equations leads to large-scale differentialalgebraic equations of special structure. For model reduction of linear systems, we employ a balanced truncation approach, whereas nonlinear systems are reduced using a proper orthogonal decomposition method combined with a discrete empirical interpolation technique. We will exploit the special structure of the underlying problem to improve the performance of the model reduction algorithms.
\end{abstract}

Keywords: Magneto-quasistatic equations, model reduction, differential-algebraic equations, balanced truncation, proper orthogonal decomposition, discrete empirical interpolation

\section{MAGNETO-QUASISTATIC SIMULATION}

The dynamic behaviour of electromagnetic devices can be described by Maxwell's equations coupled with circuit equations. In magneto-quasistatic (MQS) problems, the contribution of the displacement currents is negligible compared to the conductive currents. A finite element discretization of the resulting Maxwell equations combined with the circuit coupling equations yields a nonlinear system of differential-algebraic equations (DAEs)

$$
E \dot{x}=A(x) x+B u, \quad y=C x
$$

with the state vector $x=\left[a^{T}, i^{T}\right]^{T}$, the output $y=i$, and

$$
E=\left[\begin{array}{cc}
M & 0 \\
X^{T} & 0
\end{array}\right], \quad A(x)=\left[\begin{array}{cc}
-K(a) & X \\
0 & -R
\end{array}\right], \quad B=C^{T}=\left[\begin{array}{l}
0 \\
I
\end{array}\right],
$$

see, e.g., Schöps (2011). Here, $a \in \mathbb{R}^{n_{a}}$ is a semidiscretized vector of magnetic potentials, $i, u \in \mathbb{R}^{m}$ are vectors of currents and voltages of voltage sources, $M$ is a conductivity matrix, $K(a)$ is a curl-curl matrix, $X$ is a coupling matrix, and $R$ is a resistance matrix. Substructuring the spatial domain into conductive and non-conductive subdomains and reordering the state variables accordingly, we can partition the state vector $a$ and the matrices $M, K(a)$ and $X$ as

$a=\left[\begin{array}{l}a_{1} \\ a_{2}\end{array}\right], M=\left[\begin{array}{cc}M_{11} & 0 \\ 0 & 0\end{array}\right], K(a)=\left[\begin{array}{cc}K_{11}\left(a_{1}\right) & K_{12} \\ K_{21} & K_{22}\end{array}\right], X=\left[\begin{array}{l}X_{1} \\ X_{2}\end{array}\right]$, where $a_{1} \in \mathbb{R}^{n_{1}}, a_{2} \in \mathbb{R}^{n_{2}}, M_{11} \in \mathbb{R}^{n_{1}, n_{1}}$ is nonsingular, $K_{12}, K_{21}^{T} \in \mathbb{R}^{n_{1}, n_{2}}$ and $K_{22} \in \mathbb{R}^{n_{2}, n_{2}}$ are constant, and $X_{2} \in \mathbb{R}^{n_{2}, m}$ has full column rank. Let the columns of the matrix $Y \in \mathbb{R}^{n_{2}, n_{2}-m}$ form an orthonormal basis of the nullspace of $X_{2}^{T}$. Then for $Z=X_{2}\left(X_{2}^{T} X_{2}\right)^{-1 / 2}$, the matrix $[Z, Y]$ is orthogonal. One can show that if $Y^{T} K_{22} Y$ is

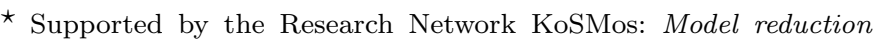
based simulation of coupled PDAE systems funded by the German Ministry of Education and Science (BMBF), grant 05M13WAA.
}

nonsingular, then the DAE (1) has index one. Considering the state transformation

$$
T x=\left[\begin{array}{ccc}
I_{n_{1}} & 0 & 0 \\
0 & Z^{T} & 0 \\
0 & Y^{T} & 0 \\
0 & 0 & I_{m}
\end{array}\right] x=\left[\begin{array}{c}
a_{1} \\
a_{2,1} \\
a_{2,2} \\
i
\end{array}\right]
$$

where $I_{k}$ denotes a $k \times k$ identity matrix, the transformed state vector can be partitioned into the differential and algebraic components given by $\left[a_{1}^{T}, a_{2,1}^{T}\right]^{T}$ and $\left[a_{2,2}^{T}, i^{T}\right]^{T}$, respectively.

Unfortunately, the numerical solution of the MQS systems requires an enormous amount of storage and large computational time. Model order reduction has proved to be an effective tool in analysis and simulation of complex systems. The goal of model reduction is to replace a largescale system by a reduced-order model which captures the dynamic behaviour of the original system and preserves essential physical properties. Model reduction of a linear part in the MQS system has been considered in Schmidthäusler et al. (2014). In this contribution, we show how the special structure of the MQS system can be exploited to construct efficient model reduction methods for linear and nonlinear problems.

\section{MODEL REDUCTION OF LINEAR SYSTEMS}

If $K_{11}(a)$ does not dependent on the potential $a$, then we obtain the linear time-invariant DAE system

$$
E \dot{x}=A x+B u, \quad y=C x .
$$

For model reduction of such a system, we can use balanced truncation as described in Stykel (2004). This approach requires the computation of the spectral projectors onto the deflating subspaces of the pencil $\lambda E-A$ corresponding to the finite and infinite eigenvalues. To overcome this difficulty, we exploit the structure of the DAE (3) and transform it to the ODE form 


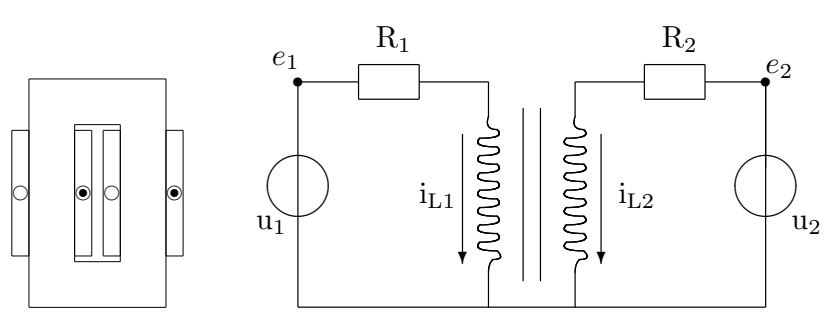

Fig. 1. 2D transformer (left) and transformer model (right).

$$
E_{1} \dot{x}_{1}=A_{1} x_{1}+B_{1} u, \quad y=C_{1} x_{1},
$$

where $x_{1}=\left[a_{1}^{T}, a_{2,1}^{T}\right]^{T} \in \mathbb{R}^{n_{1}+m}$ and $E_{1}$ is nonsingular. In balanced truncation model reduction applied to (4), we have to solve the generalized Lyapunov equations

$$
\begin{aligned}
& A_{1} P E_{1}^{T}+E_{1} P A_{1}^{T}=-B_{1} B_{1}^{T}, \\
& A_{1}^{T} Q E_{1}+E_{1}^{T} Q A_{1}=-C_{1}^{T} C_{1} .
\end{aligned}
$$

For this purpose, we can use the low-rank alternating direction implicit method, e.g., Benner at al. (2013). In this method, the computation of the matrix-vector products

$$
\left(\tau E_{1}+A_{1}\right)^{-1} v, \quad A_{1}^{-1} E_{1} v, \quad E_{1}^{-1} A_{1} v
$$

is required. Note that in contrast to the sparse matrices $E, A, B$ and $C$ in (3), the system matrices $E_{1}, A_{1}, B_{1}$ and $C_{1}$ in (4) are, in general, dense. Therefore, neither these matrices nor the inverses in (5) will be constructed explicitly. Instead, we will exploit their block structure in order to compute the products $A_{1}^{-1} E_{1} v$ and $E_{1}^{-1} A_{1} v$. Moreover, the calculation of $\left(\tau E_{1}+A_{1}\right)^{-1} v$ with $v=\left[v_{1}^{T}, v_{2}^{T}\right]^{T}$ can be reduced to solving a sparse linear system $(\tau E+A) z=\left[v_{1}^{T},\left(Z v_{2}\right)^{T}, 0\right]^{T}$.

\section{MODEL REDUCTION OF NONLINEAR SYSTEMS}

For model reduction of the nonlinear DAE (1), we use the proper orthogonal decomposition (POD) method, e.g., Volkwein (2013). This method consists in constructing a snapshot matrix $\mathcal{X}=\left[x\left(t_{1}\right), \ldots, x\left(t_{q}\right)\right]$ and computing a singular value decomposition (SVD)

$$
\mathcal{X}=\left[V, V_{0}\right] \operatorname{diag}\left(\Sigma, \Sigma_{0}\right)\left[W, W_{0}\right]^{T},
$$

where $\Sigma$ contains the dominant singular values of $\mathcal{X}$. Then a reduced-order model can be determined by projection

$$
\hat{E} \dot{\hat{x}}=\hat{A}(\hat{x}) \hat{x}+\hat{B} u, \quad \hat{y}=\hat{C} \hat{x}
$$

with $\hat{x} \in \mathbb{R}^{r}, \hat{E}=V^{T} E V, \hat{A}(\hat{x})=V^{T} A(V \hat{x}) V, \hat{B}=V^{T} B$, and $\hat{C}=C V$. The columns of $V$ are referred to as the POD basis of $\mathcal{X}$. This naive model reduction approach has several disadvantages. First note that the differential and algebraic components of the state vector $x$ are mixed in the reduced-order model (6). Secondly, it is well known that the reduction of the algebraic components and the algebraic constraints in DAEs may lead to inaccurate and physically meaningless results, see Stykel (2004).

As an example, let us consider a single-phase transformer with a nonlinear iron core shown in Fig. 1, see Schöps (2011). The upper plot in Fig. 2 presents the relative errors in the output components of the POD-reduced transformer model. In our experiments, the dimensions are $n_{a}=7821$, $n_{1}=3264, n_{2}=4557, m=2$, and the reduced dimension is $r=362$. One can see that the relative errors are quite large.
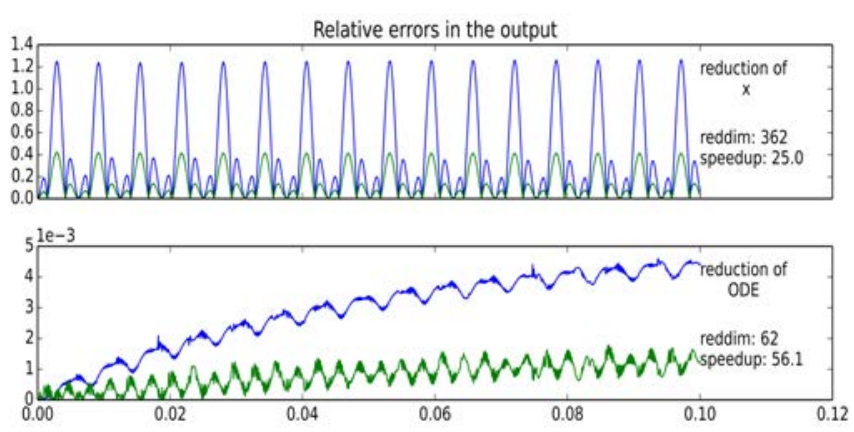

Fig. 2. Relative errors in the output components for different model reduction approaches.

In order to improve the approximation quality, we propose to transform the DAE (1) to the ODE

$$
E_{1} \dot{x}_{1}=A_{1}\left(x_{1}\right) x_{1}+B_{1} u, \quad y=C_{1} x_{1}
$$

with nonsingular $E_{1}$ and $x_{1}=\left[a_{1}^{T}, a_{2,1}^{T}\right]^{T}$ and reduce the vector $a_{1}$ remaining $a_{2,1}$ unchanged, since it usually has only a few entries. For this purpose, we first determine the snapshot matrix $\mathcal{X}$ by solving the DAE (1) and compute the POD basis $V_{a_{1}}$ of the matrix $\left[I_{n_{1}}, 0\right] \mathcal{X}$. Then the reduced-order model (6) can be obtained from (7) by projection $\hat{E}=V^{T} E_{1} V, \hat{A}(\hat{x})=V^{T} A_{1}(V \hat{x}) V, \hat{B}=V^{T} B_{1}$, and $\hat{C}=C_{1} V$ with $V=\operatorname{diag}\left(V_{a_{1}}, I_{m}\right)$.

To speed up the simulation of the reduced-order model (6), we employ discrete empirical interpolation method, see Chaturantabut et al. (2010), for the efficient evaluation of the nonlinearity $V^{T} A_{1}(V \hat{x}) V \hat{x}$.

The lower plot in Fig. 2 shows the relative errors in the output components for the reduced model of order $r=62$ computed by our model reduction approach. One can see that the reduced ODE system is about factor 6 smaller than the reduced model obtained by the naive POD method and has the relative error of 2 orders of magnitude smaller than that of the first reduced model.

\section{REFERENCES}

P. Benner, J. Saak. Numerical solution of large and sparse continuous time algebraic matrix Riccati and Lyapunov equations: a state of the art survey. GAMMMitteilungen, 36(1):32-52, 2013.

S. Chaturantabut, D. Sorensen. Nonlinear model reduction via discrete empirical interpolation. SIAM J. Sci. Comput., 32(5):2737-2764, 2010.

S. Volkwein. Proper Orthogonal Decomposition: Theory and Reduced-Order Modelling. Lecture Notes, University of Konstanz, 2013.

D. Schmidthäusler, S. Schöps, M. Clements. Linear subspace reduction for quasistatic field simulations to accelerate repeated computations. IEEE Trans. Magn., 50(2):7010304, 2014

S. Schöps. Multiscale Modeling and Multirate TimeIntegration of Field/Circuit Coupled Problems. Ph.D. thesis, Bergische Universität Wuppertal, 2011.

T. Stykel. Gramian-based model reduction for descriptor systems. Math. Control Signals Systems, 16:297-319, 2004. 


\title{
Model order reduction approaches for the optimal design of permanent magnets in electro-magnetic machines
}

\author{
A. Alla* M. Hinze* O. Lass ${ }^{* *}$ S. Ulbrich ${ }^{* *}$ \\ * Universität Hamburg, Bundesstr. 55, 20146, Hamburg, Germany \\ (e-mail: \{alessandro.alla, michael.hinze\}@uni-hamburg.de). \\ ** Technische Universität Darmstadt, Dolivostr. 15, 64293 Darmstadt, \\ Germany (e-mail: \{lass, ulbrich\}@mathematik.tu-darmstadt.de)
}

\begin{abstract}
In an electromagnetic machine with permanent magnets the excitation field is provided by a permanent magnet instead of a coil. The center of the generator, the rotor, contains the magnet. Our optimization goal consists in finding the minimum volume of the magnet which gives a desired electromotive force. This results in an optimization problem for a parametrized partial differential equation (PDE). We propose a goal-oriented model order reduction approach to provide a reduced order surrogate model for the parametrized PDE which then is utilized in the numerical optimization. Numerical tests will be provided in order to show the effectiveness of the proposed method.
\end{abstract}

Keywords: Proper Orthogonal Decomposition, Optimization, Model Order Reduction, Static Maxwell's equation

\footnotetext{
* This work is supported by the German BMBF in the context of the SIMUROM project (grant number 05M2013).
} 


\title{
Fast Simulation of Wireless Power Transfer Systems With Varying Coil Alignment
}

\author{
D. Klis* S. Burgard* O. Farle* R. Dyczij-Edlinger* \\ * Chair for Electromagnetic Theory, Saarland University, \\ Campus C63, D-66123 Saarbrücken, Germany \\ (e-mail: edlinger@lte.uni-saarland.de)
}

Keywords: Eddy currents, Finite element analysis, Homogenization, Parametric model reduction, Wireless power systems

\section{SUMMARY}

Wireless power transfer (WPT) systems have experienced a rise in popularity, because recent developments in power semiconductors have enabled high efficiency in addition to the apparent advantages of contactless systems. Most WPT systems operate in the magneto-quasistatic regime; they utilize magnetic coupling between planar coils. The present paper focuses on the inductive subsystem of a WPT as defined in the Qi standard by the Wireless Power Consortium (2012), specifically the configuration of the power transmitter and receiver coils. The efficiency of WPT systems generally depends on the inductive coupling factor and the losses present in the system. Since the operating frequency is in the upper $\mathrm{kHz}$ to $\mathrm{MHz}$ range, the direct-current (DC) losses due to the finite electric conductivity of the wires are dominated by alternating-current (AC) losses caused by eddy currents, specifically by the skin effect and the proximity effect; see Dixon, Jr (1988). Since the magnetic cores are never driven into saturation in such applications, magnetic losses and non-linearities are of minor importance. To reduce AC losses, the coils are usually wound from litz wire, which consists of bundles of thin braided strands. These are individually isolated and change position periodically within the wire. Thus, the net current carried by each of the parallel-circuited strands is expected to be the same. Typical strand diameters are in the range of $10-100 \mu \mathrm{m}$; the number of strands per wire may exceed several hundred. AC losses mainly depend on frequency, number and configuration of the coil windings, and the micro-structure of the litz wire.

The goal of this paper is to provide a numerical method for predicting the electromagnetic response as a function of frequency and the position of the receiver coil relative to the transmitter. The model must thus include both the macroscopic geometry of the overall WPT system as well as the micro-structure of the litz wire.

Thanks to its flexibility in modeling geometry and materials, the finite-element (FE) method is generally wellsuited for analyzing such systems. However, the presence of litz wires makes a brute-force approach impractical: Constructing a FE mesh that captures the exact paths of all the braided strands is unrealistic. Besides, the di-

^ Work in cooperation with RRC power solutions GmbH. Supported
by the German Federal Ministry for Economic Affairs and Energy. mension of the resulting $\mathrm{FE}$ matrices would be excessive. As a remedy, we employ a homogenization approach for the micro-structure of the litz wire and model the domain of the coil windings on the macroscopic scale by a uniform bulk of meta-material, cf. Bossavit (1994), Gyselinck and Dular (2005). The meta-material has a complex reluctivity

$$
\nu_{m}(\omega)=\nu_{m}^{\prime}(\omega)-j \nu_{m}^{\prime \prime}(\omega)
$$

which depends on the angular frequency $\omega$ in a plane tangent to the winding direction, and a real-valued reluctivity $\nu_{z}$ along the winding direction. Homogenization also introduces one series impedance $Z(\omega)$ per coil.

Using homogenization, a single FE model is solved with ease on a modern computer. However, constructing an entire response surface for a broad parameter domain is still a formidable task, because the number of sampling points and, thus, FE solutions is usually very large. In our case, the FE model for one parametric configuration leads to an $N$-dimensional linear system of the form

$$
\begin{gathered}
{\left[\mathbf{M}_{0}+\omega \mathbf{M}_{1}+\sum_{h=1}^{H}\left(\nu_{m}^{h}(\omega) \mathbf{M}_{m}^{h}+\nu_{z}^{h} \mathbf{M}_{z}^{h}\right)\right] \mathbf{x}=\mathbf{B i},} \\
\mathbf{u}=j \omega \mathbf{B}^{T} \mathbf{x}+\operatorname{diag} Z_{h}(\omega) \mathbf{i}
\end{gathered}
$$

has to be solved. The vectors $\mathbf{i}$ and $\mathbf{u}$ denote the coil currents and voltages, respectively. All $\mathbf{M}_{i} \in \mathbb{C}^{N \times N}$ and $\mathbf{B} \in \mathbb{C}^{N \times H}$ are sparse matrices, and $\mathbf{x} \in \mathbb{C}^{N}$ is the solution vector. When a single coil is excited by a unit current, $[\mathbf{i}]_{h}=1 \mathrm{~A}$, the output is the corresponding column of the impedance matrix $\mathbf{Z}$ of the system.

Methods of parametric model-order reduction (PMOR), such as Rozza et al. (2008), Panzer et al. (2010), greatly alleviate computational costs: Since the dimension of the resulting parametric reduced-order model (PROM) is much smaller than that of the underlying FE system, solution times improve by orders of magnitude. Besides, the error of the PMOR process is controllable and can be made of the same order as that of the FE solution. We employ the method from Burgard et al. (2015), which is applicable to affinely parameterized systems. Since geometry variations cause non-affine parameter dependencies in (2), the original model is replaced by an interpolated model

$$
\begin{gathered}
\left(\sum_{i, l} \phi_{i}(\omega) \Gamma_{l}(\mathbf{p}) \mathbf{M}_{i}^{l}\right) \mathbf{x}^{\prime}(\omega, \mathbf{p})=\mathbf{B}(\omega) \mathbf{i}, \\
\mathbf{u}^{\prime}(\omega, \mathbf{p})=\mathbf{B}(\omega)^{T} \mathbf{x}^{\prime}(\omega, \mathbf{p})+\mathbf{D}(\omega) \mathbf{i} .
\end{gathered}
$$




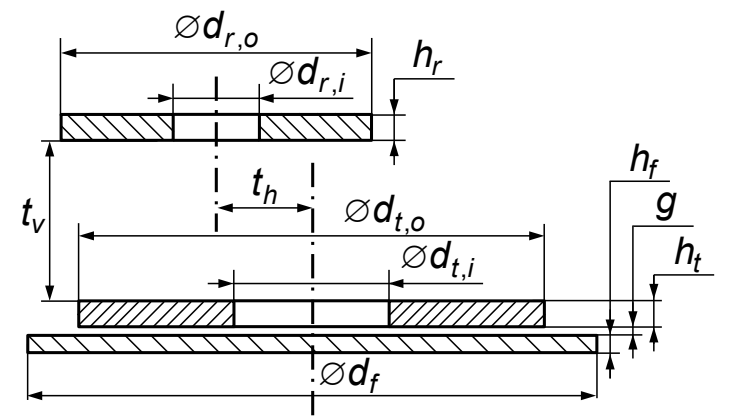

Table 1. Litz Wire Specifications

\begin{tabular}{lrrr} 
& Transmitter & Receiver & Unit \\
Coil type & $A 5$ & $\# 5$ & \\
\hline Litz diameter & 80 & 30 & $\mu \mathrm{m}$ \\
Conductivity & $5.8 \cdot 10^{7}$ & $5.8 \cdot 10^{7}$ & $\mathrm{~S} / \mathrm{m}$ \\
Filling factor & 11663 & 75917 & $\mathrm{~cm}^{-2}$ \\
Strands per wire & 105 & 240 & \\
Turns & 20 & 18 & \\
Winding layers & 2 & 2 &
\end{tabular}

Table 2. Computational Data ${ }^{a}$

\begin{tabular}{lrr} 
Model & PROM & FE model \\
\hline \hline Dimension & 8 & 65,878 \\
Model generation & $1.25 \mathrm{~h}$ & - \\
Model evaluation & $2.94 \cdot 10^{-4} \mathrm{~s}$ & $5.02 \mathrm{~s}$ \\
\hline $\begin{array}{l}\text { Simulation time for } \\
101^{3} \text { sample points }\end{array}$ & $5.05 \mathrm{~min}$ & $59,80 \mathrm{~d}^{b}$ \\
\hline Estimated avg. error in Z & $4.29 \cdot 10^{-4}$ & - \\
\hline${ }^{a}$ Performance on Intel Xeon E5620 using Matlab R2013b with \\
-singleCompThread option and Pardiso as linear solver. \\
${ }^{b}$ Extrapolated.
\end{tabular}

Herein, $\mathbf{p}$ is a parameter vector, and $\phi_{i}$ and $\Gamma_{l}$ are scalarvalued functions. The matrices $\mathbf{M}_{i}^{l}$ are constant with respect to $\mathbf{p}$.

\section{NUMERICAL EXAMPLES}

Fig. 2 shows a WPT system with two litz wire coils specified by the Wireless Power Consortium (2012): The lower one is the Power Transmitter A5 (part 1, p. 28) and the upper one is the Power Receiver \#5 (part 3, p. 18). Windings and litz wire data are presented in Table 1. The system parameters are given by the frequency $f$, the vertical displacement $t_{v}$, and the axial mismatch $t_{h}$ :

$$
\begin{aligned}
f & \in[1,300] \mathrm{kHz}, \\
t_{v} & \in[4,12] \mathrm{mm}, \\
t_{h} & \in[0,12] \mathrm{mm} .
\end{aligned}
$$

The output of the PROM is the parameter-dependent impedance matrix of the system $\mathbf{Z}(\omega ; \mathbf{p})$, with $\mathbf{p}=\left(t_{v}, t_{h}\right)$. Simulation times and model sizes are given in Table 2. The PMOR process reduces the dimension of the FE system from 65,878 to 8 . The generation time for the PROM is about one and a half hour, which is well-invested: Speed-up in evaluation time, compared to FE model, is in the order of 17,000. A parametric sweep based on $101^{3}$ sampling points for $\left(\omega, t_{v}, t_{t}\right)$ takes only five minutes with the PROM; conventional FE analysis would take nearly 60 days.

Fig. 1 shows the equivalent relative complex permeability and the skin effect impedance of the wires used in the

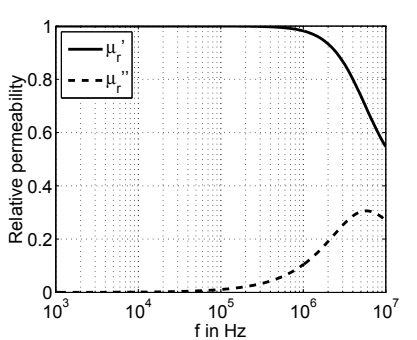

(a) Rel. permeability $\mu_{r}^{\prime}-j \mu_{r}^{\prime \prime}$.

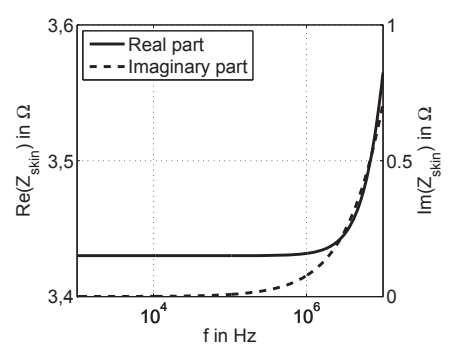

(b) Series impedance.
Fig. 1. Homogenization results for transmitter coil.

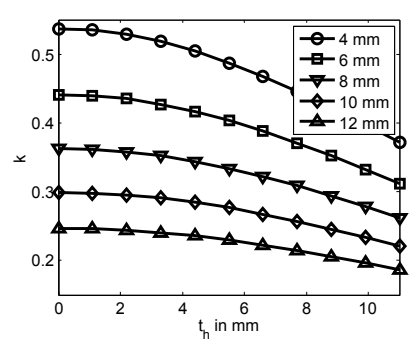

(a) PROM solution.

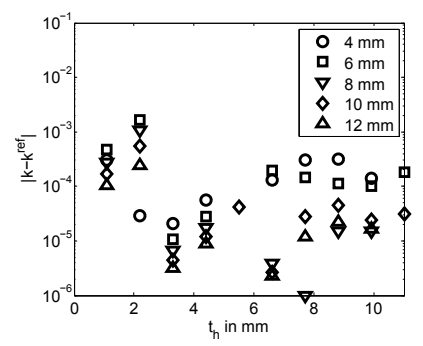

(b) Errors.
Fig. 2. Coupling factor $k$ and errors at $f=300 \mathrm{kHz}$. Parameter: vertical coil displacement $t_{v}$.

transmitter coil, obtained by homogenization on a hexagonal unit cell. To validate the reliability of the suggested approach, we compare the PROM solutions to reference values obtained by conventional $\mathrm{FE}$ simulations for a large number of parameter points. Fig. 2 gives results for the coupling factor: The maximum error is $1.7 \cdot 10^{-3}$, and typical errors lie in the range of $1 \cdot 10^{-4}$.

\section{REFERENCES}

Bossavit, A. (1994). Effective penetration depth in spatially periodic grids: a novel approach to homogenization. In Int. Symp. Electromagnetic Compatibility $E M C^{\prime} 94,859-864$.

Burgard, S., Klis, D., Farle, O., and Dyczij-Edlinger, R. (2015). Order-reduction of fields-level models with affine and non-affine parameters by interpolation of subspaces. Accepted for presentation at Mathmod 2015, Vienna.

Dixon, Jr, L.H. (1988). Eddy current losses in transformer windings and circuit wiring. In SEM600 Unitrode Seminar.

Gyselinck, J. and Dular, P. (2005). Frequency-domain homogenization of bundles of wires in 2-D magnetodynamic FE calculations. IEEE Trans. Magn., 41(5), 1416-1419.

Panzer, H., Mohring, J., Eid, R., and Lohmann, B. (2010). Parametric model order reduction by matrix interpolation. Automatisierungstechnik, 58(8), 475-484.

Rozza, G., Huynh, D., and Patera, A.T. (2008). Reduced basis approximation and a posteriori error estimation for affinely parametrized elliptic coercive partial differential equations. Arch. of Comput. Methods Eng., 15(3), 229275.

Wireless Power Consortium (2012). Qi standard, "system description wireless power transfer". Version 1.1.1. Available upon request at http://www. wirelesspowerconsortium.com. 


\title{
Interface Reduction for CMS Methods and Alternative Model Order Reduction
}

\author{
Philip Holzwarth and Peter Eberhard \\ Institute of Engineering and Computational Mechanics, University of \\ Stuttgart, Germany, (e-mail: philip.holzwarth@itm.uni-stuttgart.de, \\ peter.eberhard@itm.uni-stuttgart.de)
}

In many cases, complex dynamical systems consist of separable components. In the context of finite element substructuring techniques and elastic multibody systems, this separation is maintained in a simulation environment. With this modular setup, single components can be exchanged without having to re-model the whole system. Thus, a numerical optimization of substructures can be performed with an admissible computational effort. In order to obtain reliable simulation results, the description of the interface or port that is used for the connection is very important. The assumption of non-deformable interfaces is a simplification that generates so-called RBE2-elements, which are widely used in practice.

In many cases, however, this assumption of rigid interfaces is not valid and increases the stiffness of the overall system significantly. In this contribution, new interface definitions based on system-level snapshots, Legendre polynomials, compare Fig. 1, and the ESVDMOR approach presented in Benner and Schneider (2012) are derived. All presented methods share the basic idea that deformations of the interface are restricted to a specific set of deformation patterns, which is automatically determined by the respective interface definition algorithm. This can be considered as a generalization of the RBE2-elements, which use a rigid motion of the interface as admissible deformation pattern.

The usage of densely meshed finite element models leads to differential equations of large dimension. A reduction of the degrees of freedom is necessary to make sophisticated dynamical simulations, e.g. a time integration, computa-
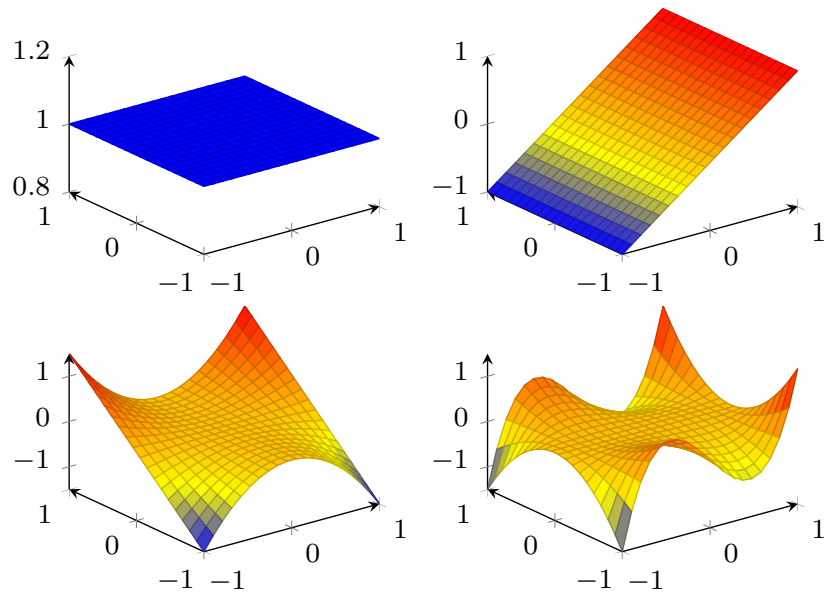

Fig. 1. Two-dimensional Legendre polynomials of orders $[0,0],[1,0],[2,1]$ and $[3,2]$. tionally affordable. The specific interface definitions must be considered in the model reduction algorithms. Nodebased reduction algorithms, such as the Craig-Bampton scheme (Craig and Bampton, 1968) as as well as the CMSGram reduction (Holzwarth and Eberhard, 2015) require an adaption if specific interface definitions are used. The CMS-Gram algorithm is a hybrid reduction method that guarantees interface compatibility and is characterized by a very fast error convergence due to the use of frequencyweighted Gramian-matrix based reduction augmented by static correction modes.

The advantages and drawbacks of the presented interface formulations are explained and demonstrated for an exemplary model, shown in Fig. 2.

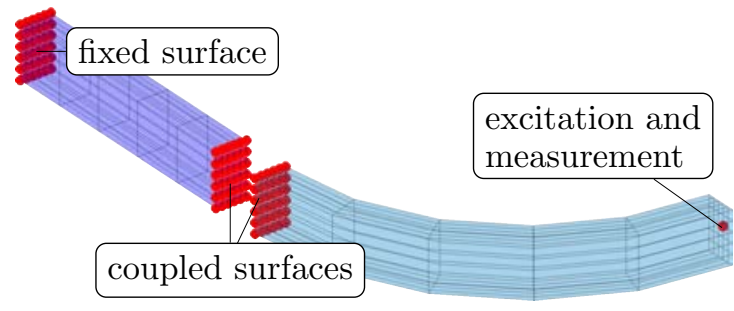

Fig. 2. Straight and curved beam (FE models) and coupling conditions.

\section{REFERENCES}

Benner, P. and Schneider, A. (2012). Model reduction for linear descriptor systems with many ports. In M. Günther, A. Bartel, M. Brunk, S. Schöps, and M. Striebel (eds.), Progress in Industrial Mathematics at ECMI 2010, volume 17 of Mathematics in Industry, 137-143. Springer-Verlag, Berlin, Germany.

Craig, R. and Bampton, M. (1968). Coupling of substructures for dynamic analyses. AIAA Journal, 6(7), 13131319.

Holzwarth, P. and Eberhard, P. (2015). SVD-based improvements for component mode synthesis in elastic multibody systems. European Journal of Mechanics A/Solids, 49, 408-418. 


\title{
A joint IMEX-MOR approach for Water Networks
}

\author{
Sara Grundel * Lennart Jansen ** \\ * Max-Planck Institute for Dynamics of Complex Technical Systems, \\ Magdeburg, Germany (e-mail: grundel@mpi-magdeburg.mpg.de). \\ ${ }^{* *}$ Heinrich Heine Univeristät Düsseldorf, Germany (e-mail: \\ lennart.jansen@uni-duesseldorf.de)
}

\begin{abstract}
Modeling and Simulation of fluids in large network is a rather challenging problem. We provide an approach combining techniques in Model Order Reduction (MOR) and implicit-explicit (IMEX) integration to create efficient and stable simulations.
\end{abstract}

Keywords: Model Reduction, Integration, Differential Equations, Networks, Directed Graphs

\section{INTRODUCTION}

The simulation of fluids within a large network of pipes poses several mathematical challenges. Typically after spatial discretization the resulting mathematical system is a nonlinear differential algebraic system. Standard techniques are often slow due to the stiffness of the equation. We will show a several step process on how to improve on the timing. A first and major step in order to achieve stable and fast simulators for these problems is what we call the decoupling step. In that step we are able to model the system as a discrete index 1 DAE. This step is only possible due to the choosen discretization we use. Next we use a combination of Model Order Reduction (MOR) methods in order to create a smaller scale index 1 Differential Algebraic Equation. And last but not least we use an implicitexplicit (IMEX) integration method to reduce the time-step for the stiff nonlinear differential equation. We will only present a simplified network here which includes pipes, reservoirs and so called demand nodes. This system will actually result in an ODE which simplifies the discussion.

\section{MODELING}

It is common to define a connected and directed simple graph $G=G(V, E)$ representing the pipe network. This allows a more compact representation of the model equations. The set $V$ are the nodes and $E$ are the edges and we will describe the different node and edge elements in the following.

\subsection{Node Elements}

Reservoirs are water sources with unlimited capacity. Thus, we assume that they have a constant pressure $p_{s}$. Furthermore no balance equation holds at a reservoir, since an arbitrary amount of water may leave or enter the reservoir.

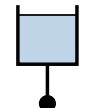

$$
p=p_{s}
$$

In contrast to reservoirs, tanks have limited capacity. Never the less, pressure can in- or decrease even though the tank is full or empty respectively. We will not talk about tanks in more details here.
A demand node has a given demand $q_{\mathrm{s}}: I \longrightarrow \mathbb{R}_{+}$. Thus, the difference between the amount of water flowing towards a node, and the amount of water flowing away from the node has to be $q$

$$
\sum_{i \in I_{\text {in }}} q_{\text {in }}^{i}-\sum_{i \in I_{\text {out }}} q_{\text {out }}^{i}=q_{\mathrm{s}}(t)
$$

with $q_{\text {in }}^{i}$ and $q_{\text {out }}^{i}$ being the incoming and outgoing flow of edges connected to the demand node, respectively. It is possible that $q_{\mathrm{s}}(t)=0$ for all $t \in I$.

\subsection{Edge Elements}

First we will discuss the pipe model. The behavior of a pipe is described by a continuity equation and an equation describing the movement inside a pipe. We consider circular pipes with diameter $D$, cross-section $A=\frac{\pi}{4} D^{2}$ and length $\ell$. The independent variables are space $x \in[0, \ell]:=\Omega \subset \mathbb{R}$ and time $t \in\left[t_{0}, T\right]:=I \subset \mathbb{R}$. The time dependent variables are the mass flow $m: \Omega \times I \longrightarrow \mathbb{R}$ and the pressure $p: \Omega \times I \longrightarrow \mathbb{R}$. The parameters $a, \rho$ and $c$ depend on material properties of the pipe and the gas. $\alpha$ is the angle of the pipe and $g$ is the gravitational constant. With this, we get the following partial differential equation, which describes the behavior in pipes

$$
\begin{array}{r}
\frac{\partial p}{\partial t}(x, t)+\frac{a^{2}}{A} \frac{\partial m}{\partial x}(x, t)=0 \\
\frac{\partial m}{\partial t}(x, t)+A \frac{\partial p}{\partial x}(x, t)+\rho A g \sin \alpha+c|m(x, t)| m(x, t)=0 \\
\text { pipe: } \quad \text { Hyperbolic PDE }
\end{array}
$$

Further edge elements are valves and pumps, which we also omit in this extended abstract.

\subsection{Network Model}

From now on we consider a network with $n_{p}$ many pipes and $n_{d}$ many demand nodes and $n_{r s}$ many reservoirs. For each pipe $i$, we get a flow $m_{i}: \Omega_{i} \times I \longrightarrow \mathbb{R}, \Omega_{i}=\left[x_{L_{i}}, x_{R_{i}}\right] \subset \mathbb{R}, I=\left[t_{0}, T\right]$ and a pressure $p_{i}: \Omega_{i} \times I \longrightarrow \mathbb{R}$ both depending on space $x \in \Omega_{i}$ and time $t \in I$. The node variables are $p_{d}: I \longrightarrow \mathbb{R}^{n_{d}}$ and 
$p_{r s}: I \longrightarrow \mathbb{R}^{n_{r s}}$, the pressures at demand nodes and reservoirs. We define

$$
\begin{gathered}
m_{L}(t)=\left(m_{i}\left(x_{L_{i}}, t\right)\right)_{i \in \mathscr{A}_{p i}}, m_{R}(t)=\left(m_{i}\left(x_{R_{i}}, t\right)\right)_{i \in \mathscr{A}_{p i}} . \\
p_{L}(t)=\left(p_{i}\left(x_{L_{i}}, t\right)\right)_{i \in \mathscr{A}_{p i}}, p_{R}(t)=\left(p_{i}\left(x_{R_{i}}, t\right)\right)_{i \in \mathscr{A}_{p i}} .
\end{gathered}
$$

$m_{L}$ being the vector with all pipe flows at their tail-node and $m_{R}$ the flow vector at their head-nodes and similarly for $p_{L}$ and $p_{R}$. Note, that the node pressures coincide with the head- and tail pressures of the pipes. We call the vector of demand and reservoir pressures by $p$.

Last we define the following incidence matrices

$A_{R}^{r s} \in \mathbb{R}^{n_{p} \times n_{r s}}\left(A_{R}^{r s}\right)_{i j}=\left\{\begin{array}{l}1 \text { if reservoir node } j \text { is head of pipe } i \\ 0 \text { else }\end{array}\right.$ $A_{L}^{r s} \in \mathbb{R}^{n_{p} \times n_{r s}}\left(A_{L}^{r s}\right)_{i j}=\left\{\begin{aligned}-1 & \text { if reservoir node } j \text { is tail of pipe } i \\ 0 & \text { else }\end{aligned}\right.$ $A_{R} \in \mathbb{R}^{n_{p} \times n_{d}},\left(A_{R}\right)_{i j}=\left\{\begin{array}{l}1 \text { if demand node } j \text { is head of pipe } i \\ 0 \text { else }\end{array}\right.$ $A_{L} \in \mathbb{R}^{n_{p} \times n_{d}},\left(A_{L}\right)_{i j}=\left\{\begin{aligned} &-1 \text { if demand node } j \text { is tail of flow } i \\ & 0 \text { else }\end{aligned}\right.$ We can combine them all and get the full incidence matrix $A$

$$
A:=\left(A_{R}^{r s}+A_{L}^{r s} A_{R}+A_{L}\right) \in \mathbb{R}^{n_{p} \times+d_{r s}+n_{d}}
$$

With the help of these matrices we can write the spatial discretized system of equations as

$$
\begin{aligned}
\dot{p}_{R}+D_{\alpha}\left(m_{L}-m_{R}\right) & =0 \\
\dot{m}_{L}+D_{\beta} A^{T} p+\gamma+G\left(m_{L}\right) m_{L} & =0 \\
A_{R} m_{R}+A_{L} m_{L} & =q_{s e t} \\
A_{R} p_{d} & =p_{R} \\
A_{L} p_{d} & =p_{L} \\
A_{R}^{r s} p_{r s} & =p_{R} \\
A_{L}^{r s} p_{r s} & =p_{L} \\
p_{r s} & =p_{\text {set }}
\end{aligned}
$$

To obtain these equation it is crucial to chose a suitable spatial discretization. In particular the time derivative of the pressure is evaluated at the right end of the pipe and the time derivative of the flux at the left end. The size of the first two equation is the number of pipes, equation (4) the number of junction, $(5,6,7,8)$ number of pipes and the last equation number of tanks. $D_{\alpha}$ is a diagonal matrix containing $\alpha_{i}=a_{i}^{2} / A_{i} / \ell_{i}$ on the diagonal and $D_{\beta}$ similar with $\beta_{i}=A_{i} / \ell_{i} . \gamma$ is a vector with $\gamma_{i}=\rho_{i} A_{i} g \sin \alpha_{i}$ and $G\left(m_{L}\right)$ is a diagonal matrix function such that $G\left(m_{L}\right)_{i}=c_{i} m_{L}^{i}$. The matrix $\mathrm{A}$ is the incidence matrix as described above. The vector $q_{\text {set }}$ has an entry for every demand node showing the given demand at that particular node given by $q_{s}(t)$ and similar is $p_{s e t}$ the vector of the given pressures $p_{s}$ at the reservoirs. In the modeling of the graph it is crucial to pick the direction of the edges such that every demand node has a right end of a pipe. This is possible for any topology as long as one of the nodes in the graph is not a demand node which means in our case it has to be a reservoir. We furthermore want all pipes the end in a reservoir to end in a left node there.

\section{DECOUPLING}

By selecting a matrix $A_{\text {select }}$ which picks one pipe for each node that has a right end in that given node we can rewrite the system of equation in the variables $p_{d}$ and $m_{L}$.

$$
\begin{array}{r}
\dot{p}_{d}+A_{\text {select }} D_{\alpha}\left(-C q_{\text {set }}+C A m_{L}\right)=0 \\
\dot{m}_{L}+D_{\beta} A^{T}\left(\begin{array}{c}
p_{d} \\
p_{\text {set }}
\end{array}\right)+\gamma+G\left(m_{L}\right) m_{L}=0
\end{array}
$$

We will explain in detail how we create the matrix $C$ which is the crucial part in this decoupling process. This resulting ODE is of size $n_{d}+n_{p}$ and has the general structure

$$
\dot{x}=T x+g(x, t)=f(x, t),
$$

where the matrix $T$ is given by

$$
T=\left(\begin{array}{cc}
0 & A_{\text {select }} D_{\text {alpha }} C A \\
D_{\beta}\left(A_{R}+A_{L}\right)^{T} & 0
\end{array}\right),
$$

and the vector $x$ is combined by $p_{d}$ and $m_{L}$.

\section{IMEX}

In order to solve this stiff and nonlinear ODE we make use of implicit-explicit (IMEX) integration methods. This allow us to deal with the stiffness in an efficient way while not having to solve large-scale nonlinear problems. First order methods are of the flavor

$$
\frac{x_{n+1}-x_{n}}{h}=(1-\gamma) T x_{n}+\gamma T x_{n+1}+g\left(x_{n}, t\right)
$$

for $\gamma \in[0,1]$ and time setp $h$, which leads to the iteration

$$
x_{n+1}=(1-h \gamma T)^{-1}\left(x_{n}+(1-\gamma) T x_{n}+h g\left(x_{n}\right) .\right.
$$

We study convergence properties of that by analyzing the matrix $T$ and the function $g$ as well as the differences for several values of $\gamma$. If $\gamma=0$ we get explicit Euler and if $\gamma=1$ we get a combination of implicit Euler for the linear part an explicit Euler for the nonlinear part. We will also show the differences within this class of methods as well as the difference to second order methods following Ascher et al. (1995).

\section{MODEL ORDER REDUCTION}

On the resulting ODE (10) we use the Model Order Reduction techniques Proper Orthogonal Decomposition (POD) together with Discrete Empirical Interpolation (DEIM) by Chaturantabut and Sorensen (2010). POD is a projection-based method where we find a projection matrix $W$ such that the solution of (10) $x \approx W \hat{x}$ for $\hat{x}$ in a lower dimensional space. The resulting low-dimensional ODE is then given by

$$
\dot{\hat{x}}=W^{T} T W \hat{x}+W^{T} g(W \hat{x}, t) .
$$

DEIM is then used to create a truely low-dimensional function approximating $W^{T} g(W \hat{x}, t)$.

\section{CONCLUSIONS}

The combination of POD-DEIM with the IMEX integration results in a significant speedup of simulation time.

\section{REFERENCES}

Ascher, U.M., Ruuth, S.J., and Wetton, B.T. (1995). Implicitexplicit methods for time-dependent partial differential equations. SIAM Journal on Numerical Analysis, 32(3), 797-823.

Chaturantabut, S. and Sorensen, D.C. (2010). Nonlinear model reduction via discrete empirical interpolation. SIAM J. Sci. Comput., 32(5), 2737-2764. doi:10.1137/090766498. 


\title{
Optimization strategy for parameter sampling in the reduced basis method
}

\author{
L. Iapichino* S. Volkwein* \\ * Department of Mathematics and Statistics, University of Konstanz, \\ Universitätsstraße 10, D-78457 Konstanz, Germany; (E-mail: \\ $\{$ Laura.Iapichino, Stefan.Volkwein\}@uni-konstanz.de).
}

\begin{abstract}
The reduced basis (RB) method is an efficient technique to solve parametric partial differential equations in a multi-query context, where the solution has to be computed for many different parameter values. The RB method drastically reduces the computational time for any additional solution (during the so-called online stage) once an initial set of basis functions has been computed (during the so-called offline stage) still retaining a certified level of accuracy. The greedy algorithm is the classical sampling strategy to select parameter values that define the set of basis functions. Here, an alternative and competitive approach for choosing the parameter values is presented. The new approach is based on an optimization problem for the parameters that allows to reduce the computational complexity of the offline stage of the RB method and improve its effectiveness.
\end{abstract}

Keywords: PDE-constrained optimization, reduced-order models, reduced basis method, greedy algorithm, parametric distributed function 


\title{
Basis generation approaches for a reduced basis linear quadratic regulator
}

\author{
Andreas Schmidt* Markus Dihlmann* Bernard Haasdonk* \\ * Institute of Applied Analysis and Numerical Simulation, University \\ of Stuttgart, Pfaffenwaldring 57, 70569 Stuttgart, Germany
}

Many technical, biological or economical phenomena can be modeled in terms of partial differential equations (PDE). Often those models depend on one or more parameter describing for example material coefficients or other design parameters. An important task in applications is the control of the systems in order to achieve certain goals such as stability. One common way to do this, is to design a linear quadratic regulator (LQR) feedback control, cf. Knobloch (1986). To apply this technique, the PDE is spatially discretized, which yields a very high dimensional system of ordinary differential equations. The solution of the control problem then requires the solution of a nonlinear high dimensional matrix equation, making the procedure very expensive and hence infeasible for multiquery scenarios. We therefore are interested in applying the Reduced Basis (RB) method to accelerate the procedure, cf. Patera and Rozza (2007).

The systems under consideration can be written as linear time invariant (LTI) systems

$$
\left\{\begin{aligned}
\frac{\mathrm{d}}{\mathrm{d} t} x(t, \boldsymbol{\mu}) & =A(\boldsymbol{\mu}) x(t, \boldsymbol{\mu})+B(\boldsymbol{\mu}) u(t) \\
x(0, \boldsymbol{\mu}) & =x_{0}(\boldsymbol{\mu}),
\end{aligned}\right.
$$

where the system matrices have the dimensions $A(\boldsymbol{\mu}) \in$ $\mathbb{R}^{n \times n}$ and $B(\boldsymbol{\mu}) \in \mathbb{R}^{n \times m}$ with a probably very large dimension $n$. The function $u(t) \in \mathbb{R}^{m}$ is called control and should be determined in such a way, that the system (1) is stable and the following cost functional is minimized

$$
\int_{0}^{\infty} x(t, \boldsymbol{\mu})^{T} Q(\boldsymbol{\mu}) x(t, \boldsymbol{\mu})+u(t)^{T} R(\boldsymbol{\mu}) u(t) \mathrm{d} t,
$$

for positive definite matrices $Q(\boldsymbol{\mu}), R(\boldsymbol{\mu})$. Furthermore the control should be a linear feedback control, i.e. it must have the form $u(t)=-K(\boldsymbol{\mu}) x(t, \boldsymbol{\mu})$. This problem can be solved by finding the unique positive definite $\operatorname{root} P(\boldsymbol{\mu})$ of the Algebraic Riccati Equation (ARE)

$$
\begin{aligned}
& A(\boldsymbol{\mu})^{T} P(\boldsymbol{\mu})+P(\boldsymbol{\mu}) A(\boldsymbol{\mu})+Q(\boldsymbol{\mu}) \\
& -P(\boldsymbol{\mu}) B(\boldsymbol{\mu}) R(\boldsymbol{\mu})^{-1} B(\boldsymbol{\mu})^{T} P(\boldsymbol{\mu})=0,
\end{aligned}
$$

that stabilizes the closed loop (all real parts of the eigenvalues are in the left open complex half plane)

$$
\dot{x}(t, \boldsymbol{\mu})=(A(\boldsymbol{\mu})+B(\boldsymbol{\mu}) K(\boldsymbol{\mu})) x(t, \boldsymbol{\mu}),
$$

with $K(\boldsymbol{\mu})=-R(\boldsymbol{\mu})^{-1} B(\boldsymbol{\mu})^{T} P(\boldsymbol{\mu})$. Equation (2) is a quadratic matrix equation with $n^{2}$ unknowns and hence

\footnotetext{
* The authors acknowledges funding by the Landesstiftung Baden Württemberg gGmbH and would like to thank the German Research Foundation (DFG) for financial support within the Cluster of Excellence in Simulation Technology (EXC 310/1) at the University of Stuttgart.
}

expensive to solve. We thus are interested in accelerating the computation by employing the Reduced Basis (RB) method. The goal of the RB method is to divide the computation in an arbitrary complex offline stage, where a problem adapted solution space is constructed from carefully chosen solution snapshots, i.e. correct solutions to the high dimensional problem. The reduced space is then spanned by the columns of a matrix $V \in \mathbb{R}^{n \times r}$. By means of another matrix $W$ with $W^{T} V=I_{r \times r}$ a Petrov-Galerkin projection of (1) is performed, resulting in

$$
\left\{\begin{aligned}
\frac{\mathrm{d}}{\mathrm{d} t} \hat{x}(t, \boldsymbol{\mu}) & =\hat{A}(\boldsymbol{\mu}) \hat{x}(t, \boldsymbol{\mu})+\hat{B}(\boldsymbol{\mu}) \hat{u}(t) \\
\hat{x}(0, \boldsymbol{\mu}) & =W^{T} x_{0}(\boldsymbol{\mu}),
\end{aligned}\right.
$$

where $\hat{A}(\boldsymbol{\mu}):=W^{T} A(\boldsymbol{\mu}) V$ and $\hat{B}(\boldsymbol{\mu}):=W^{T}(\boldsymbol{\mu})$. In the online phase the reduced ARE

$$
\begin{aligned}
& \hat{A}(\boldsymbol{\mu})^{T} \hat{P}(\boldsymbol{\mu})+\hat{P}(\boldsymbol{\mu}) \hat{A}(\boldsymbol{\mu})+\hat{Q}(\boldsymbol{\mu}) \\
& -\hat{P}(\boldsymbol{\mu}) \hat{B}(\boldsymbol{\mu}) R(\boldsymbol{\mu})^{-1} \hat{B}(\boldsymbol{\mu})^{T} \hat{P}(\boldsymbol{\mu})=0,
\end{aligned}
$$

is solved for $\hat{P}(\boldsymbol{\mu}) \in \mathbb{R}^{r \times r}$ and the approximation to the full dimensional solution is defined as $\tilde{P}(\boldsymbol{\mu}):=V \hat{P}(\boldsymbol{\mu}) V^{T}$. There are now two main questions arising naturally. How can a good basis be constructed and how big is the error between the correct solution and the reduced solution, measured by the deviation in the controlled state and the feedback gain $K(\boldsymbol{\mu})$.

We build the basis by applying a greedy-like algorithm that successively adds the worst approximated element to the basis. Two algorithms are compared. The first algorithm is a standard POD-Greedy procedure based on state snapshots. Although a good space for the uncontrolled system is constructed, we found out that this approach does not capture the feedback information in a satisfactory way. This is why we extend the algorithm by adding parts of the feedback gain matrices $K(\boldsymbol{\mu})$ to the basis, resulting in the $K$-POD-Greedy algorithm. A numerical example, based on the $2 \mathrm{D}$ heat equation, shows the improved performance of the second algorithm.

\section{REFERENCES}

Knobloch, H.W. (1986). Linear control theory. SpringerVerlag New York, Inc.

Patera, A. and Rozza, G. (2007). Reduced Basis Approximation and a Posteriori Error Estimation for Parametrized Partial Differential Equations. MIT. Version 1.0, Copyright MIT 2006-2007, to appear in (tentative rubric) MIT Pappalardo Graduate Monographs in Mechanical Engineering. 


\title{
A Certified Reduced Basis Approach for Parametrized Linear-Quadratic Optimal Control Problems with Control Constraints
}

\author{
Eduard Bader* Mark Kärcher ${ }^{* *}$ Martin Grepl ${ }^{* * *}$ \\ Karen Veroy-Grepl ${ }^{* * * *}$ \\ * Aachen Institute for Advanced Study in Computational Engineering \\ Science (AICES), RWTH Aachen University, Schinkelstraße 2, 52062 \\ Aachen, Germany (e-mail: bader@aices.rwth-aachen.de). \\ ** Aachen Institute for Advanced Study in Computational Engineering \\ Science (AICES), RWTH Aachen University, Schinkelstraße 2, 52062 \\ Aachen, Germany (e-mail: kaercher@aices.rwth-aachen.de) \\ *** Numerical Mathematics, RWTH Aachen University, \\ Templergraben 55, 52056 Aachen, Germany (e-mail: \\ grepl@igpm.rwth-aachen.de) \\ **** Aachen Institute for Advanced Study in Computational \\ Engineering Science (AICES), RWTH Aachen University, \\ Schinkelstraße 2, 52062 Aachen, Germany (e-mail: \\ veroy@aices.rwth-aachen.de)
}

\begin{abstract}
:
In this talk, we consider the efficient and reliable solution of distributed optimal control problems governed by parametrized elliptic partial differential equations involving constraints on the control. The reduced basis method is used as a low-dimensional surrogate model to solve the optimal control problem. To this end, we introduce reduced basis spaces not only for the state and adjoint variable but also for the distributed control variable and propose rigorous error bounds for the error in the optimal control. The reduced basis optimal control problem and associated a posteriori error bounds can be efficiently evaluated in an offline-online computational procedure, thus making our approach relevant in the many-query or real-time context. We present numerical results for a model problem to show the validity of our approach.
\end{abstract}

Many problems in science and engineering can be modeled in terms of optimal control problems governed by parametrized partial differential equations (PDEs). While the PDE describes the underlying system or component behavior, the parameters often serve to identify a particular configuration of the component - such as boundary and initial conditions, material properties, and geometry. In such cases - in addition to solving the optimal control problem itself - one is often interested in exploring many different parameter configurations and thus in speeding up the solution of the optimal control problem. However, using classical discretization techniques such as finite elements or finite volumes even a single solution is often computationally expensive and time-consuming, a parameter-space exploration thus prohibitive. One way to decrease the computational burden is the surrogate model approach, where the original high-dimensional model is replaced by a reduced order approximation. These ideas have received a lot of attention in the past and various model order reduction techniques have been used in this context. However, the solution of the reduced order optimal con-

\footnotetext{
* This work was supported by the Excellence Initiative of the German federal and state governments and the German Research Foundation through Grant GSC 111.
}

trol problem is generally sub-optimal and reliable error estimation is thus crucial. Besides serving as a certificate of fidelity for the sub-optimal solution, our a posteriori error bounds are also a crucial ingredient in generating the reduced basis with greedy algorithms.

A new approach for efficient computation of error bounds for unconstrained distributed control problems was proposed in Kärcher et al. (2014). This approach, however, and all other existing approaches in the literature, see e.g. Negri et al. (2013); Negri (2011); Rozza et al. (2012), are not directly applicable to the important case with additional constraints on the control.

In this work we extend the methodology presented in Zhang et al. (2014) to consider PDE-constrained optimal control problems. The authors in Zhang et al. (2014) develop a certified Reduced Basis (RB) method that provides sharp and inexpensive a posteriori error bounds for variational inequalities. In particular, the approach has advantages compared to prior work on variational inequalities with the RB method Haasdonk et al. (2012). The methodology in Zhang et al. (2014) not only (i) provides sharper error bounds that mimic the convergence rate of the RB approximation, but also (ii) does so at an 
online cost that is independent of the high dimension of the original problem.

In particular we use the approach presented in Zhang et al. (2014) $(i)$ to construct a feasible RB approximation of the control and $(i i)$ to derive efficiently computable $a$ posteriori error bounds. The main idea is to generate two RB-systems. The first one is "standard" and is used to construct low dimensional approximations for the state and the Lagrange multiplier. In the second one we construct nonnegative slack variables for the control and so can generate feasible low dimensional surrogates for the control. Finally, we extend the proof of the a posteriori error bounds from Kärcher et al. (2014) to derive efficient a posteriori bounds for the control error in the constraint case.

\section{REFERENCES}

Haasdonk, B., Salomon, J., and Wohlmuth, B. (2012). A reduced basis method for parametrized variational inequalities. SIAM J. Numer. Anal., 50(5), 2656-2676.

Kärcher, M., Grepl, M., and Veroy, K. (2014). Certified reduced basis methods for parametrized distributed optimal control problems. submitted to SIAM Journal on Control and Optimization.

Negri, F., Rozza, G., Manzoni, A., and Quarteroni, A. (2013). Reduced basis method for parametrized elliptic optimal control problems. SIAM J. Sci. Comput. Accepted for publication.

Negri, F. (2011). Reduced basis method for parametrized optimal control problems governed by PDEs. Master's thesis, Politecnico di Milano.

Rozza, G., Manzoni, A., and Negri, F. (2012). Reduction strategies for pde-constrained optimization problems in haemodynamics. In Proceedings ECCOMAS Congress, Vienna Austria.

Zhang, Z., Bader, E., and Veroy, K. (2014). A duality approach to error estimation for variational inequalities. submitted to SIAM Journal on Scientific Computing. URL http: //arxiv.org/abs/1410. 2095. 


\title{
Numerical Investigations of an Error Bound for Reduced Basis Approximations of Noncoercice Variational Inequalities *
}

\author{
Silke Glas* Karsten Urban** \\ * Chair for Energy Trading and Finance, University of Duisburg-Essen, \\ 45141 Essen, Germany (e-mail: silke.glas@uni-due.de). \\ ** Institute for Numerical Mathematics, University of Ulm, \\ 89081 Ulm, Germany (e-mail: karsten.urban@uni-ulm.de)
}

\begin{abstract}
We consider variational inequalities with different trial and test spaces and a possibly noncoercive bilinear form. Wellposedness has been shown under general conditions that are e.g. valid for the space-time formulation of parabolic variational inequalities. Using space-time formulations, we do not have a time-stepping scheme anymore, but take the time as an additional variable in the variational formulation of the problem. As an example for a parabolic variational inequality, we may think about time-dependent obstacle problems or option pricing, e.g. for American Options.
\end{abstract}

Fine discretizations for such problems resolve in large scale problems and thus in long computing times. To reduce the size of these problems, we use the Reduced Basis Method (RBM)[Patera and Rozza (2006)]. The objective of the $\mathrm{RBM}$ is to efficiently reduce discretized parametrized partial differential equations. Problems are considered where not only a single solution is needed but solutions for a range of different parameter configurations.

Combining the RBM with the space-time formulation, a residual based error estimator has been derived in [Glas and Urban (2014)]. In this paper, we provide corresponding numerical results for a parametrized heat inequality model. Particularly, we perform two experiments concerning the error estimator. In the first one, we focus on rigor and efficiency of the error estimator depending on the specific method used for the basis generation and on the shape of the obstacle. In the second one, we show the quantitative reduction using the $\mathrm{RBM}$ in this setting.

\section{REFERENCES}

Glas, S. and Urban, K. (2014). On noncoervice variational inequalities. SIAM Journal on Numerical Analysis, 52, 2250-2271.

Patera, A. and Rozza, G. (2006). Reduced Basis Approximation and A Posteriori Error Estimation for Parametrized Partial Differential Equations. Graduate Studies in Mathematics. Copyright MIT, to appear in (tentative rubric) MIT Pappalardo Graduate Monographs in Mechanical Engineering., 1.0 edition.

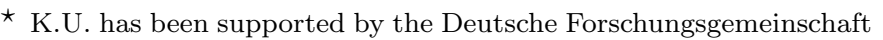
(DFG) under GrK 1100. Both authors have been supported by the DFG under SPP1324.
} 


\title{
The Empirical Cross Gramian for Parametrized Nonlinear Systems *
}

\author{
Christian Himpe* Mario Ohlberger ${ }^{* *}$ \\ * Westfälische Wilhelms Universtät, Münster, 48149 Germany \\ (e-mail: christian.himpe@uni-muenster.de). \\ ** Westfälische Wilhelms Universtät, Münster, 48149 Germany \\ (e-mail: mario.ohlberger@uni-muenster.de)
}

\begin{abstract}
The cross gramian matrix can be used for model order reduction as well as system identification of linear control systems, which are frequently used in the sciences. The empirical cross gramian is solely computed from trajectories and hence extends beyond linear state-space systems to nonlinear systems. In this work the applicability of the empirical cross gramian also for parametrized systems is demonstrated and assessed using a nonlinear benchmark problem.
\end{abstract}

Keywords: Model Reduction, Controllability, Observability, Nonlinear Systems, System Identification, Cross Gramian

\section{INTRODUCTION}

Large-scale parametrized state-space systems such as models of neuronal networks often require model order reduction to accelerate the evaluation, especially in a multiquery setting when varying parameters for example during optimization.

For a square linear control system, a linear control system with the same number of inputs and outputs,

$$
\begin{aligned}
\dot{x}(t) & =A x(t)+B u(t), \\
y(t) & =C x(t),
\end{aligned}
$$

$\operatorname{dim}(u)=\operatorname{dim}(y)$, the cross gramian matrix, reviewed in Antoulas (2005), enables the computation of a reduced order model, based on the systems controllability and observability.

The system gramians, the controllability gramian and observability gramian, used in balanced truncation, and the cross gramian classically exploit the linear control system structure for computation. The empirical gramians Lall et al. (1999) extend this concept to nonlinear systems,

$$
\begin{aligned}
& \dot{x}(t)=f(x(t), u(t)), \\
& y(t)=g(x(t), u(t)) .
\end{aligned}
$$

In this work, for parametrized square nonlinear systems:

$$
\begin{aligned}
& \dot{x}(t)=f(x(t), u(t), \theta), \\
& y(t)=g(x(t), u(t), \theta),
\end{aligned}
$$

the empirical variant of the cross gramian Himpe and Ohlberger (2014a) is demonstrated to compute a reduced order model that is valid over a given parameter space, around a steady state $\bar{x}$, exemplary for a benchmark model.

\footnotetext{
* This work was supported by the Deutsche Forschungsgemeinschaft, DFG EXC 1003 Cells in Motion Cluster of Excellence, Münster, Germany, as well as the Center for Developing Mathematics in Interaction, DEMAIN, Münster, Germany.
}

\section{CROSS GRAMIAN}

The cross gramian $W_{X}$ is defined as the product of controllability operator $\mathcal{C}(u):=\int_{0}^{\infty} e^{A t} B u(t) \mathrm{d} t$ and observability operator $\mathcal{O}\left(x_{0}\right):=C e^{A t} x_{0}$,

$$
W_{X}:=\mathcal{C} \circ \mathcal{O}=\int_{0}^{\infty} e^{A t} B C e^{A t} \mathrm{~d} t,
$$

which is classically computed as the solution of a Sylvester equation: $A W_{X}+W_{X} A=-B C$. If the underlying system is symmetric: $\mathcal{O C}=(\mathcal{O C})^{*}$, then the following property relates the cross gramian to the controllability gramian $W_{C}:=\mathcal{C C}^{*}$ and observability gramian $W_{O}:=\mathcal{O}^{*} \mathcal{O}$,

$$
\begin{aligned}
W_{X}^{2} & =\mathcal{C O} \mathcal{C O} \\
& =\mathcal{C C}^{*} \mathcal{O}^{*} \mathcal{O}=W_{C} W_{O} \\
\Rightarrow\left|\lambda\left(W_{X}\right)\right| & =\sqrt{\lambda\left(W_{C} W_{O}\right)} .
\end{aligned}
$$

The singular value decomposition of the cross gramian then yields a projection which can be truncated to generate a reduced order model similar to balanced truncation.

\section{EMPIRICAL CROSS GRAMIAN}

The empirical cross gramian from Himpe and Ohlberger (2014a) is an empirical gramian based on computing trajectories for perturbed input $u \in Q_{U}$ and initial states $x_{0} \in Q_{X}$. Additionally for parametrized systems, trajectories for perturbed parameters $\theta \in Q_{\Theta}$ can be computed:

$$
\begin{array}{r}
\widehat{W}_{X}:=\frac{1}{\left|Q_{U}\right|\left|Q_{X}\right|\left|Q_{\Theta}\right|} \sum_{h=1}^{\left|Q_{U}\right|} \sum_{i=1}^{\left|Q_{X}\right|} \sum_{j=1}^{\left|Q_{\Theta}\right|} \int_{0}^{\infty} \Psi^{h i j}(t) \mathrm{d} t, \\
\Psi_{k, i}^{h i j}(t)=\left(x_{k}^{h j}(t)-\bar{x}_{k}\right)\left(y_{h}^{i j}(t)-\bar{y}_{h}\right),
\end{array}
$$

with $x_{k}^{h j}(t)$ being the $k$-th component of the state trajectory with perturbations from $Q_{U}$ and $Q_{\Theta}$ around the steady state $\bar{x}$, and $y_{h}^{i j}(t)$ being the $h$-th component of the output trajectory with perturbations from $Q_{X}$ and 


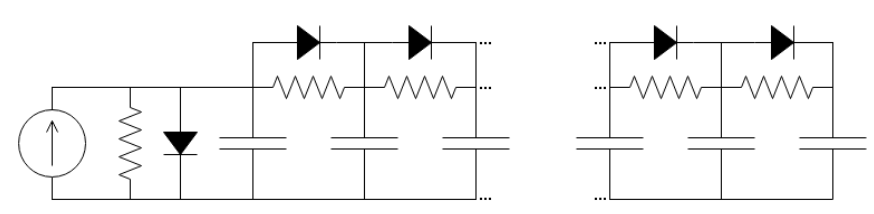

Fig. 1. Nonlinear resistor capacitor network ${ }^{1}$.

$Q_{\Theta}$ with steady state output $\bar{y}$. The number of perturbations $\left|Q_{U}\right|,\left|Q_{X}\right|,\left|Q_{\Theta}\right|$ depends on the operating region of the system. For linear systems the (linear) cross gramian equals the empirical cross gramian as shown in Himpe and Ohlberger (2014a), hence we use $\widehat{W}_{X}=W_{X}$. But, since the computation of the empirical cross gramian requires only simulations of the underlying system, this approach also extends to nonlinear systems. The (empirical) cross gramian is only applicable to square systems and exhibits its core property (1) for symmetric systems. The nonlinear extension of linear symmetric systems are gradient systems Scherpen and van der Schaft (2011), and only for gradient systems this empirical cross gramian is expected to yield workable results.

\section{NUMERICAL RESULTS}

The model reduction capabilities of the empirical cross gramian for parametrized systems are tested on a nonlinear benchmark problem listed at (MORwiki community, 2015, Nonlinear RC Ladder); a resistor-capacitor cascade with nonlinear resistors (see also the circuit schematics in Figure 1):

$$
\begin{aligned}
& \dot{x}(t)=\left(\begin{array}{c}
-g\left(x_{1}(t)\right)-g\left(x_{1}(t)-x_{2}(t)\right)+u(t) \\
g\left(x_{1}(t)-x_{2}(t)\right)-g\left(x_{2}(t)-x_{3}(t)\right) \\
\vdots \\
g\left(x_{k-1}(t)-x_{k}(t)\right)-g\left(x_{k}(t)-x_{x+1}(t)\right) \\
\vdots \\
g\left(x_{N-1}(t)-x_{N}(t)\right)
\end{array}\right), \\
& y(t)=x_{1}(t),
\end{aligned}
$$

with $g_{\theta}: \mathbb{R} \rightarrow \mathbb{R}$ given by:

$$
g_{\theta}(x)=\exp (10 x)+\theta x-1 .
$$

The linear part of the resistor network is parametrized, with $\theta_{i}$ describing the $i$-th resistor's linear resistance value. Since (2) is a SISO system, the empirical cross gramian applies here, because all SISO system are gradient systems as noted in Scherpen and van der Schaft (2011).

For the following experiments ${ }^{2}$, the empirical cross gramian (WX) is computed using the empirical gramian framework from Himpe and Ohlberger (2013); Himpe (2015) and compared to balanced truncation (BT) of the empirical controllability gramian and empirical observability gramian introduced in Lall et al. (1999). The number of states is set to $\operatorname{dim}(x)=1000$, the input signal is selected as $u(t)=e^{-t}$ and the test parameters are drawn from a uniform distribution $P(\theta)=U\left(\frac{1}{2}, \frac{3}{2}\right)$. Figure 2 shows, while both methods require nearly the same offline time to assemble the reduced order model, that the output error of the empirical cross gramian's reduced order model, is

\footnotetext{
1 Adapted from "Nonlinear RC Ladder", MORwiki http:// modelreduction.org/index.php/Nonlinear_RC_Ladder

2 The companion code to reproduce these results can be found at http://www.runmycode.org/companion/view/1084
}
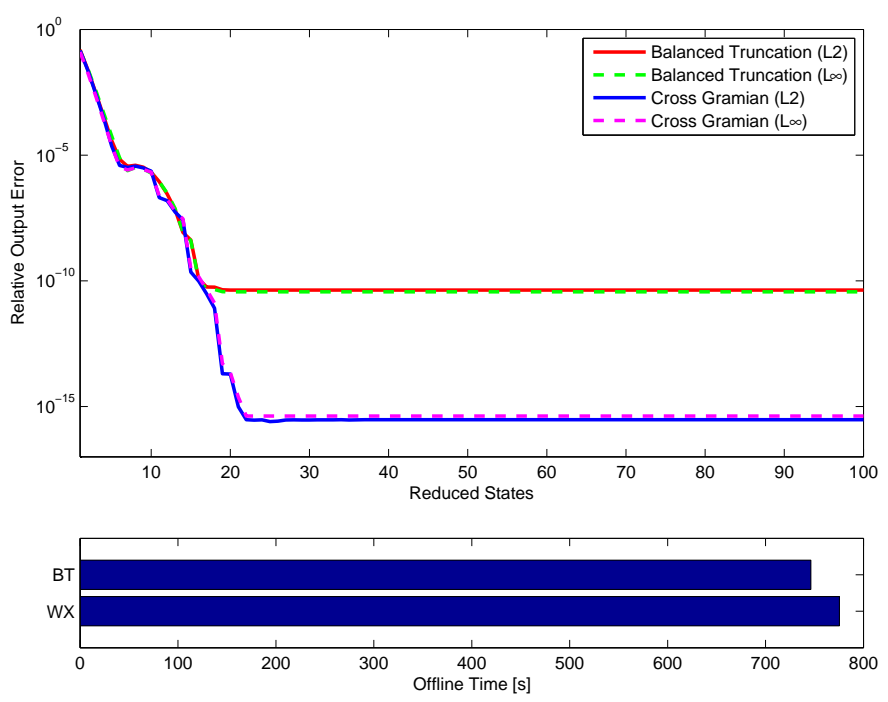

Fig. 2. Relative $L_{2}$ and $L_{\infty}$ output errors for the first hundred reduced states and offline time required to compute the associated gramians.

about five orders of magnitude below the output error of the reduced model computed by balanced truncation.

\section{REMARKS AND OUTLOOK}

As demonstrated, the empirical cross gramian can be applied to parametrized nonlinear systems with an improved performance over balanced truncation. Future extensions will include expanding the scope of the empirical cross gramian beyond symmetric linear systems as in Himpe and Ohlberger (2014b), or gradient nonlinear systems to more general (non-symmetric) configurations Himpe and Ohlberger (2015).

\section{REFERENCES}

Antoulas, A. (2005). Approximation of large-scale dynamical systems, volume 6 . SIAM.

Himpe, C. (2015). emgr - Empirical Gramian Framework. http://gramian.de.

Himpe, C. and Ohlberger, M. (2013). A Unified Software Framework for Empirical Gramians. Journal of Mathematics, 2013, 1-6.

Himpe, C. and Ohlberger, M. (2014a). Cross-Gramian Based Combined State and Parameter Reduction for Large-Scale Control Systems. Mathematical Problems in Engineering, 2014, 1-13.

Himpe, C. and Ohlberger, M. (2014b). Model Reduction for Complex Hyperbolic Networks. In Proceedings of the European Control Conference, 2739-2743.

Himpe, C. and Ohlberger, M. (2015). A Note on the Cross Gramian for Non-Symmetric Systems. Submitted. Preprint at arXiv, math.OC(1501.05519), 1-6.

Lall, S., Marsden, J., and Glavaski, S. (1999). Empirical model reduction of controlled nonlinear systems. In Proceedings of the 14th IFAC Congress, volume F, 473478.

MORwiki community (2015). Model Order Reduction Wiki. http://modelreduction.org.

Scherpen, J. and van der Schaft, A. (2011). Balanced model reduction of gradient systems. Information and Software Technology, 12745-12750. 


\title{
Interactive Simulations Using Localized Reduced Basis Methods *
}

\author{
Andreas Buhr* Mario Ohlberger* \\ * Institute for Computational and Applied Mathematics \\ University of Münster \\ Einsteinstraße 62, 48149 Münster, Germany \\ (e-mail: andreas.buhr@uni-muenster.de).
}

\begin{abstract}
An interactive simulation tool should allow its user to change the geometry of the simulation and present an updated solution within a very short time span. To achieve this, the Reduced Basis Method can be used. For problems described by parametrized partial differential equations, it allows for very fast recomputation of the solution after parameter changes. In many cases, changes in the geometry can be accounted for by parametrization. However, this approach has two drawbacks: First, not all geometric variations can be described efficiently by parametrization. Second, the parametrization and thereby the type of changes possible has to be specified before the setup phase. The user is then restricted to these.

To overcome these limitations, we propose to localize the basis generation in the Reduced Basis Method. Using basis functions having support only on a small subset of the domain, one can react to arbitrary local geometry modifications by recreating only the basis functions in an environment of the modification.
\end{abstract}

Keywords: Reduced-order models, Parametrization, Maxwell equations, Localization, Error estimation, Domain Decomposition

\section{INTRODUCTION}

For problems which can be formulated as parametrized partial differential equations, the Reduced Basis Method (RBM) has proven to be an effective tool to accelerate the simulation (see e.g. Patera and Rozza (2007)). At the cost of a long 'offline' computation, one gains the possibility to change parameters 'online' and obtain results quickly. Publications on the RBM often show speed-ups of orders of magnitude.

The changes to the simulated model which are possible during the 'online' phase are however limited to parameter changes. This implies that all modifications which should be possible during the 'online' phase have to be formulated as parametrizations of the problem. Parametrization of the geometry is possible (see e.g. Drohmann et al. (2009)), but this is not always efficient. Furthermore, these approaches often fail to describe topology changing modifications of the geometry. An even larger problem in the context of interactive simulations with the RBM is the fact that the parametrization has to be specified before the 'offline' phase and cannot be changed during the 'online' phase. In order to combine the possibility for arbitrary geometry changes with the speed of the RBM we propose to use basis functions with localized support for the reduced basis. Using basis functions which have support only in a small subset of the domain which is simulated, one can reuse a part of the basis functions after a geometry change. In case of localized changes, which are typical for many engineering workflows, most basis functions can be kept.

\footnotetext{
* This work has been supported by CST - Computer Simulation Technology AG
}

After calculating some new basis functions, an updated solution can be calculated. Because basis functions have to be regenerated after geometry changes, this approach blurs the clear separation between 'offline' and 'online' phase.

\section{EXISTING METHODS}

Many numerical multiscale methods like e.g. the Multiscale Finite Element Method as introduced in Hou and $\mathrm{Wu}$ (1997) involve the calculation of localized ansatz functions and a subsequent Galerkin projection into the space spanned by these functions. The combination of the RBM with numerical multiscale methods leads to a method suitable for parametrized multiscale problems. For problems without scale separation, an adaptive enrichment of the approximation space, steered by a-posteriori error estimators, leads to an efficient method as realized for the Generalized Multiscale Finite Element Method by Chung et al. (2014) or for the Localized Reduced Basis Multiscale Method by Albrecht and Ohlberger (2013). These methods could be extended for interactivity in our sense. Another method aiming at larger freedom for the user during the 'online' phase is the Static Condensation Reduced Basis Element Method introduced in Phuong Huynh et al. (2012). While allowing arbitrary recombination of so called 'components' online, it does not allow for arbitrary geometry changes.

\section{PROPOSED LOCALIZATION}

We consider a parametrized problem described for any parameter $\mu$ in the parameter space $\mathcal{P}$ by a sesquilinear 
form $a_{\mu}: V \times V \rightarrow \mathbb{C}$ and an antilinear form $f_{\mu}: V \rightarrow \mathbb{C}$ emerging from the weak formulation of the time harmonic Maxwell equation. We assume $a_{\mu}$ to be inf-sup stable for all parameter values with $\alpha_{L B}$ as a lower bound for the inf-sup constant:

$$
0<\alpha_{L B} \leq \inf _{\substack{\varphi_{1} \in V \\ \varphi_{1} \neq 0}} \sup _{\substack{\varphi_{2} \in V \\ \varphi_{2} \neq 0}} \frac{\left|a_{\mu}\left(\varphi_{1}, \varphi_{2}\right)\right|}{\left\|\varphi_{1}\right\|_{V}\left\|\varphi_{2}\right\|_{V}} \quad \forall \mu \in \mathcal{P} .
$$

The solution $u_{\mu} \in V$ is the solution of

$$
a_{\mu}\left(u_{\mu}, v\right)=f_{\mu}(v) \quad \forall v \in V .
$$

Here the space $V$ is a discrete ansatz space spanned by basis functions $\left\{\psi_{1}, \ldots, \psi_{N}\right\}$. Both the standard RBM and the proposed method construct a reduced space $\widetilde{V} \subset V$ as the span of constructed basis functions

$$
\widetilde{V}=\operatorname{span}\left\{\widetilde{\psi}_{1}, \ldots, \widetilde{\psi}_{\widetilde{N}}\right\}, \quad \widetilde{\psi}_{i}=\sum_{j=1}^{N} b_{j i} \psi_{j} .
$$

In the standard RBM, the space $\widetilde{V}_{R B}$ is constructed as the span of solutions to carefully selected parameter values:

$$
\widetilde{V}_{R B}:=\operatorname{span}\left\{u_{\mu}, \mu \in \Xi_{\text {snapshot }}\right\} .
$$

In contrast, in our approach, the construction of the reduced space is a four step procedure: The localization is introduced by first defining a direct decomposition of the ansatz space $V$ i.e.

$$
V=\bigoplus_{k=1}^{N_{s}} V_{k}
$$

where each $V_{k}$ is a subspace of the original ansatz space $V$. To construct the spaces $V_{k}$ we introduce a non overlapping domain decomposition and group the ansatz functions of $V$ by the domains they have support in. Then we define each space $V_{k}$ as the span of one group of ansatz functions. We also introduce projection operators $P_{k}: V \rightarrow V_{k}$ which are uniquely defined by

$$
\varphi=\sum_{k=1}^{N_{s}} P_{k}(\varphi) \quad \forall \varphi \in V .
$$

In a second step, for each space $V_{k}$ we construct a subspace

$$
\widetilde{V}_{k} \subset V_{k} \text {. }
$$

The way of constructing these subspaces is crucial for the performance of the method. The spaces are constructed iteratively by solving local problems on overlapping patches, steered by an a-posteriori error estimator. Note that the spaces $\widetilde{V}_{k}$ are subspaces of $V$.

In a third step, we mix these spaces using linear extension operators. Let $\mathcal{E}:\left\{1, \ldots, N_{s}\right\} \rightarrow \operatorname{Pow}\left(\left\{1, \ldots, N_{s}\right\}\right)$ be the function defining the extension set for each space. Let $E_{k j}: V_{k} \rightarrow V_{j}$ be linear extension operators. The extended spaces are defined as

$$
\widetilde{V}_{k}^{E}:=\operatorname{span}\left\{\varphi+\sum_{j \in \mathcal{E}(k)} E_{k j}(\varphi) \quad \mid \varphi \in \widetilde{V}_{k}\right\} .
$$

In case $\mathcal{E}(k)=\emptyset$ it holds $\widetilde{V}_{k}^{E}=\widetilde{V}_{k}$. In any case, the spaces $\widetilde{V}_{k}^{E}$ are subspaces of $V$. While this step is not necessary for the method, it leads to smaller basis sizes.

In the fourth step we combine the spaces to the resulting global solution space

$$
\widetilde{V}_{L R B}:=\bigoplus_{k=1}^{N_{s}} \widetilde{V}_{k}^{E}
$$

and then solve

$$
a_{\mu}\left(\widetilde{u}_{\mu}, \widetilde{v}\right)=f_{\mu}(\widetilde{v}) \quad \forall \widetilde{v} \in \widetilde{V}_{L R B} .
$$

Note again that $\widetilde{V}_{L R B}$ is a subspace of $V$.

\section{A-POSTERIORI ERROR ESTIMATOR}

From standard RB theory it is known that the error can be bounded using the dual norm of the residual $\mathrm{R}_{\mu}\left(\widetilde{u}_{\mu}\right) \in V^{\prime}$ :

$$
\left\|u_{\mu}-\widetilde{u}_{\mu}\right\|_{V} \leq \frac{1}{\alpha_{L B}}\left\|\mathrm{R}_{\mu}\left(\widetilde{u}_{\mu}\right)\right\|_{V^{\prime}} .
$$

The calculation of the dual norm of the residual usually involves the calculation of global Riesz representatives for all parts of an affine decomposition of the residual. To localize this step, we use localized dual space norms. One can show that for any $F \in V^{\prime}$ and therefore also for the residual $\mathrm{R}_{\mu}\left(\widetilde{u}_{\mu}\right)$ it holds

$$
\|\mathrm{F}\|_{V^{\prime}} \leq \sum_{k=1}^{N_{s}} c_{k}\|\mathrm{~F}\|_{V_{k}^{\prime}} \leq\left(\sum_{k=1}^{N_{s}} c_{k}\right)\|\mathrm{F}\|_{V^{\prime}}
$$

where the computable constants $c_{k}$ are defined by

$$
c_{k}:=\max _{\varphi \in V} \frac{\left\|P_{k}(\varphi)\right\|_{V}}{\|\varphi\|_{V}} .
$$

\section{NUMERICAL VALIDATION}

A parallel and efficient implementation of the proposed method for the two dimensional time harmonic Maxwell equation was written. It allows its user to change the geometry of the simulation interactively in any way and presents updated results quickly for localized changes.

\section{REFERENCES}

Albrecht, F. and Ohlberger, M. (2013). The localized reduced basis multi-scale method with online enrichment. Oberwolfach Reports, vol.7, 406-409.

Chung, E.T., Efendiev, Y., and Li, G. (2014). An adaptive GMsFEM for high-contrast flow problems. Journal of Computational Physics, 273, 5476.

Drohmann, M., Haasdonk, B., and Ohlberger, M. (2009). Reduced basis method for finite volume approximation of evolution equations on parametrized geometries. Proceedings of ALGORITMY, 1-10.

Hou, T.Y. and Wu, X.H. (1997). A multiscale finite element method for elliptic problems in composite materials and porous media. Journal of Computational Physics, 134, 169-189.

Patera, A. and Rozza, G. (2007). Reduced Basis Approximation and A Posteriori Error Estimation for Parametrized Partial Differential Equations. Version 1.0, Copyright MIT (2006-2007), to appear in (tentative rubric) MIT Pappalardo Graduate Monographs in Mechanical Engineering.

Phuong Huynh, D.B., Knezevic, D.J., and Patera, A.T. (2012). A static condensation reduced basis element method: approximation and a posteriori error estimation. M2AN, 47(1), 213-251. 
MATHMOD 2015 Abstract Volume 


\title{
MATHMOD 2015 ABSTRACT VOLUME
}

\author{
MATHMOD Minisymposium \\ Object-Oriented Modelling, Simulation and \\ Optimization
}


MATHMOD 2015 Abstract Volume 


\title{
Efficient Computation of State Derivatives for Multi-Rate Integration of Object-Oriented Models
}

\author{
Francesco Casella* \\ * Dipartimento di Elettronica, Informazione e Bioingegneria, \\ Politecnico di Milano, Italy (e-mail: francesco.casella@polimi.it).
}

\section{INTRODUCTION AND MOTIVATION}

State-of-the-art tools to simulate declarative, ObjectOriented (OO) models, in particular Modelica tools such as Dymola or OpenModelica, follow several steps to achieve their goal. Starting from the differential-algebraic equations (DAEs) that describe the system, they first symbolically remove trivial equations such as $a \pm b=0$ and $a= \pm b$ from the system, then apply sophisticated symbolic simplifications, including symbolic index reduction. The system model is thus brought to a simpler, index-1 DAE form

$$
F(x, \dot{x}, v, t)=0,
$$

where $F(\cdot)$ is a vector function, $x$ is the vector of state variables, $v$ the vector of algebraic (i.e., non-state) variables and $t$ is time. Efficient code is then generated to solve this system for $(\dot{x}, v)$ assuming $(x, t)$ known; thus, the system is conceptually brought into state-space form with explicit ordinary differential equations (ODEs) and output equations

$$
\begin{aligned}
& \dot{x}=f(x, t) \\
& v=g(x, t) .
\end{aligned}
$$

This code is then linked to ODE solvers to numerically compute the system transients.

State-of-the-art ODE integration algorithms employed for this purpose are currently of the single-rate type, which evaluate the entire vector of derivatives $f(x, t)$ at each time step. As the size of the system grows, this approach can become increasingly inefficient for models characterised by dynamic behaviour on different time scales and/or by a large number of loosely interacting sub-systems with uncorrelated local activity.

In both cases, assuming adaptive error control is used, when the system shows fast changes in one of its local or fast subsystems, short time steps are taken to limit the error below the given tolerance, and this requires recomputing the entire derivative vector $f(x, t)$ at very closely spaced time steps. This can be extremely inefficient, as the derivative of all other slow and/or loosely coupled sub-systems will hardly change over such a short time span, so that most of those computations will not in fact bring any new useful information to the solver.

A recent paper by Ranade and Casella (2014) proposes to introduce multi-rate algorithms in these scenarios. Multirate algorithms have been studies since the early sixties, see, e.g., Rice (1960), Gear and Wells (1984), Engstler and Lubich (1997), Savcenco et al. (2007), but haven't found use in $\mathrm{OO}$ simulation tools so far. A representative case study presented by Ranade and Casella (2014) shows that the number of evaluations of individual components of $f(x, t)$ during the simulation of a transient scales up with the square of the system size, compared to the cubic increase obtained by standard single-rate algorithms. It is then apparent that these methods can provide huge advantages in simulation speed as the size and degree of detail of the model increases.

A basic requirement for the use of multi-rate algorithms is that at each time step, only a sub-set of the state derivative vector $f(x, t)$ needs to be computed. The goal of this paper is to show that this can be accomplished by a suitable extension of the code generation techniques which are currently adopted by state-of-the-art OO tools.

\section{MAIN CONTRIBUTION}

The paper shows how to build a particular equation dependency graph starting from an index-1 DAE model of the system. From this graph, it shows how to extract all the information that is required to support multi-rate ODE solvers. In particular, it shows how to generate code to efficiently compute any sub-set of the state vector.

The proposed algorithms are applied to a simple exemplary test case, showing the dependency graph and the generated pseudo-code.

\section{REFERENCES}

Engstler, C. and Lubich, C. (1997). Multirate extrapolation methods for differential equations with different time scales. Computing, 58(2), 173-185.

Gear, C. and Wells, D. (1984). Multirate linear multistep methods. BIT 24, 484-502.

Ranade, A. and Casella, F. (2014). Multi-rate integration algorithms: a path towards efficient simulation of objectoriented models of very large systems. In Proceedings EOOLT 2014 Workshop. ACM, Berlin, Germany. doi: 10.1145/2666202.2666214.

Rice, J.R. (1960). Split runge-kutta method for simultaneous equations. JOURNAL OF RESEARCH of the National Bureau of Standards-B. Mathematics and Mathematical Physics, 64B(5), 151-170.

Savcenco, V., Hundsdorfer, W., and Verwer, J. (2007). A multirate timestepping strategy for stiff ordinary differential equations. BIT: Numerical Mathematics, 47(1), 137-155. 


\title{
Design of Feed Drives with Object-Oriented Behavior Models
}

\author{
W. Herfs, D. Özdemir, W. Lohse, C. Brecher \\ Laboratory for Machine Tools and Production Engineering (WZL) of the RWTH Aachen University \\ Aachen, Germany (e-mail: \{w.herfs,d.oezdemir,w.lohse, c.brecher\}@wzl.rwth-aachen.de)
}

Feed drives have major influence on the performance, reliability and cost of machine tools and production plants. Oversized components lead to high costs, not only with regards to purchase, but more importantly with regards to energy consumption over the lifecycle. With undersized components the machine will not be able to meet performance or reliability requirements. Moreover, feed drive components have to be chosen with regards to their dynamic properties to account for size tolerances of the work piece.

The problem of quickly comparing different feed drive design concepts and choosing adequate components remains largely unsolved. The approach that is presented in this paper aims to close this gap by leveraging object-oriented physical modelling languages in combination with optimization techniques for parameter and component determination.

The approach is based on intuitive and re-useable objectoriented behavior models in Modelica that are augmented with requirements. For automatic component selection and parameter optimization the simulation platform has been coupled with the simulation frameworks NOMAD and DAKOTA. We demonstrated that this approach is able find the optimal combination of components within a time of less than 1 minute. In comparison to existing sizing tools it is not only possible to choose a motor for a given mechanical configuration, but the motor and the mechanical system are optimized at the same time. Thus, it is easier for the engineer to design a feed drive concept that fulfills the requirements in an optimal way.

So far, three different optimization algorithms were considered, where the Mesh Adaptive Direct Search (MADS) in NOMAD is the fastest and most robust. A review showed that different algorithms from the commercial optimization framework TOMLAB perform well in comparison to other implementations (Rios \& Sahinidis, 2009). A future comparison of different solvers should therefore include this framework. For all solvers it is essential to constrain the search space by pre-selection. This manual step could be supported by a rule-based approach. Further improvements in solving the optimization can be expected from a two-step approach, where first only the algebraic design equations are considered and second the system of differential algebraic equations. To visualize results the methods developed in (Eppelt \& AlKhawli, 2014) can be used. In a subsequent paper optimization with regards to the frequency domain is discussed. To obtain the frequency response from the Modelica model we applied a method that is described in (Bünte, 2011). The approach of this paper is naturally not restricted to the design of feed drive systems. Other applications may include hydraulics, pneumatics and mechatronic systems in general.

\section{ACKNOWLDGEMENT}

The authors would like to thank the German research Foundation DFG for the support within the project "Optimierung des Systementwurfs von Maschinen und Anlagen auf Basis komponentenorientierter Verhaltensmodelle"

\section{REFERENCES}

Bünte, T. (2011). Recording of Model Frequency Responses and Describing Functions in Modelica. Proc. of the 8th Modelica Conference, 686-696.

Eppelt, U., \& Al-Khawli, T. (2014). Metamodeling of Laser Cutting. International Conference of Numerical Analysis and Applied Mathematics (ICNAAM), (in press).

Rios, L., \& Sahinidis, N. (2009). Derivative-free optimization: A review of algorithms and comparison of software implementations. Advances in Optimization II (AIChE 2009 Annual Meeting), $183 e$. 


\title{
A Modelica simulator to support the development of the control system of an autonomous All-Terrain mobile robot
}

\author{
Enzo L. D'Amelio Luca Bascetta* Davide A. Cucci ${ }^{* *}$ \\ Matteo Matteucci* Gianluca Bardaro*
* Politecnico di Milano, Dipartimento di Elettronica, Informazione e Bioingegneria, Piazza Leonardo da Vinci 32, 20133 Milano (Italy), (e-mail: \{luca.bascetta, matteo.matteucci\}@polimi.it).
** École Polytechnique Fédérale de Lausanne, Geodetic Engineering Laboratory, Station 18, CH-1015 Lausanne (Switzerland) (e-mail: davide.cucci@epfl.ch).

The aim of our research is to develop an All-Terrain Mobile Robot (ATMR), based on a commercial All-Terrain Vehicle (ATV), suitable for a wide range of different outdoor operations (Figure 1). The ATMR should be able to operate in any natural environment with a high level of autonomy. The advantage of using ATVs is represented by their good traversability potential for poorly traversable terrains, as well as by the possibility to operate in unsafe environments. On the other hand, the main disadvantage of ATVs is their low stability margin due to dynamic constraints, roll-over risk, and excessive side slip.

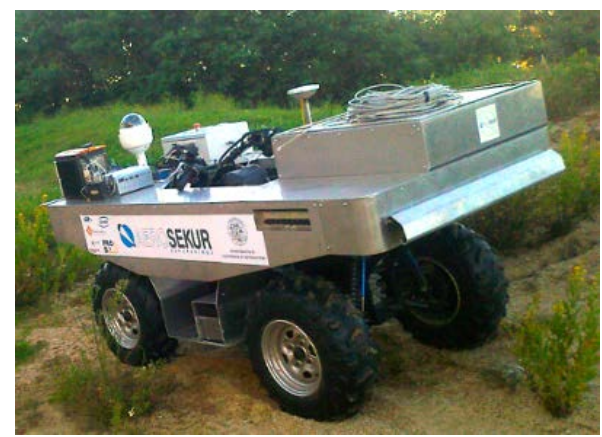

Fig. 1. The ATMR.

These characteristics call for the adoption of suitable control systems, whose aim is not only to fulfil the ATMR mission, but also to ensure the vehicle safety. When the vehicle stability is concerned, however, the design and testing of the control system is a critical task, as it may require to drive the vehicle at (or very close to) its stability limit. Nevertheless, even the experimental testing of a trajectory tracking system, though less critical, poses some difficulties. To mention just a few, it must be considered that testing the trajectory tracking controller at high velocity requires a wide area and specific safety devices in order to protect the vehicle and people who perform the experiment in case of an accident. As a consequence, in order to support the design phase and the preliminary validation of an ATMR, the development of an accurate dynamic simulator of the vehicle is of utmost importance. To this extent, a multi-body simulator, namely Modelica, has been chosen. Moreover, in order to better structure the simulator and maximise code reusability, the model has been developed starting from the Vehicle Dynamics Library.

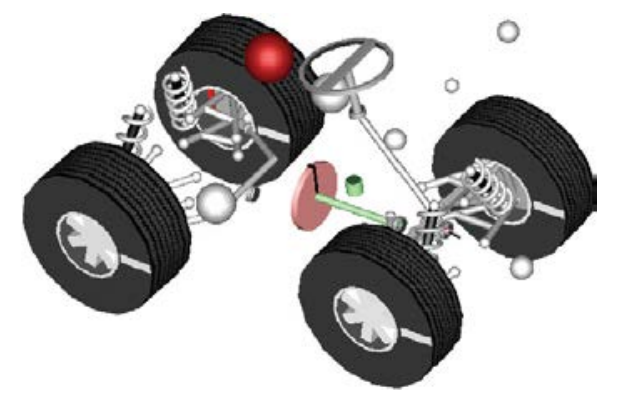

Fig. 2. The ATMR model without the cover.

The model (Figure 2) aims at replicating the behaviour of a Yamaha Grizzly 700 ATV (Figure 1), focusing on the dynamics that are relevant to the design and test of the vehicle control system.

A thorough experimental validation of the simulator, demonstrating its accuracy in reproducing the behaviour of the experimental system, is also presented (Figure 3).

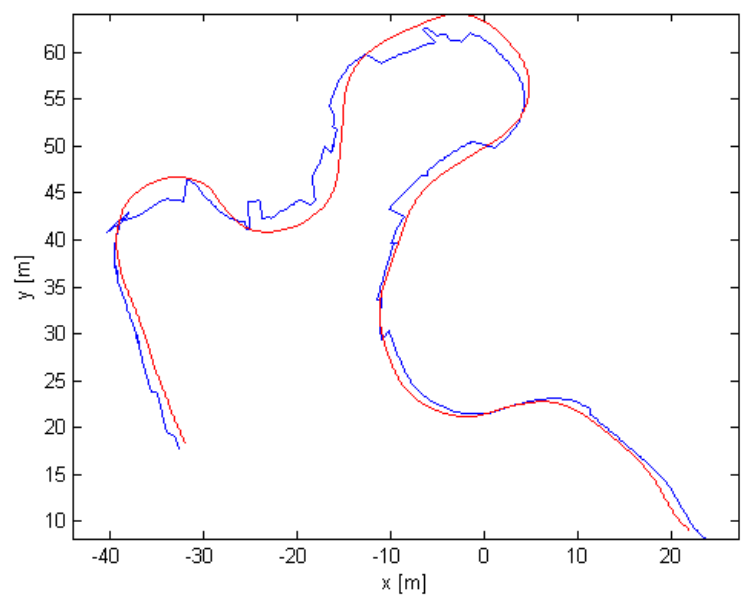

Fig. 3. The experimental vehicle path (blue line) and the simulated path (red line). 


\title{
Effects of Event-Free Noise Signals on Continuous-Time Simulation Performance
}

\author{
Franciscus L. J. van der Linden* Andreas Klöckner* \\ Dirk Zimmer* \\ * DLR German Aerospace Center, Institute of System Dynamics and \\ Control, 82234 Weßling, Germany \\ (e-mail: franciscus.vanderlinden@dlr.de).
}

\section{INTRODUCTION}

Generating stochastic input signals such as noise in physical systems is traditionally implemented using discrete random number generators based on discrete time-events. Within the Modelica community, random number generators free of time-events have recently been proposed in order to increase the performance of system simulations Klöckner et al. (2014). However, the impact of such signals on commonly used solvers, such as DASSL or Radau IIA, is still under discussion. In order to provide better understanding for modeling practitioners, we examine the influence of eventfree noise models on simulation performance. To this end, we conduct practical simulation experiments with systems of three sizes, two solvers, and different parameters. Results indicate that step-size control can handle event-free noise generators well and that they outperform sampled generators. The findings can be related to other timedependent system inputs.

\section{RESULTS}

An excerpt of the results is shown in Figure 1. It shows the number of function evaluations and the simulation time as functions of the integrator tolerance for a simple system. The results are produced using different interpolation methods for the noise signal. Dashed lines represent the sample-based noise generation method. Solid lines represent the sample-free DIRCS method proposed in prior work. The results show that the sample-free noise method reduces computational costs, especially with loose tolerances.

\section{REFERENCES}

Klöckner, A., van der Linden, F.L.J., and Zimmer, D. (2014). Noise Generation for Continuous System Simulation. In Proceedings of the 10th International Modelica Conference, 837-846. Modelica Association and Linköping University Electronic Press, Lund, Sweden. doi:10.3384/ECP14096837.
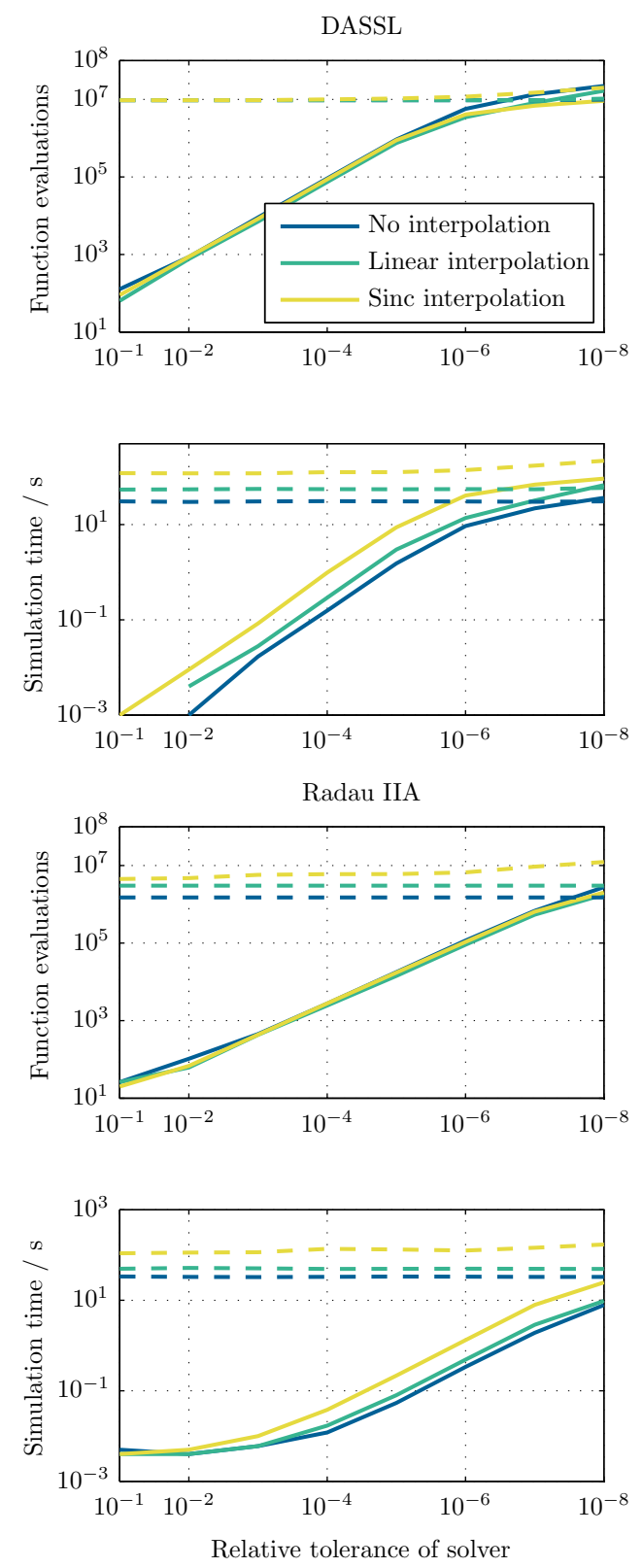

Fig. 1. Function evaluations and simulation time as a function of the integrator tolerance. Dashed lines: sample-based noise generation method. Solid lines: sample-free DIRCS method proposed in prior work 


\title{
Input design and parameter estimation with open source tools *
}

\author{
Simon Mayr* Gernot Grabmair ** Johann Reger*** \\ * Upper Austria University of Applied Sciences, Stelzhamerstraße 23, \\ 4600 Wels, Austria (e-mail: simon.mayr@fh-wels.at). \\ ** Upper Austria University of Applied Sciences, Stelzhamerstraße 23, \\ 4600 Wels, Austria (e-mail: gernot.grabmair@fh-wels.at) \\ *** Control Engineering Group, Technische Universität Ilmenau, \\ Helmholtzplatz 5,98693 Ilmenau, Germany, (e-mail: reger@ieee.org)
}

\section{INTRODUCTION}

Model-based design (MBD) is a common and wide-spread approach in various areas of application. Over time, MBD processes have received more and more attention in medium-sized and small businesses in order to improve the efficiency of the development processes. However, the pricing of existing commercial software-tools like Matlab/Simulink or Dymola may easily surpass necessary budgets, for small companies in particular. That is the rationale why we initiated to create a free alternative that may help small companies getting started in MBD processes. In Grabmair et al. (2014) an open source tool-chain for model-based design of embedded systems was presented. The focus of the contribution was on code generation. In contrast, to Grabmair et al. (2014), this work extends the free tool-chain by the generation of optimal excitation signals in combination with the solution of optimal control problems (OCP), and parameter estimation.

It is very common that trajectories for mechanical systems are generated by solving a particular optimization problem. For decreasing mechanical wear in the plant these profiles need to provide, for example, smooth (rest-to-rest) movement in accordance with restrictions in jerk, acceleration, and velocity. However, smooth trajectories tend to contain insufficient excitation information for identification. To this end, we propose to exploit a measure for the information content concerning some unknown parameters in the optimal control problem.

\section{METHODS}

Continuous-time system identification can be done by minimizing a cost function. The cost function becomes zero in the case when the deviation between measurement $\mathbf{y}_{m}$ and simulation signal $\mathbf{y}$ is zero on $\left[t_{0}, t_{e}\right]$ (undisturbed case). This is fulfilled when the model is parametrized with the correct parameter values.

To ensure an input signal with sufficient 'information' for identification we propose to combine the standard optimal control problem (OCP) with an input design approach. For analyzing the information content of a measurement we resort to the Fisher matrix $\mathbf{F}(\mathbf{u}, \mathbf{p})$, see Majer (1997).

\footnotetext{
* We gratefully acknowledge the support from the Austrian funding agency FFG in Coin-project ProtoFrame (project number 839074).
}

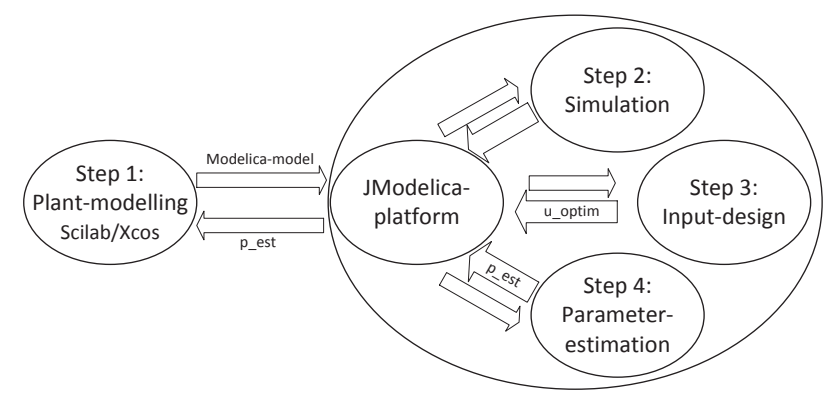

Fig. 1. Tool-chain for MBD process with focus on input design and parameter estimation

This matrix indicates how much information a number of $n_{y}$ measurable system outputs contain about $n_{p}$ unknown system parameters.

\section{SOFTWARE TOOLS}

The tool-chain we present in this paper aims to cover plant modelling (Step 1), basic model verification (Step 2), optimal excitation signal calculation (Step 3) and parameter estimation (Step 4), see Fig. 1.

For Step 1 we use the open source software Scilab/XCos. It serves as model editor. The platform for Step $2-4$ is JModelica. It is mainly used to solve the highly nonlinear optimization problem for input design and the parameter identification optimization problem.

\section{EXPERIMENT}

The whole tool-chain is tested by the well-known cart and pendulum example in gantry crane configuration in simulation with additional output noise.

\section{REFERENCES}

Grabmair, G., Mayr, S., Hochwallner, M., and Aigner, M. (2014). Model based control design - a free tool-chain. In European Control Conference (ECC). Strasbourg, France.

Majer, C.P. (1997). Parameterschaetzung Versuchsplanung und Trajektorienoptimierung fuer Verfahrenstechnische Prozesse. Ph.D. thesis, Stuttgart University, Stuttgart. 
MATHMOD 2015 Abstract Volume 


\section{MATHMOD 2015 ABSTRACT VOLUME}

MATHMOD Minisymposium
Multiscale Modeling of Transport- and Flow-Problems 
MATHMOD 2015 Abstract Volume 


\title{
Modelling of Langevin Equations by the Method of Multiple Scales
}

\author{
Jürgen Geiser* \\ * Ruhr University of Bochum, Department of Electrical Engineering \\ and Information Technology, Universitätsstr. 150, D-44801 Bochum, \\ Germany (e-mail: juergen.geiser@ruhr-uni-bochum.de).
}

\section{INTRODUCTION}

We are motivated to apply a multiscale model to solve Langevin equations. The Langevin equations are used to simulate the characteristics of Fokker-Planck equations with Coulomb collisions, see Cohen et al. (2010). Such models are applied to simulate sufficient dense plasma applications, e.g., magnetic fusion and inertial fusion problems.

In our paper, we consider a simpler model of a single particle density $f$ in a dense plasma problem and apply the appropriate description with a one-dimensional FokkerPlanck $(\mathrm{FP})$ equations in the phase space $(x, v)$ :

$$
\frac{\partial f}{\partial t}+v \frac{\partial f}{\partial x}+E(x, \epsilon) \frac{\partial f}{\partial v}=\frac{\partial}{\partial v}\left(-\gamma v f+\beta^{-1} \gamma \frac{\partial f}{\partial v}\right),
$$

where we assume to have a nonlinear and singular perturbed electric-field $E(x, \epsilon)=\epsilon \frac{2}{x^{2}}-2 x, \epsilon \in(0,1]$ and $\epsilon$ is the perturbation parameter.

The underlying Langevin-like equations of the FokkerPlanck equation (1) are given as (see also Bou-Rabee et al. (2012)):

- Ordinary differential equation (ODE)

$$
\frac{d x}{d t}=v, \frac{d v}{d t}=E(x, \epsilon)
$$

- Stochastic differential equation (SDE)

$$
\frac{d x}{d t}=0, d v=-\gamma v d t+\sqrt{2 \beta^{-1} \gamma} d W,
$$

where $W$ is a one-dimensional Brownian motion.

Based on the different time-scales of the underlying ODE (smooth time-scales) and SDE (rough time-scales), we have to consider effective multiscale methods, see Geiser (2013).

\section{MULTISCALE SPLITTING METHODS}

We propose modified splitting methods to resolve the different behaviors of the ODE and SDE parts. Splitting methods are important when we can split into different equations-parts with different behaviors, e.g., multiple time-scales, see McLachlan et al. (2002).

The proposed splitting method is based on a multiple scale method and we apply the following hierarchical ordering:

$$
x(t, \epsilon)=x_{0}(t)+\epsilon x_{1}(t)+O\left(\epsilon^{2}\right),
$$

with the initial conditions $x(0, \epsilon)=x(0)$ and $\frac{d x(0, \epsilon)}{d t}=v(0)$ and we obtain a system of first order different equations:

$$
\begin{aligned}
& d x_{0}=v_{0} d t, \\
& d x_{1}=v_{1} d t, \\
& d v_{0}=-2 x_{0} d t-\gamma v_{0} d t+\sqrt{2 \beta^{-1} \gamma} d W, \\
& d v_{1}=-2 x_{1} d t+\frac{2}{x_{0}^{3}} d t-\gamma v_{1} d t+\sqrt{2 \beta^{-1} \gamma} d W, \\
& x_{0}(0)=x(0), v_{0}(0)=v(0), x_{1}(0)=v_{1}(0)=0 .
\end{aligned}
$$

Such an extension of the original Langevin equations (2)-(3) is called multiscale Langevin equations and can be solved with respect to the different time-scales. The underlying Lie-Trotter splitting method, see Geiser (2011), is modified to an improved predictor-corrector splitting method (PC-AB splitting method), which embeds the method of multiple scales.

\section{CONCLUSION}

In the numerical experiments, we obtained a more detailed resolution of the multiscale problem with the novel splitting method. We apply different scales based on an electric-field, that covers blow ups (impact oscillators) and also oscillating scales (harmonic oscillator). Further we propose to develop general methods for realistic multiparticle collision operators of the Fokker-Planck equations.

\section{REFERENCES}

N. Bou-Rabee and E. Vanden-Eijnden. A patch that imparts unconditional stability to explicit integrators for Langevin-like equations. Journal of Computational Physics 231:2565-2580, 2012.

B. Cohen, A. Dimits, A. Friedman and R. Caflisch. Timestep considerations in particle simulation algorithms for Coulomb collisions in plasmas. IEEE Transactions on Plasma Science, 38(9):2394-2406, 2010.

J. Geiser. Iterative Splitting Methods for Differential Equations. Numerical Analysis and Scientific Computing Series, CRC Press, Chapman \& Hall/CRC , edited by Magoules and Lai, 2011.

J. Geiser. Multiscale splitting for stochastic differential equations: Applications in particle collisions. Journal of Coupled Systems and Multiscale Dynamics, American Scientific Publishers, Valencia, CA, USA, 2013.

R.I. McLachlan and G.R.W. Quispel. Splitting methods. Acta Numerica, 341-434, 2002. 


\title{
Reaction-Diffusion Approach to Somite Formation
}

\author{
A. Lemarchand ${ }^{* 1,2}$ and C. Bianca ${ }^{1,2}$ \\ 1 Sorbonne Universités, UPMC Univ Paris 06, UMR 7600, \\ Laboratoire de Physique Théorique de la Matière Condensée, \\ 4, place Jussieu, case courrier 121, 75252 Paris cedex 05, France \\ 2 CNRS, UMR 7600 LPTMC, Paris, France
}

With the aim to propose minimal models of vertebrae formation, we present two kinds of reaction-diffusion models, the first one of clock-andwavefront type [1] and the second one of Turing type [2]. A correspondence between the species of the reaction schemes and biologically relevant molecules known as morphogens will be proposed and the robustness of the spatial structures to internal fluctuations will be examined. The ability of the model of Turing type to reproduce experiments involving grafting of morphogen sources or sinks into embryos will be shown [3].

\section{References}

[1] P. Dziekan, B. Nowakowski, and A. Lemarchand, Reaction-diffusion scheme for the clock and wavefront mechanism of pattern formation. Eur. Phys. J. B 87, 77, 2014.

[2] P. Dziekan, L. Signon, B. Nowakowski, and A. Lemarchand, Reactiondiffusion approach to prevertebrae formation: Effect of a local source of morphogen. J. Chem. Phys. 139, 114107, 2013.

[3] P. Dziekan, L. Signon, B. Nowakowski, and A. Lemarchand, Effect of a local source or sink of inhibitor on Turing patterns. Commun. Theor. Phys. 62, 622-630, 2014. 


\title{
Smoothed Particle Hydrodynamics and Model-Order Reduction for Efficient Modeling of Fluid-Structure Interaction
}

\author{
Markus Schörgenhumer* Alexander Humer *,** \\ Johannes Gerstmayr *** \\ * Linz Center of Mechatronics GmbH, Linz, Austria \\ (e-mail: markus.schoergenhumer@lcm.at) \\ ** Institute of Technical Mechanics, Johannes Kepler University, Linz, \\ Austria (e-mail: alexander.humer@jku.at) \\ *** Institute of Mechatronics, University of Innsbruck, Innsbruck, \\ Austria, (e-mail: johannes.gerstmayr@uibk.ac.at)
}

\begin{abstract}
Modeling the interaction of fluids with moving, flexible structures is a major and still very challenging subject in the field of multi-physics problems. In this work, an efficient computational approach based on the coupling of modally reduced flexible multibody systems with fluids modeled by means of smoothed particle hydrodynamics is outlined.
\end{abstract}

Keywords: flexible multibody dynamics, fluid-structure interaction, generalized component mode synthesis, smoothed particle hydrodynamics, simulator coupling

\section{INTRODUCTION}

The interaction of fluids with solid structures - briefly referred to as fluid-structure interaction (FSI) - can be observed on all length scales in nature, science, and engineering ranging from biomechanics of blood cells in micro-capillares to turbulent flow around flexible composite blades of high-power wind turbines.

FSI problems are multi-scale problems in several respects. Fluids typically require a much finer spatial resolution as compared to the solid domains and also the time scales differ in several orders within both domains.

In order to tackle the complexity of FSI problems, numerous computational approaches have already been applied, in particular, during the last decade, see Hou et al. (2012). The lack of methods that are equally versatile as efficient, however, still is a significant drawback of today's approach that is addressed by the ideas presented in what follows.

Subsequently, we present an overview of a partitioned approach to FSI, in which the mechanical subsystem is embedded in the framework of flexible multibody dynamics, while the fluid part is modeled by means of the "smoothed particle hydrodynamics" (SPH) method. The focus lies both on modal reduction in the context of coupled FSI simulations, and the underlying modeling of the interaction between the components of the multibody system and the fluid continuum represented by SPH particles, see Schörgenhumer et al. (2013, 2014).

\section{COUPLING MULTIBODY SYSTEMS AND FLUIDS}

In general, a multibody system is a mechanical system consisting of rigid and flexible bodies, joints and external forces. Especially for transient simulations of complex real- world applications with flexible components and a large number of degrees of freedom, techniques for model-order reduction need to be employed to increase efficiency or to allow transient simulations in the first place.

The idea of model-order reduction is to reduce the involved degrees of freedom by projecting a high-dimensional, say finite element, model onto a lower-dimensional approximation space that captures all important features with sufficient accuracy. In multibody dynamics, a component mode synthesis approach is frequently employed for this purpose. The idea is to use a selected number of mode shapes for the representation of the flexible deformation relative to the individual bodies' rigid body motion, which is represented by a floating frame of reference. In the present approach, however, we rely on a generalized modal basis that enables the interpolation of absolute displacements avoiding a relative description and, thus, the complex structure of the inertia terms, see Pechstein et al. (2013). The linear mapping between the full set of degrees of freedom $\boldsymbol{x}_{s}$ and the generalized coordinates $\boldsymbol{q}_{r}$,

$$
\boldsymbol{x}_{s}=\boldsymbol{x}_{r e f}+\mathbf{N} \boldsymbol{q}_{r}
$$

with constant reference positions $\boldsymbol{x}_{\text {ref }}$ and transformation matrix $\mathbf{N}$, is implemented within the parallelized fluid code of our co-simulation approach. Such approach allows the computationally demanding coupling terms to be efficiently evaluated in parallel since interface positions between solid and fluid domains are immediately accessible.

Boundary conditions and the interaction of a multibody system with its environment are realized by appropriate external forces and constraints. In particular, for the interaction with a viscous fluid, the following conditions must be satisfied on the surface $\Gamma$ of any body in contact with the fluid: The kinematic boundary condition, 


$$
\boldsymbol{v}_{s}=\boldsymbol{v}_{f} \text { on } \Gamma
$$

with the velocities of solid and fluid $\boldsymbol{v}_{s}$ and $\boldsymbol{v}_{f}$, respectively, and the equilibrium of forces,

$$
\boldsymbol{\sigma}_{s} \cdot \boldsymbol{n}=\boldsymbol{\sigma}_{f} \cdot \boldsymbol{n} \text { on } \Gamma
$$

where $\boldsymbol{\sigma}_{s}$ and $\boldsymbol{\sigma}_{f}$ denote the solid and fluid stress tensor, and $\boldsymbol{n}$ the outer surface normal.

Strictly speaking, Eqs. (2) and (3) must be satisfied at all times. Depending on the numerical implementation of the coupled problem, this may be actually enforced in each discrete time step (monolithic approaches, stronglycoupled partitioned schemes), or only hold approximately (weakly-coupled partitioned schemes). The latter is the case for our co-simulation-based coupling of the multibody dynamics with the fluid dynamics.

\section{SPH AND THE FSI CONDITIONS}

In contrast to the widely-used finite volume and finite element methods of fluid dynamics, the particle-based SPH method does not depend on a spatially fixed or moving mesh. In SPH, unordered SPH particles fill the role of the volume elements or nodes from these classical meshbased approaches, i.e., they are used for the discretization of the fluid continuum and the spatial interpolation of the field variables, see, e.g., Monaghan (2005) or Liu and Liu (2010). Owing to its meshless character the strengths of SPH lie in its versatility and simplicity, particularly, in case of problems with significantly changing fluid domains, e.g, free-surface problems and FSI applications.

The coupling conditions (2) and (3) are implemented in the sense of a penalty approach by the introduction of appropriate repulsive and viscous forces, $\boldsymbol{F}_{\text {rep }}$ and $\boldsymbol{F}_{v i s c}$, which act between the boundaries and any SPH particle in range. These FSI forces are computed numerically from convolution integrals of force densities $\boldsymbol{f}_{\text {rep }}\left(\boldsymbol{r}_{\text {rel }}\right)$ and $\boldsymbol{f}_{\text {visc }}\left(\boldsymbol{r}_{\text {rel }}, \boldsymbol{v}_{\text {rel }}\right)$ over any boundary or surface of a body in contact with the fluid, cf. Schörgenhumer et al. (2013),

$$
\begin{gathered}
\boldsymbol{F}_{r e p}=\int_{\Gamma} \boldsymbol{f}_{r e p}\left(\boldsymbol{r}_{r e l}\right) \mathrm{d} S, \\
\boldsymbol{F}_{v i s c}=\int_{\Gamma} \boldsymbol{f}_{v i s c}\left(\boldsymbol{r}_{r e l}, \boldsymbol{v}_{r e l}\right) \mathrm{d} S,
\end{gathered}
$$

with $\boldsymbol{r}_{\text {rel }}$ and $\boldsymbol{v}_{\text {rel }}$ denoting the relative position and velocity of the corresponding SPH particle and the local integration point (cf. Fig. 1).

Furthermore, we are currently developing an alternative approach based on a consistent extension of the SPH formulation at boundaries.

\section{EXAMPLES AND CONCLUSIONS}

One of the main features of our approach is its flexibility: Complex mechanical systems can be efficiently modeled within the framework of flexible multibody dynamics combined with modal reduction, while $\mathrm{SPH}$ is able to capture fluid dynamics in basically arbitrarily moving or changing domains, and, moreover, is well suited for parallelization.

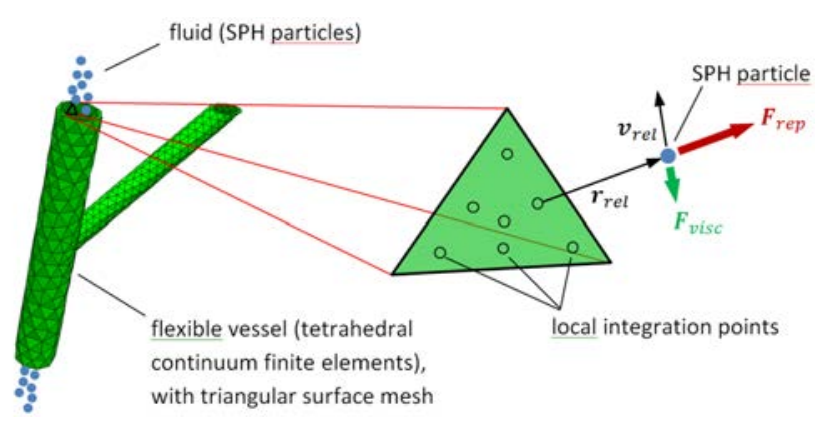

Fig. 1. Sketch of the FSI forces between a discretized surface and an SPH particle.

We have applied our approach to various examples and test problems. For illustration, Fig. 2 shows a screenshot of an impact simulation of a deformable rocket on a free water surface.

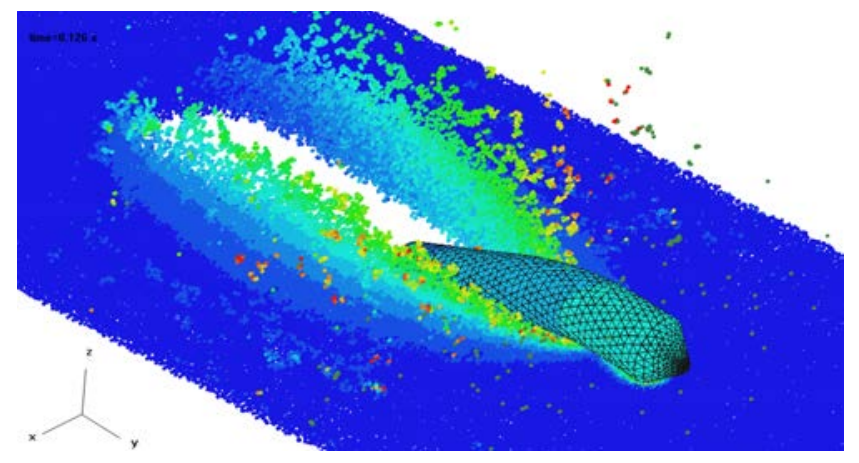

Fig. 2. Impact of a flexible rocket shell on a water surface. The particle colors correspond to the flow speed.

\section{ACKNOWLEDGEMENTS}

The authors have been supported by the Linz Center of Mechatronics (LCM) in the framework of the Austrian COMET-K2 program.

\section{REFERENCES}

Hou, G., Wang, J., and Layton, A. (2012). Numerical methods for fluid-structure interaction - a review. Commun. Comput. Phys., 12, 337-377.

Liu, M. and Liu, G. (2010). Smoothed particle hydrodynamics (SPH): an overview and recent developments. Arch. Comp. Meth. Eng., 17, 25-76.

Monaghan, J. (2005). Smoothed particle hydrodynamics. Rep. Prog. Phys., 68, 1703-1759.

Pechstein, A., Reischl, D., and Gerstmayr, J. (2013). A generalized component mode synthesis approach for flexible multibody systems with a constant mass matrix. J. Comput. Nonlinear Dyn., 8, 011019-011028.

Schörgenhumer, M., Gruber, P., and Gerstmayr, J. (2013). Interaction of flexible multibody systems with fluids analyzed by means of smoothed particle hydrodynamics. Multibody Syst. Dyn., 30, 53-76.

Schörgenhumer, M., Humer, A., and Gerstmayr, J. (2014). Efficient fluid-structure interaction based on modally reduced multibody systems and smoothed particle hydrodynamics. In E. Oñate, X. Oliver, and A. Huerta (eds.), Proc. 11th World Congress on Computational Mechanics (WCCM IX), 3080-3091. Barcelona, Spain. 


\title{
Modelling Approach for Mobile and Immobile Transport Problems with Multiple Time-Scales
}

\author{
Jürgen Geiser* \\ * Ruhr University of Bochum, Department of Electrical Engineering \\ and Information Technology, Universitätsstr. 150, D-44801 Bochum, \\ Germany (e-mail: juergen.geiser@ruhr-uni-bochum.de).
}

\section{INTRODUCTION}

We are motivated to apply a modelling approach for mobile and immobile transport problems. Such modelling problems are applied in porous media models, e.g., bioremediation, fluid-solid interactions, see Bear (1972). We apply a modelling approach after Coats et al. (1964), which allows to decouple mobile and immobile fluids in porous medium. Therefore, we can deal with different time-scales of the mobile parts (smooth scales) and immobile parts (rough scales). For such a decomposition, it is important to consider multiscale methods to resolve the various time-scales. For such methods, we propose fast iterative splitting schemes.

\section{MULTISCALE SPLITTING METHODS}

Splitting methods are important for decompositions into simpler equation parts, while each part can be solved more accurate and faster as the original equation, see Geiser (2011).

In our case, the modelling equations consists of a system of convection-diffusion-reaction equations. We apply spatial discretization methods to the spatial operators, for example with Finite Volume methods (see Eymard et al. (2000)) and obtain a system of ordinary differential equations:

$$
\frac{\partial U(t)}{\partial t}=A U(t)+\epsilon B U(t), \text { with } U\left(t^{n}\right)=U^{n},
$$

where $U^{n}=(u, g)^{t}=\left(u_{1}, \ldots, u_{m}, g_{1}, \ldots, g_{m}\right)^{t}$ is the vector of the mobile and immobile solutions. Furthermore, the small scale is given by $\epsilon=\beta$. Now, we have $A$ as an operator for the smooth time-scale of the mobile-immobile part and $B$ as an operator for the rough time-scale of between the mobile and immobile parts.

The method of multiple scales is applied and we obtain a multiscale solutions $U(t, \epsilon)$, which is given as:

$$
U(t, \epsilon)=U_{0}(t)+\epsilon U_{1}(t)+\epsilon^{2} U_{2}(t)+\ldots+\epsilon^{J} U_{J}(t),
$$

with the initial conditions $U(0, \epsilon)=U(0)$, and where $J \in \mathbb{N}^{+}$is a fixed number of iterations.

We gain an underlying hierarchical equation system, which is derived as:

$$
\frac{\partial U_{0}(t)}{\partial t}=A U_{0}(t)
$$

$$
\begin{aligned}
\frac{\partial U_{1}(t)}{\partial t} & =A U_{1}(t)+B U_{0}(t), \\
& \vdots \\
\frac{\partial U_{I}(t)}{\partial t} & =A U_{J}(t)+B U_{J-1},
\end{aligned}
$$

where the initial conditions are $U_{0}(0)=U(0)$ and $U_{j}(0)=$ $0, \forall j=1, \ldots, J$.

Such an hierarchical equation system is equal to an effective so called iterative operator splitting method, see Geiser (2008). Therefore, we could solve our multiple time-scale approach of a mobile and immobile transport problem with an effective and fast iterative scheme. Such a direct application to fast iterative schemes allows to embed multiscales and also has the benefit of fast splitting algorithms, see Geiser (2011).

\section{CONCLUSION}

In the numerical experiments, we apply the fast iterative splitting scheme to coupled transport-reaction problems with multiple scales. The results present the benefit of such fast splitting approaches and we obtained accurate solutions of the full problem. In the future, such splitting approaches allow a direct combination of multiple-time scales and iterative splitting schemes, which are important for solving large systems of convection-diffusion-reaction equations.

\section{REFERENCES}

J. Bear. Dynamics of Fluids in Porous Media. American Elsevier, New York, 1972.

K.H. Coats and B.D. Smith. Dead-end pore volume and dispersion in porous media. Society of Petroleum Engineers Journal 4(3):73-84, 1964.

R. Eymard, T.R. Gallouet, and R. Herbin. The finite volume method. Handbook of Numerical Analysis, Vol. VII, 713-1020, edited by P.G. Ciarlet and J.L. Lions, 2000.

J. Geiser. Iterative operator-splitting methods with higher order time-integration methods and applications for parabolic partial differential equations. Journal of Computational and Applied Mathematics 217:227-242, 2008.

J. Geiser. Iterative Splitting Methods for Differential Equations. Numerical Analysis and Scientific Computing Series, CRC Press, Chapman \& Hall/CRC , edited by Magoules and Lai, 2011. 


\title{
Multiscale Analysis of a Retarded Equation: From Kinetic to Macroscopic Scale
}

\author{
Carlo ianca ??, Annie Lemarchand??
}

* Sorbonne Universités, UPMC Univ Paris 06, UMR 7600, Laboratoire de Physique Théorique de la Matière Condensée, 4, place Jussieu, case courrier 121, 75252 Paris Cedex 05, France. CNRS, UMR 7600 LPTMC, Paris, France

(e-mail: bianca@lptmc.jussieu.fr, e-mail: anle@lptmc.jussieu.fr).

\begin{abstract}
This paper is devoted to the problem of linking the dynamics at kinetic scale, described by a retarded thermostatted kinetic equation, with the dynamics at macroscopic scale. Specifically the macroscopic equation is derived by considering a scaling parameter into the underlying retarded equation and considering the related convergence when the scaling parameter goes to zero. The results show the onset of diffusion at macroscopic scale.
\end{abstract}

Keywords: Diffusion, Thermostats, Nonlinearity, Scaling, Macroscopic equation 


\title{
Extended Discrete Element Method (XDEM) as an Efficient Algorithm for Applications in Multi-physics
}

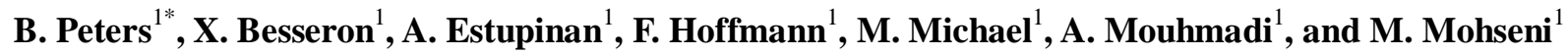 \\ ${ }^{1}$ University of Luxembourg (Faculty of Science, Technology and Computer Science, Luxembourg, Luxembourg) \\ *bernhard.peters@uni.lu
}

\begin{abstract}
A large number of engineering applications involve granular material or a particulate phase in combination with a gaseous or liquid phase. Predominant applications are as diverse as pharmaceutical industry e.g. drug production, agriculture food and processing industry, mining, construction and agricultural machinery, metals manufacturing, energy production and systems biology. In order to predict and analyze relevant phenomena accurately, tailored algorithms that resolve dominant length scales are required. A novel technique referred to as Extended Discrete Element Method (XDEM) has emerged only recently that offers a significant advancement for multi-physics applications. It is based on a coupled discrete and continuous i.e. Lagrange-Euler numerical simulation concept. XDEM treats the solid phase representing the particles and the fluid phase or a structure as two distinguished phases that are coupled through heat, mass and momentum transfer. An outstanding feature of the numerical concept is that each particle is treated as an individual entity that is described by its thermodynamic state e.g. temperature and reaction progress and its position and orientation in time and space. The thermodynamic state includes one-dimensional and transient distributions of temperature and species within the particle and therefore, allows a detailed and accurate characterization of the reaction progress. Thus, the proposed methodology provides a high degree of resolution ranging from scales within a particle to the continuum phase as global dimensions. These superior features as compared to traditional and pure continuum mechanics approaches are applied to predict drying of wood particles in a packed bed and impact of particles on a membrane as shown in fig. 1 .
\end{abstract}
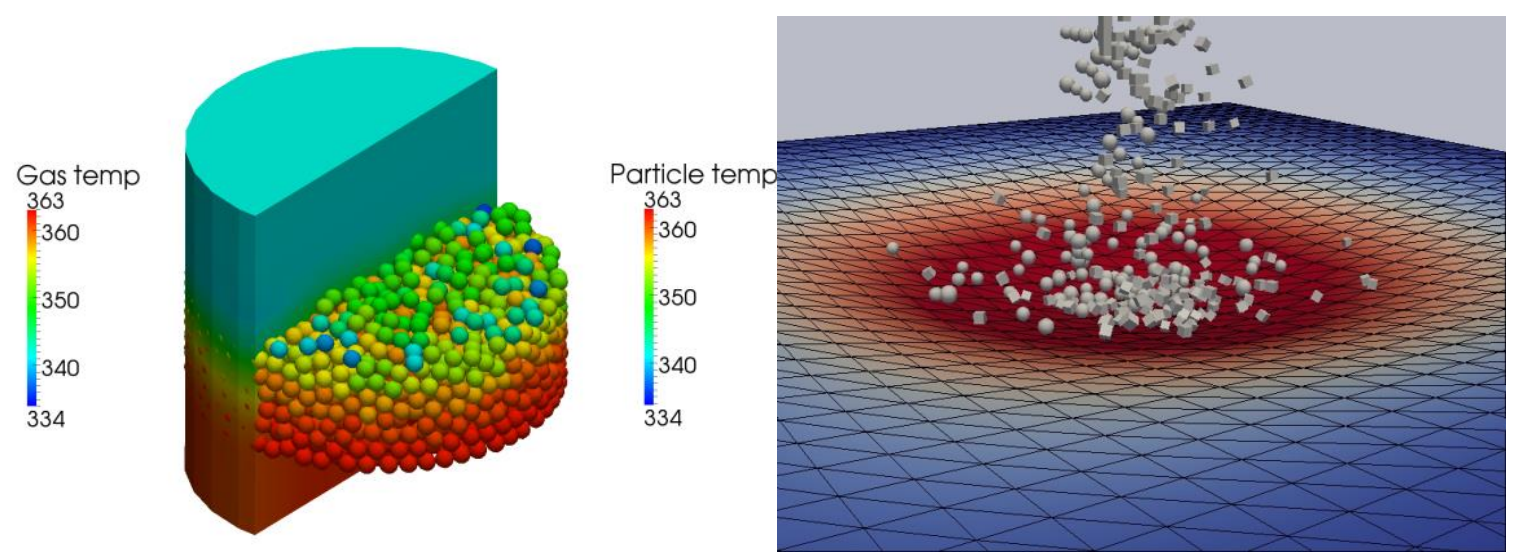

Figure 1: Particle resolved properties and air temperature during drying in a packed bed reactor and deformation of a membrane due to impacting particles

Fig. 1 shows the rather inhomogeneous drying process in the upper part of the reactor with higher temperatures around the circumference of the inner reactor wall. The latter is due to increased porosity in conjunction with higher mass flow rates than in the center of the reactor, and thus, augmented heat transfer. A further application in fig. 1 depicts the deformation of a surface under the impact of particles discharged from a hopper. Due to an increasing vertical deformation particles roll or slide down toward the bottom of the recess, where they are collected in a heap. Hence, the Extended Discrete Element Method offers a high degree of resolution avoiding further empirical correlations and extends the knowledge into the underlying physics.

Keywords: Discrete Element Method (DEM), Computational Fluid Dynamics (CFD), Finite Element Analysis (FEA), High Performance Computing (HPC) 
MATHMOD 2015 Abstract Volume 


\title{
MATHMOD 2015 ABSTRACT VOLUME
}

\author{
MATHMOD Minisymposium \\ Engineering Applications of Fractional Derivatives
}


MATHMOD 2015 Abstract Volume 


\title{
Four ways to interpret temporal memory operators in the wave equation
}

\author{
Sverre Holm \\ Department of Informatics, University of Oslo \\ (e-mail: sverre (a) ifi.uio.no).
}

\begin{abstract}
Arbitrary power law attenuation and dispersion in medical ultrasound and elastography can be modeled with temporal memory operators in the wave equation. Here four alternative interpretations are given. Power laws for attenuation with exponents other than 2 correspond to the use of convolution operators expressible as fractional derivatives. The corresponding constitutive equation can be verified by time domain measurements. A framework with multiple relaxation processes can be formulated via the complex compressibility. The constitutive equation can also be expressed with higher order derivatives.
\end{abstract}

Keywords: Wave equation, attenuation, dispersion, power law, fractional derivative.

\section{INTRODUCTION}

The viscous wave equation

$$
\nabla^{2} u-\frac{1}{c_{0}^{2}} \frac{\partial^{2} u}{\partial t^{2}}+\tau_{\sigma} \frac{\partial}{\partial t} \nabla^{2} u=0
$$

where $u(x, t)$ is compressional or shear displacement and $c_{0}$ is the zero frequency phase velocity given by the elasticity and the density, $c_{0}^{2}=E_{e} / \rho$. It results in a power law attenuation $\alpha_{k}(\omega)=\alpha_{0} \omega^{2}$ and a constant phase velocity for $\omega \tau_{\sigma} \ll 1$ in a homogeneous and isotropic medium. For high relative frequencies both the attenuation and the phase velocity are $\propto \omega^{0.5}$. This does not fit well with measurements of compressional waves in medical ultrasound at $\mathrm{MHz}$ frequencies. Neither does it describe all shear wave measurements in elastography (10-1000 Hz) (Holm and Sinkus (2010); Holm and Näsholm (2014)).

Eq. (1) can be derived from a viscous constitutive equation:

$$
\sigma(t)=E_{e}\left[\varepsilon(t)+\tau_{\sigma} \frac{\partial \varepsilon(t)}{\partial t}\right]
$$

where $\varepsilon(t)$ is strain, $\sigma(t)$ is stress, and $\tau_{\sigma}=\eta / E_{e}$ is the ratio of viscosity and elasticity. Let the compressibility be $\kappa_{0}=1 / E_{e}$, then the complex compressibility in the frequency domain is:

$$
\kappa(\omega)=\varepsilon(\omega) / \sigma(\omega)=\frac{\kappa_{0}}{1+i \omega \tau_{\sigma}}=\kappa_{0}-i \omega \frac{\tau_{s} \kappa_{0}}{1+i \omega \tau_{\sigma}} .
$$

These derivations are more fully found in Holm et al. (2013).

Here we will generalize these equations to fit better to medical ultrasound and elastography. However, the acceptance of in particular fractional models has been slow in these fields, often due to a weak link to the physics. Therefore alternative formulations will be given. The paper starts with the frequency domain with more general power laws and then moves to time and more general constitutive equations than Eq. (2). An alternative formulation can be given via more general expressions for the compressibility than Eq. (3), and finally via a formulation in terms of constitutive equations with higher order derivatives.

\section{FREQUENCY DOMAIN: POWER LAW ATTENUATION}

A wave equation with a convolution loss operator is the most versatile formulation (Mainardi (2010), Sect. 4.2.2):

$$
\nabla^{2} u-\frac{1}{c_{0}^{2}} \frac{\partial^{2} u}{\partial t^{2}}+\Phi(t) * \nabla^{2} u=0,
$$

where $\Phi(t)=[d G(t) / d t] / G_{g}$ is the rate of relaxation, $G(t)$ is the relaxation modulus, $G_{g}=G\left(0^{+}\right)$is the glass modulus, and $E_{e}=G(\infty)$ is the equilibrium modulus. One particular form of the convolution operator is a power law memory in time. The operator is given by an order parameter $m-1<\alpha<m$ where $\mathrm{m}$ is the smallest integer larger than the order:

$$
\Phi(t) * f(t)=\tau_{\sigma}^{\alpha} \frac{d^{m} f(t)}{d t^{m}} * \frac{1}{\Gamma(m-\alpha) t^{\alpha+1-m}} .
$$

This operator is of particular interest since its Fourier transform is a power law also (Podlubny (1999), sect. 2.9):

$$
\mathscr{F}\left(\frac{1}{\Gamma(m-\alpha) t^{\alpha+1-m}}\right)=(i \omega)^{\alpha-m} .
$$

This ensures that the solution has power law attenuation with an exponent in the most interesting range $(0,2]$. Due to the similarity between this Fourier transform and that of the integer order derivative, the time domain operator can also be written as a non-integer order or fractional derivative:

$$
\Phi(t) * f(t)=\tau_{\sigma}^{\alpha} \frac{\partial^{\alpha} f(t)}{\partial t^{\alpha}} .
$$

The viscous wave equation, Eq. (1) is a special case with $\alpha=1$.

The power laws motivate their inclusion in Szabo (2014), ch. 4. Similar models based on the fractional Laplacian are also used in the k-Wave MATLAB toolbox (Treeby and Cox (2014)).

\section{TIME DOMAIN: CONSTITUTIVE EQUATION}

The constitutive stress-strain relation corresponding to this generalized wave equation is the fractional Kelvin-Voigt equation:

$$
\sigma(t)=E_{e} \varepsilon(t)+G(t) * \frac{\partial \varepsilon(t)}{\partial t}=E_{e}\left[\varepsilon(t)+\tau_{\sigma}^{\alpha} \frac{\partial^{\alpha} \varepsilon(t)}{\partial t^{\alpha}}\right]
$$

This model and its generalization, the fractional Zener model with fractional derivatives on both sides of the equal sign, fit well for biological materials such as brain, human root dentin, cranial bone, liver, arteries, breast, and hamstring muscle. They also fit other materials such as metals, doped corning glass, 
rubber, and polymers (see Näsholm and Holm (2013) and references therein). Most of these references apply to low frequency rheology, i.e. shear waves.

\section{A DISTRIBUTION OF RELAXATION PROCESSES}

Acoustic media such as salt water and air can be accurately described by a viscous part plus two physical relaxation processes (Salt water: boric acid $\left(\mathrm{B}(\mathrm{OH})_{3}\right)$ and magnesium sulfate $\left(\mathrm{MgSO}_{4}\right)$. Air: nitrogen $\left(\mathrm{N}_{2}\right)$ and oxygen $\left(\mathrm{O}_{2}\right)$ ). Similar models of low order can be used to model arbitrary power law attenuation for more complex media over a limited frequency range (Tabei et al. (2003)). In that case, the parameters of the model are not taken from physical processes, but rather selected to minimize a model fit error over the desired frequency range.

In general an $N$ 'th order relaxation process consists of $N$ springdamper terms, each one with relaxation time $\tau_{v}=\eta_{v} / E_{v}$. This is the Maxwell-Wiechert model of Fig. (1) which can be expressed as a sum of compressibilities like that of Eq. (3):

$$
\kappa(\omega)=\kappa_{0}-i \omega \sum_{v=1}^{N} \frac{\kappa_{v} \tau_{v}}{1+i \omega \tau_{v}},
$$

where the compressibilities are $\kappa_{v}=1 / E_{v}$.

The fractional Zener model can be interpreted as a distribution of an infinite number of relaxation models, Mainardi (1994). That means that $N \rightarrow \infty$ in Eq. (9) and the sum can be turned into an integral. The particular weighting, $\kappa_{v}\left(\Omega_{v}\right), \Omega_{v}=1 / \tau_{v}$, of each relaxation process follows a Mittag-Leffler function as a function of relaxation frequency, $\Omega$. This function has a power law tail and thus indicates fractal properties in the medium, Näsholm and Holm (2011). These properties can also be used for optimum selection of a few number of relaxation processes when a power law is to be approximated over a limited frequency range, Näsholm (2013).

\section{HIGHER ORDER DERIVATIVES}

The Maxwell-Wiechert model of Fig. (1) is also equivalent to a linear differential equation of order N (Tschoegl (1989), sect. 3.7, Mainardi (2010), Sect. 2.4)):

$$
\sum_{n=0}^{N} p_{n} \frac{\partial^{n} \sigma(t)}{\partial t^{n}}=\sum_{n=0}^{N} q_{n} \frac{\partial^{n} \varepsilon(t)}{\partial t^{n}}
$$

This is an alternative to Eq. (8) for a generalization of Eq. (2).

When $N \rightarrow \infty$ and the coefficients are chosen using similar reasoning as in the previous section this constitutive equation

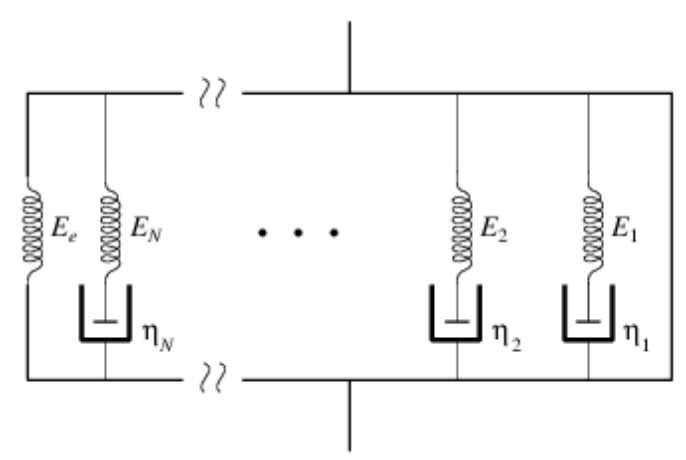

Fig. 1. Maxwell-Wiechert model (Näsholm and Holm (2013)). can describe a fractional one like Eq. (8). Higher order derivatives are conceptually simple and may offer a more intutive explanation than one using fractional derivatives.

However the advantage of the fractional description is parsimony, i.e. a minimal number of parameters. As an example Eq. (8) requires only $E_{e}, \tau_{\sigma}$ and $\alpha$ while Eq. (10) in principle requires an infinite number of parameters. In practice this number can probably be truncated, but nevertheless it will exceed three.

A fractional derivative expressed with higher order derivatives can also be found in Oldham and Spanier (1974), sect. 3.5, from a mathematical point of view.

\section{CONCLUSION}

One way to model arbitrary power law attenuation is via temporal memory operators. In order to further the understanding of this point of view, four interpretations have been given here with the hope of contributing to an increase of the acceptance of these models in applied fields.

\section{REFERENCES}

Holm, S., Näsholm, S.P., Prieur, F., and Sinkus, R. (2013). Deriving fractional acoustic wave equations from mechanical and thermal constitutive equations. Comput. Math. Appl., 66(5), 621-629.

Holm, S. and Sinkus, R. (2010). A unifying fractional wave equation for compressional and shear waves. J. Acoust. Soc. Am., 127, 542-548.

Holm, S. and Näsholm, S.P. (2014). Comparison of fractional wave equations for power law attenuation in ultrasound and elastography. Ultrasound. Med. Biol., 40(4), 695-703.

Mainardi, F. (1994). Fractional relaxation in anelastic solids. Journal of alloys and compounds, 211, 534-538.

Mainardi, F. (2010). Fractional Calculus and Waves in Linear Viscoelesticity: An Introduction to Mathematical Models, 1347. Imperial College Press, London, UK.

Näsholm, S.P. (2013). Model-based discrete relaxation process representation of band-limited power-law attenuation. $J$. Acoust. Soc. Am., 133(3), 1742-1750.

Näsholm, S.P. and Holm, S. (2011). Linking multiple relaxation, power-law attenuation, and fractional wave equations. J. Acoust. Soc. Am., 130(5), 3038-3045.

Näsholm, S.P. and Holm, S. (2013). On a fractional Zener elastic wave equation. Fractional Calculus and Applied Analysis, 16, 26-50.

Oldham, K.B. and Spanier, J. (1974). The Fractional Calculus, vol. 111 of Mathematics in science and engineering. Academic Press, New York, London.

Podlubny, I. (1999). Fractional differential equations. Academic Press, New York.

Szabo, T.L. (2014). Diagnostic Ultrasound Imaging: Inside Out, Second Ed. Academic Press.

Tabei, M., Mast, T.D., and Waag, R.C. (2003). Simulation of ultrasonic focus aberration and correction through human tissue. J. Acoust. Soc. Am., 113(2), 1166-1176.

Treeby, B.E. and Cox, B. (2014). Modeling power law absorption and dispersion in viscoelastic solids using a split-field and the fractional Laplacian. J. Acoust. Soc. Am., 136(4), 1499-1510.

Tschoegl, N.W. (1989). The phenomenological theory of linear viscoelastic behavior: an introduction. Springer-Verlag Berlin. 


\title{
Recent Results in Fractional-Order Modeling in Multi-Agent Systems and Linear Friction Welding *
}

\author{
Bill Goodwine and Kevin Leyden* \\ * Department of Aerospace 83 Mechanical Engineering, University of \\ Notre Dame, Notre Dame, IN 46556 USA (e-mail: \\ bill@controls.ame.nd.edu and kleyden@nd.edu).
}

\begin{abstract}
This abstract outlines recent results in two new applications for fractional-order modeling of engineering systems. First, fractional-order dynamics present in two types of multiagent systems are discussed; namely, a specific tree graph structure for a robotic formation control problem and fractional-order dynamics of a scale-free network of agents. Second, fractional-order dynamics present in modeling of the linear friction welding process are discussed.
\end{abstract}

Keywords: Fractional-order dynamics, multi-agent systems, manufacturing

\section{INTRODUCTION}

This abstract outlines results in fractional-order modeling for networked Cyber-Physical Systems (CPS) and Linear Friction Welding (LFW) in manufacturing which are new applications for fractional-order modeling. For distinct types of linear systems with integer-order component dynamics, the interaction among the components leads to fractional-order dynamics. Recognizing this fractionalorder nature of the dynamics is important because: 1) it leads to a deeper understanding of the system and broadens the "toolbox" of control possibilities for multi-robot systems; 2) it provides for substantial model reduction and computational savings for modeling and controlling the system; and, 3) when considering loop shaping, large frequency ranges characterized by non-integer order dynamics (non-integer magnitude slopes and non-multiple of $90^{\circ}$ phases) may need to be addressed by fractional-order control methods.

Fractional calculus has a long history which dates back to near the foundations of calculus, and it has been used in engineering applications for at least several decades. Books on the subject include Baleanu et al. (2011); Ortigueira (2011) and review articles include Ortigueira (2008); Machado et al. (2011). Other related studies include Silva et al. (2004) (walking robots), Delavari et al. (2013); Zhao et al. (2005) (flexible manipulators), Chen and Moore (2002) (time delays) and control using fractional-order PID control Zhao et al. (2005); Monje et al. (2008). Studies in other areas such as visco-elastic phenomena can be found in Heymans and Bauwens (1994); Mayes (2012).

\section{MULTI-AGENT SYSTEMS}

Consider the two systems illustrated in Figures 1 and 2 . For the first system, based on a self-similarity analysis, if there are an infinite number of generations, it is easy to

* The support of the US National Science Foundation under the CPS Large Grant No. CNS-1035655 is gratefully acknowledged.

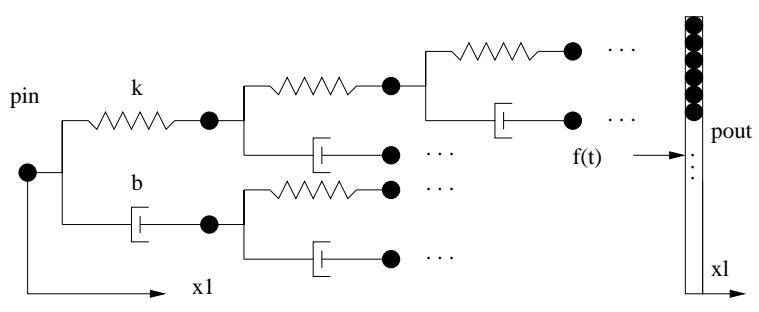

Fig. 1. Structure of robotic formation.

show that the transfer function relating the difference in applied force at the input and output levels is given by

$$
\frac{X_{1}(s)-X_{\text {last }}(s)}{F(s)}=\left(\frac{1}{\sqrt{k b}}\right) \frac{1}{\sqrt{s}} .
$$

Simulations show that this is a good approximation even for a relatively small number of generations, such as four or five generations.

Motivated by self-similarity leading to fractional-order dynamics for the tree structure, because scale free networks are also characterized by self-similarity, we have investigated whether fractional-order effects are present in such networks. For the specific scale-free network illustrated in Figure 2, if the blue node is displaced by a unit step, a graph of the response of all the other nodes is illustrated in Figure 3. In that plot, the thicker blue line represents the unit step response for the equation

$$
\frac{d^{\frac{4}{5}} x}{d t^{\frac{4}{5}}}(t)+\frac{3}{40} x(t)=\frac{3}{40} .
$$

Clearly, most of the other nodes respond in a fractional manner due to the change in the input.

\section{LINEAR FRICTION WELDING}

Friction welding is a process for joining two pieces of metal where the heat for the process is generated by friction caused by the parts rubbing together rather than 


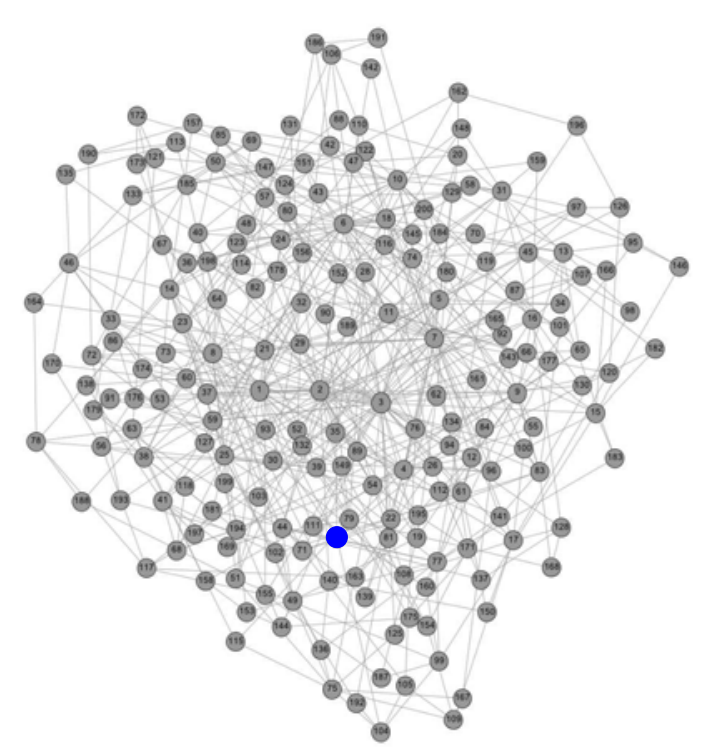

Fig. 2. Scale-free network.

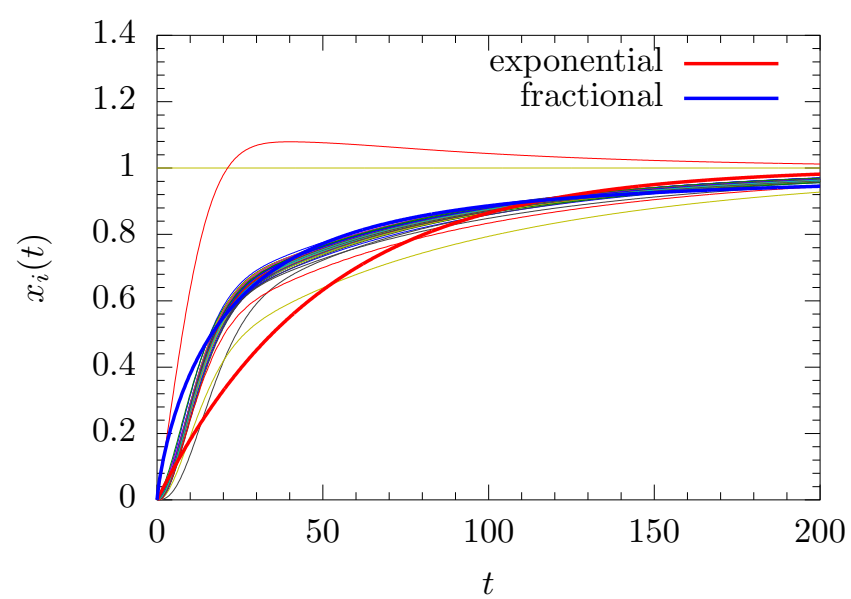

Fig. 3. Step response of scale free network.

by an external source as in traditional welding. Most friction welding is rotary, where parts rotating relative to each other are brought into contact; this requires axisymmetric parts. In contrast, in linear friction welding (LFW), the parts are brought into contact and oscillated. A complication inherent to LFW is a need for controlling actuators that, in the absence of model-based control, must be very large. This creates a cost barrier; at present, LFW is only commercially deployed for the titanium stages in jet engine compressors. Model-based control algorithms will make smaller LFW machines possible, reducing cost and enabling other industries to use the process.

Fractional-order modeling is suitable in developing such algorithms for physical reasons; at first contact between the parts, the force resulting from the relative motion is codominated by Coulomb friction and elastic effects. As the parts are heated, this force transitions to a more viscouslike effect. In other words, there is a transition from zerothorder forces to first-order forces dominating the process. The following discussion of system identification results from LFW experimental data shows that the process can be modeled effectively by fractional-order equations.
The data contain the input oscillation force, $u(t)$, and output position of the welded part, $x(t)$, both sinusoids. The median absolute value of the extrema in each plot is considered the amplitude; the median phase difference between output and input is taken as a constant, $\phi$.

Accounting for inertia, the fractional-order model is

$$
m \frac{d^{2} x}{d t^{2}}(t)+b \frac{d^{q} x}{d t^{q}}(t)=u(t)
$$

with $0 \leq q<2$ and $m, b, q$ real. In steady state, the fractional derivative of $x(t)=A \cos (\omega t+\phi)$ is given by

$$
\frac{d^{q} x}{d t^{q}}(t)=A \omega^{q} \cos \left(\omega t+\phi+\frac{\pi}{2} q\right)
$$

which, along with the second derivative of $x(t)$ and $u(t)=$ $F \cos (\omega t)$, are substituted into Equation 2. For any choice of $q$, matching amplitude and phase on both sides of Equation 2 yields $m$ and $b$. If $m$ and $b$ share sign, the equation is physically plausible. This is the case for 10 welds of varied process parameters when $0<q \leq 0.8$. Therefore, this model is a viable candidate for LFW because it offers governing equations that imply a stable process and follow from the data.

\section{REFERENCES}

Baleanu, D., Machado, J.A.T., and Luo, A.C.J. (2011). Fractional Dynamics and Control. Springer Publishing Company, Incorporated.

Chen, Y. and Moore, K.L. (2002). Analytical stability bound for a class of delayed fractional-order dynamic systems. Nonlinear Dynamics, 29(1-4), 191-200.

Delavari, H., Lanusse, P., and Sabatier, J. (2013). Fractional order controller design for a flexible link manipulator robot. Asian Journal of Control, 15, 783795.

Heymans, N. and Bauwens, J. (1994). Fractal rheological models and fractional differential equations for viscoelastic behavior. Rheologica Acta, 33, 210219.

Machado, J.T., Kiryakova, V., and Mainardi, F. (2011). Recent history of fractional calculus. Communications in Nonlinear Science and Numerical Simulation, 16(3), $1140-1153$.

Mayes, J. (2012). Reduction and Approximation in Large and Infinite Potential-Driven Flow Networks. Ph.D. thesis, University of Notre Dame.

Monje, C.A., Vinagre, B.M., Feliu, V., and Chen, Y. (2008). Tuning and auto-tuning of fractional order controllers for industry applications. Control Engineering Practice, 16(7), $798-812$.

Ortigueira, M.D. (2011). Fractional Calculus for Scientists and Engineers, volume 84 of Lecture Notes in Electrical Engineering. Springer.

Ortigueira, M. (2008). An introduction to the fractional continuous-time linear systems: the 21 st century systems. Circuits and Systems Magazine, IEEE, 8(3), 1926. doi:10.1109/MCAS.2008.928419.

Silva, M.F., Machado, J.T., and Lopes, A. (2004). Fractional order control of a hexapod robot. Nonlinear Dynamics, 38(1-4), 417-433.

Zhao, C., Xue, D., and Chen, Y. (2005). A fractional order pid tuning algorithm for a class of fractional order plants. In Proceedings of the IEEE International Conference on Mechatronics \& Automation. 


\title{
Fractional-Order Modeling of High-Pressure Fluid-Dynamic Flows: An Automotive Application
}

\author{
Paolo Lino* Guido Maione* Fabrizio Saponaro* \\ * Dipartimento di Ingegneria Elettrica e dell'Informazione, \\ Politecnico di Bari, Bari, 70125 Italy \\ (e-mail: \{paolo.lino,guido.maione\}@poliba.it, saponaro.fab@gmail.com)
}

\section{INTRODUCTION}

Efficiency, pollutant emissions, and consumptions of diesel engines are affected by the metering of the air/fuel mixture. Thus, automotive industry advanced the common rail (CR) technology for a better accuracy in fuel metering. In this context, new electro-injectors improved injection. However, an accurate model is necessary for the nonlinear fuel dynamics and for the mechanical deformation of relevant parts of injectors. The model should allow us to identify faults, analyze different configurations, but also an easy implementation of control algorithms. To this aim, a new mathematical model of the CR diesel electroinjector is proposed. First, the CR injection system and its main components are described. Then the model employs a fractional-order representation of the propagation of fuel pressure wave in the $\mathrm{CR}$ and pipes of diesel injection systems. Simulation validates the model by comparison with real data and results given by an integer-order model.

\section{FRACTIONAL-ORDER MODEL OF THE ELECTRO-IN ECTOR}

A CR diesel injection system includes a high pressure pump that sends fuel to the CR volume, which is directly connected to the electro-injectors. These elements control the amount of fuel injected into the combustion chamber and shape the flow rate, provided that an electronic controlled solenoid valve regulates the CR pressure. An electro-injector consists of a control circuit and a feeding circuit. The first includes a control chamber, whose pressure is changed by an electro-hydraulic valve. The feeding circuit ends the flow path, where the injector nozzles are located. The two circuits are connected by an annular pipe. A plunger-needle element lies between the circuits to regulate the injection flow. The plunger-needle lift depends on the difference between pressures in the control chamber and in the feeding circuit. Then the injector is opened (closed) by properly reducing (increasing) the first pressure by means of the electro-hydraulic valve.

Modeling is done by dividing the injector into different volumes. The continuity equation, the momentum equation, and the Newton's second law of motion are applied for each subsystem, resulting in lumped or distributed parameters representations. A uniform, time-varying, pressure distribution is assumed in large accumulation volumes, for which a lumped parameters model is obtained. The considered laws provide ODEs to describe the fuel pressure dynamics inside each volume. The instantaneous flow sections and volumes depend on the axial displacement of moving parts. Moreover, the plunger-needle is modeled as a series of mass-spring-damper systems.

Instead, a time-varying and nonuniform pressure distribution in a volume yields PDEs to describe pressure wave propagation. In details, a fractional-order model for the main propagation of pressure wave in the annular pipe is deduced as follows. Propagation in high-pressure pipes is expressed by the Navier-Stokes (N-S) equations, i.e. nonlinear PDEs. A linearization of the continuity and momentum equations for the flow of fluids is extrapolated from the N-S equations. Simple arrangements and substitution of the integer-order by the fractional-order time derivative lead to the fractional-order model:

$$
\frac{\partial^{2 \nu} P}{\partial t^{2 \nu}}=c^{2} \frac{\partial^{2} P}{\partial x^{2}}+\frac{R}{\rho} \frac{\partial^{\nu}}{\partial t^{\nu}}\left(\frac{\partial^{2} P}{\partial x^{2}}\right)
$$

where $P$ and $\rho$ are the fuel pressure and density, $c$ is the sound speed in the fluid along direction $x, R$ considers the fuel kinematic viscosity, and $\nu$ is the fractional order. Moreover, the annular pipe is spatially discretized into $n$ elements: the instantaneous pressure $P_{i}$ in the $i$-element depends on $P_{i+1}$ and $P_{i-1}$. Laplace transform leads to:

$$
\begin{aligned}
P_{i}(s) & =\frac{s^{\nu} R+\rho c^{2}}{s^{2 \nu} \rho x^{2}+s^{\nu} 2 R+2 \rho c^{2}} P_{i+1}(s)+P_{i-1}(s) \\
& =H(s) P_{i+1}(s)+P_{i-1}(s) .
\end{aligned}
$$

The complete fractional-order transfer function of the whole pipe is obtained by a recursive procedure. For $n=6$

$$
P_{6}=\frac{\left(1-4 H^{2}+3 H^{4}\right) H P_{A V}}{1-5 H^{2}+6 H^{4}-H^{6}}+\frac{H^{6} P_{C R}}{1-5 H^{2}+6 H^{4}-H^{6}} .
$$

The operators $s^{\nu}$ and $s^{2 \nu}$ are approximated by rational transfer functions. The nonlinear model of the injector is implemented in the Matlab/Simulink environment. Simulation results are compared with experimental data from a real injector: they show a good match and the improvement by the fractional-order model is clear.

\section{CONCLUSION}

The fractional-order model of electro-injectors for CR diesel engines well predicts the injected flow rate in several working conditions. The model is suitable for optimizing the injector configuration and parameters and for deriving better fuel rate shaping strategies. 


\title{
Optimized Identification Method for Fractional Heating Processes on Heterogeneous Materials
}

\author{
Riccardo Caponetto*, Francesca Sapuppo* \\ Vincenzo Tomasello* \\ *Dipartimento di Ingegneria Elettrica, Elettronica e dei Sistemi, Università degli Studi di Catania \\ Catania, Italy ( e-mail: riccardo.caponetto@dieei.unict.it)
}

\begin{abstract}
An enhanced model for the heating process on heterogeneous materials based on fractional order PDEs has been identified through an optimization algorithm. Experimentation has been carried out on a finite length beam filled with a composite material in order to obtain a model in the frequency domain. The obtained results confirm the fractional nature of the heating processes when diffusion occurs in not continuous composite materials.
\end{abstract}

Keywords: Fractional order systems, modeling, optimization, heat transfer, composite materials.

\section{INTRODUCTION}

The association between the heterogeneous materials and Fractional Order Systems is an actual research issue involving investigation at the micro- and macro-scale (Caponetto et al.). This research follows the increasing interest for heterogeneous and composite materials. They are becoming more common in a wide range of functional devices, particularly those involving energy transport, conversion, and storage (Srivastava et al.). Modeling and controlling such properties becomes therefore crucial for the optimal materials design. In particular the fractional order nature of heat transfer model in heterogeneous, porous, or composite materials (Sierociuk, D.et al.) (Kulish et al.) is the main focus of this work in which the advantages of optimization methods for modeling heat transfer phenomena on non-homogeneous beam heating process have been presented.

\section{FRACTIONAL MODEL}

Non-homogeneous beam heating is associated to the effect of anomalous diffusion, due to part of the heat flux dispersed into the neighbouring environment (Sierociuk et al.). The diffusion heat equation applied to a semi-infinite bar is described in (1) in the time domain. Considering the initial condition in (2), the boundary conditions in (3) and (4), and the Laplace transform it is possible to obtain the transfer function $G(x, s)$ in (5) (Sierociuk et al.). $H$ is the heat flux, $T$ the temperature, $\lambda$ is a normalized distance from the heat source.

$\frac{\partial T(x, t)}{\partial t}=a^{2} \frac{\partial^{2} T(x, t)}{\partial x^{2}}$

$T(x, t=0)=0$ temperature along the tube at $t=0(2)$

$H(x=0, t)=-K \frac{\partial T}{\partial x}($ at $x=0)$, heat flux at $x=0$ (3)

$T(x \rightarrow \infty, t=0)=0$ temperature at $x \rightarrow \infty$ (4)
$G(x, s)=\frac{T(x, s)}{H(x, s)}=\frac{T_{1}}{\left(T_{3} s\right)^{0.5}+1} e^{-\lambda\left(T_{2} s\right)^{0.5}}$

\section{EXPERIMENTATION}

Experimentation has been carried out in order to support the model identification process. The thermal system (Fig.1) consists of a metal beam filled with composite material made of 50:50 mixture of buckshot and couscous particles (Sierociuk, D.et al.). One end of the metal beam is connected to a Peltier cell which is integrated in the heat pump assembly. Such assembly contains a heat sink and the fan: they are used to control the temperature of the cell cold side and to maintain it approximately constant at the ambient temperature. The heat flux out of the Peltier module is controlled by the input current signal. Three temperature sensors (LM35DH) are positioned at fixed distances from the heat source (sensor 1 at $\mathrm{d}_{\lambda_{1}}=2.5 \mathrm{~cm}$, sensor 2 at $\mathrm{d}_{\lambda_{2}}=3.8 \mathrm{~cm}$, and sensor 3 at $\mathrm{d}_{\lambda 3}=4.9 \mathrm{~cm}$,).

\section{MODEL IDENTIFICATION}

\subsection{The Optimized Identification Process}

Identification of the model was performed in the frequency domain. Sinusoidal signals at different frequencies have been used for the model identification in the frequency domain. In particular sinusoids with frequencies $1 \mathrm{mHz}, 3 \mathrm{mHz}, 5 \mathrm{mHz}$, $7 \mathrm{mHz}, 9 \mathrm{mHz}, 10 \mathrm{mHz}, 20 \mathrm{mHz}, 30 \mathrm{mHz}, 50 \mathrm{mHz}$ where used, with a heat flux peak to peak amplitude of $80 \mathrm{~W}$.

Given the spatial dependence of the model, the identification process is performed in two steps. First, a multi-objective Nelder Mead simplex optimization algorithm was used in order to identify the model parameters by fitting the model response (module and phase) to the experimental data for sensor 1 at the normalized distance $\lambda 1$. Then, in order to validate the identified model at different distances from the heat source, the same optimized identification algorithm has been used in order to identify the $\lambda 2$ and $\lambda 3$ parameters by 
comparing the model output $(\mathrm{T})$ to the experimental temperature measured respectively at distances $d_{\lambda_{2}}$ and $d_{\lambda_{3}}$. The quality of the identified model on the spatial domain has been measured by comparing estimated distances $d_{\lambda_{2}}$ and $d_{\lambda 3}$ (corresponding to the identified $\lambda 2$ and $\lambda 3$ ) with the real ones.

\subsection{The Ideal Model Identification}

First, the identification of the ideal model (5) parameters T1, $\mathrm{T} 2$ and T3 has been performed (Fig. 2(a)) at distance $\lambda 1$. Bode diagrams of the identified model are presented in Fig.3. Then the distances $d_{\lambda 2}$ and $d_{\lambda 3}$ where estimated. Estimated $\mathrm{d}_{\lambda 2}$ was $3.19 \mathrm{~cm}$, against the real one at $3.8 \mathrm{~cm}$. Estimated $\mathrm{d}_{\lambda 3}$ was $3.9 \mathrm{~cm}$, against the real one at $4.8 \mathrm{~cm}$.

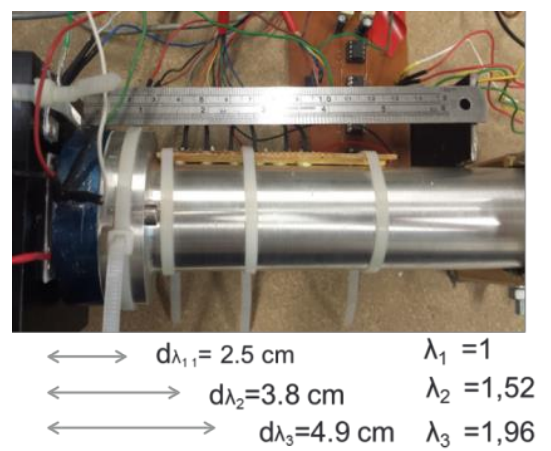

Fig. 1. Experimental setup: Beam photo.

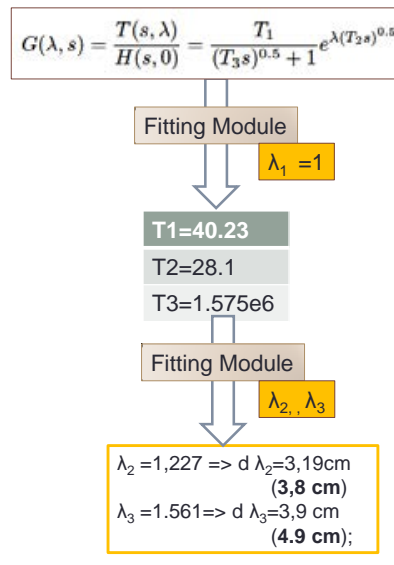

(a)

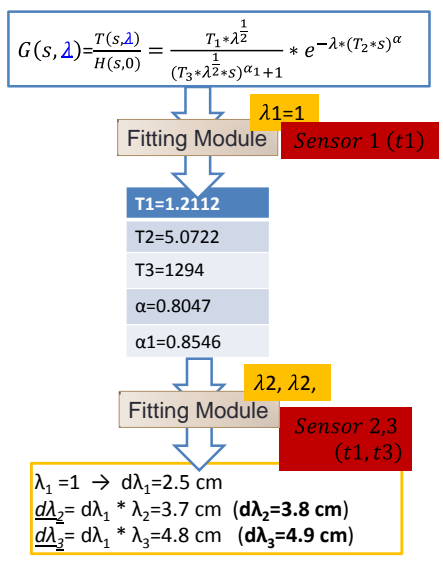

(b)
Fig. 2 Identification process. (a) ideal heat transfer model.(b) enhanced heat transfer model for heterogeneous materials

\subsection{The Enhanced Model Identification}

The ideal model in (5) was enhanced for heterogeneous materials obtaining (6) in order to better fit the experimental data for all the three sensors. In particular the fractional order of the system $\left(\alpha_{1}\right)$ was also left as parameter to be identified. First, the identification of the enhanced model (6) parameters T1, T2, T3, $\alpha_{1}, \alpha$ has been performed (Fig. 2(b)) at distance $\lambda 1$. Bode diagrams of the identified model are presented in Fig. 4.
Then the distances $d_{\lambda 2}$ and $d_{\lambda 3}$ where estimated.

$T(s, \lambda)=\frac{T_{1} * \lambda^{\frac{1}{2}}}{\left(T_{3} * \lambda^{\frac{1}{2}} * s\right)^{\alpha_{1+1}}} * e^{-\lambda *\left(T_{2} * s\right)^{\alpha}} * H(s, 0)$

The optimized model better fits the experimental data measured for sensor 2 and 3. Estimated $d_{\lambda_{2}}$ was $3.7 \mathrm{~cm}$, against the real one at $3.8 \mathrm{~cm}$. Estimated $\mathrm{d}_{\lambda 3}$ was $4.7 \mathrm{~cm}$, against the real one at $4.8 \mathrm{~cm}$.

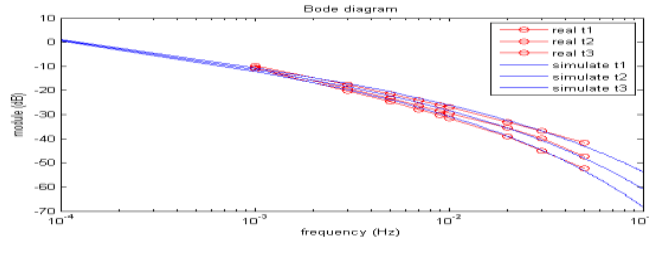

(a)

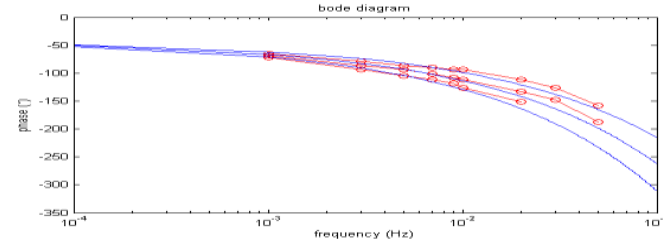

Fig. 3. Ideal Heat Transfer Model: (a) Module and (b) Phase Bode diagrams
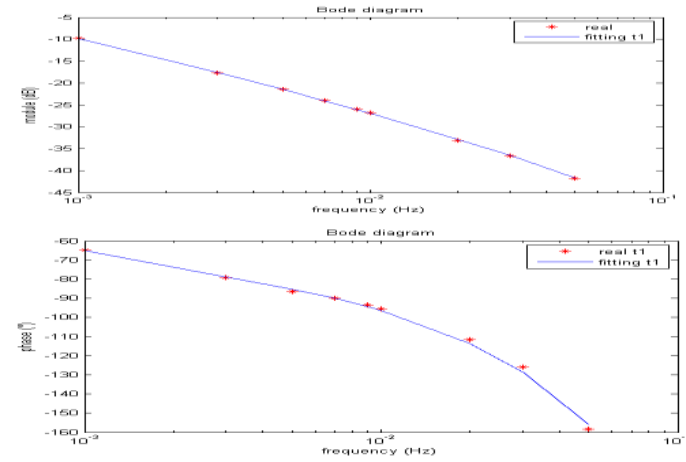

Fig. 4 Optimized Heat Transfer Model on Sensor 1 ( $\lambda 1)$ : (a) Module and (b) Phase Bode diagrams

\section{REFERENCES}

Caponetto, R., Graziani, S., Pappalardo, F. L. and Sapuppo, F.(2013), Experimental Characterization of Ionic Polymer Metal Composite as a Novel Fractional Order Element, Advances in Mathematical Physics, Volume 2013, 10 pages

Kulish, V. V. and Lage, J.L.. (2000). Fractional-Diffusion Solutions for Transient Local Temperature and Heat Flux. Transactions of the ASME, 122, page 372.

Sierociuk, D., Dzieliński, A., Sarwas, G. Petras, I. Podlubny, I. and Skovranek, T. (2013). Modelling heat transfer in heterogeneous media using fractional calculus, Philos Trans A Math Phys Eng Sci., 371(1990)

Srivastava, I., Sadasivam, S., Smith, K. C., and Fisher, T. S.(2013) Combined Microstructure and Heat Conduction Modeling of Heterogeneous Interfaces and Materials, $J$. Heat Transfer 135(6), 061603. 
MATHMOD 2015 Abstract Volume 


\title{
MATHMOD 2015 ABSTRACT VOLUME
}

\author{
MATHMOD Minisymposium \\ Distributed Parameter Systems
}


MATHMOD 2015 Abstract Volume 


\title{
Infinite Dimensional Port Hamiltonian Representation of reaction diffusion processes *
}

\author{
W. Zhou* B. Hamroun* Y. Le Gorrec ${ }^{* *}$ F.Couenne* \\ * LAGEP, LAGEP, UMR CNRS 500\%, University of Lyon1, \\ Villeurbanne, France, (e-mails: \\ \{zhou;hamroun;couenne\}@lagep.univ-lyon1.fr). \\ ** FEMTO-ST, ENSMM Besançon, Besançon, France (e-mail: \\ yann.le.gorrec@ens2m.fr)
}

\section{INTRODUCTION}

The Port Hamiltonian framework is based on a geometric description of physical systems and is particularly useful for the modeling and control of multi-physics, network and distributed parameters systems (Duindam et al., 2002). Since its definition and its extension to distributed parameter systems it has been widely and successfully applied to classical mechanical and electrical systems, both in finite and infinite dimensions. The application of Port Hamiltonian formalism to thermodynamic systems is much more tedious as in the general non isothermal case the link between energy and irreversible phenomena cannot be associated to a linear geometric structure.

The idea of this paper is to propose a proper decomposition of thermodynamic phenomena in the case of non isothermal distributed reaction diffusion systems in order to highlight the inherent interconnection structure, called Stokes Dirac structure. The irreversibility is taken into account through an appropriate non linear closure relation between extended port variables, as it can be done for purely dissipative systems.

\section{MODEL OF DIFFUSION REACTION PROCESS}

The model for the reaction diffusion system under consideration is given by:

$$
\left\{\begin{array}{l}
\rho \partial_{t} \omega_{A}=-\partial_{z} f_{d}^{A}-M_{A} \nu_{A} r \\
\rho \partial_{t} \omega_{B}=-\partial_{z} f_{d}^{B}+M_{B} \nu_{B} r \\
\rho \partial_{t} s=-\partial_{z} f^{s}-\frac{q}{T_{j}}+\sigma
\end{array}\right.
$$

where $\omega_{i}$ represents the mass fraction of the species $i$ ( $i=A, B)$ and $s$ is the entropy per mass unit. $M_{i}$ is the molar mass of species $i, T_{j}$ is the distributed temperature of the jacket, $f^{s}=\sum_{i=A, B} f_{d}^{i} s_{i}+f_{F}^{s}$ is the total entropy flux where $f_{F}^{s}=\frac{f_{F}}{T}$ is the entropy flux due to conduction $f_{F}^{s}=\frac{f_{F}}{T}$ and $s_{i}$ the partial mass entropy of species $i$. $r$ represents the chemical reaction and $\nu_{A}, \nu_{B}>0$ are the stoichiometric coefficients. $q(z)$ is the distributed heat transfer $q(z)$ between the reactor and its jacket. $\sigma$ is the irreversible production of entropy.

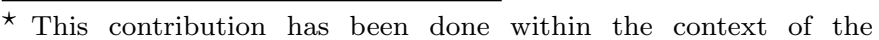
French National Research Agency sponsored projet HAMECMOPSYS (ANR-11- BS03-0002)
}

\section{PORT HAMILTONIAN FORMULATION FOR DIFFUSION REACTION PROCESSES}

The model of reaction diffusion system given in (1) can be rewritten as:

$$
\left\{\begin{array}{l}
F=J E+\mathcal{B}_{e} u \\
y=-\mathcal{B}_{e}^{\star} E
\end{array}\right.
$$

with some appropriate choices of $E$ and $F$, the extended effort and flow variables, $u=-\frac{q}{T}, y=T$ and $\mathcal{B}_{e}=\left(\begin{array}{c}\mathcal{B} \\ 0_{3} \\ 0_{5}\end{array}\right)$ where $\mathcal{B}=\left(\begin{array}{l}0 \\ 0 \\ 1\end{array}\right) . J$ is a skew-symmetric differential operator given by:

$$
J=P_{1} \partial_{z}+P_{0}
$$

with $P_{1}$ a symmetric matrix and $P_{0}$ a skew symmetric one. Proposition 1. The linear subset $\mathcal{D}$ :

$\mathcal{D}=\left\{\left(\left[\begin{array}{c}F \\ u \\ f_{\partial}\end{array}\right],\left[\begin{array}{c}E \\ y \\ e_{\partial}\end{array}\right]\right) \in \mathcal{E} \times \mathcal{F} \mid F=J E+\mathcal{B}_{e} u\right.$, $y=-\mathcal{B}_{e} E$, for some appropriate boundary port variables $\left.\left[\begin{array}{l}f_{\partial} \\ e_{\partial}\end{array}\right]_{(0, L)}\right\}$ is a Stokes Dirac structure.

Furthermore the irreversible feature of the system is defined by:

$$
-E\left(\begin{array}{ccc}
0_{3} & 0 & 0 \\
0 & 0 & 0 \\
0 & 0 & I_{4}
\end{array}\right) F>0
$$

\section{CONCLUSION}

This paper is a first attempt to the port Hamiltonian representation of a tubular reactor. It remains to show how the convection phenomenon can be integrated in such formulation. This formulation could be advantageously used for control purposes using the passivity based methods.

\section{REFERENCES}

Duindam V., Macchelli A., Stramigioli S. and Bruyninckx H. (Eds.). Modeling and Control of Complex Physical Systems: The Port-Hamiltonian Approach. Springer Verlag New York inc., 2009. 


\title{
Distributed and backstepping boundary controls to achieve IDA-PBC design
}

\author{
N.M. Trang VU* L. LEFE்VRE* R. NOUAILLETAS**
}

*Univ. Grenoble Alpes, LCIS, F-26902, France;

${ }^{*} \mathrm{CEA} / \mathrm{DSM} / \mathrm{IRFM} / \mathrm{STEP}, \mathrm{CEA}$ Cadarache, France

Keywords: distributed parameters systems, port-Hamiltonian systems, IDA-PBC control, backstepping control, plasma control

In this paper, an IDA-PBC design for infinite dimensional PHS with both finite rank distributed control and boundary control is proposed. The controls are designed in such a way that the matching error from the distributed control is compensated by the boundary control. More precisely, while keeping the geometrical interconnection structure (namely the Dirac structure) unchanged, we propose energy shaping and damping assignment to match a restricted class of closed loop port-Hamiltonian systems. Because of the system constraints on the design parameters, there is no exact solution for the matching equation. Therefore we use a finite rank distributed control in the state equation and a boundary control simultaneously. We propose an approximated solution to the matching equation such that the error (with respect to some "exact solution") may be compensated by another boundary control via a "propagation function" (or backstepping control) to be determined.

Our application case concerns the control of the radial profile of the magnetic flux in tokamaks (cf. Wesson [2004]), the experimental facility for nuclear fusion reaction. The aim to reach some specific internal radial plasma profiles in order to avoid magneto-hydrodynamic (MHD) instabilities and to improve the plasma confinement stands an outstanding challenge in tokamak control recently. In our previous works $\mathrm{Vu}$ et al. [2013], the IDA-PBC laws were derived to the finite-dimensional $\mathrm{PCH}$ system of the tokamak plasma. In this example, the infinite-dimensional $\mathrm{PCH}$ of the resistive diffusion equation (cf. Vu et al. [2014]) will make title for the control synthesis. Both boundary (coils) and distributed (antennas) actuators are indeed available. A non-inductive current injection plays the role of the finite rank distributed control while the loop voltage generated by the transformer effect of the central solenoid coil realizes the boundary control. The distributed control is finite rank since only the total incoming power and the angle for the injected waves are controlled while the radial distribution (shape) of the control action is fixed for a given actuator. This feedback distributed control is employed to match a system with a homogeneous state equation. The boundary control here is unlike the "traditional" methods where the boundary action is homogenized in system state evolution equation. This new boundary control action includes both the original boundary control and the propagation of the distributed control.

\section{REFERENCES}

F. Felici, O. Sauter, S. Coda, B.P. Duval, T.P. Goodman, J.M. Moret, J.I. Paley, and the TCV Team. Real-time physics-model-based simulation of the current density profile in tokamak plasmas. Nuclear Fusion, 51, 2011.

M. Krstic and A. Smyshlyaev. Boundary control of PDEs: A course on Backstepping designs. Advances in Design and Control, SIAM, Philadelphia, 2008.

A. Macchelli, A.J. van der Schaft, and C. Melchiorri. Port hamiltonian formulation of infinite dimensional systems. i. modeling. Proc. 50th IEEE Conference on Decisions and Control (CDC04), $2004 a$.

A. Macchelli, A.J. van der Schaft, and C. Melchiorri. Port hamiltonian formulation of infinite dimensional systems. ii. boundary control by interconnection. 43rd IEEE Conference on Decisions and Control (CDC04), 2004b.

R. Ortega and E. Garcia-Canseco. Interconnection and damping assignement passivit-based control: A survey. European Journal of Control, 110, 2003.

J. Villegas, H. Zwart, Y. Le Gorrec, and B.M. Maschke. Stability and stabilization of a class of boundary control systems. IEEE Transaction On Automatic Control, 54: 142-147, January 2009.

Trang.N.M. Vu and L.Lefèvre. Material balance and closure equations for plasmas in tokamaks. IFAC Workshop on Thermodynamic Foundations of Mathematical Systems Theory, July 13-16 2013.

Trang.N.M. Vu, L. Lefèvre, R. Nouailletas, and S. Brémond. An ida-pbc approach for the control of $1 \mathrm{~d}$ plasma profile in tokamaks. 52nd IEEE Conference on Decision and Control, Florence, Italy, December 10-13 2013.

Trang.N.M. Vu, L. Lefèvre, R. Nouailletas, and S. Brémond. Structure preserving reduction for thermomagneto plasma control model. The 21st International Symposium on Mathematical Theory of Networks and Systems, Groningen, Netherlands, July 07-11 2014.

J. Wesson. Tokamaks. Third edition. Oxford Science Publications, 2004.

H. Zwart, Y. Le Gorrec, B. Maschke, and J. Villegas. Well-posedness and regularity of hyperbolic boundary control systems on a one-dimensional spatial domain. ESAIM: Control, Optimisation and Calculus of Variations, August 2009. Published Online: DOI: $10.1051 / \mathrm{cocv} / 2009036$. 


\title{
ASYMPTOTIC STABILISATION OF DisTRIBUTED PORT-HAMILTONIAN SYSTEMS BY BOUNDARY ENERGY-SHAPING CONTROL
}

\author{
Alessandro Macchelli * Yann Le Gorrec ${ }^{* *}$ Héctor Ramírez ** \\ * University of Bologna, Dept. of Electrical, Electronic and Information Engineering (DEI), \\ viale del Risorgimento 2, 40136 Bologna, Italy (E-mail: alessandro.macchelli@ unibo.it) \\ ** FEMTO-ST Institute, AS2M department, Université de Franche-Comté, \\ 24 rue Alain Savary, F-25000 Besancon, France \\ (E-mail: hector.ramirez@ femto-st.fr, legorrec@femto-st.fr) \\ Corresponding author: A. Macchelli
}

\begin{abstract}
Most of the current research on the stability and stabilisation of distributed port-Hamiltonian systems deals with the development of boundary controllers. This task has been usually accomplished by looking at, or generating, a set of Casimir functions in closed-loop that robustly (i.e., independently from the Hamiltonian functions) relates the state of the infinite dimensional port-Hamiltonian system with the state of the controller, which is a finite dimensional port-Hamiltonian system interconnected to the boundary of the distributed parameter one. The shape of the closed-loop energy function is changed by acting on the Hamiltonian of the controller e.g. to introduce a minimum in a desired configuration. As discussed in [5,7], this procedure is the generalisation of the control by interconnection via Casimir generation (energy-Casimir method) developed for finite dimensional systems. The result is an energy-balancing passivity-based controller that is not able to deal with equilibria that require an infinite amount of supplied energy in steady state, i.e. with the so-called "dissipation obstacle."
\end{abstract}

In this paper, it is shown how to enlarge the class of boundary energy-shaping controllers beyond the dissipation obstacle by focusing on the trajectories that correspond to a particular Hamiltonian, rather than on the geometric structure (i.e., the Dirac structure), of the system only (see e.g., [3,4]). Since the state dependent control action obtained thanks to the energy-Casimir method is able to shape the Hamiltonian function, the idea is to procede in a more direct manner, i.e. by determining a feedback law that maps the open-loop trajectories into the trajectories of a target system with the same port-Hamiltonian structure (i.e., Dirac and resistive structures are not modified), but characterised by a shaped Hamiltonian with the desired stability properties. This is the same concept adopted in finite dimensions in case of stabilisation with state-modulated sources discussed in [5], or with the more general IDA-PBC control technique presented in [6].

In this paper, then, the boundary control via energy-shaping is developed for the class of linear, distributed, portHamiltonian systems presented in $[1,2]$. By transforming the original system via state feedback into a new one with an Hamiltonian function that has an isolated minimum at the equilibrium, simple stability is obtained. To have asymptotic stability, it is necessary to add damping by means of a further control loop. In this respect, another important contribution of this paper is to show that, if it is possible to impose full boundary dissipation to the port-Hamiltonian system resulting from the energy-shaping procedure, then the desired equilibrium can be proved to be asymptotically stable. It is worth noting that the proposed techniques can be easily extended to the nonlinear case: the difficult part is then to prove existence of solutions for the set of coupled PDEs and ODEs associated to the closed-loop system, and the invariance properties of the steady state trajectories determined by the damping injection loop.

[1] B. Jacob and H.J. Zwart. Linear Port-Hamiltonian Systems on Infinite-dimensional Spaces, volume 223 of Operator Theory: Advances and Applications. Birkhäuser, Basel, 2012.

[2] Y. Le Gorrec, H. Zwart, and B.M. Maschke. Dirac structures and boundary control systems associated with skew-symmetric differential operators. SIAM Journal on Control and Optimization, 44(5):1864-1892, 2005.

[3] A. Macchelli. Dirac structures on Hilbert spaces and boundary control of distributed port-Hamiltonian systems. Systems \& Control Letters, 68:43-50, Jun. 2014.

[4] A. Macchelli. Passivity-based control of implicit port-Hamiltonian systems. SIAM Journal on Control and Optimization, 52(4):2422-2448, 2014.

[5] R. Ortega, A.J. van der Schaft, I. Mareels, and B.M. Maschke. Putting energy back in control. Control Systems Magazine, IEEE, pages 18-33, Apr. 2001.

[6] R. Ortega, A.J. van der Schaft, B.M. Maschke, and G. Escobar. Interconnection and damping assignment passivity-based control of port-controlled Hamiltonian systems. Automatica, 38(4):585-596, 2002.

[7] A.J. van der Schaft. $L_{2}$-Gain and Passivity Techniques in Nonlinear Control. Communication and Control Engineering. Springer-Verlag, 2000. 


\title{
Heat Transfer with Specular Reflections in an Experimental Annealing Device*
}

\author{
L. Jadachowski ${ }^{*}$ A. Steinboeck ${ }^{* *}$ A. Kugi* \\ * Christian Doppler Laboratory for Model-Based Process Control in the Steel \\ Industry, Automation and Control Institute, Vienna University of Technology, \\ Austria, (e-mail: \{jadachowski,kugi\}@acin.tuwien.ac.at) \\ ** Automation and Control Institute, Vienna University of Technology, \\ Austria, (e-mail: steinboeck@acin.tuwien.ac.at)
}

Annealing plays an important role in the production of high-quality steels. While the temperature evolution of steel strips in continuous annealing furnaces is a well studied field, the development of experimental reheating and annealing devices is less frequently explored. This is especially true when dealing with batch-type furnaces heated by electrically driven infrared (IR) radiators, where spatial temperature distributions in a steel strip arise due to relative positions of the IR-radiators and the specimen.

In this contribution, a 1-dimensional distributed-parameter model of the spatial-temporal temperature evolution in a steel strip is proposed. A cross-section of the annealing furnace geometry under consideration is shown in Fig. 1. It mainly consists of a water-cooled housing with a steel specimen inside and two arrays of IR-lamps (horizontal and vertical) mounted on gold-coated watercooled reflectors. The lamps are separated by the quartz glass plates from the annealing chamber filled with an inert atmosphere. The steel strip is clamped by two watercooled specimen holders, which can be vertically moved. By supplying electric power to the IR-lamps, the specimen is heated by means of thermal radiation.

The goal is to derive a mathematical model, which can be used to control and maintain the temperature uniformity in the steel specimen during the heating-up process. The model takes into account heat conduction in the specimen, thermal radiation in a partially specular enclosure, free convection and in-domain heat losses in the specimen holder. Here, the vertical IR-lamps are assumed to be inactive. As a consequence, the heating chamber of the furnace is considered infinitely wide and all variables are assumed to be invariant w.r.t. the specimen width. This also justifies a 2-dimensional interpretation of the radiation problem. Moreover, halogen IR-lamps, which serve as distributed heat sources, are modeled for simplicity as infinitely long opaque cylinders. The temperature evolution within the specimen fillet is of particular interest.

For the determination of heat flows by radiation, the evaluation of view factors between surfaces of an enclosure is

\footnotetext{
^ The financial support by the Austrian Federal Ministry of Science, Research and Economy, the National Foundation for Research, Technology and Development, and voestalpine Stahl GmbH is gratefully acknowledged. The second author gratefully acknowledges financial support provided by the Austrian Academy of Sciences in the form of an APART-fellowship at the Automation and Control Institute of Vienna University of Technology.
}

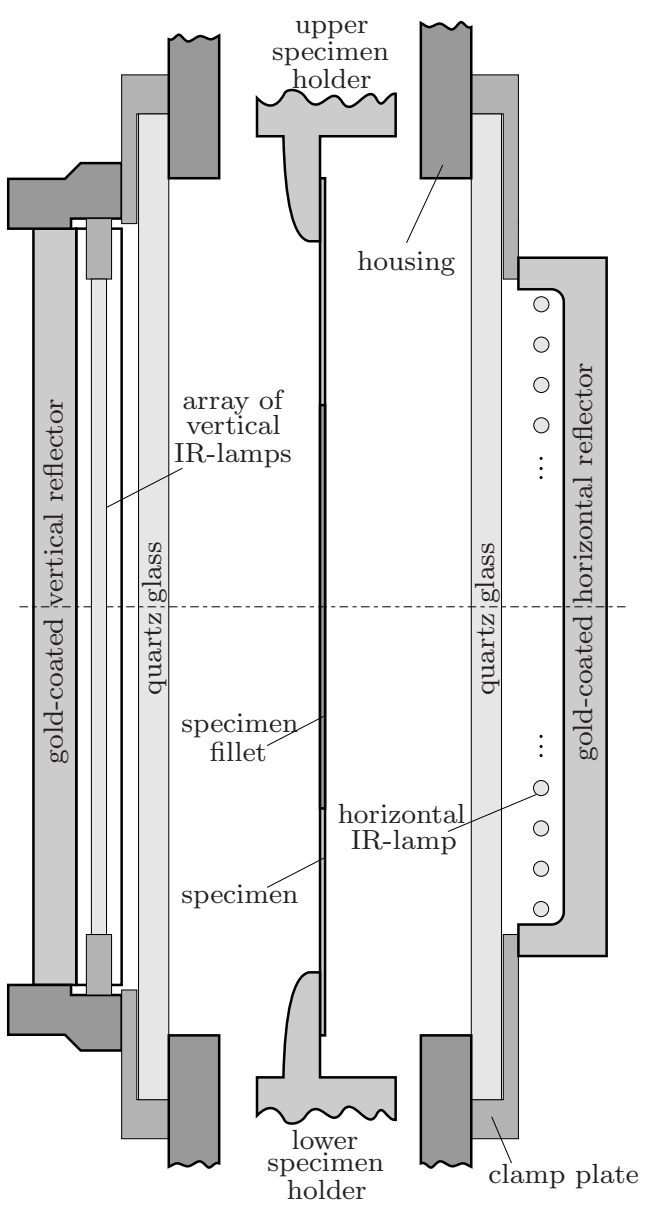

Fig. 1. Cross-section of the annealing furnace.

required. When dealing with enclosures including specular surfaces, it is essential to take into account the specific directional paths that the reflected radiation follows. This can be achieved by means of radiation exchange factors, which describe how diffuse energy leaving a surface arrives at a second surface directly and by all possible intermediate specular reflections. In enclosures with many specular surfaces, the computation of specular exchange factors can be laborious, in particular, when multiple reflections occur. Hence, in view of the complex furnace geometry, the FE software ANSYS is utilized for the computation of the exchange factors. A successful verification of the model for both temporal and spatial temperature evolution in the specimen has been carried out based on measurements. 


\title{
How to Choose the State for Distributed-Parameter Systems, a Geometric Point of View
}

\author{
K. Schlacher* M. Schöberl** \\ * Institute of Automatic Control and Control Systems Technology, \\ Johannes Kepler University Linz, 4040, Austria \\ (e-mail: Kurt.Schlacher@jku.at) \\ ** (e-mail: Markus.Schoeberl@jku.at)
}

\begin{abstract}
The choice of the state for a distributed-parameter system has some subtleties. The group of structure preserving maps or bundle morphisms, which map states to states and state and inputs to inputs is often rich enough for explicit control systems described by ODEs. In contrast the group of admissible maps is not limited to morphisms in the PDE case. The best known example is the wave equation, where two different descriptions in electrical and mechanical engineering are used, which are not linked by a bundle morphism. This contribution is limited to Hamiltonian and Lagrangian systems, where it is shown, how different descriptions can be derived from the same variational principle by standard and non standard transformations. In particular, we consider beams and plates.
\end{abstract}

Keywords: Lagrangian systems, Hamiltonian systems, variational principle, jet structures

\section{INTRODUCTION}

If one combs through the literature on geometric modeling of distributed-parameter systems, then one finds a lot of contributions related to Lagrangian or Hamiltonian systems. In contrast to the lumped or ODE case, where the picture is settled, one finds several apparently different approaches in the PDE case, see e.g Macchelli and Melchiorri (2004), Macchelli et al. (2005), Schöberl and Siuka (2013) for mechanical systems. More general approaches or other types of systems are considered e.g. in van der Schaft and Maschke (2002), Macchelli et al. (2004), Macchelli et al. (2004), Schlacher (2008), Schöberl and Siuka (2014).

The goal of this discussion contribution is to show, that some of the pictures from above have a common origin, which is related to the variation principle of classical physics. In Sec. 2 the ODE case is shortly illustrated to introduce the mathematical machinery. Sec. 3 deals with PDEs, where we show, that the same machinery offers significantly more choices. Throughout this contribution we use the language of differential geometry and apply the standard tensor notation, see e.g. in Giachetta et al. (1997).

\section{THE ODE CASE}

We consider the ODE case first to make the differences to the PDE case better visible. Therefore, we repeat the derivation of the Lagrangian equations shortly. Let $\mathcal{E} \stackrel{\pi}{\rightarrow} \mathcal{B}$ be a bundle with coordinates $(t)$ for $\mathcal{B}$ and $\left(t, x^{1}, \ldots, x^{n}\right)$ for $\mathcal{E}$. The first jet manifold of $\mathcal{E}$ is denoted by $J(\mathcal{E})$, where we use the $\left(t, x^{1}, \ldots, x^{n}, x_{1}^{1}, \ldots, x_{1}^{n}\right)$. A section $\sigma \in \Gamma(\mathcal{E})$ of $\mathcal{E}$ is a map $\sigma: \mathcal{B} \rightarrow \mathcal{E}$, which meets $\pi \circ \sigma=\operatorname{id}_{\mathcal{B}}$. Its extension to a map $j(\sigma): \mathcal{B} \rightarrow J(\mathcal{E})$ is given by $x_{1}^{i}=\partial_{1} \sigma^{i}(t)$. Let $L\left(t, x, x_{0}\right) \in C^{\infty}(J(\mathcal{E}))$ denote the Lagrangian. An admissible variation is described by a flow $\phi_{\epsilon}: \mathbb{R} \times \mathcal{E} \rightarrow \mathcal{E}$, which does not change the time. The infinitesimal generator of $\phi_{\epsilon}$ is the vector field $a=a^{i} \partial_{x^{i}}=$ $\Gamma(\mathcal{T}(\mathcal{E}))$. A section $\sigma$ is called critical, iff

$$
\left.\partial_{\epsilon} \int_{T} L \circ j\left(\phi_{\epsilon}(\sigma)\right)\right|_{\epsilon=0}=0
$$

is met. Short calculations lead to

$0=\int_{T} a^{i}\left(\partial_{x^{i}} L-d_{t}\left(\partial_{x_{t}^{i}} L\right)\right) \circ j^{2}(\sigma) \mathrm{d} t+\left.a^{i} \partial_{x_{t}^{i}} L \circ j^{1}(\sigma)\right|_{\partial T}$.

The Euler-Lagrange equations follows from the requirement that this relation holds for any $a$. Here, $d_{t}=\partial_{t}+$ $x_{1}^{i} \partial_{x^{i}}+x_{2}^{i} \partial_{x^{i}}^{1}$ is the total time derivative, $T$ denotes the interval $\left[t_{1}, t_{2}\right]$ and $\partial T$ its boundary, where the variation vanishes.

An alternative approach to the problem from above follows from the idea to hide the jet variables in the Lagrangian $^{1}$ $L$ by the substitution $x_{t}^{i}=u$. Let $\mathcal{Z} \stackrel{\rho}{\rightarrow} \mathcal{E}$ be the bundle with coordinates $\left(t, x^{1}, \ldots, x^{n}, u^{1}, \ldots, u^{n}\right)$ for $\mathcal{Z}$. An admissible variation is a flow $\varphi_{\epsilon}: \mathbb{R} \times \mathcal{Z} \rightarrow \mathcal{Z}$, which does not vary the time $t$ and the relation $x_{t}^{i}=u$. Instead of determining such a flow, we take a flow with infinitesimal generator $v=a^{i} \partial_{x^{i}}+b^{i} \partial_{u^{i}} \in \Gamma(\mathcal{T}(\mathcal{Z}))$ and use the technique of Lagrange multipliers $\lambda_{i} \in C^{\infty}(\mathcal{Z})$. After a short calculation we get the relation

$$
\begin{gathered}
0=\int_{T}\left(a^{i}\left(\partial_{x^{i}} L-d_{t}\left(\lambda_{i}\right)+b^{i}\left(\partial_{u^{i}} L-\lambda_{i}\right)\right) \circ j(\sigma) \mathrm{d} t\right. \\
\quad+\left.\left(\lambda_{i} a^{i}\right) \circ \sigma\right|_{\partial T},
\end{gathered}
$$

1 Roughly speaking we convert the classical variational problem to an optimal control problem. 
which is supposed to vanish for any $v$. Please note that one gets a set of PDEs, because the multipliers $\lambda_{i}$ are functions. Continuing this way, one comes to the Hamilton Jacobi approach. Now, we assume, the relation $p_{i}=\partial_{u^{i}} L(t, x, u)$ is solvable with respect to $u=$ $l(t, x, p)$. Let us introduce the bundle $\mathcal{P} \stackrel{\varrho}{\rightarrow} \mathcal{E}$ with coordinates $\left(t, x^{1}, \ldots, x^{n}, p_{1}, \ldots, p_{n}\right)$. We transfer the relations $d_{t}\left(\lambda_{i}\right)=\partial_{x^{i}} L, \lambda_{i}=\partial_{u^{i}} L, x_{t}^{i}=u^{i}$ from $J(\mathcal{Z})$ to $J(\mathcal{P})$ and derive the Hamilton equations

$$
\begin{aligned}
& x_{t}^{i}=\left.u\right|_{u=l(t, x, p)}=\partial_{p_{i}} H(t, x, p) \\
& p_{i}^{t}=\left.\partial_{x^{i}} L\right|_{u=l(t, x, p)}=-\partial_{x^{i}} H(t, x, p)
\end{aligned}
$$

with $H(t, x, p)=\left(p_{i} u^{i}-L\right)_{u=l(t, x, p)}$. Obviously, these equations form a system of explicit first order ODEs.

\section{THE PDE CASE}

Fortunately the PDE case is not much more involved than the ODE case concerning the Lagrangian approach. We replace the bundle from the previous section by $\mathcal{E} \stackrel{\pi}{\rightarrow} \mathcal{B}$ with coordinates $\left(X^{0}, \ldots, X^{m}\right)$ for $\mathcal{B}$ and $\left(X, x^{1}, \ldots, x^{n}\right)$ for $\mathcal{E}$, where we use the convention $X^{0}=t$. The first jet manifold of $\mathcal{E}$ is introduced analogously. We confine ourselves to first order Lagrangians, where the Lagrangian density $L \in \Gamma(J(\mathcal{E}))$ depends on $t, X, x$ and on first order jet variables $x_{\alpha}^{i}$. Performing the variation like in the previous section we get the analogous result

$$
\begin{gathered}
0=\int_{D} a^{i}\left(\partial_{x^{i}} L-d_{\alpha}\left(\partial_{x^{i}}^{\alpha} L\right)\right) \circ j^{2}(\sigma) \mathrm{d} X \\
\left.+\int_{\partial \mathcal{D}} a^{i} \partial_{x^{i}} L \circ j(\sigma) \partial_{X^{\alpha}}\right\rfloor \mathrm{d} X
\end{gathered}
$$

with the volume form $\mathrm{d} X=\mathrm{d} t \wedge \mathrm{d} X^{1} \wedge \cdots \wedge \mathrm{d} X^{m}$ defined on $\mathcal{D}=T \times \mathcal{X}$ with the spatial part $\mathcal{X}$. The total derivative into the direction $X^{\alpha}$ is denoted by $d_{\alpha}=\partial_{\alpha}+x_{\alpha}^{i} \partial_{x^{i}}+$ $x_{\alpha \beta}^{i} \partial_{x_{\beta}^{i}}$. Suitable boundary conditions have to be added for the spatial boundary $\partial \mathcal{X}$.

In contrast to the Lagrangian approach, one has more possibilities to derive certain types of Hamiltonian equations. To derive evolutionary equations in standard form, we add the relations $x_{0}^{i}=u_{0}^{i}$, where $u_{0}^{i}$ are not jet variables. Performing the variation analogously to the ODE case we derive finally the following Hamiltonian equations

$$
\begin{aligned}
x_{0}^{i} & =\partial_{p_{i}^{0}} H\left(t, x, x_{1}, \ldots, x_{m}, p^{0}\right) \\
p_{i}^{0} & =-\delta_{x^{i}} H\left(t, x, x_{1}, \ldots, x_{m}, p^{0}\right)
\end{aligned}
$$

with the variational derivative $\delta_{x^{i}}=\partial_{x^{i}}-d_{\alpha} \partial_{x^{i}}^{\alpha}$ and Hamiltonian density $H=\left(p_{i}^{0} u_{0}^{i}-L\right)_{u_{0}^{i}=l\left(\ldots, p^{0}\right)}$, where $l$ meets $p_{i}^{0}=\partial_{u_{0}^{i}} L \circ l=p_{i}^{0}$.

But there is no reason to hide time derivatives only. One gets the polysymplectic approach by hiding all derivatives. In this case we add the relations $x_{\alpha}^{i}=u_{\alpha}^{i}$ and perform the variation analogously to the ODE case. Please note, that $u_{\alpha}^{i}$ are not jet variables. If the relations $p_{i}^{\alpha}=$ $\partial_{u^{i}} L(t, X, x, u)$, where $u$ denotes the set of all $u_{\alpha}^{i}$, are solvable with respect to $u$, then we are able to introduce the $H(t, X, x, p)=\lambda_{i}^{\alpha} u_{\alpha}^{i}-L$. The polysymplectic equations, also known as De Donder-Weyl equations, follow as

$$
\begin{aligned}
x_{\alpha}^{i} & =\partial_{p_{i}^{\alpha}} H \\
p_{i, \beta}^{\alpha} \delta_{\alpha}^{\beta} & =-\partial_{x^{i}} H,
\end{aligned}
$$

where $\delta_{\alpha}^{\beta}$ denotes the Kronecker symbol. Obviously, intermediate approaches between the evolutionary and the polysymplectic ones exists.

Finally, we consider the case, that the Lagrangian density is independent of $x^{i}$. This fact allows us to perform the transformation $x_{\alpha}^{i}=q_{\alpha}^{i}$, where $q_{\alpha}^{i}$ are not jet variables. Because of $x_{\alpha \beta}^{i}=x_{\beta \alpha}^{i}$ we get the integrability conditions $q_{\alpha, \beta}^{i}=q_{\beta, \alpha}^{i}$. Again one can add this relation and perform the variation. Obviously, we get here a third type of Hamiltonian equations.

\section{CONCLUSION}

According to Sec. 2 the state of a Lagrangian system follows as $\left(x^{1}, \ldots, x^{n}, x_{1}^{1}, \ldots, x_{1}^{n}\right)$. Any change of coordinates $f: J(\overline{\mathcal{E}}) \rightarrow J(\mathcal{E})$, which preserves the jet structure preserves the Lagrangian structure, too. The transition to the Hamiltonian description $\left(x^{1}, \ldots, x^{n}, x_{1}^{1}, \ldots, x_{1}^{n}\right) \rightarrow$ $\left(x^{1}, \ldots, x^{n}, p_{1}, \ldots, p_{n}\right)$ is not structure preserving, since the jet structure is replaced by a symmplectic or more general by a Dirac one. To preserve the Hamiltonian description by coordinate changes, one has to preserve these structures. The main observation of Sec. 2 is, that the set of possible and useful transformations is richer. We will show, that at first glance different choices of states have the origin in ones, which are even related to the variation principle.

\section{REFERENCES}

Giachetta, G., Mangiarotti, L., and Sardanashvily, G. (1997). New Lagrangian and Hamiltonian Methods in Field Theory. World Scientific, Singapore.

Macchelli, A. and Melchiorri, C. (2004). Modeling and control of the timoshenko beam. the distributed port hamiltonian approach. SIAM Journal on Control and Optimization, 43, 743-767.

Macchelli, A., Melchiorri, C., and Bassi, L. (2005). Port based modelling and control of the mindlin plate. In Proceedings 44th IEEE Conference on Decision and Control (CDC), 5989-5994.

Macchelli, A., van der Schaft, A., and Melchiorri, C. (2004). Port hamiltonian formulation of infinite dimensional systems i. modeling. In Proceedings 43rd IEEE Conference on Decision and Control (CDC), 3762-3767.

Schlacher, K. (2008). Mathematical modeling for nonlinear control: a hamiltonian approach. Mathematics and Computers in simulation, 97, 829-849.

Schöberl, M. and Siuka, A. (2013). On casimir functionals for infinite-dimensional port-hamiltonian control systems. IEEE Transactions on Automatic Control, 58, 1823-1828.

Schöberl, M. and Siuka, A. (2014). Jet bundle formulation of infinite-dimensional port-hamiltonian systems using differential operators. Automatica, 50, 607-613.

van der Schaft, A. and Maschke, B. (2002). Hamiltonian formulation of distributed-parameter systems with boundary energy flow. Journal of Geometry and Physics, 42, 166-194. 


\title{
Bi-Zone Modelling and Simulation of an Extrusion Process with Variable Screw Speed
}

\author{
F. Lotero* F. Couenne ${ }^{* *}$ B. Maschke ${ }^{* *}$ D. Sbarbaro* \\ * Department of Electrical Engineering, Universidad de Concepción, \\ Chile \\ ** Laboratoire d'Automatique et Génie des Procédés, LAGEP UMR \\ CNRS 5007, Université Lyon 1, Faculté des Sciences et Technologies, \\ Villeurbanne F-69622, France, e-mail: maschke@lagep.univ-lyon1.fr
}

\section{INTRODUCTION}

In the food and chemical industry, the extrusion processes are mainly used for mixing, processing and moulding of either polymers or raw materials for products such as cereals, potato chips and cookie dough. Basically, an extruder is made of a barrel, and a screw who rotates inside and forces the product according to the Archimedes principle. At the end of the barrel, there is a die where the material comes out of the process. There exist two type of extruders, the single screw and the twin-screw extruder. Single screw extruders have poor mixing capability and also they are limited in their ability to transport high viscous materials. On the other side, twin screw extruders, are divided in two additional types: counter-rotating and co-rotating screws. The co-rotating extruders are by far, the most used type thanks to its high mixing capabilities and also because of the self-cleaning capability.

Modelling the extrusion process is very complex due to the strong interactions that exist between the different mechanisms of the process. This complexity has lead to focus most part of the studies in literature on experimental data obtained at pilot-scale (Janssen, 1998)(Garge et al., 2012).There also exist several methodologies to model the extrusion process such as a finite dimensional model based on a flow representation of a series of Continuous Stirred Tank Reactors (CSTR)(Choulak et al., 2004)(Ganzeveld et al., 1994), a quasi-three-dimensional model (Das and Ghoshdastidar, 2002), and a Dynamic Gray Box model (Iqbal et al., 2010).

This work deals with the modelling of the extrusion process as a bi-zone model with moving interface. In this particular case the extrusion process is simplified as a nonlinear infinite dimensional bi-zone model and only melt conveying is considered. A novel presentation of the finite volume method approach adapted to a moving interface system is presented in order to discretize the model. In order to illustrate the behaviour of the presented model, a simulation was considered with variable screw speed $N_{0}$ and constant feed rate $F_{i n}$. The results show the dynamics of the moving interface, filling ratio and temperature along the process with physical coherence. Also it was

\footnotetext{
* This work was supported in part by the ANR sponsored project HAMECMOPSYS under Reference Code ANR-11-BS03-0002 and by the French-Chilean ECOS/CONICYT project C12E08.
}

possible to show on the simulation that the position of the moving interface appears as a point of discontinuity. Future works will consider variable viscosity and several moving interfaces as well as the use of this model for control purposes.

\section{REFERENCES}

Choulak, S., Couenne, F., Le Gorrec, Y., Jallut, C., Cassagnau, P., and Michel, A. (2004). Genericdynamic model for simulation and control of reactive extrusion. Ind. Eng. Chem. Res, 43, 7373-7382.

Das, M.K. and Ghoshdastidar, P.S. (2002). Experimental validation of a quasi three-dimensional conjugate heat transfer model for the metering section of a single screw plasticating extruder. Journal of Materials Processing Technology, 120(1-3), 397-411.

Ganzeveld, K., Capel, J., van der Wal, D.J., and Janssen, L. (1994). The modelling of counter-rotating twin screw extruders as reactors for single-component reactions. Chemical Engineering Science, 49(10), 1639-1649.

Garge, S.C., Wetzer, M.D., and Ogunnaik, B.A. (2012). Control-relevant model identification of reactive extrusion processes. Journal of Process Control, 22(8), 14571467.

Iqbal, M.H., Sundararaj, U., and Shah, S.L. (2010). New approach to develop dynamic gray box model for a plasticating twin-screw extruder. Industrial and Engineering Chemistry Research, (49), 648-657.

Janssen, L.P.B.M. (1998). On the stability of reactive extrusion. Polymer Engineering and Science, 38(12), 2010-2019. 


\title{
Dynamical Models of Axially Moving Rods with Tensile and Bending Stiffness *
}

\author{
A. Steinboeck ${ }^{*}$ M. Baumgart ${ }^{\dagger}$ G. Stadler $^{\dagger}$ M. Saxinger ${ }^{\dagger}$ \\ A. Kugi ${ }^{\dagger}$ \\ * Automation and Control Institute, Vienna University of Technology, \\ Gußhausstraße 27-29, 1040 Vienna, Austria \\ (e-mail: andreas.steinboeck@tuwien.ac.at) \\ $\dagger$ Christian Doppler Laboratory for Model-Based Control in the Steel \\ Industry, Automation and Control Institute, Vienna University of \\ Technology, Gußhausstraße 27-29, 1040 Vienna, Austria \\ (e-mail: \{baumgart,stadler,saxinger,kugi\}@acin.tuwien.ac.at)
}

\section{INTRODUCTION}

The analysis of vibrations in axially moving rods (strings or beams) has a long tradition in many industrial applications like saw blades and continuous production systems for paper or strip steel. There exist different mathematical modeling approaches. Two-degree-of-freedom (2-DOF) models are obtained if axial displacements $u$ and transversal displacements $w$ of the rod are considered. Simpler 1-DOF models capture only transversal displacements $w$. Chen (2005) and Marynowski and Kapitaniak (2014) provided overviews of modeling strategies for axially moving continua, i. e., strings, beams, and plates.

In view of the various existing modeling strategies, the following research questions are posed:

(a) What are the conditions that render the use of 1-DOF models tenable?

(b) What are the differences between the derivation of such models by reducing a 2-DOF model and their direct formulation using just 1 DOF?

Answers to these questions are the main findings that are presented in this paper.

\section{METHODOLOGY}

A geometrically nonlinear 2-DOF model of an axially moving rod with tensile and bending stiffness is developed. For the derivation, shear rigidity is assumed and the balance of momentum is used. It is then demonstrated that Hamilton's Principle yields the same model. In this model, some forces can be attributed to the superimposed axial motion of the rod. Moreover, the coupling effect between axial displacements $u$ and transversal displacements $w$ crystallizes in the form of the tensile force $N$ in the rod.

\footnotetext{
* The first author gratefully acknowledges financial support provided by the Austrian Academy of Sciences in the form of an APART fellowship at the Automation and Control Institute of Vienna University of Technology. The other four authors gratefully acknowledge financial support provided by the Austrian Federal Ministry of Science, Research and Economy, the National Foundation for Research, Technology and Development, and voestalpine Stahl GmbH.
}

It is shown that usually the stiffness against axial displacements $u$ is significantly higher than the stiffness against transversal displacements $w$. This is the reason why the solution of $N$ can be derived by integration a-priori. Together with boundary conditions for longitudinal displacements $u$, this facilitates the elimination of $u$ from the equations yielding a 1-DOF model. However, if $u$ is of interest, it can still be computed ex post. Depending on the boundary conditions, the deduced 1-DOF model can have the form of an integro-differential equation.

Alternative 1-DOF models are directly derived based on assumptions concerning the tensile force $N$ and the corresponding axial strains. However, in these models, the axial displacement $u$ is not explicitly used, meaning that a second DOF is avoided in the model right from the beginning. The axial strain can either be modeled as GreenLagrangian strain or Cauchy strain, which gives models with a similar structure. Finally, a third option for directly deriving a 1-DOF model based on Hamilton's principle is demonstrated. All directly derived 1-DOF models are simple boundary value problems and do not involve integrodifferential equations.

An example problem is solved using the obtained 2-DOF and 1-DOF models in both steady-state and transient numerical analyses. The accuracy of the models is assessed by comparison with a known steady-state analytical solution, i. e., the catenary.

\section{REFERENCES}

Chen, L.Q. (2005). Analysis and control of transverse vibrations of axially moving strings. Applied Mechanics Reviews, 58(2), 91-116.

Marynowski, K. and Kapitaniak, T. (2014). Dynamics of axially moving continua. International Journal of Mechanical Sciences, 81, 26-41. 


\title{
Modeling the Dynamics of a Flexible Belt Drive Using the Equations of a Deformable String with Discontinuities
}

\author{
Yu. Vetyukov* V. Eliseev ${ }^{* *}$ M. Krommer ${ }^{* * *}$ \\ * Johannes Kepler University Linz, Institute of Technical Mechanics, \\ Altenbergerstr.69, 4040 Linz, Austria (e-mail: yury.vetyukov@jku.at) \\ ** St. Petersburg State Polytechnical University, Faculty of Mechanics \\ and Mechanical Engineering, Polytechnicheskaya ul. 29, 195251 \\ St.Petersburg, Russia (e-mail: yeliseyev@inbox.ru) \\ *** Vienna University of Technology, Institute of Mechanics and \\ Mechatronics, Karlsplatz 13, 1040 Vienna \\ (e-mail: michael.krommer@tuwien.ac.at)
}

\section{INTRODUCTION}

In the present paper we numerically study transient dynamics of a belt drive based on the model, which has earlier been presented by the authors in (Eliseev, 2009; Eliseev and Vetyukov, 2012). Velocity and strain of the extensible belt vary between the tight and the slack sides of the drive, which leads to the sliding friction between the belt and the pulleys. This model of creep of the belt near the points, where it leaves the pulleys, was first studied by Reynolds (1874) and is adopted in the engineering and technical literature. Common assumptions ignore dynamic effects due to the acceleration of the belt and involve assumptions concerning tension forces at the slack and the tight sides.

Rubin (2000) used a model of extensible string for describing steady operation of a belt drive in the framework of the creep theory. He consistently analyzes the fields of velocity and strain of the belt in three qualitatively different domains: at the free spans, at the zones of perfect contact between the belt and the pulley, as well as at the zones of sliding friction contact. Resulting relations for the rotational moments at the pulleys, their angular velocities and coefficient of efficiency of the belt drive are shown to be different from the results in the technical literature.

\section{ANALYSIS}

Eliseev (2009) suggested a novel approach to analyzing an extensible belt, whose particles are moving along a given contour. The equations are transformed from the Lagrangian description with the material coordinate $s$ to the Eulerian form with the spatial coordinate $\sigma$ (see Fig. 1). Relatively simple treatment of unsteady regimes of motion in both numerical and analytical contexts is possible with the assumed idealized model of perfect contact of the belt with the pulleys. The corresponding conditions of jumps of the field of velocities and concentrated forces in the points 1 and 3, where the belt leaves the pulleys, are new for the analysis of belt drives dynamics; see Irschik (2007).

\footnotetext{
* Support of Yury Vetyukov from the Austrian COMET-K2 programme of the Linz Center of Mechatronics (LCM) is gratefully acknowledged.
}

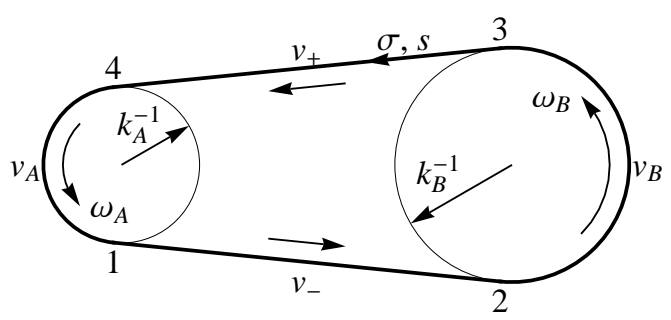

Fig. 1. Scheme of a two-pulley drive with the domains of motion of the belt: 12,34 - straight free spans (slack span and tight span respectively), 41, 23 - regions of contact with the driving pulley $A$ and with the driven one $B$; the single arc coordinate $\sigma$ is counted from the point $4 ; s$ is the material coordinate

In the present paper, we present the complete mathematical model including the quasilinear wave equations at the free spans, the coupling conditions based on the transport of the material particles by the pulleys and equations of dynamics of the pulleys. Finite difference discretization allows numerical analysis of the resulting system of partial differential equations with delays. Example solution for the case of start up and accelerated motion of a friction belt drive is presented and discussed.

\section{REFERENCES}

Eliseev, V. and Vetyukov, Y. (2012). Effects of deformation in the dynamics of belt drive. Acta Mechanica, 223, $1657-1667$.

Eliseev, V. (2009). A model of elastic string for transmissions with flexible coupling (in Russian). Scientific and technical bulletin of St. Petersburg State Polytechnical University, 84, 192-195.

Irschik, H. (2007). On rational treatments of the general laws of balance and jump, with emphasis on configurational formulations. Acta Mechanica, 194, 11-32.

Reynolds, O. (1874). On the efficiency of belts or straps as communicators of work. The Engineer, 38, 396.

Rubin, M. (2000). An exact solution for steady motion of an extensible belt in multipulley belt drive systems. Journal of Mechanical Design, 122, 311-316. 


\title{
Lagrangian and Port-Hamiltonian formulation for Distributed-parameter systems
}

\author{
M. Schöberl* K. Schlacher* \\ * Johannes Kepler University Linz, Institute of Automatic Control and \\ Control Systems Technology, Austria \\ (e-mail: markus.schoeberl@jku.at, kurt.schlacher@jku.at)
}

The structural analysis of dynamical systems can be performed in various ways and two prominent possibilities are the Lagrangian and the Hamiltonian perspective. When a systems allows to be analyzed in either of these two representations this has desirable benefits regarding the modeling since then the physical properties are rediscovered in the structure of the equations in a remarkable way, but also for the control of the system a Lagrangian or Hamiltonian representation can be beneficial. This observation holds independent of the concrete character of the system, may it be linear or nonlinear, described by ordinary differential equations (odes) or by partial differential equations (pdes), see e.g. Giachetta et al. (1997); van der Schaft (2000); Maschke et al. (2000); van der Schaft and Maschke (2002); Macchelli and Melchiorri (2004); Schlacher (2008); Schöberl and Siuka (2013) and references therein.

In this paper we will focus on distributed-parameter systems described by pdes that allow for a variational formulation, hence they are described by their Lagrangian density. From the Lagrangian density one can derive the partial differential equations on the domain and the boundary conditions. However, the extraction of the boundary conditions has to be performed with due care since it makes a big difference whether the Lagrangian depends on firstorder derivatives (first-order field theory) or on higher ones (higher-order field theory). In this contribution we will focus on second-order field theory and we wish to demonstrate how one can extract the physical meaningful boundary conditions by posing extra conditions on the set of possible boundary terms. The main difficulty in the higher-order case is the fact that repeated integration by parts cannot be performed uniquely for higher-order mixed partial derivatives. This is a well-known problem in mathematical physics and has the consequence that the Cartanform approach in the geometric version of the calculus of variations is only unambiguous for first-order field theories, see e.g. Giachetta et al. (1997). A possible (non-intrisic) extension to the higher-order case based on the so-called extended Cartan-form can be found in Ennsbrunner (2006) which makes use of the geometric shape of the boundary. We will also use the geometric shape of the boundary to be able to present a different (simpler) solution for the case of second-order derivatives in the Lagrangian density.

Besides the Lagrangian formulation of field theories it is also of interest to investigate possible Hamiltonian counterparts, see e.g. Olver (1986); van der Schaft and
Maschke (2002); Macchelli and Melchiorri (2004); Ennsbrunner (2006); Schöberl et al. (2008); Schöberl and Siuka (2013, 2014). Also in the Hamiltonian scenario the case of higher-order Hamiltonians causes the same mathematical difficulties as in the Lagrangian approach, but fortunately one is able to apply the same machinery also in the Hamiltonian setting to extract the boundary terms that may lead to boundary ports.

\section{REFERENCES}

Ennsbrunner, H. (2006). Infinite-dimensional EulerLagrange and Port Hamiltonian Systems. University Linz, PhD-Thesis.

Giachetta, G., Mangiarotti, L., and Sardanashvily, G. (1997). New Lagrangian and Hamiltonian Methods in Field Theory. World Scientific, Singapore.

Macchelli, A. and Melchiorri, C. (2004). Modeling and control of the timoshenko beam. the distributed port hamiltonian approach. SIAM Journal on Control and Optimization, 43, 743-767.

Maschke, B., Ortega, R., and van der Schaft, A. (2000). Energy-based lyapunov functions for forced hamiltonian systems with dissipation. IEEE Transactions on Automatic Control, 45, 1498-1502.

Olver, P. (1986). Applications of Lie Groups to Differential Equations. Springer-Verlag, New York.

Schlacher, K. (2008). Mathematical modeling for nonlinear control: a hamiltonian approach. Mathematics and Computers in simulation, 97, 829-849.

Schöberl, M., Ennsbrunner, H., and Schlacher, K. (2008). Modelling of piezoelectric structures - a hamiltonian approach. Mathematical and Computer Modelling of Dynamical Systems, 14, 179-193.

Schöberl, M. and Siuka, A. (2013). On casimir functionals for infinite-dimensional port-hamiltonian control systems. IEEE Transactions on Automatic Control, 58, 1823-1828.

Schöberl, M. and Siuka, A. (2014). Jet bundle formulation of infinite-dimensional port-hamiltonian systems using differential operators. Automatica, 50, 607-613.

van der Schaft, A. (2000). L2-Gain and Passivity Techniques in Nonlinear Control. Springer-Verlag, New York.

van der Schaft, A. and Maschke, B. (2002). Hamiltonian formulation of distributed-parameter systems with boundary energy flow. Journal of Geometry and Physics, 42, 166-194. 


\section{MATHMOD 2015 ABSTRACT VOLUME}

MATHMOD Minisymposium

Modelling of Dynamics and Control for Mobile Robots 
MATHMOD 2015 Abstract Volume 


\title{
Swimming and crawling motility at microscopic scales: from biological templates to bio-inspired devices
}

\author{
Antonio DeSimone* Giancarlo Cicconofri ${ }^{* *}$ \\ * SISSA-International School for Advanced Studies, Via Bonomea 265, \\ 34136 Trieste, Italy (e-mail: desimone@sissa.it). \\ ** SISSA-International School for Advanced Studies, Via Bonomea \\ 265, 34136 Trieste, Italy (e-mail: giancarlo.cicconofri@sissa.it).
}

\begin{abstract}
We discuss the mechanical bases of cellular motility by swimming and crawling. Special emphasis is placed on the connections between low Reynolds number swimming and Geometric Control Theory, and on the geometric structure of the underlying equations of motion. We examine some concrete examples, taken from the case studies that have been recently considered by our group. These include reverse engineering of the euglenoid movement, self-propelled droplets of active fluids, and one-dimensional models of slender crawlers.
\end{abstract}

Keywords: Modelling Applications in Mechanics, Mechatronics, and Robotics; Control Systems and Real-Time Applications; Biology, Physiology and Medicine; Self-propelled locomotion; Unicelluar swimmers; Biological Crawlers; Soft bio-inspired robotics.

\section{INTRODUCTION}

Motility of cells is at the root of many fundamental processes in biology: from sperm cells swimming to fertilize an egg cell, to leukocytes migrating towards newly opened wounds to activate the response of the immune system, to metastatic tumor cells crawling to invade nearby tissues. These are achieved by cells whose size is a few tens of microns at most.

While the idea of trying to mimic such remarkable capabilities is quite natural, much remains to be done before we can turn this idea into working engineering practice. For this purpose, we still need to learn many things on how to control and power these microscopic devices. It is the aim of this communication to report on recent progress done in our group to meet some of the challenges on the way to make artificial motility at the micron scale a concrete engineering possibility.

\section{FROM LOW REYNOLDS NUMBER SWIMMERS TO CRAWLERS ON VISCOUS SURFACES}

In order to discuss the mechanical bases of cellular motility by swimming and crawling, it is very instructive to start from the mechanics of swimming of unicellular organisms. Due to the small sizes and velocities involved, inertia is negligible, and the relevant hydrodynamics is the one at low Reynolds numbers (Re). In fact, we take $R e=0$, so that the Stokes system is used instead of the NavierStokes equations for the motion of the fluid surrounding the swimmer.

As explained in detail in [1], a clear geometric structure emerges when the swimming problem is looked at from the perspective of Geometric Control Theory. This geometric structure can be used to rationalise also the mechanics of crawlers on viscous substrates, and it allows us to discuss such crawlers with exactly the same tools we developed in the context of low Re swimmers.

To illustrate this point, we consider a one-dimensional crawler on a flat surface, whose kinematics is described by the position $x_{1}(t)$ of one of the two ends (the left one), and by the function $s(X, t)$ giving the current distance at time $t$ of the material point $X$ from the left end. We assume that the function $s(X, t)$, giving the shape of the crawler, can be prescribed at will. We are interested in calculating the motion $x_{1}(t)$ resulting from a prescribed sequence of shapes $s(X, t)$.

The equation of motion determining $x_{1}(t)$ is the force balance

$$
F(t)=\int_{0}^{L} f(s(X, t), t) s^{\prime}(X, T) d X=0
$$

where $f$ describes the mechanical interactions between the crawler and the surface, a force density per unit current length, and $s^{\prime}(X, T)$ is the derivative of $s$ with respect to $X$, necessary to convert the integral over the current configuration into an integral over the reference configuration, given by the interval $[0, L]$. We assume that $f=f(s, t)$ is given at point $s$ and time $t$ by $f(s, t)=$ $-\mu v(s, t)$, where $v(s, t)$ is the velocity of point $s$ at time $t$ and $\mu>0$ is a viscosity coefficient.

Equation (1), which expresses Newton's law in the inertialess degenerate case, turns out to be a non-holonomic constraint relating position to shape. In fact, observing that $v(s(X, t), t)=\dot{x}_{1}(t)+\dot{s}(X, t)$, where a superposed dot denotes time differentiation, we can solve eq. (1) for $\dot{x}_{1}$ obtaining 


$$
F(t)=0 \Rightarrow \dot{x}_{1}(t)=-\frac{1}{l(t)} \int_{0}^{L} \dot{s}(X, t) s^{\prime}(X, t) d X
$$

Following [2], to which we refer the reader for further details, we consider the special case in which the body of the crawler is divided into two segments, to the left and the right of a central point $x^{*}$, in each of which $s^{\prime}(X, t)$ is affine. Thus, the two segments have lengths $l_{1}(t), l_{2}(t)$ which oscillate in time between the two values $0<a<b$. We obtain in this case

$$
F(t)=0 \Rightarrow \dot{x}^{*}=\frac{l_{1}}{2\left(l_{1}+l_{2}\right)} \dot{l}_{1}-\frac{l_{2}}{2\left(l_{1}+l_{2}\right)} \dot{l}_{2} .
$$

It is convenient to introduce the notation

$$
\dot{x}^{*}=\mathbf{V}(\mathbf{l}) \cdot \mathbf{i}=V_{1}\left(l_{1}, l_{2}\right) \dot{l}_{1}+V_{2}\left(l_{1}, l_{2}\right) \dot{l}_{2}
$$

where

$$
V_{1}\left(l_{1}, l_{2}\right):=\frac{l_{1}}{2\left(l_{1}+l_{2}\right)}, \quad V_{2}\left(l_{1}, l_{2}\right):=-\frac{l_{2}}{2\left(l_{1}+l_{2}\right)} .
$$

A stroke is a closed curve in the space of shapes $\left(l_{1}, l_{2}\right)$, obtained as the image of the map $t \mapsto \mathbf{l}(t)$, where $t$ varies in some time interval $[0, T]$ and $\mathbf{l}(0)=\mathbf{l}(T)$. The image $\Gamma$ of this map can be seen as the boundary $\partial \Omega$ of a region $\Omega$ in the space of shapes. The net displacement $\Delta x^{*}$ at the end of one stroke (tracing the curve $\Gamma=\partial \Omega$ once in the counter-clockwise orientation) is then obtained by integrating eq. (4) over $[0, T]$ and using Stokes theorem:

$$
\Delta x^{*}=\int_{0}^{T} \dot{x}^{*}(t) d t=\int_{\Omega} \operatorname{curl} \mathbf{V}\left(l_{1}, l_{2}\right) d l_{1} d l_{2}
$$

where

$$
\operatorname{curl} \mathbf{V}=\frac{\partial V_{2}}{\partial l_{1}}-\frac{\partial V_{1}}{\partial l_{2}}=\frac{1}{2\left(l_{1}+l_{2}\right)} .
$$

Just as in the case of low Re swimmers, the resulting displacement is a geometric quantity, namely, it only depends on the path traced in the space of shapes, and not on the speed at which this path is traversed.

\section{DISCUSSION}

The geometric structure of the equations of motion, common to low Re swimmers and crawlers on viscous substrates, allows us to discuss them in unified framework.

We will illustrate the advantages of such a unified perspective by examining some concrete examples, taken from the case studies that have been recently considered by our group. These include reverse engineering of the euglenoid movement [3], one-dimensional models of slender crawlers [4], droplets of active fluids [5].

The lessons learned in the context of biological cell motility will then be re-examined, with the aim of building a dictionary of elementary motility mechanism to be used in prototypes of bio-inspired motile micro-robots. Again, case studies from our recent research will be illustrated, such as crawlers on directional substrates [6,7], and artificial swimmers moving thanks to the active control of their geometry $[8,9]$.

\section{ACKNOWLEDGEMENTS}

Financial support by the European Research Council through the Advanced Grant 340685-MicroMotility is gratefully acknowledged. This paper draws from joint work with P. Gidoni and G. Noselli.

\section{REFERENCES}

[1] F. Alouges, A. DeSimone, A. Lefebvre. Optimal strokes for low Reynolds number swimmers: an example. $J$. Nonlinear Sci., 18:277-302, 2008.

[2] A. DeSimone, A. Tatone. Crawling motility through the analysis of model locomotors: two case studies. Europ. Phys. Journal E, 35:85, 2012.

[3] M. Arroyo, L. Heltai, D. Milan, and A. DeSimone. Reverse engineering the euglenoid movement. Proc. Nat. Acad. Sci. USA, 109: 1787, 2012.

[5] A. DeSimone, F. Guarnieri, G. Noselli, A. Tatone. Crawlers in viscous environments: linear vs. nonlinear rheologies. International J. Nonlinear Mechanics, $56: 132-141,2013$.

[5] L.Giomi and A. DeSimone. Spontaneous division and motility in active nematic droplets. Phys. Rev. Lett., 112:147802, 2014.

[6] P. Gidoni, G. Noselli, A. DeSimone. Crawling on directional surfaces. Int J Nonlinear Mech., 61: 65 , 2014.

[7] G. Noselli and A. DeSimone. A robotic crawler exploiting directional frictional interactions: experiments, numeric, and derivation of a reduced model. Proc Roy Soc A, 470:20140333, 2014.

[8] F. Alouges, A. DeSimone, L. Giraldi, M. Zoppello: Selfpropulsion of slender micro-swimmers by curvature control: N-link swimmers. International J. Nonlinear Mechanics, 56, 142-147, 2013.

[9] M. Arroyo, A. DeSimone. Shape control of active surfaces inspired by the movement of euglenids. $J$. Mech. Phys. Solids, 62:99, 2014. 


\title{
Limbless locomotion on solid surfaces: a case study in soft bio-inspired robotics
}

\author{
Giancarlo Cicconofri* Antonio DeSimone** \\ * SISSA-International School for Advanced Studies, Via Bonomea 265, \\ 34136 Trieste, Italy (e-mail: giancarlo.cicconofri@sissa.it) \\ ** SISSA-International School for Advanced Studies, Via Bonomea \\ 265, 34136 Trieste, Italy (e-mail: desimone@sissa.it).
}

\begin{abstract}
We examine the problem of snake-like locomotion by studying a model system consisting of a planar inextensible elastic rod that is able to control its spontaneous curvature. Using Cosserat theory we derive the equations of motion for two special cases: one in which the system is confined inside a channel with frictionless walls, and one in which the system is placed on an anisotropic frictional environment with an infinite contrast between longitudinal and transversal friction, so that the rod can slide longitudinally along its axis, but cannot slip laterally (i.e., in the transversal direction). The results obtained by solving these equations of motion are reminiscent of classical experimental results in the biological literature, and provide a scheme to rationalise the observed behaviour.
\end{abstract}

Keywords: Modelling Applications in Mechanics, Mechatronics, and Robotics; Control Systems and Real-Time Applications; Biology, Physiology and Medicine; Self-propelled locomotion; Limbless undulatory locomotion; Snake locomotion; Soft bio-inspired robotics.

\section{INTRODUCTION}

Soft bio-inspired robotics is a new and recent paradigm in robotic science whereby inspiration is sought from nature to endow robots with new capabilities in terms of dexterity and adaptability $[1,2]$. Examples include the manipulation abilities of an elephant trunk or of an octopus arm, and the ability of snakes to handle unexpected interactions with unstructured environments and move successfully on uneven terrains by adapting their gait to ground properties that change from place to place in an unpredictable way.

Snake-like robotic locomotion is one of the first examples of soft bio-inspired robotics, thanks to the pioneering work of Hirose [3]. The way snakes move has been the subject of seminal works by Gray [4] who described the mechanics underlying snake locomotion inside closely fitting channels and, more broadly, snake propulsion on a surface in the presence of external push-points. More recently, the focus has turned to the importance of frictional anisotropy of snake ventral skin on flat surfaces as a mean of selfpropulsion, stimulating both experimental and theoretical research $[5,6]$.

Our approach to snake-like locomotion is based on modelling the system (either snake or robot) as a planar inextensible elastic rod that is able to control its spontaneous curvature [7]. We use a Cosserat model, and derive the equations of motion for two special cases: one in which the system is confined inside a channel with frictionless walls, and one in which the system is placed on an anisotropic frictional environment with an infinite contrast between longitudinal and transversal friction, so that the rod can slide longitudinally along its axis, but cannot slip laterally (i.e., in the transversal direction).

\section{A WARM-UP CASE: SLIDING INSIDE A CHANNEL WITH FRICTIONLESS WALLS}

In order to discuss the key elements of our approach, we focus here on the case of motion inside a tightly fitting, frictionless channel.

We consider an inextensible rod of length $L$, which backbone curve is described at every time $t$ by a planar curve $\mathbf{r}(\cdot, t)$ parametrised by arc-length. We associate to the system a potential energy $\mathcal{U}$ and a kinetic energy $\mathcal{T}$ given by the following expressions

$\mathcal{U}=\frac{E J}{2} \int_{0}^{L}(k(s, t)-\alpha(s, t))^{2} d s, \mathcal{T}=\frac{1}{2} \int_{0}^{L} \rho\left|\frac{\partial \mathbf{r}}{\partial t}(s, t)\right|^{2} d s$.

In the previous definitions $E J>0$ denotes the bending stiffness of the rod, $\rho>0$ is the mass density per unit length, $k(s, t)$ is the curvature of $\mathbf{r}$ at reference coordinate $s$ and time $t$, and $\alpha(s, t)$ is an assigned history of spontaneous curvatures.

We place our rod inside a curved channel fitting exactly its body. We model such a setting by imposing the backbone curve $\mathbf{r}$ to be forced inside the graph of the planar curve $\boldsymbol{\Gamma}$, which we interpret as the central line of the channel. Suppose $\boldsymbol{\Gamma}$ is parametrised by arc-length. The constrained system can be described with only one degree of freedom, namely, the curvilinear coordinate $s_{0}(t)$ relative to $\boldsymbol{\Gamma}$ of the first end of the rod. In particular we have $\mathbf{r}(s, t)=$ $\boldsymbol{\Gamma}\left(s_{0}(t)+s\right)$ and $k(s, t)=k_{\Gamma}\left(s_{0}(t)+s\right)$, where $k_{\Gamma}$ is the curvature of $\boldsymbol{\Gamma}$. As a consequence, also the potential and kinetic energies of the constrained system can be reduced to functions depending only on $s_{0}$, its time derivative $\dot{s}_{0}$, and the time variable $t$. The same holds for the Lagrangian function $\mathcal{L}$ of the system, which is defined as $\mathcal{L}:=\mathcal{T}-\mathcal{U}$. 
The time-evolution of the (only) 'generalised coordinate' of the system $s_{0}$ is then given by the Euler-Lagrange equation

which can be rewritten as

$$
\frac{d}{d t} \frac{\partial \mathcal{L}}{\partial \dot{s}_{0}}=\frac{\partial \mathcal{L}}{\partial s_{0}}
$$

$$
\begin{aligned}
\rho L \ddot{s}_{0}(t)= & \frac{E J}{2}\left(k_{\Gamma}^{2}\left(s_{0}(t)\right)-k_{\Gamma}^{2}\left(s_{0}(t)+L\right)\right) \\
& +E J \int_{0}^{L} \alpha(s, t) \frac{\partial k_{\Gamma}}{\partial s}\left(s_{0}(t)+s\right) d s .
\end{aligned}
$$

The equation above reduces the dynamic of the active rod to that of a point particle of mass $\rho L$, subject to a passive potential force of geometric origin (the difference of curvature of the curve $\Gamma$ at the two ends of the rod), and by an active term generated by the spontaneous curvature.

Let us consider only the passive term by setting $\alpha=0$. The system described in this case is a passive elastic rod with straight rest configuration placed inside a curved channel with no frictional interactions. Because of inextensibility, the driving force on the rod depends only on the curvature of the channel at the two ends of the body. Moreover, the sign of this force is such that the rod is always pushed towards the region of smaller curvature. As an example, consider the case of a spiral-shaped channel, where $k_{\Gamma}$ is a strictly decreasing function. If we thread the rod inside the spiral we need to perform a positive work since we have to increase the curvature at every point of the body. If we then release the rod it will accelerate towards the exit of the channel, moving towards a 'straighter' configuration, decreasing its elastic energy and therefore increasing its kinetic energy.

Let us suppose now that $\alpha \neq 0$. The active force term can assume any value if we suppose that we have no restrictions in the choice of $\alpha$, and the active elastic rod can slide inside the spiral without need of external pushing. More generally, an active rod can achieve motion in a predetermined direction when placed inside any channel which does not present circular or straight sections of length greater than $L$. This last result is reminiscent of theoretical and experimental findings of J. Gray in his study (4) of snake undulatory locomotion. Using an energy balance argument, he concludes that it is possible for a snake to slide inside a channel closely fitting its body only provided such a channel exhibits a variation of curvature along its track. He then shows experimentally that snakes are able to move in sinusoidal closely fitting channels, but not in straight (closely fitting) ones.

\section{DISCUSSION}

The full discussion of the locomotion problem requires more than what it has been sketched in the previous section. In particular, it is of crucial importance to determine the forces transmitted by the rod (snake or robot) to the environment, and to correlate them to muscular or motor activity (the spontaneous curvature $\alpha(s, t)$ ), and to the geometry of the trajectory. For this purpose, it is necessary to formulate and solve the full system of three differential equations (balance of linear momentum and of angular momentum) governing the dynamics of the Cosserat rod.
Using Cosserat theory, we derive the equations of motion for the two special cases of interest, namely, one in which the system is confined inside a channel with frictionless walls and one in which the system is placed on an anisotropic frictional environment with an infinite contrast between longitudinal and transversal friction, so that the rod can slide longitudinally along its axis, but cannot slip laterally (i.e., in the transversal direction).

The presence of constraints leads in both settings to nonstandard boundary conditions. These allow us to close the equations of motion and reduce them to a differential and an integro-differential equation, respectively, for one end point (the tail) of the active rod. In both cases we also provide analytic solutions for a special class of "serpentine" motions.

We highlight the role of the spontaneous curvature in providing the driving force (and the steering, in the freepath case) needed for locomotion. By solving the equation of motions in some specific examples, we obtain results that are also reminiscent of the experimental findings that are presented in the zoological literature. Thus, our analysis provides a scheme to rationalise the observed behaviour.

\section{ACKNOWLEDGEMENTS}

Financial support by the European Research Council through the Advanced Grant 340685-MicroMotility is gratefully acknowledged. We also gratefully acknowledge helpful discussions with P. Gidoni and G. Noselli.

\section{REFERENCES}

[1] D. Trivedi, C.D. Rahn, W.M. Kier, I.D. Walker. Soft robotics: Biological inspiration, state of the art, and future research. Applied Bionics and Biomechanics, 5.3: 99-117, 2008.

[2] S. Kim, C. Laschi, B. Trimmer. Soft robotics: a bioinspired evolution in robotics. Trends in Biotechnology, 31.5: 287-294, 2013.

[3] S. Hirose. Biologically inspired robots: snake-like locomotors and manipulators. Oxford University Press, 1993.

[4] J. Gray. The mechanism of locomotion in snakes. Journal of Experimental Biology 23.2:101-120, 1946.

[5] Z.V. Guo, L. Mahadevan. Limbless undulatory propulsion on land. Proceedings of the National Academy of Sciences USA, 105.9: 3179-3184, 2008.

[6] D.L. Hu, J. Nirody, T. Scott, M.J. Shelley. The mechanics of slithering locomotion. Proceedings of the National Academy of Sciences USA106.25:1008110085, 2009.

[7] G. Cicconofri, A. DeSimone. A study of snake-like locomotion through the analysis of a flexible robot model. Submitted for publication, 2014. 


\title{
Simple Modelling of the Rowing Process
}

\author{
Sergey Karmanov*. Felix L. Chernousko* \\ *Institute for Problems in Mechanics of the Russian Academy of Sciences, \\ 101-1 Vernadsky Ave, Moscow 119526 Russia \\ (e-mail: karmanovsp@gmail.com,chern@ipmnet.ru)
}

\section{ABSTRACT}

The dynamics of the rowing boats have been considered in a number of papers (Cabrera et al., 2006, Formaggia et al., 2009, Formaggia et al., 2010, Wingard, 1991). In these papers, several models of rowing boats are introduced and described, complex models of oar blade are considered.

In the paper, a simple model of a boat equipped with a pair of two identical oars and moving in a fluid is considered (Fig. 1). The oars perform periodic translational motions relative to the main body. The masses of oars are considered negligible.

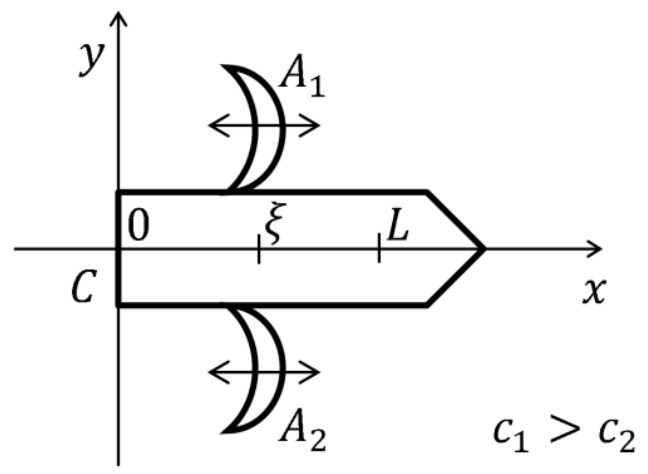

Fig. 1. Mechanical model.

Resistance forces acting upon the moving bodies are directed opposite to their velocities and proportional to the squared velocities. The resistance coefficients of the oars for the direct and reverse strokes are different (for the direct stroke they are greater than for the reverse stroke). The relative velocity of the oars is assumed to be constant during both the direct and reverse strokes (Fig. 2).

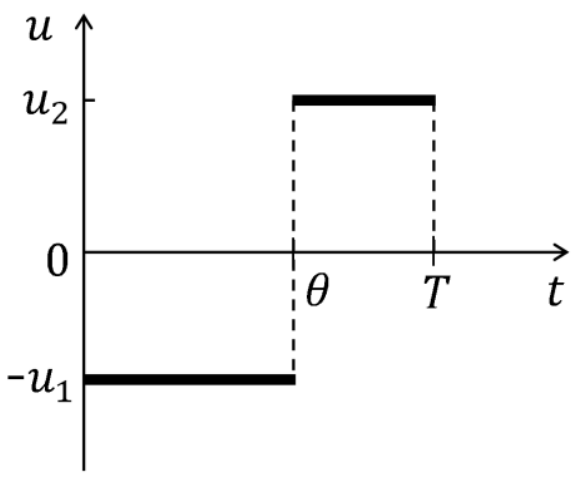

The equations of motion are analyzed and solved. Certain general properties of the rowing locomotion are established.

Series of numerical simulations are carried on. It is observed that the velocity of the main body tends to the periodic regime.

A special attention is given to cyclical motions in which the velocity of the body changes periodically in time. The conditions on the system parameters that ensure the existence of cyclical motions are obtained.

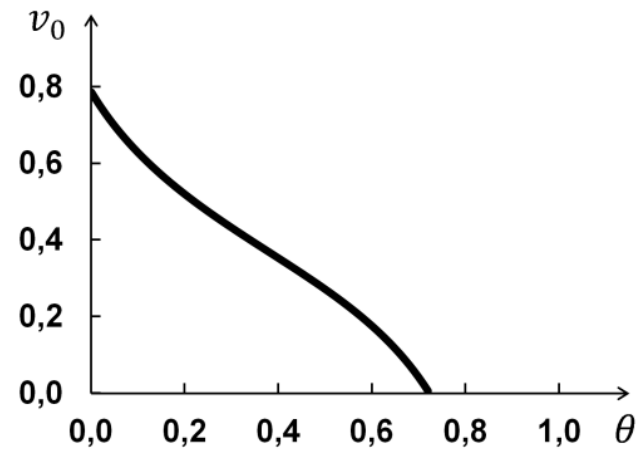

Fig. 3. Initial velocity vs the moment of the switch for the cyclical motion $(\mathrm{T}=1)$.

Acknowledgements. This study is supported by the Russian Science Foundation (Grant no.14-11-00298).

\section{REFERENCES}

Cabrera, D., Ruina, A., Kleshnev, V. (2006). A simple 1+ dimensional model of rowing mimics observed forces and motions. Human Movement Science, 25, 192-220.

Formaggia, L., Miglio, E., Mola, A., Montano, A. (2009). A model for the dynamics of rowing boats. International Journal for Numerical Methods in Fluids, 61(2), 119143.

Formaggia, L., Mola, A., Parolini, N., Pischiutta, M. (2010). A three-dimensional model for the dynamics and hydrodynamics of rowing boats. Proceedings of the Institution of Mechanical Engineers, Part P: Journal of Sports Engineering and Technology, 224(1), 51-56.

Wingard, P.A. (1991). Improving the Rowing Process with a Computer Model and Optimal Control Theory. Massachusetts Institute of Technology.

Fig. 2. Relative velocity of the oars 


\title{
Modelling of quasi-static motions for three-body mobile robot
}

\author{
Felix L. Chernousko* Tatiana Yu. Figurina* \\ * Institute for Problems in Mechanics of the Russian Academy of \\ Sciences, 101-1 Vernadsky Ave, Moscow 119526 Russia (e-mail: \\ chern@ipmnet.ru,t_figurina@mail.ru)
}

\begin{abstract}
Quasi-static motion of a three-body mobile robot along a horizontal plane in the presence of dry friction is considered. The control forces are due to pairwise interaction between the bodies. For the quasi-static motion, the control forces should be chosen so that the equilibrium conditions hold for each body and, hence, for the entire robot. The quasi-static motions, for which one of the bodies is moving, while the other two bodies are in a state of rest, are described. It is shown that if the products of the weight of each body by the corresponding friction coefficient satisfy the triangle inequalities, then each body of the robot can be quasistatically moved to any prescribed position in the plane, whereas the other two bodies are at rest. Thus, quasi-static controllability of the robot, subject to the aforementioned assumptions about the parameters, is proved. An optimal control problem for the moving body is solved, and the shortest (minimizing the work against friction) trajectory is shown to be a two-link broken line or a straight line segment. An algorithm for transferring the mobile robot to a given state is presented. The results obtained can be used for designing control strategies for mobile robotic systems.
\end{abstract}

Keywords: multibody mechanism, control, optimization, dry friction, robotics.

\section{EQUATIONS OF QUASI-STATIC MOTION}

Let us consider the mobile robot consisting of three bodies $M_{i}$ modelled as points with masses $m_{i}$ (bodies' sizes are neglected with respect to the distances between the bodies), $i=1,2,3$, on a horizontal rough plane (Figure 1 ). etween each pair of bodies $M_{i}$, control forces act, changing the distances between the bodies. Such mobile robot can move along a plane when changing its configuration due to the friction forces between the bodies and the plane. We study possible quasi-static motions of the robot, i.e. the motions with infinitesimal velocities and accelerations of the bodies.

Dry friction force $\mathbf{F}_{i}$ exerted upon body $M_{i}$ is given by equations

$$
\mathbf{F}_{i}=-k_{i} m_{i} g \frac{\mathbf{v}_{i}}{\left|\mathbf{v}_{i}\right|}, \mathbf{v}_{i} \neq 0, \quad\left|\mathbf{F}_{i}\right| \leq k_{i} m_{i} g, \mathbf{v}_{i}=0
$$

where $\mathbf{v}_{i}$ is the velocity of body $M_{i}, k_{i}$ is the friction coefficient, and $g$ is the gravity acceleration. For any pair of indices $i \neq j$, we have the interaction control force $\mathbf{f}_{i j}$ acting from body $M_{i}$ upon body $M_{j}$ and directed along $M_{i} M_{j}$

$$
\mathbf{f}_{i j}=-\mathbf{f}_{j i}, \quad \mathbf{f}_{i j} \times\left(\mathbf{r}_{i}-\mathbf{r}_{j}\right)=0, \quad i, j=1,2,3, \quad i \neq j(2)
$$

where $\mathbf{r}_{i}$ is the radius-vector of point $M_{i}$. We consider only such control forces $\mathbf{f}_{i j}$ that lead to quasi-static motion of each body $M_{i}$. In such motions, the equilibrium equations hold

* This study was partly supported by the Russian Science Foundation (project 14-11-00298).

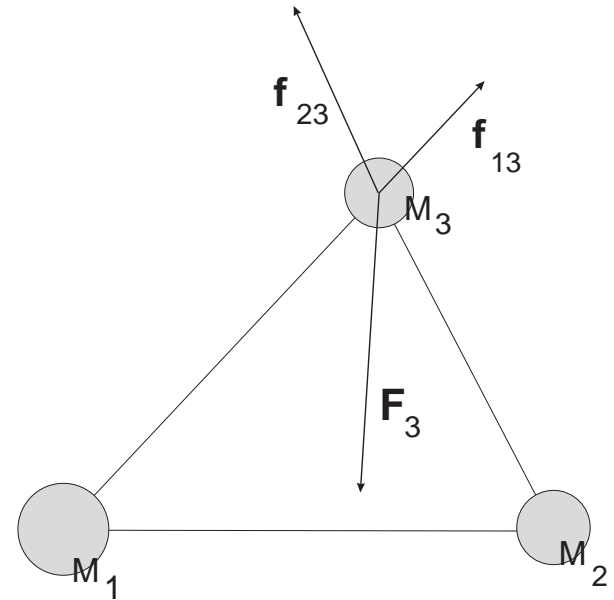

Fig. 1. Three-body system

$$
\mathbf{F}_{i}+\mathbf{f}_{j i}+\mathbf{f}_{k i}=0, \quad\{i, j, k\}=\{1,2,3\}
$$

From equations (3), it follows

$$
\sum_{i=1}^{3} \mathbf{F}_{i}=0, \quad \sum_{i=1}^{3} \mathbf{r}_{i} \times \mathbf{F}_{i}=0 .
$$

It is shown that if forces $\mathbf{F}_{i}$ satisfy equations (4), and points $M_{1}, M_{2}, M_{3}$ do not belong to a straight line, then the uniquely defined forces $\mathbf{f}_{i j}$ exist such that equations (3) hold. So we solve equations (4), instead of (3), taken together with relations (1) and (2). We suppose that the parameters of the system satisfy the following triangle inequalities 


$$
k_{\alpha} m_{\alpha}+k_{\beta} m_{\beta} \geq k_{\gamma} m_{\gamma}, \quad\{\alpha, \beta, \gamma\}=\{1,2,3\}
$$

It can be shown that each of the three inequalities (5) is a necessary condition for the possibility to move body $M_{\gamma}$.

\section{QUASI-STATIC MOTIONS WITH ONE MOVING ODY}

Let us find all quasi-static motions such that two bodies of the system (for example, $M_{1}$ and $M_{2}$ ) do not move, whereas the third body $\left(M_{3}\right)$ moves. Let $D$ be the intersection of the circles with centers in points $M_{1}$ and $M_{2}$ and radii $a k_{1} m_{1} /\left(k_{3} m_{3}\right), a k_{2} m_{2} /\left(k_{3} m_{3}\right)$, respectively, where $a=\left|\mathbf{r}_{1}-\mathbf{r}_{2}\right|$ is the distance between points $M_{1}$ and $M_{2}$. Set $D$ is not empty, according to inequality (5) with $\gamma=3$.

\section{Proposition 1}

Quasi-static motion of body $M_{3}$, with bodies $M_{1}$ and $M_{2}$ fixed, is possible iff the straight line containing point $M_{3}$ and parallel to its velocity intersects set $D$ (Figure 2).

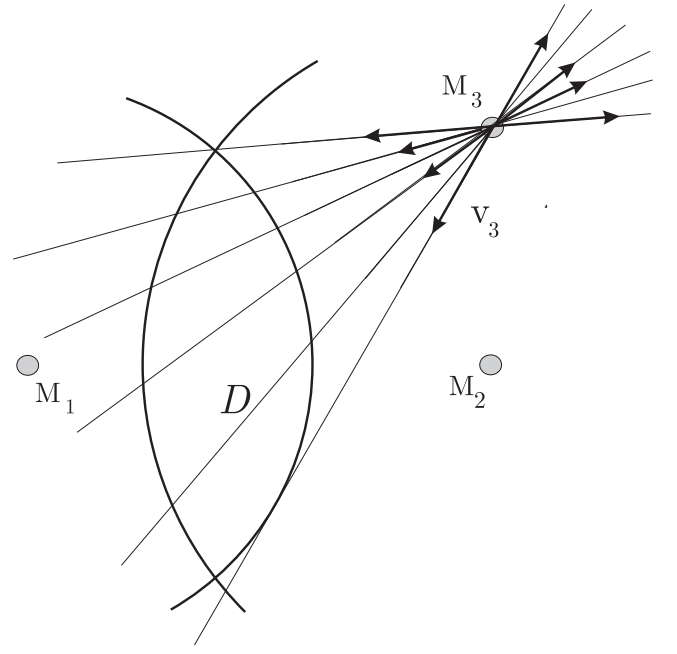

Fig. 2. Possible directions of body $M_{3}$ motion

\section{Corollary}

Under conditions (5), the three-body mobile robot is quasistatically controllable, i.e., can be quasi-statically brought to any preset position.

\section{OPTIMAL QUASI-STATIC MOTIONS.}

Let us consider optimal control problem for the moving body. Let two points $A$ and $B$ be fixed on the plane. It is required to move quasi-statically body $M_{3}$ from point $A$ to point $B$ with minimal work against friction, i.e., to construct the trajectory of quasi-static motion for body $M_{3}$ with the minimum length.

\section{Proposition 2}

If the straight line $A B$ intersects set $D$, then the trajectory with the minimum length for body $M_{3}$ quasi-statically moving from point $A$ to point $B$ is the segment $A B$. If the the straight line $A B$ does not intersect set $D$, then the trajectory with the minimum length is two-link broken line $A C B$ with links $A C$ and $B C$ belonging to the supporting straight lines of set $D$. Here, point $A$ and set $D$ are located on different sides of straight line $B C$, and point $B$ and set $D$ are located on different sides of straight line $A C$ (Figure $3)$.

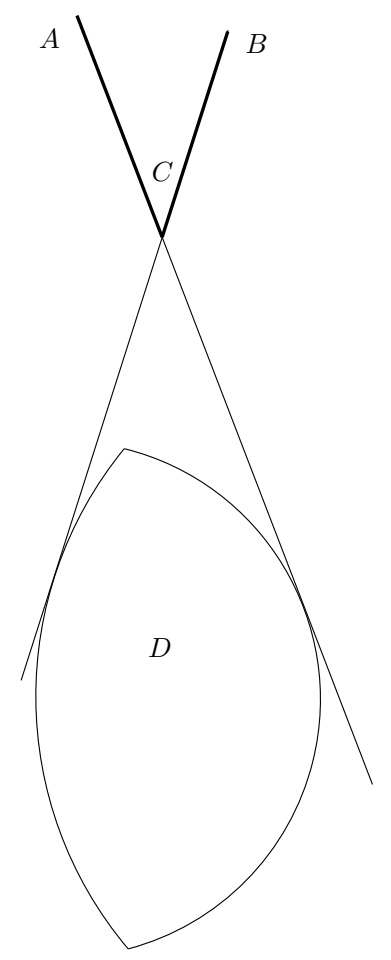

Fig. 3. Optimal trajectory

The algorithm for the displacement of the three-body mobile robot from any initial position to any given terminal one is presented. At any moment, only one body is moving, and each body moves only during one time interval. There exist six ways of displacement, corresponding to six possible permutations of bodies' numbers. If permutation $(i, j, k)$ is chosen, then bodies $M_{i}, M_{j}, M_{k}$ move one after another, while two other bodies are at rest. During each time interval, the moving body follows along the shortest trajectory which is either a straight line or a two-link broken line, according to Proposition 2. Comparing these six ways of displacement, the algorithm corresponding to the minimum work can be chosen.

\section{CONCLUSION}

Quasi-static motions of a three-body robot on a horizontal plane in the presence of dry friction is analyzed. Interaction forces between each pair of bodies are considered as controls. The case in which one body is moving whereas two others are at rest is considered. It is shown that, if the triangle inequalities (5) for the masses and friction coefficients holds, then the moving body can be relocated from any initial position to any terminal one. Optimal trajectory (minimizing the work against friction along the trajectory) is found and is proved to be a a polygonal chain of one or two line segments. Quasi-static controllability of the system is established. The algorithm for relocating of the system to the given state is presented. The case where the distances between the bodies are bounded from above and/or below is to be studied. 


\title{
Control of a Chain-like Locomotion System Subject to Undulatory Excitation
}

\author{
Nikolay Bolotnik* Mikhail Pivovarov ${ }^{* *}$ Igor Zeidis ${ }^{* *}$ \\ Klaus Zimmermann ${ }^{* *}$ \\ * Institute for Problems in Mechanics of the Russian Academy of \\ Sciences, 101-1 Vernadsky Ave, Moscow 119526 Russia (e-mail: \\ bolotnik@ipmnet.ru) \\ ** Ilmenau University of Technology, PF 1005 65, D-98684 Ilmenau, \\ Germany,(e-mail: zeidis.igor@tu-ilmenau.de)
}

The motion of a chain of bodies along a horizontal straight line in a resistive environment is studied. The motion is excited and controlled by changing the distances between the adjacent bodies. Such systems imitate the locomotion of worms that do not have extremities and move due to redistribution of mass within their bodies and contact with the environment. Therefore, investigation of the behavior of such systems may contribute to more profound understanding of the principles of limbless locomotion of living beings. On the other hand, these principles of motion can be used for some kinds of mobile robots. The advantage of such worm-like robots in comparison with the conventional mobile mechanisms is that robots of this type do not require special propelling devices (wheels, legs or caterpillars) and transmissions, are simple in design and easy to miniaturize. For a consistent presentation of recent research results related to the worm-like locomotion, see, e.g., the books by Zimmermann et al. (2009) and Steigenberger and Behn (2012).

In our paper, we consider a chain of three identical bodies depicted in Fig. 1. The bodies are modeled by particles of mass $M$ each. The coordinates of the particles in a fixed reference frame are denoted by $x_{1}, x_{2}$, and $x_{3}$, respectively; $l_{1}=x_{2}-x_{1}, l_{2}=x_{3}-x_{2}$. Between the bodies of the chain and the surface along which the system moves, dry Coulomb's friction acts. The quantities $l_{1}$ and $l_{2}$ are specified by explicit $T$-periodic functions of time $t$ that are related by

$$
l_{2}(t)=l_{1}(t+\tau), \quad|\tau|<T .
$$

For this type of excitation, the relative motion of the bodies of the chain resembles a wave traveling with velocity $1 /|\tau|$ leftward (from body 3 to body 1 ), if $\tau>0$, or rightward (from body 1 to body 3 ), if $\tau<0$.

We assume that the forces of friction between the bodies of the system and the surface along which it moves are small in comparison with the forces of interaction of the chain's bodies. We introduce a small parameter and approximate the equation of motion of the system's center of mass by the averaged equation obtained by averaging the primary equation with respect to the explicit time. Based on the averaged equation, we investigate the motion of the center

\footnotetext{
‡ This study was partly supported by the German Research Society (DFG) (projects ZI 540/19-1 and SFB 622) and the Russian Foundation for Basic Research (project 14-01-00061 and 15-51-12381).
}

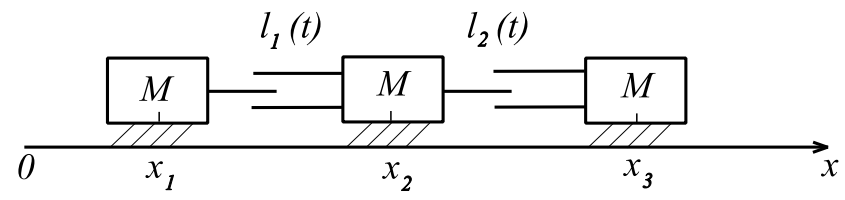

Fig. 1. A three-body limbless locomotion system

of mass of the chain for the case where $l_{1}(t)$ and $l_{2}(t)$ are specific piecewise linear or piecewise quadratic functions.

For these excitation modes, we established that the system can start moving from a state of rest only if the propagation velocity of the excitation wave exceeds a threshold value. The chain can move only in the direction opposite to that of the wave propagation. The velocity of the center of mass of the chain approaches a steady-state value, as time elapses. The steady-state motion of the system is asymptotically stable with respect to the velocity of the center of mass. The steady-state velocity continuously depends on the wave propagation velocity. The steady-state velocity is close to zero, if the wave propagation velocity is close to the threshold value or approaches infinity, and has a maximum for some value of the wave propagation velocity. The piecewise quadratic excitation is inferior to the piecewise linear excitation in the range of the wave propagation velocities at which the system can start moving from a state of rest for both types of excitation. In this range, the piecewise linear excitation mode provides higher value for the velocity of the steady-state motion of the chain's center of mass. The threshold values of the excitation wave propagation velocity, until achieving which the system cannot start moving, are the same for both excitation modes. However, unlike the piecewise quadratic mode, the piecewise linear mode has an upper bound for the wave propagation velocity, beyond which the motion of the system becomes impossible.

\section{REFERENCES}

J. Steigenberger and C. Behn. Worm-like Locomotion Systems: an Intermediate Theoretical Approach. Oldenbourg Wissenschaftsverlag, Munich, 2012.

K. Zimmermann, I. Zeidis, and C. Behn. Mechanics of Terrestrial Locomotion with a Focus on Nonpedal Motion Systems. Springer, Heidelberg, 2009. 


\title{
An Approach to the Dynamics of Vibration-driven Robots with Bristles
}

\author{
Felix Becker \\ Technische Universität Ilmenau, Technical Mechanics Group, PF 100565 \\ 98693 Ilmenau, Germany (Tel: +49 367769 1845; e-mail: felix.becker@tu-ilmenau.de).
}

\begin{abstract}
In this paper, a rigid body model of vibration-driven robots with bristles is presented and studied numerically. Bristles provide asymmetric friction properties to transform the vibrations of internal masses to a directed locomotion. The aim of this study is to investigate the motion principle of robots with bristles based on mechanical models and to find the influence of system parameters to the locomotion velocity.
\end{abstract}

Keywords: Mobile robots, Vibration, Robot dynamics, Bristle, Anisotropic Coulomb friction.

\section{INTRODUCTION}

Vibration-driven robots are excited by periodic oscillations of internal masses. Asymmetric characteristics of the system are required to transform the internal motion to a directed locomotion. A common realization in nature and engineering are bristles or spikes. They can mechanically interlock to the surface and provide asymmetric friction forces. Zimmermann et al. (2009), Behn and Steigenberger (2012), Bolotnik et al. (2013) and Chernousko (2014) studied models of worm-like locomotion systems. They consist of several bristle-attached segments, which can move relative to each other in the direction of motion. The impact of the bristles is modelled by Coulomb friction with the coefficient $\mu$, with different values for the forward (+) and backward (-) motions: $\mu_{+} \neq \mu_{-}$. Ioi (1999), DeSimone and Tatone (2012), Giomi and Hawley-Weld (2013) analysed vibration-driven robots taking into account elastic properties of the bristles. The present study follows the same approach for singlesegmented robots.

\section{MODEL}

To study the dynamics of vibration-driven robots with bristles, the two-dimensional rigid body model, presented in Fig. 1, is developed. Two Cartesian coordinate systems are introduced - the fixed reference frame $(0 x y)$ and

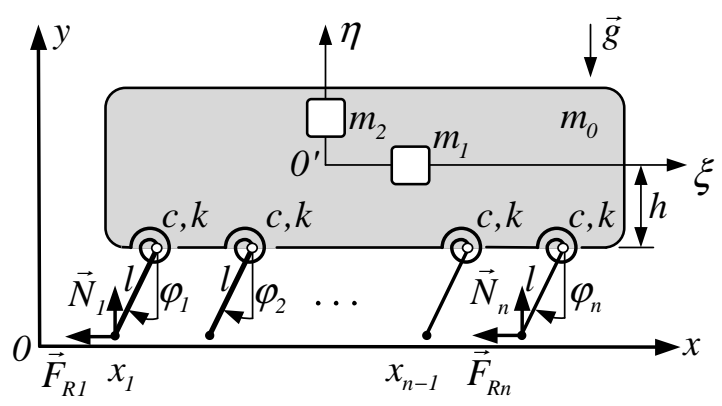

Fig. 1: Model of a vibration-driven robot with bristles the body-attached frame $\left(0^{\prime} \xi \eta\right)$. The model consists of a main body with the mass $m_{0}$ and two internal point masses $m_{1}$ and $m_{2}$. They are excited kinematically with the periodic functions $\xi(t)$ and $\eta(t) . m_{1}$ vibrates in the horizontal direction and $m_{2}$ in the vertical direction. To model the bristles, a number $n$ of weightless support elements with the length $l$ are connected to the main body by rotational springs $c$ and dampers $k . \varphi_{1}, \ldots, \varphi_{n}$ are inclination angles of the bristles to the vertical direction. The endpoints have constant contact to the surface. The system cannot rotate in the plane ( $0 x y)$. Therefore,

$$
y=h+l \cos \varphi
$$

where $\varphi=\varphi_{i}$, with $i=1, \ldots, n$.

All contact points have the same velocity $\dot{\tilde{x}}=\dot{x}-l \dot{\varphi} \cos \varphi$, where $\dot{x}$ is the velocity of the system. Coulomb friction forces are modelled as

$$
F_{R}=-\mu|N| \operatorname{sign}(\dot{\tilde{x}}) .
$$

The coefficient of friction is determined by

$$
\mu= \begin{cases}\mu_{-}, & \text {if } \dot{x}-l \dot{\varphi} \cos \varphi<0, \\ \mu_{+}, & \text {if } \dot{x}-l \dot{\varphi} \cos \varphi>0 .\end{cases}
$$

With $m_{R}=m_{0}+m_{1}+m_{2}$ as the overall mass of the robot, the normal force is determined by

$$
N=m_{R}\left(g-l \ddot{\varphi} \sin \varphi-l \dot{\varphi}^{2} \cos \varphi\right)+m_{2} \ddot{\eta} .
$$

Using the Lagrange equations of the second kind, the equations of motion can be given as

$$
\ddot{x}+\frac{m_{1}}{m_{R}} \ddot{\xi}=-\mu\left[g-l \ddot{\varphi} \sin \varphi-l \dot{\varphi}^{2} \cos \varphi+\frac{m_{2}}{m_{R}} \ddot{\eta}\right] \operatorname{sign}(\dot{\tilde{x}}),
$$




$$
\begin{array}{r}
{\left[\ddot{\varphi} \sin \varphi+\dot{\varphi}^{2} \cos \varphi-\frac{g}{l}-\frac{m_{2}}{m_{R} l} \ddot{\eta}\right][\sin \varphi+\mu \cos \varphi \operatorname{sign}(\dot{\tilde{x}})]} \\
+\frac{n k}{m_{R} l^{2}} \dot{\varphi}+\frac{n c}{m_{R} l^{2}}\left(\varphi-\varphi_{0}\right)=0 .
\end{array}
$$

$\varphi_{0}$ is the bristle angle in the unloaded state.

\section{SIMULATIONS}

The dynamics of the system is studied for two exemplary model configurations (see Fig. 2) with the values of parameters given in Table 1. Model A is a system with spikes and horizontal excitation. $N$ remains constant. The locomotion is achieved by the anisotropy of $\mu$. Model B represents a system with long, elastic bristles, which deflect considerably while the locomotion. It is excited by the vertical vibration of $m_{2}$. The locomotion depends on the periodic normal force. The displacement $x$ of the system, the deflection $\varphi$ of the bristles, the friction force $F_{R}$ and the normal force $N$ are plotted over the time $t$ in Fig. 2.

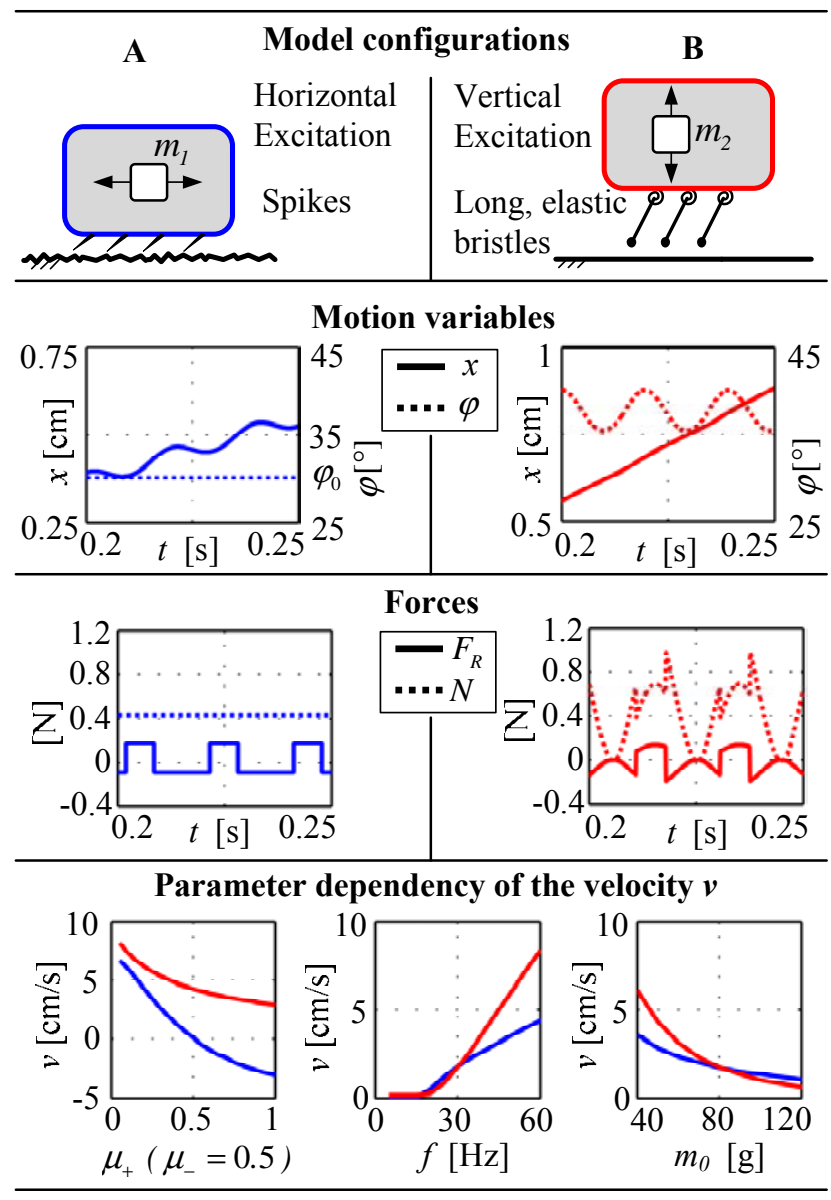

Fig. 2: Model configurations and results of the simulations

Numerical parameter studies have been performed to find the dependency of the locomotion velocity on the anisotropy of the friction coefficient, the excitation frequency and the mass of the main body. As it is assumed that the system has constant contact to the surface, the ranges of parameters are limited by the condition $N \geq 0$. Fig. 2 shows that the decrease of the system's mass or the increase of the excitation frequency leads to a faster locomotion. While the motion direction of Model A is determined only by friction conditions, Model B is more robust against surrounding conditions. It can move forward, even if the friction coefficient in the backward direction is considerably smaller. Further results and comparison with experiments are given in detail in Becker et al., 2014 (a). A prototype for the inspection of pipelines is presented in Becker et al., 2014 (b).

Table 1. Model parameters for the simulations

\begin{tabular}{|c|c|c|c|}
\hline & & Model A & Model B \\
\hline Overall mass & $m_{R}$ & \multicolumn{2}{|c|}{$44 \mathrm{~g}$} \\
\hline Internal masses & $m_{1}=m_{2}$ & \multicolumn{2}{|c|}{$2 \mathrm{~g}$} \\
\hline Length & $l$ & $1 \mathrm{~mm}$ & $10 \mathrm{~mm}$ \\
\hline Unloaded angle & $\varphi_{0}$ & \multicolumn{2}{|c|}{$30^{\circ}$} \\
\hline Stiffness & $n \cdot c$ & $0.2 \mathrm{Nm}$ & $0.02 \mathrm{Nm}$ \\
\hline Damping & $n \cdot k$ & $2 \cdot 10^{-4} \mathrm{~kg} \mathrm{~m}^{2} / \mathrm{s}$ \\
\hline \multirow{2}{*}{ Friction } & $\mu_{+}$ & \multicolumn{2}{|c|}{0.2} \\
\cline { 2 - 4 } & $\mu_{-}$ & 0.4 & 0.2 \\
\hline \multirow{2}{*}{ Excitation } & $\xi(t)$ & $A \sin (\omega t)$ & 0 \\
\cline { 2 - 4 } & $\eta(t)$ & 0 & $A \sin (\omega t)$ \\
\cline { 2 - 4 } & $A$ & \multicolumn{2}{|c|}{$5 \mathrm{~mm}$} \\
\cline { 2 - 4 } & $\omega$ & \multicolumn{2}{|c|}{$2 \pi \cdot 50 \mathrm{~Hz}$} \\
\hline
\end{tabular}

\section{REFERENCES}

Becker, F., Börner, S., Lysenko, V., Zeidis, I. and Zimmermann, K. (2014). On the Mechanics of Bristlebots - Modeling, Simulation and Experiments. Proc. of ISR / Robotik 2014, pp. 15-20, VDE Verlag, Berlin.

Becker, F., Börner, S., Kästner, T., Lysenko, V., Zeidis, I. and Zimmermann, K. (2014). Spy Bristle Bot - A Vibration-driven Robot for the Inspection of Pipelines. Proc. of $58^{\text {th }}$ IWK, Int. Scient. Coll., Ilmenau.

Behn, C. and Steigenberger, J. (2012). Worm-like Locomotion Systems, Oldenbourg, München.

Bolotnik, N., Pivovarov, M., Zeidis, I. and Zimmermann, K. (2013). The Undulatory Motion of a Chain of Particles in a Resistive Medium in the Case of a Smooth Excitation Mode. ZAMM 93 (12) pp. 895-913.

Chernousko, F. L. (2014). Dynamics and Optimization of Multibody Systems in the Presence of Dry Friction. Constructive Nonsmooth Analysis and Related Topics, 87, pp. 71-100, Springer, New York.

DeSimone, A. and Tatone, A. (2012). Crawling Motility through the Analysis of Model Locomotors: Two Case Studies. Eur. Phys. J. E., 35 (85).

Giomi, L. and Hawley-Weld, L. (2013). Swarming, Swirling and Stasis in Sequestered Bristle-bots. Proc. R. Soc. A 469, 20120637, pp. 1-21.

Ioi, K. (1999). A Mobile Micro-robot using Centrifugal Forces. Proc. Int. Conf. Adv. Intell. Mech., pp. 736-741.

Zimmermann, K., Zeidis, I. and Behn, C. (2009). Mechanics of Terrestrial Locomotion, Springer, Berlin. 


\title{
DYNAMICS AND CONTROL OF A SUCTION-TYPE WALL-CLIMBING ROBOT
}

\author{
Yunafi'atul Aniroh*, Andika P. Yudha*, Hung-Chyun \\ Chou*,Chung-Hsien Kuo*, Felix L. Chernousko**, V.G. \\ Gradetsky** and Nikolay Bolotnik** \\ *Department of Electrical Engineering, National Taiwan \\ University of Science and Technology, Taipei, Taiwan, ROC (e-mail: \\ $\{m 10007808, m 10207813, d 9907308, c h k u o\} @ m a i l . n t u s t . e d u . t w)$ \\ **The Institute for Problems in Mechanics RAS, Moscow, Russia \\ (e-mail: \{chern,gradet,bolotnik\}@ipmnet.ru)
}

\begin{abstract}
This paper presents the trajectory tracking approach for a wall-climbing robot by using adaptive control schemes. The most important consideration for controlling the wall-climbing robot is to make sure that the wheels can be always well contacted to the wall regardless of the slope conditions without sacrificing robot's mobility. To consider different slope conditions of the wall, this paper proposes adaptive control schemes to alter the vacuum force so that different gravity effects can be properly dealt with. Practically, pressure and IMU sensors are used to provide the vacuum force and spatial posture information for realizing adaptive control schemes. Finally, MATLAB simulations and real tests for dealing with different surface slope conditions were performed with the triangle trajectories.
\end{abstract}

Keywords: Wall climbing robot, adaptive control, trajectory tracking, inertial measurement unit

\section{INTRODUCTION}

The wall climbing robots are able to perform dangerous operation, such as the inspection of high-rise building, spray painting and sand blasting of gas tanks, maintenance of nuclear facilities, surveillance and reconnaissance,...etc. The robotics team of the City College of New York (CCNY) has developed the wall climbing robot with the function of transit from two different surfaces, such as grounds, walls, and ceilings.

This paper proposes an adaptive control schemes to alter the vacuum force, and different gravity effects can be properly dealt with. In order to implement the adaptive controller, the dynamic model of the robot as friction, slip, and weight needs to be determined.

Traditional adaptive control is not sufficiently mature to solve control problems with challenges which the strict performance and the guarantees of robustness are required, because set of unknown constants need to be estimated. The parameter estimation needs an approach that explicitly accounts for robust performance and stability specifications. To achieve this goal, this paper proposes a robust adaptive control approach, sliding controller. The sliding controller is expected to avoid the slips of wheels, as well as to reduce the power consumptions of the wheel motors and the suction motor. The sliding controller design provides a systematic approach to solve the problem of maintaining stability and consistent performance while facing to the modeling imprecision.

\section{DYNAMIC MODELLING}

The wall climbing robot has parameter variations and uncertainties which are caused by friction, slip, and weight. The suitable controller of the robot is determined according to the dynamics model. In the project, the wall climbing robot is capable to move on the surface with different slopes.

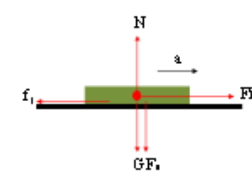

(a)

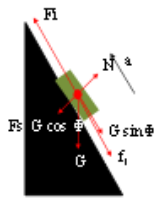

(b)

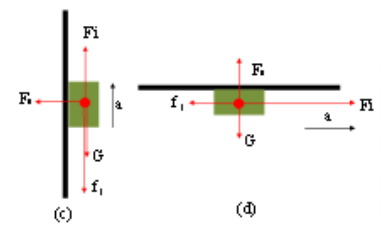

(d)
Fig. 1. The cases of different surfaces, (a) on the floor. (b) on the slope. (c) on the wall. (d). on the ceiling

The differential equations is expressed as

$$
\left[\begin{array}{c}
m \ddot{x} \\
m \ddot{y} \\
J \ddot{\theta}
\end{array}\right]=\alpha \boldsymbol{P}(\theta) \boldsymbol{U}(t)-2 \beta\left[\begin{array}{c}
\dot{x} \\
\dot{y} \\
d^{2} \dot{\theta}
\end{array}\right]
$$

With

$$
\begin{aligned}
& P(\theta)=\left[\begin{array}{ccc}
-\sin \theta & \sin \theta & \sin \theta \\
\cos \theta & -\cos \theta & -\cos \theta-\frac{G \sin \phi \cos ^{2} \theta}{2 f_{1}} \\
1 & 1 & -\operatorname{sgn}(\theta) \frac{M_{r}}{2 d f_{1}}
\end{array}\right] \\
& \boldsymbol{U}(t)=\left[\begin{array}{c}
U_{1}(t) \\
U_{2}(t) \\
\frac{2 f_{1}}{\alpha}
\end{array}\right]
\end{aligned}
$$

Where $\mathrm{d}$ is the distance of the tracked wheel from $\mathrm{CM}$; $\mathrm{G}$ is the weight of the robot; $\mathrm{m}$ is the mass of the robot; $f_{i}$ is the rolling friction between tracked wheel and surface; $M_{r}$ is the resistance moment produced by friction 
on surface. $\alpha(\mathrm{N} / \mathrm{V})$ can be determined as $\alpha=\frac{k_{t}}{J R_{a} r_{g}}$, where $\mathrm{R}_{\mathrm{a}}$ is the armature resistance of the motors; $r_{g}$ is the gear ratio; $k_{t}$ represents the torque coefficient; $J$ denotes the moment of inertia; $U_{i}$ is the voltage which applies to the $i$ th motor, and $v_{i}$ is the velocity of the $i$-th wheel.

\section{SLiding CONTROLler}

This part describes the control of nonlinear systems of the form which is derived in chapter II where the models are imprecise. By defining the vector $\ddot{Z}=\left[\begin{array}{lll}\ddot{x} & \ddot{y} & \ddot{\theta}\end{array}\right]^{T}$, the dynamic model (1) is rewritten as the following state space equations:

$$
M \ddot{Z}=b U-f_{1}-f_{2} \cdot g_{1}-f_{3} \cdot g_{2}
$$

Where :

$$
\begin{aligned}
& b=\alpha\left[\begin{array}{cc}
-\sin \theta & \sin \theta \\
\cos \theta & -\cos \theta \\
d & d
\end{array}\right] ; f_{1}=B \dot{Z} ; f_{3}=\sin \phi ; \\
& B=\left[\begin{array}{ccc}
2 \beta & 0 & 0 \\
0 & 2 \beta & 0 \\
0 & 0 & 2 \beta d^{2}
\end{array}\right] ; g_{1}=\left[\begin{array}{c}
\sin \theta \\
-\cos \theta \\
-\operatorname{sgn}(\dot{\theta})
\end{array}\right] ; g_{2}=\left[\begin{array}{c}
0 \\
-G \cos ^{2} \theta \\
0
\end{array}\right] ; \\
& M=\left[\begin{array}{ccc}
m & 0 & 0 \\
0 & m & 0 \\
0 & 0 & J
\end{array}\right] ; f_{2}=\left[\begin{array}{ccc}
2 f & 0 & 0 \\
0 & 2 f & 0 \\
0 & 0 & M r
\end{array}\right] ; \text { and } \beta=\frac{k_{e} k_{t}}{J R_{a}}
\end{aligned}
$$

Where $e$ is equal to $Z-Z_{d} ; k_{e}$ denotes the backemf coefficient; $\theta$ is $x y$ plane attitude; $\phi$ is slop attitude and the desired differentiable trajectory is described by $Z_{d}=\left[\begin{array}{lll}x_{d} & y_{d} & \theta_{d}\end{array}\right]^{T} . \lambda$ is a strict constant. The controller design is translated in terms of finding a control law for the vector $\mathbf{U}$ that verifies the individual sliding conditions of the differentiable form of vector $\mathbf{s}$ :

$$
\dot{s}=\left[-M^{-1} f_{1}-M^{-1} \hat{f}_{2} g_{1}-M^{-1} \hat{f}_{3} g_{2}-\ddot{Z}_{d}+\lambda \dot{e}\right]-M^{-1} \Delta \hat{f}_{2} g_{1}-M^{-1} \Delta \hat{f}_{3} g_{2}+M^{-1} b U
$$

$\lambda$ is a strict constant. To eliminate the chatter, term $\operatorname{sgn}(\mathrm{s})$ of $\boldsymbol{U}$, is replaced by sat $(s / \Phi)$.

$$
\begin{aligned}
& \text { 1. If }|s|>\Phi \\
& U=-M b^{-1}\left\{\left[-M^{-1} f_{1}-M^{-1} \hat{f}_{2} g_{1}-M^{-1} \hat{f}_{3} g_{2}-\ddot{Z}_{d}+\lambda \dot{e}\right]+\eta_{1}(s / \Phi)\right\}
\end{aligned}
$$

2. If $|s|<\Phi$

$U=-M b^{-1}\left\{\left[-M^{-1} f_{1}-M^{-1} \hat{f}_{2} g_{1}-M^{-1} \hat{f}_{3} g_{2}-\ddot{Z}_{d}+\lambda \dot{e}\right]+\eta_{1} \cdot \operatorname{sgn}(s)\right\}$

let's pick :

$$
\eta_{1}=\alpha_{1}+\alpha_{2}+\eta \quad ; \eta>0
$$

\section{EXPERIMENTS RESULT}

Fig. 2 shows experimental and simulation result of climbing robot triangle trajectory. The parameters in the simulations are selected from datasheet as followings: $\alpha=$ $131.5363 \mathrm{~N} / \mathrm{v} ; \beta=71.1087 \mathrm{Kg} / \mathrm{s} ; \mathrm{U}_{\max }=24.0 \mathrm{~V} ; \mu=0.4 ; \mathrm{m}$ $=1.7 \mathrm{Kg} ; \mathrm{G}=16.6 \mathrm{~N} ; 1=58 \mathrm{~mm} ; \mathrm{d}=11.5 \mathrm{~cm} ; \rho_{\mathrm{r}}=333.8$ $\mathrm{N} / \mathrm{m} ; \mathrm{J} \approx 1.08 .10^{-6} \mathrm{Kgm}^{2} ; \Phi=5000 ; \lambda=5000 ; \eta=$ $\operatorname{diag}\{0.1,0.1,0.1\}$, and suppose the actual values for uncertain parameters are $f_{2_{1}} \in[0,88], \hat{f}_{2_{1}}=44 \Delta \hat{f}_{2_{1}}=\alpha_{1}=44$, $f_{2_{2}} \in[0,88], \hat{f}_{2_{2}}=44, \Delta \hat{f}_{22}=\alpha_{2}=44, f_{23} \in[0,88], \hat{f}_{2_{3}}=44$, $\Delta \hat{f}_{23}=\alpha_{3}=44, f_{3} \in[-1,1], \hat{f}_{3}=0, \Delta \hat{f}_{3}=\delta=1$.

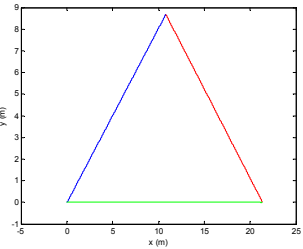

(a)

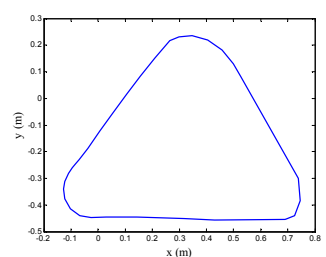

(c)

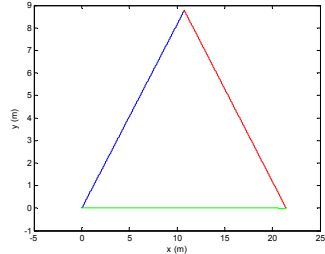

(b)

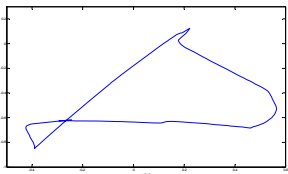

(d)
Fig. 2. Triangle tracking performance Result of the robot on the different slope (a) Simulation Result on the wall, (b) on ceiling, (c) Experimental result with $\lambda=24000$, (d) Experimental result with PID Controller.

\section{CONCLUSION}

This paper presents a wheeled wall climbing robot robust adaptive control method of trajectory tracking. The second-order nonlinear dynamic model with two unknown parameters and bounded uncertainties are constructed via the Newtonian second motion law. Based on this dynamic model, the adaptive robust controller is synthesized via adaptive gain smooth sliding controller and achieves both trajectory tracking and stabilization. A smooth sliding controller with adaptively update the gains for the switching functions is proposed in order to control the nonlinear systems. Simulation and experiment confirm the theoretical results and conform to the expected robot trajectory planning.

\section{REFERENCES}

A. Filipescu, L. Dugard, J-M. Dion, “Adaptive gain sliding observer based sliding controller for uncertain parameters nonlinear systems. Application to flexible joint robots.

Decision and Control", IEEE Conference, Volume 4, Issue, 9-12 Dec. 2003 Page(s): 3537-3542.

R. Yue, J. Xiao, S. Wang, S. L. Joseph, "Modeling and Path Planning of the City_climber Robot Part I : Dynamic Modeling", Proc. Of the 2009 IEEE Int. Conf. on Robotics and Biomemetics, Gulin, China, December 19-23, 2009. 


\title{
Cascaded Nonlinear Control of a Duocopter with Disturbance Compensation by an Unscented Kalman Filter
}

\author{
Harald Aschemann, Thomas Meinlschmidt* \\ * Chair of Mechatronics, University of Rostock, D - 18059 Rostock, \\ Germany, (e-mail: Harald.Aschemann@uni-rostock.de).
}

\section{EXTENDED ABSTRACT}

A cascaded control strategy for an innovative Duocopter test rig as depicted in Fig. 1 - a helicopter with two rotors combined with a guiding mechanism - is presented in this paper. The guiding mechanism consists of a rocker arm with a sliding carriage that enforces a planar workspace of the Duocopter. The Duocopter is attached to the carriage by a rotary joint and offers 3 degrees of freedom. The derived system model has similarities with a PVTOL and a planar model of a quadrocopter but involves additional terms due to the guiding mechanism. In the paper, a model-based cascaded control strategy is proposed: in the outer MIMO control loop, sliding mode techniques are employed to control both the horizontal and the vertical Duocopter position. The rotation angle of the Duocopter is controlled in a linear inner control loop using flatnessbased techniques. An additional feedforward control takes into account all known parts of the coupling forces between the carriage and the rocker. The control structure is extended by an unscented Kalman filter (UKF), see Julier and Uhlmann (1997) and van der Merwe (2004), that provides estimates for the state vector and, moreover, estimates for remaining errors concerning the feedforward coupling forces. The sum of the feedforward part and the estimated part can be used to accurately compensate for the impact of the guiding mechanism on the motion of the Duocopter frame. The efficiency of the proposed control strategy is demonstrated by experiments. As shown in Fig. 2, it provides a precise tracking of desired trajectories for the Duocopter position in both horizontal and vertical direction that outperforms the results achieved in Meinlschmidt and Aschemann (2014).

\section{REFERENCES}

Julier, S. and Uhlmann, J. (1997). A New Extension of the Kalman Filter to Nonlinear Systems. In Proceedings of the SPIE - Int. Soc. Opt. Eng. (USA), volume 3068. Orlando, FL.

Meinlschmidt, T. and Aschemann, H. (2014). Cascaded Flatness-Based Control of a Duocopter Subject to Unknown Disturbances. In IEEE Intl. Conference on Methods and Models in Automation and Robotics MMAR 2014. Miedzyzdroje, Poland.

van der Merwe, R. (2004). Sigma-Point Kalman Filters for Probabilistic Inference in Dynamic State-Space Models. Ph.D. thesis, OGI School of Science \& Engineering, Oregon Health \& Science University.
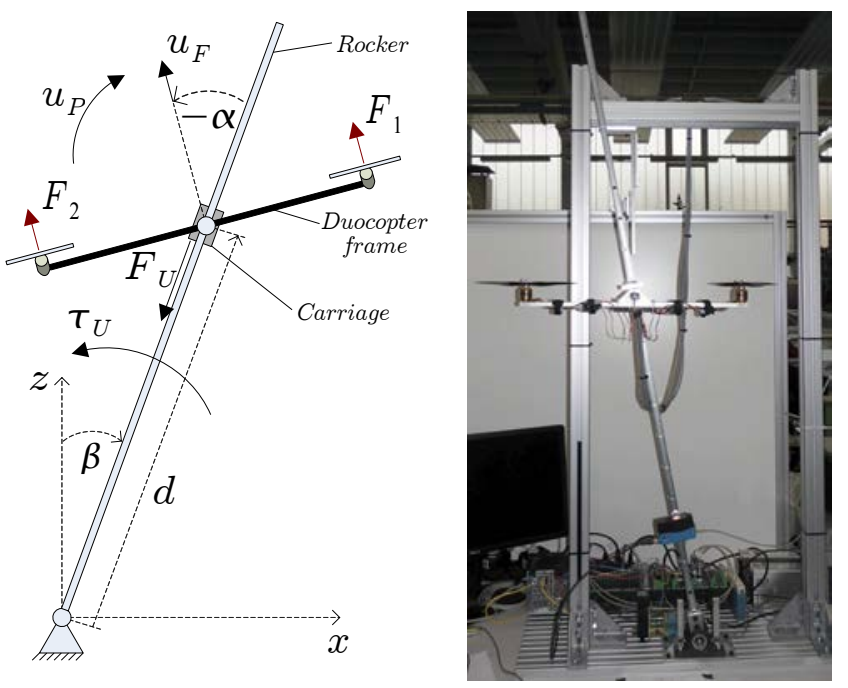

Fig. 1. Mechanical structure and corresponding test rig.

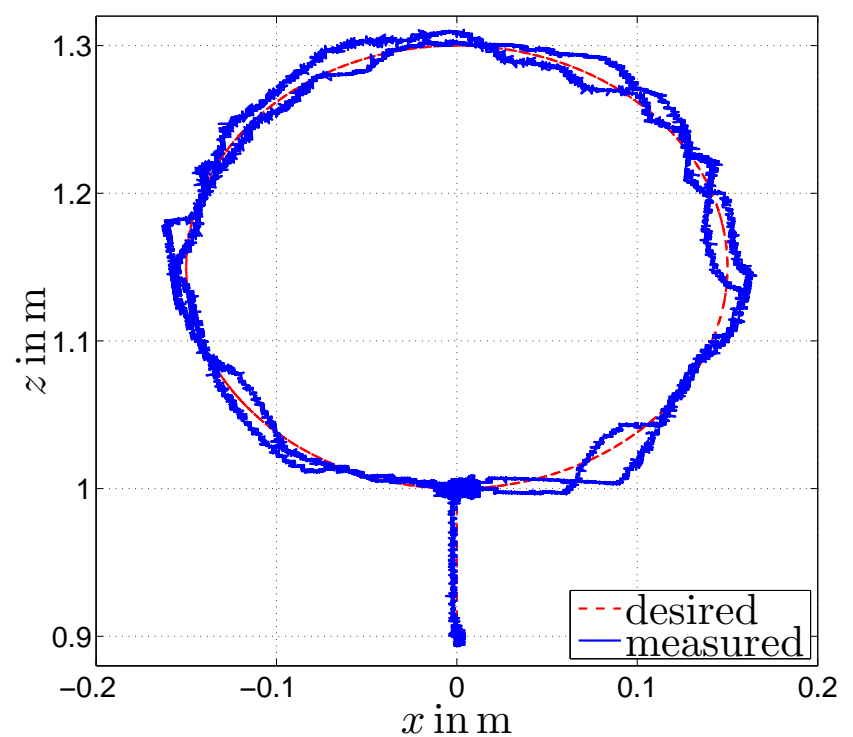

Fig. 2. Trajectory and tracking performance in the $x, z$ plane: experimental results using the proposed sliding mode control. 


\title{
Mathematical and dynamic model of the lower limb exoskeleton and synthesis of anthropomorphic gait *
}

\author{
Anton P. Aliseychik* Igor A. Orlov* Vladimir E. Pavlovsky* \\ Alexander K. Platonov* Alexander A. Ptakhin ${ }^{* *}$ \\ * Keldysh Institute of Applied Mathematics (Russian Academy of \\ Sciences), Russian Federation (e-mail: \{aliseychik, \\ i.orlov\}@keldysh.ru,vlpavl@mail.ru,akp31mail@gmail.com). \\ ** Lomonosov Moscow State University, Moscow, Russian Federation \\ (e-mail:aptakhin@gmail.com).
}

\begin{abstract}
Work deals with biped mechanical systems, so called exoskeletons. General purpose of this work is to construct control for exoskeleton motion similar to human walking. In the work the following problems are considered: kinematics synthesis based on motion capture technology, solving direct and inverse dynamics problems for finding control torques and forces, full control system elaborating and verifying constructed system in the program package "Universal Mechanism", which is intended for simulation of kinematics and dynamics of planar and spatial mechanical systems.
\end{abstract}

Keywords: mathematical and physical modeling, direct and inverse dynamics problems, pulse width modulation (PWM) control, pneumatic actuator, biomechatronics, exoskeleton.

Work deals with special biped system, also called the exoskeleton. Exoskeleton is a device designed primarily to increase manpower by an external skeleton. Exoskeleton designed as well to restore people locomotion function for those persons who were without movement for a long time and can't control his legs. Purpose of this work is to construct and verify control for exoskeleton motion which is similar to human walking. First it is required to find the law of natural human movement. The result of algorithms work is the full kinematics scheme of movement which in some sense repeats the walking man. In this work it is considered a multilink model of the exoskeleton in which we can control torques at its joints. Using kinematics it is possible to solve direct problem of dynamic i.e. find the necessary torques. The resulting control scheme verified in the program package "Universal Mechanism". Model have to follow specified motion law, reacting to disturbance forces.

According to technology of video capture of motion recording and processing of natural walking of the person (operator) is made. Recording is made by means of two and more Kinect cameras making a movement snaps from different foreshortenings. Computer vision algorithms solves the problem of filtering image noise, combining information from different cameras, detection of body parts, as well as determining the position and orientation of parts of the body during movement. This part is implemented by program motion capture system iPi Recorder and iPi Mocap Studio with enhancements made by authors. The anthropomorphous kinematic scheme of motion is result

\footnotetext{
* This research is supported by the Russian Foundation for Basic Research (grant 13-01-12037 ofi_m) and the Russian Science Foundation (grant 14-39-00008)
}

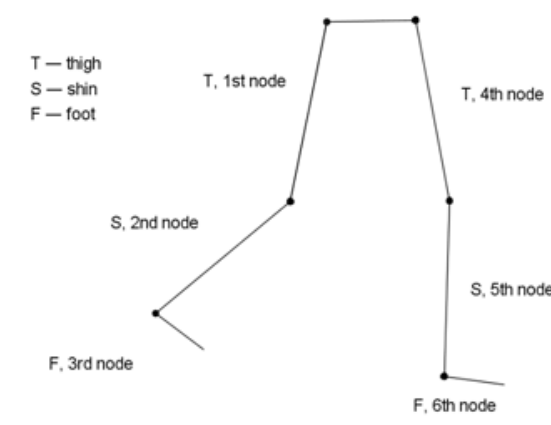

Fig. 1. General human kinematic model

of work of algorithms. The output is a file with the structure of the multilink model in BVH file format, which is widely used as motion capture data representation. This data contain small measuring noise which at calculation of the second derivative turns into noise of considerable amplitude. Convolution - Fourier's window transformation with Hann window function is used, it cleans this noise:

$$
\omega(n)=\frac{1}{2}\left[1-\cos \left(\frac{2 \pi n}{N-1}\right)\right]
$$

Consider the simplified general model (Fig. 1). Each leg is plain one and consists of 3 nodes: thigh, shin, foot. Both legs are attached to the fixed node which is human pelvis.

Control torques were calculated using recursive NewtonEuler equations (Fig. 2). Computations is implemented in Python program using SciPy library. Below is a pseudocode program, implemented this equations: 


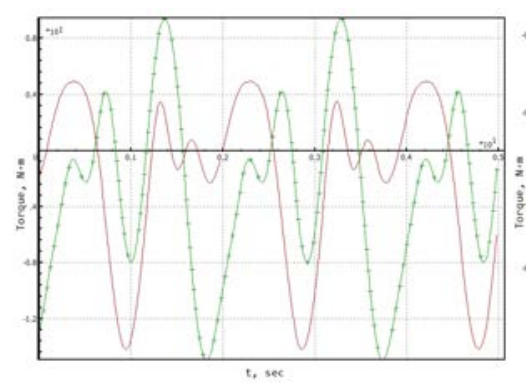

(a) Thigh

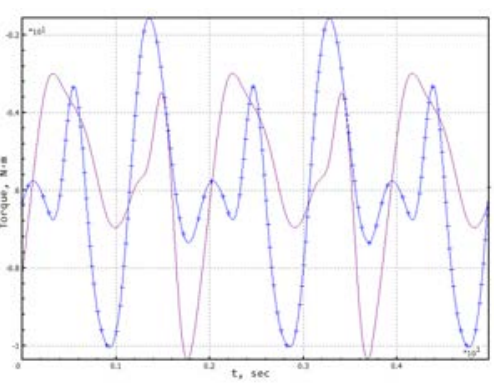

(b) Shin

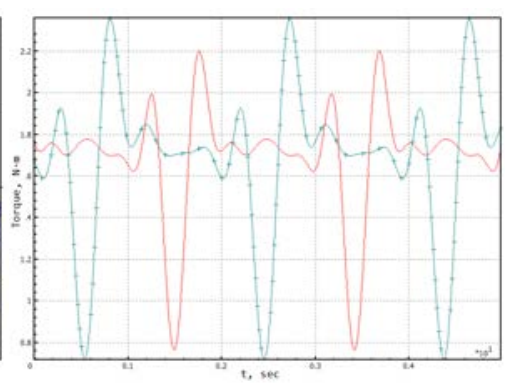

(c) Foots

Fig. 2. Torques. Without marker for left leg. With + marker for right leg

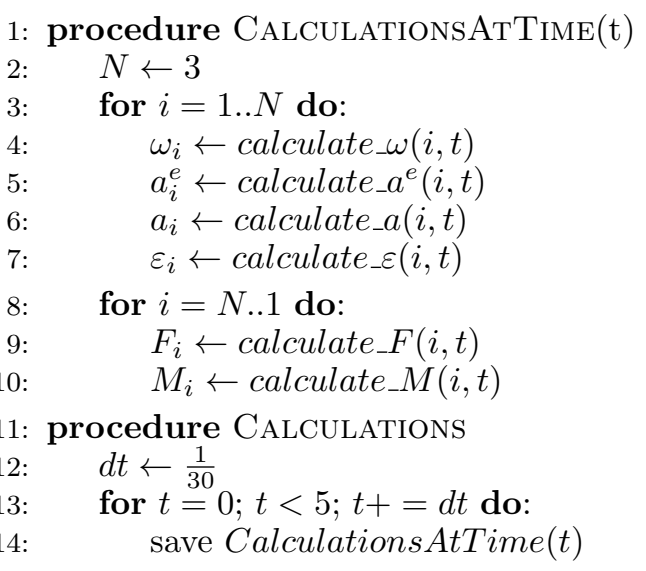

We'll construct a complete control for the human model. Introduce $\boldsymbol{e}(t)$ the error between program position and actual one:

$$
\boldsymbol{e}(t)=\boldsymbol{q}^{p}(t)-\boldsymbol{q}(t)
$$

Control law is constructed with partitioned controller scheme. In general $\boldsymbol{u}$ :

$$
\boldsymbol{u}=A \boldsymbol{f}+\boldsymbol{\beta}
$$

Where $\boldsymbol{\beta}=\boldsymbol{b}(\boldsymbol{q}, \dot{\boldsymbol{q}})+\boldsymbol{c}(\boldsymbol{q}), \boldsymbol{f}=\ddot{\boldsymbol{q}}^{\boldsymbol{p}}+K_{v} \dot{\boldsymbol{e}}+K_{p} \boldsymbol{e}$.

Finally, substituted:

$$
\boldsymbol{u}=A(\boldsymbol{q})\left(\ddot{\boldsymbol{q}}^{p}+K_{v} \dot{\boldsymbol{e}}+K_{p} \boldsymbol{e}\right)+\boldsymbol{b}(\boldsymbol{q}, \dot{\boldsymbol{q}})+\boldsymbol{c}(\boldsymbol{q})
$$

Same design principles was implemented in the special device - lower limb exoskeleton made for helping patients who have lost the mobility of the lower limbs or for working with athletes or astronauts on different stages of rehabilitation. This exoskeleton design has significant differences between from existing since the system does not have its own joints, which imitate or follow human joints. Due to this, the problem is solved by an exact repetition of the kinematics of the particular patient, moreover, by software. Exoskeleton has a modular design, and some joints can be easily added or removed to create a more rigid or flat design. The basic assembly exoskeleton consists of five hard parts - seat, attachments to the leg and thigh, interconnected cylinders. Most pneumatic cylinders are attached to the rigid parts of assembly using two- or threefold hinges. So, the structure may have six degrees of freedom of the hip mounting related to the seat and six degrees of freedom of the thigh mount related to the hip and seat. After the typical trajectories of pattern and the laws of motion of the representative points on them are built, they are performed as the laws of motion of the
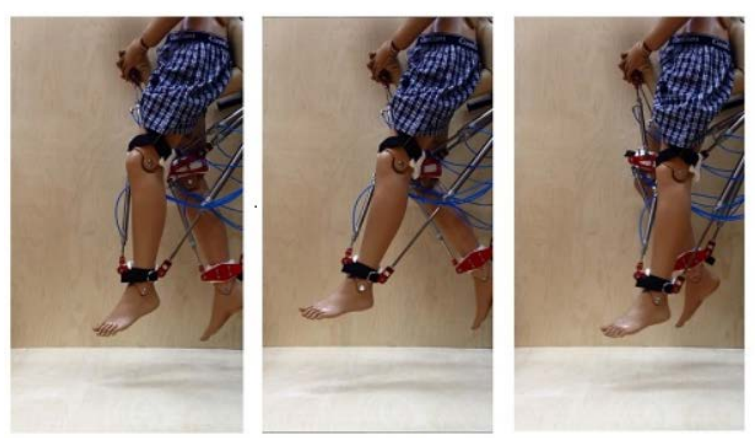

Fig. 3. Biomechatronic complex walking

drive cylinder unit. This conversion can be done on the basis of cyclic calculation of the inverse kinematic problem for the exoskeleton, which is not a trivial one because of the redundancy and complexity of its kinematic scheme.

For a complete calculation for the direct dynamic problem to control the force, exerted by clamping cylinders, full dynamic model of a complex software package "Universal Mechanism" (Universal Mechanism) combined with means package Matlab SIMULINK is realized. Resulting trajectory motion of cylinders in the modular microcontroller system "Robocon" is implemented. This system has a complete library of the lower level to control the drives and to input the analog or digital sensors. Top-level programs are executed on an external computer, which control the complex and displays the received telemetry. Real exoskeleton motion shown on figure 3.

It is implemented a set of solutions for the exoskeleton control problem. Kinematics scheme was built with motion capture technology. It is proved computational efficiency of nonlinear control servo system when it is implemented on microcomputers. The schemes of calculations are given with choosing adequate parameters of the servo system. In the software environment "Universal Mechanism" it is checked that the chosen control effectively realizes the given program motion at impact of external disturbancies.

\section{REFERENCES}

I.A. Orlov, A.P. Aliseychik, A.A. Ptakhin, A.K. Platonov, V.E. Pavlovsky, Biomechatronic Neurorehabilitation Complex: Design, Models and Control. Proceedings of the 5th IEEE RAS \& EMBS International Conference on Biomedical Robotics and Biomechatronics, Brazil, Sao Paulo, August 12-15, 2014, pp. 791-796. 


\title{
Finite Element Approaches for Real-Time Control and Observer Design of Flexible Rack Feeder Systems
}

\author{
Andreas Rauh* Julia Warncke* Georgy V. Kostin ** \\ Vasily V. Saurin** Harald Aschemann* \\ * Chair of Mechatronics, University of Rostock, Rostock, Germany \\ (e-mail: $\{$ Andreas.Rauh, Julia. Warncke, \\ Harald.Aschemann\}@uni-rostock.de) \\ ** A. Ishlinsky Institute for Problems in Mechanics of the Russian \\ Academy of Sciences, Moscow, Russia, (e-mail:
}

\{kostin,saurin\}@ipmnet.ru)

\section{OVERVIEW: MODELING OF ELASTIC SYSTEMS}

The basis for the design of real-time capable control and state estimation strategies for mechanical systems with distributed parameters is the derivation of suitable system models. The dimensionality of these models has to be limited to a reasonable size to make sure that the resulting controllers and state estimators can be implemented in real time. Therefore, a huge amount of work has been performed in recent years which is related to early lumping techniques to replace the governing partial differential equations by sets of ordinary differential equations. In this paper, both local and global approximations of the system dynamics are compared in simulations. These approximations are determined either by using a finite element (FE) representation that is based on the Method of Integrodifferential Relations (MIDR) or, alternatively, by a coarse global Ritz ansatz (Kersten et al., 2014; Kostin et al., 2012). Especially the MIDR allows for the derivation of accurate models which exactly fulfill energy conservation laws as well as boundary and inter-element conditions. In this paper, the elastic rack feeder system depicted in Fig. 1 is considered as a benchmark scenario. During modeling, the original double beam structure is reduced to a single Bernoulli beam model with modified boundary conditions. In addition to this elastic component, two rigid bodies (point masses of the vertically movable cage and the pulley

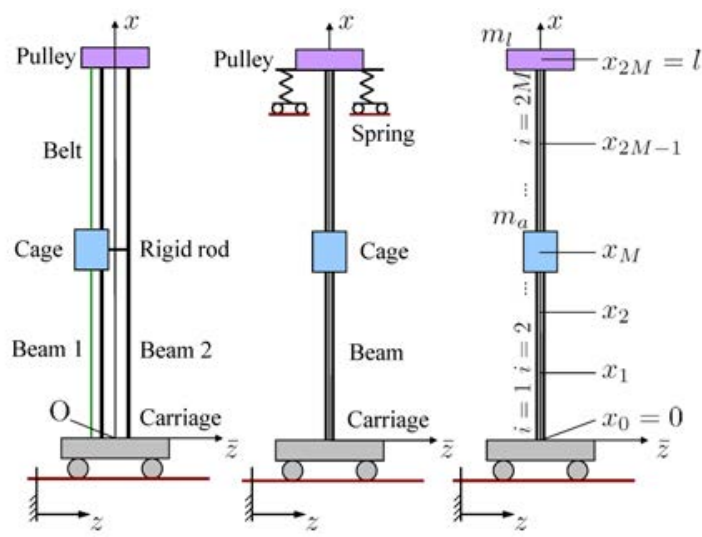

Fig. 1. Modeling of the high-speed rack feeder: Reduction to a single beam and definition of the FE mesh. at the beam tip) are included in the model together with a mathematical representation for the lag behavior of the underlying carriage velocity control.

\section{SELECTED SIMULATION RESULTS}

Based on the MIDR, the following simulations have been performed with a total number of $2 M=4 \mathrm{FEs}$ and different polynomial orders $N$ for the representation of the simulated rack feeder and for the control design model:

$S 1$ Plant with $N=3$, control/ observer design for $N=3$ S2 Plant with $N=4$, control/ observer design for $N=3$ S3 Plant with $N=4$, control/ observer design for $N=4$ S4 Plant with $N=4$, Ritz ansatz (control/ observer)

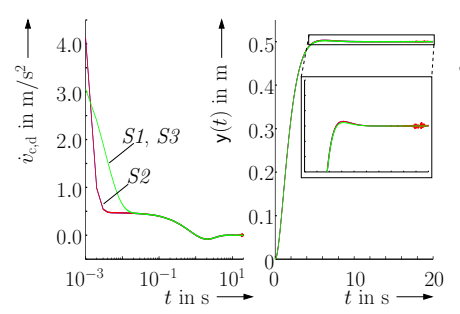

(a) Input and output for $S 1-S 3$.

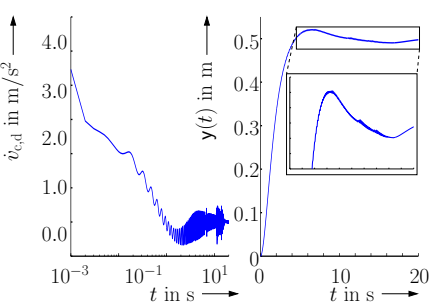

(b) Input and output for $S_{4}$.
Fig. 2. Simulation of the observer-based state feedback controller with a desired horizontal cage position of $0.5 \mathrm{~m}, \dot{v}_{\mathrm{c}, \mathrm{d}}$ : commanded carriage acceleration (system input), y: horizontal cage position (output).

\section{REFERENCES}

Kersten, J., Rauh, A., and Aschemann, H. (2014). Finite Element Modeling for Heat Transfer Processes Using the Method of Integro-Differential Relations with Applications in Control Engineering. In Proc. of IEEE Intl. Conference on Methods and Models in Automation and Robotics MMAR 2014. Miedzyzdroje, Poland.

Kostin, G.V., Rauh, A., Saurin, V.V., and Aschemann, H. (2012). Optimal Real-Time Control of Flexible Rack Feeders Using the Method of Integro-Differential Relations. In Proc. of 7th Vienna Intl. Conference on Mathematical Modelling MATHMOD 2012. Vienna, Austria. Available at IFAC-PapersOnLine.net. 


\title{
MATHMOD 2015 ABSTRACT VOLUME
}

\author{
MATHMOD Minisymposium \\ Mathematical Modelling and Control of \\ Bio-Chemical Processes
}


MATHMOD 2015 Abstract Volume 


\title{
Development and analysis of a mathematical model for a synthetic biological cell
}

\author{
Eugenia Schneider* Michael Mangold ${ }^{* *}$ \\ * Max-Planck-Institute for Dynamics of Complex Technical Systems, \\ 39106 Magdeburg, Germany (e-mail: \\ eschneider@mpi-magdeburg.mpg.de) \\ ** Max-Planck-Institute for Dynamics of Complex Technical Systems, \\ 39106 Magdeburg, Germany (e-mail: \\ mangold@mpi-magdeburg.mpg.de)
}

\begin{abstract}
Synthetic biology as a new discipline is driven by progresses made in the understanding of microbiological processes and its mathematical description by system biological models. In spite of existing mathematical models there is a need for further theoretical analysis and mathematical modeling for a deepened insights into functional device interactions. Most of the existing models may be structured into three functional devices: a container forming the boundary of the cell, a metabolism generating the building blocks of the cell, and a programming part containing genetic information and regulating the processes inside the cell.

The topic of this work is the definition of a structure that masters the synchronization of those three devices. The dependence of the functional devices is well exemplified by the Chemoton model described by Gánti which comprises three functional self-reproducing devices. The Chemoton model displays self-sustained oscillations. This behavior could be verified by our own simulations. However, if we wanted to reduce the complexity of the model structure these oscillations were not necessarily synchronized with cell growth. The task of this work is to find a model structure with less complexity in comparison to the Chemoton and with an inherent mechanism guaranteeing the synchronization of container, metabolism and program for a wide range of kinetic parameter values. The minimal cascade model for the mitotic oscillator proposed by Goldbeter provides such a model structure. By combining the functional devices as proposed in the Chemoton model with the less complex structure of Goldbeter's minimal cascade we developed a model of a self-reproducible artificial cell. The proposed model structure establishes a close interaction between the activation of the metabolism and the replication of the polymer. This interaction forces a synchronization between metabolism and polymerization. In the first instance, the volume changes of the mother cell are neglected. The system shows an autonomous oscillatory behavior with a stable limit cycle. The self-reproduction study shows that the quantities of conversed materials are sufficient to initiate a new cell cycle of daughter cell. Consequently, the model offers a robust self-replicating state. Furthermore, we can show that the model is robust with respect to parameter uncertainties. In addition, we consider the model taking into account volume changes. In this case the system shows stable periodic oscillations in the intracellular components of the mother cell as well in spite of the dilution due to the volume increase.

A further question, that we want to study in the future, is how the Chemoton model of Gánti can be modified and reduced to accomplish similar synchronization of the concentration changes with the volume change as the model described in this work. In addition we want to expand the model described here with respect to the polymerization subsystem similar to the Chemoton. In this way we expect to gradually approach these two models in order to better understand the synchronization mechanism of the Chemoton model.
\end{abstract}




\title{
On stability analysis of genetic regulatory networks represented by delay-differential equations
}

\author{
Laurie Haustenne ${ }^{*}$ Georges Bastin ${ }^{* *}$ Jean-Michel Coron ${ }^{* * *}$ \\ Laetitia Fontaine* Pascal Hols* \\ * Biochimie et Génétique Moléculaire Bactérienne, \\ Institut des Sciences de la Vie, \\ Université catholique de Louvain, 1348 Louvain-la-Neuve, Belgium. \\ ** Department of Mathematical Engineering, \\ ICTEAM, Université catholique de Louvain, \\ 4, Avenue G. Lemaitre, 1348 Louvain-La-Neuve, Belgium. \\ *** Laboratoire Jacques-Louis Lions, Université Pierre et Marie Curie, \\ Institut Universitaire de France, \\ 4, Place Jussieu, 75252 Paris Cedex, France.
}

\section{INTRODUCTION}

This paper deals with continuous time models of genetic networks when time delays are included to allow for the time required for transcription, translation, and transport. The network dynamics are therefore represented by delaydifferential equations whose solutions evolve in the positive orthant. It is well known that these systems may have multiple steady-states or equilibria. A critical issue is to determine the stability of these equilibria. The purpose of this paper is to give a concise, but instrumental, presentation of a general method which can be used for the stability analysis of the steady-states of genetic regulatory networks represented by delay-differential equations. Two complementary ways are investigated: a frequency domain approach which applies to linearized models and gives necessary and sufficient conditions for the local steady-state stability, a Lyapunov approach derived from the viewpoint of hyperbolic PDE systems which gives sufficient conditions for global stability. These methods are illustrated with the example of the "toggle switch". The advantage of the frequency domain method is to give necessary and sufficient conditions but the stability is local. In contrast, the Lyapunov method may give global stability results but the stability conditions are only sufficient and can be conservative in some instances. An application to the genetic network of the basic mechanism for the competence development in Streptococcus thermophilus is in progress and will be presented at the conference.

\footnotetext{
* GB and JMC are partially supported by the ERC advanced grant 266907 (CPDENL) of the 7th Research Framework Programme (FP7). GB is also partially supported by the Belgian Programme on Interuniversity Attraction Poles (IAP VII/19).
}

\section{RESULTS}

We consider a genetic regulatory network which involves $n$ genes interconnected through activators or repressors and whose dynamics are represented by the following model:

$$
\begin{aligned}
& \frac{d M_{i}(t)}{d t}=b_{i}+h_{i}\left(P_{k}\left(t-\tau_{k}\right)\right)-\delta_{i} M_{i}(t), \\
& \frac{d P_{i}(t)}{d t}=\alpha_{i} M_{i}\left(t-\tau_{n+i}\right)-\beta_{i} P_{i}(t),
\end{aligned}
$$

where, at time $t, M_{i}(t)$ is the density of mRNA molecules and $P_{i}$ the density of proteins expressed by the $i$-th gene.

For the frequency domain stability analysis, the model is linearized about a steady-state and the characteristic equation is computed using Laplace transform. The steady-state is locally exponentially stable if the roots of the characteristic equation have strictly negative real parts and are bounded away from zero. Since the characteristic equation involves time delays, the stability analysis is performed using the Walton-Marshall procedure. The methodology is illustrated with the example of the Toggle Switch.

For the time domain analysis, the time delays as represented by hyperbolic transport partial differential equations. In this framework, it is then shown how the use of an appropriate quadratic Lyapunov function can be used to derive instrumental sufficient conditions for the global stability of steady-states. 


\title{
Symmetry Principles in Optimization \\ Problems : an application to Protein Stability Prediction
}

\author{
Fabrizio Pucci ${ }^{a}$, Katrien Bernaerts ${ }^{a, b}$, Fabian Teheux ${ }^{a}$, \\ Dimitri Gilis ${ }^{a}$, Marianne Rooman ${ }^{a}$ \\ ${ }^{a}$ Department of BioModeling, BioInformatics \& BioProcesses, \\ Université Libre de Bruxelles, \\ Avenue F.D. Roosevelt 50, 1050 Bruxelles, BE \\ ${ }^{b}$ BioBased Materials, Faculty of Humanities and Sciences, \\ Maastricht University, \\ P.O. Box 616, 6200 MD Maastricht, The Netherlands \\ (e-mail:mrooman@ulb.ac.be)
}

We show how the adequate use of the intrinsic symmetry of a system when setting up its model structure can avoid unwanted biases in the parameter optimization phase. The playground of our analysis is the prediction of protein thermodynamic stability changes upon single amino acid substitutions (point mutations). The optimization of protein stability is of utmost importance in the design of modified proteins, with applications in a wide series of bioprocesses.

Using a simple artificial neural network (ANN), sixteen different energy-like contributions are combined to predict the change in folding free energy between the mutant (mut) and wild-type $(w t)$ proteins $\left(\Delta \Delta G=\Delta G^{m u t}\right.$ $\left.\Delta G^{w t}\right)$. Since the folding free energy is a state function, $\Delta \Delta G$ has to be antisymmetric under inverse mutations, namely $\Delta \Delta G($ mut $\rightarrow w t)=-\Delta \Delta G(w t \rightarrow m u t)$. Here we show that the presence of terms violating this symmetry in the model structure induces a bias towards the dataset on which the ANN is trained, even if a strict $n$-fold crossvalidation procedure is performed.

Among the sixteen energy contributions, thirteen maintain the symmetry under inverse mutations. They correspond to statistical mean force potentials, extracted from frequencies of observation of certain sequence-structure associations in a dataset of native protein structures, using the inverse Boltzmann law. In the original model (called PoPMuSiC v.2.1) there are three additional terms, i.e. two volume contributions and an independent term, which break explicitly the symmetry under inverse mutations.

The terms of the model structure are combined with sigmoid weights that depend on the solvent accessibility of the mutated residue. The parameters are estimated using an artificial neural network (ANN) with as cost function the root mean square error $\sigma$ between the predicted and experimental $\Delta \Delta G$ values in a given learning set.

To test the independence of the model from the dataset on which the ANN is trained, we construct three datasets: the direct dataset (DIR) composed of 2648 experimentally characterized mutations, the inverse dataset (INV) constructed from all the inverse mutations of the DIR dataset, and the balanced dataset (BAL) that is the combination of the DIR and INV datasets.

We identified the parameters in turn on these three sets, and used the models so obtained to predict the impact on $\Delta \Delta G$ of the mutations belonging to the DIR dataset, using a 10 -fold cross validation procedure. As seen in Fig. (a), the model structure with sixteen terms show a severe bias towards the training datasets. After a carefully analysis, we figured out that the three terms that break the symmetry under the inverse mutations are responsible for this bias. Indeed, after removing such terms and introducing a new completely symmetric free energy functional, the model gives predictions that are slightly less efficient in terms of root mean square error with respect to the experimental $\Delta \Delta G \mathrm{~s}$, but appear to be basically independent of the training dataset (Figure (b)) and are thus more satisfactory.

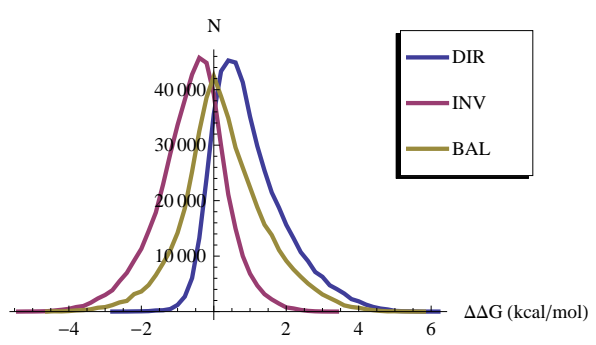

(a)

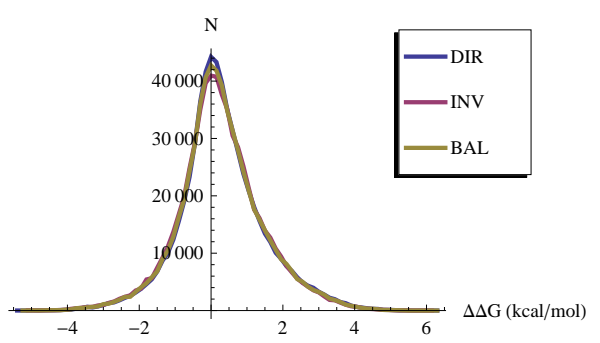

(b) 


\title{
Metabolic Flux Analysis of hybridoma cells: underdetermined network and influence of batch and perfusion operating modes.
}

\author{
Sofia Fernandes* Georges Bastin ** Alain Vande Wouwer* \\ * Service d'Automatique, University of Mons, 31 Boulevard Dolez, \\ 7000 Mons, Belgium (e-mail: sofia.afonsofernandes and \\ alain.vandewouwer@umons.ac.be) \\ ** Cesame, Université catholique de Louvain, av. G. Lemaitre 4, B1348 \\ Louvain-La-Neuve, Belgium (e-mail: Georges.Bastin@uclouvain.be)
}

\section{ABSTRACT}

Knowledge of intracellular fluxes is of critical importance in the process of investigating and understanding cell metabolism. Metabolic Flux Analysis (MFA) appears as a tool to determine intracellular fluxes from extracellular measurements, such as cell density, substrates and products concentrations. In this work, metabolic flux analysis (MFA) is applied to determine the flux distribution within a metabolic network representing the metabolism of a hybridoma cell line. Even though a relatively large set of extracellular measurements are available, mass balancing leads to an underdetermined system and a unique solution cannot be computed. To tackle this problem, a convex analysis approach is therefore used to compute the metabolic fluxes as positive bounded intervals, with the help of the toolbox METATOOL. Our main objective is to investigate the influence of the number of extracellular measurements and the culture operating mode, i.e., batch or perfusion, on the flux distribution. Our study is based on sets of experimental data from hybridoma HB58 cell batch/perfusion cultures.

\section{REFERENCES}

Stephanopoulos, G., Aristidou, A. A., and Nielsen, J. (1998). Metabolic engineering: principles and methodologies. Academic press.

Provost, A. and Bastin, G. (2004). Dynamic metabolic modelling under the balanced growth condition. Journal of Process Control, 14(7), 717:728.

Pfeiffer, T., Nu, J., Montero, F., Schuster, S., et al. (1999). Metatool: for studying metabolic networks. Bioinformatics, 15(3), 251:257.

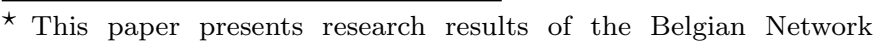
DYSCO (Dynamical Systems, Control, and Optimization), funded by the Interuniversity Attraction Poles Programme initiated by the Belgian Science Policy Office.
} 


\title{
Structural identifiability analysis of the Anaerobic Digestion Model No. 1 using a local algebraic observability approach
}

\author{
Joost Lauwers, Philippe Nimmegeers, Filip Logist, \\ Jan Van Impe ${ }^{\star}$
$K U$ Leuven, Department of Chemical Engineering, Chemical and Biochemical Process Technology and Control (BioTeC), $W$. de Croylaan 46, B-3001 Leuven, Belgium. \\ (e-mail: jan.vanimpe@cit.kuleuven.be).
}

Dynamic models are essential tools for model-based control and optimisation of bioprocesses. Due to the complexity of the processes, however, these models generally contain large numbers of $(i)$ non-linear ordinary differential or differential algebraic equations (ODEs or DAEs) and $(i i)$ parameters which have to be estimated. The latter issue raises questions with respect to the structural identifiability of the model, i.e., the possibility to correctly estimate the model's parameters, under perfect conditions.

In this work the large-scale Anaerobic Digestion Model No. 1 (ADM1) (Batstone et al., 2002), depicted in Figure 1, is subjected to a structural identifiability analysis. ADM1 has 29 differential and 10 algebraic states. It is assumed that 73 parameters and all initial conditions are unknown and have to be estimated. Different measurement scenarios are investigated: all states are measured and each state is measured separately. This is done for two situations: $(i)$ the liquid inflow rate and the concentrations in the inflow are the input variables and ( $i i)$ the liquid inflow rate is the only input with the concentrations in the inflow fixed and non-zero.

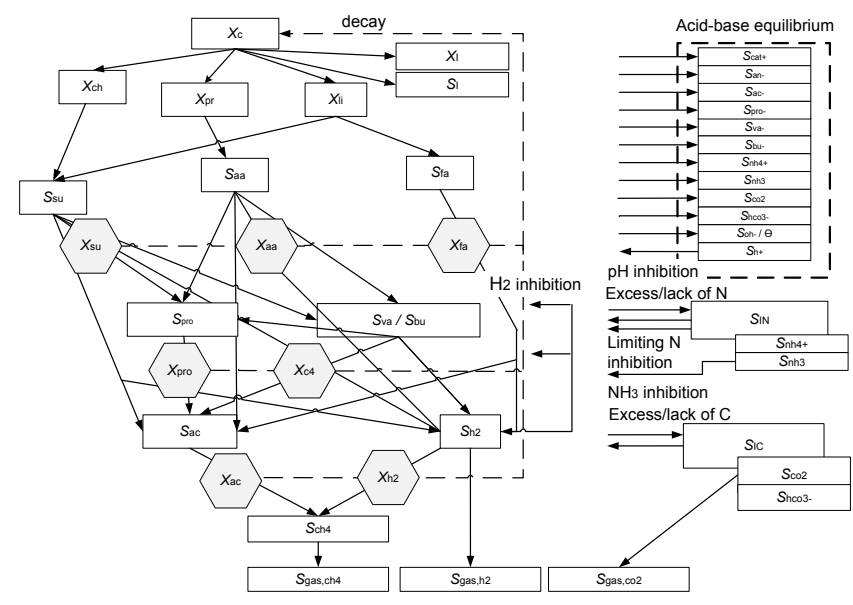

Fig. 1. The ADM1 reaction network.

In this work the local algebraic observability (Sedoglavic, 2002) is investigated, which guarantees structural local identifiability, i.e., the possibility of identifying a finite number of parameter values that explain the model's input-output behaviour. This involves the calculation of the Jacobian of successive Lie-derivatives. Although this approach is in general computationally extremely intensive, the use of a probabilistic semi-numerical algorithm presented by Sedoglavic (2002) renders problems of the size of ADM1, feasible.

It was found that the results with fixed concentrations of the inflowing components were identical to those in which these concentrations were allowed to vary. Thus, excitations of the system by the liquid inflow with fixed concentrations, provide in principle sufficient excitation for identifying the parameters and initial conditions.

The analysis showed also that in case all states are measured, all but 2 parameters are observable. These parameters pertain to the inerts in the reaction scheme. In regard to the structure and equations of ADM1, this result is not surprising, since the inerts are dead ends in the degradation process. In the case any state excluding inerts, cations or anions is measured, all states and parameters except those pertaining to inerts, cations and inerts are observable. Again, the non-observability of the parameters can be logically explained from the reaction scheme.

Because several non-observable parameters/states were found, observable parameter combinations where sought that involve the non-observable parameters/states. Although mathematical techniques exist to identify these combinations automatically, there is no need to apply them here. It is relatively easy to propose parameter/state combinations and check the observability of these proposed combinations. In this manner, 3 observable parameter combinations were found.

\section{REFERENCES}

Batstone, D., Keller, J., Angelidaki, I., Kalyuzhnyi, S., Pavlostathis, S., Rozzi, A., Sanders, W., Siegrist, H., and Vavilin, V. (2002). The IWA anaerobic digestion model No. (ADM1). Water Science and Technology, 45, 65-73.

Sedoglavic, A. (2002). A probalistic algorithm to test local algebraic observability in polynomial time. Journal of Symbolic Computation, 33, 735-755.

\footnotetext{
* Corresponding Author.
} 


\title{
Kriging based iterative parameter estimation procedure for biotechnology applications with nonlinear trend functions
}

\author{
Lars Freier*, Eric von Lieres *;** \\ * IBG-1: Biotechnology, Forschungszentrum Jülich, Germany \\ **e.von.lieres@fz-juelich.de
}

Empirical and mechanistical modeling approaches are often used in order to analyze functional relationships between process factors and system response and to identify process optima. The Kriging method allows to integrate both modeling approaches by combining statistical information on a given data set with a priori defined trend functions. However, trend functions from biotechnology applications are typically nonlinear with respect to the model parameters, which is not supported by standard Kriging. In this paper, we present an extension of the Kriging method for handling nonlinear trend functions by a Taylor based linearization approach which leads to an iterative parameter estimation procedure. 


\title{
Set-membership identification of an agro-ecosystem from a small data set: The case of ammonia volatilisation in a flooded rice field
}

\author{
Nurulhuda Khairudin ${ }^{* * * * *}$, Paul C. Struik**, Karel J. Keesman*** \\ *Biological and Agricultural Engineering, Faculty of Engineering, Universiti Putra Malaysia, \\ Serdang, Malaysia (Tel: +31 061614 7916; e-mail: nurul.khairudin@wur.nl). \\ **Centre for Crop Systems Analysis, Wageningen University, \\ Wageningen, The Netherlands, (e-mail: paul.struik@wur.nl) \\ ***Biobased Chemistry and Technology, Wageningen University, \\ Wageningen, The Netherlands, (e-mail: karel.keesman@wur.nl)
}

\begin{abstract}
Uncertainty analysis is an essential part of systems modelling. Preferably, the uncertainty analysis should start with a parameter estimation step. However, for agroecosystems, available data sets are typically small. Therefore, probabilistic parameter estimation techniques are not adequate. Alternatively, a set-membership or bounded-error approach can be used. In this paper, we demonstrate a set-membership approach to estimate the unknown parameters in a biochemical model from a small data set. As a case study, a biochemical model developed to estimate ammonia volatilisation in a flooded rice field was used. A total of 741 feasible parameter-vectors were found from 4000 simulated parameter-vectors. Eigenvalue decomposition of an ellipsoidal outer-bounding set indicated that the model was most sensitive to the growth rate of the rice plants, $\beta$. Visual inspection of the feasible parameter-vectors showed that the estimates of the nitrogen uptake rate $K_{\text {wupt }}$ are hyperbolically related to the estimates of $\beta$.
\end{abstract}

Keywords: Set-membership approach, parameter estimation, uncertainty analysis, small data set, ammonia volatilisation 


\title{
Parameter Identification of the Droop Model using Optimal Experiment Design
}

\author{
Micaela Benavides* \\ Dries Telen, Joost Lauwers, Filip Logist, Jan Van Impe ${ }^{\dagger}$ \\ Alain Vande Wouwer $\ddagger$ \\ * BioSys, University of Mons, 7000 Mons, Belgium, (e-mail: \\ micaela.benavides@umons.ac.be). \\ $\dagger$ Dept. of Chemical Engineering, KU Leuven, 3001 Leuven, Belgium \\ (e-mail: Dries.Telen@cit.kuleuven.be,Joost.Lauwers@cit.kuleuven.be \\ Filip.Logist@cit.kuleuven.be, Jan.VanImpe@cit.kuleuven.be) \\ $\ddagger$ BioSys, University of Mons, 7000 Mons, Belgium, (e-mail: \\ alain.vandewouwer@umons.ac.be)
}

\section{INTRODUCTION}

The Droop model is a classical model used to describe substrate limitation in cultures of micro-algae in photobioreactors. In this study, we address the questions of structural identifiability as well as practical identifiability. Optimal experiment design based on the M-criterion is used to determine a small set of experiments that would allow an accurate estimation of the model parameters.

Considering that the parameters of Droop model are $\rho_{m}$ $\mu_{m}, K_{s}$ and $Q_{0}$, the structural identifiability of Droop model is first checked using differential algebra implemented in the software DAISY (Bellu et al., 2007). Then, practical identifiability is investigated on the basis of a simulation study. To this end, two continuous experiments are designed, in order to provide sufficient information for the identification of the 4 model parameters. The specific experiments are therefore designed to target 3 out of the 4 parameters. The first experiment allows minimizing the uncertainty on the parameters $\rho_{m}$ and $\mu_{m}$, while the second one targets parameter $K_{s}$. These two optimal experiments have been designed using a direct optimal control approach. To this end, the infinite dimensional optimal control, or dynamic optimization problem, has been converted in a large-scale nonlinear optimization problem. The resulting nonlinear optimization problem has been solved using the interior point routine IPOPT (Wächter and Biegler, 2006). The first optimal experiment design suggests as initial conditions, $X_{(0)}=65, S_{(0)}=100$, $Q_{(0)}=0.08$, and a time-varying dilution rate $D(t)$ as shown in Figure 1.

To test the proposed approach, parameter identification is achieved from simulation results, where the collected measurements are corrupted by Gaussian white noise. The results are analyzed in terms of parametric sensitivities, confidence intervals and cross validation. The confidence intervals of the estimated parameters are calculated using the inverse of the Fisher information matrix. Figure 2 shows the relative error for each parameter and for all experiments considering a $95 \%$ confidence interval.

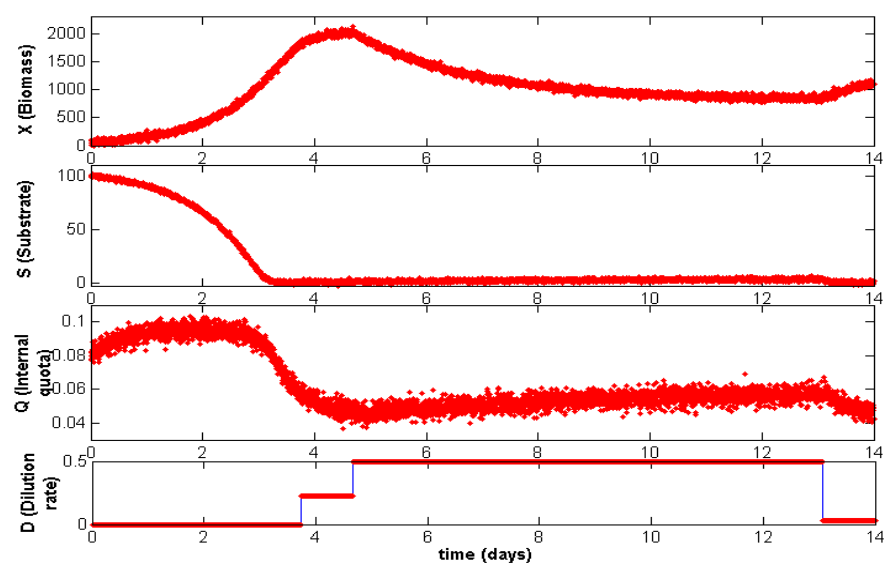

Fig. 1. First optimal experiment for estimating $\mu$ and $\rho$ accurately.

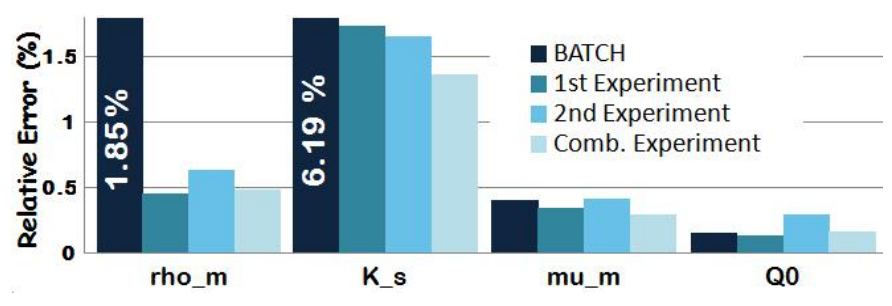

Fig. 2. Relative error of the estimated parameters for all experiments.

The accuracy of the parameters is much improved as compared to what would have been obtained using data collected from standard batch experiments.

\section{REFERENCES}

Bellu, G., Saccomani, M.P., Audoly, S., and D'Angiò, L. (2007). Daisy: A new software tool to test global identifiability of biological and physiological systems. Computer Methods and Programs in Biomedicine, 88(1), $52-61$.

Wächter, A. and Biegler, L.T. (2006). On the implementation of an interior-point filter line-search algorithm for large-scale nonlinear programming. Mathematical Programming, 106(1), 25-57. 


\title{
Macroscopic Modelling of Intracellular Reserve Carbohydrates Production during Baker's Yeast Cultures
}

\author{
A. Richelle* and Ph. Bogaerts* \\ *3BIO-BioControl,Université Libre de Bruxelles, Brussels, Belgium \\ (Tel: +32-6506608; e-mail: arichell@ulb.ac.be, philippe.bogaerts@ulb.ac.be).
}

\section{INTRODUCTION}

Over the years, the culturing conditions of commercial baker's yeast have been optimized in order to obtain a high carbohydrates content (trehalose and glycogen). Indeed, trehalose and glycogen, through their accumulation or mobilization, are key metabolites in the adaptation of Saccharomyces cerevisiae to its environment. These energy storage compounds are crucial for maintaining cell viability and improving the physiological activities of yeast as final product. Both metabolites are significantly involved in the general metabolism of yeast via complex pathways that are not yet fully understood. Carbohydrates metabolism seems to be directly related to the glycolysis and the high turnover of these metabolites would ensure a continuous influx of glucose units in carbon metabolism. Hence, the understanding of the formation and the accumulation of carbohydrates has become a hot topic in baker's yeast industry, as well as in the winemaking and brewing industries (Guillou et al., 2004, Jørgensen et al., 2002).

Quite surprisingly, these metabolites have been little studied from a modelling standpoint (van Dijck et al., 1995). Some models of trehalose metabolism exist in the literature but, to the knowledge of the authors, none for glycogen. Hence, this study proposes to extend the macroscopic model linking the nitrogen and glucose consumption in yeast cultures developed by Richelle et al. (2014a) to the reserve carbohydrates metabolism.

\section{MODEL EXTENSION DEVELOPMENT}

To test all possibilities of trehalose and glycogen introduction into the model of Richelle et al. (2014a), a parametric estimation procedure taking into account consumption and synthesis of trehalose and glycogen by each reaction was performed (introduction of 16 pseudo-stoichiometric parameters). Based on these results, the number of parameters has been reduced to take into account only the most significant ones (6 stoichiometric parameters). The proposed reaction scheme is consistent with the literature.

\section{MODEL IDENTIFICATION AND EXPERIMENTAL VALIDATION}

The model parameters were obtained via nonlinear least squares identification. The proposed model extension was validated with experimental data of six yeast fed-batch cultures. This experimental field is composed by the four experiments used for the model identification in Richelle et al. (2014a) and by two other experiments which have been performed in optimal operating conditions for maximizing biomass production (Richelle et al., 2014b). The validation results show that the model successfully predicts the dynamics of accumulation and mobilization of trehalose and glycogen during all periods of the six cultures, even in crossvalidation.

\section{CONCLUSIONS}

An extension of the model by Richelle et al. (2014) has been proposed to describe the reserve carbohydrates metabolism during yeast cultures. On the one hand, this model extension allows the quantitative description of the storage carbohydrates metabolism in yeast cultures; on the other hand, it will be valuable for the determination of culture conditions aiming at maximizing yeast productivity while guaranteeing the accumulation of a required amount of trehalose and glycogen and for the development of control tools aiming at maintaining yeasts in this specific physiological state through proper management of the supplied substrates. This extension is a contribution in the management of cellular physiology from extracellular culture environment (substrates concentrations).

\section{REFERENCES}

Guillou, V., Plourde-Owobi, L., Parrou, J.L., Goma, G., and François, J. (2004). Role of reserve carbohydrates in the growth dynamics of Saccharomyces cerevisiae. FEMS Yeast Research, 4, 773-787.

Jørgensen, H., Olsson, L., Rønnow, B., and Palmqvist, E.A. (2002). Fed-batch cultivation of baker's yeast followed by nitrogen or carbon starvation: effects on fermentative capacity and content of trehalose and glycogen. Applied Microbiology Biotechnology, 59, 310-317.

Richelle, A., Fickers, P., Bogaerts, Ph. (2014a). Macroscopic modelling of baker's yeast production in fed-batch cultures and its link with trehalose production. Computers \& Chemical Engineering, 61, 220-233.

Richelle, A., Bogaerts, Ph. (2014b). Off-line optimization of baker's yeast production process. Chemical Engineering Science, 119, 40-52.

van Dijck, P., Colavizza, D., Smet, P., and Thevelein, J.M. (1995). Differential importance of trehalose in stress resistance in fermenting and nonfermenting Saccharomyces cerevisiae cells. Applied and Environmental Microbiology, 61(1), 109-115. 


\title{
Validation of a Simple Fouling Model for a Submerged Membrane Bioreactor
}

\author{
G. Araujo Pimentel ${ }^{*, * *}$ M. Dalmau ${ }^{* * * *}$ A.Vargas ${ }^{* * *}$ \\ J. Comas ${ }^{* * *}$ I. Rodriguez-Roda ${ }^{* * * *, \dagger}$ A. Rapaport ${ }^{* *}$ \\ A. Vande Wouwer* \\ * University of Mons, Automatic Control Laboratory, Bd. Dolez 31, \\ 7000 Mons, Belgium \\ \{guilherme.araujopimentel;alain.vandewouwer\}@umons.ac.be \\ ** Equipe Projet INRIA MODEMIC, UMR MISTEA, Montpellier, \\ France.rapaport@supagro.inra.fr \\ *** Lab. for Research on Advanced Water Treatment Processes, \\ Instituto de Ingeniería, Universidad Nacional Autónoma de México, \\ Blvd. Juriquilla 3001, Juriquilla, Querétaro, 76230, México. \\ avargasc@ii.unam.mx \\ **** LEQUiA, Laboratory of Chemical and Environmental Engineering, \\ University of Girona, Campus de Montilivi, E-17071 Girona, \\ Catalonia, Spain \{montse.dalmau; quim; ignasi\}@lequia.udg.cat \\ † ICRA (Catalan Institute for Water Research), Scientific and \\ Technological Park of the University of Girona, H2O Building c/Emili \\ Grahit 101, E17003 Girona, Catalonia, Spain irodriguezroda@icra.cat
}

\section{MOTIVATION}

The aerobic submerged membrane bioreactor (sMBR) has been increasingly applied to wastewater treatment, due to its high effluent quality (regarding solid matters), footprint reduction and the decoupling of the hydraulic and solid retention times. In its simple configuration, an sMBR combines the function of an activated sludge system with secondary and tertiary filtration in a single tank. One of the main drawbacks of the sMBR process is the fouling phenomenon, caused by particles that attach to the membrane surface decreasing process efficiency and increasing energy consumption. Most of the published submerged membrane bioreactor models have been proposed for process description and gain of insight, resulting in a large number of parameters to estimate from experimental data. These models are usually too complex for process control, and there is a need for simple, dedicated, dynamic models. In this study, attention is focused on the fouling phenomenon, which hampers the efficient operation of sMBRs.

\section{SMBR FOULING MODEL}

The proposed dynamic model uses the resistance-in-series model $R_{\text {total }}=R_{m}+\delta_{R}+R_{\text {cake }} . R_{m}$ is the membrane intrinsic resistance (assumed constant); $\delta_{R}$ is the total membrane resistance disturbance, resulting from poreblocking, biofilm, concentration polarization and scaling; $R_{\text {cake }}$ is the sludge cake resistance where $R_{\text {cake }}=\rho \frac{m+m_{0}}{A}$

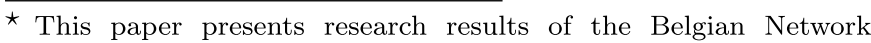
DYSCO (Dynamical Systems, Control, and Optimization), funded by the Interuniversity Attraction Poles Programme, initiated by the Belgian State, Science Policy Office. Alejandro Vargas has been beneficiary of a post-doc grant from Belgium Science Policy Office (BELSPO). with the specific cake resistance $\rho$, the initial cake mass $m_{0}$ and the membrane area $A$. The cake mass $m$ is model as follows:

$$
\left\{\begin{array}{l}
\frac{d \beta}{d t}=\gamma \beta \\
\frac{d m}{d t}=Q_{o u t} X-J_{\text {air }} \beta \frac{m^{2}}{K_{a i r}+m}
\end{array}\right.
$$

where $\beta$ is long-term fouling evolution, $Q_{\text {out }}$ is the permeate flow rate and $J_{\text {air }}$ is the air scour rate, $X$ is the TSS and $K_{\text {air }}$ and $\gamma$ are model parameters. It is important to remember that sMBR plants are exposed to daily temperature variations which influence the temperature and the apparent viscosity of the water, resulting in TMP variations. The influence of water temperature into the trans-membrane pressure is modeled as TMP = $\frac{Q_{\text {out }}}{A} R_{\text {total }} A_{1} e^{\frac{A_{2}}{\text { Temp }}}$.

\section{MODEL VALIDATION}

The model is validated using a large data base collected from a pilot plant and to ease the identification procedure, a separation of the time scales of the process in slow and fast dynamics is exploited. The results show that the model can be used to predict the trans-membrane pressure behavior in a medium-term, about 10 days ahead.

\section{REFERENCES}

Dalmau, M., Rodríguez-Roda, I., Ayesa, E., Odriozola, J., Sancho, L., and Comas, J. (2013). Development of a decision tree for the integrated operation of nutrient removal MBRs based on simulation studies and expert knowledge. Chemical Engineering Journal, 217, $174-184$.

Pimentel, G.A., Vande Wouwer, A., Harmand, J., and Rapaport, A. (2015). Design, analysis and validation of a simple dynamic 


\title{
Thermodynamic behavior-rules for a bacterial individual-based model to study the denitrification process
}

\author{
Pablo Araujo ${ }^{1,2}$, Anna Gras ${ }^{2}$ and Marta Ginovart ${ }^{3}$ \\ ${ }^{1}$ Chemical Engineering Faculty, Central University of Ecuador. ${ }^{2}$ Department of Agri-Food Engineering and Biotechnology. \\ ${ }^{3}$ Department of Applied Mathematics III, Universitat Politècnica de Catalunya - Barcelona Tech. \\ Corresponding author: Pablo Araujo, Department of Agri-Food Engineering and Biotechnology, Universitat Politècnica de \\ Catalunya-Barcelona Tech, Barcelona - Spain; mail: paraujo@iquce.edu.ec/pablo@araujo.ec
}

Denitrification is the process in which bacteria, for instance Paracoccus denitrificans, one of the frequently chosen species for biochemistry studies, use nitrate as a final electron acceptor and carry out respiratory metabolism in anaerobic conditions. Denitrification reduces the nitrate content of soil, so that fewer nitrates can leach downwards and root uptake may be hindered. Denitrification is also a source of environmental burden in agricultural soils; nitrous oxide $\left(\mathrm{N}_{2} \mathrm{O}\right)$ emissions are very important due to the large amount of $\mathrm{N}$-fertilizer in crops and soil organic matter mineralization. To study the effects of denitrification on the nitrogen balance in agricultural systems controlled experiments in bioreactors and simulation models can be helpful tools.

An interesting modeling approach paradigm which is an alternative to the population-level approach [2] is called "individualbased modeling" (IBM). Microbial IBMs provide some advantages over the traditional population-level models [3]. We have developed a model for denitrifying bacteria called INDISIM-Paracoccus. It is a bacterial IBM used to simulate continuous or batch cultures under diverse substrate-dependent cell growth of the bacterium $P$. denitrificans under aerobic and anaerobic conditions. The model assumes a culture medium containing succinate as a carbon source, ammonium as a nitrogen source and various electron acceptors such as oxygen, nitrate, nitrite, nitric oxide and nitrous oxide. It embeds thermodynamic properties in individual cells thorough the Thermodynamic Electron Equivalents Model (TEEM2) [4] for bacterial growth prediction within the IBM INDISIM [1] to obtain stoichiometric reactions that are an intracellular model for generating the microorganism behavior-rules. In the INDISM-Paracoccus framework, the objectives of this study are to: i) show how balanced energy reactions are incorporated into the behavior-rules for cellular maintenance and for biomass synthesis following a thermodynamic approach, and ii) implement the model on NetLogo and test two hypotheses about the order in which the reactions are followed by the bacteria while the denitrification process occurs. Temporal evolutions of some system variables will be analyzed and compared.

The energy reactions consider that succinate and some electron acceptors were obtained according to TEEM2 for aerobic and anaerobic cellular maintenance. The stoichiometric coefficients, for a metabolic pathway, were obtained from Gibbs free energy for a half-reaction with an assigned $\varepsilon$ value in the range proposed by McCarty (2007) and others. The simulator INDISIM-Paracoccus offers the possibility of interpreting and investigating the dynamics of $P$. denitrificans growing in controlled conditions. When converting the reactions that represent metabolic pathways into a balanced chemical equation by applying the TEEM2, the individual growth yield obtained is higher than published population yields, but the population growth yield is in accordance with reported $P$. denitrificans values. The hypothesis that the reactions in the bacterium occur according to their standard Gibbs energy does not seem plausible, because the NO production reaches higher values than those reported by experimentalists, but it was useful in the first steps of our investigation to develop and parameterize the model.

Keywords: denitrification, Paracoccus denitrificans, bacterial yield prediction, individual-based model, TEEM2, INDISIM.

[1] Ginovart, M., López, D., \& Valls, J. (2002). INDISIM, an individual-based discrete simulation model to study bacterial cultures. Journal of Theoretical Biology, 214(2), 305-19. doi:10.1006/jtbi.2001.2466

[2] Grimm, V. (1999). Ten years of individual-based modelling in ecology: what have we learned and what could we learn in the future? Ecological Modelling, 115(2-3), 129-148. doi:10.1016/S0304-3800(98)00188-4

[3] Kreft, J.-U., Plugge, C. M., Grimm, V., Prats, C., Leveau, J. H. J., Banitz, T., ... Hellweger, F. L. (2013). Mighty small: Observing and modeling individual microbes becomes big science. Proceedings of the National Academy of Sciences of the United States of America, 110(45), 18027-8. doi:10.1073/pnas.1317472110

[4] McCarty, P. L. (2007). Thermodynamic electron equivalents model for bacterial yield prediction: modifications and comparative evaluations. Biotechnology and Bioengineering, 97(2), 377-388. doi:10.1002/bit

Acknowledgments: Financial support was received from the Ecuador National Secretary of Science and Technology (SENESCYT) and the Plan Nacional I+D+i of the Spanish Ministerio de Educación y Ciencia (MICINN, CGL201020160 . 


\title{
Modelling freezing processes of high concentrated systems
}

\author{
E. Lopez-Quiroga, R. Wang, O. Gouseti, P.J. Fryer, S. Bakalis
}

\author{
School of Chemical Engineering, University of Birmingham, Edgbaston, B15 2TT \\ UK (Tel:+44 (0)121414 5383; e-mail: e.lopez-quiroga@bham.ac.uk).
}

\section{INTRODUCTION}

Controlling ice crystal formation is critical in determining the microstructure and quality of frozen foods in processes such as freezing and freeze-drying (Petzold and Aguilera, 2009). As recent trends in food processing head towards the manufacturing of highly concentrated foodstuff, efficient performance of such industrially relevant crystallisation processes has become increasingly difficult. The upcoming challenges therefore rely not only in developing successful processing protocols but also in the understanding the insights of these systems. In this framework, mathematical modelling can be employed to obtain an accurate description of the system behaviour as well as to help in the comprehension of the process dynamics.

\section{MATERIALS AND METHODS}

Sucrose was mixed (magnetic stirrer) with distiller water under heating ( $\max 50{ }^{\circ} \mathrm{C}$ ) in closed container to prepare solutions in a range of concentrations(20\%, 50\%, 60\%). Differential scanning calorimeter (DSC) experiments have been conducted in order to characterise the crystallisation kinetics of water during freezing. In addition, a series of seeding experiments (secondary nucleation) in a $60 \% \mathrm{w} / \mathrm{w}$ sucrose solution have been performed too.

\section{MATHEMATICAL MODELLING}

In this work, two first principles-based models describing freezing processes are presented. They are aimed at helping in the study and analysis of the different crystallisation phenomena (e.g. secondary and primary homogeneous) which can occur during the freezing of sucrose solutions with a high content in solids $(20 \%-60 \% \mathrm{w} / \mathrm{w})$. The first of them couples heat and mass transfer mechanisms, while the second one combines a heat transfer problem with ice crystal nucleation and growth kinetics.

\section{RESULTS AND DISCUSSION}

The proposed models have been implemented and solved in a commercial FEM software (COMSOL Multiphysics). For the secondary nucleation case, a $60 \% \mathrm{w} / \mathrm{w}$ sucrose solution has been considered for numerical purposes. Overall model and experiments correlate well, although the model systematically overestimates crystal growth rates. It must be noted that the simulated results correspond to a system in equilibrium, while experiments must have been performed presumably under non-equilibrium conditions. The corresponding model for primary homogeneous crystallisation, which couples heat transfer problem with ice crystal nucleation and growth kinetics, has been solved for $20 \%$ and $50 \%$ w/w sucrose solutions, and different air fractions. It has been found that the addition of air tends to delay the crystal formation, as can be seen in Figure 1 for the $50 \%$ solution. This is understood as a direct consequence of the insulating effect of air within the product, which slows down the heat transfer through the sample. The relationship between water diffusivity and front position in (Miyawaki 2001) has been employed to estimate the mean crystal size resulting from this homogeneous process, and a correlation between higher air fractions and larger crystals has been established.

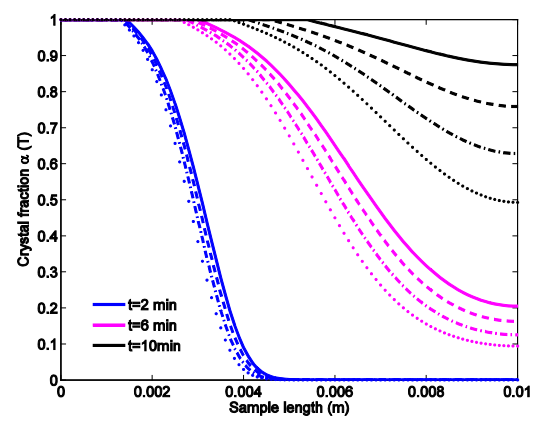

Fig. 1. Comparison of the crystal fraction $\alpha(\mathrm{T})$ along the sample between the non-aerated $50 \% \mathrm{w} / \mathrm{w}$ sucrose solution (solid), and the aerated solution with air fractions $\varepsilon=0.1$ (dashed), $\varepsilon=0.2$ (dashed-dot) and $\varepsilon=0.3$ (dotted).

\section{CONCLUSIONS}

A first approach to the modelling of crystallisation phenomena (both primary and secondary mechanisms) in highly concentrated systems has been presented. This constitutes an important contribution towards the design of targeted frozen product microstructure, which is a key factor not only for the quality of frozen foods but also in other freeze-related processes such as freeze concentration and freeze drying.

\section{ACKNOWLEDGEMENTS}

The authors acknowledge financial support received from EPSRC (grant no. EP/K011820/1) and Innovate UK (grant no. TS/K003909/1), as well as contributions made by Mondelèz International.

\section{REFERENCES}

Miyawaki, O. (2001). Analysis and control of ice crystal structure in frozen food and their application to food processing. Food Science and Technology Research (7), 1-7.

Petzold, G. and Aguilera, J.M. (2009). Ice morphology: fundamentals and technological applications in foods. Food Biophysics 4, 378-396. 


\title{
MATHMOD 2015 ABSTRACT VOLUME
}

\author{
MATHMOD Minisymposium \\ Dynamics of Machines and Structures
}


MATHMOD 2015 Abstract Volume 


\title{
Optimal Deployment of a Tethered Satellite using Tension Control
}

\author{
Alois Steindl* \\ * Institute for Mechanics and Mechatronics, Vienna University of \\ Technology, Vienna, Austria (e-mail: Alois.Steindl@tuwien.ac.at).
}

\begin{abstract}
We investigate the deployment of a tethered satellite from the space station, which moves along a Keplerian elliptic orbit with small eccentricity. The control of the satellite's motion is performed by applying a restricted tension force along the connecting tether. The goal of the control is to steer the satellite from a periodic state close to the local vertical position to a periodic motion farther away from the space station in shortest time.
\end{abstract}

Keywords: Tension control, Time optimal control, Pontryagin's Maximum Principle, Elliptic orbit

\section{INTRODUCTION}

The deployment and retrieval of satellites from space ships are very demanding tasks in space missions, because the possible control actions are just tension forces along the tethers, if one doesn't want to use control rockets for this purpose (Barkow et al. (2003); Jin and $\mathrm{Hu}$ (2006); Williams (2008)). Usually Kissel's law is used for controlling the deployment of satellites. It leads to a slow decay of the pendulum oscillations and takes a lot of time. In order to estimate, how much time could be saved at all by applying some faster strategy, we searched the time-optimal control. It turned out, that this control took significantly less time, but on the other hand it uses bangbang control, which could lead to strong oscillations in the tether configuration.

Nevertheless we could show, that applying that control strategy, the subsatellite could be steered from the initial vertical stationary state close to the space station to another steady state in greater distance within a few revolutions.

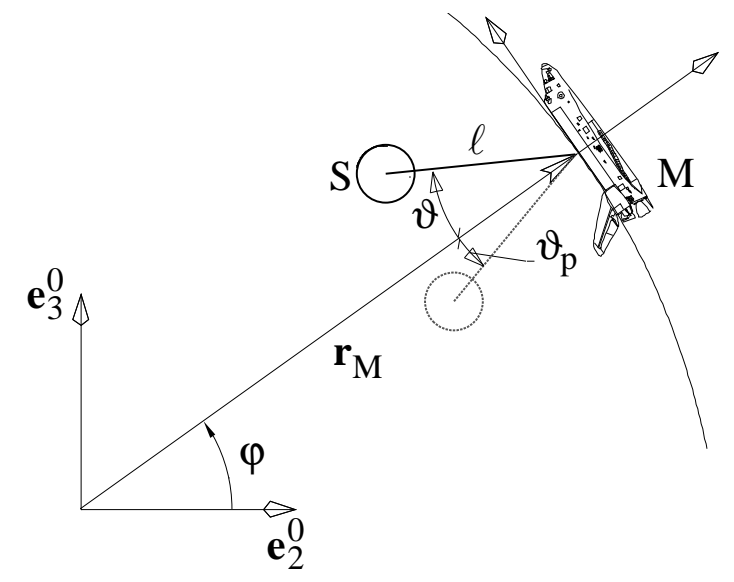

Fig. 1. Space station ("M") on an elliptic orbit and subsatellite ("S") swinging about the small periodic orbit $\vartheta_{p}(t)$.
One of the shortcomings in previous investigations was the restriction to perfectly circular orbits of the space station. Therefore we assume, that the space station moves on an Keplerian ellipse with small eccentricity. In the initial state the subsatellite performs a tiny periodic motion close to the local vertical direction. At the end the satellite should reach another periodic solution farther apart from the space station.

In the limiting case of zero eccentricity the small initial and final periodic solutions agree with the radial rest positions and we can use the trajectories obtained in our previous investigations. Applying a continuation from the circular problem to larger eccentricities we want to study the evolving shape of the optimal paths.

\section{MECHANICAL MODEL AND GOVERNING EQUATIONS}

In order to derive manageable equations of motion, we state the following assumptions:

- The space station and the subsatellite move in the equatorial plane and the gravity potential is perfectly symmetric.

- The mass of the space station is negligible compared to the mass of the earth, but is significantly larger than the mass of the sub-satellite. Therefore the motion of the sub-satellite has no influence on the space station's orbit.

- The tether is massless, straight and inextensible. The control is applied by pulling at one end of the cable.

- The eccentricity of the elliptic orbit is very small $e \ll 1$.

- The maximum length of the tether is small compared to the radius $\ell / r_{M} \ll 1$.

Of course, most of these assumptions are quite simplifying and should be carefully inspected in a real application.

In dimensionless quantities the dynamics of the space station is governed by the well known equations for a body in a central field 


$$
\begin{aligned}
\ddot{r}_{M} & =-1 / r_{M}^{2}+r_{M} \dot{\varphi}^{2}, \\
\ddot{\varphi} & =-2 \dot{r}_{M} \dot{\varphi} / r_{M} .
\end{aligned}
$$

The constant angular momentum $p_{\varphi}=r_{M}^{2} \dot{\varphi}$ is taken to be 1 , such that the circular orbit is given by $r_{M} \equiv 1$ and $\varphi=\varphi_{0}+t$. The rescaled time $t$ corresponds to orbital time.

The equations for the subsatellite can be written as

$$
\begin{aligned}
\ddot{\vartheta}= & \ddot{\varphi}-\frac{2 \dot{\ell}(\dot{\vartheta}-\dot{\varphi})+2 \dot{r}_{M} \dot{\varphi} \cos \vartheta+\ddot{r}_{M} \sin \vartheta}{\ell} \\
& -\frac{r_{M}}{\ell}\left(\sin \vartheta / r_{1}^{3}+\dot{\varphi}^{2} \cos \vartheta+\ddot{\varphi} \sin \vartheta\right), \\
\ddot{\ell}= & -u+\ddot{r}_{M} \cos \vartheta-2 \dot{r}_{M} \dot{\varphi} \sin \vartheta-\ell\left(1 / r_{1}^{3}-(\dot{\vartheta}-\dot{\varphi})^{2}\right) \\
& +r_{M}\left(\cos \vartheta / r_{1}^{3}-\dot{\varphi}^{2} \cos \vartheta-\ddot{\varphi} \sin \vartheta\right),
\end{aligned}
$$

where $r_{1}^{2}=r_{M}^{2}-2 r_{m} \ell \cos \vartheta+\ell^{2}$ and the control force $u(t)$ satisfies

$$
0 \leq u \leq u_{\max }
$$

Using Pontryagin's Maximum Principle (Leitman (1981)) we search the optimal control $u(t)$, which minimizes the penalty function

$$
C=\int_{0}^{T}\left(1+c_{v} \vartheta^{2} / 2\right) d t
$$

and steers the satellite from the initial state

$$
\ell(0)=\ell_{0}, \quad \dot{\ell}(0)=0, \quad \vartheta(0)=\vartheta_{0}(0), \dot{\vartheta}(0)=\dot{\vartheta}_{0}(0)
$$

to the final position

$$
\ell(T)=\ell_{1}, \quad \dot{\ell}(T)=0, \quad \vartheta(T)=\vartheta_{1}(T), \dot{\vartheta}(T)=\dot{\vartheta}_{1}(T),
$$

where $\vartheta_{i}(t)$ are the periodic solutions close to the radial direction with constant $\ell=\ell_{i}$. Since the frequencies of the small oscillations $\vartheta_{i}(t)$ differ by a small amount, these have to be calculated additionally.

The coefficient $c_{v}$ in (6) serves to avoid too large intermediate lateral deviations from the radial direction.

\section{PRELIMINARY RESULTS}

In order to calculate the optimal solutions for eccentric orbits, we apply a continuation method (Seydel (1984)) starting from the available solutions for circular orbits. For the solution of the boundary value problem with switching conditions the program Bndsco (Oberle et al. (1985)) is used. In Fig. 2 the evolution of the state variables $\ell(t)$ (scaled) and $\vartheta(t)$ is displayed for different values of the eccentricity $\varepsilon$. It is clearly visible, that the amplitude of the solutions and the time period to reach the final state depend strongly on $\varepsilon$. The time-optimal path of the sub-satellite in a co-rotating frame is displayed in Fig. 3. Dashed segments indicate free flight paths $(u=0)$, whereas solid segments show paths with applied tension $\left(u=u_{\max }\right)$. For these solutions the ratio tether length has been scaled, such that the final tether length corresponds to 700 metres. Since the eccentricities are very small, the periodic near-axial motions are invisible. Nevertheless the eccentricity has a remarkable influence on the shape of the obtained trajectories.

During the talk the dependence of the optimal solutions on the eccentricity, the final tether length and the parameter $c_{v}$ will be studied in greater detail.

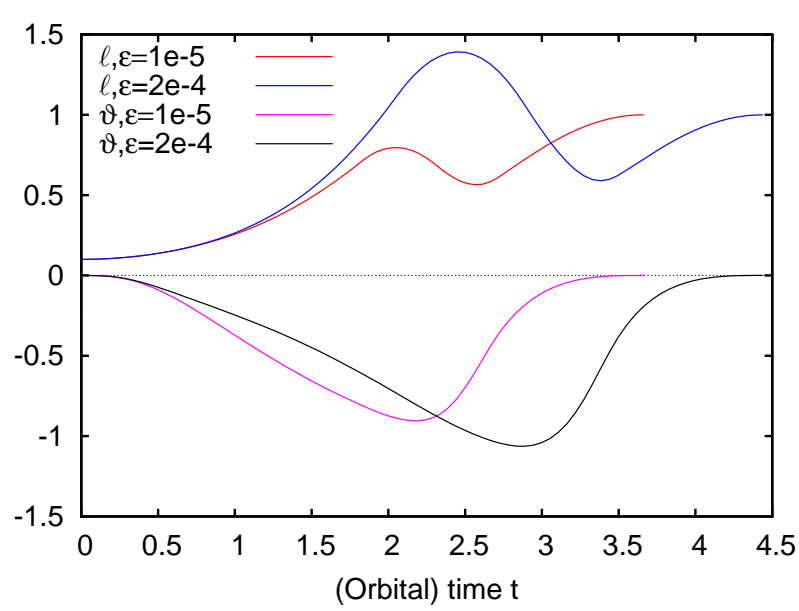

Fig. 2. Variation of the state variables $\ell(t)$ (scaled) and $\vartheta(t)$ for different values of eccentricity $\varepsilon$

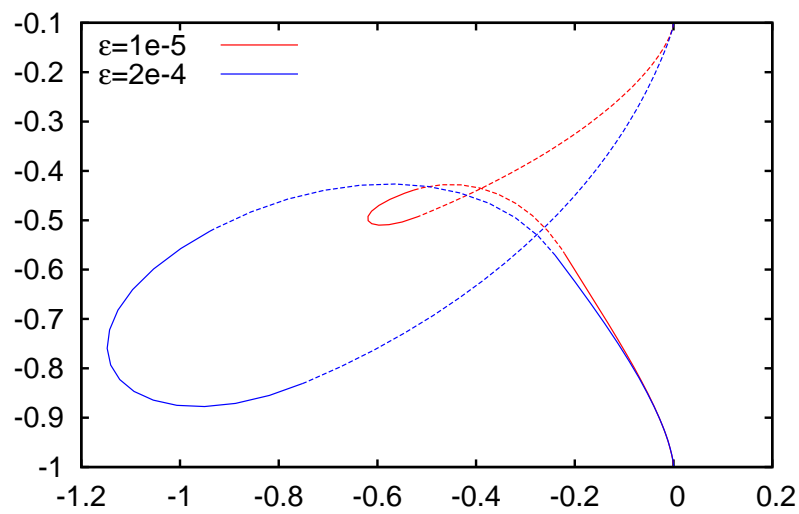

Fig. 3. Comparison of trajectories for different values of the eccentricity $\varepsilon$

\section{REFERENCES}

Barkow, B., Steindl, A., Troger, H., and Wiedermann, G. (2003). Various methods of controlling the deployment of a tethered satellite. Journal of Vibration and Control, $9,187-208$.

Jin, D.P. and Hu, H.Y. (2006). Optimal control of a tethered subsatellite of three degrees of freedom. Nonlinear Dynamics, 46, 161-178.

Leitman, G. (1981). The Calculus of Variations and Optimal Control - An Introduction. Plenum Press.

Oberle, H.J., Grimm, W., and Berger, E. (1985). BNDSCO, Rechenprogramm zur Lösung beschränkter optimaler Steuerungsprobleme. Benutzeranleitung M 8509, Techn. Univ. München.

Seydel, R. (1984). A continuation algorithm with step control. In Numerical methods for bifurcation problems. ISNM 70. Birkhäuser.

Williams, P. (2008). Optimal deployment/retrieval of tethered satellites. Journal of Spacecraft and Rockets, $25(2), 324-343$. 


\title{
Bicycle Rider Control Modelling for Path Tracking
}

\author{
Johannes Edelmann Martin Haudum Manfred Plöchl \\ Institute of Mechanics and Mechatronics, Vienna University of \\ Technology,Vienna, Austria (e-mail: johannes.edelmann@tuwien.ac.at; \\ martin.haudum@tuwien.ac.at; manfred.ploechl@tuwien.ac.at)
}

\section{INTRODUCTION}

The need to understand the steering behaviour of a bicycle rider may result from an engineering requirement as well as from scientific interest. For vehicle dynamics simulations - with focus on the bicycle rather than on the rider a 'virtual test rider' is needed to stabilize the motion of the bicycle and to track a demanded trajectory. From a more general point of view, insight into human steering behaviour of bicycles and conclusions thereof are desirable.

Thus, a growing number of scientific papers addresses the dynamics of the bicycle and the rider. An overview of papers on the dynamics of both motorcycles and bicycles is given by Limebeer and Sharp (2006), a comprehensive, recent review on bicycle dynamics and rider control literature is given by Schwab and Meijaard (2013). However, most of the research in rider control is dedicated to the rider of the motorcycle, see for instance the review by Popov et al. (2010), and more recent Massaro et al. (2012).

To distinguish driver/rider models from 'automatic driving controllers', at least some human key demands, for instance preview, prediction/anticipation, adaptation/learning or planning capabilities need to be mapped, see e.g. Plöchl and Edelmann (2007).

In the full paper, stability properties as well as basic dynamic characteristics of the passive (uncontrolled) bicyclerider system and consequences on the rider control modelling are addressed. In particular, the unstable motion of the system at low velocities and bandwidth limitations caused by non-minimum phase dynamics are emphasized. To analyse the effectiveness of the steering torque and the lean torque as possible rider's inputs to control the dynamics of the bicycle, a controllability analysis of the bicycle-rider system has been performed. Finally, a new bicycle rider control model considering basic human rider properties is presented.

\section{RIDER CONTROL MODELLING}

The rider modelling approach presented in the paper is based on findings of Donges (1978) on automobile driving. Donges concluded from measurements on two levels of control action of the driver. A feed-forward control represents the guidance level of steering activity and mimics the anticipatory response to the desired path curvature. A compensatory feedback control compensates for estimated, prospective deviations from the desired trajectory due to external disturbances and lack of knowledge of the driver/rider on the dynamics of the vehicle. We assume the rider to set a desired roll angle of the bicycle from experience for both the guidance level and the compensatory level by applying the necessary steering torque to the handlebar, Figure 1. Due to the inherently unstable motion of the bicycle within a respective velocity range, in addition to path tracking, the rider needs to stabilize the motion of the bicycle at these velocities. For that purpose, the steering torque applied to the handlebar to obtain the desired roll angle is derived in a third level, the stabilizing feedback level. The proposed structure of the rider control model allows for feed-forward control at the bicycle guidance level and maps the rider's ability to stabilize the bicycle, without aiming to track a certain trajectory.

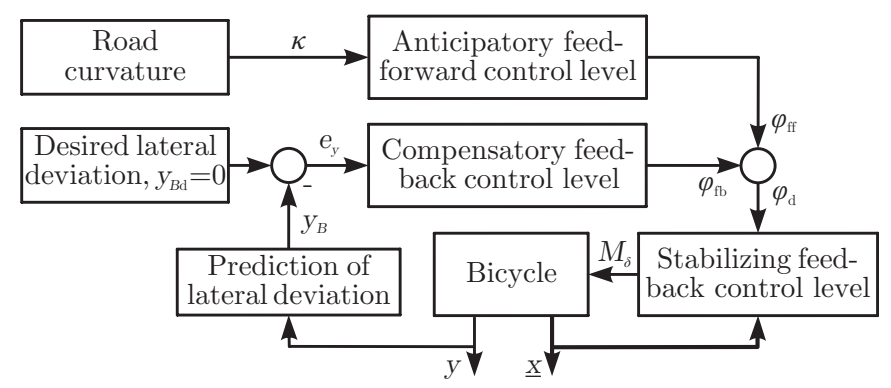

Fig. 1. Schematic diagram of the bicycle rider control model.

\section{REFERENCES}

E. Donges. A two-level model of driver steering behaviour. Human factors, 20(6):691-707, 1978.

D.J.N. Limebeer and R.S. Sharp. Bicycles, motorcycles, and models. IEEE Control Systems Magazine, 26:34-61, 2006.

M. Massaro, R. Lot, and V. Cossalter. A virtual motorcycle driver to simulate real manoeuvres from experimental data. Proc. IMechE Part D: J. Automobile Engineering, 226(9):1211-1219, 2012.

M. Plöchl and J. Edelmann. Driver models in automobile dynamics application. Vehicle System Dynamics, 45(78):699-741, 2007.

A.A. Popov, S. Rowell, and J.P. Meijaard. A review on motorcycle and rider modelling for steering control. Vehicle System Dynamics, 48(6):775-792, 2010.

A.L. Schwab, J.P. Meijaard. A review on bicycle dynamics and rider control. Vehicle System Dynamics, 51(7): 1059-1090, 2013.

R.S. Sharp. On the stability and control of the bicycle, Applied Mechanics Reviews, 61:060803-1-24, 2008. 


\title{
Efficient simulation of railway pantograph/ catenary interaction using pantograph-fixed coordinates
}

\author{
Daniel Ritzberger * Emir Talic* Alexander Schirrer* \\ * Vienna University of Technology, Austria, (e-mail: \\ daniel.ritzberger@gmail.com).
}

In this paper, a novel formulation of the railway catenary and pantograph dynamics is proposed with the goal to make a real-time simulation feasible. The predominant system formulation found in the literature consists of a resting catenary, which is described by a set of coupled partial differential equations, and a moving pantograph. The catenary is modelled as a whole with fixed wire positions at both ends. Since the pantograph moves over the catenary, the length of the system has to be at least the size of the travelled distance during the simulation and has to be increased even further for unphysical reflections at the boundary to not disturb the solution. This consequently leads to large numerical systems that require great computational effort. To make this problem real-time solvable modal superposition methods have been used at the cost of model accuracy.

The formulation proposed in this paper addresses the aforementioned problems and introduces a coordinate transformation so that the pantograph is at rest with respect to the new coordinate and the catenary moves over the pantograph. The computational domain is then truncated at a length of one span of the catenary. At the boundary of the computational domain, absorbing boundary conditions are applied to reduce unphysical reflections. This leads to a reduction in variables that have to be calculated each time step. Furthermore the length of the system is independent from the simulation time.

The small-scale moving system and a large reference system with fixed catenary and moving pantograph are compared in numerical results. It shows that the computation time is greatly reduced and the solution gives a good approximation of the system dynamics. 


\title{
Co-Simulation of Matlab and FlightGear for Identification and Control of Aircraft
}

\author{
Guilherme Aschauer* Alexander Schirrer* Martin Kozek* \\ * Inst. of Mechanics 8 Mechatronics, Vienna University of Technology, \\ Austria \\ e-mail: guilherme.aschauer@tuwien.ac.at
}

The paper outlines the development of a co-simulation solution of Matlab and FlightGear in which the communication between these programs is done via UDP without needing further toolsets. In order to obtain high flexibility, Simulink and especially the Aerospace Toolbox have not been used.

The simulation and rendering is done by FlightGear and flight measurement signals (at least altitude and heading) are sent to Matlab, which in turn sends back actuator input values (throttle, elevator, aileron, and rudder) computed by a flight control system. A simplified outline of the communication is shown in Figure 1.

This approach benefits from the high degree of realism provided by FlightGear which already uses well-established, realistic flight dynamic models, which are based on the nonlinear equations of motion and therefore avoids the use of potentially over-simplified flight dynamics models. Also, different planes and flying objects can be used.

After establishing a working connection between Matlab and FlightGear, identification runs are made for longitudinal and lateral motion independent of each other for different altitudes with a chirp signal overlaid with low-pass filtered white noise as input data to guarantee sufficient excitation in the relevant frequency areas. With this input/ output data a model estimation process is performed using gray-box models.

Using the so obtained dynamic models, three different reference tracking controllers (heading and altitude) are designed: a model predictive controller (MPC), an LQR controller, and a scheduled LQR controller.

Additionally, two state observers are designed, assuming that only the altitude and heading are measurable, but corrupted by noise. A block diagram of the structure of an integrated LQR controller utilizing a state observer is shown in Figure 2.

The models and controllers are then validated by flying through turbulent weather conditions with reference test signals including steps and ramps.

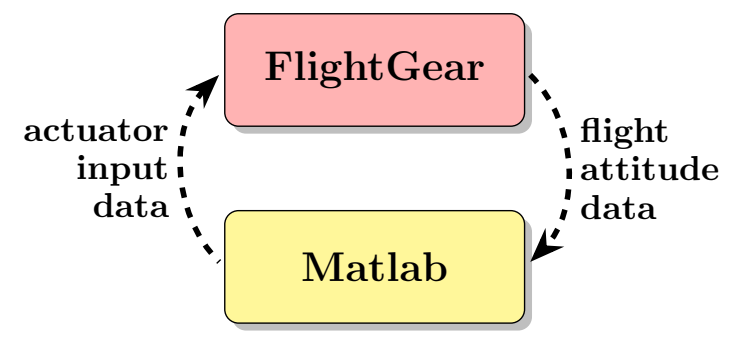

Fig. 1. UDP communication block diagram

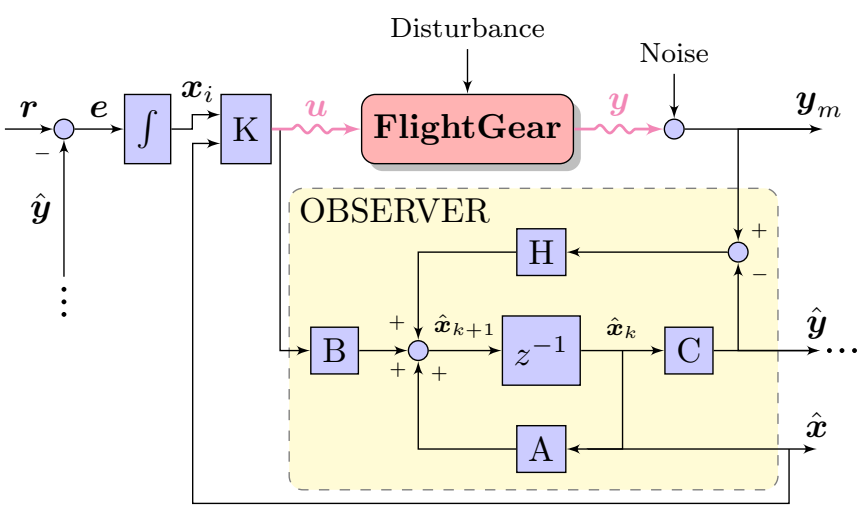

Fig. 2. Integrating LQR controller with state observer 


\title{
Data-based and analytic modelling for model-based control of a flexible beam
}

\author{
Christian Dullinger* Alexander Schirrer* Martin Kozek* \\ * Vienna University of Technology, Austria (e-mail: \\ christian.dullinger@tuwien.ac.at).
}

\begin{abstract}
This paper evaluates and compares data-based and analytic modelling for modelbased active vibration control based on the performance of the closed loop system for a simply supported flexible beam. The thin beam is controlled utilizing collocated piezo patch actuators and sensors. An electrodynamic shaker introduces a disturbance force in the direction of oscillation. The data-based design plant is identified using N4SID. The analytically obtained design plant is based on a one-dimensional finite element (FE) model. The comparison of the two entirely different modelling approaches is validated using a variety of different optimal \& robust multi-input multi-output (MIMO) control design methods.
\end{abstract}

\section{INTRODUCTION}

Optimizing a structure for low weight generally leads to decreased overall stiffness and lower natural frequencies of structural vibrations. Because of low damping, occurring vibrations can show large amplitudes. For these reasons, so called "smart structures" are increasingly used. By utilizing integrated actuators, sensors and a controller, the dynamic properties of the structure can be improved. Novel so-called macro fiber composites (MFC) piezo patches are well suited as actuators/sensors for structural control applications and exhibit almost hysteresis-free, linear behavior. Classic PID control can not adequately address the structural control task, this can be done efficiently by model-based feedback control. For flexible structures with simple geometry analytical/numerical modelling approaches like the finite element method (FEM) are able to provide accurate dynamic models. However, for modelbased control the input-output behavior is of fundamental importance. Therefore, a direct, data-based identification of the input-output behavior is a powerful approach.

This paper evaluates and compares these two entirely different modelling approaches specifically in their capability to produce design models for control design using the specific example of a beam with collocated piezo patch actuators/sensors and an electrodynamic shaker as disturbance source shown schematically in Figure 1, see also Dullinger et al. (2014). The comparison of the modelling approaches is validated based on the performance of the closed loop system using a variety of different control design methods. Therefore, the answer to the question - "Which modelling approach provides the better design plant?" - is valid not only for a specific controller, but provides a more general statement.

\section{METHODOLOGY}

\subsection{System modelling}

Identified (ID) plant model In order to directly include the physical couplings between inputs and outputs

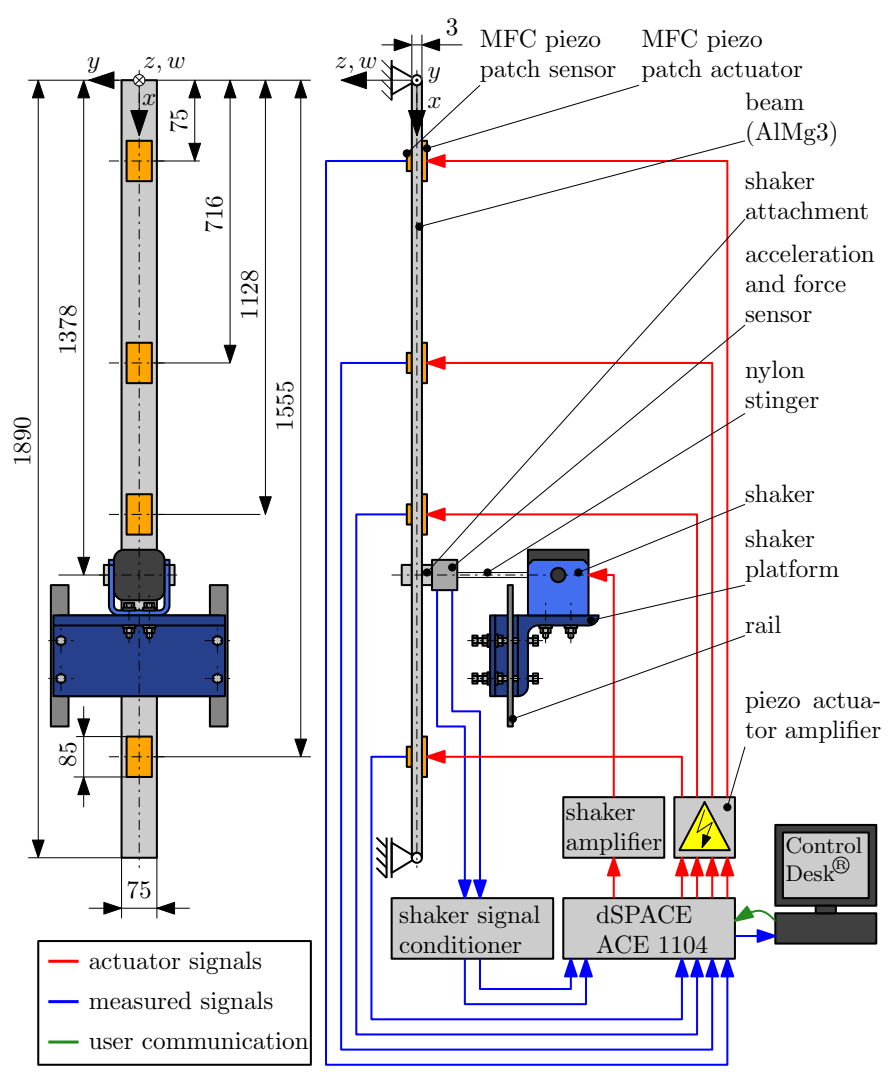

Figure 1. Schematic sketch of the experimental set-up

a MIMO identification procedure had to be used. To concentrate the excitation on the modes below $50 \mathrm{~Hz}$ low-pass filtered discrete white noise signals (i.e. Gaussian random amplitudes at the chosen sampling frequency of $5 \cdot 10^{3} \mathrm{~Hz}$ ) were applied to the four piezo actuators and the shaker. Subsequently, the data were filtered and down-sampled to $100 \mathrm{~Hz}$. Mean values and linear trends were removed. Then N4SID (see Ljung (1987)) was applied to identify stable, linear state-space models in discrete-time. 
Finite element (FE) model For spatial discretization of the bending beam 100 Hermitian beam elements (i.e. Euler-Bernoulli beams with Hermitian shape functions) with two translational and two rotatory degrees of freedom were used to describe the displacement $w$ in transversal $z$ direction. This lead to a nodal model in nodal coordinates represented by the second-order matrix differential equation in terms of the nodal displacement vector $\boldsymbol{q}$ :

$$
\begin{aligned}
\boldsymbol{M} \ddot{\boldsymbol{q}}+\boldsymbol{D} \dot{\boldsymbol{q}}+\boldsymbol{K} \boldsymbol{q} & =\boldsymbol{B} \boldsymbol{u} \\
\boldsymbol{y} & =\boldsymbol{C}_{\mathrm{q}} \boldsymbol{q}
\end{aligned}
$$

The global mass and stiffness matrix $\boldsymbol{M}$ respectively $\boldsymbol{K}$ of the beam were obtained by assembling the local matrices of the Hermitian beam element. A Rayleigh Damping matrix $\boldsymbol{D}=\alpha \boldsymbol{M}+\beta \boldsymbol{K}$ was chosen. Information of the identified model of the beam without shaker was used for computing $\alpha$ and $\beta$. The piezo patch actuators were approximated by two opposite single moments, one at each end of a patch. The shaker excitation force was modeled as a single transversal force. The inputs are collected in $\boldsymbol{u}$. $\boldsymbol{B}$ represents the input matrix. For the four piezo sensors bending strain outputs were formulated and collected in $\boldsymbol{y} . \boldsymbol{C}_{\boldsymbol{q}}$ represents the output matrix. By transforming the nodal model to modal coordinates it was feasible to replace the fist five modal damping coefficients by those of the identified model of the pure beam. In order to enable a selection of the modes of interest the modal model in modal coordinates was transformed to a state-space system in modal form, see Gawronski (2004). Beside the before mentioned benefit the modal form enabled an efficient modelling of parametric uncertainty. The fact that longitudinal displacements were not modeled together with the collocation of the piezo patch actuators and sensors required a state-space feed through matrix. The influence of a mounted shaker was modelled by a feedback loop utilizing the force input and an additional acceleration and displacement output at the shaker position.

\subsection{Investigated optimal \& robust control design methods}

LQG control with modal weighting is well suited to directly address the damping of undesirable eigenmodes. However, robustness can not directly be tuned or guaranteed. Thus, model errors can be highly restrictive particularly in the presence of lightly damped structural modes. This motivates optimal \& robust $\mathcal{H}_{\infty}$ control design methods for structural control which provide robustness guarantees for norm-bounded model uncertainties. Utilizing frequency-dependent design weights, the potential performance improvements by $\mathcal{H}_{\infty}$ control heavily depend on an adequate uncertainty description. Mixed-sensitivity $\mathcal{H}_{\infty}$ optimal control has implicit robustness properties. A more complex design method called $\mathrm{D}(\mathrm{G}) \mathrm{K}$-iteration allows for an explicit uncertainty modelling and is therefore potentially even more powerful. However, a $D(G) K$ design requires more effort in tuning, its solution demands sophisticated numerical tools, and often it produces a large dynamic system order of the synthesized controller, see Skogestad and Postlethwaite (2005).

\section{VALIDATION OF CONTROL PERFORMANCE}

Utilizing the methods from above a variety of controllers were designed, implemented on the dSPACE platform and

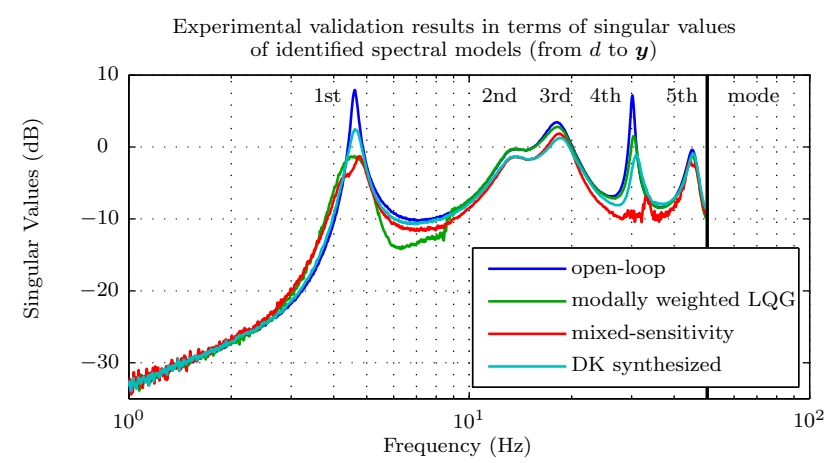

Figure 2. Experimentally validated (disturbance rejection) performance; design plant: "ID model"

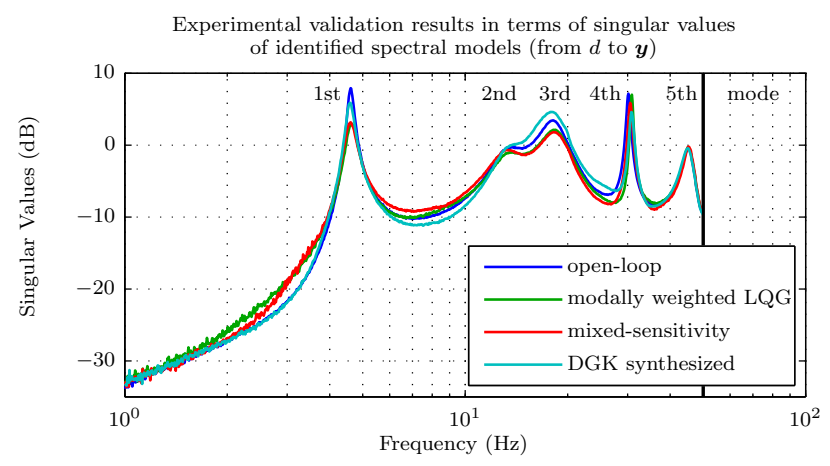

Figure 3. Experimentally validated (disturbance rejection) performance; design plant: "FE model"

validated on the test bed using a noise signal as disturbance signal $d$. In Figure 2 and Figure 3 the experimental results are compared in terms of singular values of spectral, non-parametric models for the identified respectively the analytical model as design plant. For the FE model the natural frequency of the lowest-damped, fourth mode stated a critical model error leading to difficulties when designing controllers to improve the damping of this mode in the experiment. This task was best accomplished by a DGK-synthesized controller applying specific uncertainty modelling on the fourth mode.

\section{CONCLUSIONS}

The data-based modelling approach clearly provided the better design plant. No increased effort in controller design/uncertainty modelling was necessary for the identified model. Nevertheless, it has been shown that also an FE model utilizing a one-dimensional finite element for spatial discretization can actually be used as design plant.

\section{REFERENCES}

Dullinger, C., Schirrer, A., and Kozek, M. (2014). Advanced control education: Optimal \& robust mimo control of a flexible beam setup. In 19th IFAC World Congress, Cape Town, volume 19, 9019-9025.

Gawronski, W.K. (2004). Advanced Structural Dynamics an Active Control of Structures. Springer.

Ljung, L. (1987). System identification: theory for the user. Prentice-Hall information and system sciences series. Prentice-Hall.

Skogestad, S. and Postlethwaite, I. (2005). Multivariable Feedback Control. Wiley. 


\title{
Analysis of time-varying mechanical systems using the method of impulsive parametric excitation
}

\author{
T. Pumhössel ${ }^{*}$ P. Hehenberger ${ }^{* *}$ \\ * Johannes Kepler University Linz, Austria (e-mail: \\ thomas.pumhoessel@jku.at). \\ ** Johannes Kepler University Linz, Austria (e-mail: \\ peter.hehenberger@jku.at).
}

In this contribution, mechanical systems with time-varying parameters are investigated by replacing the original system of continuous differential equations by a set of difference equations using the method of impulsive parametric excitation of C.S. Hsu, see Hsu (1972); Hsu and Cheng (1973). This description allows to analyze the effect of time-varying parameters to the modal energy content, and moreover, provides a basis for applying parametric excitation in order to affect the energy content in a desired manner. It is assumed, that the equations of motion of a mechanical system are of the form

$$
\mathbf{M} \mathbf{x}^{\prime \prime}(\tau)+\mathbf{C x}^{\prime}(\tau)+(\mathbf{K}+p(\tau) \mathbf{G}) \mathbf{x}(\tau)=\mathbf{0},
$$

where $\mathbf{x}$ represents the $n$-dimensional displacement vector, and $\mathbf{M}, \mathbf{C}$ and $\mathbf{K}$ the constant and symmetric mass, damping and stiffness matrices. The term $p(\tau) \mathbf{G}$ represents a time-varying stiffness, with $p(\tau)$ being any arbitrary function of time $\tau$, and $\mathbf{G}$ a constant and symmetric matrix. The function $p(\tau)$ is approximated by a series of DiracDelta functions. Therefore, the sequence of instants of time $\bar{\tau}_{0}<\bar{\tau}_{1}<\ldots<\bar{\tau}_{k-1}<\bar{\tau}_{k}<\bar{\tau}_{k+1}<\ldots<\bar{\tau}_{K}$ is introduced, where $\bar{\tau}_{k}-\bar{\tau}_{k-1}, k=1,2, \ldots K$, is constant. For each interval $\left[\bar{\tau}_{k-1}, \bar{\tau}_{k}\right], p(\tau)$ is replaced by an impulsive function of Dirac-Delta type located at the center $\tau_{k}=\left(\bar{\tau}_{k}-\bar{\tau}_{k-1}\right) / 2$ of the interval and with a strength $\varepsilon_{k}$ which is approximated by $\varepsilon_{k}=p\left(\tau_{k}\right)\left(\bar{\tau}_{k}-\bar{\tau}_{k-1}\right)$. Applying a modal transformation $\mathbf{x}=\mathbf{\Phi} \mathbf{y}$, where $\boldsymbol{\Phi}$ comprises the natural modeshapes of the undamped mechanical system without parametric excitation, the equations of motion are replaced approximately by

$$
\mathbf{M}^{*} \mathbf{y}^{\prime \prime}+\mathbf{C}^{*} \mathbf{y}^{\prime}+\mathbf{K}^{*} \mathbf{y}=-\left\{\sum_{k=1}^{K} \varepsilon_{k} \delta\left(\tau-\tau_{k}\right) \mathbf{G}^{*}\right\} \mathbf{y}
$$

where $\delta\left(\tau-\tau_{k}\right)$ represents the Dirac-Delta function. The effect of a parametric impulse of Dirac-Delta type to the state-vector is given by

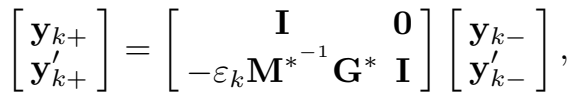

where the -/+-sign denotes quantities just before/after the instant of time the parametric impulse is applied. Therewith, the variation of the energy $E_{i}$ of a mode $i$, caused by a parametric impulse can be written in the form

$$
\Delta E_{i, k}=-\varepsilon_{k} \beta_{i, k-}\left[y_{i, k-}^{\prime}-\frac{1}{2 \mu_{i}} \varepsilon_{k} \beta_{i, k-}\right],
$$

where $\beta_{i, k-}=\sum_{j=1}^{n} \gamma_{i j} y_{j, k-}$. Based on Eq. (4), parametric impulses can be applied, to affect the modal energy

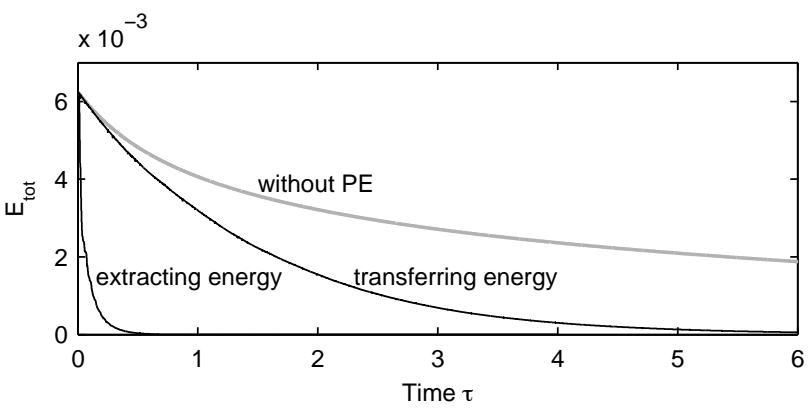

Fig. 1. Comparison of total energy content for transferring and extracting vibration energy by impulsive parametric excitation, with the case where no parametric excitation is present.

content of a mechanical system in a targeted manner. One application is the transfer of energy across modes. Therefore, the overall number of $n$ modes is divided in a set $A$ of lower, and a set $B$ of higher modes. It can be shown, that a certain impulsive strength $\varepsilon_{k}$ exists, which allows to shift vibration energy from set $A$ to set $B$ of modes. Therewith, the damping properties of the mechanical system can be utilized much more effectively, and hence, a faster decay of vibrations than without parametric excitation is observed. Another possibility is the extraction of energy from mechanical systems with parametric impulses. Figure 1 shows a comparison of the decay of the total energy $E_{\text {tot }}$ of a mechanical system with three degrees of freedom, where no parametric excitation is present, with the cases where energy is transferred from mode set $A=\{1,2\}$ to set $B=\{3\}$, respectively, energy is extracted from the overall mechanical system. Extracting energy, as well as transferring energy to higher modes, results in a much faster decay of the energy content of the mechanical system, compared to the case without parametric excitation, where only natural damping is present.

\section{REFERENCES}

Hsu, C. (1972). Impulsive parametric excitation: Theory. Journal of Applied Mechanics, 39(2), 551-558.

Hsu, C. and Cheng, W. (1973). Applications of the theory of impulsive parametric excitation and new treatments of general parametric excitation problems. Journal of Applied Mechanics, 40(1), 78-86. 


\title{
MATHMOD 2015 ABSTRACT VOLUME
}

\author{
MATHMOD Minisymposium \\ Free Boundary Problems in Applications
}


MATHMOD 2015 Abstract Volume 


\title{
Numerics of contact line motion for thin films
}

\author{
Dirk Peschka * \\ * Weierstrass Insitute, Mohrenstr. 39, 10117 Berlin, Germany \\ (e-mail: dirk.peschka@wias-berlin.de)
}

\begin{abstract}
We introduce an algorithm for the explicit treatment of contact line motion for thinfilm problems and compare its solutions with exact source-type solutions and their asymptotic behavior near the contact line.
\end{abstract}

Keywords: thin fluid films, free boundary problems, numerical algorithms, self-similar solutions

\section{MOTIVATION \& ALGORITHM}

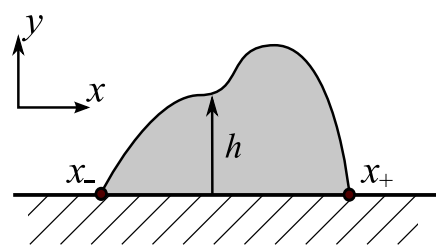

The spreading of a viscous liquid droplet of height $h(t, x)$ on a solid substrate is governed by the differential equation

$$
\begin{aligned}
& \dot{h}+\left(|h|^{n} h_{x x x}\right)_{x}=0, \\
& h(0, x)=h_{0}(x),
\end{aligned}
$$

where we use the notation $\dot{h}=\partial_{t} h$. The mobility exponent $n$ depends on the type of friction with the substrate, where usually one has $0<n \leq 3$ as it is discussed by Eggers (2004). Additionally we assume that the initial support is an interval $\left(x_{-}, x_{+}\right):=\operatorname{supp} h_{0}$, where $x_{ \pm}$evolve with time. As boundary conditions we consider a zero contact angle and specify a kinematic condition, so that for $t>0$

$$
\begin{aligned}
& h_{x}\left(t, x_{ \pm}\right)=0, \\
& \dot{x}_{ \pm}=\lim _{x \rightarrow x_{ \pm}}\left(|h|^{n-1} h_{x x x}\right) .
\end{aligned}
$$

For $n>1$ the kinematic condition (1d) implies $h_{x x x} \rightarrow \infty$ as $x \rightarrow x_{ \pm}$for the contact line to move with a finite velocity. For the free-boundary problem above existence of solutions in weighted Hölder spaces was shown by Giacomelli and Knüpfer (2010).

Numerical algorithms for this problem mainly rely on global solutions for this problem, i.e. algorithms which solve for $h(t, x)$ for $x \in \mathbb{R}$ and preserve non-negativity outside $\left(x_{-}, x_{+}\right)$in a sense, see e.g. the works by Zhornitskaya and Bertozzi (1999); Grün and Rumpf (2000). Here we go a different route and do not look for global solutions but rather seek solutions of the free-boundary problem (1). Therefore we use finite elements and seek spatially discrete, piecewise linear functions $\dot{h}, \pi$ that satisfy

\footnotetext{
* Financial support by DFG in the MATHEON project C10 and by Einstein Center for Mathematics in Berlin ECMath in project OT1
}

$$
\begin{aligned}
& \int_{x_{-}}^{x_{+}}\left(\dot{h} \phi+|h|^{n} \pi_{x} \phi_{x}\right) \mathrm{d} x=0 \\
& \int_{x_{-}}^{x_{+}}\left(\pi \varphi-\tau \dot{h}_{x} \varphi_{x}\right) \mathrm{d} x=\int_{x_{-}}^{x_{+}} h_{x} \varphi_{x} \mathrm{~d} x
\end{aligned}
$$

for all $\phi, \varphi$. No essential boundary conditions are imposed on solutions or test functions and all appearances of $h$ are treated explicitly. In order to arrive at (2) we introduced a new variable $\pi=-h_{x x}$ and split (1a) in two second order equations. In (2b) defining $\pi$ we replaced $h$ by $h+\tau \dot{h}$ where $\tau=t^{k+1}-t^{k}$ to obtain a stable method similar to a (semi)-implicit Euler method. For any given $h$ defined on $\left(x_{-}, x_{+}\right)$this gives us the time-derivative $\dot{h}$ in the Eulerian reference frame. However, we need to specify a method to compute $x_{ \pm}$and $h$ at time $t^{k+1}$ from the corresponding data at time $t^{k}$. Here we use the fact that in a reference frame moving with velocity $\dot{\psi}$ time derivatives of $H(t, y)=h(t, \psi(t, y))$ simply transform according to $\dot{H}=\dot{h}+\dot{\psi} h_{x}$. If we choose $\psi(t, y)=x_{-}(t)+y\left(x_{+}(t)-\right.$ $\left.x_{-}(t)\right)$ with $y=\left(x-x_{-}\left(t^{k}\right)\right) /\left(x_{+}\left(t^{k}\right)-x_{-}\left(t^{k}\right)\right)$ then we can determine $\dot{\psi}, \dot{H}$ entirely and uniquely from $\dot{h}$ and $h_{x}$ at $t^{k}$. Then we can move the vertices of the decomposition and the nodal values according to

$$
\begin{aligned}
& x^{k+1}=x^{k}+\tau \dot{\psi}\left(x^{k}\right) \\
& h^{k+1}=h^{k}+\tau \dot{H}
\end{aligned}
$$

which concludes the algorithm.

\section{REFERENCES}

Eggers, J. (2004). Toward a description of contact line motion at higher capillary numbers. Physics of Fluids (1994-present), 16(9), 3491-3494.

Giacomelli, L. and Knüpfer, H. (2010). A free boundary problem of fourth order: classical solutions in weighted Hölder spaces. Communications in Partial Differential Equations, 35(11), 2059-2091.

Grün, G. and Rumpf, M. (2000). Nonnegativity preserving convergent schemes for the thin film equation. $\mathrm{Nu}$ merische Mathematik, 87(1), 113-152.

Zhornitskaya, L. and Bertozzi, A.L. (1999). Positivitypreserving numerical schemes for lubrication-type equations. SIAM Journal on Numerical Analysis, 37(2), 523555 . 


\title{
Sharp interface limits of an anisotropic phase field model for solid-state dewetting *
}

\author{
Marion Dziwnik* Andreas Münch ${ }^{* *}$ Barbara Wagner ${ }^{*, * * *}$ \\ * Technische Universität Berlin, Institute of Mathematics, Straße des \\ 17. Juni 136, 10623 Berlin, Germany (e-mail: \\ dziwnik@math.tu-berlin.de) \\ ** Mathematical Institute, University of Oxford, 24-29 St. Giles', \\ Oxford OX1 3LB,UK (e-mail: muench@maths.ox.ac.uk) \\ *** Weierstrass Institute, Mohrenstraße 39, 10117 Berlin, Germany \\ (e-mail: bwagner@math.tu-berlin.de)
}

\begin{abstract}
We propose a phase field model for solid state dewetting where the surface energy is weakly anisotropic. The evolution is based on the Cahn-Hilliard equation with degenerate mobility and a free boundary condition at the film-substrate contact line. We derive the corresponding sharp interface limit via matched asymptotic analysis involving multiple inner layers. The resulting sharp interface model is consistent with the pure surface diffusion model. In addition, we show that the natural boundary conditions, as indicated from the first variation of the total free energy, imply a contact angle condition for the dewetting front, which, in the isotropic case, is consistent with the well-known Young's equation.
\end{abstract}

Keywords: phase field model, matched asymptotic expansions, sharp interface model, free boundaries, dewetting solid films

\section{INTRODUCTION}

Dewetting of solid films is one of the important processes used for nanostructuring and functionalizing surfaces for a variety of technological applications, such as for example in thin film solar cells and other optoelectronic devices.

Since the dynamical dewetting process usually involves a succession of topological transitions of the thin dewetting film the phase field framework provides an adequate modeling approach for a continuum description.

Similar as in the isotropic case, studied by Jiang et al. (2012), the evolution is based on the Cahn-Hilliard equation with a degenerate mobility, where the appropriate choice of the degeneracy significantly affects the corresponding sharp interface limit, as pointed out by Lee et al. (2014). We additionally include anisotropic surface energies, as introduced by Kobayashi (1993), and investigate dewetting from a solid substrate, which implies corresponding boundary conditions at triple junctions for the anisotropic Cahn-Hilliard equation.

In the present paper we propose a phase field model for solving the anisotropic surface diffusion dewetting problem and derive the corresponding sharp-interface limit in the weakly anisotropic case and inside the model domain which confirms the approach of surface diffusion for the present choice of mobility $M$ and free energy $F$. Moreover we deal with the corresponding boundary condition at the triple junction and apply an appropriate asymptotic

^ MD and BW gratefully acknowledges the support by the Federal Ministry of Education (BMBF) and the state government of Berlin (SENBWF) in the framework of the program Spitzenforschung und Innovation in den Neuen Ländern (Grant Number 03IS2151). method in order to derive the anisotropic contact angle boundary condition.

\section{MODELING}

We consider a phase-field function $u=u(\mathbf{x})$ that lives on the domain $\Omega$, where $u(\mathbf{x})=0$, has been defined such that $u(\mathbf{x}) \equiv 1$ represents the film and $u(\mathbf{x}) \equiv-1$ the vapor phase. For this phase-field variable the total free energy $W$ combines the Ginzburg-Landau free energy density for the film-vapor system and the energy density of the wall

$$
W^{\epsilon}=\int_{\Omega} f_{F V} d \Omega+\int_{\gamma_{w}} f_{w} d \Gamma_{w} .
$$

Following the approach of Kobayashi (1993), we consider an anisotropic free energy functional of the form

$$
f_{F V}(u, \nabla u)=\lambda\left(F(u)+\frac{\epsilon^{2}}{2} \gamma(-\nabla u)^{2}|\nabla u|^{2}\right),
$$

and

$$
f_{w} \equiv \frac{\gamma_{V S}+\gamma_{F S}}{2}-\frac{u\left(3-u^{2}\right)}{4}\left(\gamma_{V S}-\gamma_{F S}\right),
$$

where $F(u)$ is the homogeneous free energy, $\gamma: \mathbb{R}^{2} \rightarrow \mathbb{R}^{+}$ is the anisotropic interface energy between film and vapor, $\gamma_{V S}$ is the interface energy of the vapor-surface and $\gamma_{F S}$ of the fluid-surface interface and $\lambda$ represents the mixing energy density.

We assume that the order parameter $u$ is conserved and define the mass flux of $u$ to be

$$
\mathbf{j}=M(u) \nabla \mu,
$$

where the chemical potential $\mu$ is the first variational derivative of $W^{\epsilon}$ with respect to $u$ 


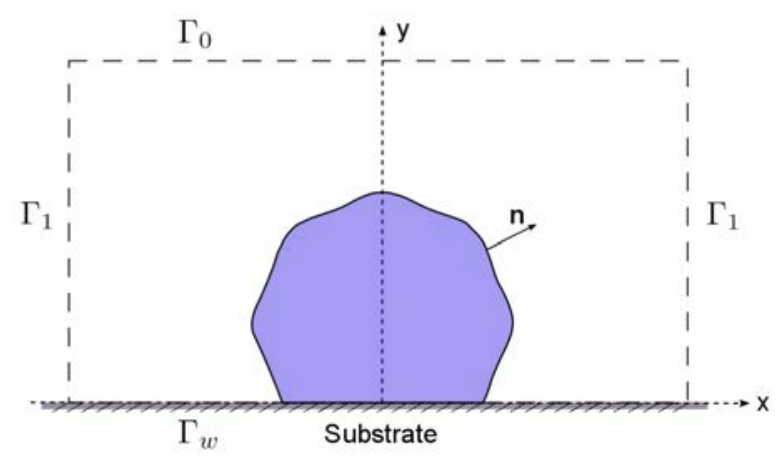

Fig. 1. A sketch of the model domain.

$$
\mu(u):=F^{\prime}(u)-\epsilon^{2} \nabla\left(\gamma \gamma^{\prime}\left(\begin{array}{c}
-u_{y} \\
u_{x}
\end{array}\right)+\gamma^{2} \nabla u\right)
$$

and we chose the biquadratic diffusional mobility $M(u)$ and free energy $F(u)$ to be

and

$$
M(u)=\left(1-u^{2}\right)^{2},
$$

$$
F(u)=\frac{1}{2}\left(1-u^{2}\right)^{2}
$$

Fick's second law then yields the anisotropic Cahn-Hilliard equation

$$
\begin{aligned}
\partial_{t} u & =\nabla \cdot M(u) \nabla \mu, \\
\mu & =F^{\prime}(u)-\epsilon^{2} \nabla\left(\gamma \gamma^{\prime}\left(\begin{array}{c}
-u_{y} \\
u_{x}
\end{array}\right)+\gamma^{2} \nabla u\right),
\end{aligned}
$$

subject to the following natural boundary conditions

$$
\begin{array}{r}
\epsilon \mathbf{m} \cdot\left[\gamma(\theta) \gamma^{\prime}(\theta)\left(\begin{array}{c}
-u_{y} \\
u_{x}
\end{array}\right)+\gamma(\theta)^{2} \nabla u\right]+\frac{f_{w}^{\prime}}{\lambda}=0, \\
\mathbf{m} \cdot(M(u) \nabla \mu)=0,
\end{array}
$$

on $\Gamma_{w}$ and

$$
\begin{array}{r}
\mathbf{m} \cdot \nabla u=0, \\
\mathbf{m} \cdot(M(u) \nabla \mu)=0,
\end{array}
$$

on $\partial \Omega \backslash \Gamma_{w}$.

\section{SHARP INTERFACE LIMITS}

\subsection{Away from the solid boundary}

We apply the method of matched asymptotic expansions in order to study the long time behaviour of (8) in the limit $\epsilon \rightarrow 0$ and capture the contribution from surface diffusion. Motivated by Lee et al. (2014), we consider three layers, an outer layer away from the contact line, an interior layer about the interface, and furthermore, a second interior layer where $u$ is below and arbitrarily close to 1 . The reason for considering this second interior layer is, that in inner coordinates about the interface, the solution is similar to a tanh function, which differs from its asymptotic value -1 as the inner coordinate tends to $-\infty$ by a small amount $\epsilon$. Taking the exponential representation of tanh this distance can be measured to be of $O(\ln (1 / \epsilon))$, which means that the free boundary, where $u=1$, is asymptotically far away from the interface. Consequently a second interior layer must match between the classic outer and inner solution. Finally we obtain the sharp interface problem which correctly describes the anisotropic evolution due to surface diffusion

$$
\begin{aligned}
& \mu_{1}=\frac{2}{3}\left(\gamma_{0}+\gamma_{0}^{\prime \prime}\right) \kappa, \\
& v_{n}=\left(\frac{2}{3}\right)^{2} \partial_{s s}\left(\left(\gamma_{0}+\gamma_{0}^{\prime \prime}\right) \kappa\right),
\end{aligned}
$$

where $\mu_{1}$ is the first order outer correction for the chemical potential, $\gamma_{0}$ the leading order of the anisotropy function, $\kappa$ the mean curvature, $v_{n}$ the normal velocity and $s$ the arclength.

\subsection{On solid boundaries}

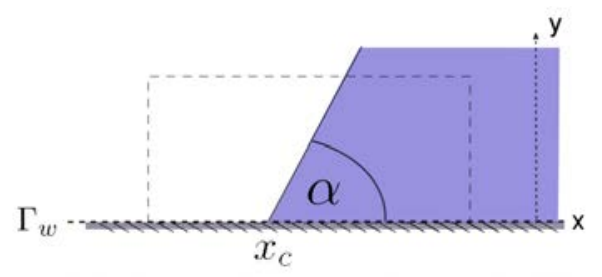

Fig. 2. A sketch of the local domain.

We study the behavior of the anisotropic Cahn-Hilliard equation (8) in a local domain around the contact point $x_{c}$ with boundary condition (8c). Motivated by Owen et al. (1990) we study the behaviour of $u$ in a box around the contact point $x_{c}$. Introducing a boundary layer and an interior layer which imply corresponding matching conditions, we show that the leading order system of (8) with boundary condition (8c) leads to the contact angle boundary condition

$$
\gamma_{V S}-\gamma_{F S}=-\gamma_{0}^{\prime} \sin \alpha+\gamma_{0} \cos \alpha,
$$

which is referred to Young-Herring condition in the literature (Min and Wong (2006)).

\section{CONCLUSION}

In the present work we introduced a phase field model for anisotropic surface diffusion dewetting and derived the corresponding asymptotic sharp interface limit. We confirmed that the present phase field model describes motion through pure surface diffusion.

Moreover we derived a boundary condition for the moving boundary at the solid substrate and verified that this condition corresponds to a contact angle boundary condition in the sharp interface model.

\section{REFERENCES}

Jiang, W., Bao, W., Thompson, C.V., and Srolovitz, D.J. (2012). Phase field approach for simulating solid-state dewetting problems. Acta Mater., 60, 5578- 5592.

Kobayashi, R. (1993). Modeling and numerical simulations of dendritic crystal growth. Physica D, 63, 410-423.

Lee, A.A., Münch, A., and Süli, E. (2014). Sharp interface limits of the Cahn-Hilliard equation with degenerate mobility. Submitted to SIAM Journal of Applied Mathematics, unknown, unknown.

Min, D. and Wong, H. (2006). The effect of strong surface energy anisotropy on migrating grain-boundary grooves. Jour. Appl. Phys., 100, 053523.

Owen, N., Rubinstein, J., and Sternberg, P. (1990). Minimizers and gradient flows for singularly perturbed bistable potentials with a dirichlet condition. Proc. $R$. Soc. Lond. A, 429, 505-532. 


\title{
A free boundary problem for interfaces in forward-backward lattice diffusion
}

\author{
Michael Helmers *,** \\ * Institute for Applied Mathematics, University of Bonn, Endenicher \\ Allee 60,53115 Bonn, Germany (e-mail: helmers@iam.uni-bonn.de) \\ ** Joint work with Michael Herrmann, Institute for Computational and \\ Applied Mathematics, University of Münster
}

The forward-backward diffusion equation

$$
\partial_{\tau} U=\partial_{\xi}^{2} P, \quad P=\Phi^{\prime}(U)
$$

where $\Phi^{\prime}$ is a bistable nonlinearity as in Figure 1 is in general ill-posed due to the decreasing branch of $\Phi^{\prime}$. A microscopic regularization of (1) is given by the lattice ODE

where

$$
\dot{u}_{j}(t)=\Delta p_{j}(t), \quad p_{j}=\Phi^{\prime}\left(u_{j}\right)
$$

$$
\Delta p_{j}=p_{j+1}-2 p_{j}+p_{j-1},
$$

provided that the macroscopic variables are introduced by the parabolic scaling

$$
\tau=\varepsilon^{2} t, \quad \xi=\varepsilon j, \quad U_{\varepsilon}(\tau, \xi)=u_{j}(t)
$$

with $\varepsilon>0$ being a small parameter. Other known regularizations are the viscous approximation and the CahnHilliard model, which add $\varepsilon^{2} \partial_{\xi}^{2} \partial_{\tau} U$ and $-\varepsilon^{2} \partial_{\xi}^{4} U$, respectively, to the right hand side of the first equation in (1).

A key feature of the dynamics of (1) and any regularization are phase interfaces which separate space-time regions where $U$ takes values in one of the two stable phases, that is, in the different connected components of $\left\{u: \Phi^{\prime \prime}(u)>0\right\}$. Heuristic arguments and numerical simulations indicate that the effective dynamics of the lattice as $\varepsilon \rightarrow 0$ is for a large class of initial data described by a free boundary problem with hysteresis. In the case of a single interface located at $\xi^{*}(\tau)$, this limit combines bulk diffusion

$$
\partial_{\tau} U=\partial_{\xi}^{2} P
$$

for $\xi \neq \xi^{*}(\tau)$ with the Stefan condition

$$
\frac{\mathrm{d} \xi^{*}}{\mathrm{~d} \tau}\|U\|+\left\|\partial_{\xi} P\right\|=0=\|P\|
$$

and the hysteretic flow rule

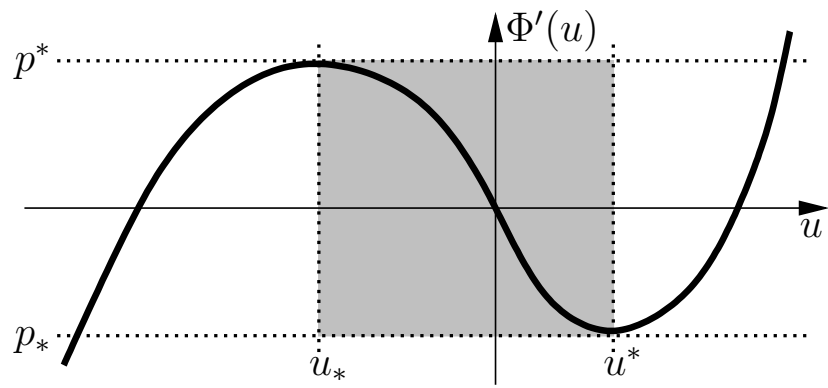

Fig. 1. Bistable nonlinearity with the stable regions $\left(-\infty, u_{*}\right) \cup\left(u^{*}, \infty\right)=\left\{u: \Phi^{\prime \prime}(u)>0\right\}$ and the unstable branch $\left(u_{*}, u^{*}\right)$ where $\Phi^{\prime}$ decreases.

$$
\begin{aligned}
& \left.\frac{\mathrm{d} \xi^{*}}{\mathrm{~d} \tau} \| U\right]<0 \quad \Longrightarrow \quad P=p^{*}, \\
& \frac{\mathrm{d} \xi^{*}}{\mathrm{~d} \tau}\|U\|>0 \quad \Longrightarrow \quad P=p_{*}
\end{aligned}
$$

at $\xi=\xi^{*}(\tau)$. Here $\|\cdot\|$ denotes the jump across the interface and $p^{*}, p_{*}$ are the inflection points of $\Phi$. See Figure 2 for an illustration.

Since the dynamics of a moving interface in the lattice contain several relevant time scales, a rigorous justification of the above limit model for arbitrary potentials is currently out of reach. However, for the piecewise quadratic potential

$$
\Phi(u)=\frac{1}{2}\left(u^{2}-2|u|+1\right), \quad \Phi^{\prime}(u)=u-\operatorname{sign} u
$$

the analysis simplifies significantly and the limit model follows from careful inspection of the microscopic dynamics and the representation formula

$$
p_{j}(t)=\sum_{k \in \mathbb{Z}} g_{j-k}(t) p_{k}(0)-2 \sum_{k \geq 1} \chi_{\left[t_{k}^{+}, \infty\right)}(t) g_{j-k}\left(t-t_{k}\right)
$$

where $\left(g_{j}\right)_{j \in \mathbb{Z}}$ denotes the discrete heat kernel and $\chi_{I}$ is the indicator function of the time interval $I$.

In this contribution, we review the major aspects of the microscopic dynamics as well as the difficulties they pose for passing to the limit as $\varepsilon \rightarrow 0$. We then use the insight gained from heuristic arguments and the results for the case (2) to discuss ideas for the rigorous treatment of general potentials.

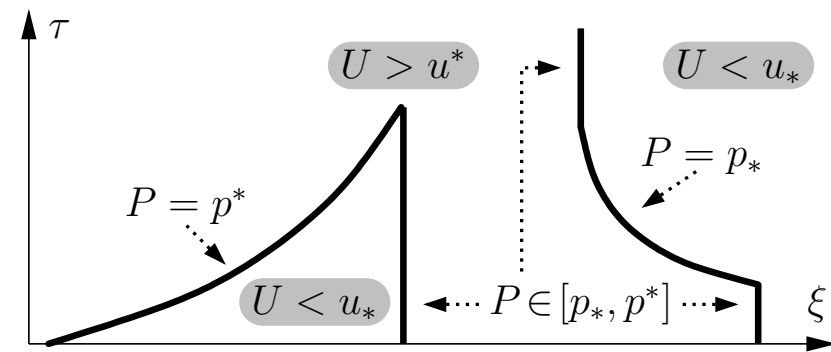

Fig. 2. Sketch of three interfaces. The left interface moves until it collides with the one in the middle; the right interface is initially at rest, then starts to move and later stops again. 


\title{
Sharp Interface Limits of the Cahn-Hilliard Equation with Degenerate Mobility
}

\author{
Alpha A Lee* Andreas Münch ${ }^{* *}$ Endre Süli ${ }^{* * *}$ \\ * Mathematical Institute, University of Oxford, Andrew Wiles Building, \\ Woodstock Road, Oxford (e-mail: alpha.lee@maths.ox.ac.uk) \\ ** Mathematical Institute, University of Oxford, Andrew Wiles \\ Building, Woodstock Road, Oxford (e-mail: \\ andreas.muench@maths.ox.ac.uk) \\ *** Mathematical Institute, University of Oxford, Andrew Wiles \\ Building, Woodstock Road, Oxford (e-mail: endre.suli@maths.ox.ac.uk)
}

Phase field models are a common framework to describe the mesoscale kinetics of phase separation and patternformation. Replacing a sharp interface by a diffuse order parameter profile, phase field models avoid the numerically challenging interface tracking, and are versatile enough to capture topological changes. Although models can be constructed starting from a systematic coarse-graining of the microscopic dynamics, their use as a numerical tool to solve free boundary problems requires careful consideration of what their correct asymptotic long-time sharp interface limits are.

One of the simplest models for spinodal demixing of binary alloys is the Cahn-Hilliard equation, where the order parameter $u$ is conserved and satisfies

$$
\begin{aligned}
u_{t} & =-\nabla \cdot \mathbf{j}, \quad \mathbf{j}=-M(u) \nabla \mu, \\
\mu & =-\epsilon^{2} \nabla^{2} u+f^{\prime}(u), \\
f(u) & =\frac{1}{4}\left(1-u^{2}\right)^{2},
\end{aligned}
$$

where $M(u)$ is the mobility function; $\mathbf{j}$ is the flux; $\mu$ is the chemical potential; $\epsilon$ is the interfacial tension which determines the width of the interface, and $f(u)$ is the bulk free energy.

Whilst the asymptotic sharp interface limit $\epsilon \rightarrow 0$ for $M(u)=$ const has been shown by Pego (1989) (and proven rigorously in Alikakos et al. (1994)) to reduce to the Mullins-Sekerka problem (Mullins and Sekerka, 1963) at the long time scale $t=O(1 / \epsilon)$, the sharp interface limit for a degenerate mobility function, which vanishes at the pure phases, e.g. $M(u)=\frac{1}{2}\left(1-u^{2}\right)$, has been recently a matter of controversy.

A body of prior works (e.g. Bhate et al. (2000); Yeon et al. (2006); Jiang et al. (2012)) used the Cahn-Hilliard equation with degenerate mobility as a basis for solving the surface diffusion free boundary problem, where the interface velocity is proportional to the surface Laplacian of the mean curvature, i.e.

$$
v_{n}=\mathcal{M} \Delta_{s} \kappa
$$

with $\mathcal{M}$ a constant and $\kappa$ the mean curvature. This phase field approach to surface diffusion model has been the key framework for modelling a myriad of complex physical processes such as electromigration in metals (Mahadevan and Bradley, 1999), heteroepitaxial growth (Rätz et al.,

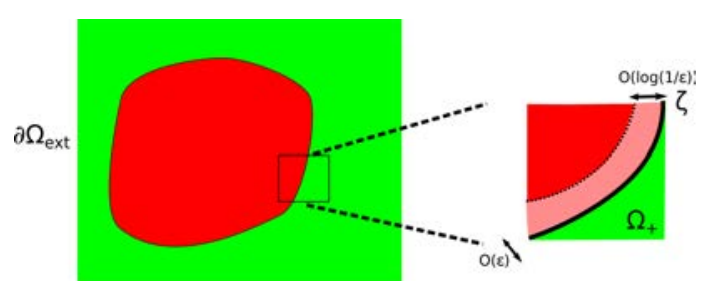

Fig. 1. Illustration of the asymptotic structure of the degenerate mobility case. The dotted line indicates the contact line free boundary beyond which $u=1$, and the solid line denotes the interface $\zeta$. There are interior layers about the contact line and interface respectively, and an outer layer outside the precipitate (the green region $\Omega_{+}$).

2006) and more recently solid-solid dewetting (Jiang et al., 2012). Heuristically, the degeneracy of the mobility function at the pure phases suppresses the flux normal to the interface and therefore the diffusion from or into the bulk. However, recently, Gugenberger et al. (2008) have suggested an inconsistency in the matching condition used and cast this "conventional wisdom" into doubt.

To resolve this conundrum, we revisit the matched asymptotic analysis of Equation (1). Our analysis reveals several aspects of the asymptotic structure of the solution which were not taken into account previously. First, unlike the constant mobility case, degeneracy in the mobility introduces a free boundary where the order parameter reaches $u= \pm 1$. The dynamics of the free boundary "contact line" couples with the dynamics of the interface, and requires an $O(\epsilon \log (1 / \epsilon))$ small contact line region to complete the asymptotic analysis via exponential asymptotics (see Figure 1 for the asymptotic structure of the solution).

Second, we found that with the commonly used quadratic mobility $M(u)=\frac{1}{2}\left(1-u^{2}\right)$, mass flux from and into the bulk region (where $u= \pm 1$ ) still appears in the leading order dynamics. As a result of that, the interface velocity in the sharp interface model has two contributions: one from surface diffusion (Equation (2)) which is local to the interface, and another contribution from nonlinear bulk diffusion. More precisely, our analysis leads to the sharp interface limit 


$$
\begin{aligned}
\nabla \cdot\left(\mu_{1} \nabla \mu_{1}\right) & =0, \quad \text { in } \Omega_{+}, \\
\mu_{1} & =\frac{2}{3} \kappa, \quad \text { on } \zeta, \\
\nabla_{n} \mu_{1} & =0, \quad \text { on } \partial \Omega_{\mathrm{ext}}, \\
v_{n} & =\frac{2}{3} \Delta_{s} \kappa+\frac{1}{4} \mu_{1} \nabla_{n} \mu_{1} \quad \text { on } \zeta,
\end{aligned}
$$

with the definition of $\Omega_{+}, \Omega_{\text {ext }}$ and $\zeta$ identical to Figure 1. Unlike pure surface diffusion, the mass flux contribution to normal velocity in Equation (3), which satisfies a porousmedium equation, couples precipitates with each other. This results in coarsening where larger precipitates grow at the expense of smaller ones.

Third, not all degenerate mobilities are the same. For higher order mobilities $M_{\alpha}(u)=\left(1-u^{2}\right)^{\alpha}, \alpha>1$, our asymptotics suggests that the normal mass flux does not enter into $v_{n}$ at leading order, so that the surface diffusion model is recovered in the asymptotic limit. (Note that $M_{\alpha}^{\prime}(1)$ diverges for $\alpha<1$, hence we restrict our attention to the case $\alpha>1$.)

Fourth, the sharp interface limit is dependent on the precise form of the free energy. Our asymptotic matching relies on a continuous free energy function, which can be derived via a Landau-Ginzburg expansion based on symmetry arguments for a second order phase transition. The Landau-Ginzburg formalism assumes that the free energy is an analytic function. Indeed, the double obstacle free energy, which is the low temperature limit of the lattice gas entropy and diverges at the pure phases, was shown to give surface diffusion flow even for $M_{1}(u)=\left(1-u^{2}\right)$ (Cahn et al., 1996). This outlines an underappreciated piece of physics: different free energy functions for phase transitions, though being in the same equilibrium universality class, may have drastically different dynamics.

Numerical solutions of Equation (1) agree well with our asymptotic results. Taken together, our asymptotic framework conclusively closes the conundrum in the literature and proposes the phase field approximation to a new free boundary problem involving coupled surface diffusion and nonlinear bulk diffusion. Phase field approaches to surface diffusion cannot be realised with the Cahn-Hilliard equation with a degenerate mobility $M(u)=1-u^{2}$ and polynomial free energy, as was repeatedly assumed in the literature - a higher order degeneracy in the mobility or a double obstacle free energy maybe needed.

\section{REFERENCES}

Alikakos, N.D., Bates, P.W., and Chen, X. (1994). Convergence of the Cahn-Hilliard equation to the Hele-Shaw model. Archive for Rational Mechanics and Analysis, 128(2), 165-205.

Bhate, D.N., Kumar, A., and Bower, A.F. (2000). Diffuse interface model for electromigration and stress voiding. Journal of Applied Physics, 87(4), 1712-1721.

Cahn, J.W., Elliott, C.M., and Novick-Cohen, A. (1996). The Cahn-Hilliard equation with a concentration dependent mobility: motion by minus the laplacian of the mean curvature. European Journal of Applied Mathematics, 7(3), 287-302.

Gugenberger, C., Spatschek, R., and Kassner, K. (2008). Comparison of phase-field models for surface diffusion. Physical Review E, 78(1), 016703.
Jiang, W., Bao, W., Thompson, C.V., and Srolovitz, D.J. (2012). Phase field approach for simulating solid-state dewetting problems. Acta Materialia, 60(15), 55785592 .

Mahadevan, M. and Bradley, R.M. (1999). Phase field model of surface electromigration in single crystal metal thin films. Physica D: Nonlinear Phenomena, 126(3), 201-213.

Mullins, W.W. and Sekerka, R.F. (1963). Morphological stability of a particle growing by diffusion or heat flow. Journal of Applied Physics, 34(2), 323-329.

Pego, R.L. (1989). Front migration in the nonlinear CahnHilliard equation. Proceedings of the Royal Society of London. A. Mathematical and Physical Sciences, 422(1863), 261-278.

Rätz, A., Ribalta, A., and Voigt, A. (2006). Surface evolution of elastically stressed films under deposition by a diffuse interface model. Journal of Computational Physics, 214(1), 187-208.

Yeon, D.H., Cha, P.R., and Grant, M. (2006). Phase field model of stress-induced surface instabilities: Surface diffusion. Acta materialia, 54(6), 1623-1630. 


\title{
A Gradient Flow Approach for Dissipative Equations on a Free Domain
}

\author{
Martijn M. Zaal* \\ * Institut für Angewandte Mathematik, Universität Bonn, Germany \\ (e-mail:mzaal@iam.uni-bonn.de).
}

In this paper, we will study a gradient flow approach for proving existence of solutions to dissipative equations with a free boundary. For the class of problems we consider, the movement of the boundary is determined by its mean curvature and the equation in the domain. The most important example is the following problem:

$$
\left\{\begin{aligned}
\frac{\partial u}{\partial t} & =\kappa \Delta u, & & \text { for } x \in \Omega_{t}, t>0, \\
-\kappa \frac{\mathrm{d} u}{\mathrm{~d} \mathbf{n}} & =u v, & & \text { for } x \in \partial \Omega_{t}, t>0, \\
v & =H+u, & & \text { for } x \in \partial \Omega_{t}, t>0,
\end{aligned}\right.
$$

where $\mathbf{n}$ denotes the outward normal to $\partial \Omega_{t}$, and the normal velocity $v$ and mean curvature $H$ of $\partial \Omega_{t}$ are signed accordingly, that is, $H=-2$ for the unit sphere in $\mathbb{R}^{3}$. This problem was proposed as a simplified model for cell swelling by osmosis. In this model, the free domain $\Omega_{t}$ should be interpreted as being a single cell submerged in pure water. The quantity $u$ is the concentration density of a particular solute, e.g. salt, that is trapped inside the cell. From this point of view, the first boundary condition can be interpreted as a non-permeability condition: it is not difficult to show that this condition means that there is no flux of solute through the boundary. The boundary of the domain, now representing the membrane of the cell, moves by two counteracting effects: the surface tension, trying to minimize the surface area of the membrane, and the osmotic force arising from the difference in concentration of solute across the membrane. This is modeled by the equation for the normal velocity $v$.

It appears that Problem (1) can be seen as a gradient flow: a first indication is the existence of a Lyapunov functional

$$
\Phi(\Omega, u)=|\partial \Omega|+\int_{\Omega} u \log u \mathrm{~d} x .
$$

Note that both terms of $\Phi$ can be interpreted: the first is the surface area of the membrane, and the second one is the negative Boltzmann entropy. Moreover, we will see that the osmotic force, represented by the term $u$ in the equation for $v$, arises from the coupling.

As Problem (1) seems to have traits of a gradient flow, it makes sense to try to apply the theory of gradient flows and gradient flow-like systems. A strategy to prove existence of gradient flow-like systems is based on the backward Euler scheme for approximating gradient flows in $\mathbb{R}^{n}$. More precisely, an approximation of the gradient flow $\dot{x}=-\nabla V(x)$ can be obtained by repeatedly solving

$$
\min _{y \in \mathbb{R}^{n}} V(y)+\frac{1}{2 h}\left|y-x\left(t_{k}\right)\right|^{2}
$$

where $t_{k}=k h$ for some discretization parameter $h>0$. Note that the problem (2) is much easier to generalize to, for example, a metric space as it does not involve any notion of gradient.

It is already know that the above scheme can be used to obtain solutions of the diffusion equation. The problem that has to be solved at every time step is

$$
\min _{v \in \mathcal{P}_{2}\left(\mathbb{R}^{n}\right)} \int_{\mathbb{R}^{n}} v \log v \mathrm{~d} x+\frac{1}{2 h} W_{2}^{2}\left(u\left(t_{k}\right), v\right),
$$

where $W_{2}$ is the standard Wasserstein metric on the space $\mathcal{P}_{2}\left(\mathbb{R}^{n}\right)$ of probability measures with finite second moment.

Similar results are also available for the mean curvature flow: if the functional

$$
|\partial F|+\frac{1}{h} \int_{E\left(t_{k}\right) \triangle F} d\left(x, \partial E\left(t_{k}\right)\right) \mathrm{d} x
$$

is minimized over the space of all sets of finite perimeter, it can be shown that, as $h \downarrow 0$, this results in a solution of the mean curvature flow under the condition that no multilayering takes place. Note that the second term of this functional is not the square of a metric.

We will show that a well-chosen combination of these two approaches can be used to obtain solutions of Problem (1). The main technical difficulty is to find an appropriate way of including the non-permeability condition into the formulation. It is, however, not necessary to include an extra term to account for the osmotic force, that is, the term $u$ in the equation for the normal velocity $v$.

If the diffusion equation in (1) is replaced by another (nonlinear) equation, the same approach can be used if the functional that is minimized on each time step is adjusted appropriately. It will be argued that this time discretization scheme converges, and that it formally solves a free boundary problem related to (1). 


\title{
Modelling, Simulation and Stability of Free Surface and Bulk Nanobubbles in Hydrogen Electrolysis
}

\author{
Sven-Joachim Kimmerle* \\ * University of the German Federal Armed Forces Munich, \\ Institute of Mathematics and Computer Applications (LRT-1), \\ 85577 Neubiberg/München, Werner-Heisenberg-Weg 39, Germany \\ (e-mail: sven-joachim.kimmerle@unibw.de)
}

\section{INTRODUCTION}

We consider two examples for precipitation by phase transitions, (i) nanobubbles arising in hydrogen electrolysis and (ii) liquid arsenic rich droplets in semi-insulating gallium arsenide (GaAs) crystals. In this talk, we focus on sharp-interface models and present the derivation of macroscopic evolution equations in detail for model (i), using formal homogenization techniques. The resulting nonlinear dynamical systems are simulated. We discuss stationary solutions and their stability with focus on model (ii). This allows to validate our models.

\section{HYDROGEN NANOBUBBLES}

Lifetimes of surface nanobubbles are observed experimentally that are orders of magnitude longer than one may expect from classical theory (Zhang et al. (2008)). Nanobubbles are encountered in a variety of applications, having desired or undesired effects. One important example for the latter is the generation of hydrogen nanobubbles using polymer electrolyte membrane electrolysers, where the surface nanobubbles (nanocaps) cover the Pt electrode, where the electrolysis is catalyzed. Several ansatzes have been suggested in order to explain the unexpected stability of nanocaps, e.g. (a) considering a dynamical equilibrium with steady in-/outflux over the phase interface, (b) thin films with impurities around the precipitates, and (c) interaction between multiple bubbles, including several nanocaps and also bulk nanospheres. It is important to consider effect (c) since most experiments have to deal inevitably with a large number of nanobubbles.

We consider here pure hydrogen nanobubbles, being spherical nanobubbles within water and spherical nanocaps sitting on the boundary with the electrode. We study approach (c) only and we propose a new model for the evolution of precipitates within this process. Using among other things the Young-Laplace and the Henry law, we may formulate an elliptic boundary value problem for the quasistatic evolution of the hydrogen concentration within the bubbles and the liquid in-between. This is coupled with ordinary differential equations for the free boundaries at the gas-liquid interface, i.e. the radii of nanospheres and the curvature radii of nanocaps, denoted by $\mathcal{R}_{i}$ for the corresponding bubble $i=1, \ldots \mathcal{N}(t)$. This coupled ODEPDE problem may be simplified by formal homogenization techniques exploiting typical scales. It turns out that in the liquid only the mean field concentration, that may be represented by an equivalent radius $\bar{R}$, has to be considered. Finally, we obtain a DAE system of the following form

$$
\begin{aligned}
& \frac{\partial}{\partial t} \mathcal{R}_{i}=C_{D}\left(1 / \overline{\mathcal{R}}-1 / \mathcal{R}_{i}\right), \quad i=1, \ldots \mathcal{N}, \\
& \bar{R}=\left(1-\eta \sum_{i=1}^{\mathcal{N}} \omega_{i} \mathcal{R}_{i}^{3}\right) /\left(1 / \hat{R}-\eta \sum_{i=1}^{\mathcal{N}} \omega_{i} \mathcal{R}_{i}^{2}\right),
\end{aligned}
$$

with certain non-negative constants $C_{D}, \hat{R}, \eta$ (encoding a volume fraction scale), and $\omega_{i}$, being the ratio between the volume of a nanocap and a nanosphere, if bubble $i$ is a cap, and being 1 otherwise.

We solve this problem numerically, in doing so it is crucial that our algorithm takes care of the vanishing of bubbles with time. With exception of the largest bubbles all precipitates disappear, in particular all spheres dissolve. However, we observe several meta-stable bubble radii, corresponding to caps, for times of $10^{-3}-10^{-1} \mathrm{~s}$. This fits to experimental observations and shows that (c) contributes to the observed stability of nanobubbles. However, this explanation of the long lifetimes depends e.g. sensitively on the typical bubble spacing, that is not known precisely.

\section{FURTHER ASPECTS}

For model (ii), the evolution of nanodroplets in the production of GaAs wavers during a final heat treatment, we encounter a mathematical system of similar type as (1) $\&$ (2). We present results on stationary radii and their stability in this context, since in this model we deal with spherical precipitates in the bulk only. The latter results may be extended to hydrogen nanobubbles. Note that the results of the GaAs model also fit well to experiments. For a mean field model for (ii) and numerical optimal control of the resulting macroscopic equations, see Kimmerle (2013).

\section{REFERENCES}

S.-J. Kimmerle. Optimal control of mean field models for phase transitions. In I. Troch, F. Breitenecker, editors, 7th Vienna International Conference on Mathematical Modelling, Vienna, Austria, February 14-17, 2012; IFAC Mathematical Modelling, volume 7/1, pages 1107-1111, 2013.

X.H. Zhang, A. Quinn, and W.A. Ducker. Nanobubbles at the interface between water and a hydrophobic solid. Langmuir, 24:4756-4764, 2008. 


\title{
Towards Topology Optimization in Quasi-Static Elastoplasticity
}

\author{
Stephan Schleicher * Roland Herzog ** \\ * University of Technology, Chemnitz, 09111 Germany (e-mail: \\ stephan.schleicher@mathematik.tu-chemnitz.de) \\ ** University of Technology, Chemnitz, 09111 Germany (e-mail: \\ roland.herzog@mathematik.tu-chemnitz.de)
}

Keywords: shape optimization

\section{INTRODUCTION AND MOTIVATION}

Topology optimization is a powerful tool in modern applied mathematics, e.g., to design lightweight structures or machine parts. Our goal is to develop a mathematical framework for the application of topology optimization techniques for models of elastoplasticity. Our objective functional may depend on the state variables $\boldsymbol{u}$ and $\boldsymbol{p}$ i.e., the displacement and the plastic strains respectively. These state variables satisfy a variational inequality related to the model of elastoplasticity in use. One possible application area could be the design of shock absorbers, i.e., structures $\Omega \subset \mathbb{R}^{3}$ which absorb given or random forces.

\section{THE FORWARD PROBLEM}

Before outlining the topology optimization problem we address the forward problem, following Han and Reddy (1999). The forward problem with linear kinematic hardening in its primal (stress-based) formulation reads as follows.

Given volume forces $\boldsymbol{f}: \Omega \times(0, T) \rightarrow \mathbb{R}^{3}$, find $\boldsymbol{w}=(\boldsymbol{u}, \boldsymbol{p}):(0, T) \rightarrow V \times Q_{0}$ with $\boldsymbol{w}(0)=(\mathbf{0}, \mathbf{0})$

such that for almost all $t \in(0, T)$,

$b(\boldsymbol{u}, \boldsymbol{v})-c(\boldsymbol{p}, \boldsymbol{v})=\langle\ell, \boldsymbol{v}\rangle \quad \forall \boldsymbol{v} \in V$

$j(\boldsymbol{q})-j(\dot{\boldsymbol{p}})-c(\boldsymbol{q}-\dot{\boldsymbol{p}}, \boldsymbol{u})+d(\boldsymbol{p}, \boldsymbol{q}-\dot{\boldsymbol{p}}) \geq 0 \quad \forall \boldsymbol{q} \in Q_{0}$.

where

$$
\begin{aligned}
V & =H_{0}^{1}(\Omega)^{3}, \\
Q_{0} & =\left\{\boldsymbol{q} \in L^{2}\left(\Omega ; \mathbb{R}^{3 \times 3}\right), \boldsymbol{q}_{i j}=\boldsymbol{q}_{j i}, \operatorname{tr} \boldsymbol{q}=0\right\}, \\
b(\boldsymbol{u}, \boldsymbol{v}) & =\int_{\Omega} \mathbb{C} \boldsymbol{\varepsilon}(\boldsymbol{u}): \boldsymbol{\varepsilon}(\boldsymbol{v}) \mathrm{d} \boldsymbol{x}, c(\boldsymbol{q}, \boldsymbol{v})=\int_{\Omega} \mathbb{C} \boldsymbol{q}: \boldsymbol{\varepsilon}(\boldsymbol{v}) \mathrm{d} \boldsymbol{x}, \\
d(\boldsymbol{p}, \boldsymbol{q}) & =\int_{\Omega} \mathbb{C} \boldsymbol{p}: \boldsymbol{q}+k \boldsymbol{p}: \boldsymbol{q} \mathrm{d} \boldsymbol{x}, j(\boldsymbol{q})=\int_{\Omega} \tilde{\sigma}|\boldsymbol{q}(\boldsymbol{x})| \mathrm{d} \boldsymbol{x} \\
\text { and }\langle\ell(t), \boldsymbol{v}\rangle & =\int_{\Omega} \boldsymbol{f}(t) \cdot \boldsymbol{v} \mathrm{d} \boldsymbol{x} .
\end{aligned}
$$

Here we use a model of plasticity containing linear elasticity $(\mathbb{C}$ is the fourth order elasticity tensor with Lamé parameters $\lambda$ and $\mu$ ) and linear kinematic hardening with the von Misses yield condition. Thus, we have only one hardening parameter $k$, the hardening modulus of the material. In that case the dissipation functional is given by $j($.) with the yield stress $\widetilde{\sigma}$. Throughout, $|\boldsymbol{q}|$ denotes the Frobenius norm of a matrix $\boldsymbol{q}$. We note that the boundary conditions may be more general.

In (Han and Reddy, 1999, chapter 7.2) we can find a proof of the existence and uniqueness of a solution of (EP).

\subsection{Solution Algorithm}

Using an implicit (backward) Euler scheme, we can reformulate (EP) as a sequence of minimization problems, in which we seek the update $\Delta \boldsymbol{w}_{n}$ at time $t=t_{n}$ (hence $\left.\boldsymbol{w}_{n+1}=\Delta \boldsymbol{w}_{n}+\boldsymbol{w}_{n}\right)$ which solves the following problem:

$$
\min _{\Delta \boldsymbol{w} \in V \times Q_{0}} \mathcal{L}_{n}(\Delta \boldsymbol{w})=\frac{1}{2} a(\Delta \boldsymbol{w}, \Delta \boldsymbol{w})+j(\boldsymbol{q})-\left\langle\boldsymbol{L}_{n}, \Delta \boldsymbol{w}\right\rangle,
$$

where $\Delta \boldsymbol{w}=(\Delta \boldsymbol{u}, \Delta \boldsymbol{p})$ and with $\boldsymbol{z}=(\boldsymbol{v}, \boldsymbol{q})$

$$
a(\Delta \boldsymbol{w}, \boldsymbol{z})=b(\Delta \boldsymbol{u}, \boldsymbol{v})-c(\Delta \boldsymbol{p}, \boldsymbol{v})-c(\boldsymbol{q}, \Delta \boldsymbol{u})+d(\Delta \boldsymbol{p}, \boldsymbol{q})
$$

and

$$
\left\langle\boldsymbol{L}_{n}, \Delta \boldsymbol{w}\right\rangle=\left\langle\ell\left(t_{n}\right), \Delta \boldsymbol{w}\right\rangle-a\left(\boldsymbol{w}_{n}, \Delta \boldsymbol{w}\right)
$$

depends on the forcing term and the state at the previous time step, see (Han and Reddy, 1999, chapter 12) for details.

The classical approach to solve this minimization problem is a predictor/corrector algorithm. First we seek the displacement update $\Delta \boldsymbol{u}_{n}$, afterwards we solve an additional minimization problem to get the update for the plastic strains $\Delta \boldsymbol{p}_{n}$.

We use the so called elastic predictor which solves a linear elasticity problem for the unknown displacement update estimation with volume forces given by previous iterations. The predictor step at time $t_{n}$ (we drop the time index for quantities of the current time) is:

Seek $\Delta \hat{\boldsymbol{u}}^{i} \in V$ such that

$$
b\left(\Delta \hat{\boldsymbol{u}}^{i}, \hat{\boldsymbol{v}}\right)=\left\langle R^{i}, \hat{\boldsymbol{v}}\right\rangle \quad \forall \hat{\boldsymbol{v}} \in V
$$

where

$$
\left\langle R^{i}, \hat{\boldsymbol{v}}\right\rangle=-b\left(\Delta \boldsymbol{u}^{i}, \hat{\boldsymbol{v}}\right)+c\left(\Delta \boldsymbol{p}^{i}, \hat{\boldsymbol{v}}\right)+\left\langle\ell_{n}^{1}, \hat{\boldsymbol{v}}\right\rangle,
$$

with $\left\langle\ell_{n}^{1}, \hat{\boldsymbol{v}}\right\rangle=\int_{\Omega} \boldsymbol{f}\left(t_{n}\right) \cdot \hat{\boldsymbol{v}}-\boldsymbol{\sigma}_{n-1}: \boldsymbol{\varepsilon}(\hat{\boldsymbol{v}}) \mathrm{d} \boldsymbol{x}$, where $\boldsymbol{\sigma}_{n-1}=$ $\mathbb{C}\left(\boldsymbol{\varepsilon}\left(\boldsymbol{u}_{n-1}\right)-\boldsymbol{p}_{n-1}\right)$ is the elastic stress of the previous time step. The right hand side of $(\mathrm{Pr})$ is the residual of the first 
equation in (EP) representing the not yet balanced forces. The quantities $\Delta \boldsymbol{u}^{i}$ and $\Delta \boldsymbol{p}^{i}$ are known from the previous predictor/corrector iteration. The next estimate for $\Delta \boldsymbol{u}_{n}$ is $\Delta \boldsymbol{u}^{i+1}=\Delta \hat{\boldsymbol{u}}^{i}+\Delta \boldsymbol{u}^{i}$.

With that estimate in hand we can start the corrector step, i.e., find a new estimate $\Delta \boldsymbol{p}^{i+1} \in Q_{0}$ for $\Delta \boldsymbol{p}_{n}$ satisfying

$$
\begin{array}{r}
j(\boldsymbol{q})-j\left(\Delta \boldsymbol{p}^{i+1}\right)+d\left(\Delta \boldsymbol{p}^{i+1}, \boldsymbol{q}-\Delta \boldsymbol{p}^{i+1}\right) \geq\left\langle\ell_{n}^{2}, \boldsymbol{q}-\Delta \boldsymbol{p}^{i+1}\right\rangle \\
+c\left(\boldsymbol{q}-\Delta \boldsymbol{p}^{i+1}, \Delta \boldsymbol{u}^{i+1}\right) \quad \forall \boldsymbol{q} \in Q_{0}, \quad(\mathrm{Co})
\end{array}
$$

where

$$
\left\langle\ell_{n}^{2}, \boldsymbol{q}\right\rangle=\int_{\Omega}\left(\boldsymbol{\sigma}_{n-1}+k \boldsymbol{p}_{n-1}\right): \boldsymbol{q} \mathrm{d} \boldsymbol{x} .
$$

Since $\Delta \boldsymbol{u}^{i+1}$ and therefore $\boldsymbol{\varepsilon}\left(\Delta \boldsymbol{u}^{i+1}\right)$ are given from the predictor step, we can reformulate the inequality (Co) in a pointwise sense. After some arrangements we arrive at the following solution of (Co) (see (Alberty et al., 1999, chapter 7)):

$$
\Delta \boldsymbol{p}^{i+1}=\frac{(|\operatorname{dev} \boldsymbol{A}|-\tilde{\sigma})_{+}}{2 \mu+k} \frac{\operatorname{dev} \boldsymbol{A}}{|\operatorname{dev} \boldsymbol{A}|},
$$

where

$$
\begin{aligned}
\boldsymbol{A} & :=\boldsymbol{\sigma}_{n-1}+k \boldsymbol{p}_{n-1}+\mathbb{C} \boldsymbol{\varepsilon}\left(\Delta \boldsymbol{u}^{i+1}\right), \\
\operatorname{dev} \boldsymbol{A} & :=\boldsymbol{A}-(1 / 3 \operatorname{tr} \boldsymbol{A}) \boldsymbol{I}, \\
(f)_{+} & :=\max (f, 0)
\end{aligned}
$$

and $\mu$ is the shear modulus. Hence, the main work in each predictor/corrector iteration is the solution of the displacement problem $(\mathrm{Pr})$.

The algorithm below shows a summary of the described techniques.

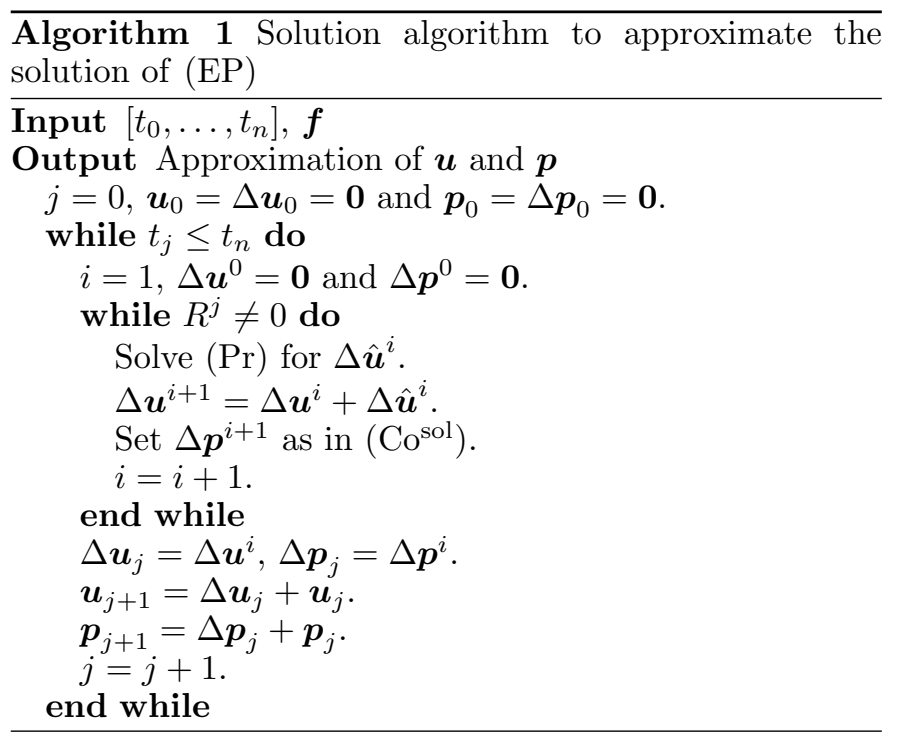

\section{TOWARDS TOPOLOGY OPTIMIZATION}

In the previous section we discussed the solution of the plastic forward problem with the goal to get an approximation of the displacement $\boldsymbol{u}: \Omega \times[0, T] \rightarrow \mathbb{R}^{3}$ and the plastic strain $\boldsymbol{p}: \Omega \times[0, T] \rightarrow \mathbb{R}_{\mathrm{sym}}^{3 \times 3}$ for a given body $\Omega \subset \mathbb{R}^{3}$ and given forces $f: \Omega \rightarrow \mathbb{R}^{3}$.

The aim of topology optimization is to find the optimal distribution of a given material whose parameters are given by $\mathbb{C}_{0}, k_{0}$ and $\widetilde{\sigma}_{0}$, see Bendsøe and Sigmund (2004) or Schwarz et al. (2001) for example. The distribution can be modeled with a pseudo density $\rho(\boldsymbol{x}): \Omega_{0} \rightarrow[0,1]$, where $\Omega_{0}$ is the completely filled initial body and $\rho\left(\Omega_{0}\right) \subset \mathbb{R}^{3}$ is a modified one. If $\rho(\boldsymbol{x})=0$ holds in a point $\boldsymbol{x} \in \Omega_{0}$ there will be no material in the modified body. Assuming that the intermediate material can be described by using scaled material parameters, i.e., $\rho(\boldsymbol{x}) \mathbb{C}_{0}$ in place of $\mathbb{C}_{0}$, and similarly for $k$ and $\tilde{\sigma}$, we arrive at the following optimization problem for the density $\rho$ :

$$
\begin{aligned}
& \min _{\rho: \Omega_{0} \rightarrow[0,1]} J(\boldsymbol{u}, \boldsymbol{p}, \rho, t) \\
& \text { s.t. } \boldsymbol{u} \text { and } \boldsymbol{p} \text { solve }(\mathrm{EP}) \\
& \qquad \begin{aligned}
\text { with } \mathbb{C} & =\rho(\boldsymbol{x}) \mathbb{C}_{0}, \\
k & =\rho(\boldsymbol{x}) k_{0} \text { and } \\
\tilde{\sigma} & =\rho(\boldsymbol{x}) \widetilde{\sigma}_{0},
\end{aligned}
\end{aligned}
$$

where $J$ is the goal functional.

It is often desirable to allow only the values near 0 and 1 for the density. A common approach to enforce this is to employ the SIMP-model ('solid isotropic material with penalization'), in which $\rho(\boldsymbol{x})^{p} \mathbb{C}_{0}$ is used for the elasticity tensor in (TO) for some $p>1$, and similarly for $k$ and $\widetilde{\sigma}$.

Problem (TO) has been considered in Schwarz et al. (2001) and numerical results are also included there. These were obtained by adjoint-based gradients and then applying the so-called optimality-criterion (OC) method. The latter can be viewed as a fixed-point iteration applied to the optimality conditions, which is rather slow to converge.

\section{OUTLOOK}

Studying the existence of a minimizer and deriving optimality conditions for a suitably regularized version of (TO) are problems which we intend to address in the future. We also plan to apply modern semismooth optimization frameworks to this problem. We will utilize our previous experience on regularized formulations of elastoplasticity, see, e.g., Herzog et al. (2012).

\section{REFERENCES}

Alberty, J., Carstensen, C., and Zarrabi, D. (1999). Adaptive numerical analysis in primal elastoplasticity with hardening. Computer Methods in Applied Mechanics and Engineering, 171, 175-204. doi:10.1016/S00457825(98)00210-2.

Bendsøe, M.P. and Sigmund, O. (2004). Topology optimization : Theory, methods, and applications. Springer, Berlin, 2. ed., corrected print. edition.

Han, W. and Reddy, B.D. (1999). Plasticity. Springer, New York.

Herzog, R., Meyer, C., and Wachsmuth, G. (2012). Cstationarity for optimal control of static plasticity with linear kinematic hardening. SIAM Journal on Control and Optimization, 50(5), 3052-3082. doi: $10.1137 / 100809325$.

Schwarz, S., Maute, K., and Ramm, E. (2001). Topology and shape optimization for elastoplastic structural response. Computer Methods in Applied Mechanics and Engineering, 190(15-17), 2135 - 2155. doi: 10.1016/S0045-7825(00)00227-9. 


\title{
A method to model impulsive Multi-Body-Dynamics using Riemann-Stieltjes-Integrals
}

\author{
Johannes Michael* Matthias Gerdts** \\ * Universität der Bundeswehr, Fakultät für Luft- und \\ Raumfahrttechnik, Institut für Mathematik und Rechneranwendung, \\ München, Germany (e-mail: johannes.michael@unibw.de) \\ ** Universität der Bundeswehr, Fakultät für Luft- und \\ Raumfahrttechnik, Institut für Mathematik und Rechneranwendung, \\ München, Germany (e-mail: matthias.gerdts@unibw.de)
}

A wide range of simulation and optimization methods for smooth dynamical systems exist, that provide as accurate results as desired. But when there exist discontinuities in the dynamics there is still a lack of widely spread integration schemes that are easy to use without further knowledge. The simplest example one can think of is a ball bouncing on a surface. For the calculation of the impact dynamics it is possible to formulate the ball in a complex way using finite elements and elasticity and plasticity, but when you are just interested in the macroscopic behaviour, the user might want to use a simpler model of a mass point. When omitting the complex body interaction the contact can be modelled by applying an instantaneous change in velocity using Newton's impact law. This formulation leads to a complementarity system with an additional constraint on the post impact velocity, namely

$$
\begin{aligned}
0 \leq g(q(t)) \perp \lambda(t) & \geq 0, \\
\lambda(q(t)) \cdot\left(\dot{q}\left(t^{+}\right)+\mathcal{S} \dot{q}\left(t^{-}\right)\right) & =0 .
\end{aligned}
$$

One possibiliy to model this type dynamic system is to use hybrid automata using one vector field describing the continuous dynamic and one reset map defining the post impact state in the gap is closed, see Lygeros et al. (2003). In this work we demonstrate an alternative formulation using Riemann-Stieltjes-Integrals, see e.g. Natanson and Bögel (1977), that leads to a closed formula using functions of bounded variation for the impact behaviour. The integral system can be written as

$$
\begin{aligned}
x(t) & =x_{a}+\int_{a}^{t} F(x(\tau)) d \tau+\int_{a}^{t} G(x(\tau)) d \alpha(x)(\tau), \\
x_{a} & =x(a) .
\end{aligned}
$$

Here the instantaneous change in velocity is modelled using the function $\alpha(x)(\tau)$. This will turn out to be a step function of bounded variation with steps at the time instants of the impact points.

In further research this formulation will be used to calculate derivatives needed for a descend algorithm in the calculation of optimal controls for impulsive mechanical multi-body-systems. Having this in mind the RiemannStieltjes formulation delivers theoretical properties that can be exploited to obtain differentiability results under certain restrictions.

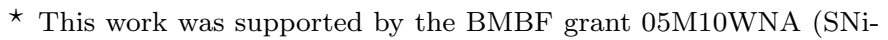
MoRed).
We show a general energetically consistent method to calculate the post impact state or in other words the step height of $\alpha(x)(\tau)$ of the system under Coulomb friction, see Bowling et al. (2009). This formulation results in a convex nonlinear minimization problem that has to be solved at each impact to calculate the velocity update of the underlying system.

The solution of the system is calculated with a fixed step size using an adapted version of Newton-Cotes formulas to handle the integration of the Stieltjes integral as it is explained in Tortorella (1990). Examples of different systems like an impulsive halfcar model demonstrate how to model multiple restrictions and verify the abilities of the proposed model.

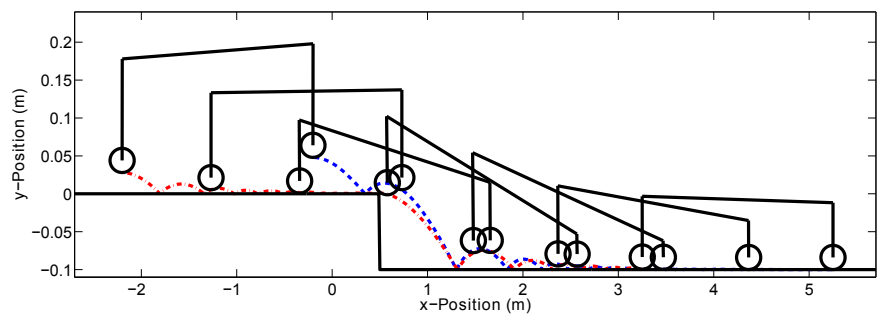

Fig. 1. Trajectories of the front (dashed) and the rear (dash-dotted) wheel of the halfcar model

\section{REFERENCES}

Bowling, a., Flickinger, D., and Harmeyer, S. (2009). Energetically consistent collisions in simulation of multibody systems. IEEE International Conference on Robotics and Automation, 1303-1308.

Lygeros, J., Johansson, K., Simic, S., and Sastry, S. (2003). Dynamical properties of hybrid automata. IEEE Transactions on Automatic Control, 48(1), 2-17.

Natanson, I. and Bögel, K. (1977). Theorie der Funktionen einer reellen Veränderlichen. Mathematische Lehrbücher und Monographien: Mathematische Lehrbücher. Akademie-Verlag.

Tortorella, M. (1990). Closed newton-cotes quadrature rules for stieltjes integrals and numerical convolution of life distributions. SIAM Journal on Scientific and Statistical Computing, 11(4), 732-748. 
MATHMOD 2015 Abstract Volume 


\title{
MATHMOD 2015 ABSTRACT VOLUME
}

\author{
MATHMOD Minisymposium \\ Interdisciplinary Data Based Modelling and Simulation \\ in Health Systems Research
}


MATHMOD 2015 Abstract Volume 


\title{
Data Wrangling: Making data useful again
}

\author{
Florian Endel ${ }^{*}$ Harald Piringer ${ }^{* *}$ \\ * University of Technology Vienna (florian.endel@tuwien.ac.at) \\ ** VRVis Research Center, Vienna, Austria
}

\begin{abstract}
Data analysis has become an everyday business and advancements of data management routines open up new opportunities. Nevertheless, transforming and assembling newly acquired data into a suitable form remains tedious. It is often stated, that data cleaning is a critical part of the overall process, but also consumes sublime amounts of time and resources. Data Wrangling is not only about transforming and cleaning procedures. Many other aspects like data quality, merging of different sources, reproducible processes, and managing data provenance have to be considered. Although various tools designed for specific tasks are available, software solutions accompanying the whole process are still rare.

In this paper, some aspects of this first phase of most data driven projects, also known as data wrangling, data munging or janitorial work are described. Beginning with an overview on the topic and current problems, concrete common tasks as well as selected software solutions and techniques are discussed.
\end{abstract}

Keywords: Data acquisition, Databases, Bad data identification, Data wrangling.

\section{INTRODUCTION}

The terms "data science", "datafication", "business analytics" and "big data" (Ayankoya et al. (2014); Cukier and Mayer-Schoenberger (2013); Larson (2013); Lohr (2012)) were coined based on many different developments in data retrieval, storage and analysis during the last years. Although tools and technologies evolve constantly, understanding and preparing a newly acquired dataset for further usage still requires much time and effort. This initial and very fundamental process of examining and transforming data into a usable form is known as "data wrangling", "data munging" or even "janitorial work".

As foundation of the data wrangling process, a broad and deep understanding of the content, structure, quality issues and necessary transformations as well as appropriate tools and technological resources are needed. The whole wrangling procedure needs to be very efficient, especially for small projects or unique datasets, where the effort to automate and document does not seem to be achievable, although necessary.

Altogether, data cleaning accounts for 50 percent to 80 percent (Kandel et al. (2011); Dasu and Johnson (2003); Lohr (2014)) of the time and costs in analytic or data warehousing projects respectively.

Different challenges and possible solutions are described as basis for further discussion. The focus is set primarily on short-term projects, directed by a tight schedule and (naturally) merely adequate resources.

Recognising the practical significance and describing applicable workflows for data wrangling, while keeping in mind the necessity to focus real-world problems and constraints, is the main scope of this discussion.

\section{MOTIVATION}

Collecting data from more or less structured sources and preparing it for visualization, modelling or permanent storage is a necessity of our projects. As described above, this work can be tedious and consumes an inordinate amount of time. An overview of common topics, routines and challenges alongside with achievable solutions is collected to speed up and improve structuring of future work.

\section{APPROACH}

Based on published literature and gathered experiences, several key aspects concerning data wrangling are identified and discussed. Understanding data preparation as iterative, multidisciplinary process, the focus is set on realistically applicable routines and solutions for most common sources of error.

\subsection{Example scenarios}

Two diverse, real world exemplary scenarios are presented. By reference to problems, applied solutions, and lessons learned during these projects, several aspects of the wrangling process are described.

First, a huge collection of several undocumented data files have to be prepared and cleaned. In the end a highly structured database storing the foundation for further analysis as well as a reproducible process of the whole transformation is needed.

Second, complex but well known data is extracted from another database and put into a format that can easily be used for visualization. Additionally, auxiliary information from additional sources have to be merged into the exported file. 


\subsection{Challenges}

A collection of main challenges appearing in nearly every data driven project are summarized. Next, solutions for each objective and ideally, for a model process covering at least typical requirements have to be discussed.

Basic Features Approaching a new dataset for the first time, many basic aspects like size, encoding, and format have to be explored.

Data Quality is a central aspect of almost every data driven project. Several dimensions of data quality and metrics to quantify them are defined in literature. Ideally, data quality is monitored and documented throughout the whole lifetime of a dataset.

Merging \& Linking Integration of data is often needed to complete the picture. Therefore, merging and linking of divergent structures, coding conventions, formats and data models are an important task when preparing data for further utilization. Varying scenarios can be identified based on the variety of sources, type of data, linkage method, objective and reason for data linkage.

Reproducibility \& Documentation are important but often neglected components of every scientific research. Handling changes of data and methods over time as well as the recreation of previously acquired results are common demands which can be hard to fulfil, especially in connection with manual interaction.

Big Data Generally, large amounts of data can be complicated to analyse. Although the volume of data which is labelled as being big depends on the application at hand, managing and analysing millions or billions of datasets or several gigabytes / terabytes respectively, does require special treatment and technologies (e.g. Hu et al. (2014)).

Dirty Data Despite all efforts conducted during data quality assessment processes, it is not always clear how knowledge about quality issues can be handled appropriately. In some cases further feedback loops with data sources and providers are viable and cleaning up flaws is possible. Otherwise, strategies to work with dirty data have to be implemented. Depending on the severity of the errors, the resulting effects can range from minor disturbances to the necessity to completely re-engineer analytical processes.

Uncertainty Data quality does not only occur in discrete statuses, i.e. clean and faulty. In Skeels et al. (2008) five different types of uncertainty are classified: measurement precision, completeness, inference, disagreement, and credibility. Reasons for uncertainty range from measurement errors, processing errors as far as intentionally introduced inaccuracies e.g. due to privacy concerns. Visualization (e.g. Correa et al. (2009)) can help to intuitively present uncertainty.

Error tolerance Known and unknown errors as well as uncertainties should be expected when using real world data (e.g. Haug et al. (2011)). Therefore, data storage (e.g. Benjelloun et al. (2007)), analytic routines, and algorithms need to tolerate different kinds of errors.

Transformation \& Editing Although the whole data wrangling process is about transforming some kind of input into a usable form, the concrete implementation of these transformations including all additional constraints (e.g. quality, reproducibility) is still an open issue. Many software solutions exists with advantages and drawbacks.

\section{RESULTS}

Summarizing, it can be shown that data wrangling is still a defiant process. Although a variety of solutions for single problems and special situations exist, hardly any of them cover all raised considerations.

Concepts and tools facilitating the struggle of data wrangling require additional research. Additionally, further requirements as well as the meaningfulness of presented objectives have to be discussed and refined.

\section{REFERENCES}

Ayankoya, K., Calitz, A., and Greyling, J. (2014). Intrinsic relations between data science, big data, business analytics and datafication. In Proceedings of the Southern African Institute for Computer Scientist and Information Technologists Annual Conference 2014 on SAICSIT 2014 Empowered by Technology, SAICSIT' 14, 192:192-192:198. ACM, New York, NY, USA. doi: $10.1145 / 2664591.2664619$.

Benjelloun, O., Sarma, A.D., Halevy, A., Theobald, M., and Widom, J. (2007). Databases with uncertainty and lineage. Technical Report 2007-26, Stanford InfoLab. URL http: //ilpubs.stanford.edu:8090/811/.

Correa, C., Chan, Y.H., and Ma, K.L. (2009). A framework for uncertainty-aware visual analytics. In IEEE Symposium on Visual Analytics Science and Technology, 2009. VAST 2009, 51-58. doi:10.1109/VAST.2009.5332611.

Cukier, K.N. and Mayer-Schoenberger, V. (2013). The rise of big data. Foreign Affairs, (May/June 2013).

Dasu, T. and Johnson, T. (2003). Exploratory Data Mining and Data Cleaning. John Wiley \&amp; Sons, Inc., New York, NY, USA, 1 edition.

Haug, A., Zachariassen, F., and Liempd, D.v. (2011). The costs of poor data quality. Journal of Industrial Engineering and Management, 4(2), 168-193. doi: 10.3926/jiem..v4n2.p168-193.

Hu, H., Wen, Y., Chua, T.S., and Li, X. (2014). Toward scalable systems for big data analytics: A technology tutorial. IEEE Access, 2, 652-687. doi: 10.1109/ACCESS.2014.2332453.

Kandel, S., Heer, J., Plaisant, C., Kennedy, J., van Ham, F., Riche, N.H., Weaver, C., Lee, B., Brodbeck, D., and Buono, P. (2011). Research directions in data wrangling: Visualizations and transformations for usable and credible data. Information Visualization, 10(4), $271-288$.

Larson, E.B. (2013). Building trust in the power of big data research to serve the public good. JAMA, 309(23), 2443-2444. doi:10.1001/jama.2013.5914.

Lohr, S. (2012). Big data's impact in the world. The New York Times.

Lohr, S. (2014). For big-data scientists, janitor work is key hurdle to insights. The New York Times.

Skeels, M., Lee, B., Smith, G., and Robertson, G. (2008). Revealing uncertainty for information visualization. In Proceedings of the Working Conference on Advanced Visual Interfaces, AVI '08, 376-379. ACM, New York, NY, USA. doi:10.1145/1385569.1385637. 


\title{
Reproducible Database Queries in Privacy Sensitive Applications
}

\author{
Stefan Pröll* Rudolf Mayer* Andreas Rauber** \\ *SBA-Research, Vienna, Austria (e-mail: sproell@sba-research.org, \\ rmayer@sba-research.org) \\ ** Institute of Software Technology and Interactive Systems at the \\ Vienna University of Technology, Austria (e-mail: \\ rauber@ifs.tuwien.ac.at)
}

\begin{abstract}
Research databases are an important building block in eScience and computational science investigations. For enabling reproducible research, an approach is needed which supports the identification and citation of the exact data (sub)sets utilized in experiments. While this itself is a challenge, in many cases the data stored in databases is sensitive and needs to be protected. Due to the increasing complexity of eScience investigations, data is often integrated from different sources, potentially stemming from competing data owners. In order to achieve the research goals, the data needs to be combined and analysed as a whole. As data owners of such sources may have potential conflicts of interest in certain aspects, a mechanism is needed which prevents the retrieval and or recombination of privacy related data while still full access to own data must be granted at all times.
\end{abstract}

Keywords: Reproducibility, Relational databases, Data handling systems, Data privacy, Data sets

\section{INTRODUCTION}

Data is a crucial element in computational science. On the one hand, data is often used as input for research, e.g. in statistical machine learning. Also in mathematical models that themselves do not directly learn from data, data observations might still be utilized in an earlier step, to obtain and estimate fitting parameter settings for the models. On the other hand, complex data can also be the output of an experiment or simulation. Important decisions are made on the basis of such data, for example, whether an experiment was considered successful or a model is considered valuable is often settled on the result sets delivered from statistical analysis of data. As data is not only a final product of research investigations, but is transformed, updated or changed in the many processing steps that form an experiment, each intermediate result needs to be traceable and identifiable. Hence it is a fundamental requirement to know which exact data was involved in the research, and its intermediate steps. Data citation tackles the problem of uniquely identifying, referencing and citing datasets and their subsets in order to make them retrievable at a later point in time. The goal of citing data is to attach a persistent identifier to each dataset retrieved from a data source, and use this identifier as a handle which allows to retrieve the exact same dataset again. Thus data citation enables the examination, revision or analysis of data and therefore constitutes evidence for decisions and how they have been made.

In most settings, it is not a feasible approach to export each individual dataset and store it as data dump in

\footnotetext{
^ Parts of this work are supported by the project DEXHELPP.
}

an archive, as such a solution does not scale well for large data volumes, caused either by large data itself, or many and repeated processing steps. Further, without additional metadata the management of data files becomes a challenge. Verifying whether a data set is actually the one required can be achieved by applying fingerprinting and checksum mechanisms, but without the knowledge how the data was derived and how a specific subset was selected, the reproducibility of data driven experiments is limited.

In many cases the datasets contain sensitive data and privacy protocols need to be applied. Implementing thorough permission schemes is essential, but the goal of providing a secure eScience environment becomes more challenging if several stakeholders with potentially competing interests need to exchange data. All participating partners obviously need access to the data they contributed, but providing access to aggregated results and compiled result sets is a further requirement. The system needs to support analysis of the data but it needs to prevent the creation of datasets which contain sensitive data or allow the deduction of such information via skilful querying (Dwork and Smith (2010)).

\section{DATA CITATION IN VOLATILE SOURCES}

In many scenarios, data is not just static but it can also be highly dynamic. New records can be created, some data may get updated whereas older records may be deleted. For this reason the full history of all operations, which either added, altered or deleted any record in the database system, needs to be traced. Still, only versioning of databases itself does not yet allow to retrieve a specific result set from a given point in time. Most modern rela- 
tional database management systems (RDBMS) support point in time recovery (PITR), which can be used for rolling back the data to a specific date. Although this method allows querying on top of the data as they were at any given moment in time, the approach is not feasible for retrieving historical data in an convenient fashion. This is due to the potentially costly rollback operation to a specific data version, which cannot be reused for other queries. It is to mention that the term version may be misleading in the context of data citation. By a version we understand a specific state of the records in a result set, we do not refer to an export of data which is assigned a version number. The same query for instance will produce different versions of result sets, whenever a single record contained in the result was changed between two executions of a query.

To achieve versioning, all events performed on each record need to be stored with a timestamp in order to create an audit trail of all changes which have been introduced into the database. The system needs to provide information if a record was inserted, updated or a deleted. This data can be stored in a history table and allows retrieving a specific state of the database records at any given time. Additionally to the provenance of any record in the database, the actual query which was used for retrieving the dataset needs to be stored with additional metadata.

For storing the queries with their execution metadata, the concept of the query store was developed by Proell and Rauber (2014). The goal of the query store is to attach persistent identifiers to query results and allow retrieving the same data again by re-executing a query against historical data. A persistent identifier (PID) uniquely identifies a resource for the long term by utilizing a managed infrastructure providing additional services which can be used for accessing the metadata and the object itself in a reliable way. Instead of attaching a PID directly to the exported dataset, the PID references the query which ultimately produces the dataset. The Query Store needs to detect whether a issued query is already stored persistently or if a new query needs to be inserted. In order to validate the re-executed query result for its correctness, a hash key is computed. A result set is only considered correct if and only if all records are included in the same sequence and ordering as in the original query. When querying sensitive data, several security policies need to be applied which need to prevent unauthorized access and impermissible queries. The knowledge necessary for applying such queries needs to be preserved, maintained and re-applied for the re-executed queries retrieving historical data.

\section{REQUIREMENTS IN PRIVACY SENSITIVE APPLICATIONS}

Many scientific projects are collaborative and involve different organizations working together and exchanging data. Although the stakeholders pursue a common goal, it is not necessarily true that all exchanged data should be available to all project partners without limitations. Research data often contains information which needs to be protected from unauthorized access and privacy needs to be maintained. This is especially true for projects that involve data which can be attributed to individuals and may even contain highly sensitive data such as health records.

Whenever such data is exchanged, the database management handling the data needs to ensure that privacy is maintained within the whole data life cycle. Providing a secure database for several stakeholders with potentially conflicting interests goes beyond the implementation of permission schemes for individual tables. The system must preserve the privacy of the data at all times and may also prevent data leakage through clever queries with could reveal individual details.

Furthermore, a mechanism is needed which allows tracing all executed queries and logs the activities on the system. Researchers and administrators need to be able to reproduce a specific query which was issued against the database and retrieve the very same dataset again. There are several reasons for this requirement. Researchers, as mentioned earlier, need to have the possibility retrieving the data again which was used a model in order to verify and rerun the experiment. Moreover, the project administrators and managers require to verify that the data was retrieved in compliance with the ethical policies defined in a project. Therefore, a mechanism is required which allows auditing the queries and the resulting datasets for their privacy compliance.

To this end, the above introduced data citation approach needs to be adapted to cope with this more complex setting. For example, the re-execution of a query also requires to maintain the permission rights of the records as access to a specific portion of the data may be granted or revoked. This information needs to be captured and stored in the query store in order to reproduce who retrieved sensitive data and prove that access to a specific dataset was granted. Additionally, the query store can also serve as an audit trail and needs to be protected from manipulation.

\section{CONCLUSIONS}

Reproducibility is a key requirement for computational research and therefore the complete workflow from experimental setup to results needs to be traceable and understandable retrospectively. Data citation enables peers to verify the data used in each step of an experiment, thus it constitutes significantly to reproducibility of experiments. In turn, reproducibility provides documentation which fosters reuse of results and intermediate research products. It is clear that data is a fundamental basis for most experiments, therefore the creation process of a dataset needs to be reproducible as well. For obvious reasons, reproducibility includes privacy preserving methods which hinder disclosure of sensitive data.

\section{REFERENCES}

Dwork, C. and Smith, A. (2010). Differential privacy for statistics: What we know and what we want to learn. Journal of Privacy and Confidentiality, 1(2).

Proell, S. and Rauber, A. (2014). A Scalable Framework for Dynamic Data Citation of Arbitrary Structured Data. In 3rd International Conference on Data Management Technologies and Applications (DATA2014). Vienna, Austria. 


\title{
Identifying Structural Changes in Austrian Social Insurance Data
}

\author{
Thomas Ortner* Peter Filzmoser* Gottfried Endel** \\ * Department of Statistics and Probability Theory, Vienna University \\ of Technology, Austria, (e-mail: thomas.ortner@tuwien.ac.at, \\ p.filzmoser@tuwien.ac.at) \\ ** Main Association of Austrian Social Security Institutions, Austria \\ (e-mail: gottfried.endel@hvb.sozvers.at).
}

\begin{abstract}
:
Testing for structural changes is a well studied field. Classical tests for breakpoint detection utilize F-statistics which depend on independent and identically normal distributed residuals. In general, this condition is not satisfied which leads to distorted test results when the p-values of classical tests are close to the significance level. Thus, permutation tests are used to properly estimate the critical values.

Based on the accounting data of Austrian hospitals, collected by the Austrian social insurance institutions, specific observations (hospital stays) which are connected to pre-defined diseases are analysed. For those groups of observations we use characteristic factors, to test for structural changes from different perspectives. The first test analyses the temporal trend and identifies breakpoints, caused by changes in the underlying system between years. The second analysis focuses on identifying differences between hospitals. Both implemented tests ensure the often ignored aspect of a homogeneous data base for further analysis.
\end{abstract}

Keywords: Breakpoints, Structural Change, Permutation Test, Cut Off Value, Time Series

\footnotetext{
* This work has been partly funded by the K-project DEXHELPP through COMET - Competence Centers for Excellent Technologies, supported by BMVIT, BMWFI and the province Vienna. The COMET program is administrated by FFG.
} 


\title{
Visual Analysis of Disease Prevalence Based on Health Accounting Data
}

\author{
Harald Piringer*
}

\author{
*VRVis Research Center, Donau-City-Strasse 1, 1220 Vienna, Austria \\ (Tel: 0043-1-20501-30100; e-mail: hp@vrvis.at).
}

\begin{abstract}
This paper describes some initial results of analyzing Austrian population and disease prevalence data using interactive visualization. The goal is to convey a first idea of available data in the GAP-DRG database, formulate hypotheses regarding a geographic distribution of disease prevalence, and describe some potential data quality issues.
\end{abstract}

Keywords: DEXHELPP, Visualization, Health, Decision

\section{INTRODUCTION}

The consortium of the K-Project DEXHELPP brings together scientists from many different areas in order to attain a better understanding of the Austrian health care system and to provide methods that support decision making in this area. Data exploration is a crucial task in this context in order to gain more knowledge about how the health system works. This paper aims to provide an overview of a subset of the underlying data and to formulate preliminary hypotheses as result of an initial analysis. Methodologically, interactive visualization was used as an effective approach to exploratory data analysis. This paper thus focuses on showing visual rather than textual results to illustrate respective benefits.

\section{DATA BASIS}

The analysis is based on an excerpt from GAP-DRG, a database comprising personal (yet pseudo-anonymized) accounting data of the Austrian health system from the years 2006 and 2007. The focus of the analysis was on the characterization of the 121 Austrian political districts from 2006 and 2007 regarding (1) the gender of patients, (2) the age of patients in 10-year cohorts, (3) time in quarterly years, and (4) the relative distribution of diseases as represented by ICD-9 codes. The information about ICD-9 codes is based on a mapping from ATC codes which uniquely identify medications. This mapping was the result of a previous project (see [1], [2]) and has a varying degree of uncertainty for different ICD-9 codes.

\section{RESULTS}

This section describes the application of techniques from multivariate visualization to the described data.

\section{Overall Distribution of Diagnoses by Age and District}

Fig. 1 shows the distribution of diagnoses by age group.

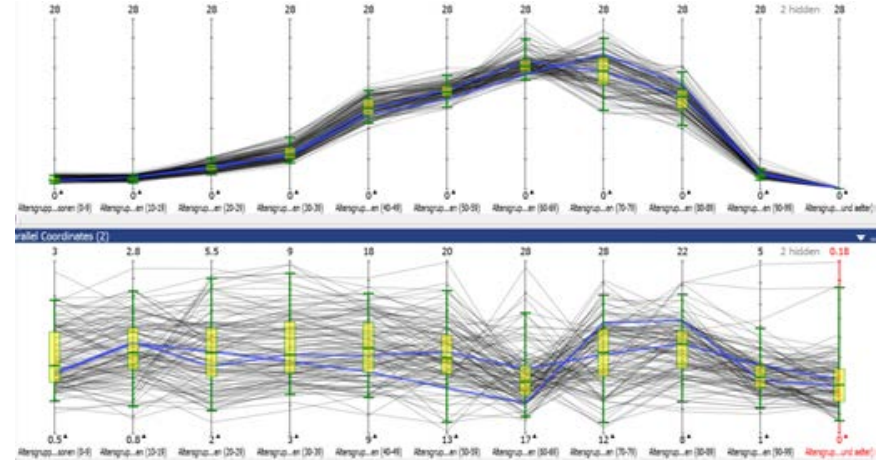

Fig. 1.: Relative frequencies of diagnoses by 10-year cohorts (one per axis) per district (i.e., each district corresponds to one line-strip) as Parallel Coordinate Plots.

Equally scaled axes (top) show that most diagnoses belong to patients between the age of 40 and 89 . Unequally scaled axes (bottom) reveal bundles of districts with a similar age distribution of diagnoses. Applying K-Means Clustering (5 clusters) to this data shows a distinct regional cohesion.

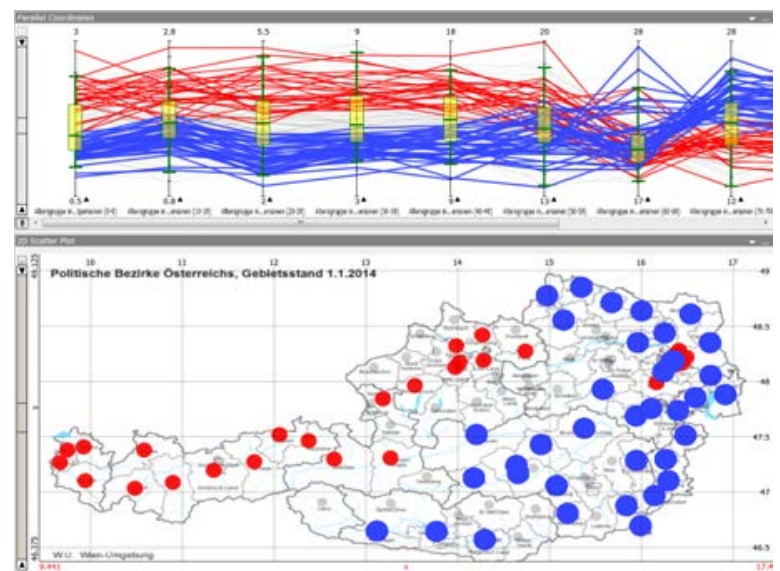

Fig. 2. Comparison of two (out of five) clusters regarding the relative age distribution of diagnoses.

For example, one cluster roughly corresponds to western Austria and parts of Vienna as having a different relative age 
distribution of diagnoses than most of eastern Austria (see Fig. 2). Interestingly, federal capitals formed another cluster.

\section{Regional distribution of ICD-9 diagnoses}

The analysis also revealed a distinct regional coherence for the relative prevalence of many ICD-9 codes. For example, some ICD-9 codes are more frequent in some parts of Austria by a factor of $2-3$ than in others or show peaks in adjacent districts. There are also districts with an outlying behavior, e.g., Hollabrunn, as shown in Fig. 3.

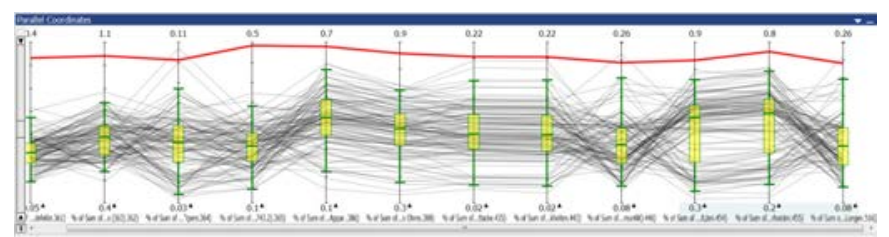

Fig. 3: The distribution of the relative frequencies of 12 ICD-9 codes per district. The red line represents Hollabrunn.

Performing a K-Means clustering ( 9 clusters) for the 100 ICD-9 codes with the highest overall prevalence reflects the aforementioned regional coherence (see Fig. 4). Some clusters even coincide with the federal states of Austria surprisingly well, e.g., Northern Tyrol forms one cluster.
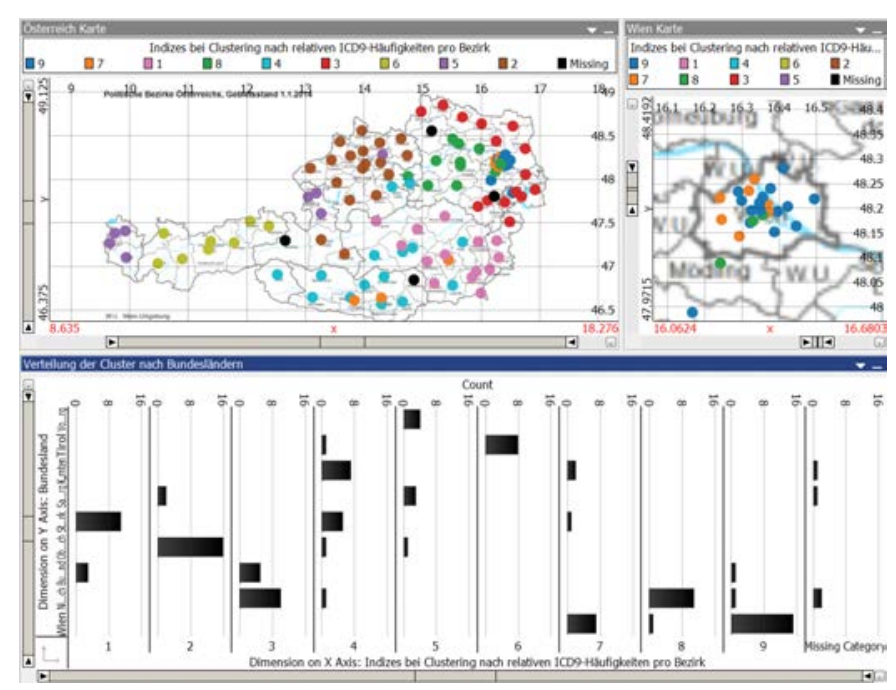

Fig. 4: Result of clustering Austria'a political districts by the relative frequencies of the 100 most frequent ICD-9 codes using K-means (9 clusters). The upper part of the figure shows the cluster membership by color for entire Austria and Vienna. The lower part shows the number of districts by cluster (columns) and federal state (rows).

\section{Correlation between ICD-9 Codes}

The relative frequencies of many ICD-9 codes per district correlate with each other. Fig. 5 shows a correlation matrix of the most prevalent ICD-9 codes, where a red background indicates a positive correlation while blue means negative correlation. Interestingly, strong correlations also exist between ICD-9 codes which belong to different ICD-9 groups. Furthermore, the analysis showed that negative correlations might be related to the different age distribution of districts.

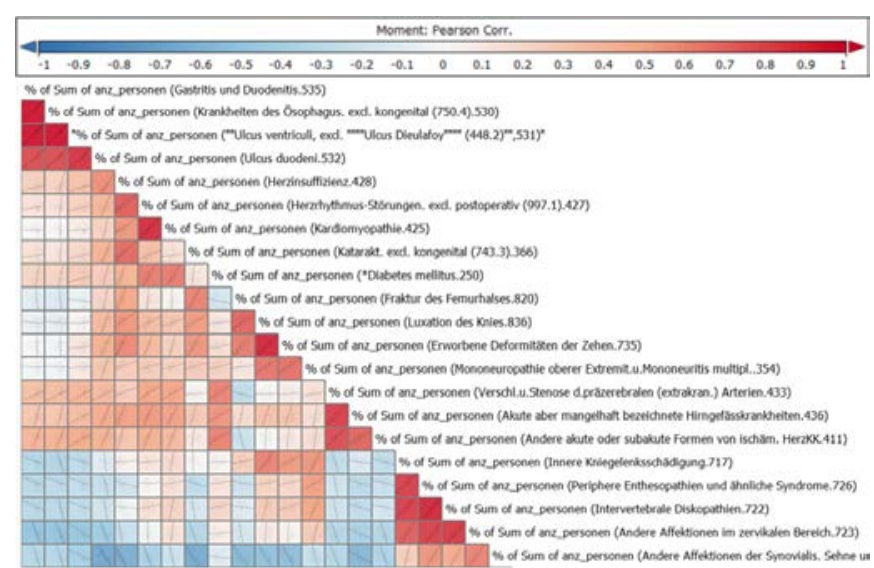

Fig. 5: Correlation matrix of ICD-9 codes.

\section{Additional Findings Regarding Anomalies}

Additional analyses (not illustrated in this paper) revealed many empty ("null") values in the gender entry, which are comparatively unevenly distributed over federal states. While Upper Austria has about 7\% and Salzburg about 5\% of "null" values, Styria has less than $1 \%$. Another abnormality is that one of the districts in Vienna (Margareten) has an unrealistically large number of diagnoses as compared to other districts and also shows an outlying behaviour for the temporal distribution of diagnoses. The analysis also revealed further issues like potential artefacts of the ATC to ICD-9 mapping, e.g., male patients classified as having "complications that mostly occur in combination with pregnancy" (ICD-9 codes 640 - 648).

\section{CONCLUSION AND FURTHER ANALYSIS}

Visualization is not only an effective means for communication, but has also great potential for exploring data and discovering unexpected patterns. This makes visualization especially well-suited for data plausibilization and hypothesis generation. In this context, interactive visualization is especially powerful to answer numerous questions in a short time and to overcome limitations of static diagrams regarding data size and complexity.

While this paper described a small number of possible analyses, numerous factors like socio-economic aspects have not yet been taken into account. There thus seems to be a huge potential in conducting more visual analyses for this data and for data from other areas of DEXHELPP for a better understanding and gaining more insight.

\section{REFERENCES}

[1] A. Weisser, G. Endel, P. Filzmoser, and M. Gyimesi, "ATC -> ICD - evaluating the reliability of prognoses for ICD-10 diagnoses derived from the ATC-Code of prescriptions," BMC Health Serv. Res., vol. 8, no. Suppl 1, p. A10, 2008.

[2] P. Filzmoser, A. Eisl, and F. Endel, "ATC - ICD: Determination of the reliability for predicting the ICD code from the ATC code," Hauptverband der Österreichischen Sozialversicherungsträger, EBM Bericht, Dec. 2009. 


\title{
Simulation of Physiologic Ectopic Beats in Heartbeat Intervals to Validate Algorithms
}

\author{
Martin Bachler ${ }^{*, * *}$ Matthias Hörtenhuber ${ }^{* *}$ Martin Frank ${ }^{* *}$ \\ Siegfried Wassertheurer* Christopher Mayer* \\ * AIT Austrian Institute of Technology GmbH, Health E Environment \\ Department, Biomedical Systems, Donau-City-Str. 1, 1220 Vienna, \\ Austria (e-mail: \{martin.bachler, siegfried.wassertheurer, \\ christopher.mayer\}@ait.ac.at) \\ ** Vienna University of Technology, Institute for Analysis and \\ Scientific Computing, Wiedner Hauptstr. 8-10, 1040 Vienna, Austria \\ (e-mail: \{martin.bachler, e0927120\}@student.tuwien.ac.at, \\ martin.frank@alumni.tuwien.ac.at)
}

\section{INTRODUCTION}

Diseases of the heart and the cardiovascular system are the leading cause of death in developed countries (Nichols et al., 2012). The heart rate variability is a promising marker for the early diagnosis of these diseases and shows a significant relationship to cardiovascular mortality, but it is easily disrupted by ectopic heart beats (American Heart Association Inc.; European Society of Cardiology, 1996; Sethuraman et al., 2010). Numerous methods of ectopic beat correction exist to counteract this problem, but they need to be validated using comprehensive data sets with customizable features (Jung et al., 1996). The best way of acquiring data for validation is to artificially generate them, but existing interbeat-interval simulators cannot reproduce regulatory mechanisms of the heart rate in all their complexity. Therefore, a method to add simulated ectopic beats to prerecorded physiologic heartbeat interval data is presented in this work.

\section{METHODS}

The development of the ectopic beat simulator is based on physiologic data acquired from Physionet.org (Goldberger et al., 2000), a free online archive of physiological signals. In the first step, measurements of real ectopic beats are analyzed and a model is built from their statistical characteristics. It describes the prolongation of the interbeatintervals before $\left(I_{B}\right)$ and their shortening after $\left(I_{A}\right)$ the ectopic beat as a function of the heart rate $(H R)$ :

$$
\begin{aligned}
I_{B} & =-0.0053 \cdot H R+b \text { and } \\
I_{A} & =-0.0125 \cdot H R+a, \\
\text { for } b & \in[0.9474,1.1966] \text { and } \\
a & \in[1.8956,2.1024], \text { chosen randomly. }
\end{aligned}
$$

In the next step, this model is incorporated in a simulator used to add generated ectopic beats to ectopic-free heart rate variability data sequences. It is capable of simulating supraventricular contractions and premature ventricular contractions in user-defined combinations and intensities.

\section{RESULTS AND DISCUSSION}

The results are inspected using qualitative and quantitative comparisons between the simulator and real heart rate variability data by visual inspection and analysis of covariance. The characteristics of bigeminy, supraventricular arrhythmia, and single supraventricular contractions, seem to be rendered very well by the simulator. The results of the quantitative inspection show that there is no statistically significant difference between the model of simulated and real ectopic beats.

\section{CONCLUSION}

The presented simulator for adding ectopic beats to heartbeat interval data shows a reasonable reproduction of ectopic beats, enabling comprehensive validation of ectopic beat correction methods.

\section{REFERENCES}

American Heart Association Inc.; European Society of Cardiology (1996). Guidelines - heart rate variability. European Heart Journal, 17, 354-381.

Goldberger, A.L., Amaral, L.A.N., Glass, L., Hausdorff, J.M., Ivanov, P.C., Mark, R.G., Mietus, J.E., Moody, G.B., Peng, C.K., and Stanley, H.E. (2000). Physiobank, physiotoolkit, and physionet: Components of a new research resource for complex physiologic signals. Circulation, 101(23), e215-e220.

Jung, J., Heisel, A., Tscholl, D., Fries, R., Schieffer, H., and Özbek, C. (1996). Assessment of heart rate variability by using different commercially available systems. The American Journal of Cardiology, 78(1), 118-120.

Nichols, M., Townsend, N., Luengo-Fernandez, R., Leal, J., Gray, A., Scarborough, P., and Rayner, M. (2012). European cardiovascular disease statistics 2012. European Heart Network, Brussels, European Society of Cardiology, Sophia Antipolis, P104.

Sethuraman, G., Ryan, K.L., Rickards, C.A., and Convertino, V.A. (2010). Ectopy in trauma patients: cautions for use of heart period variability in medical monitoring. Aviation, space, and environmental medicine, 81(2), 125-129. 


\title{
Modeling Methods Development for Routine Data based Screening Evaluation: Decision Making for Organized Abdominal Aortic Aneurysm Screening
}

\author{
G. Zauner*, C. Urach**, G. Endel***, I. Wilbacher*** \\ *dwh GmbH simulation services, 1070 Vienna, Austria, \\ (Tel:01-526-5526; e-mail: guenther.zauner@dwh.at) \\ **Vienna University of Technology, Institute for Analysis and Scientific Computing, \\ 1040 Vienna,Austria, and dwh GmbH simulation services, 1070 Vienna, Austria, \\ (e-mail: christoph.urach@dwh.at) \\ ***Main Association of Austrian Social Security Institutions, 1030 Vienna, \\ Austria, (e-mail: Gottfried.endel@hvb.sozvers.at, Ingrid.wilbacher@hvb.sozvers.at)
}

\section{INTRODUCTION}

Screening technologies are under discussion in many health care systems of industrialized countries. Besides cancer screening a growing list of other health problems are getting in focus. This work presents a dynamic simulation based decision support for the evaluation of organized screening interventions concerning patients with abdominal aortic aneurysms (AAA). The interdisciplinary subject required the collaboration of experts in different fields, which were medical doctors, Health Technology Assessment (HTA) experts and modelers. The here presented modular model structure was constructed in such a flexible way that it allows adaptation on screening research questions for similar diseases. Another focus of the work was integration of risk factors and how this boundaries and restrictions influence the choice of modeling methods to use, fitting the real world structure using a parameterize able implementation. This is especially because of the steadily increasing knowledge about treatment or improved treatment of AAA which could cause the necessity of a periodic evaluation.

\section{RESULTS}

By inclusion of several patient specific properties, the model does not only provide comparison of current state with organized screening of defined age groups, but also the elaboration of alterations of population characteristics, for incidence changing in smoking behavior, and its consequences on AAA cases. In the tornado diagram (Fig. 1) exemplarily results for ruptures and the influence of single parameters are shown.

\section{CONCLUSIONS}

The realized work fits to the given demand in the Austrian setting. It is data driven using Austrian billing data of health insurance institutions, especially for parameterization of number of cases and treatment. The combination of data evaluation strategies and advanced dynamic modelling, in this case Agent Based Modeling, lead to higher flexibility in comparison to methods used in literature, especially Markov Models. Of course poor statistical models are not capable to deal with the given complexity and the changing scenarios of interest. The developed framework is used to compare organized screening strategies but also allows assessment of changes of other factors (e.g. smoking) which have an impact on the growth of the aorta.

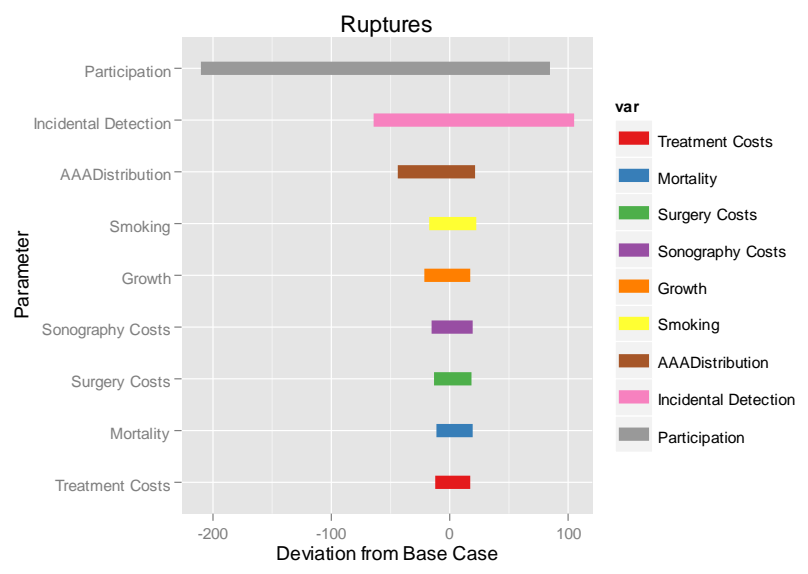

Fig. 1. Tornado diagram for identification of main influence factors realized by univariate variation in the $95 \%$ confidence intervals of the patient parameters of interest.

Its modular design allows integration of further knowledge on AAA for additional analysis and evaluation of screening strategies. The structure also supports an objective discussion and is an important decision support tool, the fundamental idea can be used for other screening questions of not communicable diseases in the Austrian setting.

Actual and further steps in refinement of the interfaces of the model modules are under development in DEXHELPP.

\section{ACKNOWLEDGEMENT}

The research project DEXHELPP.(Decison Support for Health Policy and Planning: Methods, Models and Technologies based on Existing Health Care Data) is in the frame of COMET-Competence Centers for Excellent Technologies. DEXHELPP is supported by BMVIT, BMWFW and the state Vienna. The COMET program is transacted by the FFG. 
MATHMOD 2015 Abstract Volume 


\section{MATHMOD 2015 ABSTRACT VOLUME}

MATHMOD Minisymposium Computational Methods in Modelling and Simulation of Fluid Power Systems 
MATHMOD 2015 Abstract Volume 


\title{
Multi-phase and Multi-component CFD Analysis of a Load - Sensing Proportional Control Valve
}

\author{
E. Bigliardi*, M. Francia*, M. Milani*, \\ L. Montorsi*, F. Paltrinieri*, M. Stefani* \\ * Department of Sciences and Methods for Engineering, University of Modena and Reggio Emilia, \\ Reggio Emilia, Italy, (e-mail: massimo.milani@unimore.it)
}

\section{INTRODUCTION}

Numerical analysis is gaining an important role in the design of hydraulic components and systems. In particular, multidimensional simulation is increasingly applied to the investigation of the fluid dynamics behavior of hydraulic component in order to broaden and complete the experimental campaigns. Moreover, the human and computational resources to be involved in the numerical analysis are now not only acceptable, but also advantageous, due to the continuous development of computational platforms and CFD tools.

In literature, many examples are available concerning the advantages gained by using the numerical analysis. Among them, several applications on the study of hydraulic valves can be pointed out; for instance, in Yang (2004 and 2005) the CFD analysis was used to study the flow field and the flowinduced forces in hydraulic valves, as well as in Borghi et al. (2000, 2005 and 2005) and in Del Vescovo et al (2003) and in Franzoni et al. (2008), theoretical approaches and experimental investigations have been compared to CFD predictions for the hydraulic valves design and optimization.

In this paper the metering characteristics of a closed center load - sensing proportional control valve are investigated using the multi-phase multi-component numerical simulation. The CFD tool is used to simulate the effects of different operating conditions on the valve flow characteristics. In particular, several opening displacements and pressure drops are analysed and the onset regions for aeration and cavitation are addressed.

The hydraulic valve performance is evaluated in terms of overall discharge coefficient, efflux angle, flow forces and pressure and velocity distributions in the critical region.

\section{CONCLUSION}

A multi-phase multi-component CFD approach has been applied to investigate the flow through the metering edge of a load - sensing proportional control valve. The numerical simulation included models for cavitation, gas absorption and release and turbulence. Different operating conditions as well as spool displacements were analysed in terms of overall discharge coefficient, efflux angle, flow forces and pressure and velocity distributions in the critical region.

The numerical results demonstrated to be in good agreement with the experimental measurements in terms of total pressure drop across the valve as well as in terms of discharge coefficients.

Significant differences were outlined between the numerical value and the experimental estimation when evaluating the flow forces. In particular, the latter method for determining the forces exerted by the fluid on the spool surfaces neglects the effects of the shear stresses on the spool wall. Moreover, the assumption of the total valve pressure drop acting on the entire inlet and outlet surfaces on the spool resulted to be limiting, in particular for the large apertures and flow rates. In these cases, the experimental estimation proved the underestimate the total flow forces.

Finally, the onset areas for aeration and cavitation were investigated. The simulation proved that these regions are located downstream of the metering edge on the outer radius where flow recirculation takes place. Nevertheless, the aeration region resulted to be smaller than the low-pressure zone due to a degasification effect of the onset area, which released almost the entire content of air dissolved in the operating fluid thus preventing further aeration even though the pressure remained below the saturation value.

\section{REFERENCES}

Borghi, M., Milani, M., Paoluzzi, R. (2000) Stationary axial flow force analysis on compensated spool valves. International Journal of Fluid Power 1 No. 1 p.p. 17-25.

Borghi, M., Milani, M., Paltrinieri, F. (2004). The Influence of the Notch Shape and Number on Proportional Directional Control Valves Metering Characteristics. SAE Paper 2004-01-2619.

Borghi, M., Milani, M., Paoluzzi, R. (2005). Influence of Notch Shape and Number on the Metering Characteristics of Hydraulic Spool Valves. International Journal of Fluid Power. ISSN 1439-9776 - Vol. 6, N. 2 pp. 5-18.

Brennen, C.E. (1995) Cavitation and Bubble Dynamics, Oxford University Press.

Del Vescovo, G., Lippolis, A. (2003). Three Dimensional analysis of flow forces on directional control valves. International Journal of Fluid Power. Vol.4 Number 2.

Franzoni, F., Milani, M., Montorsi, L., (2008) Cavitating Flows in Hydraulic Multidimensional CFD Analysis, SAE Paper 2008-01-678.

Yang, R. (2004) Improving Spool Valve Operating Effort Using CFD, SAE Paper 2004-01-2620. 


\title{
A Combined Methodology for Studying the Axial Balancing Mechanism of Orbit Annular Hydraulic Machines
}

\author{
Elisa Bigliardi*, Marco Francia**, Massimo Milani***, \\ Luca Montorsi $* * * *$, Fabrizio Paltrinieri $* * * * *$, Matteo Stefani $* * * * * *$ \\ * DISMI - University of Modena and Reggio Emilia, Reggio Emilia, 42122 \\ Italy (e-mail: elisa.bigliardi@unimore.it) \\ **DISMI - University of Modena and Reggio Emilia, Reggio Emilia, 42122 \\ Italy (e-mail: marco.francia@unimore.it) \\ *** DISMI - University of Modena and Reggio Emilia, Reggio Emilia, 42122 \\ Italy (Tel: +390522 52 3501; e-mail: massimo.milani@unimore.it) \\ **** DISMI - University of Modena and Reggio Emilia, Reggio Emilia, 42122 \\ Italy (Tel: +390522 52 3502; e-mail: luca.montorsi@unimore.it) \\ ***** DISMI - University of Modena and Reggio Emilia, Reggio Emilia, 42122 \\ Italy (Tel: +390522 52 3503; e-mail: fabrizio.paltrinieri@unimore.it) \\ ****** DISMI - University of Modena and Reggio Emilia, Reggio Emilia, 42122 \\ Italy (e-mail: matteo.stefani@unimore.it)
}

\section{INTRODUCTION}

Technical and technological developments are continuously giving strong impulse to the performance improvement of hydraulic systems for industrial and mobile applications. Among others design aspects, particular attention has been devoted to the specific power and the overall efficiency increasing of the positive displacement units. This attention has deeply conditioned the design process in the field of mobile equipment applications, particular care has been dedicated to axial piston, external and internal gear pumps and motors. As well known, this design evolution determined a continuous increase in supply pressure operating level and a parallel decreasing of the units weight and volume.

The hydraulic machines designers have to face two main contrasting requirements: on one hand, the increasing of the motor supply mean operating pressure, on the other hand the contemporarily overall efficiency optimization.

Nowadays, hydraulically balanced orbit machines represent a good design compromise in solving these problems and, thanks to the high density of power, they are commonly employed in many mobile applications.

During the last twenty years many contributions in this field have been proposed by several authors. For example, a simplified numerical model useful in determining the pressure transients in external gear pumps is presented and validated in Mancò et al. (1989) and in Mancò et al. (1993), while their journal bearing performance, their suction capabilities and the pressure transients within their meshing teeth are investigated in Gutés et al. (2000), Poy Ferrer et al. (2002) and Edge et al. (2001).

Besides, in Koç et al. (1997a), Koç et al. (1997b) and Koç et al. (1997c) the lubrication mechanisms of positive displacement motors and pumps sealing gaps are illustrated, with particular attention given to the numerical and to the experimental analyses of the high pressure pumps bush-type bearings. These studies highlight the importance of the main motors and pumps components proper design, and in particular of the pressure balanced bearing blocks. The fluid dynamics behaviour of the sealing and bearing gaps are also deepened in many papers, where the attention has been devoted to the study both of the microscopic and macroscopic gap geometry (Lasaar (2000)) and of the thermal (Olems (2000)) and EHD (Huang (2002)) effects influence on energy dissipation. Finally, a reliable numerical method for the evaluation of the axial force in pressure compensated gears pumps and motors is presented in Bonacini et al. (1987).

In this paper an innovative CAE methodology based on both 2D CFD and lumped and distributed parameters numerical simulations has been built up and applied for studying and analyzing the operation of hydraulic roller motors. More in details, the predictive capabilities of the numerical procedure has been clearly demonstrated through a detailed comparison between computational results and experimental measurements gained from a testing campaign performed for a wide range of operating conditions in terms of both exerted torque levels and rotational speeds. Besides, the application of a customized 2D CFD model, based on the numerical integration of the Reynolds equation, permits to investigate the lubrication mechanism interesting the lateral clearances and to estimate the internal leakages of actual rotor-stator group of orbit roller motors. Then, the numerical results gathered from these CFD simulations have been adopted for calibrating the lumped and distributed parameters numerical model and thus for increasing the accuracy of the overall CAE procedure.

\section{CONCLUSIONS}

In this paper an innovative computational approach, useful for predicting the performance of orbit annular machines, has been proposed and validated through a detailed numerical versus 
experimental comparison. More in details, an iterative procedure, based on both lumped and distributed numerical modelling and 2D CFD simulations, has been built up and applied to the study of a typical hydraulic roller motor for a wide range of operating conditions, in terms of both rotational speed and exerted torque.

The numerical results obtained for convergent solutions have been found in very good agreement with the experimental measurements and percentage errors always lower than $3 \%$ clearly demonstrate the consistency of this novel approach. Moreover, the analysis of the 2D CFD pressure distributions acting on the stator, rotor and rollers lateral surfaces have been very useful in order to estimate the motor internal leakages and to understand its axial balancing mechanism. In this way, the predictive capability of the lumped and distributive numerical model have been increased and optimized geometrical solutions for the main machine inner components can be designed and verified.

\section{REFERENCES}

Babbone, R., Bottazzi, D., Cagni, G., Grasselli, F., and Milani, M. (2011). CAE design of orbit annular machines. $12^{\text {th }}$ Scandinavian International Conference on Fluid Power, Tampere (Finland), May 18-20, 2011.

Bassani, R. and Piccigallo, B. (1992). Hydrostatic lubrification. Elsevier.

Bonacini, C. and Carra, R. (1987). A numerical method to evaluate axial force in pressure compensated gears pumps and motors. Proceedings of the $43^{\text {rd }}$ Annual Meeting, National Conference on Fluid Power, NFPA, Chicago (USA), October 11-13, pp. 403-408.

Edge, K., Keogh, P., and Eaton, M. (2001). Modelling and simulation of pressures within the meshing teeth of gear pumps. International Conference on Recent Advances in Aerospace Actuation Systems and Components, Toulouse (France), June 13-15, 2001.

Gutés, M., Gámez Montero, P.J., Castilla, R., and Codina Macià, E. (2000). Journal bearing performance in gear pumps. Proceedings of the $1^{\text {st }}$ International FPNI Ph.D. Symposium on Fluid Power, Hamburg (Germany), September 20-22, 2000, pp. 259-269.

Huang, C. (2002). Gap flow investigation of the piston cylinder assembly in axial piston pumps considering EHD effects. Proceedings of the $2^{\text {nd }}$ International FPNI Ph.D. Symposium on Fluid Power, Modena (Italy), July 3-6, 2002.

Ivantysyn, J. and Ivantysynova, M. (2003). Hydrostatic pumps and motors. TBI Edition.

Koç, E., Kurbant, A.O., and Hooke, C.J. (1997). An analysis of the lubrication mechanisms of the bush-type bearings in high pressure pumps. Tribology International, Vol. 30 (Num. 8), pp. 553-560.

Koç, E. and Hooke, C.J. (1997). An experimental investigation into the design and performance of hydrostatically loaded floating wear plates in gear pumps. Wear, Num. 209, pp. 184-192.
Koç, E. and Hooke, C.J. (1997). Considerations in the design of partially hydrostatic slipper bearings. Tribology International, Vol. 30 (Num. 11), pp. 815-823.

Kreysig, E. (1993). Advanced engineering mathematics. John Wiley \& Sons.

Lasaar, R. (2000). The influence of the microscopic and macroscopic gap geometry on the energy dissipation in the lubricating gaps of displacement machines. Proceedings of the $1^{\text {st }}$ International FPNI Ph.D. Symposium on Fluid Power, Hamburg (Germany), September 20-22, 2000, pp. 101-116.

Mancò, S. and Nervegna, N. (1989). Simulation of an external gear pump and experimental verification. JHPS International Symposium on Fluid Power, Tokio (Japan), March 1989, pp. 139-152.

Mancò, S. and Nervegna, N. (1993). Pressure transients in an external gear hydraulic pump. JHPS International Symposium on Fluid Power, Tokio (Japan), March 1993, pp. 221-227.

Merrit, H.E. (1966). Hydraulic control system. John Wiley \& Sons.

Olems, L. (2000). Investigations of the temperature behaviour of the piston cylinder assembly in axial piston pumps. International Journal of Fluid Power, Num. 1, pp. 27-38.

Poy Ferrer, M. and Codina Macià, E. (2002). Suction capability of gear pumps. Proceedings of the $2^{\text {nd }}$ International FPNI Ph.D. Symposium on Fluid Power, Modena (Italy), July 3-6, 2002.

Saad, Y. (1996). Iterative methods for sparse linear equations. PWS Publishing Company.

Wieczorek, U. (2000). Simulation of the gap flow in the sealing and bearing gaps of axial piston machines. Proceedings of the $1^{\text {st }}$ International FPNI Ph.D. Symposium on Fluid Power, Hamburg (Germany), September 20-22, 2000, pp. 493-507. 


\title{
A study on mesh refinement in OpenFOAM for wave propagation problems in fluid power systems
}

\author{
Clemens Fries* Bernhard Manhartsgruber ** \\ * Johannes Kepler University, Linz, 4040 Linz (e-mail: \\ clemens.fries@jku.at). \\ ** Johannes Kepler University, Linz, 4040 Linz (e-mail: \\ bernhard.manhartsgruber@jku.at).
}

\begin{abstract}
Due to rising importance of wave propagation effects in fluid power systems, CFD (computational fluid dynamics) codes take also on greater significance. These highly sophisticated codes have the capability to calculate the full resolution of a pressure field as well as of a velocity field in arbitrarily complex geometries. But this brilliant development brings also a huge disadvantage, namely enormous computational costs. Because of this reason CFD software often disqualifies itself as first choice application. However, considering a whole computational domain and it's physics the idea comes up, that there are regions which require a higher spatial resolution than others. Mesh refinement is a common practice to get a higher resolution in regions of greater interest while reducing the overall number of cells and hence the simulation time. This paper presents results of simulations with OpenFOAM, where mesh refinement has been done with polyhedral elements. As a simple benchmark system a pipe with a cross-sectional jump has been used. First the optimal CFD result for this system has been determined by doubeling the number of elements in each direction for each additional simulation until the variations in the outcome has vanished. This simuation result has been compared to a set of simulations, where mesh refinement has only adapted to the region after the cross-sectional jump, where the higher number of cells has been considered as a benefit.
\end{abstract}

Keywords: OpenFOAM, Computational Fluid Dynamics, Hydraulics, Pipelines, Transmission lines, Discretization, Mesh, Refinement

\section{INDRODUCTION}

The rising demand for accuracy of system responses as well as the greater range of operating frequencies in fluid power systems, makes CFD (computational fluid dynamics) codes more and more popular in the hydraulic community. Given reasons require a high resolution in space and time, which in many cases leads to a very long calculation time. At the latest for overall system simulations the duration of calculation pushes all borders. Because of this, different efforts have been made to reduce simulation time. A coupling of CFD codes for more complex geometries with transmission line models for simple pipes has for example been published in [Fries and Manhartsgruber, 2014]. A different approach to reduce calculation time can be found in mesh generation. Obviously there are certain regions within a computational domain, which require a higher spatial resolution than others. This for instance can be done by introducing a certain grading, meaning a definite aspect ratio between cell size in a certain direction. If the initial mesh is a hexahedral one, a different approach would be to split particular cells in each direction once. In other words, considering a two dimensional square and applying this strategy on it, the result would be four squares as it can be seen in Fig. 1. Or considering a cube in the three dimensional space the result would be eight new cubes. Such a multiblock/multilevel mesh refinement strategy has been published in Teigland and Eliassen [July 2001] and has also been considered as possible mesh refinement procedure in the work of Andrew et al. [1983]. As can be found on http://www.openfoam.com/ [2014], the open source CFD software package OpenFOAM supports unstructured meshes of cells of any shape, where the number of faces of the cells as well as the number of edges of the face can be arbitrary, meaning the use of polyhedral cells is feasible. It's also possible to connect one face of one cell with more faces of another cell, meaning OpenFOAM has the potential to manage calculations with multiblock refined meshes.

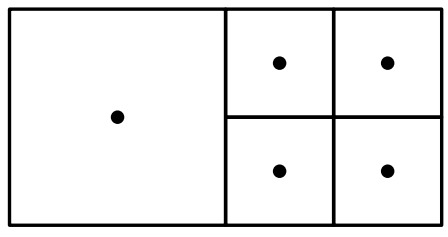

Fig. 1. mesh refinement strategy

Goal of this work is to verify the possibilty of OpenFOAM to handle such multiblock refined meshes and to evaluate 


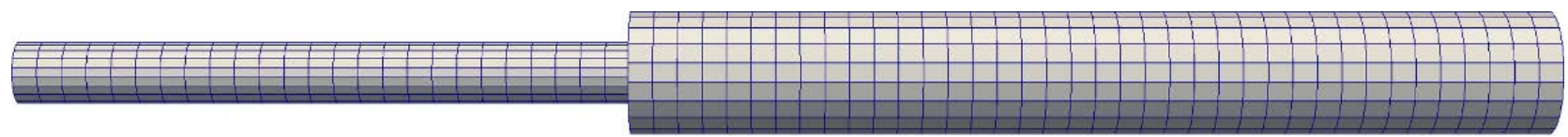

Fig. 2. computational domain axial direction

the benefit with respect to accuracy as well as calculation time. For this reason a cross-sectional, axisymmetric expansion of a duct, which can be seen in Fig. 2, has been chosen as a simple benchmark system. Altough this geometry seems fairly ordinary, the flow in such a system can already get considerably complex. Another reason for the selection of this part can be found in the widespread use in industry.

\section{SIMULATION}

Before a CFD simulation can be executed, the flow domain has to be discretized. The mesh of the geometry can be seen in Fig. 2. The left side in Fig. 2 represents the inlet, whereas the right side represents the outlet. As boundary condition on the inlet a parabolic velocity profile has been chosen, while the pressure on the other side, the outlet, has been set to a constant value.

In a first step a mesh fineness study has been accomplished in order to determine the convergence of the CFD result. The velocity profile on the point of the step has been determined as the simulation result to take a look at and therefore has been recorded during the simulation. Beginning with a rather coarse mesh with approximately 5000 cells a number of simulations have been run, whereby the cells of the mesh have always been halved in each direction for any additional run-through. In Fig. 3 the first three iterations of this procedure are shown.

In a second step the mesh has been refined in the way described above in a small region around the cross-sectional step and simulations with the same conditions have been carried out. The partial refinement has been adopted for two, four and eight times the original cell length in both directions of the step one and two times. A comparison of the velocity profiles at the diameter step can be seen in Fig. 4. Obviously it does not matter how long the refinement area considering this small range of length is. Although it can be seen that this kind of mesh refinement strategy used with OpenFOAM works quite well. All of the results obtained with this partial mesh refinement are closer to the next overall refined case. Apparently, the small region around the expansion has been too less to resolve the rather complicated part of the recirculation area, reaching to the reattachment point.

\section{ACKNOWLEDGEMENTS}

This work has been carried out at LCM GmbH as part of a K2 project. K2 projects are financed using funding from the Austrian COMET-K2 programme. The COMET K2 projects at LCM are supported by the Austrian federal government, the federal state of Upper Austria, the Johannes Kepler University and all of the scientific partners which form part of the K2-COMET Consortium.

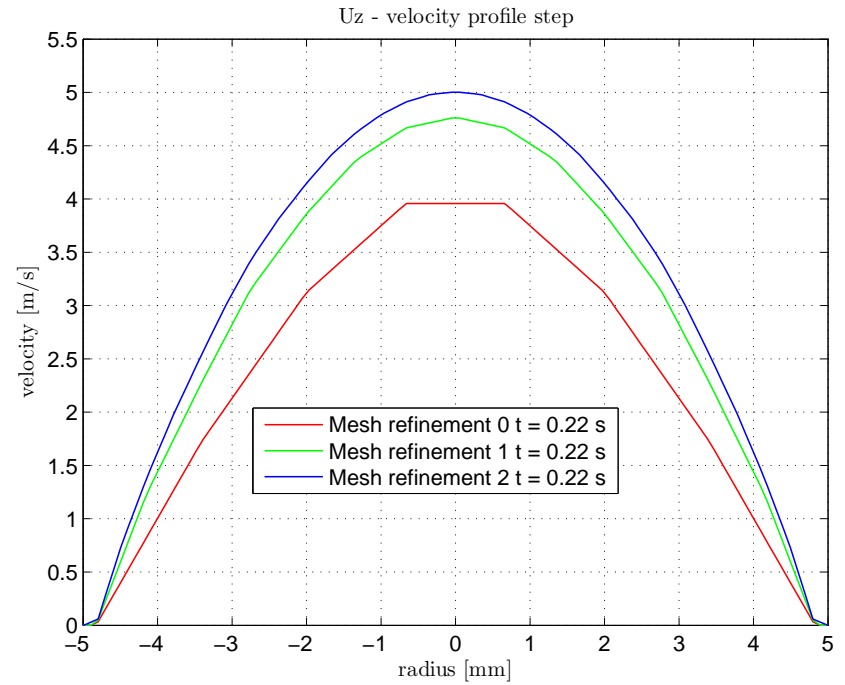

Fig. 3. $U_{z}$ using overall mesh refinement

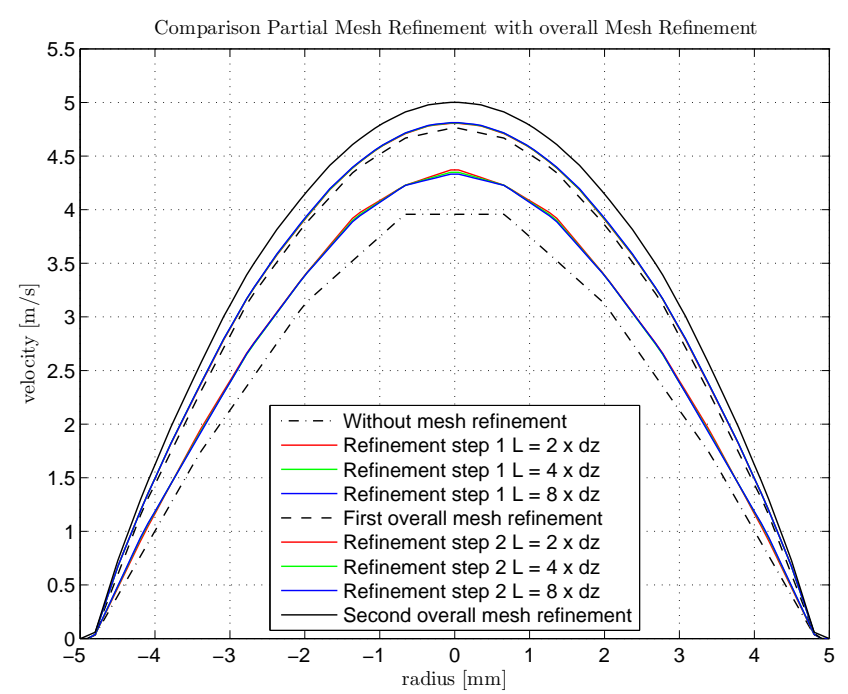

Fig. 4. $U_{z}$ using partial mesh refinement

\section{REFERENCES}

Andrew, R.B., Sherman, A.H., and Weiser, A. (1983). Some refinement algorithms and data structures for regular local mesh refinement.

Fries, C. and Manhartsgruber, B. (2014). Dynamics of transmission line junctions - comparison of cfd results against measurements. In Proceedings of FPNI 2014 PhD Symposium on Fluid Power, Laapeenrante, FIN, June 10-13. doi:10.1115/FEDSM2013-16592.

http://www.openfoam.com/ (2014). http: //www. openfoam.com/.

URL

Teigland, R. and Eliassen, I.K. (July 2001). A multiblock/multilevel mesh refinement procedure for cfd computations. International Journal for Numerical Methods in Fluids. doi:10.1002/fld.143. 


\title{
Low Frequency Correction of a Multi-degrees-of-freedom Model for Hydraulic Pipeline Systems
}

\author{
Gudrun Mikota* \\ *Institute of Machine Design and Hydraulic Drives, Johannes Kepler University Linz, \\ 4040 Linz, Austria (Tel: +43-732-2468-6538; e-mail: gudrun.mikota@jku.at)
}

The transient laminar flow of a compressible Newtonian fluid in a straight circular pipeline has been modelled by rational fraction modal approximations. Such models were published by various authors; they can be used for time-domain simulation and are well suited to study the dynamic behaviour of individual pipelines. Compared to the transcendental model by D'Souza et al. (1964), Kojima et al. (2002) encountered large errors when they combined modal approximations for the simulation of compound pipeline systems; they therefore suggested to calculate transcendental transfer functions of the entire system and approximate the result in a second step.

For a closed-end pipeline, injected flow rate excitations and resulting pressure responses, Mikota (2013) derived the modal decomposition of the transcendental pipeline model. Transcendental modal transfer functions were approximated by rational fraction expressions, which lead to a multidegrees-of-freedom description of the pipeline. Mikota (2014) used this model to investigate a specific pipeline network and experienced similar problems as Kojima et al. (2002). However, by comparing transcendental and approximated transfer functions of the network, it became clear that these problems were located in the low frequency range. They were explained by the fact that for damped pipeline systems, the modal approximation of an individual pipeline is rather inaccurate in the frequency range below the first pipeline resonance. If the pipeline becomes part of a network, this frequency range will contain one or more network resonances, for which the approximation will be wrong.

In this paper, the modal approximations from Mikota (2013) are modified in a way that corrects the low frequency errors for a predefined pipeline system. Compared to Mikota (2014), the hydraulic pipeline system model is rebuilt from a modal description in which some natural frequencies and damping ratios are corrected. Although the underlying multidegrees-of-freedom model of an individual pipeline features proportional damping, this is not necessarily the case for the assembled multi-degrees-of-freedom model of the hydraulic pipeline system. To keep within the framework of proportional damping, the eigenvectors of the corrected system are assumed to be real and can therefore be taken from the undamped version of the pipeline system model. In the low frequency range, natural frequencies and damping ratios are calculated from single frequency approximations of the individual pipelines. Each of these approximations is accurate in the vicinity of a pipeline system resonance.
Higher natural frequencies and the respective damping ratios are taken from the viscous damping approximaton as used by Mikota (2014).

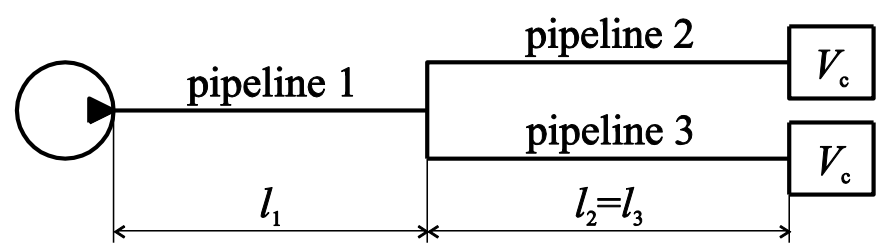

Fig. 1. Hydraulic pipeline network.

The new method is applied to the pipeline network from Mikota (2014), see Fig. 1. It leads to a significant improvement of the multi-degrees-of-freedom pipeline system model. This model is described by a system of linear second order differential equations with constant coefficients and is therefore suited for time-domain simulation. Apart from that, it gives insight into the modal properties of the pipeline system, which helps understanding the dynamic system behaviour.

\section{REFERENCES}

D'Souza, A.F. and Oldenburger, R. (1964). Dynamic response of fluid lines. Transactions of the ASME Journal of Basic Engineering, Vol. 86, pp. 589-598.

Kojima, E., Shinada, M., and Yu, J. (2002). Development of accurate and practical simulation technique based on the modal approximations for fluid transients in compound fluid-line systems. International Journal of Fluid Power, Vol. 3, No. 2, pp. 5-15.

Mikota, G. (2013). Modal analysis of hydraulic pipelines. Journal of Sound and Vibration, Vol. 332, pp. 27943805 .

Mikota, G. (2014). A multi-degrees-of-freedom model for hydraulic pipeline systems. In The 9th International Fluid Power Conference, Aachen, Germany. 


\title{
A reference model for modal approximations of linear transmission line dynamics
}

\author{
Bernhard Manhartsgruber \\ Institute of Machine Design and Hydraulic Drives, Johannes Kepler \\ University, Linz, Austria, (e-mail: bernhard.manhartsgruber@jku.at)
}

\begin{abstract}
Transmission line dynamics is an important subject for the simulation of fluid power systems. In the case of moderate amplitudes, linear models are widely applied. While the frequency domain offers a very compact and straightforward description of the inputoutput behaviour, the implementation of transmission line models in standard simulation software using a linear, time-invariant, finite-dimensional state space system description poses an approximation problem that has been treated by a large number of authors in the fluid power literature. This paper presents the solution of the approximation problem in an optimal way and gives a set of reference data.
\end{abstract}

The transient flow of a weakly compressible liquid in a straight, rigid-walled transmission line of circular cross section can be desribed as in D'Souza and Oldenburger (1964)

$$
\left[\begin{array}{c}
\hat{Q}_{0} \\
\hat{p}_{1}
\end{array}\right]=\underbrace{\left[\begin{array}{cr}
\frac{\tanh (s Z)}{Z}-\frac{1}{\cosh (s Z)} \\
\frac{1}{\cosh (s Z)} Z \tanh (s Z)
\end{array}\right]}_{\mathbf{G}(s)}\left[\begin{array}{l}
\hat{p}_{0} \\
\hat{Q}_{1}
\end{array}\right]
$$

with

$$
Z=Z_{0} \sqrt{-\frac{J_{0}\left(\sqrt{-\frac{s}{\nu}} R\right)}{J_{2}\left(\sqrt{-\frac{s}{\nu}} R\right)}}, \gamma=\frac{s L}{c_{0}} \sqrt{-\frac{J_{0}\left(\sqrt{-\frac{s}{\nu}} R\right)}{J_{2}\left(\sqrt{-\frac{s}{\nu}} R\right)}}
$$

and

$$
Z_{0}=\frac{\sqrt{K \rho}}{R^{2} \pi}, \quad c_{0}=\sqrt{\frac{K}{\rho}} .
$$

This model describes the input-output behaviour between the two ends of a line of length $L$ and internal radius $R$ filled with a liquid of density $\rho$, kinematic viscosity $\nu$, and bulk modulus $K$. The pressure $p_{0}$ at one end and the flow rate $Q_{1}$ at the opposing end are taken as input variables, while the dual quantities $Q_{0}$ and $p_{1}$ serve as output signals.

If the implementation of the model is limited to linear, time invariant systems in state-space form like

$$
\begin{array}{r}
\dot{\mathbf{x}}=\mathbf{A} \cdot \mathbf{x}+\mathbf{B} \cdot\left[\begin{array}{c}
p_{0} \\
Q_{1}
\end{array}\right] \\
{\left[\begin{array}{c}
Q_{0} \\
p_{1}
\end{array}\right]=\mathbf{C} \cdot \mathbf{x}+\mathbf{D} \cdot\left[\begin{array}{l}
p_{0} \\
Q_{1}
\end{array}\right]}
\end{array}
$$

the approximation problem reduces to choosing a system order and finding the coefficients in the matrices $\mathbf{A}, \mathbf{B}, \mathbf{C}$ and $\mathbf{D}$. The presented approach has already been proposed
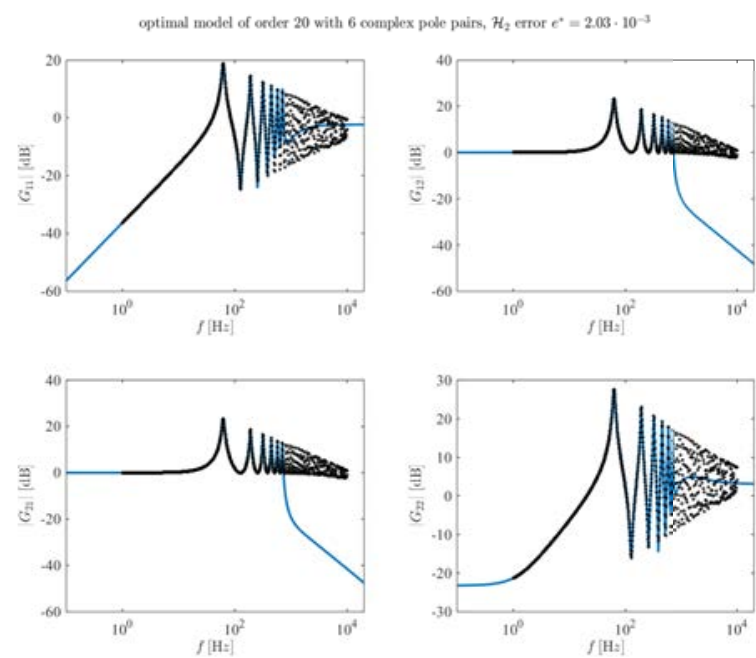

Fig. 1. Magntiutde plots, optimal model of order 20.

in Manhartsgruber $(2005,2006)$, however due to the high demand on computational resources the method was not investigated further. Now, after a decade of advance in computer power, the method is revisited in the present paper.

\section{REFERENCES}

D'Souza, A. and Oldenburger, R. (1964). Dynamic response of fluid lines. Trans. ASME, J. Basic Engng, 86, 589-598.

Manhartsgruber, B. (2005). Reduced order, passive models for liquid transmission lines (invited paper). In Proceedings of $3 r d$ IASME/WSEAS International Conference on Fluid Mechanics and Aerodynamics, August 20-22, 2005, Corfu, Greece.

Manhartsgruber, B. (2006). Reduced order modelling of the hydraulic wave converter. WSEAS Transactions on Fluid Mechanics, 1(6), 647-654. ISSN: 1790-5087. 
MATHMOD 2015 Abstract Volume 


\title{
MATHMOD 2015 ABSTRACT VOLUME
}

\author{
MATHMOD Minisymposium \\ Systems of Systems Modelling, Simulation and \\ Optimization
}


MATHMOD 2015 Abstract Volume 


\title{
Challenges in the Modelling and Operation of Physically Coupled Systems of Systems *
}

\author{
David Kampert and Ulrich Epple
}

\author{
Chair of Process Control Engineering, RWTH Aachen University, \\ Aachen, Germany (e-mail: \{kampert,epple\}@plt-rwth.aachen.de)
}

\begin{abstract}
The DYMASOS project focuses on the control of large-scale "System of Systems" that are coupled by exchange of matter and energy. A key challenge with regard to control is that each system has some degree of autonomy in its respective decisions and behavior, which disallows centralized control methods. This paper provides an overview on modeling and optimization techniques that are applied in DYMASOS and points out the general challenges that arise when applying them to real-world case studies.
\end{abstract}

Keywords: Systems of Systems, Systems Engineering, Systems Design, Automatic Control Systems, Control Engineering, DYMASOS.

A "System of Systems (SoS)" is commonly understood as a network of interconnected large-scale technical systems that are geographically distributed and have operational and managerial independence, even though the systems are serving a common purpose (see e.g. Maier (1998) for a detailed definition). The project DYMASOS (DYnamic MAnagement of physically coupled Systems Of Systems, cf. DYMASOS Project (2013)) addresses SoSs that are not only coupled by exchange of information, but also by flows of matter and/or energy, e.g. electrical power grids with multiple energy producers and consumers. The Goal of DYMASOS is to investigate new methods of control, simulation and operation of SoSs, which go along with corresponding SoS models. Due to the technological heterogeneity of SoSs, there is currently no universal method of SoS modeling and operation (Luzeaux (2013)).

The goal of this paper is to provide an overview on modeling and optimization techniques that are applied in DYMASOS and to discuss the challenges that occur when these techniques are applied in real-world case studies.

\section{CASE STUDIES AND SOLUTION CONCEPTS}

The DYMASOS case studies originate from the chemical industry and from electrical power grids. Concerning the chemical industry, the systems that constitute the SoS are production plants that are, in principle, operated individually. However, the plants share a common infrastructure in the form of distribution grids for product exchange and energy supply. In the case studies regarding electrical power grids, systems are producers and consumers of electrical energy, for example a power grid with connected charging stations for electric vehicles. In both cases the use of a common infrastructure is the subject of SoS optimization.

\footnotetext{
* The authors gratefully acklowledge funding for the DYMASOS project by the European Community's Seventh Framework Programme under grant agreement no FP7-ICT-2013-10-611281.
}

\subsection{SoS Control Strategy 1: Mean Field Approach}

In electrical grids, each energy consumer seeks for an energy consumption plan that leads to minimum costs while satisfying the energy needs (e.g. keeping within the temperature range of a refrigerator). Future scenarios such as "smart grid" suppose that energy prices depend on the dynamic load of the grid, which in turn depends on the individual consumption profiles. The task then is to find feasible consumption modes that reduce load fluctuations. This problem can be solved by mean field game theory.

If the state and input parameters of each consumer $i$ are denoted as $x_{i}$ and $u_{i}$, each consumer chooses the proximate state $x_{i}^{+}$by minimizing its cost function. Provided that the number of consumers is sufficiently big, the cost function can be modeled as $J_{i}\left(x_{i}, u_{i} \mid \bar{x}\right)$, where $\bar{x}$ is the "mean state" of all $x_{i}$ (i.e. $x_{j \neq i}$ not need to be known to i). Though the optimization requires $\bar{x}$ to be computed in a centralized way and to be communicated with all consumers, the planning of $x_{i}^{+}$takes place decentralized. The procedure can be applied iteratively by minimizing $J_{i}$ for each consumer to find $x_{i}^{+}$, followed by computing and broadcasting $\bar{x}^{+}$as the proximate mean state. For certain $J_{i}$, the procedure leads to unique optimum solutions that constitute a Nash equilibrium (Ma et al. (2010)).

\subsection{SoS Control Strategy 2: Coalition Approach}

In a case study regarding electric vehicles, the goal is to optimize the capacity utilization of the charging stations and the electrical grid. In contrast to the previously discussed scenario, car drivers cannot be assumed to apply certain optimization techniques or be forced to use the charging stations at a specific time. The most promising way to produce the requested behavior is by controlling charging prices. However, the prices depend on the effective total costs and hence on the behavior of the car drivers. The challenge is to find a pricing scheme that takes this retroactivity into account and that is just and reasonable towards the car drivers. A recently discussed 
strategy to solve this type of problem is the application of coalition game theory (see e.g. Muros Ponce et al. (2014)).

Coalitional games are a concept of game theory, where disjoint sets of $n$ total players cooperate in order to maximize their common gain. In this case study, this results in coalitions of car drivers, charging stations and the electrical grids that they are connected to. Each player in a coalition has influence on the charging price; the car drivers, for example, gain influence by being able to choose the charging station. Given that a cost function $c$ exists that maps each possible coalition $S$ to real-valued costs, the charging price for driver $i$ can be calculated by the Shapley value $p_{i}$, which is

$$
p_{i}(c)=\sum_{S} \frac{|S| !(n-|S|-1) !}{n !}(c(S \cup i)-c(S)) .
$$

The Shapley value guarantees that the total effective costs are distributed, that $p_{i}$ increases linear with $c$ and that players (car drivers) who cause the same costs for the coalition are also charged the same price.

\subsection{SoS Control Strategy 3: Market-Based Approach}

In the case study originating from the chemical industry, the plants constitute an SoS that has a fixed structure and known dynamics. To this extent, the situation is simpler than in the other case studies. The challenge is that each plant plans its respective production singlehandedly. However, the dependencies that are induced by the common infrastructure produce strong side conditions. The procedure to find a feasible overall production plan involves an iterative process of global SoS simulation and negotiating plant demands until a solution is found. These negotiations are based on an artificial market where each plant can bid for the utilization the shared resources.

Let $\mathbf{y}_{i}$ denote the products of plant $i, D_{i}$ the corresponding supply demand vector of the plant and $P_{i}$ a diagonal penalty matrix for violating demands. Furthermore, $\mathbf{p}_{r, i}$ are resource prices, $\mathbf{u}_{i}$ is the utitlization of shared resources for plant $i, \mathbf{p}_{t}$ are prices for using the shared infrastructure and $A_{i}^{+}$is the mapping of resource use to infrastructure use. The global optimization problem then is the minimization of

$$
\sum_{i}\left(\mathbf{y}_{i}-D_{i}\right)^{T} P_{i}\left(\mathbf{y}_{i}-D_{i}\right)+\mathbf{p}_{r, i}^{T} \mathbf{u}_{i}+\mathbf{p}_{t}^{T} A_{i}^{+} \mathbf{u}_{i}
$$

yielding a choice of $\mathbf{u}_{i}$ that will both satisfy the production demands and minimize costs. As side conditions, the plant dynamics, the relation between $\mathbf{y}_{i}$ and the plant states, restrictions on $\mathbf{u}_{i}$ and the balancing of the shared resources need to be taken into account. By applying the alternating direction method of multipliers (Gabay and Mercier (1976)) the problem can be solved simultaneously for each plant. This enables the iterative process of price negotiation between central coordinator and plants.

\section{OPEN CHALLENGES}

The given outlines of solution concepts can only provide a rough idea of the complete solutions. Indeed, the preparation of the solution methods is a subject of ongoing research. But there are nonetheless more challenges and open questions than merely finding solutions for the previously described problems. Solving a problem for an operating SoS is accompanied by additional practical side conditions than when it is solved for a system with a centralized organization. Some of these aspects are itemized in Figure 1. Unfortunately, issues of this kind are not systematically

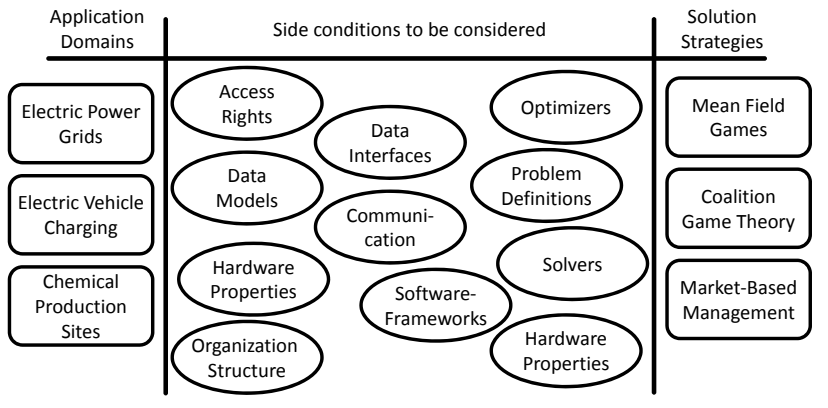

Fig. 1. Open challenges in the application of SoS management methods.

addressed by researchers. The reasons are as follows:

(1) The respective tasks and responsibilities are not conjointly agreed.

(2) There is no theory behind it.

(3) The affected fields are addressed by different communities that each have their best practices.

(4) Too many variations exist and interdependencies are manifold.

(5) The prevailing situation is often not documented.

The result is a growing gap between theory and practice that hinders working concepts from being practically applied in operating SoSs. For this reason, the development of engineering tools for SoSs constitutes a discrete task in DYMASOS. Along with the evaluation of the discussed solution concepts, the project results will also cover concepts for the solutions' application. However, it is an open question to which degree SoS engineering methods can be unified and harmonized. Addressing this question is an essential goal of SoS research.

\section{REFERENCES}

DYMASOS Project (2013). Dynamic Management of Physically Coupled Systems of Systems. URL www. dymasos.eu.

Gabay, D. and Mercier, B. (1976). A dual algorithm for the solution of nonlinear variational problems via finite element approximation. Computers \& Mathematics with Applications, 2(1), 17 - 40.

Luzeaux, D. (2013). Systems of Systems, chapter 10, 321362. John Wiley \& Sons, Inc.

Ma, Z., Callaway, D., and Hiskens, I. (2010). Decentralized charging control for large populations of plug-in electric vehicles: Application of the nash certainty equivalence principle. In Control Applications (CCA), 2010 IEEE International Conference on, 191-195.

Maier, M.W. (1998). Architecting principles for systemsof-systems. Systems Engineering, 1(4), 267-284.

Muros Ponce, F.J., Maestre, J.M., Algaba Durn, E., Alamo, T., and Camacho, E.F. (2014). An Iterative Design Method for Coalitional Control Networks with Constraints on the Shapley Value. In Proceedings of the 19th IFAC World Congress, 1188-1193. 


\title{
Mean Field Modeling of Large-Scale Energy Systems
}

\author{
Basilio Gentile, Sergio Grammatico and John Lygeros
}

\begin{abstract}
This work proposes mean field game-type models for two instances of largescale energy systems, namely plug-in electric vehicles and thermostatically controlled loads. Theoretical and numerical analysis show that both systems possess an equilibrium configuration which is optimal for the individuals and beneficial for the overall population.
\end{abstract}

Keywords: large-scale systems, energy systems, electric vehicles, thermostatically-controlled loads, game theoretical methods, demand response methods.

\section{INTRODUCTION}

Recent years have witnessed a renewed interest and a change of perspective in the study of energy systems, as a consequence of several reasons. Among these, the introduction of a large number of plug-in electric vehicles $(\mathrm{PEVs})$ is foreseen to significantly impact the power grid in the nearby future. Their aggregate energy demand poses new challenges that can be addressed in several ways, as for instance by macroscopic incentives that allow coordination of the charging schedules. Another emerging opportunity arises from the recent deployment of millions of smart meters, sensors, and control devices in electric power distribution grids down to residential and commercial buildings; the availability of this technology enables the supply and demand sides to interact by exchanging price and demand information. In fact when provided with sufficient incentives, thermostatically controlled loads (TCLs) may be willing to adjust their energy usage to tradeoff between comfort and energy consumption.

We model these two large-scale systems via mean field games techniques, focusing on the interaction between the aggregate behavior of the population and the individual agent, which trades-off its own good with the interest of the overall system [1]. The analysis of such models seeks to find equilibrium configurations by exploiting the large number of agents involved in the game.

\section{MODELING OF ENERGY SYSTEMS}

\subsection{Plug-in Electric Vehicles}

We first investigate the problem of coordinating the charging schedule of a large population of PEVs, introduced in [2] and extended in [3]. For each $\mathrm{PEV} i \in \mathbb{Z}[1, N]$, we consider the discrete-time linear dynamics

$$
x_{t+1}^{i}=x_{t}^{i}+b_{i} u_{t}^{i}
$$

in the interval $\mathbb{Z}[0, T]$, where $x_{t}^{i} \in[0,1]$ and $u_{t}^{i} \in[0,1]$ are the state of charge and the charging control input at time $t$; the parameter $b^{i}>0$ represents the charging efficiency.

\footnotetext{
1 The authors are with the Automatic Control Laboratory, ETH Zurich, Switzerland. E-mail addresses: \{grammatico, gentileb, parisef, lygeros\}@control.ee.ethz.ch

Research partially supported by the European Commission under project DYMASOS (FP7-ICT 611281).
}

Each PEV $i$ needs to have its battery charged by the amount $\gamma_{i} \in[0,1]$ during the charge interval $\mathbb{Z}[0, T]$; such requirement can be formulated as the charging constraint $\sum_{t=0}^{T-1} u_{t}^{i}=\mathbf{1}^{\top} \boldsymbol{u}^{i}=\gamma_{i}$. The interest of each vehicle is to minimize its charging cost $\sum_{t=0}^{T-1} p_{t}(\cdot) u_{t}^{i}=\boldsymbol{p}(\cdot)^{\top} \boldsymbol{u}^{i}$, where $\boldsymbol{p}(\cdot)^{\top}=\left(p_{0}(\cdot), \ldots, p_{T-1}(\cdot)\right)^{\top}$ is the electricity price function at different time instants. We consider a dynamic pricing, where the price of electricity at time $t$ depends on the overall demand at time $t$; the overall demand is the sum of the inflexible demand and the aggregate PEV demand. The price is here modeled to be the affine function $\boldsymbol{p}(\boldsymbol{z}):=2(a \boldsymbol{z}+\boldsymbol{c})$, where $\boldsymbol{z}$ and $\boldsymbol{c} \geq \mathbf{0}$ are the PEV and non-PEV demands, while the parameter $a>0$ represents the inverse of the price elasticity. Since such linear cost leads to optimal solutions which are discontinuous with respect to the aggregate demand $\boldsymbol{z}$, following [2], [3] we introduce a quadratic relaxation term.

Given the average PEV demand $\boldsymbol{z}=\left(z_{0}, \ldots, z_{T-1}\right)$, the optimal charging control $\boldsymbol{u}^{i \star}$ for vehicle $i \in \mathbb{Z}[1, N]$ is

$$
\begin{aligned}
\boldsymbol{u}^{i \star}(\boldsymbol{z}):= & \arg \min _{\boldsymbol{u} \in \mathbb{R}^{T}} \delta\|\boldsymbol{u}-\boldsymbol{z}\|^{2}+2(a \boldsymbol{z}+\boldsymbol{c})^{\top} \boldsymbol{u} \\
& \text { subject to: } \mathbf{0} \leq \boldsymbol{u} \leq \boldsymbol{U}^{i}, \mathbf{1}^{\top} \boldsymbol{u}=\gamma_{i},
\end{aligned}
$$

where $\delta>0$ should be chosen small to approximate the original linear cost $2(a \boldsymbol{z}+\boldsymbol{c})^{\top} \boldsymbol{u}^{i}[3$, Section V].

\subsection{Thermostatically Controlled Loads}

As a second example of large-scale energy system we describe here a population of TCLs. By considering the discrete-time counterpart of [4], in [5] we model the switching dynamics of each TCL $i \in \mathbb{Z}[1, N]$ as

$$
x_{t+1}^{i}=\left\{\begin{array}{ll}
a_{i}\left(x_{t}^{i}-x_{\mathrm{OFF}}^{i}\right)+x_{\mathrm{OFF}}^{i} & \text { if } \bar{u}_{t}^{i}=0 \\
a_{i}\left(x_{t}^{i}-x_{\mathrm{ON}}^{i}\right)+x_{\mathrm{ON}}^{i} & \text { if } \bar{u}_{t}^{i}=1
\end{array},\right.
$$

where $x_{t}^{i} \in \mathbb{R}$ is the temperature deviation from the setpoint 0 at time $t, \bar{u}_{t}^{i} \in\{0,1\}$ represents the ON/OFF reference value of the TCL, $a_{i} \in(0,1)$ denotes the heating rate, $x_{\mathrm{OFF}}^{i}<0<x_{\mathrm{ON}}^{i}$ are the steady state temperature deviations. To make the problem treatable, we convexify the control inputs by allowing $u_{t}^{i}$ in $[0,1]$ as in [4].

Define $\boldsymbol{z}=\frac{1}{N} \sum_{i=1}^{N}\left(\boldsymbol{x}^{i} ; \boldsymbol{u}^{i}\right)$ as the vector containing the average temperature and the average power consumptions. 
Given a fixed $\boldsymbol{z}$, each TCL $i \in \mathbb{Z}[1, N]$ selects the input $\boldsymbol{u}=\left\{u_{t}\right\}_{t=0}^{T-1}$ to minimize its cost function $J_{i}$ defined as

$$
\begin{array}{r}
J_{i}(\boldsymbol{x}, \boldsymbol{u}, \boldsymbol{z}):=\sum_{t=0}^{T-1} q_{i}^{2}\left|x_{t+1}-\bar{x}_{t+1}^{i}\right|^{2}+r_{i}^{2}\left|u_{t}-\bar{u}_{t}^{i}\right|^{2}+ \\
2\left(C_{t}\left[\begin{array}{c}
z_{t}^{x} \\
z_{t}^{u}-\frac{1}{2}
\end{array}\right]+c_{t}\right)^{\top}\left(\begin{array}{c}
x_{t+1} \\
u_{t}-\frac{1}{2}
\end{array}\right),
\end{array}
$$

where $z_{t}:=\left[z_{t}^{x}, z_{t}^{u}\right]^{\top}$; the decision variables are $\boldsymbol{x}=$ $\left(x_{1}, \ldots, x_{T}\right)^{\top}$ and $\boldsymbol{u}=\left(u_{0}, u_{1}, \ldots, u_{T-1}\right)^{\top}$. The quantities $\overline{\boldsymbol{x}}^{i}=\left(\bar{x}_{1}^{i}, \ldots, \bar{x}_{T}^{i}\right)^{\top} \in \mathbb{R}^{T}$ and $\overline{\boldsymbol{u}}^{i}=\left(\bar{u}_{0}^{i}, \ldots, \bar{u}_{T-1}^{i}\right)^{\top} \in$ $\{0,1\}^{T}$ are the desired temperatures and ON/OFF inputs in absence of external incentives; the remaining parameters satisfy $q_{i}, r_{i}>0, C_{t} \in \mathbb{R}_{\geq 0}^{2 \times 2}$ and $c_{t} \in \mathbb{R}$ for all $t$.

The cost $J_{i}$ in (3) consists of two main contributions. The term $q_{i}^{2}\left|x_{t+1}-\bar{x}_{t+1}^{i}\right|^{2}+r_{i}^{2}\left|u_{t}-\bar{u}_{t}^{i}\right|^{2}$ penalizes the deviation from the typical ON/OFF evolution $\left\{\left(\bar{x}_{t+1}^{i} ; \bar{u}_{t}^{i}\right)\right\}_{t=0}^{T-1}$. The term $2\left(C_{t} z_{t}+c_{t}\right)^{\top}\left(\begin{array}{c}x_{t+1} \\ u_{t}\end{array}\right)$ couples every single TCL to the overall population through the vector $\boldsymbol{z}$ and represents an incentive for the TCL to participate in grid services. Specifically, each TCL has an incentive to turn OFF during a power demand peak and to turn ON during a power demand valley (analogously for the temperature).

The optimal response of TCL $i$ with respect to a reference signal from the utility is given by the mapping

$$
\begin{array}{cl}
\left(\boldsymbol{x}^{i \star}(\boldsymbol{z}) ; \boldsymbol{u}^{i \star}(\boldsymbol{z})\right):=\underset{\boldsymbol{x}, \boldsymbol{u}}{\arg \min } & J_{i}(\boldsymbol{x}, \boldsymbol{u}, \boldsymbol{z}) \\
\text { subject to: } & \boldsymbol{x} \in \mathcal{X}^{i} \\
& \boldsymbol{u} \in \mathcal{U}^{i} \\
& (\boldsymbol{x}, \boldsymbol{u}) \in \mathcal{D}^{i},
\end{array}
$$

where $\mathcal{X}^{i}:=\mathcal{X}_{1}^{i} \times \cdots \times \mathcal{X}_{T}^{i} \subseteq \mathbb{R}^{T}$ denotes convex state constraints, for instance in terms of comfort boundaries $\mathcal{X}_{t}^{i}:=$ $\left[\underline{x}_{t}^{i}, \bar{x}_{t}^{i}\right], \mathcal{U}^{i}:=[0,1]^{T} \subset \mathbb{R}^{T}$ is the set of convexified control inputs, and $\mathcal{D}^{i}:=\left\{\left(\left\{x_{t}\right\}_{t=1}^{T},\left\{u_{t}\right\}_{t=0}^{T-1}\right) \in \mathbb{R}^{2 T} \mid(2)\right.$ holds $\}$.

\section{NUMERICAL ANALYSIS}

The model (1) of a population of PEVs and the model (4) of a population of TCLs both result in a convex optimization problem, with a quadratic cost function whose linear part depends on the average behavior $\boldsymbol{z}$. The two models aim to describe large-scale populations, hence their analysis cannot be performed via standard game theoretical techniques. However, using operator theory concepts it is shown in [6] that the two systems possess an $\varepsilon$-Nash equilibrium configuration, with $\varepsilon$ going linearly to zero as the population size $N$ grows. Moreover, [6] proposes an iterative algorithm that steers the population towards such equilibrium configuration. In Figure 1 and 2 we report the average population behaviors at the equilibrium configurations found via the algorithmic procedure. Both figures reveal that the coupling between the individual's interest and the population's average behavior is beneficial for the overall system. In fact Figure 1 shows that the coordination of the charging schedule of the PEVs results in filling the overnight gap of the non-PEV demand; such aggregate behavior ensures that large excursions in demand are not likely to happen. Similarly, Figure 2 indicates that the TCLs on average may fail to follow their ON/OFF reference signals to avoid aggregate demand peaks or valleys, thus improving system stability (for instance, in terms of frequency synchronization [4]).

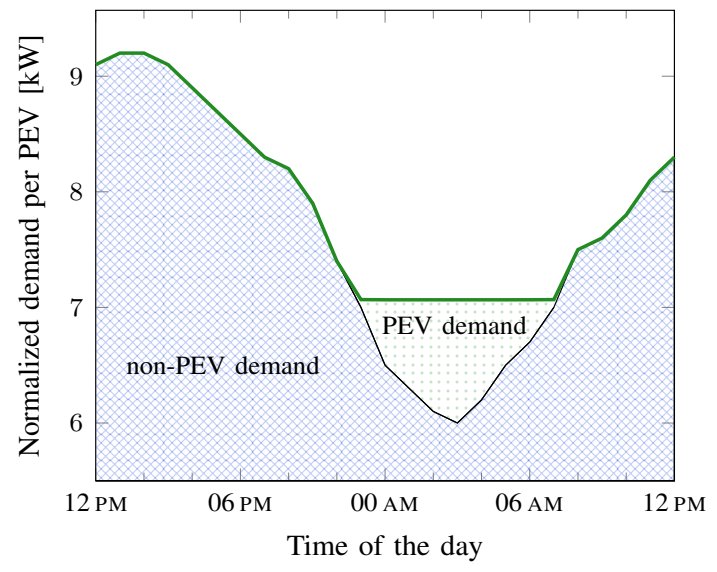

Fig. 1. The average PEV energy demand at the equilibrium fills the overnight valley of the non-PEV demand.

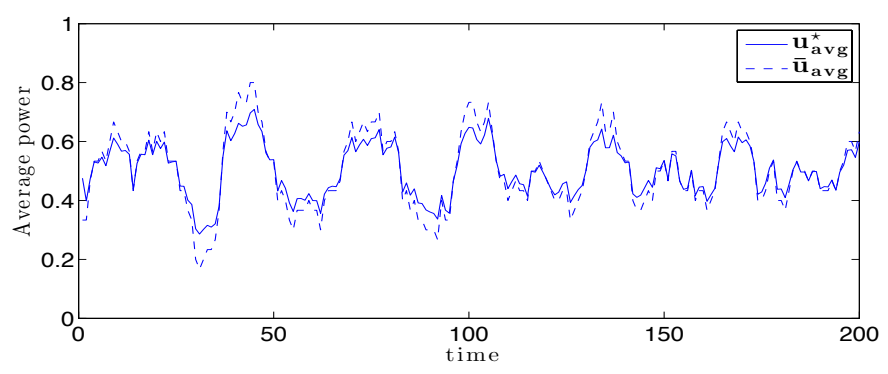

Fig. 2. The average TCL power consumption (solid) and the average reference TCL consumption (dashed). The average consumption smooths the demand peaks.

\section{REFERENCES}

[1] M. Huang, P. Caines, and R. Malhamé, "Largepopulation cost-coupled LQG problems with nonuniform agents: Individual-mass behavior and decentralized $\varepsilon$-Nash equilibria," IEEE Trans. on Automatic Control, vol. 52, no. 9, pp. 1560-1571, 2007.

[2] Z. Ma, D. Callaway, and I. Hiskens, "Decentralized charging control of large populations of plug-in electric vehicles," IEEE Trans. on Control Systems Technology, vol. 21, no. 1, pp. 67-78, 2013.

[3] F. Parise, M. Colombino, S. Grammatico, and J. Lygeros, "Mean field constrained charging policy for large populations of plug-in electric vehicles," in Proc. of the IEEE Conference on Decision and Control, Los Angeles, California, USA, 2014.

[4] F. Bagagiolo and D. Bauso, "Mean-field games and dynamic demand management in power grids," Dynamic Games and Applications, vol. 4, no. 2, pp. 155-176, 2014.

[5] S. Grammatico, B. Gentile, F. Parise, and J. Lygeros, "A mean field control approach for demand side management of large populations of thermostatically controlled loads," in European Control Conference (submitted), June 2015.

[6] S. Grammatico, F. Parise, M. Colombino, and J. Lygeros, "Decentralized convergence to Nash equilibria in constrained mean field control," submitted to IEEE Trans. on Automatic Control., 2014. 


\title{
A Modelica-based Modeling and Simulation Framework for Large-scale Cyber-physical Systems of Systems
}

\author{
S. Nazari*, C. Sonntag**, S. Engell* \\ * Process Dynamics and Operations Group, Department of Biochemical and Chemical Engineering, Technische Universität \\ Dortmund, Dortmund, Germany (email: shaghayegh.nazari@bci.tu-dortmund.de, sebastian.engell@,bci.tu-dortmund.de) \\ **euTeXoo GmbH, Dortmund, Germany (e-mail: christian@eutexoo.de)
}

\begin{abstract}
This paper proposes a modeling, simulation, and validation framework for large-scale technical Cyber-physical Systems of Systems with distributed management architectures. The goal of the framework, which is based on the freely available Modelica language for object-oriented heterogeneous modeling, is to reduce the (currently very large) engineering effort for distributed management architectures while improving the quality of the designed system. The framework allows engineers to equip the management system with standardized interfaces, which will significantly increase re-usability of newly developed and legacy models. Furthermore, the overall CPSoS model, including the communication architecture, is generated automatically, making tedious manual implementation superfluous while reducing the potential of modeling errors, and the standardized, generic interfaces to which model components must connect to will provide a straightforward avenue for the deployment of management solutions to industrial hardware systems.
\end{abstract}

Keywords: Modeling, Simulation, Distributed Management, Modelica, Cyber-physical Systems of Systems, Complex Systems, Distributed Management, Distributed Control

\section{INTRODUCTION}

Modern large-scale, complex technical systems, such as electric power networks, railway systems, or large industrial production sites, consist of many, partly autonomous, physical and cyber-components with complex interactions. The automation/management architecture of such Cyberphysical Systems of Systems $(C P S o S)^{l}$ reflects this complexity in that it is highly distributed and consists of several interacting hierarchical layers. In particular, many of the components are controlled locally (e.g. production plants in a chemical production site) while global coordination is needed to drive the complete CPSoS towards the fulfilment of global performance and safety criteria (such as coordination of the steam and energy generation in chemical production sites).

Model-based engineering is essential to guide and validate the design of these systems, to engineer and test automation architectures, to determine performance measures, to design and validate safety measures, and to train operators. Consequently, the use of model-based technologies is now well established in industrial practice. While powerful modeling and simulation languages and tools are available, the lack of frameworks that are tailored to CPSoS with distributed management architectures poses severe limitations. The complex automation and communication architectures of management systems, including their connections to models of the technical system, must be implemented manually. This:

1. Leads to a large engineering effort (and, consequently, financial) overhead,

2. May introduce additional errors into the model,

\footnotetext{
${ }^{1}$ See http://www.cpsos.eu
}

3. Makes it very difficult to re-use existing model components, and

4. Complicates the testing of an existing management scheme on different technical systems (or even different models of the same technical system) since manual implementations usually focus on a specific system model due to the lack of pre-defined standard interfaces for the automation system.

In this contribution, a new modeling and simulation framework for CPSoS with distributed management is presented that is currently developed within the European research and innovation project DYMASOS ${ }^{2}$ and that provides automated support for the model-based design and validation of CPSoS under distributed management.

\section{THE MODELING AND SIMULATION FRAMEWORK}

The modeling and simulation framework will provide a structured approach to the implementation of the large-scale CPSoS models. It is based on the freely available, objectoriented Modelica language which was specifically designed for heterogeneous systems modeling, see e.g. [1, 2], which is a very rich language for equation-based modeling that has achieved wide adoption in a variety of industrial branches.

The framework represents each subsystem using one of four model components, as shown in Fig. 1. The management architecture consists of communicating local coordination algorithms and an (optional) global coordination algorithm.

2 DYMASOS: Dynamic Management of Physically Coupled Systems of Systems. Supported by the European Commission under the FP7-ICT Programme (contract no. 611281), http://www.dymasos.eu 
The local coordination algorithms optionally have access to a design model to determine a locally optimal subsystem operation with respect to a local problem formulation. The local coordination algorithms perform real-time control of the physical CPSoS that is represented by a set of validation models, which are detailed models that accurately represent the real physical subsystems and their interconnections.

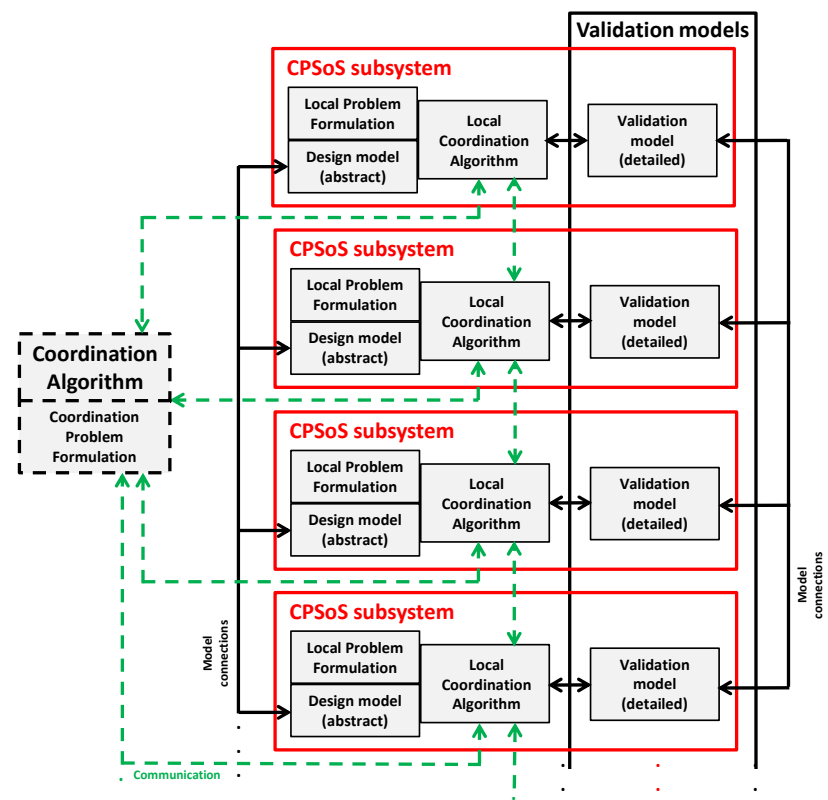

Fig. 1: Structure of a CPSoS model in the modelling and simulation framework.

The framework provides standardized interfaces for the connection of physical process model components (i.e. design and validation models) as well as automation components (i.e. local and global coordinators). In order to implement the interconnections of the model structure shown in Fig. 1, four interfaces must be specified, namely the interfaces between global and local coordination algorithms, between local coordination algorithms, between local coordination algorithms and validation models, and between validation model components. Here, the interface between two validation models is implemented using the standard Modelica interfaces while dedicated, generic interface definitions are developed for the connections within the distributed management system, and for the connection of the management system to the validation models. All interfaces support time-discrete as well as event-driven communication. In addition to these two types of communication, the interface between the validation models and the local coordinators also supports continuous communication. This option is considered for cases where the local coordinator contains a simple low-level controller, e.g. a PI controller.

The framework will enable a fully automatic generation of interconnections and of the communication structure of the CPSoS in Modelica. The information on the communication structure and the parameterization of the interfaces (i.e. numbers, names, types and dimensionalities of the variables that can be transmitted via the interfaces) are provided to the model generator via an XML configuration file. Based on this information and repositories of white-box and black-box model and automation components (the latter of which can be connected by co-simulation via the Functional Mockup Interface/FMI), the automatic model generator will first verify the structural correctness of the interconnections, generate the required communication structure of the CPSoS model, and integrate all model components into a consistent model of the complete CPSoS. The workflow of the generation process of the automated CPSoS model is shown in Fig 2. The interaction of all the model components takes place via a Modelica-based model management engine that allows management components to communicate (e.g. in the beginning of the simulation coordinators can define their parameters such as execution delay, sampling time, etc., via the interfaces). During the simulation, they can use the interfaces to send data requests to other coordinators or retrieve information about the model structure.

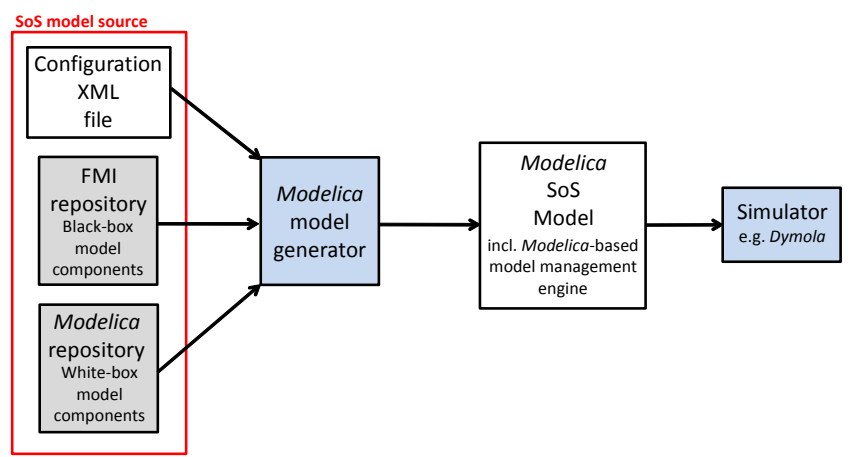

Fig. 2: Workflow for the generation and execution of automated CPSoS models.

This plug-and-play approach makes model development much more efficient and less error-prone, makes model components easily re-usable in different scenarios, allows to easily test and validate different management algorithms on an (existing) model of an industrial CPSoS, and simplifies the direct deployment of new management architectures to the automation hardware of the real-world CPSoS due to the availability of generic interfaces that can be directly connected to hardware systems.

The framework will be illustrated on an industrial application example, the CPSoS model of petrochemical production complex where different plants are interconnected by networks of mass and energy.

\section{REFERENCES}

[1] P. Fritzson and P. Bunus: Modelica - A General ObjectOriented Language for Continuous and Discrete-Event System Modeling and Simulation. In Proc. Annual Simulation Symposium, 2002, 365-380.

[2] Modelica and the Modelica Association, http://www.modelica.org. 


\title{
LEVELS OF DETAIL AND APPROPRIATE MODEL TYPES FOR VIRTUAL COMMISSIONING IN MANUFACTURING ENGINEERING
}

\author{
P. Puntel Schmidt, A. Fay \\ Helmut-Schmidt-University Hamburg, Germany
}

\begin{abstract}
Corresponding author: P. Puntel Schmidt, Helmut-Schmidt-University, Department of Automation
22065 Hamburg, Holstenhofweg 85, Germany, philipp. puntelschmidt @ hsu-hh. de
\end{abstract}

\begin{abstract}
Introduction. Virtual commissioning (VC) is used to test control code deployed on Programmable Logical Controllers. Simulation models of a plant are the basis for simulation approaches covering virtual commissioning. However, it is often unclear what granularity these simulation models should have and how standardized levels of detail and particular model types should be defined. The approach presented herein identifies appropriate levels of detail and model types based on a hierarchical perception of automated manufacturing plants. The aim of this contribution is to systematize modelling approaches, especially the automatic generation of manufacturing plants' models.
\end{abstract}

Content. Manufacturing plants can be simulated at any level of detail demanded by a customer, leaving a wide range of possible simulation models to use during VC. The granularity of a simulation (what elements of a plant are modelled at which level of detail by which model types) can be seen as a key aspect and takes a high impact on the complexity of the simulation model: the more variations of specific elements exist, the more difficult it is to realise a simulation model. This applies particularly to an adaptive level of detail, where by principle $n$ elements can be described by $m$ models. A clear and systematic solution for this problem is missing at the moment. To approach a systematic definition of sensible levels of detail for simulation models used for VC, the appropriate granularity of simulation models should be clarified by means of appropriate model types. Basically, automated plants are structured hierarchically, describing the structure and arrangement of elements like sensors, actors and others. The data model of the Digital Factory [1] describes this structure (as is or projected to be on the field). Pursuing a hierarchical perception of the data model and its included structure, the specific plant can be divided into different hierarchy levels, one being subsystems. Subsystems describe function groups that can be found in nearly every equipment. From a mechatronic point of view regarding to [2], subsystems can be described "[...] as concurrent integration of various mechanical modules [...]". Modules comprise of components that describe the typical elements of a mechatronic system: the basic system, defined as a structure (either mechanical, electromechanical, hydraulic or pneumatic or combination of these), sensors and actors. Two fundamental model types can be identified: A simulation of a whole module as an integrated mechatronic model of the mechatronic domains mechanic and electronic and/or models of components, granular fine decomposed, modelling individual components of the mechatronic system. Besides the two identified basic model types and the appropriate granularity of simulation models, additional models types (extending the predefined basic model types) should be defined to harmonize possible VC approaches. The identification and definition of these model types should thereby be guided by general design principles (exemplary described by [3]), and can be identified as follows:

- Dead time models on module level: Simple simulations models based on time-based behaviour of modules

- Simple device- and kinematic-models on component level: Simple simulations models based on timebased behaviour of a module that comprises components

- Device- and kinetic-models on component level: Simulation models with physical behaviour but restricted movement of goods

- Complex device- and kinetic-models on component level: Simulation models with physical behaviour and free movement of goods in space

These model types are included into a pattern that is inspired by traffic flow simulations and that describes 4 different levels of detail: macroscopic, mesoscopic not dynamical, mesoscopic dynamical and microscopic level of detail. The definition of appropriate levels of detail and corresponding model types made in this contribution contributes to an automatic generation of simulation models of automated plants and VC approaches overall.

Summary. The defined levels of detail and their appropriate model types already showed their appropriateness at first applications. Modelling and validation effort could be reduced while simulation results were not influenced. The systematic definition of few but applicable levels of detail and their appropriate models benefits approaches since model size and intricacy could be reduced to an appropriate level. Future improvements should cover industrial applications, especially with deployment of a physical/dynamical 3D-simulation. Current research of the authors is focused on a method to systematically identify the appropriate level of detail needed for the respective application or use case.

[1] VDI4499 (2008): Digital Factory. VDI-Verlag, Düsseldorf.

[2] VDI2206 (2004): Design methodology for mechatronic systems. VDI-Verlag, Düsseldorf

[3] Haberfellner, R., de Weck, O., Fricke, E., Vössner, S. (2012): Systems Engineering. Grundlagen und Anwendungen. Orell Füssli Verlag AG, Zürich. 


\title{
Equipment Interconnection Models in Discrete Manufacturing
}

\author{
Sten Grüner* Peter Weber ${ }^{* *}$ Constantin Wagner* \\ Ulrich Epple* \\ * Chair of Process Control Engineering, RWTH Aachen University, \\ Aachen, Germany (e-mail: \\ \{s.gruener, c.wagner,epple\}@plt.rwth-aachen.de). \\ ** ABB Corporate Research, Ladenburg, Germany (e-mail: \\ peter.weber@de.abb.com)
}

\begin{abstract}
Information about product flow paths is important for tasks in factory automation. The common CAD/CAE models do not explicitly provide information about product paths. A model for product flows in discrete manufacturing is briefly discussed in this paper.
\end{abstract}

Keywords: Models, Product flow, Manufacturing systems

\section{INTRODUCTION}

Knowledge about product flows (these are the currently available and possible product paths) inside a plant is valuable for many tasks in automation engineering e.g. generation of interlocks. The most commonly known model type for discrete manufacturing systems is the CAD/CAE (CAx) model. These models are very detailed and can be used for various tasks ranging from a manufacturing plant design to a physically-correct plant simulation. However, when product flows are the main focal point, the CAx model reach its limits: Addressing the question whether a product will (or will not) pass a certain equipment unit if placed on a certain conveyor is non-trivial and requires a resource-intensive simulation of the entire plant model. The second disadvantage of CAx models is their late availability: the complete 3D models are only available when the utilized equipment has been identified or, in some cases, only shortly before the plant's construction. The same goals can be achieved with less modelling and simulation effort by using lower fidelity models that concentrate on a specific plant aspect.

A specialized model aiming to close the discussed gap called Product Flow Exchange (PFX) was presented in Grüner et al. (2014) (the original name Product Flow Diagram was abandoned due to a name collision). PFX is aimed take on a same role in factory automation as Pipe and Instrumentation Diagrams (P\&ID) in process industry. In this discussion paper we present PFX and illustrate its advantages with the help of an example.

\section{MODEL DESCRIPTION}

The model itself and its machine-readable format discussed in Section 3 are more complex than the graph projection presented in this section and includes e.g. possibilities of hierarchical element composition. The simplified graph interpretation, however, is sufficient to demonstrate the advantages of equipment interconnection models over
CAx data. A comprehensive formal model of PFX including device classes, ports and flow allowance functions can be easily adopted from the product flow model for P\&ID as presented in a paper by Quirós et al. (2009).

A manufacturing plant $P$ that is modelled in PFX can be simplistically considered as an undirected attributed graph with partitioned sets of vertices and edges, i.e.

$$
P=\left(F_{V}, C_{V}, P_{E}, H_{E}, C_{E}, S_{E}, a t t r\right)
$$

where the sets of vertices and edges are given by $V:=$ $F_{V} \bullet C_{V}$ and $E:=P_{E} \bullet H_{E} \bullet C_{E} \bullet S_{E}$, respectively. For an undirected graph we require $(u, v) \in E \Leftrightarrow(v, u) \in E$. The set of vertices is partitioned into the set of plant functions $F_{V}$ and the set of Process Control Engineering Requests (PCE-requests) $C_{V}$ as defined in IEC 62424 by IEC (2009). The set of edges is composed of product connections $P_{E} \subseteq F_{V} \times F_{V}$, handling connections $H_{E} \subseteq F_{V} \times F_{V}$, control connections $C_{E} \subseteq\left(F_{V} \times C_{V}\right) \cup\left(C_{V} \times F_{V}\right)$ and signal connections $S_{E} \subseteq C_{V} \times C_{V}$. An attribute function attr $:(V \cup E) \rightarrow 2^{\left(\Sigma^{+} \times \Sigma^{+}\right)}$is given that maps an edge or a vertex to a set of key-value string pairs. $\Sigma^{+}$stands for the set of finite, non-empty strings over an alphabet $\Sigma$.

Plant functions $F_{V}$ represent the plant equipment's functions regarding the product flow e.g. transport, switches, junctions etc. or changing of product properties e.g. a work station for drilling. PCE-requests $C_{V}$ are used to show the sensing and actuating capabilities of plant functions and to map them to signals e.g. a conveyor may be equipped with an I/O input for its belt drive or light-barrier that is able to sense the presence of a product.

The edges have no physical counterpart and model the logical connections between objects. Product connections are used to model product flow through the plant during its operation. Two transporting functions (representing conveyors) can e.g. be connected by a product connection to denote the falling of a product from one conveyor onto another. Handling connections are distinguished types of product connections that are not active by default and need to be activated explicitly. A prominent example 
of such product interconnections are robot pickers that can transport products within a certain range. Control connections assign plant functions to PCE-requests and, finally, control connections interconnect PCE-requests to denote control loops and other functions.

The attribute function is used to describe further properties of functions and PCE-requests e.g. the conveyor's length and signal name. Moreover, attributes can be placed on edges and are used, for example, to denote the position of a sensor, the operating range of a picker or the primary direction of a conveyor.

An advantage of graph representation of a PFX models is the possibility of algorithmic analysis. For example, a task of product flow exploration can be solved by using a standard graph algorithms e.g. a breadth first search.

\section{A MACHINE-READABLE FORMAT FOR PFX}

PFX is implemented as a role class library for a data Computer Aided Engineering Exchange (CAEX) format that is standardized in IEC (2009). CAEX has been developed to save topology information by means of hierarchically organized objects. Objects are instantiated from object classes that are also defined in CAEX. Classes have attributed interfaces, which are in turn connected by links.

The current library includes basic transporting functions (conveyors and conveyor segments), handling elements (pickers), switches, junctions, different sensor and work station types. Role classes for PCE-Requests and control functions like closed-loop controllers are also provided.

The implementation of PFX as a CAEX library has several advantages. Firstly, any PFX diagram can be saved in the canonical CAEX XML exchange format. Secondly, the new diagram type can be directly supported by the available tools and embedded into AutomationML profiles (see Drath et al. (2008)). Thirdly, the coexistence of different CAEX libraries e.g. PandIX P\&ID models (see Schüller and Epple (2012)) with PFX can be realized seamlessly for modelling hybrid plants such as a filling station. Finally, due to the inheritance mechanisms of CAEX, the provided library is extendible to cover further equipment classes.

\section{EXAMPLE OF PFX MODELLING}

The symbols from VDI 2860 by VDI (1990), a German mechanical engineering guideline, are used to denote PFX models visually. These symbols are known to German mechanical engineers. This fact contributes to the intuitive readability of PFX. Unfortunately, there is no English translation of the guideline that we currently know of.

Figure 1 shows a 3D model of a sorting facility consisting of conveyors and a picker. Figure 2 shows the PFX of the same plant. The following advantages can be seen immediately: The direction of conveyors is indicated graphically, conveyors $C_{1}$ and $C_{3}$ are subdivided into segments to define the operating range of the gripper $H_{1}$ (handling connections are shown as dashed lines), products fall from conveyor $C_{1}$ to $C_{2}$ (production connections are shown as solid lines). Furthermore, PCE-requests for $C_{1}$ 's belt (N1) and a light barrier $(G 1)$ are indicated. The requests for the remaining functions are amended for legibility reasons.

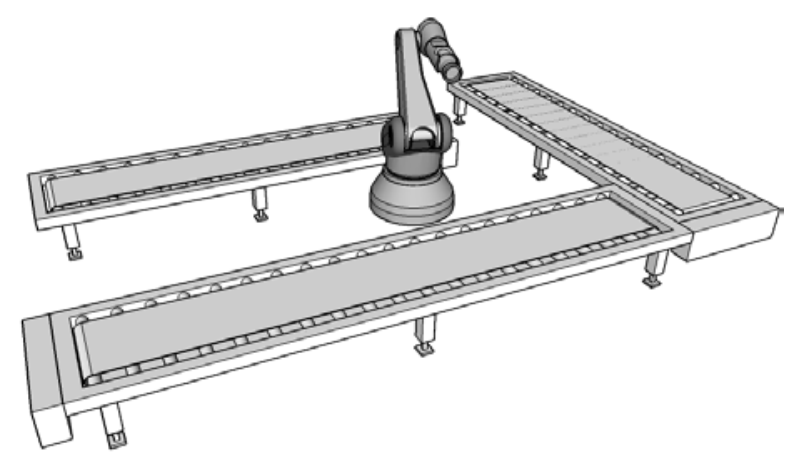

Fig. 1. A CAx model of a simple sorting facility.

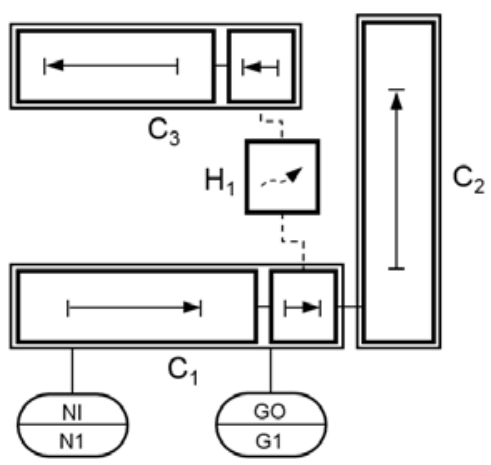

Fig. 2. A corresponding PFX model.

\section{CONCLUSION}

An equipment interconnection model for tracing product flow in manufacturing plants was briefly presented. The model can be mapped to a graph in order to allow its algorithmic analysis. Additionally, a machine readable format and a graphical representation are provided.

\section{REFERENCES}

Drath, R., Lüder, A., Peschke, J., and Hundt, L. (2008). AutomationML - the glue for seamless automation engineering. In IEEE International Conference on Emerging Technologies and Factory Automation, 2008., 616-623.

Grüner, S., Weber, P., and Epple, U. (2014). A Model for Discrete Product Flows in Manufacturing Plants. In ETFA 2014: IEEE 19th International Conference on Emerging Technologies and Factory Automation.

IEC (2009). IEC 62424: Specification for representation of process control engineering requests in P\&I Diagrams and for data exchange between P\&ID tools and PCECAE. International Electrotechnical Commission.

Quirós, G., Jorewitz, R., and Epple, U. (2009). Modelbased safety monitoring of product flow paths. In MATHMOD Vienna 09: 6th Vienna Conference on Mathematical Modelling February 11 - 13, 2009, Austria, ARGESIM report; 35, 251-260.

Schüller, A. and Epple, U. (2012). PandIX - Exchanging P\&I diagram model data. In ETFA 2012: IEEE 17th International Conference on Emerging Technologies and Factory Automation.

VDI (1990). VDI 2860: Handhabungsfunktionen, Handhabungseinrichtungen; Begriffe, Definitionen, Symbole (Handling Functions, Handling Facilities: Terms, Definitions, Symbols). Verein Deutscher Ingenieure (Association of German Engineers). 


\title{
Modeling and Transformation of systems of systems using Linked Data
}

\author{
Markus Graube, Leon Urbas \\ Chair for Control Systems Engineering \\ Technische Universität Dresden \\ Dresden, Germany \\ markus.graube@tu-dresden.de,leon.urbas@tu-dresden.de
}

\begin{abstract}
A system of systems can only works successfully if the single systems can understand each other. The necessary syntactic compatibility can be achieved by a common data model which has to be flexible in order to reflect changes in the world. Linked Data is a set of suitable technologies and best practises for this task. Furthermore Linked Data has a semantic information model and SPARQL as a powerful query language.

This article presents an approach to use SPARQL in order to transform information models into each other staying in the Linked Data world. Thus, a semantic interoperability between systems can be achieved. A prototypical implementation shows the feasibility of the approach.
\end{abstract}

Keywords: Semantic networks, information systems, information integration, Models, Graph theoretic models, Graph theory, Graphs, Transformations

\section{INTRODUCTION}

Automation is characterized by a large set of vendor, application and domain specific systems with partial models which have to be integrated during engineering and operation. The transformation of models between different systems is challenging. Reasons are proprietary interfaces, incompatible data models and lack of synchronization mechanisms. The attempts addressing these problems which make use of complex world models (eg ISO 10303), interface models (eg ISO 15926) or information models based on a set of common core models (eg CAEX). However, they were satisfactory only in a few areas. Lack of extensibility, high randomness in the actual implementation or lack of interoperability with existing tools always leads to high initial investment while providing low direct benefit.

\section{LINKED DATA AS MODELING FRAMEWORK}

The Semantic Web offers a set of proven tools and methods for these challenges. Linked Data (LD), as a back-end for the Semantic Web, provides a simple but easily expandable stack ready to model complex information structures in a consistent and semantic format. It offers a secure linking of different information sources and thus suitable for industrial use (see Graube, M. et. al. (2012)).

The data model RDF (Resource Description Framework) allows the resolution of ambiguities in a network of distributed information. Information is described as a set of semantic statements. They consist of subject, predicate and object and thus are also called triples:

<subject> <predicate> <object> .

* The work leading to this publication was funded by the German federal ministry of education and research under grant number 01IS14006C.
Every part of a statement is identified by a URI allowing to be referenced in other statements and forming a network of statements. This can be interpreted as mathematical graph. These networks can be further structured by using Named Graphs which contain a set of statements and are themselves also identified by an URI. This way named graphs form a fourth element in the statement tuple.

Ontologies define concepts used in the semantic network also in RDF which easily allows computer processing. $S P A R Q L^{1} \quad$ (SPARQL Protocol and Query Language) is the most popular query language in the LD universe. It provides a simple interface to triplestores ${ }^{2}$ for reading and writing data. SPARQL is similar to SQL and supports all main concepts of LD like triples and named graphs.

A major benefit of LD over other semantic information systems is the amount of supporting tools and its big community. LD is intensively pushed forwards both by research projects, academic institutions as well as industrial companies. LD allows the simple creation of decentral information systems using standard technologies of the Internet like HTTP allowing a wide reuse of existing frameworks and libraries. The integration of LD does not imply the exchange of existing specialized tools (CAE tools like Comos). These legacy system fulfill a specific need in a good way and will just be extended by some adapters which synchronize relevant information from the tool with the information model in the LD cloud. The

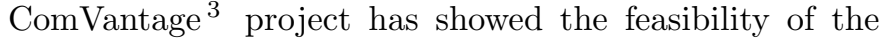
Linked Data approach for industrial applications (Münch, T. et. al. (2013)) with adapters for industrial systems like Comos, PCS 7 and OPC.

\footnotetext{
1 http://www.w3.org/TR/sparql11-overview/

2 Specialised databases for Linked Data like Virtuoso (http:// virtuoso.openlinksw.com/dataspace/doc/dav/wiki/Main/)

3 http://www . comvantage.eu
} 

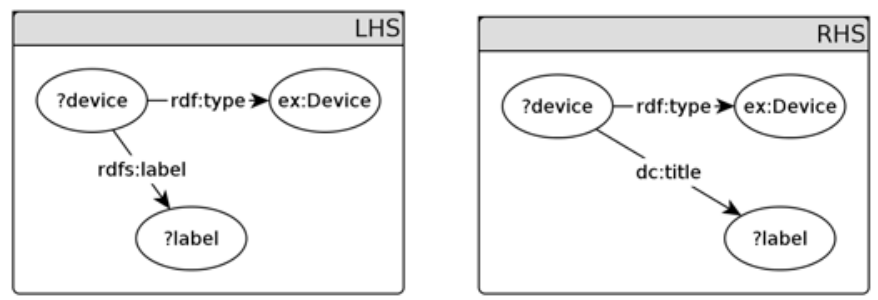

Fig. 1. Graph Transformation Rule to replace the predicate rdfs:label with dc:title

\section{MODEL TRANSFORMATION IN LINKED DATA}

Systems usually use different information models tailored for specific tasks. An integration of systems requires them to understand the information from other systems both in a syntactic and a semantic way. Adapters are semantically lifting the data from the legacy systems to LD and thus already covering syntactic homogeneity. Semantic compatibility usually requires some kind of transformation. A good traceability and maintenance of transformations can be achieved when they are formally described. Graph grammars are very powerful transformation approaches (see Taentzer, G. et. al. (2005)). They are suitable for transforming LD leveraging the fact that LD can be interpreted as mathematical graph.

A model transformation consists of a set of transformation rules. They replace all matchings of a specified subgraph in the Left-Hand-Side (LHS) model by another subgraph and hence create a new Right-Hand-Side (RHS) model. The subsequent application of a set of rules can transforms one model into another. For example, the rule depicted in figure 1 searches all devices of type ex:Device and replaces all their label properties of type rdfs:label by labels of type dc:title (prefixes ex, dc, rdfs and rdf are omitted due to brevity reasons). The free variables? device and ?label connect LHS and RHS.

SPARQL provides a graph grammar engine completely integrated in the LD universe. Each transformation rule can be expressed as a single SPARQL query. Thus, the corresponding SPARQL rule for figure 1 is shown in listing 1 . The WHERE clause (lines 7-10) contains the LHS with all triples which have to be matched in order to apply the rule. The INSERT clause (lines 1-3) and DELETE clause (lines 4-6) represent the RHS of the rule. First one is for adding information and the latter one for deleting information. Thus, one can easily distinct between deleting rules and adding rules which allows a simple check for monotony of the graph grammar. Furthermore, Negative Assertion Conditions (NACs) can be expressed by filter operations in SPARQL. Value operations and assignments can be done via LET constructs in SPARQL.

Most graph transformation tools (AGG, Fujaba, Atom3, eMoflon, Progress) rely on type-based rules which is not necessary in LD. However, this constraint has advantages in some cases and can be implemented like following. Instantiations are common edges with the URI rdf:type as predicate allowing to define these as a simple filter (as ex:Device in the example above).

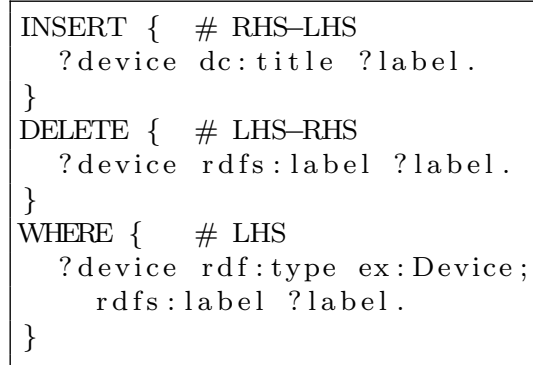

Listing 1. SPARQL representation of rule in figure 1

\section{IMPLEMENTATION}

The SPARQL graph transformation system can be implemented both as web services or on local machines. Apache Jena $^{4}$ is a popular open source Java framework for the Semantic Web and was successfully used for a prototypical implementation of the transformation engine. The system was successfully applied to the example of Königs (2005) which transforms UML class diagrams into SQL schemas and vice versa. This test cases showed the general feasibility of the approach.

The presented approach is used in recent projects for the automatic generating human-machine-interfaces, following the autoHMI concept from Obst, M. et. al. (2012), and for converting of engineering data between different customizings of CAE systems. First experiences show a good scalability and that the focus on one formal language for queries and transformations reduce the effort for familiarizing with the system for new users.

\section{CONCLUSION AND OUTLOOK}

Linked Data is a promising technology for integrating information across different systems while keeping them safe and secure relying on standard Internet technologies. The broad tool support makes the semantic lifting to LD from legacy system easy. The proposed transformation approach using SPARQL allows to stay in the LD world leveraging all its advantages. A prototypical implementation shows the feasibility while a suitable and user friendly front-end is still required for the creation, execution and tracing of SPARQL graph transformation rule sets.

\section{REFERENCES}

Graube, M. et. al. (2012). Linked data as integrating technology for industrial data. IJDST, 3(3), 40-52.

Königs, A. (2005). Model transformation with triple graph grammars. In Model Transformations in Practice Satellite Workshop of MODELS.

Münch, T. et. al. (2013). An innovative virtual enterprise approach to agile micro and SME-based collaboration networks. In Collaborative Systems for Reindustrialization, volume 408, 121-128. Springer Berlin Heidelberg.

Obst, M. et. al. (2012). Integriertes HMI-engineering. In Tagungsband Automation 2012, 227-230.

Taentzer, G. et. al. (2005). Model transformation by graph transformation: A comparative study. In Proc. Workshop Model Transformation in Practice.

\footnotetext{
4 http://jena.apache.org/
} 
MATHMOD 2015 Abstract Volume 


\title{
MATHMOD 2015 ABSTRACT VOLUME
}

\author{
MATHMOD Minisymposium \\ Mathematical Modeling for Nanoelectronic \\ Coupled Problems
}


MATHMOD 2015 Abstract Volume 


\title{
A Fractional Step Method for the Dynamic Electro-Thermal Modelling of Device Structures
}

\author{
David Duque * Sebastian Schöps* \\ * Institut für Theorie Elektromagnetischer Felder and Graduate School \\ of Computational Engineering, Schlossgartenstraße 8, Dolivostraße 15, \\ Darmstadt Germany (e-mail: \{duque,schoeps\}@gsc.tu-darmstadt.de)
}

\begin{abstract}
Device design in nano-electronics typically leads to large multi-physics problems in time-domain that are characterised by strong feedback coupling. The classical monolithic method results in huge linear systems that must be solved for all time steps. In this contribution a fractional time-stepping scheme is employed in the context of electro-thermal problems in nano-electronics that solves the system without sacrificing the convergence order in time.
\end{abstract}

Keywords: Coupled Electro-Thermal Problems, Finite Integration Technique, Index-1 Differential-Algebraic Equations, Operator Splitting, Fractional Step Methods.

\section{INTRODUCTION}

Computer aided device design in nano-electronics typically leads to large multi-physics problems. The continuous miniaturisation of modern integrated circuits has given rise to higher power densities, and thus the coupling between the electrical and thermal fields increases. Hence, the influence of temperature in device structures becomes a primary concern during the design phase.

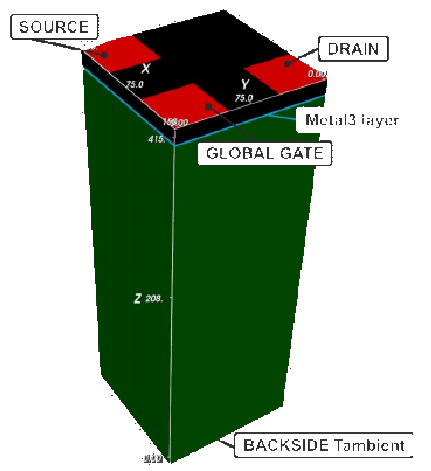

Figure 1. Part of the finger structure in a typical device of a power transistor. Image from ter Maten et al. (2014)

The FP7 project nanoCOPS derives methods for the simulation of those multi-physical problems and aims for establishing them in industrial workflows, ter Maten et al. (2014). With nanoCOPS, new designs will be possible due to the improvements of the simulation workflow, e.g., improved life-time performance is also of concern by predicting the ageing of the device. This paper describes a

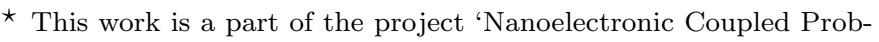
lems Solutions' (nanoCOPS) funded by the European Union within FP7-ICT-2013 (grant no. 619166). The second author is also supported by the 'Excellence Initiative' of the German Federal and State Governments and the Graduate School of Computational Engineering at Technische Universitaet Darmstadt.
}

simple approach for solving electro-thermal systems often encountered in device structure design. The approach is based on the finite integration technique (FIT), developed by Weiland (1996), and employs a fractional step method to accomplish the solution. This paper is organised as follows: In Section 2, we formulate the electro-thermal problem, and in Section 3 we numerically solve the resulting system by means of fractional step and an error correction strategy. The resulting error can then be used to improve the solution on the fly.

\section{DYNAMIC ELECTRO-THERMAL MODELLING OF DEVICES STRUCTURES}

\subsection{Problem Statement}

Figure 1 shows a typical structure of interest, which consists of dielectrics, vias, contacts, and metal interconnects. For these configurations, we aim at determining the overall voltage drop and associated temperature distribution under given conditions. We tackle this electro-thermal problem in an ab-initio consistent manner; the relevant equations are

$$
\begin{aligned}
\nabla \cdot \mathbf{J} & =-\frac{\partial \varrho_{\mathrm{e}}}{\partial t}, & \mathbf{J} & =\sigma \mathbf{E}, \\
\mathbf{E} & =-\nabla \phi-\frac{\partial \mathbf{A}}{\partial t}, & \varrho_{\mathrm{e}} & =-\nabla \cdot(\varepsilon \mathbf{E}),
\end{aligned}
$$

where $\mathbf{J}$ is the current density, $\mathbf{A}$ is the magnetic potential, $\mathbf{E}$ is the electric field, $\phi$ is the electric potential, $\varepsilon$ and $\sigma$ are the permittivity and conductivity of the medium, and $\varrho_{\mathrm{e}}$ is the electric charges density. Equations (1)-(2) are complemented with the heat equation, viz.

$$
\rho c_{\mathrm{e}} \frac{\partial T}{\partial t}=\nabla \cdot(\kappa \nabla T)+\dot{q}_{\mathrm{i}}
$$

where $\rho c_{\mathrm{e}}$ is the thermal capacitance, $\kappa$ is the thermal conductivity of the medium, $T$ is the temperature, and $\dot{q}_{\text {i }}$ is any heat source. The system (1)-(3) is coupled through 
the Joule losses $\dot{q}_{\mathrm{i}}$, and the temperature dependence of $\sigma$, and $\kappa$.

\subsection{Numerical Solution}

The solution of (1)-(3) is attained by FIT, see Weiland (1996). We neglect the effect of local variation of charges, i.e., $\partial_{t} \varrho_{\mathrm{e}} \equiv 0$. and by taking $\dot{q}_{\mathrm{i}}=\mathbf{E} \cdot \mathbf{J}$, we arrive at

$$
\begin{aligned}
\tilde{\mathbf{S}} \mathbf{M}_{\sigma} \tilde{\mathbf{S}}^{\top} \Phi= & 0, \\
\mathbf{M}_{\mathrm{c}} \frac{\partial}{\partial t} \mathbf{T}= & -\tilde{\mathbf{S}} \mathbf{M}_{\kappa} \tilde{\mathbf{S}}^{\top} \mathbf{T} \\
& +\sum_{i} \mathbf{P}\left(\mathbf{1}_{i}^{\top} \mathbf{M}_{\sigma}^{\frac{1}{2}} \tilde{\mathbf{S}}^{\top} \Phi \Phi^{\top} \tilde{\mathbf{S}} \mathbf{M}_{\sigma}^{\frac{1}{2}} \mathbf{1}_{i}\right) \mathbf{1}_{i},
\end{aligned}
$$

where $\Phi$ and $\mathbf{T}$ are the potential and temperature vectors, $\mathbf{M}_{\sigma}, \mathbf{M}_{\mathrm{c}}$, and $\mathbf{M}_{\kappa}$ are diagonal matrices, which may non-linearly depend on $\mathbf{T}$, denoting electric conductivity, thermal capacitance, and thermal conductivity; $\mathbf{P}$ is a projection matrix, see e.g. Kaufman et al. (2014) and Alotto et al. (2010); $\mathbf{1}_{i}$ is the $i$-th basis column vector ${ }^{1}$, and the sum is carried out over all primary edges of the grid Weiland (1996); Clemens et al. (2000); Clemens and Weiland (2001). $\Phi$ and $\mathbf{T}$ are then partitioned into the degrees of freedom $\Phi_{\mathrm{d}}$ and $\mathbf{T}_{\mathrm{d}}$, and the discrete imposed boundary potentials and temperatures $\Phi_{\mathrm{b}}$ and $\mathbf{T}_{\mathrm{b}}$. This leads to the compact form of the electro-thermal system, viz.

$$
\begin{aligned}
\tilde{\mathbf{S}}_{\mathrm{d}} \mathbf{M}_{\sigma} \tilde{\mathbf{S}}_{\mathrm{d}}^{\top} \Phi_{\mathrm{d}}= & -\tilde{\mathbf{S}}_{\mathrm{d}} \mathbf{M}_{\sigma} \tilde{\mathbf{S}}_{\mathrm{b}}^{\top} \Phi_{\mathrm{b}} \\
\mathbf{M}_{\mathrm{c} ; \mathrm{d}} \frac{\partial}{\partial t} \mathbf{T}_{\mathrm{d}}= & -\tilde{\mathbf{S}}_{\mathrm{d}} \mathbf{M}_{\kappa} \tilde{\mathbf{S}}_{\mathrm{d}}^{\top} \mathbf{T}_{\mathrm{d}}-\tilde{\mathbf{S}}_{\mathrm{d}} \mathbf{M}_{\kappa} \tilde{\mathbf{S}}_{\mathrm{b}}^{\top} \mathbf{T}_{\mathrm{b}} \\
& +\Phi_{\mathrm{d}}^{\top} \mathbf{H}_{\mathrm{dd}} \Phi_{\mathrm{d}}+\Phi_{\mathrm{d}}^{\top} \mathbf{H}_{\mathrm{db}} \Phi_{\mathrm{b}}
\end{aligned}
$$

where $\mathbf{H}_{\mathrm{dd}}$ and $\mathbf{H}_{\mathrm{db}}$ are heat tensors ${ }^{2}$

The system (4)-(5) is an index-1 differential-algebraic equation (DAE) that can be solved by an implicit Euler scheme or similarly to Clemens et al. (2000). However, exploiting the fractional step approach from Vijalapura et al. (2005) one can still retain the temporal convergence order albeit decoupling the systems, i.e.,

$$
\begin{aligned}
\left(\frac{\mathbf{M}_{\mathrm{c} ; \mathrm{d}}}{h}+\tilde{\mathbf{S}}_{\mathrm{d}} \tilde{\mathbf{M}}_{\kappa}^{(n+1)} \tilde{\mathbf{S}}_{\mathrm{d}}^{\top}\right) \tilde{\mathbf{T}}_{\mathrm{d}}^{(n+1)} & =\frac{\mathbf{M}_{\mathrm{c} ; \mathrm{d}}}{h} \tilde{\mathbf{T}}_{\mathrm{d}}^{(n)} \\
& -\tilde{\mathbf{S}}_{\mathrm{d}} \tilde{\mathbf{M}}_{\kappa}^{(n+1)} \tilde{\mathbf{S}}_{\mathrm{b}}^{\top} \mathbf{T}_{\mathrm{b}}^{(n+1)} \\
& +\left(\tilde{\Phi}_{\mathrm{d}}^{\left(n+\frac{1}{2}\right)}\right)^{\top} \tilde{\mathbf{H}}_{\mathrm{dd}}^{(n+1)} \tilde{\Phi}_{\mathrm{d}}^{\left(n+\frac{1}{2}\right)} \\
& +\left(\tilde{\Phi}_{\mathrm{d}}^{\left(n+\frac{1}{2}\right)}\right)^{\top} \tilde{\mathbf{H}}_{\mathrm{db}}^{(n+1)} \Phi_{\mathrm{b}}^{(n+1)}
\end{aligned}
$$

and the fractional step

$$
\tilde{\mathbf{S}}_{\mathrm{d}} \tilde{\mathbf{M}}_{\sigma}^{\left(n+\frac{1}{2}\right)} \tilde{\mathbf{S}}_{\mathrm{d}}^{\top} \tilde{\Phi}_{\mathrm{d}}^{\left(n+\frac{1}{2}\right)}=-\tilde{\mathbf{S}}_{\mathrm{d}} \tilde{\mathbf{M}}_{\sigma}^{\left(n+\frac{1}{2}\right)} \tilde{\mathbf{S}}_{\mathrm{b}}^{\top} \Phi_{\mathrm{b}}^{\left(n+\frac{1}{2}\right)}
$$

where the upper index $(n)$ refers to the time step $t_{2 n}=$ $2 n h$ and $\mathbf{M}_{\star}^{(n)}$ is short-hand for a nonlinear material dependency $\mathbf{M}_{\star}\left(\mathbf{x}^{(n)}\right)$ evaluated at time $t_{2 n}$. Next, we define the decoupling errors $e_{\Phi}=\Phi_{\mathrm{d}}-\tilde{\Phi}_{\mathrm{d}}$ and $e_{\mathbf{T}}=\mathbf{T}_{\mathrm{d}}-$ $\tilde{\mathbf{T}}_{\mathrm{d}}$, with governing equations obtained from the above expressions and (4)-(5) by defining the error matrices and

\footnotetext{
1 The column vector containing zero in all its entries but in the position $i$-th.

${ }_{2}^{2} \mathbf{H}_{\mathrm{dd}}$ and $\mathbf{H}_{\mathrm{db}}$ are tridimensional arrays of depth according to $\Phi_{\mathrm{d}}$, the subscripts dd and db specify the number of rows and columns, respectively.
}

tensors $\mathbf{E}_{\kappa} \equiv \mathbf{M}_{\kappa}-\tilde{\mathbf{M}}_{\kappa}, \mathbf{E}_{\sigma} \equiv \mathbf{M}_{\sigma}-\tilde{\mathbf{M}}_{\sigma}, \mathbf{E}_{\mathrm{dd}} \equiv \mathbf{H}_{\mathrm{dd}}-$ $\tilde{\mathbf{H}}_{\mathrm{dd}}$, and $\mathbf{E}_{\mathrm{db}} \equiv \mathbf{H}_{\mathrm{db}}-\tilde{\mathbf{H}}_{\mathrm{db}}$. The resulting error is then solved and added to the approximate solutions $\tilde{\Phi}_{\mathrm{d}}$ and $\tilde{\mathbf{T}}_{\mathrm{d}}$

\section{NUMERICAL SOLUTION}

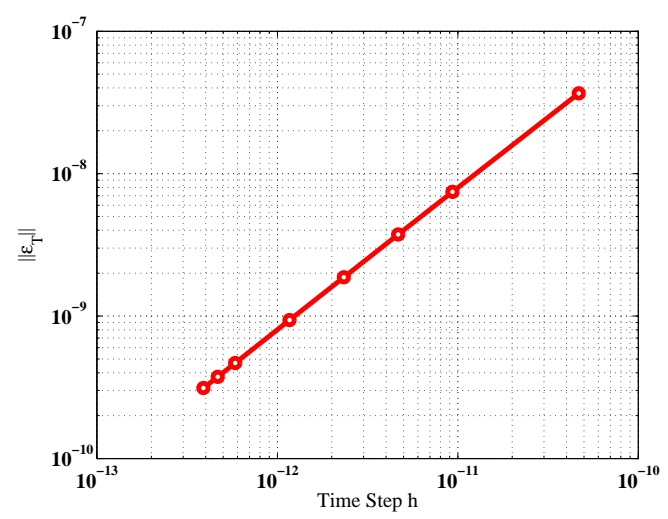

Figure 2. Temperature error $\left\|\epsilon_{\mathbf{T}}\right\|$ versus the time step $h$.

We show the correction temperature error $\left\|\epsilon_{\mathbf{T}}\right\|$ (the potential error $\epsilon_{\boldsymbol{\Phi}}$ exhibits similar trend) versus the time step $h$ in Fig. 2 of a representative electro-thermal problem in which the temperature dependence of the grid material matrices $\mathbf{M}_{\kappa}$ and $\mathbf{M}_{\sigma}$, and heat tensors $\mathbf{H}_{\mathrm{dd}}$ and $\mathbf{H}_{\mathrm{db}}$, is modelled with polynomials $\Pi_{\kappa ; \sigma}\left(\mathbf{T}, \mathbf{T}_{\mathrm{o}}\right)$ with $\mathbf{T}_{\mathrm{o}}$ the reference temperature. Both systems have been solved by means of an implicit Euler scheme with iterative refinement.

\section{REFERENCES}

Alotto, P., Freschi, F., and Repetto, M. (2010). Multiphysics problems via the cell method: The role of tonti diagrams. IEEE Trans. Magn., 46(8), 2959-2962. doi: 10.1109/TMAG.2010.2044487.

Clemens, M., Gjonaj, E., Pinder, P., and Weiland, T. (2000). Numerical simulation of coupled tansient thermal and electromagnetic fields with the finite integration method. IEEE Transaction on Magnetics, 36, 14481452.

Clemens, M. and Weiland, T. (2001). Discrete electromagnetism with the finite integration technique. Progress in Electromagnetics Research, 32, 65-87.

Kaufman, C., Günther, M., Klagges, D., Knorrenschild, M., Richwin, M., Schöps, S., and ter Maten, E.J. (2014). Efficient frequency-transient co-simulation of coupled heat-electromagnetic problems. Journal of Mathematics for Industry, 4, 1-13.

ter Maten et al., J. (2014). nanocops: Nanoelectronic coupled problem solutions. ECMI newsletter, 56. To appear.

Vijalapura, P.K., Strain, J., and Govindjee, S. (2005). Fractional step methods for index-1 differentialalgebraic equations. J. Comput. Phys., 203, 305-320.

Weiland, T. (1996). Time domain electromagnetic field computation with finite difference methods. International Journal of Numerical Modelling: Electronic Network, Devices and Fields, 9, 295-319. 


\title{
Model Order Reduction of an Electro-Thermal Package Model ${ }^{\star}$
}

\author{
N. Banagaaya ${ }^{*}$ L. Feng * P. Meuris ${ }^{* *}$ W. Schoenmaker ${ }^{* *}$ \\ P. Benner* \\ * Max Planck Institute for Dynamics of Complex Technical Systems, \\ Magdeburg, Germany \\ (e-mail: banagaaya,feng, benner@mpi-magdeburg.mpg.de) \\ ** MAGWEL NV, Leuven, Belgium \\ (e-mail: wim.schoenmaker, peter.meuris@magwel.com)
}

\begin{abstract}
Model order reduction of an electro-thermal package model is discussed. This model is an electro-thermal coupled problem with geometrical variation. Due to the coupling and parameter variation, the package model leads to a system of nonlinear parametric differentialalgebraic equations (DAEs). This model can be very large-scale which makes simulation computationally expensive. This calls for the application of model order reduction techniques, particularly for parametric model order reduction (pMOR) methods. However, many pMOR methods are restricted to linear parametric models. This motivates us to propose an approach which involves first decoupling the nonlinear parametric DAEs into differential and algebraic parts. Then, the differential and algebraic parts can be reduced separately.
\end{abstract}

Keywords: differential-algebraic equations, nonlinear parametric model, model order reduction

\section{INTRODUCTION}

An electro-thermal package can be modeled as an electrothermal coupled problem. For general complex geometries, an accurate, physical model is desired. Spatial discretization using the finite-element method (FEM), finite volume method (FVM), or finite integration technique (FIT) leads to a large-scale parametric DAE model.

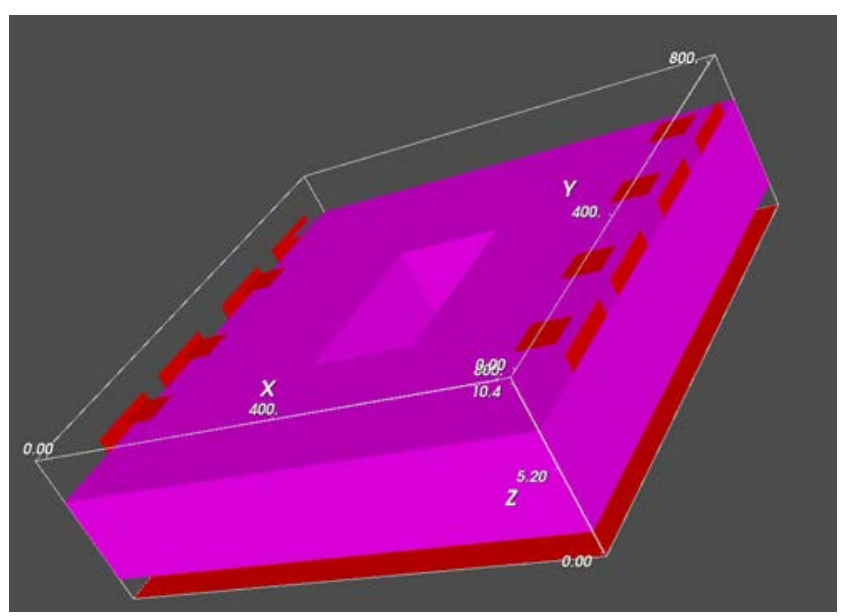

Fig. 1. Electro-thermal package

In Fig. 1, a FIT model of an electro-thermal package is shown. If we consider meshes that are topologically equivalent for different package thicknesses $p$, the parametric dependence will take the form

\footnotetext{
* This work is supported by collaborative project nanoCOPS, Nanoelectronics COupled Problems Solutions, funded by the European Union within FP7-ICT-2013-11 programme (grant No. 619166).
}

$$
\mathbf{M}(p)=\mathbf{M}_{0}+p \mathbf{M}_{1}+\frac{1}{p} \mathbf{M}_{2},
$$

where $\mathbf{M}_{i}$ are constant coefficient matrices or tensors. If the parameter $p$ symbolically appears in the package model, then its mathematical model can be written as a parametric nonlinear DAE of the form

$$
\begin{aligned}
\mathbf{E}(p) \frac{\mathrm{d} \mathbf{x}}{\mathrm{d} t} & =\mathbf{A}(p) \mathbf{x}+\mathbf{x}^{\mathrm{T}} \mathcal{F}(p) \mathbf{x}+\mathbf{B}(p) \mathbf{u}, \\
\mathbf{y} & =\mathbf{C}(p) \mathbf{x}+\mathbf{D}(p) \mathbf{u},
\end{aligned}
$$

where the matrix $\mathbf{E}(p) \in \mathbb{R}^{n \times n}$ is singular for every parameter $p, \mathbf{A}(p) \in \mathbb{R}^{n \times n}, \mathbf{B}(p) \in \mathbb{R}^{n \times m}, \mathbf{C}(p) \in \mathbb{R}^{\ell \times n}, \mathbf{D}(p) \in$ $\mathbb{R}^{\ell \times m}$, and the tensor $\mathcal{F}(p) \in \mathbb{R}^{n \times n \times n}$ can be written in the form (1). This tensor is a 3-D array of $n$ matrices. The state vector $\mathbf{x}=\mathbf{x}(t, p) \in \mathbb{R}^{n}$ includes the nodal voltages $\mathbf{x}_{v} \in \mathbb{R}^{n_{v}}$ and the nodal temperatures $\mathbf{x}_{T} \in \mathbb{R}^{n_{T}}$, i.e., $\mathbf{x}=\left(\mathbf{x}_{v}^{\mathrm{T}}, \mathbf{x}_{T}^{\mathrm{T}}\right)^{\mathrm{T}} \cdot \mathbf{u}=\mathbf{u}(t) \in \mathbb{R}^{m}$ and $\mathbf{y}=\mathbf{y}(t, p) \in \mathbb{R}^{\ell}$ are the inputs and the desired outputs, respectively. In order to obtain accurate models, fine meshes must be used which lead to very large $n$ compared to the number of inputs $m$ and desired outputs $\ell$. Despite the ever increasing computational power, simulation of these systems in acceptable time is very difficult because of the storage requirements and expensive computations, in particular if multi-query tasks are required. This calls for the application of parametric model order reduction (pMOR) methods. PMOR replaces (2) by a reduced-order model

$$
\begin{aligned}
\mathbf{E}_{r}(p) \frac{\mathrm{d} \mathbf{x}_{r}}{\mathrm{~d} t} & =\mathbf{A}_{r}(p) \mathbf{x}_{r}+\mathbf{x}_{r}^{\mathrm{T}} \mathcal{F}_{r}(p) \mathbf{x}_{r}+\mathbf{B}_{r}(p) \mathbf{u}, \\
\mathbf{y}_{r} & =\mathbf{C}_{r}(p) \mathbf{x}_{r}+\mathbf{D}_{r}(p) \mathbf{u}
\end{aligned}
$$

where $\mathbf{E}_{r}(p), \mathbf{A}_{r}(p) \in \mathbb{R}^{r \times r}, \mathbf{B}_{r}(p) \in \mathbb{R}^{r \times m}, \mathbf{C}_{r}(p) \in$ $\mathbb{R}^{\ell \times r}, \mathbf{D}_{r}(p) \in \mathbb{R}^{\ell \times m}$ and the tensor $\mathcal{F}_{r}(p) \in \mathbb{R}^{r \times r \times r}$ can 
also be written in the form (1), so that the parameter $p$ is symbolically preserved in the reduced-order model. The dimension $r \ll n$ of the reduced-order model (3) is now much smaller than that of the original model (2). A good reduced-order model is one with small approximation error $\left\|\mathbf{y}-\mathbf{y}_{r}\right\|$ in a suitable norm $\|$.$\| for every arbitrary input$ $\mathbf{u}(t)$ and parameter $p$. There are many existing pMOR methods such as the robust pMOR algorithm in Benner and Feng (2014), based on implicit moment matching. However, these methods are limited to linear parametric models, i.e., when (2) has no tensor $\mathcal{F}(p)$. With the tensor included it is difficult to use the existing methods in their current form. This motivates us to propose a new approach which involves first decoupling the nonlinear parametric DAE model (2) into differential and algebraic parts. Then, the two parts can be reduced separately. We note that this approach has the same underlying ideas as index-aware MOR methods in Banagaaya (2014), but here different decoupling approaches must be used.

\section{MODEL ORDER REDUCTION OF AN ELECTRO-THERMAL PACKAGE MODEL}

In this section, we introduce a new reduction technique which can be used to reduce electro-thermal package models of the form (2). This is done by first decoupling (2) into differential and algebraic parts. Taking advantage of the natural structure of the matrices $\mathbf{E}(p)=$ $\left(\begin{array}{cc}\mathbf{0} & \mathbf{0} \\ \mathbf{0} & \mathbf{E}_{T}(p)\end{array}\right), \mathbf{A}(p)=\left(\begin{array}{cc}\mathbf{A}_{v}(p) & \mathbf{0} \\ \mathbf{0} & \mathbf{A}_{T}(p)\end{array}\right)$ and the tensor $\mathcal{F}(p)$ in (2), we obtain the following decoupled algebraic (electrical) and differential (thermal) parts,

$$
\begin{aligned}
\mathbf{0} & =\mathbf{A}_{v}(p) \mathbf{x}_{v}+\mathbf{B}_{v}(p) \mathbf{u} \\
\mathbf{E}_{T}(p) \frac{\mathrm{d} \mathbf{x}_{T}}{\mathrm{~d} t} & =\mathbf{A}_{T}(p) \mathbf{x}_{T}+\mathbf{x}_{v}^{\mathrm{T}} \mathcal{F}_{T}(p) \mathbf{x}_{v}+\mathbf{B}_{T}(p) \mathbf{u}, \\
\mathbf{y} & =\mathbf{C}_{v}(p) \mathbf{x}_{v}+\mathbf{C}_{T}(p) \mathbf{x}_{T}+\mathbf{D}(p) \mathbf{u}
\end{aligned}
$$

where $\mathbf{A}_{v}(p) \in \mathbb{R}^{n_{v} \times n_{v}}, \mathbf{B}_{v}(p) \in \mathbb{R}^{n_{v} \times m}, \mathbf{E}_{T}(p) \in \mathbb{R}^{n_{T} \times n_{T}}$ is a nonsingular matrix for every parameter $p, \mathbf{A}_{T}(p) \in$ $\mathbb{R}^{n_{T} \times n_{T}}, \mathbf{B}_{T}(p) \in \mathbb{R}^{n_{T} \times m}, \mathbf{C}_{v}(p) \in \mathbb{R}^{\ell \times n_{v}}, \mathbf{C}_{T}(p) \in$ $\mathbb{R}^{\ell \times n_{T}}$, and $\mathcal{F}_{T}(p) \in \mathbb{R}^{n_{v} \times n_{v} \times n_{T}}$ is a nonzero part in the tensor $\mathcal{F}(p)$. We note that the total dimension of the decoupled system (4) is equal to the dimension of (2), that is, $n=n_{v}+n_{T}$. From, (4), we observe that the initial condition of (2) must be a consistent initial condition, that is, $\mathbf{x}(0, p)=\left(\mathbf{x}_{v}(0, p)^{\mathrm{T}}, \mathbf{x}_{T}(0, p)^{\mathrm{T}}\right)^{\mathrm{T}}$, where $\mathbf{x}_{v}(0, p)=-\mathbf{A}_{v}(p)^{-1} \mathbf{B}_{v}(p) \mathbf{u}(0)$ and $\mathbf{x}_{T}(0, p)$ can be chosen arbitrarily. The next step is to apply pMOR to (4a) and (4b), separately. Considering the series expansion of $x_{v}$ w.r.t the parameter $p$ in (4a), the implicit momentmatching pMOR method proposed in Benner and Feng (2014) can be applied to compute a projection matrix $\mathbf{V}_{v}$ and to get a reduced parametric algebraic model of (4a). If we first reduce (4a), this reduction induces a reduction in the differential part (4b). We note that pMOR of (4a), introduces an approximation to the nonlinear term in (4b). However, the dimension of (4b) is unreduced but the dimension of the matrix blocks of the tensor $\mathcal{F}_{T}(p)$ is reduced. In order to reduce the dimension of the differential part (4b), the same pMOR approach in Benner and Feng (2014) can be applied by ignoring the nonlinear term (coupled term) to construct another projection matrix
$\mathbf{V}_{T} \in \mathbb{R}^{n_{T} \times r_{2}}$. The two separate reduced-order models are further coupled into a single system which leads to a reduced-order model of the electro-thermal package model given by

$$
\begin{aligned}
\mathbf{0}= & \mathbf{A}_{v_{r}}(p) \mathbf{x}_{v_{r}}+\mathbf{B}_{v_{r}}(p) \mathbf{u}, \\
\mathbf{E}_{T_{r}}(p) \frac{\mathrm{d} \mathbf{x}_{T_{r}}}{\mathrm{~d} t}= & \mathbf{A}_{T_{r}}(p) \mathbf{x}_{T_{r}}+\mathbf{V}_{T^{\mathrm{T}}}^{\mathrm{T}} \mathbf{x}_{v_{r}}^{\mathrm{T}} \mathcal{F}_{T_{r}}(p) \mathbf{x}_{v_{r}} \\
& +\mathbf{B}_{T_{r}}(p) \mathbf{u}, \\
\mathbf{y}_{r}= & \mathbf{C}_{v_{r}}(p) \mathbf{x}_{v}+\mathbf{C}_{T_{r}}(p) \mathbf{x}_{T_{r}}+\mathbf{D}(p) \mathbf{u},
\end{aligned}
$$

where $\mathbf{A}_{v_{r}}(p) \in \mathbb{R}^{r_{1} \times r_{1}}, \mathbf{B}_{v_{r}}(p) \in \mathbb{R}^{r_{1} \times m}, \mathbf{E}_{T_{r}}(p), \mathbf{A}_{T_{r}}(p) \in$ $\mathbb{R}^{r_{2} \times r_{2}}, \mathbf{B}_{T}(p) \in \mathbb{R}^{r_{2} \times m}, \mathbf{C}_{v_{r}}(p) \in \mathbb{R}^{\ell \times r_{1}}, \mathbf{C}_{T_{r}}(p) \in \mathbb{R}^{\ell \times r_{2}}$, and a tensor $\mathcal{F}_{T_{r}}(p) \in \mathbb{R}^{r_{1} \times r_{1} \times n_{T}} . r_{1} \ll n_{v}$ and $r_{2} \ll n_{T}$ is the reduced dimension of $(4 \mathrm{a})$ and $(4 \mathrm{~b})$, respectively.

\section{NUMERICAL RESULTS}

We consider an electro-thermal package model from MAGWEL NV. It is a system of the form (2) with dimension $n=9193, m=34$ inputs and $\ell=68$ outputs. The system is excited by the input $\mathbf{u}(t)=\left(\mathbf{u}_{1}(t), \ldots, \mathbf{u}_{34}(t)\right)^{\mathrm{T}}$, where $\mathbf{u}_{1}(t)=1, \mathbf{u}_{2}(t)=\cdots=\mathbf{u}_{17}(t)=0$,

$\mathbf{u}_{18}(t)=\left\{\begin{array}{ll}75 \times 10^{8} t+75 & \text { If } t \leq 10^{-8} \\ 150 & \text { If } t>10^{-8}\end{array}\right.$,

$\mathbf{u}_{19}(t)=\cdots=\mathbf{u}_{34}(t)=75$. We use $\mathbf{x}_{T}(0, p)=75$ as the initial condition for the thermal part. We were able to decouple this system into the form (4) leading to an algebraic part and a differential part of dimension $n_{v}=1122$ and $n_{T}=8071$, respectively. Using the approach discussed in Sec. 2, we have reduced the dimension of the algebraic part and that of the differential part to $r_{1}=32$ and $r_{2}=577$, respectively. The reduced-order model can be written into the form (5) of dimension $r=r_{1}+r_{2}=609$ and it leads to an accurate solution with output error $\left\|\mathbf{y}-\mathbf{y}_{r}\right\|_{2} /\|\mathbf{y}\|_{2} \leq 3.3 \times 10^{-6}$. As an illustration we compare in Fig. 2, the output solution of $y_{48}(t)$ computed by full and reduced simulation, respectively.

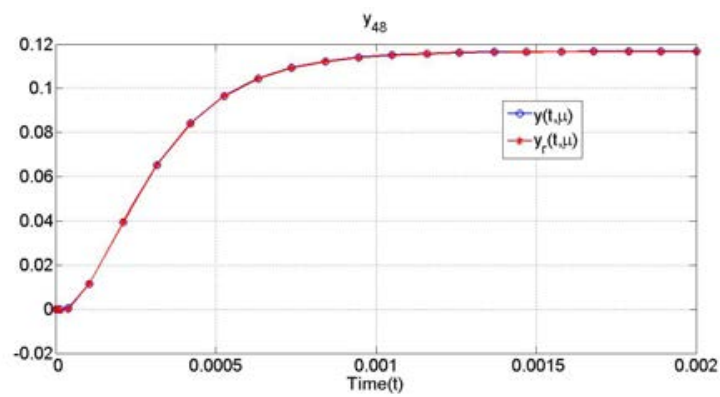

Fig. 2. Output comparison of $y_{48}(t)$.

\section{REFERENCES}

Banagaaya, N. (2014). Index-aware model order reduction methods. Ph.D. thesis, Eindhoven University of Technology, Eindhoven, Netherlands.

Benner, P. and Feng, L. (2014). A robust algorithm for parametric model order reduction based on implicit moment matching. In A. Quarteroni and G. Rozza (eds.), Reduced Order Methods for modeling and computational reduction, volume 8, 159-185. Springer MS \& A series, Springer International Publishing Switzerland. 


\title{
Measurement Setup for Identifying Parameters of the Encapsulated Bond Wires
}

\author{
J. Petrzela*, R. Sotner*, T. Gotthans*, J. Drinovsky*, T. Kratochvil*, A. Wieers** \\ *Department of Radio Electronics, Faculty of Electrical Engineering and Communication, Brno University of Technology, \\ Technicka 12, Brno 616 00, Czech Republic (Tel: 421-54114-6561; e-mail: petrzelj@feec.vutbr.cz). \\ **ON Semiconductor, Westerring 15, 9700 Oudenaarde, Belgium (e-mail: Aarnout.Wieers@onsemi.com)
}

\begin{abstract}
This short paper describes the experimental measurement setup for extraction of dynamical parameters of the encapsulated bond wires. Based on the proposed method a current carrying capability as well as fatigue process can be quantified and statistically evaluated. The integrated circuits with bonding wires and Kelvin probes were fabricated by ON Semiconductor Company in Belgium.
\end{abstract}

Keywords: Electronic system, power supplies, precision measurement, switching networks.

\section{INTRODUCTION}

The essential part of the proposed experimental setup is an internal PCI card 6251 of National Instruments which comprises of an analog output waveform for generation of the pulse driving signal, 8 bit digital port for multiplexing the individual bonding wires and an analog input 10 bits $\mathrm{A} / \mathrm{D}$ converters for measuring the necessary voltages. Referring to this, the experimental setup can be divided into two parts, namely software which directly controls this PCI card and PCB with practical implementation of the multi-channel switching power source.

Software part of the project consists of two executable Matlab scripts supplemented with a GUI. First is called Generator.m which creates a text file with analog driving signal. Driving force can be derived for non-inverting as well as inverting mosfet drivers. Up to nine bursts, with arbitrary time window and duty cycle, can be created with cooling time delays placed between these bursts. This file is loaded by the second program Measurement.m which is capable to do the rest, i.e. choose bonding wires to be measured, specify voltages for the individual measurements and accordingly to this change gain factors for digital data capture and to save them by using a text format, see Fig. 1 .

Hardware section must reflect all requirements for bond wire parameter identification. The individual bonding wires are addressed by analog multiplexer HEF4051, i.e. three bits are used to select one power channel from six possibilities. It means that PCB contains six drivers, six power switches (mosfet transistors IRFZ044) and the same amount of the amplifiers connected to bond wires via Kelvin probes. These integrated amplifiers have differential voltage inputs and nonsymmetrical output with digitally controlled gain. Since very large final gain is necessary two stage cascade connection is utilized. Up to four bits can be used to set a final gain factor; these bits can be directly set via Matlab script. This control is necessary to make use of as wide $\pm 10 \mathrm{~V}$ dynamical range of A/D converter as possible and to lift the signal from noise floor but kept it below saturation. If all address nodes of multiplexer are low or high no bond wire will be active. As shown in Fig. 2 power transistors have sources connected to a single sensing resistor. It is advantageous since the dominant current will be from power branch of the active bond wire while contributions from other bond wires will be negligible.

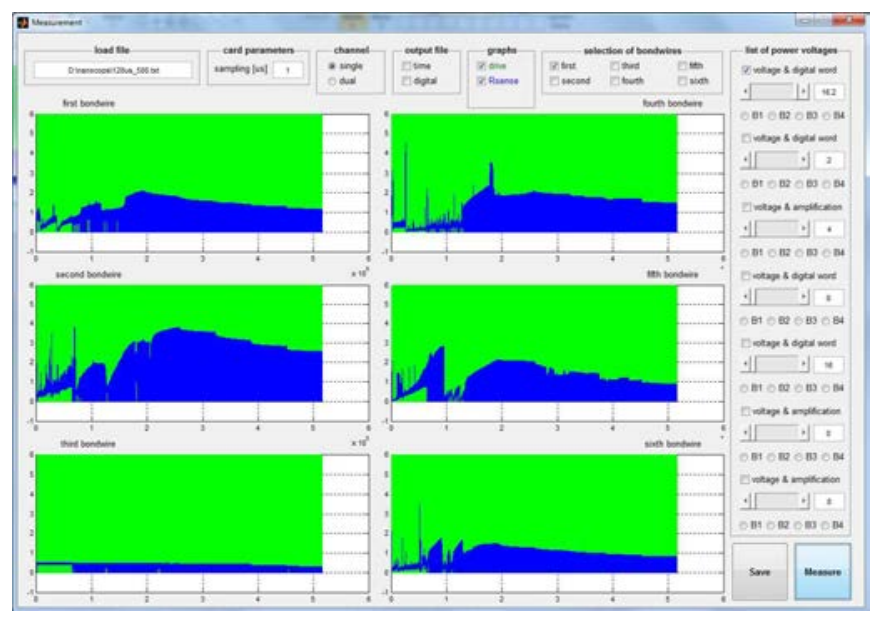

Fig. 1: Matlab program for automated bond wire fusing measurement with plotting achieved and stored results.

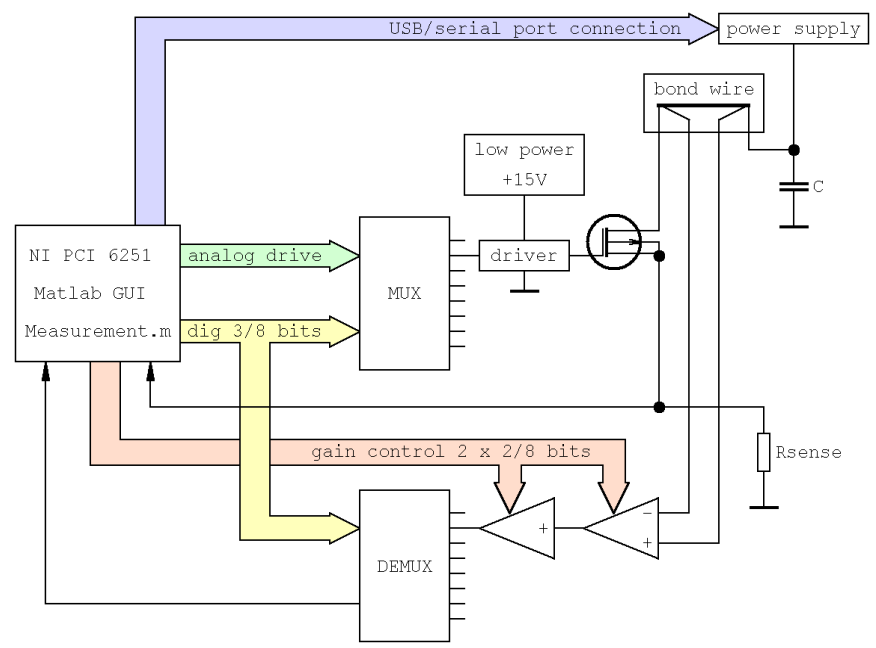

Fig. 2: Fractional block diagram of the realized PCB. 


\section{EXPERIMENTAL BACKGROUND}

The photo of experimental setup is shown in Fig. 3. Devices necessary to observe and quantify bond wire fusing scenarios are two digital oscilloscopes Agilent DSO-X 2012A, power supply Manson 16V/60A and stabilized dual-channel voltage source $30 \mathrm{~V} / 4 \mathrm{~A}$.

In order to accelerate the aging process we decide to make several tests also in the temperature cabin. Measurements are done at ambient temperature and on temperatures of $100^{\circ} \mathrm{C}$ and $180^{\circ} \mathrm{C}$. Unfortunately placing the integrated circuit in an oven and feed the PCB by long bus-type cable introduces relatively large inductance causing voltage overshoots when switch on or off. This unwanted behavior can be reduced to acceptable levels by adding a two terminal RC device between drain and source of mosfet. The time constant for this stabber can be manually adjustable via a variable resistor.

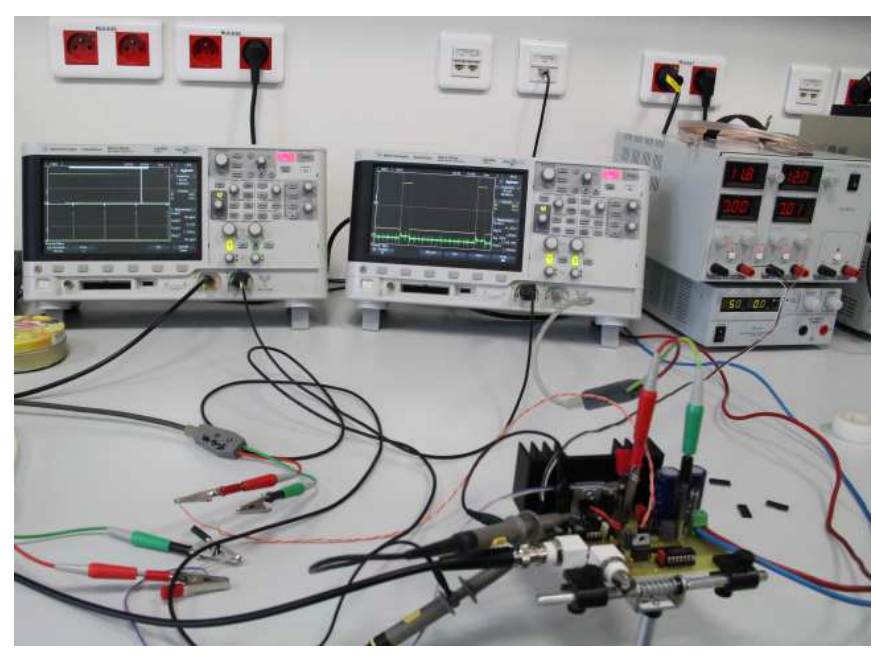

Fig. 3: Laboratory setup for bond wire fusing measurement.

\section{MEASUREMENT}

Many integrated circuits with encapsulated bond wires have been destroyed so far as demonstrated in Fig. 4. It seems that fused bond wire does not mean immediate open loop; various degradation processes have been intercepted by delayed time base feature of the oscilloscopes. Few screenshots are given in Fig. 5, Fig. 6 and Fig. 7.

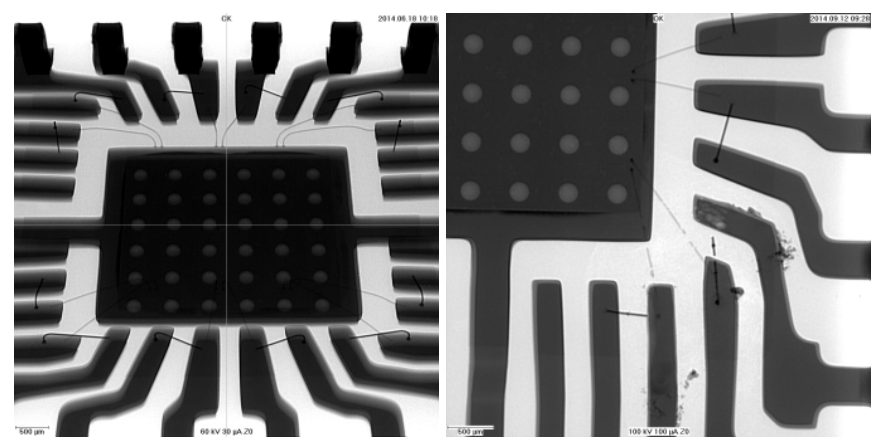

Fig. 4: X-ray of bond wires before (left) and after (right) successive fusing.
Further work in this area will be focused on precise PCB design; special attention will be paid on two-stage amplifier where each stage is controlled by two-bits. It is supposed that $1,10,100$ and 1000 gain of AD8253 will be combined with 1, 2, 5, 10 gain of AD8250.

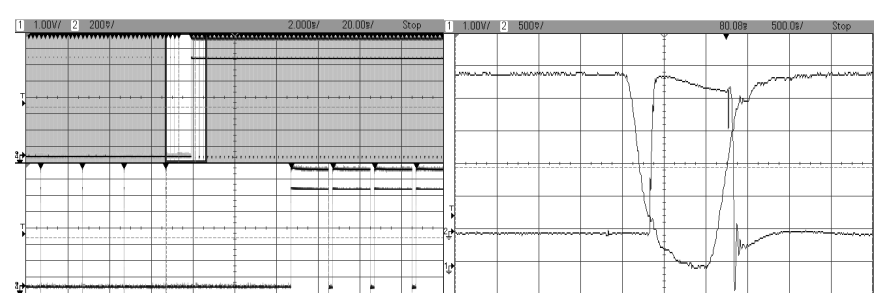

Fig. 5: Generated waveforms with different consequent pulse widths measured by digital oscilloscope (left screenshot), driving force for inverting driver and voltage across $1 \Omega / 20 \mathrm{~W}$ sensing resistor for sharp $1 \mu$ s pulse width (right screenshot).

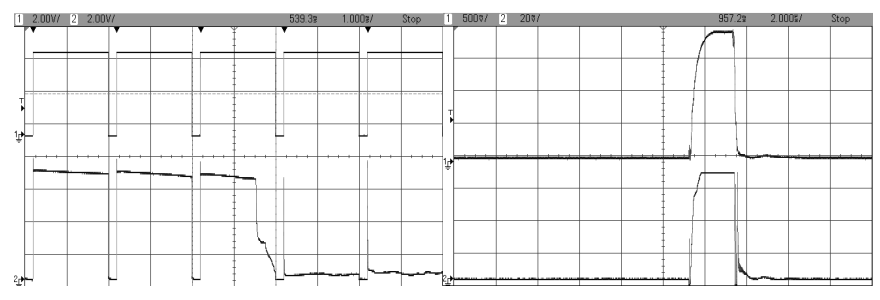

Fig. 6: Successive fusing of bonding wire (left screenshot), voltage across sensing resistor and differential voltage sensed by Kelvin probes for determination of bond wire resistance (right screenshot).

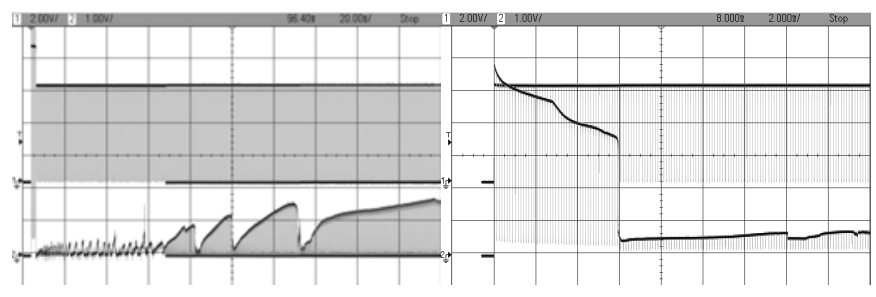

Fig. 7: Rich dynamical behavior of bond wire after fusing (left screenshot), input sequence and voltage across sensing resistor zoomed to the beginning (right traces).

\section{ACKNOWLEDGEMENT}

This paper was supported by the European project FP7nanoCOPS no. 619166 and by the Ministry of Education, Youth and Sports (MEYS) of the Czech Republic national project WICOMT no. CZ.1.07/2.3.00/20.0007. Described research was performed in laboratories supported by the SIX project; no. CZ.1.05/2.1.00/03.0072, the operational program Research and Development for Innovation.

\section{REFERENCES}

[1] MALLIK, A., STOUT, R. Simulation methods for predicting fusing current and time for encapsulated wire bonds. IEEE Transactions of Electronics Packaging Manufacturing, vol. 33, no. 4, 2010, pp. 255-264. 


\title{
Uncertainty Quantification in Electro-Thermal Coupled Problems based on a Power Transistor Device *
}

\author{
Piotr Putek ${ }^{*, * * *}$ Peter Meuris ** Michael Günther* \\ Jan ter Maten* Roland Pulch*** Aarnout Wieers**** \\ Wim Schoenmaker ${ }^{* *}$ \\ * Bergische Universität Wuppertal, Chair of Applied Mathematics and \\ Numerical Analysis, Gaußstraße 20, D-42119 Wuppertal, Germany, \\ (e-mail: \{putek,termaten,guenther\}@math.uni-wuppertal.de), \\ ** Magwel NV, Martelarenplein 13, B-3000 Leuven, Belgium \\ (e-mail: \{Peter.Meuris, Wim.Schoenmaker\}@magwel.com), \\ *** Ernst-Moritz-Arndt-Universität Greifswald, Institute for \\ Mathematics and Computer Science, Walther-Rathenau-Straße 47, \\ 17489 Greifswald, Germany, \\ (e-mail: Roland.Pulch@uni-greifswald.de). \\ **** ON Semiconductor Belgium, Westerring 15, 9700 Oudenaarde, \\ Belgium, (e-mail: Aarnout.Wieers@onsemi.com),
}

\begin{abstract}
In many applications, used in power electronic systems, the physical domain and/or material parameters cannot be determined precisely. Such geometrical variations, which have directly impact on yield and performance, are primarily caused by both lithography proximity effects and process imperfections. Consequently, the mathematical modeling results in a stochastic, coupled, system of equations, which first can be partitioned into subsidiary problems, and then solved numerically by co-simulation techniques or a monolithic approach. In the formulation and the implementation of our particular problem the Spectral Collocation Method (SCM) with a Polynomial Chaos Expansion (PCE) were used. Furthermore, we applied uncertain analysis for the power transistor device, which is of importance for the automotive industry.
\end{abstract}

Keywords: stochastic modeling, uncertainty quantification, polynomial chaos, electronics, power device.

\section{INTRODUCTION}

Power devices play a crucial role in efficiently using resources and energy in power electronics with respect to not only energy harvesting and distribution, but also to the application for automotive industry. Since power devices comprise several thousands of parallel channel devices developed in both CMOS and bipolar technology, the physical domain cannot be determined precisely, due to the uncertain geometry related to lithography proximity and several process variations. To be more specific, among many variation issues, the imperfections in manufacturing processes related to sub-wavelength lithography, lens aberration, and chemical-mechanical polishing, see, e.g., Ban et al. (2011), are the most important ones, which directly influence both yield and performance. They have also a strong impact on acceptability, reliability and profitability of power electronic systems, which depends mainly on variation tolerances, e.g., Mohanty et al. (2014). In commercial simulations tools, the assessment of the reliability and robustness of design with respect to uncertain param-

\footnotetext{
^ The project nanoCOPS (Nanoelectronic COupled Problems Solutions) is supported by the European Union in the FP7-ICT-2013-11 Program under the grant agreement number 619166.
}

eters from manufacturability, or for parameters varying within some range is usually done by setting up a type of Monte Carlo method. However, the production of statistics obtained in this way can be prohibitively expensive, especially while considering the transient simulation of the 3-D electro-thermal coupled problem. Another approach for the investigation of the impact of input variations on the power device output characteristic is based on the perturbation method, e.g., Ban et al. (2010). In our work, we apply the spectral approach based on (generalized) Polynomial Chaos Expansion in order to calculate efficiently not only the desired statistics but also the variance-based sensitivity information by exploiting rapidly converging expansion using the SCM. This solution has an additional advantage, i.e., it yields directly a response surface model, which can easily be incorporated in a robust topology optimization. Specifically, the problem under consideration comes from the automotive industry, where there is a need to handle the demanding electro-thermal operational constraints to design both components and systems. Thus, the problem with geometry variations, shown on Fig. 1(a), was formulated as the first attempt to cope with a more general problem related to the Power-MOS-electro-thermal-stress coupling, whose structure is depicted in Fig. 1(b). 


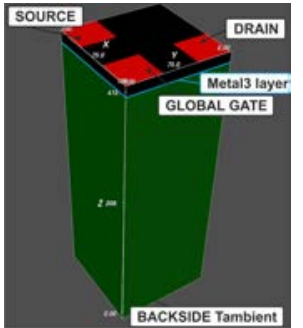

(a) Part of the finger structure of device.

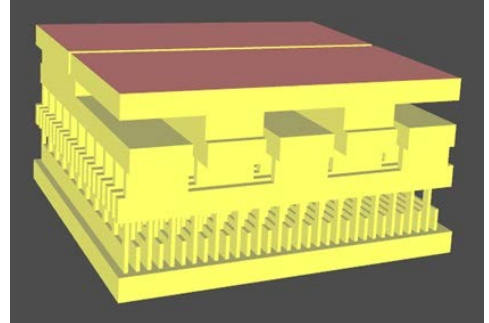

(b) Case I: Typical layout of a power transistor.
Fig. 1. The geometry of a power MOS transistor(Magwel. (2014)).

\section{STOCHASTIC MODELING USING SCM}

For uncertainty quantification (UQ), some parameters $\epsilon, W_{k}, \sigma_{k}, C_{v}$ involved in the model (1) were replaced by $v=\left(\epsilon(\omega), W_{k}(\omega), \sigma_{k}(\omega), C_{v}(\omega)\right)$ independent random variables, defined on some probabilistic space $(\Omega, \mathcal{F}, \mathcal{P})$ with a joint density. Then, the direct problem is governed by the coupled stochastic PDEs on a bounded domain $\mathrm{D} \subset \mathbb{R}^{3}$

$$
\left\{\begin{array}{l}
\nabla \cdot(\epsilon \nabla V)=\rho, \\
\nabla \cdot J=0, \\
J_{(n, p)}=q(n, p) \mu_{(n, p)} \cdot \nabla V \pm q D_{(n, p)} \nabla(n, p), \\
C_{v} \partial_{t} T=\nabla \cdot \lambda(T) \nabla T+\sigma(T)\|\nabla V\|^{2},
\end{array}\right.
$$

equipped with suitable boundary conditions, where $\rho, \epsilon$ and $q$ denote the charge density, the permittivity and the elementary charge, respectively. $V:=V(x, t, \omega)$ is the electric scalar potential. The concentration of holes and electrons is represented by $n$ and $p$, while $(n, p)$ describes compactly the equations for electrons and holes. Thus, $D_{n}$, $D_{p}, \mu_{n}, \mu_{p}, J_{n}:=J_{n}(x, t, \omega), J_{p}:=J_{p}(x, t, \omega)$ are the diffusion, mobilities and currents densities of electrons and holes. In (1), $T=T(x, t, \omega), C_{v}$ and $\lambda(T)$ are temperature, the heat capacitance and the thermal conductivity. The conductivity of $k$-th layer can be described by $\sigma=W_{k} \sigma_{k}$, where $W_{k}$ denotes the size of the layer, for example, the thickness and $\sigma_{k}=f\left(q, n, p, \mu_{(n, p)}\right)$.

Consequently, the solution of (1) becomes a time-dependent random process. Now, assuming that each component of $v_{i}$ exhibits a finite second moment, this process can be expanded in the truncated polynomial chaos (PC) series

$$
y(t, v)=\sum_{i=0}^{N} \mathrm{v}_{i}(t) \Phi_{i}(v)
$$

with orthonormal polynomial basis $\left(\Phi_{i}\right)_{i \in \mathbb{N}}$ corresponding to the probability distribution and unknown a priori coefficient functions $\mathrm{v}_{i}$. For the calculation of $\mathrm{v}_{i}$, the SCM proposed by Xiu. (2007) was used, where the deterministic problem is computed in each quadrature grid point $v^{k}$ $k=0, \ldots, K$. Thus, finally we approximate statistical moments, for example,

$$
\mathbb{E}[y(t, v)]=\mathrm{v}_{0}(t), \quad \operatorname{Var}[y(t, v)]=\sum_{i=1}^{N}\left|\mathrm{v}_{i}(t)\right|^{2}
$$

by using a multi-dimensional quadrature rule with weights $w_{k}$ ( Xiu. (2007))

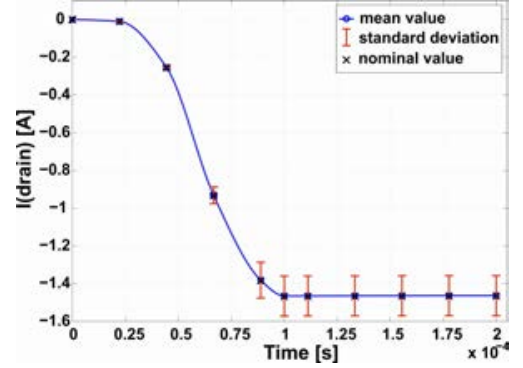

Fig. 2. UQ analysis for the thickness of the Metal3 layer modeled by a Gaussian distribution with $10 \%$ variation around a mean $1 \mu \mathrm{m}$ and the Hermite PC.

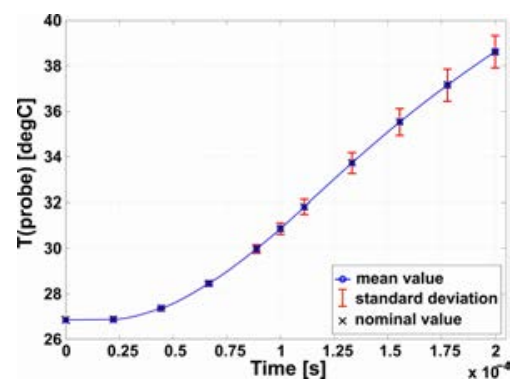

Fig. 3. UQ analysis for $\sigma_{k}$ (of the Metal3 layer) modeled by a uniform distribution with $15 \%$ variation around a mean $2 \mathrm{e} 7 \mathrm{~S} / \mathrm{m}$ and the Legendre PC.

$\mathrm{v}_{i}(t):=\left\langle\mathrm{y}(t, v), \Phi_{i}(v)\right\rangle \approx \sum_{k=0}^{K} w_{k} \mathrm{y}\left(t, v^{(k)}\right) \Phi_{i}\left(v^{(k)}\right)$.

\section{PRELIMINARY RESULTS AND CONCLUSION}

The SCM allows for reusing the existed, deterministic solver, what is an advantage in the comparison to the spectral Galerkin method. However, the number of required runs grows very quickly, even when the Smolyak algorithm has been used. On the other hand, the complexity of the SCM with the Stroud-3 formula is comparable to the worst-case analysis but allows for the better estimation of the variance. Additionally, it yields the sensitivity information. According to the SCM, the electro-thermal coupled problem defined by (1) was solved by the deterministic solver (Magwel. (2014)) for every quadrature grid point. Results of the UQ, depicted on Figs. 2 and 3, show that the output is not too much sensitive to the input variations.

\section{REFERENCES}

Ban Y. and Pan D. Z. (2011). Modeling of Layout Aware Line-Edge Roughness and Poly Optimization for Leakage Minimization. IEEE Journal on Emerging and Selected Topics in Circuits and Systems, 1:150-159.

Ban Y., Sundareswaran S., and Pan D. Z. (2010) Total sensitivity based DFM optimization of standard library cells. Proc. Int. Symp. Phys. Design, 113-120.

Mohanty S. P. and Kougianos E. (2014). Incorporating Manufacturing Process Variation Awareness in Fast Design Optimization of Nanoscale CMOS VCOs. IEEE Trans. on Semiconductor. Manuf., 27:22-31.

Xiu D. (2007) Efficient Collocational Approach for Parametric Uncertainty Analysis, Communication in Computational Physics, 2:293-309.

MAGWEL NV (2014) http://www.magwel.com/. 


\title{
Interface Model Integrating Full-Wave Maxwell Simulation Models into Modified Nodal Equations for Circuit Simulation *
}

\author{
Christian Strohm* Caren Tischendorf** \\ * Humboldt University of Berlin, Dept. of Mathematics, 10099 Berlin, \\ Germany (e-mail: strohmch@math.hu-berlin.de). \\ ** Humboldt University of Berlin, Dept. of Mathematics, 10099 Berlin, \\ Germany (e-mail: tischendorf@math.hu-berlin.de)
}

\begin{abstract}
Circuit simulators used in semiconductor industry are based on lumped element models described in form of net lists. In order to be able to incorporate the mutual electromagnetic influence of neighboring elements (e.g. cross talking), one needs refined models based on a sufficiently exact discretization of the full Maxwell equations. Here, we present an interface model for such refined models that can be used for lumped circuit net lists.
\end{abstract}

Keywords: circuit simulation, full-wave Maxwell equations, interface model, modified nodal analysis, net lists

\section{CIRCUIT EQUATIONS BASED ON NET LISTS}

The common approach for simulating circuits in semiconductor industry is the modified nodal analysis. It bases on the Kirchhoff's laws described by

$$
A \boldsymbol{i}=0, \quad \boldsymbol{v}=A^{\top} \boldsymbol{e}
$$

with the incidence matrix $A$ mapping branches to nodes of the circuit. The circuit variables are the vector $\boldsymbol{i}$ of all branch currents, the vector $\boldsymbol{v}$ of all branch voltages and the vector $\boldsymbol{e}$ of all nodal potentials. They are completed by the constitutive element equations

$$
\boldsymbol{i}_{1}=\frac{\mathrm{d}}{\mathrm{d} t} q\left(\boldsymbol{v}_{1}, t\right)+g\left(\boldsymbol{v}_{1}, t\right), \quad \boldsymbol{v}_{2}=\frac{\mathrm{d}}{\mathrm{d} t} \phi\left(\boldsymbol{i}_{2}, t\right)+r\left(\boldsymbol{i}_{2}, t\right)
$$

for lumped current and voltage controlling elements, respectively. Notice, all basic types as capacitances, inductances, resistances and sources are covered by a suitable choice of the functions $q, g, \phi$ and $r$. Performing the modified nodal analysis, we get the following reduced equation system having only the nodal potentials $e$ and the currents $i_{2}$ of the voltage controlling elements, see Estévez Schwarz and Tischendorf (2000):

$$
\begin{array}{r}
A_{1} \frac{\mathrm{d}}{\mathrm{d} t} q\left(A_{1}^{\top} \boldsymbol{e}, t\right)+A_{1} g\left(A_{1}^{\top} \boldsymbol{e}, t\right)+A_{2} \boldsymbol{i}_{2}=0, \\
\frac{\mathrm{d}}{\mathrm{d} t} \phi\left(\boldsymbol{i}_{2}, t\right)+r\left(\boldsymbol{i}_{2}, t\right)-A_{2}^{\top} \boldsymbol{e}=0,
\end{array}
$$

where the incidence matrix $A=\left(A_{1}, A_{2}\right)$ is split with respect to the current and voltage controlling elements. The equations (3)-(4) are generated automatically from net lists providing the node to branch element relation (for entries of $A_{1}$ and $A_{2}$ ) as well as the element related functions $q, g, \phi$ and $r$.

In order to be able to integrate electromagnetic field element models into such a net list description we are aiming at a description in a form of (2) for such models.

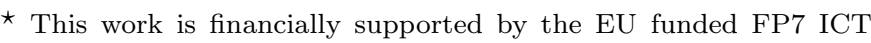
project nanoCOPS GA619166.
}

\section{FULL-WAVE MAXWELL EQUATIONS}

The electromagnetic fields can be described by the fullwave Maxwell's equations in potential formulation, see e.g. Meuris et al. (2001)

$$
\begin{aligned}
\nabla \cdot\left(\varepsilon \nabla \varphi+\varepsilon \partial_{t} \boldsymbol{A}\right) & =-\varrho \\
\nabla \times(\nu \nabla \times \boldsymbol{A})+\partial_{t}\left(\varepsilon \nabla \varphi+\varepsilon \partial_{t} \boldsymbol{A}\right) & =\boldsymbol{J}
\end{aligned}
$$

with the scalar potential $\varphi$ and the vector potential $\boldsymbol{A}$ as well as the pseudo-canonical momentum $\Pi=\partial_{t} \boldsymbol{A}$ to avoid the second-order time derivative. The material dependent parameters $\varepsilon$ and $\mu=1 / \nu$ are the permittivity and the magnetic permeability. The charge $\varrho$ and the current density $\boldsymbol{J}$ are given by the following model equations:

$$
\varrho= \begin{cases}0 & \text { for metal and isolator } \\ q\left(n-p-N_{D}\right) & \text { for semiconductor }\end{cases}
$$

and

$$
\boldsymbol{J}= \begin{cases}\sigma \boldsymbol{E} & \text { for metal } \\ \boldsymbol{J}_{\boldsymbol{n}}+\boldsymbol{J}_{\boldsymbol{p}} & \text { for semiconductor } \\ 0 & \text { for isolator }\end{cases}
$$

with the electron and hole current densities $\boldsymbol{J}_{\boldsymbol{n}}$ and $\boldsymbol{J}_{\boldsymbol{p}}$ as well as the electron and hole concentrations $n$ and $p$ satisfying

$$
\begin{aligned}
q_{e} \partial_{t} n-\nabla \cdot \boldsymbol{J}_{\boldsymbol{n}}+q_{e} R(n, p) & =0 \\
q_{e} \partial_{t} p+\nabla \cdot \boldsymbol{J}_{\boldsymbol{p}}+q_{e} R(n, p) & =0
\end{aligned}
$$

with

$$
\boldsymbol{J}_{\boldsymbol{n}}=q_{e} D_{n} \nabla n+q_{e} \mu_{n} n \boldsymbol{E}, \quad \boldsymbol{J}_{\boldsymbol{p}}=-q_{e} D_{p} \nabla p+q_{e} \mu_{p} p \boldsymbol{E} .
$$

The material depending parameters $N_{D}, \sigma, \mu_{n}$ and $\mu_{p}$ describe the doping concentration, the conductivity, the mobility of electrons and the mobility of holes. The function $R$ gives the recombination rate for electrons and holes. Finally, $q_{e}$ is the elementary charge and $D_{n}, D_{p}$ are the diffusion coefficients.

Note that the semiconductor current density model reflects the drift-diffusion model Markowich (1986) and should be 
extended by an additional current density part caused by the self-induced Lorentz force in case of circuits with fasttransient signals, see Schoenmaker et al. (2014).

\section{INTERFACE MODEL}

We assume the interface between the electromagnetic field model and the lumped circuit model to be perfectly electric conducting such that $\boldsymbol{B} \cdot n_{\perp}=0$ and $\boldsymbol{E} \cdot n_{\|}=0$ with $n_{\perp}$ and $n_{\|}$being the outer unit normal vectors transversal and parallel to the contact boundary. This motivates the boundary conditions, cf. Baumanns (2012),

$$
(\nabla \times \boldsymbol{A}) \cdot n_{\perp}=0, \quad(\nabla \varphi) \cdot n_{\|}=0 .
$$

Denoting by $\Gamma_{k}$ the $k$-th contact of the electromagnetic field model element with $\Gamma_{0}$ being the reference contact we get the current through $\Gamma_{k}$ as

$$
\boldsymbol{i}_{k}=\int_{\Gamma_{k}}\left[\boldsymbol{J}-\partial_{t}(\varepsilon(\nabla \varphi+\Pi))\right] \cdot n_{\perp} \mathrm{d} \sigma
$$

Note that equation (8) and the boundary condition (11) guarantee that the sum of all contact currents equals zero, that means

$$
\sum_{k} \boldsymbol{i}_{k}=0
$$

This model property is necessary for all lumped element descriptions in order to preserve the Kirchhoff's current law. In order to reveal the relation to the voltages $\boldsymbol{v}_{k}$ between the contact $\Gamma_{k}$ and the reference contact $\Gamma_{0}$, we express the potential $\varphi$ as

$$
\varphi(x, t)=\varphi_{b i}(x)+\varphi_{c}(x, t)
$$

with the contact potential

$$
\varphi_{c}(x, t)= \begin{cases}\boldsymbol{v}_{k} & \text { if } x \in \Gamma_{k} \\ 0 & \text { else. }\end{cases}
$$

Here, we assumed the reference contact $\Gamma_{0}$ to be the mass node for simplicity. The potential $\varphi_{b i}$ describes the position dependent built-in potential arising by varying doping concentrations and bonding different materials. Following the idea of the semiconductor circuit coupling description in Baumanns et al. (2014), we introduce for each contact $k$ a continuous auxiliary function $\omega_{k}$ satisfying the equation system

$$
\begin{gathered}
\nabla \cdot\left(-\varepsilon \nabla \omega_{k}\right)=0 \quad \text { on } \Omega_{m} \cup \Omega_{s} \cup \Omega_{i} \\
\left.\nabla \omega_{k} \cdot n_{\perp}\right|_{\Gamma_{n}}=0,\left.\omega_{k}\right|_{\Gamma_{c}}(x)= \begin{cases}1 & \text { if } x \in \Gamma_{k} \\
0 & \text { else }\end{cases} \\
\gamma_{m}\left(\left.\varepsilon \nabla \omega_{k}\right|_{\Omega_{m}}\right) \cdot n_{\perp}+\gamma_{s}\left(\left.\varepsilon \nabla \omega_{k}\right|_{\Omega_{s}}\right) \cdot n_{\perp}=0 \text { on } \Gamma_{m s} \\
\gamma_{s}\left(\left.\varepsilon \nabla \omega_{k}\right|_{\Omega_{s}}\right) \cdot n_{\perp}+\gamma_{i}\left(\left.\varepsilon \nabla \omega_{k}\right|_{\Omega_{i}}\right) \cdot n_{\perp}=0 \text { on } \Gamma_{s i} \\
\gamma_{i}\left(\left.\varepsilon \nabla \omega_{k}\right|_{\Omega_{i}}\right) \cdot n_{\perp}+\gamma_{m}\left(\left.\varepsilon \nabla \omega_{k}\right|_{\Omega_{m}}\right) \cdot n_{\perp}=0 \text { on } \Gamma_{i m}
\end{gathered}
$$

with the open domains $\Omega_{m}$ for metal regions, $\Omega_{s}$ for semiconducter regions and $\Omega_{i}$ for isolator regions as well as the interfaces $\Gamma_{m s}, \Gamma_{s i}$ and $\Gamma_{i m}$ between these regions. The contact boundary is denoted by $\Gamma_{c}=\sum_{k} \Gamma_{k}$ and the remaining boundary is called $\Gamma_{n}=\partial \Omega \backslash \Gamma_{c}$ with

$$
\Omega=\Omega_{m} \cup \Omega_{s} \cup \Omega_{i} \cup \Gamma_{m s} \cup \Gamma_{s i} \cup \Gamma_{i m} .
$$

The functions $\gamma_{m}, \gamma_{s}$ and $\gamma_{i}$ denote the trace operators mapping the functions on $\Omega_{m}, \Omega_{s}$ and $\Omega_{i}$ to functions on $\partial \Omega_{m}, \partial \Omega_{s}$ and $\partial \Omega_{i}$, respectively. The equation (13) does not have to be fulfilled for the following model description but it can be used to generate such auxiliary functions $\omega_{k}$ with help of the same discretization as used for the space discretization of the electromagnetic field equations.
Similarly to the proof in Baumanns et al. (2014), one can show that

$$
\begin{aligned}
\boldsymbol{i}_{k}= & \partial_{t} \boldsymbol{v}_{k} \int_{\Omega} \varepsilon \nabla \omega_{k} \cdot \nabla \omega_{k} \mathrm{~d} x \\
& +\partial_{t} \int_{\Omega} \varepsilon \Pi \cdot \nabla \omega_{k} \mathrm{~d} x-\int_{\Omega} \boldsymbol{J} \cdot \nabla \omega_{k} \mathrm{~d} x .
\end{aligned}
$$

The proof bases on two crucial observations. First, equation (6) provides

$$
\nabla \cdot\left(\partial_{t}(\varepsilon \nabla \varphi+\varepsilon \Pi)-\boldsymbol{J}\right)=0
$$

Secondly, the contact potential satisfies

$$
\varphi_{c}(x, t)=\sum_{k} \omega_{k}(x) \boldsymbol{v}_{k}(t)
$$

which implies

$$
\partial_{t}(\varepsilon \nabla \varphi)=\sum_{k} \partial_{t}\left(\varepsilon \nabla \omega_{k} \boldsymbol{v}_{k}\right)
$$

Introducing the matrix $M=\left(M_{k \ell}\right)$ and the vectors $\pi(t)=$ $\left(\pi_{k}(t)\right)$ and $j(t)=\left(j_{k}(t)\right)$ with

$$
\begin{aligned}
M_{k \ell} & :=\int_{\Omega} \varepsilon(x) \nabla \omega_{k}(x) \cdot \nabla \omega_{\ell}(x) \mathrm{d} x \\
\pi_{k}(t) & :=\int_{\Omega} \varepsilon(x) \Pi(t, x) \cdot \nabla \omega_{k}(x) \mathrm{d} x \\
j_{k}(t) & :=\int_{\Omega} \boldsymbol{J}(t, x) \cdot \nabla \omega_{k}(x) \mathrm{d} x
\end{aligned}
$$

we get from (18) the desired lumped description

$$
\boldsymbol{i}=\frac{\mathrm{d}}{\mathrm{d} t}(M \boldsymbol{v})+\frac{\mathrm{d}}{\mathrm{d} t} \pi-j .
$$

The equation (19) provides a lumped description for circuit elements described by the full Maxwell equations. In order to compute the volume integrals involved one can use any suitable space discretization of the full Maxwell equations in potential formulation, e.g. FIT discretization, cf. Clemens and Weiland (2002).

\section{REFERENCES}

Baumanns, S. (2012). Coupled Electromagnetic Field / Circuit Simulation. Modeling and Numerical Analysis. Logos Verlag, Berlin.

Baumanns, S., Jansen, L., Selva-Soto, M., and Tischendorf, C. (2014). Analysis of semi-discretized differential algebraic equation from coupled circuit device simulation. Computational and Applied Mathematics, 1-23.

Clemens, M. and Weiland, T. (2002). Magnetic field simulation using conformal FIT formulations. Magnetics, IEEE Transactions on, 38(2), 389-392.

Estévez Schwarz, D. and Tischendorf, C. (2000). Structural analysis of electric circuits and consequences for MNA. Internat. J. Circ. Theor. Appl., 28(2), 131-162.

Markowich, P. (1986). The Stationary Semiconductor Device Equations. Springer, Wien.

Meuris, P., Schoenmaker, W., and Magnus, W. (2001). Strategy for electromagnetic interconnect modeling. Computer-Aided Design of Integrated Circuits and Systems, IEEE Transactions on, 20(6), 753-762.

Schoenmaker, W., Chen, Q., and Galy, P. (2014). Computation of self-induced magnetic field effects including the Lorentz force for fast-transient phenomena in integrated-circuit devices. Computer-Aided Design of Integrated Circuits and Systems, IEEE Transactions on, 33(6), 893-902. 


\section{MATHMOD 2015 ABSTRACT VOLUME}

MATHMOD Minisymposium Modeling in Sport and Human Movement Science 
MATHMOD 2015 Abstract Volume 


\title{
Constants of Movement of Muscular Activation and Force-Velocity Relationship from nonlinear Optimization
}

\author{
H. Penasso* \\ * University of Graz, Institute of Sport Science, \\ 8010 Graz, Mozartgasse 14, Austria, harald.penasso@edu.uni-graz.at
}

\section{INTRODUCTION}

Just as a car needs an engine humans need skeletal muscles. With a better motor the car drives faster and optimized muscle properties lead to enhanced athletic performance. Whereas engines are exactly designed by engineers, human muscles mainly are trained without knowledge of the individual muscle parameters. To determine such parameters in vivo and non-invasively we have to convert externally observed values to internal ones. This can only be achieved with an appropriate transfer function and a mathematical muscle model. Some parameters in this model are measured directly. To determine the remaining parameter values we use nonlinear optimization strategies to match observed data and model data. Therefore, the model has to be robust and simple enough to guarantee a unique solution and it must incorporate the most important anatomical and physiological facts.

\section{METHODS}

The original approach was introduced by Sust (1987) and an improved model is described in Siebert et al. (2007). This method is extended by automatized input data corrections, standardized data selection, repeated minimization attempts from random initial values, and a smooth activation function that considers constant muscular pre-activation (Penasso, 2014).

\subsection{Model}

The model of a single leg-extension consists of an anthropometric-geometric function $G(X)$ that transfers external values to internal ones and of an activatable forceproducing muscle $f_{m}\left(v_{m}\right)$. Its force is determined by the force-velocity relation only (Hill, 1938). $G(X)$ depends on position data $X$ (distance between greater trochanter and lateral malleolus) and a simplified patellofemoral joint whereas the force of the muscle depends on contraction velocity $v_{m}$. Assuming that all muscular energy is transferred to the outside without any loss we get

$$
v_{m}=\dot{X} \cdot G(X)
$$

Ignoring all effects of mass we get a conversion from measured (external) force $F_{\text {meas }}$ and (external) velocity $\dot{X}$ to the internal muscle force $f_{m}$ :

$$
F_{\text {meas }}=G(X) \cdot f_{m}(X, \dot{X}) \text {. }
$$

To obtain muscle parameters measured data (from isometric and dynamic contractions) and model data must be in accordance. Including $N$ datasets of measured data per optimization attempt, measured values $c_{m, n}$, the function of activation dynamics $A(t)$, and the constants to obtain from optimization $c_{1 \ldots N+5}$ represent the cost function:

$$
\begin{aligned}
& G\left(X, c_{m, 1 \ldots 4}\right) \cdot A\left(t, c_{1 \ldots N+2}\right) \cdot f_{m}\left(X, \dot{X}, c_{N+3 \ldots N+5}\right) \\
& -F_{\text {meas }} \rightarrow \min .
\end{aligned}
$$

\subsection{Measured Input}

$F_{\text {meas }}$ is measured at an inclined leg-press (Tetra ${ }^{\circledR}$ Ilmenau) with a Kistler ${ }^{\circledR}$ force plate $(500 \mathrm{~Hz})$. The force plate is mounted onto a sledge whose position $X$ is recorded by a high frequency position sensor $(500 \mathrm{~Hz})$. Additional data from a goniometer placed lateral at the right knee $(500 \mathrm{~Hz})$ and data from a Vicon system with markers on both legs $(250 \mathrm{~Hz})$ are synchronized. The anthropometric lengths of the leg $c_{m, 1 \ldots 4}$ are manually measured with an accuracy of $\pm 5 \mathrm{~mm}$ with a tape measure.

From eq. 3 we see that the model strongly depends on position data. Therefore, the accuracy of the origin of ordinates must be monitored. First $X$ is shifted to match $X_{\text {(legs stretched) }}=c_{m, 1 \text { (length_thigh) }}+c_{m, 2 \text { (length_shank) }}$ and via the cosine rule a position mark is set to enforce an initial knee angle of $120 \mathrm{deg}$ for each contraction. Afterwards the goniometer is matched to this condition. All further deviations of these two systems must be due to a displacement of the subject or to a malfunction. To control that, the Vicon data is used to monitor displacements.

The data selection per contraction is first annotated manually by setting events in DEWESoft (the data acquisition software). The starting points of the contractions are set to a close prior period with constant force and remain unchanged at further processing. Endpoints of dynamic movements are automatically set when the force drops below the force at onset. For isometric contractions the data is cut where the force drops below $97 \%$ of the maximum of the actual contraction.

\subsection{Optimization}

All calculations are performed in Matlab ${ }^{\circledR}$. The optimization uses values from physiology as constraints for activation dynamics (Duchateau and Enoka, 2011) and force-velocity relation (Thaller and Wagner, 2004). To check the uniqueness of the minimum first an interior point algorithm (fmincon) searches the minimum from random initial guesses. Then a simplex algorithm (fminsearchcon, D'Errico (2012)) is started from random values within \pm 10 
$\%$ of the final values of the interior point (constraints are not narrowed down). This is repeated 20 times where the median of each parameter $c_{1 \ldots N+5}$ should have stabilized. An attempt is only accepted if its normalized mean squared error of the fit $(\in[-\inf , 1])$ is greater than the maximum of the previous values minus 0.01 ( $1 \%$ resp.). Finally the median of each $c_{1 \ldots N+5}$ is assumed to be the proper value.

\subsection{Statistics}

The goodness of the fit, $F_{\text {opt }}$ vs. $F_{\text {meas }}$, is assessed by the normalized mean squared error.

The statistics of the final set of parameters are based on D'Errico (2011, 2007); MathWorks (2014); Gavin (2013), and Yuen (2010, pp. 257-262). The following steps are performed:

(1) Computing the confidence interval of each $c_{1 \ldots N+5}$ :

(a) Degrees of freedom dof (sum of datapoints $N D$ ):

$$
d o f=N D_{F_{\text {meas }}}-(N+5)
$$

(b) Standard deviation of residuals $s d r$ :

$$
s d r=\sqrt{\sum\left(\left(F_{\text {meas }}-F_{\text {opt }}\right)^{2}\right) \cdot d o f^{-1}}
$$

(c) Jacobian matrix $\mathbf{J}$ from the model parameterized with $c_{1 \ldots N+5}$ (jacobianest.m, D'Errico (2011))

(d) Variance matrix $\boldsymbol{\sigma}^{2}$ :

$$
\boldsymbol{\sigma}^{2}=s d r^{2} \cdot\left(\mathbf{J}^{T} \cdot \mathbf{J}\right)^{-1}
$$

(e) The standard deviation of each parameter is calculated with the square root of the primary diagonal of $\boldsymbol{\sigma}^{2}$ :

$$
\sigma^{\star}=\left(\sqrt{\left(\sigma_{i j}^{2}\right)_{i, j=1 \ldots N D}}\right)^{T}
$$

(f) Ambiguous minima are considered by comparing $\sum \sigma^{\star}$ and $\sum \tilde{\sigma}$, which is calculated from repeated minimization attempts. Furthermore, only the greater of the both is used: $\sigma=\max \left\{\sum \sigma^{\star}, \sum \tilde{\sigma}\right\}$.

(g) The confidence interval is set to $5 \%$. To compute the quantile of each parameter $q_{c_{1 \ldots N+5}}$ we use a probability of $P=1-0.05 / 2=0.975$ with $V=N+5-1$ degrees of freedom as inputs for the Student's t inverse cumulative distribution function tinv.m.

(h) Finally the lower and upper bounds of each parameters' confidence interval $C I_{c_{1 \ldots N+5}}$ are computed with:

$$
C I_{c_{1 \ldots N+5}}=c_{1 \ldots N+5} \pm q_{c_{1 \ldots N+5}} \cdot\left(\frac{\sigma}{\sqrt{N+5}}\right)
$$

(2) Converting the three parameters from the forcevelocity relation $c_{1 \ldots N+5}$ to a set of other parameters describing this relation is done regarding the law of error propagation for standard deviations.

(3) Calculation of the parameter-correlation-matrix:

(a) Hessian matrix $\mathbf{H}$ from the model parameterized with $c_{1 \ldots N+5}$ (hessian.m (D'Errico, 2011)).

(b) The Covariance matrix Cov is the inverse matrix of the hessian $\mathbf{C o v}=\mathbf{H}^{-1}$ : (Yuen, 2010, p. 256). (c) Correlation matrix Cor (MathWorks, 2014):

$$
\operatorname{Cor}_{i, j}=\left\{\frac{\operatorname{Cov}_{i, j}}{\operatorname{Cov}_{i, i} \cdot \operatorname{Cov}_{j, j}}\right\}
$$

(4) The variance of each $i^{\text {th }}$ residual $\sigma_{i}$ of the fit is calculated according to Gavin (2013) Im.m line 287:

$$
\sigma_{i}^{2}=\left(\mathbf{J}_{i j}\right)_{i=i, j=1, \ldots, J} \cdot \operatorname{Cov} \cdot\left(\mathbf{J}_{i j}\right)_{i=i, j=1, \ldots, J}^{T}
$$

\section{DISCUSSION AND OUTLOOK}

This procedure provides a detailed insight into the optimization and its final parameters where the automatized routine itself ensures objectivity. Thus, we are able to determine individual muscle properties and it is now possible to use these results to address more complex questions. For example we will investigate the impact of fatigue and the influence of caffeine on the muscle activation parameters.

D'Errico, J. (2007). Standard errors of fminsearch parameters. URL http://www.mathworks.com/ matlabcentral/newsreader/view_thread/157530. Online: 17.03.2014.

D'Errico, J. (2011). Adaptive robust numerical differentiation. URL http: //www.mathworks.com/matlabcentral/ fileexchange/13490-adaptive-robust- $\backslash$ numerical-differentiation. Online: 12.03.2014.

D'Errico, J. (2012). fminsearchbnd, fminsearchcon. URL http://www.mathworks.com/matlabcentral/ fileexchange. Online: 11.03.2014.

Duchateau, J. and Enoka, R.M. (2011). Human motor unit recordings: origins and insight into the integrated motor system. Brain research, 1409, 42-61.

Gavin, H.P. (2013). The levenberg-marquardt method for nonlinear least squares curve-fitting problems. URL http://people.duke.edu/ \{\}hpgavin/ce281/ lm.pdf. Online: 17.03.2014.

Hill, A.V. (1938). The heat of shortening and the dynamic constants of muscle. Proceedings of the Royal Society (B), 126, 136-195.

MathWorks (2014). stats::correlationmatrix. URL http: //www . mathworks.de/de/help/symbolic/ mupad_ref/stats-correlationmatrix.html. Online: 17.03.2014.

Penasso, H. (2014). Activating muscles from pre-activation to MVC. In A. De Haan, C.J. De Ruiter, and E. Tsolakidis (eds.), BOOK OF ABSTRACTS, volume 19, 241. European College of Sport Science, Amsterdam, The Netherlands.

Siebert, T., Sust, M., Thaller, S., Tilp, M., and Wagner, H. (2007). An improved method to determine neuromuscular properties using force laws - from single muscle to applications in human movements. Human Movement Science, 26, 320-341.

Sust, M. (1987). Beitrag zum Aufbau einer axiomatischen Theorie der Biomechanik und Beispiele ihrer Anwendung. Habilitation, Friedrich-Schiller-Universitaet Jena.

Thaller, S. and Wagner, H. (2004). The relation between Hill's equation and individual muscle properties. Journal of theoretical Biology, 231, 319-332.

Yuen, K. (2010). Bayesian Methods for Structural Dynamics and Civil Engineering. Wiley. URL http://books. google.at/books?id=5Kr6ZvBpAHUC. 


\title{
Normalized and xPower to Generate Pacing Strategies in Road Cycling
}

\author{
T. Dahmen and D. Saupe
}

\author{
University of Konstanz, 78457 Konstanz, Germany.
}

\begin{abstract}
Normalized power and xPower have become standard measures for metabolic demand with road cycling. In contrast to average power these quantities account for the additional exertion resulting from time-variations of the pedaling power. In this contribution, we develop a method to use a slightly modified xPower to generate pacing strategies for road cycling time trials, where determining the optimal variations of the pedaling power is the ultimate goal. We tackle severe numerical issues related to singular arcs in optimal control, derive the necessary condition and use the Chebfun software system with its essential and unique capability to compute with Fréchet derivatives automatically.
\end{abstract}

Keywords: Pacing Strategy, Road Cycling, Optimal Control, Normalized Power, xPower

\section{INTRODUCTION}

Normalized power, Allen and Coggan [2005], and xPower are essential quantities in popular training analysis software such as TrainingPeaks and Golden Cheetah. In contrast to average power, these quantities are intended to account for the additional metabolic demand due to the variability of pedaling power during a ride.

The 3-Parameter Critical Power model (3PCPM), Morton [1996], has been used in conjunction with a mechanical bicycling model, Martin et al. [1998] to compute individual minimum-time pacing strategies for cycling time trials on a track with given slope profile, Dahmen [2012]. However, the 3 PCPM has been validated and is widely used to describe how long a cyclist can hold an arbitrary but timeconstant power only. The generalization to variable power required for the optimization is a venturous and unrealistic assumption.

If normalized power and xPower are beneficial replacements for average power when describing the metabolic effort of a ride, then they should be beneficial as replacement for pedaling power in the 3PCPM when generating optimal strategies.

In Dahmen [2012], it was pointed out that using the 3PCPM for the optimization leads to singular optimal control problems and a regularization of the model was necessary to obtain a solution using general-purpose optimal control software (GPOPS). These problems remain in this contribution. Therefore, we manually derive the necessary condition for the optimal control problem. Then we use a customized version of the Chebfun software system, Driscoll et al. [2014]. Its unique feature of automatic Fréchet differentiation, adopted from Chebfun, allows us to overcome these numerical issues properly.

\section{PROBLEM DEFINITION}

The kinetic energy $e_{\text {kin }} \in \mathbb{L}^{2}\left(\left[0, t_{f}\right]\right)$ and the remaining anaerobic resources $e_{\text {an }} \in \mathbb{L}^{2}$ are functions of time $t \in$ $\left[0, t_{f}\right]$, with $t_{f} \in \mathbb{R}$ being the final time. We put the variables into the vector $\boldsymbol{y}=\left(e_{\mathrm{kin}}, e_{\mathrm{an}}, t_{f}\right)^{\mathrm{T}}$ and seek

$$
\hat{\boldsymbol{y}}=\arg \min _{\boldsymbol{y} \in \mathcal{D}} t_{f}
$$

subject to the domain subspace $\mathcal{D}$. This subspace is defined by the equality constraints $\mathbf{L}(\hat{\boldsymbol{y}})(t)=0$ with

$$
\mathbf{L}=\left(\begin{array}{c}
\dot{e}_{\mathrm{kin}}+\dot{e}_{\mathrm{an}}+\mathrm{P}_{\text {res }}\left(x, e_{\mathrm{kin}}\right)-\mathrm{P}_{\mathrm{c}} \\
{\left[e_{\text {kin }}\right]^{0}} \\
{\left[e_{\text {an }}\right]^{0}-\mathrm{E}_{\text {an }}} \\
\int_{0}^{t_{f}} \sqrt{\frac{2 e_{\mathrm{kin}}}{\mathrm{m}}} \mathrm{d} t-\mathrm{x}_{f}
\end{array}\right),
$$

as well as the inequality path constraints $\mathbf{N}(\hat{\boldsymbol{y}})(t) \geq \mathbf{0}$ with

$$
\mathbf{N}=\left(\begin{array}{c}
e_{\mathrm{an}} \\
\dot{e}_{\mathrm{an}}+\mathrm{P}_{\max }-\mathrm{P}_{\mathrm{c}}
\end{array}\right)
$$

Note that $\mathrm{L}_{1}$, the first component of $\mathbf{L}$ represents a path equality constraint whereas the last three components represent functionals.

We parametrize the problem using the kinetic energy $e_{\text {kin }}$ as the variable function instead of the speed $v=$ $\sqrt{2 e_{\mathrm{kin}} / \mathrm{m}}$, thus avoiding a singularity that arises when $\dot{v}=\left(P-P_{\text {res }}\right) /(m v)$ is undefined for $v=0$.

The differential constraint $\mathrm{L}_{1}$ comprises both the mechanical model $\dot{e}_{\text {kin }}=P-P_{\text {res }}$ and the physiological model $\dot{e}_{\text {an }}=\mathrm{P}_{\mathrm{c}}-P$. Thereby, we have eliminated the pedaling power $P$.

The mechanical model stems from Martin et al. [1998]. We have $\mathrm{P}_{\text {res }}=F_{\text {res }} v$ with the resistance force

$$
F_{\text {res }}=\operatorname{mgh}^{\prime}(x)+\mathrm{k}_{1}+\mathrm{k}_{2} v+\mathrm{k}_{3} v^{2} .
$$

The constants $\mathrm{m}, \mathrm{g}$, and $\mathrm{h}(x)$ represent the mass of the cyclist and the bike, gravity, and the slope profile, respectively. The fixed parameters in $\mathbf{k}$ are coefficients quantifying friction and areal drag.

The physiological model $\dot{e}_{\mathrm{an}}=\mathrm{P}_{\mathrm{c}}-P$ originates from Morton [1996]. It quantifies the anaerobic resources $e_{\text {an }}$ decreasing at a rate equal to the amount by which the pedaling power $P$ exceeds the critical power $\mathrm{P}_{\mathrm{c}}$. 
The functional constraints $\mathrm{L}_{2}-\mathrm{L}_{4}$ enforce zero initial kinetic energy, initial anaerobic resources equal to $\mathrm{E}_{\mathrm{an}}$ (characteristic for the cyclist), and that the total distance covered at the final time be equal to the length of the track, $\mathrm{x}_{f}$.

Furthermore, $\mathbf{N}$ ensures nonnegative anaerobic resources throughout the ride and limits the pedaling power $P=$ $\mathrm{P}_{\mathrm{c}}-\dot{e}_{\text {an }}$ by some fixed maximum power, $\mathrm{P}_{\max }$.

To compute normalized power $N \in \mathbb{R}$ referring to any section $t \in\left[t_{a}, t_{b}\right]$ of a ride, the $\tau$-moving average $Q_{N} \in \mathbb{L}^{2}$ of the power function with $\tau=30 \mathrm{~s}$ is raised to the fourth power and then the fourth root of the average of the result is defined as normalized power:

$$
N=\sqrt[4]{\int_{t_{a}}^{t_{b}} \frac{Q_{N}^{4}(t)}{t_{b}-t_{a}} \mathrm{~d} t} .
$$

With the modification xPower $X \in \mathbb{R}$, the average $Q_{X}(t)$ is exponentially weighted with the time constant $\tau$. Mathematically, xPower has the advantage that $\dot{Q}_{X}=P-Q_{X}$ applies.

We modify xPower further by changing the order of averaging and raising to the fourth power to obtain $\dot{Q}(t)=$ $P^{4}(t)-Q(t)$ with $Q \in \mathbb{L}^{2}$ being instantaneous modified $x$ Power. We add $Q$ to the variable vector $\boldsymbol{y}=$ $\left(e_{\mathrm{kin}}, e_{\mathrm{an}}, Q, t_{f}\right)^{\mathrm{T}}$ and modify the differential equality constraint operator:

$$
\mathrm{L}_{1}=\dot{Q}-\dot{e}_{\text {an }}+\mathrm{P}_{\mathrm{c}}-\left(\dot{e}_{\text {kin }}+P_{\text {res }}\left(e_{\text {kin }}\right)\right)^{4},
$$

Moreover, we have to add the functional

$$
\mathrm{L}_{5}=[Q]^{0}
$$

to ensure that the cyclist is rested at the beginning.

\section{NECESSARY CONDITION AND IMPLEMENTATION}

Note that the cost functional can be expressed as $t_{f}=$ $\int_{0}^{t_{f}} \mathrm{~F} \mathrm{~d} t$ with the trivial running cost $\mathrm{F}=1$. Generally, if $\hat{\boldsymbol{y}}$ is optimal, then the direction of steepest ascent of the running cost $\mathrm{F}(\hat{\boldsymbol{y}})$ must necessarily be perpendicular to the hyper-surfaces $\mathbf{L}(\hat{\boldsymbol{y}})=0$. In other words the direction of steepest ascent of $\mathrm{F}$ and each component of $\mathbf{L}$ must be co-linear. In addition, for each point in time, the inequality constraints can either be active or inactive, i.e., $\mathbf{N}(\hat{\boldsymbol{y}})(t)=0$ or $\mathbf{N}(\hat{\boldsymbol{y}})(t)>0$. If a component of $\mathbf{N}$ is active, then the direction of steepest ascent of $\mathrm{F}$ must be co-linear with the corresponding component of $\mathbf{N}$, too.

We introduce the adjoint variables $\lambda$ with $\lambda_{1} \in \mathbb{L}^{2}$ and $\left(\lambda_{1}, \lambda_{2}, \lambda_{3}\right)^{\mathrm{T}} \in \mathbb{R}^{3}$ as well as $\boldsymbol{\nu} \in\left(\mathbb{L}^{2}\right)^{2}$ and form the augmented cost functional $\int_{0}^{t_{f}} \mathrm{H} \mathrm{d} t$ with the Hamiltonian operator

$$
\mathrm{H}=\mathrm{F}+\boldsymbol{\lambda}^{\mathbf{T}} \mathbf{L}+\boldsymbol{\nu}^{\mathbf{T}} \mathbf{N}
$$

The Hamiltonian operator $\mathrm{H}$ comprises all information of our problem. Note that for the functional derivatives $\delta \mathrm{H} / \delta \boldsymbol{\lambda}=\mathbf{L}$ and $\delta \mathrm{H} / \delta \boldsymbol{\nu}=\mathbf{N}$ apply by construction. Thus, $\mathbf{L}$ and $\mathbf{N}$ determine the sensitivity of $\mathrm{H}$ with respect to their corresponding adjoint variable.

Similarly the adjoint operators $\delta \mathrm{H} / \boldsymbol{\delta} \boldsymbol{y}=\left((\mathbf{L}, \mathbf{N})^{\mathrm{T}}\right)^{*}$ determine the sensitivity of $\mathrm{H}$ with respect to the primal variables.
If a component $\mathrm{N}_{i}$ is inactive on some interval, then $\nu_{i}=0$ on that same interval, otherwise $\nu_{i}>0$. In other words $\mathrm{N}_{i}(\hat{\boldsymbol{y}})$ and $\nu_{i}$ are complementary $\mathrm{N}_{i}(\hat{\boldsymbol{y}}) \perp \nu_{i}$. We now seek the dual solution that in addition satisfies the adjoint constraints $\delta \mathrm{H} / \delta \hat{\boldsymbol{y}}(t)=\mathbf{0}$.

Generally, the dual problem represents a multipoint boundary value problem with split boundary conditions. In contrast to our original problem, the dual problem has the property that it is complete. I.e., if split at the junctions where either $\mathrm{N}_{1}$ or $\mathrm{N}_{2}$ change from active to inactive or vice versa, on each interval between the junctions, it is guaranteed that the number of functional constraints to determine the solution is correct.

Discretizing a complete linear boundary value problems using spectral collocation results in a well-posed linear equations system that has a unique solution. Our nonlinear problem may be solved by a sequence of NewtonKantorovich iterations. This approach is taken by the Chebfun software system, Driscoll et al. [2014], which we make use of. Its unique feature of automatic Fréchet differentiation, is essential for the computations.

\section{CONCLUSIONS}

We emphasize that this approach is still work in progress since we strive to integrate our manual derivation into the automatic differentiation of the Chebfun system. Moreover, our Newton-Kantorovich iteration is not yet as robust as Chebfun's original implementation since we have not yet integrated the step size control.

Clearly, it remains to implement the algorithm for the original definition of xPower and Normalized Power. Those approaches are more complex since they involve delay differential equation. Eventually, we plan to perform tests on our bicycling simulator to assess if using any of the variants of normalized and xPower is beneficial for the generation of minimum-time pacing strategies in practice.

Our bicycle simulator is already capable of showing the open-loop optimal strategy to the athlete, but as we expect that an athlete cannot always follow exactly a strategy that is at the limit of his physical capacity, a closed loop feedback control based on model predictive control is desirable for practical use in future.

\section{REFERENCES}

Allen, H. and Coggan, A.R. (2005). Training and Racing with a Power Meter. velopress.

Dahmen, T. (2012). Optimization of pacing strategies for cycling time trials using a smooth 6-parameter endurance model. In Pre-Olympic Congress on Sports Science and Computer Science in Sport. IACSS Press.

Driscoll, T.A., Hale, N., and Trefethen, L.N. (2014). Chebfun Guide. Pafnuty Publications.

Martin, J.C., Milliken, D.L., Cobb, J.E., McFadden, K.L., and Coggan, A.R. (1998). Validation of a mathematical model for road cycling power. 14, 276-291.

Morton, R.H. (1996). A 3-parameter critical power model. $39(4), 611$. 


\title{
Towards Data-Based Assessment of Individual Tactics Skills in Team Sports Based on Fuzzy Petri Nets
}

\author{
Andrea Serafini*, Roland Leser**, \\ Thomas Hoch***, Bernhard Moser***, Arnold Baca** \\ *Research Institute of Symbolic Computation, Hagenberg, Austria; \\ (e-mail: andrea.erafini@scch.at). \\ **Centre of Sport Science and University Sports, Universtiy of Vienna (e-mail: roland.leser@univie.ac.at) \\ *** Software Competence Center Hagenberg GmbH, \\ Austria, (e-mail: bernhard.moser,thomas.hoch@scch.at)
}

\begin{abstract}
The problem of objectively assessing activity patterns of individual players in team sports in terms of tactics skills is addressed by employing concepts from fuzzy logics and state-transition graph models. This approach aims at providing high level descriptions of activity patterns based on local positioning measurements. Mackenzie \& Cushion (2013) conclude in their critical review about current performance analysis (PA) research that variables are often investigated as a result of availability rather than to develop a deeper understanding of performance. Therefore, PA studies often fail to provide useful information for the coaching practice due to the multifaceted, complex and largely unpredictable behaviour in team sports like football. In the author's opinion holistic approaches are needed that include more naturalistic and qualitative methods such as case studies and interviews. The overall goal is to develop a data-based approach based on position and tracking data provided by local positioning measurement systems (see, e.g., Leser et al. (2011), Mackenzie \& Cushion (2013), Ogris et al. (2012)) that allows the qualification of assessments based on measurements. Tactical assessments are typically semi-structured and, above all, expressed in qualitative, vague and imprecisely defined linguistic concepts. Moreover, often such assessment statements rely on implicit common-sense and intuitive reasoning assumptions. Therefore, we postulate that the knowledge representation has to satisfy the following properties: K1) the vagueness and imprecision related to temporal, spatial and spatio-temporal relationships have to be expressed in a form that is appropriate for human experts and users; K2) the knowledge base design should allow a hierarchical representation of the scenarios at different abstraction levels; K3) the design has to be based on a well-defined formalism and semantics that allows a formal analysis of different spatial, temporal and spatio-temporal relationships among the objects and conclusions based thereupon. Further, from the point of view of feasibility and practical usefulness, we postulate that the design of the knowledge base allows D1) a modular, object-oriented programming paradigm based approach in the sense that the overall knowledge base results from designing the knowledge bases for each player independently and, in a second step, the interaction options between the players, D2) an efficient evaluation of hypotheses for real-time applications, D3) to visualize at least small or moderate-sized parts of the knowledge base by intuitive graphical representation models.
\end{abstract}

Our approach is inspired by the philosophy of Carl Adam Petri (Petri (1962)) by looking for ways to decompose complex processes into a scheme of less complex state-transition process units. Mathematically, this approach led to the introduction of Petri nets and its variants, which are directed bipartite graphs made up by state and transition nodes. For example, in a chemical process chemical substances are represented by states while chemical reactions are treated as transitions between the states. The dynamics of such nets are modelled by so-called tokens which are handed over from states to other states via transition rules. As a formal, graphical, executable technique Petri nets are used for the specification, modelling and analysis of concurrent, discrete-event dynamic systems (Rozenburg \& Engelfriet (1998)). For an overview of applications see, e.g., Chen et al. (1990), Christensen \& Kristensen (2003) or Girault \& Valk (2003). Petri nets and its variants (Jensen (1991), Cardoso et al (1996), Pan $\&$ Sun (2007), Jensen et al. (2007)) meet the outlined requirements [K1]-[K3] and [D1]-[D3]. However, such a discrete event dynamic system formalism can only be applied in a reasonable way when the systems behaviour can be decomposed into separate states with well-defined conditions for state transitions. This means that all states, transitions and conditions need to be known in advance and be well defined. This brings us to the central question what are adequate states and transitions in the context of team sport. Let us point out that the discreteness of events is also a matter of the abstraction level and the context. For example, at the level of ball possession we can easily decompose the scenario into the players' positions (states), e.g., player A possesses ball approaching the goal, player B is approaching player A, some interactions (transition), e.g. duel between player A and $\mathrm{B}$, which leads to new states, e.g., B wins the ball. On this level the process of possessing, losing and winning the ball can be adequately described by a Petri net model and one can make statistical evaluations by e.g. counting the number of successful duels of a player. Obviously, such evaluations become more informative when taking circumstances and spatio-temporal characteristics of the duels into account, e.g., whether there is much pressing or not; duration of the duel; speed of the players; location on the field etc. Pressing is an example for an attribute whose occurrence is not crisply defined rather it is fuzzy (Zadeh (1965), Zadeh (1975)). Also, though spatio-temporal variables like location, duration, speed etc. are crisp in nature, for statistical evaluation and interpretation purposes clusters like "short versus long duel" are more informative in order to provide 
discrete distinguishable descriptions of situations. This motivates the introduction of fuzzy spatio-temporal relations. Ribaric \& Hrkac (2012) proposes a high-level Petri net in the context of robot soccer where the states are qualified by evaluating fuzzy spatio-temporal relations. We extend this concept by taking fuzzy context attributes into account and discuss its feasibility and usefulness. In particular, we address the question whether such a context-sensitive spatio-temporal modelling approach is feasible for real soccer scenarios from the point of view of setting up and running such models from a practical engineering point of view and meeting the requirements [K1]-[K3] and [D1]-[D3]. On a theoretical level, however, the feasibility issue also comprises questions regarding verifiability, completeness, stability, expressiveness and model-calibration. In a first step we tackle these questions by specifying experimental test scenarios that take local positioning measurement data and human trainer based assessments into account. In a second step we conduct a semi-formal analysis based on simulations. For this purpose, oneversus-one football games with defined game situations are considered in order to generate a representative quantity of data sets. Simultaneously, expert oriented judgements of individual player performances are collected from coaches. By means of interviews and content analyses the criteria for the expert judgements are gathered (Tenenbaum \& Driscoll (2005)). As an example, for the action "pass" the criteria preparation, decision and quality as well as the difficulty of the situation (defined by the opponent pressing and the size of the passing gap) are considered.

Keywords: Discrete and Discrete-Event Systems, Discretisation of Continuous Systems, Modelling Fuzzy Systems and Fuzzy Modelling, Petri Nets, Fitting Models to Real Processes, Uncertainties in Systems and in Modelling, Multiscale Modelling

\section{REFERENCES}

Alvarez-Alvarez, A., Trivino, G., Cordon, O. (2012). Human Gait Modeling Using a Genetic Fuzzy Finite State Machine. In Proc. of 2012 IEEE Transactions on Fuzzy Systems, 20(2), pp. 205-223.

Christensen, S., Kristensen, L.M. (2003). State Space Analysis of Hierarchical Coloured Petri Nets. In W.v.d. Aalst, J.-M. Colom, F. Kordon, G. Kotsis, and D. Moldt. Petri Net Approaches for Modelling and Validation. LINCOM Studies in Computer Science, No. 1, pp. 1-16.

S. M. Chen, J. S. Ke, J. F. Chang (1990). Knowledge representation using fuzzy Petri nets, IEEE Trans. Knowledge and Data Engineering, vol. 2, no. 3, pp. 311-319.

J. Cardoso, R. Valette, D. Dubois (1996). Fuzzy Petri net: An overview, In Proc. 13th IFAC World Congr., pp. $443-448$.

Jensen, K. (1991). Coloured Petri Nets: A High-level Language for System Design and Analysis. In G. Rozenberg (Ed.): Advances in Petri Nets 1990, Lecture Notes in Computer Science, vol. 483, Springer-Verlag, pp. 342-416.

Jensen, K., Kristensen, L. M., Wells, L. M. (2007). Coloured Petri Nets and CPN Tools for Modelling and Validation of Concurrent Systems, International Journal on Software Tools for Technology Transfer, vol. 9, nr. 3/4, pp. $213-254$.

C. Girault, R. Valk (2003). Petri Nets for Systems Engineering. Springer-Verlag.

Leser, R., Baca, A., Ogris, G. (2011). Local Positioning Systems in (Game) Sports. Sensors, 11, pp. 9778-9797.

Mackenzie, R., Cushion, C. (2013). Accuracy of the LPM tracking system considering dynamic position changes. Journal of Sports Sciences, 31, pp. 639-676.

Ogris, G., Leser, R., Horsak, B., Kornfeind, P., Heller, M., Baca, A. (2012). Accuracy of the LPM tracking system considering dynamic position changes. Journal of Sports Sciences, 30, pp. 1503-1511.

Petri, C.A. (1962). Kommunikation mit Automaten. Dissertation, Institut für instrumentelle Mathematik der Universität Bonn.

H. Pan, J. Sun (2007): Complex Knowledge System Modeling Based on Hierarchical Fuzzy Petri Net. In Proc. 2007 IEEE/WIC/ACM International Conferences on Web Intelligence and Intelligent Agent Technology - Workshops (WI-IATW '07). IEEE Computer Society, Washington, DC, USA, pp. 31-34.

G. Rozenburg, J. Engelfriet (1998). Elementary Net Systems, in: W. Reisig, G. Rozenberg (Eds.), Lectures on Petri Nets I: Basic Models - Advances in Petri Nets, volume 1491 of Lecture Notes in Computer Science, Springer, pp. 12-121.

S. Ribaric, T. Hrkac (2012). A model of fuzzy spatio-temporal knowledge representation and reasoning based on high-level Petri nets, Information Systems, Volume 37, Issue 3, pp. 238-256.

Tenenbaum, G., Driscoll, M. (2005). Methods of Research in Sport Sciences: Quantitative and Qualitative Approaches. Oxford: Meyer \& Meyer.

Zadeh, L. A. (1965). Fuzzy Sets. Information and Control, 8(3), pp. 338-353.

Zadeh, L. A. (1975). Fuzzy Logic and Approximate Reasoning, Synthese, 30(3-4), pp. 407-428. 


\title{
Analysis of tactical defensive behavior in team handball by means of artificial neural networks
}

\author{
M. Tilp*, N. Schrapf* \\ *University of Graz, Institute of Sports Science, Mozartgasse 14, 8010 Graz, Austria, \\ (Tel.+43(0)3163808332; Email: markus.tilp@uni-graz.at; norbert.schrapf@uni-graz.at)
}

Keywords: Game analysis, sports, tactics, sports games

\section{INTRODUCTION}

Besides the physical development of athletes, the analysis of tactics in team sports is an important factor for success. In the last decades, sports scientists have developed several systems to determine successful team tactics. A generic approach to assess the tactical behaviour of a player or a team is to count specific actions. This can be made manually by simple tally counts or with the help of a computer system. Typical results from such analyses are absolute or relative frequencies of actions or success rates respectively. Such results are helpful for athletes and coaches for feedback or tactical decisions respectively. However, the recording of single actions does not allow getting insight into the context of the specific game situation which very often is a key point to success. Another challenge in the analysis of team sports is to get enough data entries in a specific category (of single actions) due to the numerous different possibilities of game situations, e.g. an action of the same soccer player in the exactly same position on the court is highly improbable. Thus, classic statistical methods have difficulties to analyse similar but not identical data during the analyses of sports games. A possible solution for this is the use of fuzzy logic or artificial neural networks to be able to detect patterns in the tactical behaviour. In previous own studies we focused on the identification of offensive playing patterns in team handball by means of artificial neural networks (Schrapf \& Tilp, 2013). Patterns were found in the offensive action sequences which consisted of position data of the shot and its five previous passes. However, offensive behavior is always related to the defensive behavior of the opponent team. In order to analyse the defensive behaviour of handball teams we used a similar approach to find patterns based on the position data of the defending team at the instant of the shot attempt of the opponent team.

\section{METHODS}

Data was retrieved from twelve handball games of the European Under-18 Men Championship in 2012. We used an eight-camera-system to record videos of the games which were analysed in a post hoc process with the help of custom-made software. The basis of the analysis was position data of the defensive players at the instant of the shot and the shot position of the offensive player. Accurate metric positions of the team handball players were obtained by a plane-to-plane projective coordinate transformation (homography). A detailed description of the measurement system and its validity is presented elsewhere (Rudelstorfer et al., 2014). In summary, 728 actions were annotated. Each of these 728 data sets consists of a vector with the player coordinates. In a subsequent step the data was analysed by artificial neural network software (Perl, 2002) in order to find different position patterns. To obtain sufficient entropy, data was multiplicated to a quantity of 7280 datasets, applying a noise of $5 \%$ and subsequently permutated to minimize unwanted learning effects. The neural network had a dimension of 400 neurons. In the training process of the network data sets are related to specific neurons. Similar data sets are related to the same neurons based on the predefined parameters tolerance and similarity resolution. The tolerance defines the similarity within single neurons while the similarity resolution defines the similarity within clusters which are groups of neighbouring neurons. A cluster represents similar defensive behaviour. Based on 
previous analyses tolerance was set to 9.0 and similarity resolution was set to 75 .

\section{RESULTS}

In the training process the artificial neural network determined 18 clusters and 3 single neurons which could not be assigned to a cluster. Thus, we found 21 different patterns of defensive behaviour (Fig. 1).

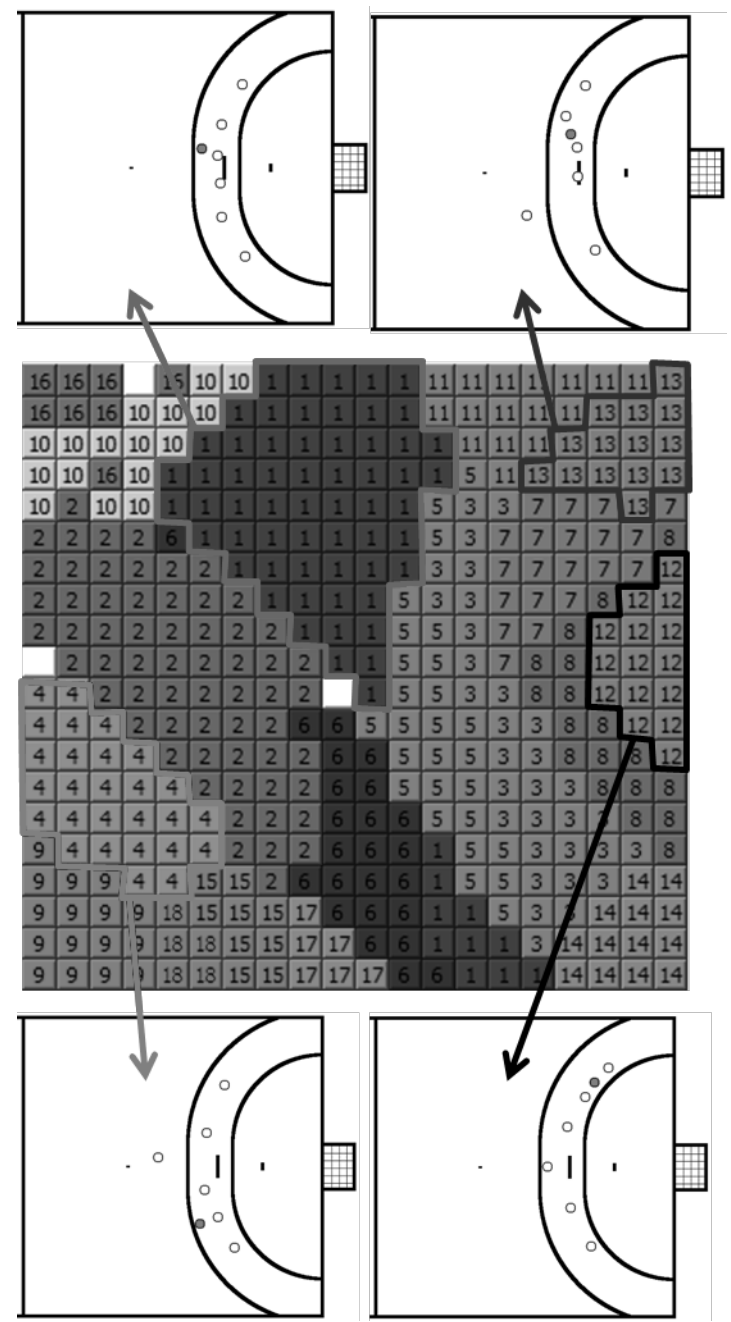

Fig. 1. Result of the training process. The clusters in the output layer of the network are represented by neurons with same number. Exemplarily, positions of the defensive players (open circles) and the position of the shot (closed circle) representing clusters $1,4,13$, and 14 are presented. Clusters 13 and 4 represent a pattern with one defender taking over an offensive role (e.g. for a counter-attack).

The determined patterns coincide with well-known defensive behaviour which is affected by the position areas of the shot. We determined two different patterns against shots from the right wing and left back position, three from the left wing, the right back, and the pivot position, and five for shots from the centre back. Furthermore, defensive formations with or without an offensive acting defensive player were discriminated.

\section{DISCUSSION}

It could be shown that artificial neural networks are capable of determining defensive patterns in team handball based on player's position. The analysis revealed that clusters represent defensive behaviour against different shot positions, distance of the defending team to the own goal, and width of the defensive

In a future project we intend to combine active and passive patterns to determine interaction between defensive and offensive teams (Fig. 2).

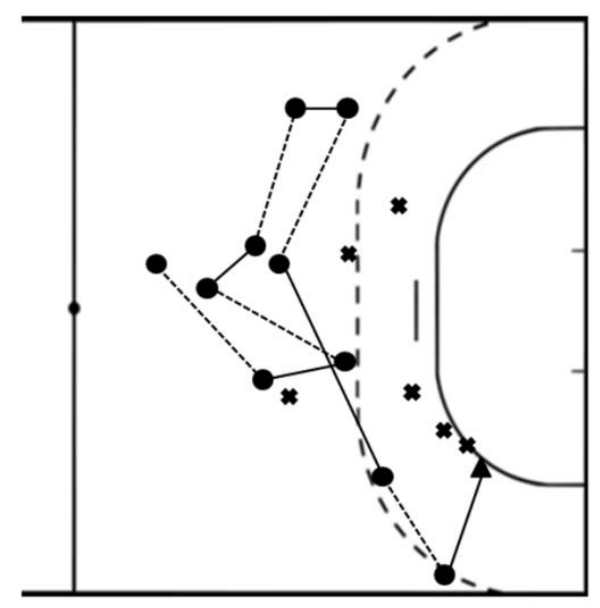

Fig. 2. Combination of an offensive action sequence, represented by the shot position (triangle) and previous five passes (circles, dashed and solid lines represent ball path and running path resp.) and defensive position at the instant of the shot (crosses).

\section{REFERENCES}

Schrapf, N. and Tilp, M. (2013). Action sequence analysis in team handball, Journal of Human Sport and Exercise, 8(3), 615-621.

Rudelsdorfer, P., Schrapf, N., Possegger, H., Mauthner, T., Bischof, H. and Tilp M. (2014). A novel method for the analysis of sequential actions in team Handball. International Journal of Computer Science in Sports, (13)/1. 69-84.

Perl, J. (2002). Game analysis and control by means of continuously learning networks. International Journal of Performance Analysis in Sports, 2. 2135. 


\title{
Recurrence Analysis of golfers' performances on the PGA TOUR
}

\author{
Michael Stöckl \\ Institute of Sport Science, University of Vienna, Auf der Schmelz 6a, \\ 1150 Vienna (e-mail: michael.stoeckl@univie.ac.at).
}

\section{INTRODUCTION}

In many sports athletes, experts, and supporters believe that "success breeds success and failure breeds failure" (Bar-Eli et al., 2006, p. 525). In particular, the psychologial concept of momentum expects an athlete to perform quite well during a certain period and better than this athlete is expected to be able to perform. There are several studies which investigated the existence of this phenomenon in different sports, amongst others, golf which is analyzed in this study. Golf itself is appropriate to analyze whether 'hot hands' or streaks of performance independent of the quality exist. Golfers are not allowed to interfere the performance of opponents and each golfer is in control of his/her performance. Hence, it is reasonable to think that factors such as momentum exist which can lead to streaks of (exceptional) performance.

To date, there are several studies investigating the existence of performance streaks on the US PGA TOUR in golf (e.g. Clark , 2005; James , 2007; Livingston , 2012), but there is only weak support for the concept of streakiness in professional golf. However, all those studies were limited to round or hole scores due to the data available. Since 2003 information on nearly every shot taken on PGA TOUR tournaments is stored in the ShotLink ${ }^{\mathrm{TM}}$ database. The existence of data describing individual shots played on the PGA TOUR led to the development of new models, such as the ISOPAR Method (Stöckl et al., 2012), which allow assessing the quality of individual shots. The ISOPAR Method provides the performance indicator Shots Saved which quantifies the quality of a stroke based on its the difficulty. Therefore, Shots Saved provides the opportunity to analyze the concept of momentum in golf on a stroketo-stroke basis.

The approach of the ISOPAR method is based on "a systems perspective which has been empirically applied to many levels of analyses of human movement and performance" (Stöckl et al., 2012, p.11). Amongst others, this approach involves the idea that human beings behave as complex dynamical systems. Hence, other than in previous studies on streakiness in golf, in this study a non-linear analysis tool is used - recurrence quantification analysis (RQA). RQA is based on the idea of recurrence plots (RP), a tool which was developed to analyze the behavior of complex dynamical systems (for an overview see Marwan et al., 2007).

\section{METHODS}

The study is based on data provided by the ShotLink ${ }^{\text {TM }}$ database. Data from 31 PGA TOUR tournaments from 2011 were used to analyze the performances of the golfers $(n=2158)$ playing four rounds in those tournaments.

The analyses were conducted in two steps using MATLAB. First, for each tournament Shots saved values were calculated for each shot taken during those tournaments using the ISOPAR Method (Stöckl et al., 2012). For each tournament for each golfer the Shots Saved values from the tournament constitute a stroke series - those stroke series contain either all shots of a golfer or only shots from a certain shot category (drives, long approach shots, or putts) of a golfer. Other shot categories such as around the green shots or short approach shots were not considered because for those categories not enough shots existed to calculate reliable RPs.

In a second step, recurrence plots (using time delay $\tau=$ 2 , embedding dimension $\mathrm{m}=2$, and threshold $\epsilon=0.14$ ) were calculated based on each golfer's stroke series. Subsequently, RQAs were performed to analyze the structure of each RP. Vertical lines in a RP indicate that the dynamical system behaved similar for a certain time (Marwan et al., 2007). In terms of golf, a vertical line represents a streak of performances similar to each other lasting for a certain time represented by the line length. The following RQA measures were used for the analyses:

- Recurrence Rate (RR) describes the percentage of recurrence points in a $R P$.

- Laminarity (LAM) is the percentage of recurrence points which form vertical lines.

- Trapping Time (TT) is the average length of vertical lines.

- Maximum vertical line length $\left(\mathrm{V}_{\max }\right)$

- Recurrence time of first type $\left(\mathrm{T}^{1}\right)$ describes the average number of strokes until a performance recurs if it recurs at all

- The percentage of not recurring performances $\left(\left(\mathrm{T}^{1}{ }_{\text {not }}\right)\right.$

Finally, those measures were statistically analyzed using SPSS 22 to gain insight into whether streakiness in golf exists on a stroke-to-stroke basis independent of the quality of those strokes.

\section{RESULTS \& DISCUSSION}

Fig. 1 shows an example RP of K.J. Choi, winner of THE PLAYERS Championship 2011, considering all his shots 


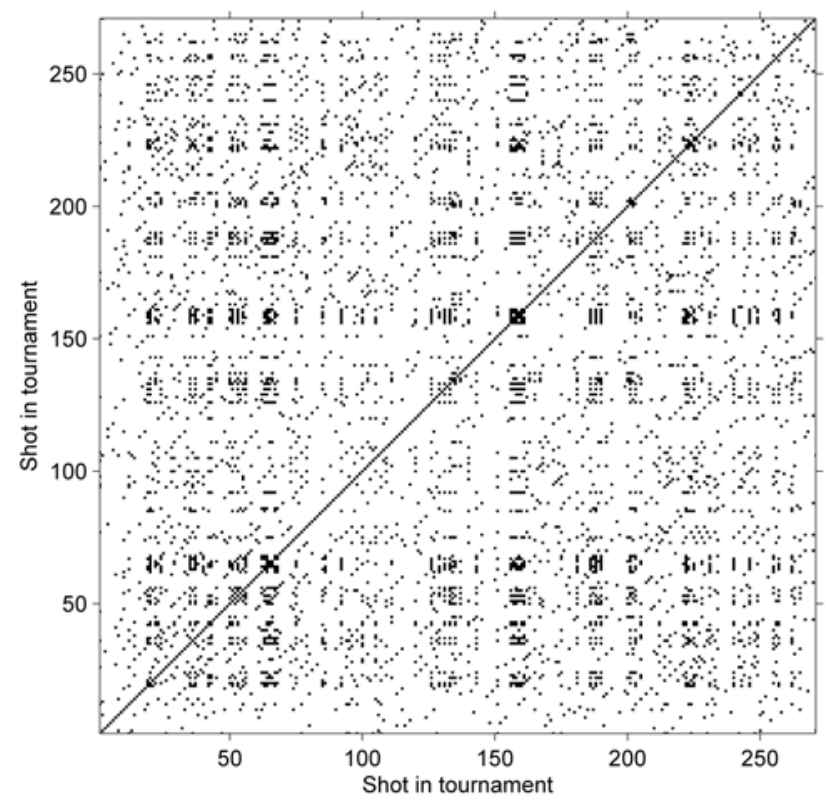

Fig. 1. Recurrence plot of K.J Choi, winner of THE PLAYERS Championship 2011

from this tournament. A black dot represents the similarity of two states, otherwise they are not similar. Based on such RPs RQAs were conducted for different stroke series, either comprised by all shots of a golfer or by all shots from a certain shot category of a golfer.

The descriptive results of the analysis considering all shots are listed in table 1 . They indicate that there are not too many recurring performances, however, a fifth of those form vertical lines. The vertical lines are short on average (mean TT $=2.3$ ), although there are longer streaks of similar performance which occur rarely. On average it took 10.3 strokes until a performance recurred if it recurred at all (9.1\% of a golfer's performances did not recur during a tournament).

Table 1. Descriptive results of the used RQA measures considering all shots

\begin{tabular}{lcccc} 
& Mean & SD & Min & Max \\
\hline $\mathrm{RR}$ & $8.0 \%$ & $1.3 \%$ & $4.0 \%$ & $14.0 \%$ \\
$\mathrm{LAM}$ & $21.1 \%$ & $5.3 \%$ & $7.0 \%$ & $40.0 \%$ \\
$\mathrm{TT}$ & 2.3 & 0.2 & 2.0 & 3.1 \\
$\mathrm{~V}_{\max }$ & 5.87 & 1.7 & 2 & 16 \\
$\mathrm{~T}^{1}$ & 10.3 & 1.6 & 5.9 & 18.2 \\
$\mathrm{~T}^{1}{ }_{\text {not }}$ & $9.1 \%$ & $2.0 \%$ & $3.0 \%$ & $18.0 \%$ \\
\hline
\end{tabular}

Furthermore, Spearman correlations between the tournament rank of the golfers and each of the RQA measures illustrate this behavior is shown by all golfers indepenent of their rank in the leaderboard (either $p>.05$ or $-0.07 \leq \rho \leq 0.04)$.

A second goal of this study was to analyze the golfers' performances within certain shot categories which are characterized by different movement patterns. Table 2 illustrates the most important descriptive results with respect to the shot categories tee shots (TS), long approach shots (LA), and putts $(\mathrm{P})$. The results indicate that the golfers showed performance patterns during tee shots which were very similar as the patterns considering all shots. There are recurring performances, which are not frequently, and about a fifth of them form vertical lines which are about as long as those considering all shots. The results are different for long approach shots and putts. Whereas performances recur less frequently performing long approach shots and performance streaks occur rarely, golfers showed the most "stable" performance pattern during putting. RPs for putting show a RR of $15 \%$ and a fourth of those performances form vertical lines, hence, were performed in a row. Moreover, performance streaks for putting are longer on average than for tee shots and long approach shots.

Table 2. Descriptive results of the used RQA measures with respect to the shot categories tee shots (TS), long approach shots (LA), and putts $(\mathrm{P})$

\begin{tabular}{lcccccc} 
& \multicolumn{3}{c}{ Mean } & & & SD \\
& TS & LA & P & TS & LA & P \\
\hline $\mathrm{RR}$ & $8.0 \%$ & $5.4 \%$ & $15.2 \%$ & $2.2 \%$ & $1.7 \%$ & $3.4 \%$ \\
$\mathrm{LAM}$ & $18.9 \%$ & $12.4 \%$ & $24.2 \%$ & $7.8 \%$ & $8.6 \%$ & $9.1 \%$ \\
$\mathrm{TT}$ & 2.2 & 2.0 & 2.8 & 0.2 & 0.6 & 0.5 \\
$\mathrm{~V}_{\max }$ & 3.5 & 2.3 & 5.58 & 1.2 & 1.0 & 2.0 \\
$\mathrm{~T}^{1}$ & 8.6 & 9.2 & 4.6 & 1.9 & 2.1 & 0.9 \\
$\mathrm{~T}^{1}{ }_{\text {not }}$ & $22.2 \%$ & $43.2 \%$ & $18.9 \%$ & $7.0 \%$ & $12.5 \%$ & $4.3 \%$ \\
\hline
\end{tabular}

As for the analyses considering all shots, there were no correlations found between the tournament rank and each RQA measure of the different shot categories.

\section{CONCLUSION}

In this study the performances of PGA TOUR golfers were analyzed looking for stable patterns. Similar to former studies, the results indicate that golfers show quite variable performance patterns. However, streaks of similar performance, independent whether the performances were good, average, or bad, occur rarely and usually do not last longer than for two holes. In total, in professional golf there is at maximum only little evidence of the existence of stroke-to-stroke streakiness.

\section{REFERENCES}

Bar-Eli, M., Avugos, S., and Raab, M. (2003). Twenty years of hot hand research. Psychology of Sport and Exercise, 7:525-553.

Clark III, R. D. (2005). An examination of the hot hand in professional golfers. Perceptual and Motor Skills, 101: 935-942.

James, N. (2007). The statistical analysis of golf performance. Annual Review of Golf Coaching 200\%, 231-248.

Livingston, J. A. (2012). The hot hand and the cold hand in professional golf. Journal of Economic Behavior \& Organization, 81:172-184.

Marwan, N., Romano, M. C., Thiel, M., and Kurths, J. (2007). Recurrence plots for the analysis of complex systems. Physics Reports, 438:237-329.

Stöckl, M., Lamb, P. F., and Lames, M. (2012). A Model for Visualizing Difficulty in Golf and Subsequent Performance Rankings on the PGA Tour. International Journal of Golf Science, 1:10-24. 


\title{
Modeling soccer matches by recurrence analyses
}

\author{
M. Lames \& D. Plück \\ Chair of Performance Analyses and Computer Science in Sport, TU \\ München, Germany (e-mail: martin.lames@tum.de, \\ denise.plueck@tum.de).
}

\begin{abstract}
The study aims at modeling soccer matches with recurrence quantification analysis (RQA). Positional data of 12 soccer matches from German Bundesliga out of the season 2009/2010 are used to produce global and local recurrence plots (RP) and perform the RQA. It is shown that RPs are representations of the soccer matches and the RQA parameters deliver descriptions of the matches.
\end{abstract}

Keywords: Nonlinearity, Fitting Models to Real Processes, Dynamic systems, Recurrence Quantification Analysis, Game sports

\section{BACKGROUND}

In soccer, the nature of the game is characterized essentially by the spatio-temporal interaction between the teams. Conceptual models interpret a soccer match as a dynamical self-organizing system Lames \& McGarry (2007). Many dynamic processes in physics, meteorology, biology and neuroscience were modeled by recurrence analyses (RQA) in recent years Marvan et al. (2007) So far, there was no approach to apply this tool in team sport and specifically in soccer. This study aims at exploring the appropriateness of recurrence analyses to describe common and individual patterns of soccer matches. Qualitatively, it was examined whether certain events (goals, freekicks, corners) could be identified in recurrence plots Pecar (2004). Quantitatively, RQA parameters were calculated for soccer matches and validated whether they help to better understand the match.

\section{METHODS}

Positional data from 22 players in 12 soccer matches from German Bundesliga season 2009/2010 provided by AMISCO were used for recurrence analyses.

The data in csv format contain the $\mathrm{x}, \mathrm{y}$-coordinates on the pitch for each field player, the goal keeper and the substitutes. The original sampling rate is $10 \mathrm{~Hz}$. X,y-coordinates of the substitutes were introduced at the appropriate time stamp in the position data of the substituted players. If a change in the team line-up after the substitution was recognized by video analysis, position data of the concerned players were exchanged accordingly if possible. The data of each game were mirrored in the second half in order to preserve playing direction. Data were aggregated to $1 \mathrm{~Hz}$. Pre-processing, such as reading, mirroring and filtering, computation of RPs and RQA was realized by an application (Microsoft Visual Studio 2013 Professional).

Global and local plots for the 12 soccer matches were created. Therefore, the Euclidean distances of all field players in each second to any other second was used as recurrence measure. Global plots, also called distance plots, are color-coded versions of RPs and image the distance values without a threshold value. Red and orange shades signal low distance values, whereas blue and purple shades show high distance values. Local plots use a distance threshold to indicate recurrence states with black points in the RPs. If the actual lineup of the players is approximately equal (depending on the distance threshold) to a former lineup, a black dot occurs. Otherwise the pixel is white. RQA parameters (RR, DET, L , LAM, TT, Lmax, Vmax and ENTR) published by Marvan et al. (2007) were automatically computed with the just mentioned application. A moving windowed RQA for soccer matches was also developed. According to considerations by Iwanski (1998) and March (2005) no embedding was used.

Recurrence threshold was fixed using the method suggested by Marwan (1999). The relation between the rate of recurrent points (DET), which build diagonal lines in the matrix, and the variability of their lengths (ENTR) was consulted. Seen over all matches, this relation reached its maximum at a value of about 9 meters. The least length of the diagonal lines (lmin) is strong related to the DETENTR relation. Due to that the distribution of diagonal lines related to an increasing recurrence threshold was considered and an appropriate value for lmin (3) was fixed. The values of the main diagonal (LOI), which is a specific characteristic of recurrence plots, were excluded from the calculation of RR, DET, L and TT Marvan et al. (2007). 
The mean radius of the LOI width for each game was used to fix the area which is obviously determined.

For the qualitative analyses remarkable pattern in the plots were localized manually and their meaning in the game with the video stream was affirmed. The sum of recurrence points per second in each game was calculated, which helps to detect rarely or frequently repeating situations in the match Marvan et al. (2007).

Quantitatively, the total number of recurrence points per second were compared for typical events like free-kick, shot at goal and corner kick and for position play. Important events of the games were obtained from game protocols in a public football database (www.kicker.de; www.bundesliga.de). The relation between these game statistics and the RQA parameters was considered. The windowed RQA was used to find differences between the RQA parameters of certain match phases like for example the initial phase of the game.

\section{RESULTS}

Visual inspection of recurrence plots showed common and individually patterned representations of the matches. Qualitatively it is possible to identify distinguished color bands in the global plots corresponding to certain events like free and corner kicks as well as goals. Blue colored lines indicate standard situations in the match. Red and orange transversing pattern signify that the players behavior is recurrent to a former situation in the match. The local plots show typical pattern like diagonal and vertical lines of recurrence plots and further specific characteristics of soccer games. For example, sequences of standard situations cause white bands in the plots. Further, a reorganization in the team lineup due to their playing tactics reduce the recurrence density in the RPs. So, the development in the density of recurrence points per second over the course of game is a fingerprint of the match. Standard situations in the game receive significant lower recurrence points in the seconds around the event than position play does. Partially significant correlations are recorded between the game statistics and the RQA parameters. The parameters also show differences in the game phases. For instance, the initial game phase averaged over the matches reaches significant higher $\mathrm{RR}$ values than the following match phase.

\section{DISCUSSION AND CONCLUSIONS}

Recurrence analysis is a new and appropriate method to model soccer games. Recurrence plots are representations of soccer matches that show common and individual characteristics of the matches. Special events in the matches can be identified and localized in the recurrence plots. Further, recurrence parameters deliver meaningful descriptions of soccer matches. Higher recurrence rates in the initial match phase may be caused by tactical efforts due to surprise the opponent and score an early goal. Significant correlations between match statistics and recurrence parameters prove validity of modeling soccer with recurrence analyses. Thus, there is an improved description of soccer as a complex system with dynamic interactions and emerging behavior based on recurrence analyses.

\section{REFERENCES}

Eckmann, J.-P., Kamphorst, O. S., \& Ruelle, D. (1987) Recurrence Plots of Dynamical Systems. Europhysics Letters, 4 (9), 973-977.

Iwanski, J.S., Bradley, E. (1998) Recurrence plots of experimental data: to embed or not to embed? Chaos, 8 (4) 861871.

Lames, M., McGarry, T. (2007) On the search for reliable performance indicators in game sports. International Journal of Performance Analysis in Sport, 7(1), 62-79.

March, T.K., Chapman, S.C., Dendy, R.O. (2005) Recurrence plot statistics and the effect of embedding. Physica D 200, (12) 171184.

March, T.K., Chapman, S.C., Dendy, R.O. (1999) Untersuchung der Klimavariabilitt in NW Argentinien mit Hilfe der quantitativen Analyse von Recurrence Plots. Masters Thesis, Dresden University of Technology.

Marwan, N., Romano, M. C., Tiel, M., \& Kurths, J. (2007) Recurrence Plots for the Analysis of Complex Systems. Physics Reports, 438(5-6), 237-329.

Pecar, B. (2004) Visual recurrence analysis as an alternative framework for time series characterization. Computer Finance and its Applications.-WIT Press., 241252 . 


\title{
MATHMOD 2015 ABSTRACT VOLUME
}

\author{
MATHMOD Minisymposium \\ Modeling and Simulation to Support Sustainable Energy \\ Systems and Water Treatment
}


MATHMOD 2015 Abstract Volume 


\title{
Comparison case between Modelica and specialized tools for building modelling
}

\author{
Daniel Fernández González* Luis J. Yebra** \\ * CIESOL Mixed Centre, Master of Solar Energy, Campus of \\ University of Almería, 04120 Almería, Spain. (e-mail: \\ danielfergon33@gmail.com) \\ ** CIEMAT Research Centre, Dept. of Energy, PSA, 04200 Tabernas, \\ Almería, Spain (e-mail: luis.yebra@\{ciemat,psa\}.es)
}

\begin{abstract}
This document presents a comparison between the simulation results of a basic building obtained from two different sets of modelling tools. The first set are object-oriented general purpose modelling tools based on the Modelica language, for which Dymola(c) and SystemModeler(c) have been used. The second group is based on DesignBuilder(c) as a special purpose building simulation tool. The building's model is presented and the simulation results explained.
\end{abstract}

Keywords: Object-oriented dynamic modelling, thermodynamic, buildings.

\section{INTRODUCTION}

Thermodynamic modelling of buildings is gaining importance in the search for higher energy efficiencies. Architectural, heating, cooling and control systems design are several disciplines in which buildings dynamic models could be applied. As in many other disciplines (electronics, electricity, mechanics, fluids, thermodynamics,...) there is a choice between special purpose simulators for each discipline or more general dynamic modelling techniques, see Cellier (1991).

Several specialized simulation tools for building may be mentioned: TRNSYSC, LIDERC, EnergyPlus(c), DOE2(C), DesignBuilder(c),... Some of these tools don't always shows the approximations and methods used for the modelling and simulation of the system, denying the users access to more detailed information.

Modelica is a general purpose object-oriented language for physical systems modelling Fritzson (2010), and a its evolution is described in Åström et al. (1998).

In this work the authors present the modeling and simulation of a simple building, with DesignBuilder as the special purpose tool, and Dymola and SystemModeler as the Modelica general purpose tools. For both Modelica tools used, the Modelica Standard Library (MSL) has been used. Modelling uncertainties of the system are presented and justified when no information was provided by the special purpose tool.

In Figure 1 the temperatures simulated for January are shown, with the results with Modelica tools in red and with DesignBuilder in blue.

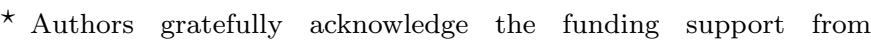
POWER, INNPACTO-HIBIOSOLEO and HYSOL research projects.
}

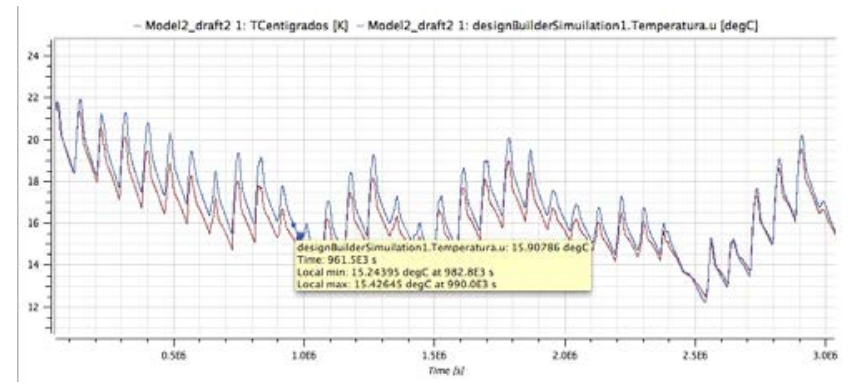

Fig. 1. Air inside temperature simulation results with DesignBuilder (blue) and Modelica (red). Month: January.

\section{CONCLUSION}

A comparison between two modelling tool sets is presented. The first one is a specific simulation package for buildings, DesignBuilder, and the other set is formed by Dymola and SystemModeler as object oriented modelling tools based on Modelica language and MSL. For the comparison a small building system is chosen, and the model and approximations are presented. At last, simulation results are presented with both tool sets and the comparison between them is performed for a complete year.

\section{REFERENCES}

Cellier, F.E. (1991). Continuous System Modeling. Springer-Verlag.

Fritzson, P. (2010). Principles of object-oriented modeling and simulation with Modelica 2.1. John Wiley \& Sons.

Åström, K.J., Elmqvist, H., and Mattsson, S.E. (1998). Evolution of Continuous-Time Modeling and Simulation. In R. Zobel and D. Moeller (eds.), Proceedings of the 12th European Simulation Multiconference, ESM'98, 9-18. Society for Computer Simulation International, Manchester, UK. 


\title{
Environmental Impacts and Benefits of Smart Home Automation: Life Cycle Assessment of Home Energy Management System
}

\author{
Jean-Nicolas Louis *, Antonio Caló ${ }^{* *}$, Kauko Leiviskä ${ }^{* *}$, \\ Eva Pongrácz ${ }^{* * * *}$ \\ * Centre for Northern Environmental Technology, Thule Institute, \\ University of Oulu, Oulu P.O.Box 7300, Finland (Tel: +358 294487 \\ 60\%; e-mail: jean-nicolas.louis@oulu.fi). \\ ** Centre for Northern Environmental Technology, Thule Institute, \\ University of Oulu, Oulu P.O.Box 7300 University of Oulu, Finland \\ (e-mail: antonio.calo@oulu.fi) \\ *** Control Engineering Laboratory, Dept. of Process and \\ Environmental Engineering, University of Oulu, Oulu P.O.Box 7300 \\ University of Oulu, Finland (e-mail: kauko.leiviska@oulu.fi) \\ **** Centre for Northern Environmental Technology, Thule Institute, \\ University of Oulu, Oulu P.O.Box 7300 University of Oulu, Finland \\ (e-mail: eva.pongracz@oulu.fi)
}

\section{INTRODUCTION}

Environmental impact assessment of technology has been recurrent and fully used for the EuP Directive. Quantifying the environmental impact of the smart grid is an on-going discussion (Hledik (2009)) as well as the role of smart metering in carbon management (Darby (2013)). In order to fully implement a Home Energy Management System (HEMS), multiple sensors are necessary for recording climatic data, or the energy consumption within the house precisely enough to know where are the energy leaks and accurately assess the potential for energy reduction and decrease in emission levels. The system has been built according to the ISO 16484-2 (CEN (2004)) and a previous study on the evaluation of the environmental impact of three types of home energy management systems (van Dam et al. (2013)).

\section{OBJECTIVES OF THE RESEARCH}

This paper discusses the life-cycle environmental impact of Home Energy Management System (HEMS), in terms of their potential benefits and detrimental impacts. It is the expectation that adapting smart home automation (SHA) would lead to reduced electricity usage in the household and overall environmental advantages. The purpose of this research was to quantify the negative environmental impacts of SHA and balance them with their benefits. The evaluation of SHA has been done by conducting a generic life cycle assessment study using SimaPro programme and the EcoInvent database.

$\star$ The Thule Institute Doctoral Programme is acknowledged for financing this research.

\section{RESULTS}

The LCA study concluded that the largest environmental impact of HEMS is the use-phase electricity consumption of home automation devices. The paper concludes that the energy payback time of home automation in term of the electricity consumption of the devices is negative by 1.6 years. The largest part of this is due to the energy consumption of smart plugs.

Overall, $99.4 \%$ of the emissions occur during the assembly and the use-phase, where the use-phase represents $84 \%$. Therefore, the paper concludes that in terms of home automation, we need to find the balance between what we actually need to control and the resulting energy consumption of the control system.

\section{REFERENCES}

CEN (2004). Building automation and control systems (BACS) - Part 2: Hardware.

Darby, S.J. (2013). The role of smart meters in carbon management. Carbon Management, 4(2), 111-113. doi: $10.4155 / \mathrm{cmt} .13 .8$.

Hledik, R. (2009). How Green Is the Smart Grid? The Electricity Journal, 22(3), 29-41. doi: 10.1016/j.tej.2009.03.001.

van Dam, S.S., Bakker, C.A., and Buiter, J.C. (2013). Do home energy management systems make sense? Assessing their overall lifecycle impact. Energy Policy, 63(C), 398-407. doi:10.1016/j.enpol.2013.09.041. URL http://dx.doi.org/10.1016/j.enpol.2013.09.041. 


\title{
The role and the impact of electric vehicles on the local power grid within the Northern Finland climatic conditions
}

\author{
First A. Author*. Second B. Author, Jr.** \\ Third C. Author*** \\ *National Institute of Standards and Technology, Boulder, CO 80305 \\ USA (Tel: 303-555-5555; e-mail: author@boulder.nist.gov). \\ **Colorado State University, Fort Collins, CO 80523 USA (e-mail: \\ author@lamar.colostate.edu) \\ *** Electrical Engineering Department, Seoul National University, \\ Seoul, Korea, (e-mail: author@snu.ac.kr)\}
}

Large scale electrification of the transportation sector is generally considered a promising strategy for improving efficiency and reducing greenhouse gases emissions. Within a broader decarbonization strategy, the development of the electric vehicles sector, including its integration within a smarter energy network, it is considered one of the cornerstones of the future energy and transportation development strategy. On the other hand, feasibility and sustainability studies on the performance of electric vehicles are performed without taking in full consideration the overall impact on the energy infrastructure that so much depends on specific local environmental and climatic conditions.

In our work, we assessed the impacts electric vehicles could have on the local (smarter) power network and the infrastructure underlying critical issues in the systems within the Northern Finland winter conditions. Considering the case study of the city of Oulu, we developed a MATLAB based simulation assessing the role a fleet of electric vehicles could play within a smart grid based power system. Analysis results strongly suggest that, within the current logistical and environmental limitations, electric vehicle cannot play an active role on the flattening of the network peaks loads. Furthermore, unless a number of coordinated efforts for the improvement of the existing urban infrastructure are considered, they are likely to significantly increase the overall power consumption opening new questions related to the necessary increase of power production. 


\title{
Evaluation of Fluidized Bed Condition by Image Analysis and Modelling
}

\author{
Mika Liukkonen*. Teri Hiltunen** \\ Yrjö Hiltunen*** \\ *Department of Environmental Science, University of Eastern Finland, P.O. Box 1627 \\ 70211 KUOPIO (Tel: 358-40-3510644; e-mail: mika.liukkonen@uef.fi). \\ **Foster Wheeler Ltd,, P.O. Box 201, 78201 Varkaus, Finland \\ ***Department of Environmental Science, University of Eastern Finland \\ P.O. Box 1627, 70211 KUOPIO
}

\section{INTRODUCTION}

Varying fuel quality and co-combustion of challenging and possibly low-grade fuels, added to the growing demands for process efficiency and the efforts to reduce harmful emissions, are the primary reasons for today's need for monitoring energy conversion processes such as fluidized bed combustion more exhaustively than before. This is because the use of difficult fuels like biomass may inflict problems such as ash sintering or agglomeration, which may lead to even unintended boiler shutdowns.

It is probable that these kinds of problems could be detected by monitoring the grain size of bed material. In addition to the fact that monitoring the coarseness of bed material would provide information on problematic phenomena such as agglomeration, another purpose for monitoring material coarseness is the optimization of bed change interval. Because there are not any direct methods available for measuring bed coarseness, this is often done indirectly by manually sieving the bottom ash which is being removed from the process, which is laborious, expensive and susceptible to errors. We have already shown that it is possible to monitor the grain size using machine vision (Liukkonen et al. 2012). In this paper we present a costefficient measurement and a further developed, indirect modelling scheme for evaluating fluidized bed condition.

\section{MATERIALS AND METHODS}

The case process is a $385 \mathrm{MW}_{\text {th }}$ large-scale industrial boiler fired by biomass and peat. The plant produces steam and electricity for a pulp and paper plant and electricity and district heat for an energy company. The digital images were taken from the bottom ash conveyor during one month.

The main stages for determining the grain size using a digital image sample are a) capturing a grayscale image, b) contrast adjustment, c) conversion to binary image, d) detection of connected objects, e) calculation of sizes of individual objects, and f) calculation of the shares of selected grain size classes $(0.5 \mathrm{~mm}, 1 \mathrm{~mm}$, and $1.5 \mathrm{~mm}$ in this case).

Presentation of analysis results is important in a monitoring system. A size distribution is a relatively simple but visual way of presenting the condition of grainy material (See Fig.).
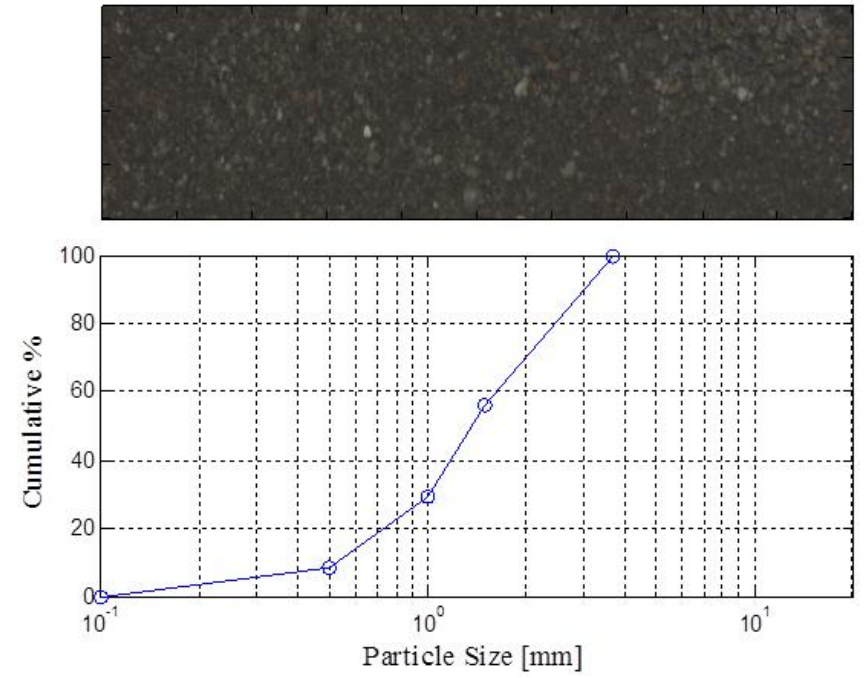

Fig. Bed condition monitor using models derived from digital images and showing a typical particle size distribution at a single moment of time.

\section{CONCLUSIONS}

The results of this study show that this kind of imaging system combined with indirect modelling methodology can be useful in detecting material coarsening automatically, thus avoiding manual sieving work. The grain size distribution obtained from digital images offers one possible and economical way of evaluating bed condition, diagnosing the reasons for changes when combined with corresponding process data, and creating a warning system for detecting sudden and long-term changes in material coarseness. Based on the data analysis it also seems that there are dependences between ash coarseness and certain process measurements, which suggests that it could be possible to create a data-based soft sensor for bed material coarseness.

\section{REFERENCES}

Liukkonen, M., Huhtinen, J., Hiltunen, T., Hälikkä, E., and Hiltunen, Y. (2012). Low-cost camera system for online estimation of grain size in fluidized bed. In: Troch I., Breitenecker F. (eds.), IFAC papers online, Proc. Mathmod 2012 - 7th Vienna International Conference on Mathematical Modelling, Vol. 7, pp. 964-967. 


\title{
Multivariate analysis models for wood properties combined with Open Modelica model for process performance monitoring
}

\author{
Jan Skvaril*, Konstantinos Kyprianidis**, Anders Avelin ${ }^{* * *}$, Monica Odlare ${ }^{* * * *}$, Erik Dahlquist****** \\ *School of Business Society and Engineering, Mälardalen University, Högskoleplan 1, 72123 Västerås, Sweden \\ (Tel: +46-21-151738; e-mail: jan.skvaril@ mdh.se) \\ **(Tel: +46-21-151765; e-mail: konstantinos.kyprianidis@mdh.se) \\ ***(Tel: +46-21-151798; e-mail: anders.avelin@mdh.se) \\ ****(Tel: +46-21-101611; e-mail: monica.odlare@mdh.se) \\ ****(Tel: +46-21-151768; e-mail: erik.dahlquist@mdh.se)
}

\section{INTRODUCTION}

To perform advanced model based control it is important to know what is fed into a system such as a waste or biomass fired boiler or a pulp digester. In this paper, we present correlations between the lignin content of different types of wood chips and their Near-infrared (NIR) spectra. The Principal Component Regression (PCR) method is used for deriving the correlation, as well as selecting certain wave lengths. Analysis is made including different parts of the spectra in the wave length range $700-2500 \mathrm{~nm}$. The model is then used as input to an Open Modelica pulp digester model to tune the reactivity constant of the dissolution of lignin. The lignin content of wood-chips is determined on-line through the NIR measurement at the feed to the digester. Simulations are carried out to determine the content of residual lignin on fibers at the exit (continuous digester) or at the end of a cook (batch digester). By comparing the deviation between predicted values and actual measured values the reactivity constant of the lignin is determined. The regression can be made to the NIR spectrum aside of the lignin content as such. The original content of lignin together with reactivity may then be used for optimized on-line control of the digester. It can also be used for diagnostic purposes with regard to process issues like hang-ups or channeling, as well as possible sensor faults and data reconciliation.

\section{METHODOLOGY}

In this paper, we mostly focus on combining statistical analyses with regression models and a physical simulation model. A case study will be used to show the potential use of the integrated model in a fiber-line.

We used 13 wood samples, one sample of white paper and one sample of hardwood from the pulp and paper plant (BillerudKorsnäs) to develop the NIR prediction model. We acquired NIR spectra by using a grating spectrometer. The NIR spectra were recorded at wavelengths between $700 \mathrm{~nm}$ and $2500 \mathrm{~nm}$ at 2-nm intervals. For determination of lignin content samples were mixed with $3 \mathrm{~mL}$ of $72 \%$ of sulfuric acid for 1 hour. 84 $\mathrm{mL}$ of deionized water was added and the samples were autoclaved for 1 hour at $121 \pm 3^{\circ} \mathrm{C}$. After cooling and filtering the acid soluble lignin (ASL) was determined by analyzing 25 $\mathrm{mL}$ of the eluate spectrophotometrically at $205 \mathrm{~nm}$. The acid insoluble lignin (AIL) was determined by measuring the amount of lignin captured in the filter after drying at $105^{\circ} \mathrm{C}$ for 2 hours (Avelin, Skvaril, Aulin, Odlare, \& Dahlquist, 2014).

We employed Principal component analysis (PCA) in order to identify the correlation between NIR spectra and solid biomass properties. We then utilized PCR and Partial least squares (PLS) regression methods for developing prediction models. Furthermore, we applied Savitzky-Golay smoothing and differentiation algorithms to the data using commercially available software (Savitzky \& Golay, 1964).

\section{RESULTS AND DISCUSSION}

According to the literature, bands around 1410, 1417, 1690 and $2200 \mathrm{~nm}$ may be assigned to lignin content (Soukupova, Rock, \& Albrechtova, 2002). The corresponding peaks, or valleys, in the 1st derivative of the absorption spectra is therefore expected at slightly lower, or higher, bands, respectively. Results show that we have a reasonably good correlation between total lignin and the absorption at $1654 \mathrm{~nm}$. The same holds true for acid insoluble lignin although the correlation with absorption at $1712 \mathrm{~nm}$ is less good, although reasonable if a few of the samples are excluded. It is always a problem carrying out this type of analysis as the variability in the chemical composition between different species is unknown. Some indication of the difference was given from how the acid insoluble part behaved during filtration. Another aspect is how much the spectra varies depending on the size of the sample when using NIR spectroscopy. We also prepare the samples in different ways before the NIR-spectrum analysis i.e. grinded material, mixture of wood chips and fine material and wood chips.

During the first step, we used univariate regression for total lignin versus $1^{\text {st }}$ derivative at $1654 \mathrm{~nm}$ and achieved an $\mathrm{R}^{2}$ of 0.92 and RMSE of 3.1. Especially for some of the wood species the deviation is significant between the lignin content and the $1^{\text {st }}$ derivative of the absorption at $1654 \mathrm{~nm}$, which is attributed to variations in the coarseness of the particles. We then used multivariate PCR for the entire NIR wavelength spectrum from 700 to $2500 \mathrm{~nm}$ and achieved an improved $\mathrm{R}^{2}$ of 0.95 and RMSE of 2.6.

To refine the models further, more samples are needed. We have analyzed the same samples many times, taking samples 
from different parts of a batch, comparing grinded samples to wood chips etc. We see that there are differences in the NIR spectra between different samples depending on how the sample was prepared; samples comprising only wood chips can improved the models. The lignin content has been derived by analyzing double samples. It is expected that the reliability of these results could be improved mildly if we analyze triple samples, or more. This is an on-going activity.

We have used a physical correlation between different operating conditions in a continuous digester at BillerudKorsnäs in Gavle, Sweden and a couple of SAPPI mills in South Africa (Jansson, Grobler, Avelin, \& Dahlquist, 2009).

$$
\frac{d L}{d t}=C_{1} \cdot[O H]^{a} \cdot[H S]^{b} \cdot e^{A-\frac{B}{T}}
$$

In equation (1), $d L / d t$ is the dissolution rate of lignin per time unit. This rate is dependent on the concentration of hydroxide $(\mathrm{OH})$, hydrogen sulfide (HS) and temperature (T) as given by the Arrhenius expression. $C_{1}$ is a reactivity constant relating to wood dimensions, how dense the chip is and the type of lignin. $a$ and $b$ are constants both set typically to 0.5 . $A$ and $B$ are constants in the Arrhenius expression used for characterizing the influence of temperature for the selected wood species. Values for these constants for particular wood types may be obtained from the literature, or calculated from process data (Jansson, et al., 2009). $d L / d t$ is multiplied by the residence time in the reactor to determine how much of the original lignin that is predicted to be dissolved. Then we performed the simulation in Open Modelica for the digester (in this case run as a batch digester).

\section{CASE STUDY}

Furthermore, we have used NIR spectra to follow how the swing between softwood and hardwood goes through a continuous digester. The NIR spectrophotometer has been in operation for many years (Axrup, Markides, \& Nilsson, 2000), and is a robust installation. In the past it has been used primarily for moisture determination.

We have observed that there are variations in absorption which are not directly related to the swing i.e., that is it is not only the "formal" feed that is varying between soft wood and hardwood, but also the properties of wood vary within each grade. This is where we attempt to develop more knowledge about these variations by building prediction models with respect to actual lignin content and also reactivity for "batches" of wood being fed to the digester, in the order of 510 tons of wood chips at a time.

From a sensitivity point of view, it can be interesting to use the simulation model to build an understanding of how much a change of only a few percent in lignin content in the feed stock can affect the final process result with respect to Kappa number, if we have assume that all other conditions/parameters remain constant.

For example, let's assume spruce with 30,31 and $34 \%$ of total lignin. Let's also assume a residence time of 3.5 hours in the cooking zone. The $\mathrm{OH}$ concentration is $0.55 \mathrm{M}$ and HS $0.2 \mathrm{M}$ and the temperature $443 \mathrm{~K}$. The reactivity constant is assumed to be $4.1 \cdot 10^{-6}$ and using formula (1) we get for $A=43.7$ and
$\mathrm{B}=16133$ that $\mathrm{dL} / \mathrm{dt}=0.002083$ (\% of dissolved lignin per second). For 3.5 hours of cooking, the dissolved lignin is then is $26.5 \%$. If we start therefore with $30 \%$ lignin content the residual lignin will be $3.75 \%$, while for $31 \%$ it will be $4.75 \%$ and for $34 \%$ it will be $7.75 \%$. If we use 5 Kappa number units for each \% of lignin, this means 18.8, 23.8 and 38.8 Kappa number units, respectively, in the fibers leaving the digester. If the reactivity varies this can give both positive and negative effects as well as operating conditions with respect to temperature, chemical concentrations and residence time. If the moisture content of the incoming wood chips increases this also means that the chemical concentration affecting the dissolution decreases, and thus moisture content is of interest to measure (by NIR). Different wood species have quite different temperature dependency. Some softwood such as Pine from Australia require a digestion temperature of $145^{\circ} \mathrm{C}$ while it is common to use a temperature around $160{ }^{\circ} \mathrm{C}$ for Pine from Scandinavia, which is principally a similar type of softwood.

\section{CONCLUSIONS}

We conclude that measuring the properties of incoming wood is important, and this methodology can be used to increase the knowledge and give tools to improve the operations and control. By comparing the prediction from the simulation to measured values we can refine the determination of the reactivity for different wood species, but also for different variants within each type of wood specie. By combining statistical models for the quality aspects like lignin content and Kappa number with physical models we can get better results and tools than if we use only one or the other.

\section{REFERENCES}

Avelin, A., Skvaril, J., Aulin, R., Odlare, M., \& Dahlquist, E. (2014). Forest biomass for bioenergy productioncomparison of different forest species. Energy Procedia, 61, 1820-1823.

Axrup, L., Markides, K., \& Nilsson, T. (2000). Using miniature diode array NIR spectrometers for analysing wood chips and bark samples in motion. Journal of Chemometrics, 14, 561-572.

Jansson, J., Grobler, F., Avelin, A., \& Dahlquist, E. (2009). On-line simulation of continuous pulp digester. Cellulose (kg/m3), 300, 160.

Savitzky, A., \& Golay, M. J. (1964). Smoothing and differentiation of data by simplified least squares procedures. Analytical chemistry, 36, 1627-1639.

Soukupova, J., Rock, B., \& Albrechtova, J. (2002). Spectral characteristics of lignin and soluble phenolics in the near infrared-a comparative study. International Journal of Remote Sensing, 23, 3039-3055. 


\title{
Monitoring of water processes using intelligent condition indicators
}

\author{
Mika Liukkonen* Yrjö Hiltunen** \\ Ilkka Laakso*** Petri Juntunen**** \\ *Department of Environmental Science, University of Eastern Finland, P.O. Box 1627, 70211 KUOPIO (Tel: 358-40-3510644; \\ e-mail:mika.liukkonen@uef.fi). \\ **Department of Environmental Science, University of Eastern Finland, P.O. Box 1627, 70211 KUOPIO \\ ***Stora Enso Fine Paper, Oulu, Finland \\ ****Kuopio Waterworks, Kuopio, Finland
}

\begin{abstract}
Water quality is an increasingly important issue, because water is the mostly used raw material in the world and poorquality water causes many difficult issues in societies and ecosystems. Industrial wastewater treatment, for instance, is facing big challenges concerning fulfilment of both general and plant-specific regulations concerning their effluents and cost management of treatment plants. The wastewater coming from the pulp and paper industry contains substances such as nutrients (phosphorus, nitrogen) and solid organic material, which in large quantities are considered harmful to the ecosystem, so competent treatment is required and the treatment efficiency has to be monitored and controlled carefully and continuously. Nonetheless, it seems that the overall operation of the treatment plants needs to be improved, if the water industry is to satisfy regulations for increased efficiency (O’Brien et al., 2011).
\end{abstract}

Monitoring and control of wastewater treatment relies a great deal on the availability of accurate and reliable sensor information. Because of the difficult environment, only a part of the process variables can be measured continuously using relatively simple and inexpensive physical sensors, whereas the determination of certain quantities of interest requires costly laboratory analyses which cannot be performed online (Valentin \& Denoeux, 2001). Therefore, it is obvious that forecasting process outputs such as discharge would offer a way of achieving better control of wastewater treatment and computational elements such as soft sensors can provide valuable tools for process monitoring. Model-predictive control of dissolved oxygen in the activated sludge process, for example, has been observed to provide significant benefits compared with the previously applied control system (Holenda et al., 2008; O’Brien et al., 2011). However, it seems that controllability is still an open issue in the water industry and due to the lack of other opportunities the common focus will be on aeration control (O'Brien et al., 2011).

A generic piece of software for monitoring water treatment processes has been presented earlier (Liukkonen et al., 2013). One possibility to perform model-based process monitoring would be to combine the basic online monitoring software with an offline modelling tool (See Fig. 1), which can be used for creating model-derived condition indicators to be shown on the screen of monitoring tool. The modelling tool makes it possible to add computational condition indicators to predict the trends of discharge concentrations or variables indicating the quality of treated water, and show them on the screen also. These intelligent condition indicators can then be followed in the monitoring tool just like the other measurements. The system is demonstrated in a wastewater treatment environment by using process measurements from an activated sludge treatment plant, which is part of a pulp and paper plant. The plant treats $300-500 \mathrm{l} / \mathrm{s}$ of waste water on an average.

The modelling tool involves currently a regression-based variable selection module, which can be used for creating variable subsets to be used in predictive models. After this procedure, the five selected variables are used for creating a model to predict the future COD rate (See Fig. 2). The developed condition indicator can then be added to the monitoring tool, as is shown in Fig. 3. It is remarkable that although multiple regression is used here as an example, the model can basically be of any type.

In this example, the gained empirical model for the instantaneous chemical oxygen demand (COD) is as in (1) (See Fig. 2):

$\mathrm{COD} \approx 0.577 * \mathrm{COD}_{0}+0.017 * \mathrm{~F}_{\mathrm{c} 1}+0.003 * \mathrm{C}_{\mathrm{CB}}+0.051 * \mathrm{~F}_{\mathrm{EC}}-$ $0.102 * \mathrm{O} 22_{\mathrm{A}}-1.815$

where $\mathrm{COD}_{0}$ denotes the previous chemical oxygen demand $(\mathrm{t} / \mathrm{d}), \mathrm{F}_{\mathrm{C} 1}$ is the symbol for the total wastewater flow in channel $1(1 / \mathrm{s}), \mathrm{C}_{\mathrm{CB}}$ is the conductance in the batch cooking channel $(\mathrm{mS} / \mathrm{m}), \mathrm{F}_{\mathrm{EC}}$ stands for the flow of the condensates from the evaporator $(1 / \mathrm{s})$, and $\mathrm{O} 2_{\mathrm{A}}$ denotes the amount of dissolved oxygen in the aeration pool $(\mathrm{mg} / \mathrm{l})$.

Challenges such as tightening legislation regarding to pollutants, pressure to increase the energy efficiency of treatment, and influents that are increasingly demanding in terms of stable and efficient treatment, are typical for industrial wastewater treatment. Monitoring systems capable of alerting when there is a drift towards an undesired or dangerous condition are useful, but a monitoring system of today should be able to assist the personnel to interpret the prevailing, and more importantly, the future risk level from the measurements. 


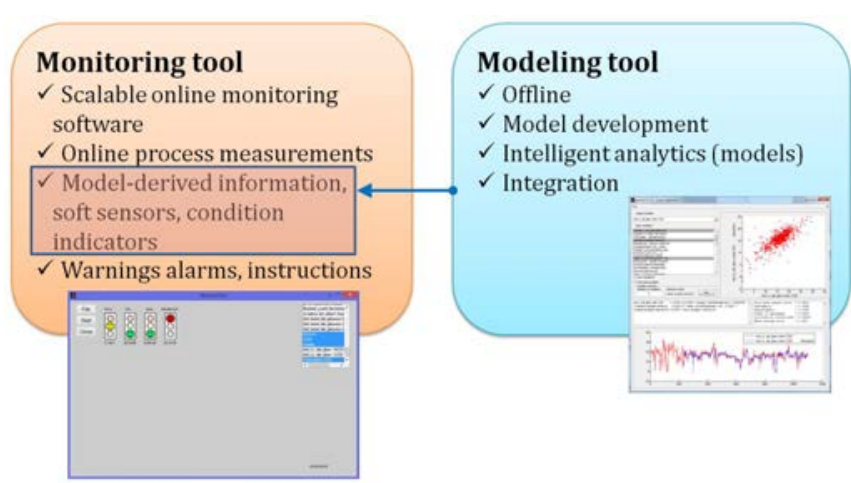

Fig. 1. The combination of monitoring and modelling tools.

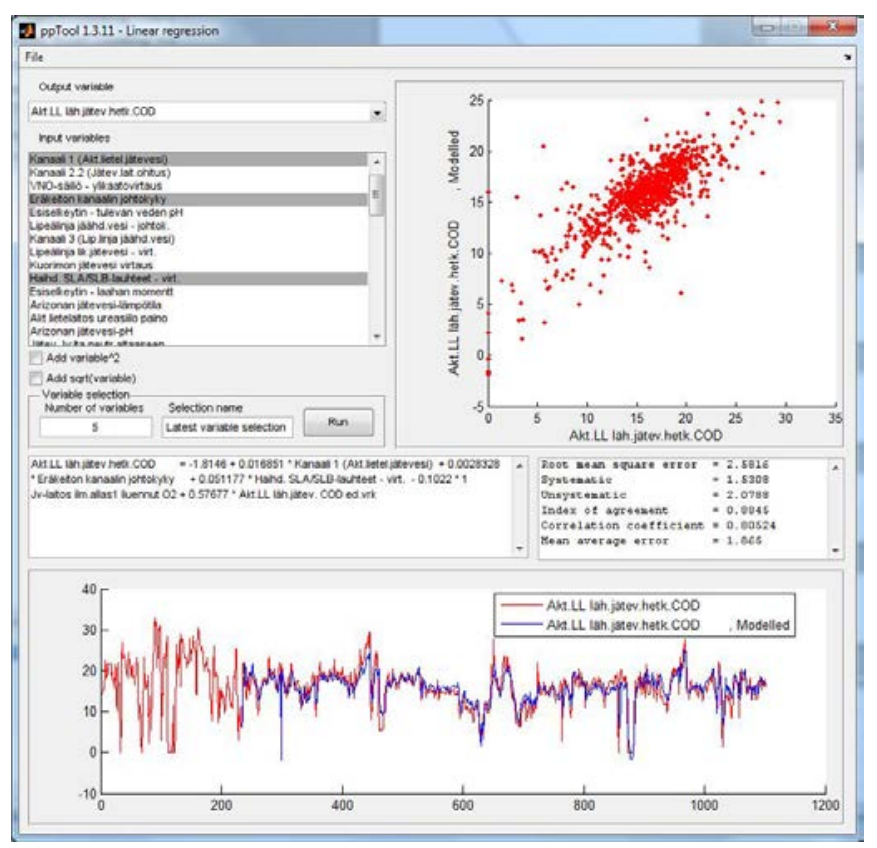

Fig. 2. The developed COD model in the model tool using the selected variables.

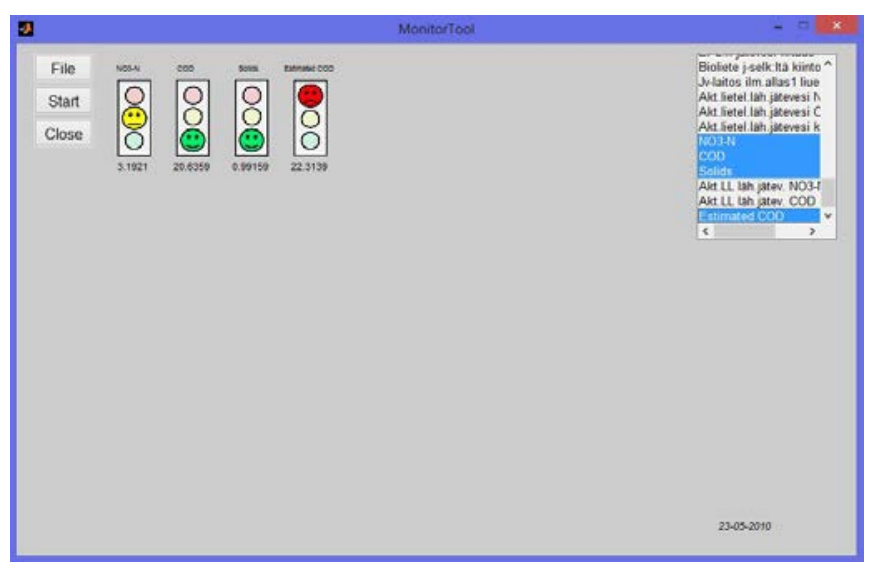

Fig. 3. The monitoring tool showing condition indicators for nitrate-N (kg/d), COD ( $\mathrm{t} / \mathrm{d})$, suspended solids $(\mathrm{t} / \mathrm{d})$ and the estimated future COD rate $(\mathrm{t} / \mathrm{d})$.

A potential future application of the software is presented in Fig. 4. Intelligent condition indicators would improve the efficiency and flexibility of monitoring in wastewater treatment plants.

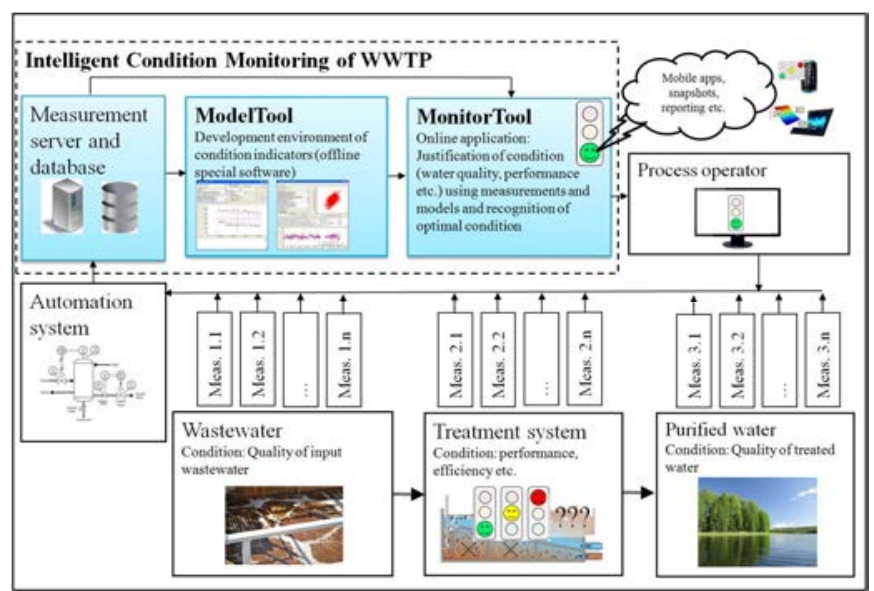

Fig. 4. Future scenario of the intelligent condition monitoring of wastewater treatment.

In summary, industrial wastewater treatment is a challenging process to control and requires sophisticated monitoring tools that can provide more advanced diagnosis and prediction capabilities. A sophisticated monitoring tool able to perform analyses and visualization of multivariable measurement data, alert in problematic situations, and provide instructions in problematic occurrences is essential for the management of water treatment. A model-based monitoring system based on specifically designed knowledge extraction and diagnostic techniques can support a decision-maker by analyzing recent process history and extracting the essential information, which can be used in predictive model-based monitoring.

\section{ACKNOWLEDGMENTS}

The research was funded by the Finnish Funding Agency for Technology and Innovation (TEKES) within the projects Measurement, Monitoring and Environmental Assessment (MMEA) and Intelligent Software and Service Concept of the Industrial Internet (InDiGO!).

\section{REFERENCES}

Holenda, B., Domokos, E., Rédey, Á., and Fazakas, J. (2008). Dissolved oxygen control of the activated sludge wastewater treatment process using model predictive control. Computers and Chemical Engineering, 32, 1270-1278.

Juntunen, P., Liukkonen, M., Lehtola, M., Hiltunen, Y. (2014). Characterization of Alum Floc in Water Treatment by Image Analysis and Modeling. Cogent Engineering, 1(1), article 944767.

Liukkonen, M., Juntunen, P., Laakso, I., Hiltunen, Y. (2013). A Software Platform for Process Monitoring: Applications to Water Treatment. Expert Systems with Applications, 40(7), 2631-2639.

O'Brien, M., Mack, J., Lennox, B., Lovett, D., and Wall A. (2011). Model predictive control of an activated sludge process: A case study. Control Engineering Practice, 19, 54-61.

Valentin, V. and Denoeux, T. (2001). A Neural Based Software Sensor for Coagulation Control in a Water Treatment Plant. Intelligent Data Analysis, 5, 23-39. 


\title{
MATHMOD 2015 ABSTRACT VOLUME
}

\author{
MATHMOD Minisymposium \\ Regularization Techniques for DAEs to Improve Model \\ Equations for the Numerical Treatment
}


MATHMOD 2015 Abstract Volume 


\title{
Regularization of nonlinear DAEs based on Algebraic Analysis *
}

\author{
Lena Scholz* Andreas Steinbrecher** \\ * Technische Universität Berlin, Institut für Mathematik, \\ 10623 Berlin, Germany (e-mail: lscholz@math.tu-berlin.de). \\ ** Technische Universität Berlin, Institut für Mathematik, \\ 10623 Berlin, Germany (e-mail: anst@math.tu-berlin.de).
}

Differential-algebraic equations (DAEs) arise naturally in many technical and industrial applications. In particular, in automatic modeling using coupling of modularized subcomponents, the coupling of subcomponents is usually described by algebraic constraints which lead to DAEs as model equations. Unfortunately, the direct numerical integration of DAEs in general is not feasible due to socalled hidden constraints which are contained in the DAE but not explicitly stated as equations. The occurrence of hidden constraints leads to difficulties in the numerical integration as instabilities or order reduction can occur. In order to avoid these difficulties one has to force the hidden constraints also for the numerical solution, i.e., one has to ensure that the approximate solution stays on the solution manifold. Thus, a regularization of the system equations, where all constraints contained in the system are explicitly stated, is to be preferred for numerical computations.

In this paper we review a remodeling or regularization techniques for general nonlinear DAEs based on an algebraic analysis that has been developed in Kunkel and Mehrmann (2001). This technique can be used to improve the model equations in order to ensure a robust and stable numerical integration of the DAE. In particular, we consider nonlinear DAEs of the form

$$
F(t, x, \dot{x})=0,
$$

with a sufficiently smooth function $F: \mathbb{I} \times \mathbb{D}_{x} \times \mathbb{D}_{\dot{x}} \rightarrow \mathbb{R}^{m}$ on a compact time interval $\mathbb{I} \subset \mathbb{R}$ and open sets $\mathbb{D}_{x}, \mathbb{D}_{\dot{x}} \subset$ $\mathbb{R}^{n}$. Together with an initial condition

$$
x\left(t_{0}\right)=x_{0}, \quad t_{0} \in \mathbb{I}, x_{0} \in \mathbb{D}_{x}
$$

we obtain an initial value problem consisting of (1) and (2). Since algebraic constraints (and in particular hidden constraints) restrict the solutions of the initial value problem (1), (2) onto a specific solution manifold, the solution behavior of a DAE is quite different to the behavior of an ordinary differential equation. Unfortunately, in general, a parametrization of the constraint manifold is not easy to obtain.

A regularization approach first presented in Campbell (1980) is based on the idea to add all derivatives of the system equations up to a certain order to the original DAE (1) until all hidden constraints are revealed. Based on this idea a general theory for nonlinear DAEs has be developed in Kunkel and Mehrmann $(2001,2006)$ that provides a unified concept for modeling, simulation, control and

* The authors have been supported by the European Research Council through ERC Advanced Grant MODSIMCONMP. optimization of DAEs. We review the main ideas of this algebraic approach and show how a regularization based on this approach can be derived.

The second part of the paper is concerned with the numerical integration of general nonlinear DAEs. In general, in the numerical solution of DAEs many difficulties arise due to the algebraic constraints, in particular due to the hidden constraints which arise in problems with a higher index. It may happen that the solution of the discretized equations is not unique, although the DAE has a unique solution. Also explicit methods cannot be used directly, since an explicit method would require the solution of a linear system with a (typically) singular coefficient matrix. Many DAEs behave in some respect like stiff differential equations which forces one to use methods with good stability properties. Another effect that occurs is that due to discretization errors, the numerical solution may drift off from the analytical solution if the constraints are not explicitly forced during the integration. Thus, in order to avoid these effects, the numerical solution has to be forced to lie on the constraint manifold. With this regard a regularized formulation is to be preferred to the original formulation (1) for the numerical solution, since it can be guaranteed that all constraints are satisfied using suitable integration methods. Thus, instead of the direct discretization of a higher index DAE (1) we can discretize an equivalent formulation of the problem in which an explicit parametrization of the constraint manifold is available such that the numerical solution can be forced to lie on this manifold.

\section{REFERENCES}

Campbell, S.L. (1980). Singular Systems of Differential Equations. Pitman, San Francisco, CA.

Kunkel, P. and Mehrmann, V. (2001). Analysis of overand underdetermined nonlinear differential-algebraic systems with application to nonlinear control problems. Mathematics of Control, Signals and Systems, 14, 233256.

Kunkel, P. and Mehrmann, V. (2006). DifferentialAlgebraic Equations - Analysis and Numerical Solution. EMS Publishing House, Zürich, Switzerland. 


\title{
Regularization of nonlinear DAEs based on Structural Analysis
}

\author{
R. McKenzie ${ }^{*}$ J. Pryce ${ }^{* *}$ N. Nedialkov ${ }^{* * *}$ G. Tan ${ }^{* * * *}$ \\ * Cardiff University, UK (e-mail: mckenzier1@cardiff.ac.uk) \\ ** Cardiff University, UK (e-mail: prycejd1@cardiff.ac.uk) \\ *** McMaster University, Canada (e-mail: nedialk@mcmaster.ca) \\ **** McMaster University, Canada (e-mail: tgn3000@msn.com)
}

\begin{abstract}
Differential algebraic equations (henceforth DAEs), arise from the equation based modelling of physical systems, such as those found in engineering or physics applications, with problems specifically arising from chemical distillation (Washington and Swartz (2011)), electronic circuits (Brenan et al. (1996)) and robotics (Campbell and Griepentrog (1995)) . Models are now frequently built interactively using different components from large libraries in environments such as GPROMS, MAPLESIM, SIMULINK and an assortment of tools that use the Modelica language. This way of modelling systems can lead to large scale DAEs (Fritzson (2015)). A common notion is the differentiation index - which is equal to the number of times all or part of the system has to be differentiated in order to solve the problem as an ODE. It is well known that solving a high index (larger than one) DAE directly is numerically difficult, hence modelling tools usually perform some structural analysis to determine the index of the problem. This talk will outline different ways of using this structural analysis in the solution and regularization of DAEs. In particular the Signature Matrix method (Pryce (2001)), Dummy Derivative method (Mattsson and Söderlind (1993)), Structural-Algebraic method (Scholz and Steinbrecher (2013)) and a new approach called Structural Analysis based Dummy Derivatives will be considered.
\end{abstract}

Keywords: DAE Systems, Numerical and Symbolical Methods for Modelling

\section{INTRODUCTION}

Frequently when one wishes to model a physical system various different modelling languages, each with their own in built libraries can be used. Typically a large scale model, say an automotive vehicle, is built using pre-existing model classes, for example one may need engine, clutch, gearbox and various other smaller classes to model a car. Modelling a physical system in such a way typically produces a differential algebraic equation (DAE). Usually in the DAE literature one likes to talk about a DAEs differentiation index i.e. the number of times all or part of the system has to be differentiated in order to solve the problem as an ODE. Consider for example the simple pendulum:

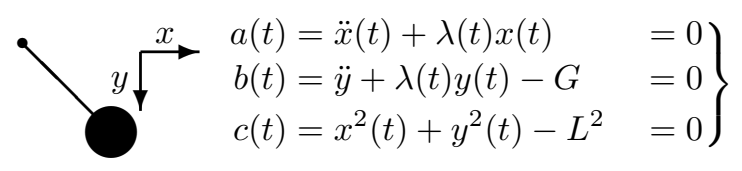

which has differentiation index 3 - one must differentiate equations $a$ and $b$ once and $c$ three times to get an ODE for $x^{\prime \prime \prime}, y^{\prime \prime \prime}$ and $\lambda^{\prime}$. For a DAE arising from a physical system it is usually non trivial to find the differentiation index, thus most solvers perform some structural analysis as a means to estimating the index. Most solvers can handle index 1 DAEs directly, so the task is usually to reduce the differentiation index to 1 , or 0 - an ODE.

\section{SIGNATURE MATRIX METHOD}

One such method of structural analysis is The Signature Matrix method (Pryce (2001)). One can use the structural information found in this method to form a Taylor series and projection based solution process - differentiating parts of the DAE in order to satisfy the so called hidden constraints of the problem and then solving for necessary Taylor coefficients (Nedialkov and Pryce (2008)). For example, the Signature Matrix method would yield the following structural information:

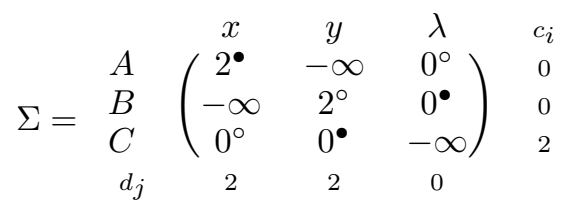

where • and $\circ$ indicate highest value transverals (HVTs), see (Pryce (2001)). The Signature Matrix gives us a solution scheme via Taylor series as shown in Table 1.

\begin{tabular}{|l|l|l|}
\hline Stage & Equations being used & Taylor coefficients found \\
\hline-2 & $C$ & $x_{0}, y_{0}$ \\
\hline-1 & $\dot{C}$ & $x_{1}, y_{1}$ \\
\hline 0 & $A, B, \ddot{C}$ & $x_{2}, y_{2}, \lambda_{0}$ \\
\hline 1 & $\dot{A}, \dot{B}, \dddot{C}$ & $x_{3}, y_{3}, \lambda_{1}$ \\
\hline$\ldots$ & $\ldots$ & $\ldots$ \\
\hline
\end{tabular}

Table 1. Stages for the simple pendulum 


\section{DUMMY DERIVATIVE METHOD}

As stated above, more frequently one will try to reduce the index of the DAE rather than solve the high index (larger than one) problem. Software generally performs an index reduction algorithm (e.g. Dummy Derivatives (Mattsson and Söderlind (1993))) to form a new equivalent index 1 DAE.

The Dummy Derivative (DD) method takes an $n \times n$ smooth DAE and adds the hidden constraint equations to the system directly. This yields an over-determined system. To return to a square system the Dummy Derivative method adds dependent variables for each constraint equation, specifically the method finds a subset of appearing derivatives to be considered algebraic. The advantage of this approach is that by solving the resulting system we satisfy all constraint equations automatically-i.e. we no longer have to worry about drift from constraints during integration, and projection techniques to rectify this. This approach can be costly for large scale systems as there is frequently a need to choose a resulting index $1 \mathrm{DAE}$ dynamically throughout the solution process by re-doing the Dummy Derivative method. For example, the simple pendulum above has two different valid index 1 Dummy Derivative systems, given by equations (2) and (3). We take $x^{\prime}$ to mean a dummy derivative for $\dot{x}$ and so on, i.e. $x^{\prime}$ is considered an algebraic variable to be solved for and not as the first time derivative of $x$. One must use the following index 1 DAE if $|x|$ is small:

$$
\left.\begin{array}{rlrl}
A(t) & =x^{\prime \prime}(t)+\lambda(t) x(t) & & =0 \\
B(t) & =\ddot{y}+\lambda(t) y(t)-G & & =0 \\
C(t) & =x^{2}(t)+y^{2}(t)-L^{2} & & =0 \\
\dot{C}(t) & =2 x x^{\prime}+2 y \dot{y} & & =0 \\
\ddot{C}(t) & =2 x x^{\prime \prime}+2 \dot{x}^{2}+2 y \ddot{y}+2 \dot{y}^{2} & & =0
\end{array}\right\}
$$

and if $|y|$ is small one must use:

$$
\left.\begin{array}{rlrl}
A(t) & =\ddot{x}(t)+\lambda(t) x(t) & & =0 \\
B(t) & =y^{\prime \prime}(t)+\lambda(t) y(t)-G & & =0 \\
C(t) & =x^{2}(t)+y^{2}(t)-L^{2} & & =0 \\
\dot{C}(t) & =2 x \dot{x}+2 y y^{\prime} & & =0 \\
\ddot{C}(t) & =2 x \ddot{x}+2 \dot{x}^{2}+2 y y^{\prime \prime}+2 y^{\prime 2} & & =0
\end{array}\right\}
$$

elsewhere either system can be used. This choice seems trivial here, but in large scale applications there is frequently a very large potential number of systems to use and a need to frequently and dynamically change between selected systems (called pivoting) - due to the number and size of these systems in practice it is usually not possible (or at least very expensive) to store all possible systems or check all systems for potential use when a pivot is needed.

\section{OTHER METHODS}

To reduce the number of potential dummy derivative systems the authors have developed a method called Structural Analysis based Dummy Derivatives that exploits a sparsity pattern inherent in the Signature Matrix method to reduce the choices one has when selecting dummy derivatives dynamically. One way it identifies necessary DDs is the following algorithm:

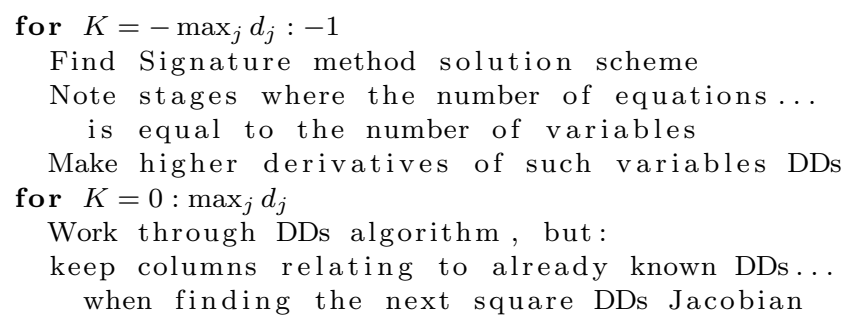

This has the advantage of reducing problem size when doing the Dummy Derivative algorithm. By exploiting a block triangular form inherent in the Signature method we can also reduce the number of index 1 systems needed to consider when performing a pivot whilst solving the DAE numerically.

The authors are also aware of a combined StructuralAlgebraic approach for the regularization of coupled DAE systems (Scholz and Steinbrecher (2013)) that identifies the hidden constraints and adds algebraic variables to the system resulting in a solvable index $1 \mathrm{DAE}$. This technique is very similar to the Dummy Derivative method, but does not have the issue of the added bookkeeping required for dynamic selection of dummy derivatives.

\section{THE TALK}

The talk will give an overview of the Signature Matrix, Dummy Derivative and Structural-Algebraic approaches, giving advantages and disadvantages for each method (illustrated via several examples) with the aim of being accessible to those without a background in DAEs. It will then proceed to introduce the new method of Structural Analysis based Dummy Derivatives and draw parallels to the Structural-Algebraic approach.

\section{REFERENCES}

Brenan, K., Campbell, S., and Petzold, L. (1996). Numerical Solution of Initial-Value Problems in DifferentialAlgebraic Equations. SIAM, Philadelphia, second edition.

Campbell, S.L. and Griepentrog, E. (1995). Solvability of general differential algebraic equations. SIAM J. Sci. Comput., 16(2), 257-270.

Fritzson, P. (2015). Principles of Object-Oriented Modeling and Simulation with Modelica 3.3: A Cyber-Physical Approach. Wiley-IEEE Press, second edition.

Mattsson, S.E. and Söderlind, G. (1993). Index reduction in differential-algebraic equations using dummy derivatives. SIAM J. Sci. Comput., 14(3), 677-692.

Nedialkov, N.S. and Pryce, J.D. (2008). Solving differential-algebraic equations by Taylor series (III): The DAETS code. JNAIAM, 3(1-2), 61-80. ISSN 17908140.

Pryce, J.D. (2001). A simple structural analysis method for DAEs. BIT, 41(2), 364-394.

Scholz, L. and Steinbrecher, A. (2013). A combined structural-algebraic approach for the regularization of coupled systems of DAEs. Technical report, Institut für Mathematik, TU Berlin.

Washington, I. and Swartz, C. (2011). On the numerical robustness of differential-algebraic distillation models. In 61st Canadian Chemical Engineering Conference. London, Ontario, Canada. 


\title{
Regularization of Quasi-Linear Differential-Algebraic Equations
}

\author{
Andreas Steinbrecher* \\ * TU Berlin, Insitut für Mathematik, D-10623 Berlin, GERMANY \\ (e-mail: anst@math.tu-berlin.de).
}

\section{INTRODUCTION}

The complete virtual design of dynamical systems, e.g., mechanical systems, electrical circuits, flow problems, or whole production processes, plays a key role in our technological progress. Therefore, the interconnection of automatic modeling tools with efficient and robust simulation tools is of growing interest. Automatic modeling using coupling of modularized subcomponents are widely used in industry. Here the coupling of subcomponents often is described by algebraic constraints (here short constraints) which leads to differential-algebraic equations (DAEs) as model equations. In general, additionally to constraints explicitly occurring in the DAE the solution is restricted by so-called hidden constraints. Those constraints are hidden in the DAE, i.e., they are not explicitly stated as equations but only obtainable by differentiating of (parts of) the DAE. The occurrence of hidden constraints impose additional consistency conditions on the initial values and provoke severe difficulties in the direct numerical integration of DAEs as instabilities or order reduction can occur. Therefore, it is necessary to regularize or remodel the model equations before a robust numerical integration is possible.

In this article we will discuss regularization methods for quasi-linear DAEs in view of its numerical treatment.

\section{ANALYSIS OF QUASI-LINEAR DAES}

In this Section we discuss an approach for the analysis of an initial value problem for quasi-linear DAEs of the form

$$
E(x(t), t) \dot{x}(t)=k(x(t), t) .
$$

consisting of $n$ equations and $n$ unknowns in $x$. We review a procedure as a tool for the analysis of quasi-linear DAEs and concluding results with respect to the analytical properties of quasi-linear DAEs, like the hidden constraints and the array of constraints

$$
0=\mathfrak{C}(x, t)
$$

containing all constraints including the hidden constraints. These are of great interest for the development of regularization techniques and numerical methods.

\section{REGULARIZATION OF QUASI-LINEAR DAES}

As mentioned above, the existence of hidden constraints leads to difficulties in the numerical treatment. Therefore,

\footnotetext{
* The authors have been supported by European Research Council through ERC Advanced Grant "Modeling, Simulation and Control of Multi-Physics Systems" MODSIMCONMP.
}

a DAE not containing any hidden constraints but with the same set of solutions is suited and preferable for the numerical treatment. We define such a DAE as regularization of the quasi-linear DAE (1).

Based on this definition, in this Section, we discuss several regularization methods for quasi-linear DAEs (1) based on the results of the procedure mentioned above.

In particular, we present the projected strangeness-free formulation

$$
\begin{aligned}
S_{D}(x, t) E(x, t) \dot{x} & =S_{D}(x, t) k(x, t), \\
0 & =\mathfrak{C}(x, t)
\end{aligned}
$$

which corresponds to a regularization of the same size as the original DAE. Here $S_{D}$ is called dynamic selector and determines a minimal set of differential equations modeling the dynamic in the system.

Furthermore, we discuss the minimally extended formulation

$$
\begin{aligned}
\widetilde{E}_{1}^{0}(x, t) \Pi_{1} \bar{\Pi}_{1} \dot{x} & =\widetilde{k}_{1}^{0}(x, t)-\widetilde{E}_{1}^{0}(x, t) \Pi_{2} w, \\
0 & =\mathfrak{C}(x, t)
\end{aligned}
$$

which corresponds to a regularization with increased size. Here $\bar{\Pi}_{1}$ determines a minimal set of variables describing the dynamic in the system.

As last we present the overdetermined formulation

$$
\begin{aligned}
E(x, t) \dot{x} & =k(x, t), \\
0 & =\mathfrak{C}(x, t)
\end{aligned}
$$

which corresponds to a regularization with increased number of equations but the same state $x$.

Beside other well known regularizations, these three regularizations above are suited for further analysis, in particular, for numerical simulation. This comes from the fact that these regularizations do not contain any hidden constraints which is of great advantages for numerical treatments.

\section{NUMERICAL TREATMENT}

At the end we give some remarks on the numerical treatment of the presented regularizations.

The projected-strangeness-free formulation (2) as well as the minimally extended formulation (3) can serve as basis for the numerical integration by use of numerical algorithms suited for stiff ODEs.

For the numerical integration of the overdetermined formulation (4) the numerical approaches or codes have to be adapted. For that we roughly present the code GEOMS suited for the numerical treatment of multibody systems and the code QUALIDAES suited for the numerical treatment of overdetermined quasi-linear DAEs. 


\title{
Index Reduction and Regularisation Methods for Multibody Systems
}

\author{
C. Pöll ${ }^{*}$ I. Hafner** \\ * Institute for Analysis and Scientific Computing, Vienna University of \\ Technology, Austria (e-mail: carina.poell@tuwien.ac.at). \\ ** dwh GmbH, Simulation Services, Vienna, Austria (e-mail: \\ irene.hafner@dwh.at).
}

\section{INTRODUCTION}

The use of object-oriented simulation tools for modelling of physical or mechanical systems leads to systems of differential-algebraic equations (DAEs) with a high differential index. The differential index indicates the minimal number of differentiations of the system which are necessary to extract a system of ordinary differential equations from the differentiated system. Especially in mechanics the use of a global coordinate system to describe the different occurring states leads to a DAE that usually has differential index three. In general the numerical solution of DAEs with high index by conventional solution methods for ordinary differential equations is very complex. Therefore methods for solving this problem are necessary, which leads to the so-called index reduction. In the following seven methods, where most of them are discussed in detail in Hairer, and Wanner (2002), are considered.

\section{DIFFERENTIATION AND SUBSTITUTION OF THE CONSTRAINTS}

In this approach the way of reducing the index is to differentiate the constraints $g(x)=0$ and substitute the constraints by their derivatives, until the system has differential index one. The problem with this method is that due to the differentiation there is a loss of information and so the necessary initial values for the back-integration are unknown and so the numerical "drift-off" occurs.

\section{BAUMGARTE-METHOD}

The Baumgarte-Method can only be used for DAEs with differential index three. The initial point of this method is the index-1-formulation of the DAE with index three. The constraint equations $\ddot{g}(x)=0$ are substituted by a linear combination of $g, \dot{g}$ and $\ddot{g}$ of the form where the parameters $\alpha$ and $\beta$ occur and have to be chosen so that the differential equation is asymptotically stable. The problem of this approach is the exact choice of the parameters $\alpha$ and $\beta$.

\section{PANTELIDES-ALGORITHM}

This algorithm solves the DAE using the so-called "Dummy Derivatives", i.e. if there is a constraint equation an integrator which is connected with the constraint is eliminated by the replacement of a derivative by a dummy variable. On the one hand the algorithm may create a lot of variables and equations, on the other hand the differential index has not to be known for using this method.

\section{STABILISATION BY PROJECTION}

If the numerical solution does not fulfill the constraints after an integration step, the numerical solution is projected onto the solution manifold, which is given by the constraints $g(x)=0$ and some of their derivatives with respect to $t$. A certain procedure leads to a system of differential equations $\dot{y}=f(t, y)$ on the manifold. There are two methods using projection, the orthogonal projection method and the symmetric projection method.

\section{METHODS BASED ON LOCAL STATE SPACE TRANSFORMATION}

The DAE is not solved on the whole state space, but on a manifold. The obtained system of differential equations on the solution manifold (see section 5) which is solved by the introduction of local coordinate transformations. The difficulty of this method is to find suitable coordinates.

\section{GEAR-GUPTA-LEIMKUHLER FORMULATION}

The Gear-Gupta-Leimkuhler formulation aims to include the description of the solution manifold by the constraint equations into the equation system. This leads to an overdetermined system, so a correction term is introduced. This DAE has differential index two and can be solved for example with BDF methods or implicit Runge-Kutta methods.

\section{CONCLUSION AND OUTLOOK}

In this paper, all in all seven methods for the regularisation of DAEs with differential index three were presented. Every method can be applied to solve the DAEs resulting from the equations of motion of mechanical systems. In further studies these methods will be tested by means of several case studies and compared regarding the distance of the numerical solution to the solution manifold, their numerical accuracy and applicability for different tasks. Additionally, methods suitable for DAEs of arbitrary index will be tried out on other than mechanical systems.

\section{REFERENCES}

Hairer, E. and Wanner, G. (2002). Solving ordinary differential equations II. Springer, Germany. 


\title{
Regularization of Electrical Circuits *
}

\author{
Caren Tischendorf* \\ * Humboldt University of Berlin, Dept. of Mathematics, 10099 Berlin, \\ Germany (e-mail: tischendorf@math.hu-berlin.de)
}

\begin{abstract}
We present a little overview about different regularization approaches for electrical circuits. Most of them treat model equations arising from the modified nodal analysis (MNA). They are of the most practical relevance since the well established circuit simulators used in semiconductor industry apply the MNA procedure to generate the circuit equations. Additionally, we discuss some hybrid modeling approaches that are proved to provide more stable model formulations than the standard MNA approach.
\end{abstract}

Keywords: regularization, electrical circuits, index reduction, modified nodal analysis, hybrid modeling

\section{CIRCUIT EQUATIONS}

The basic circuit equations are the Kirchhoff's laws that can be written as

$$
A \boldsymbol{i}=0, \quad \boldsymbol{v}=A^{\top} \boldsymbol{e}
$$

with the incidence matrix $A$ mapping branches to nodes of the circuit. The vectors $\boldsymbol{i}, \boldsymbol{v}$ and $\boldsymbol{e}$ consist of all branch currents, all branch voltages and all nodal potentials, respectively. They Kirchhoff equations are completed by the constitutive element equations

$$
\boldsymbol{i}_{1}=\frac{\mathrm{d}}{\mathrm{d} t} q\left(\boldsymbol{v}_{1}, t\right)+g\left(\boldsymbol{v}_{1}, t\right), \quad \boldsymbol{v}_{2}=\frac{\mathrm{d}}{\mathrm{d} t} \phi\left(\boldsymbol{i}_{2}, t\right)+r\left(\boldsymbol{i}_{2}, t\right)
$$

for lumped current and voltage controlling elements. The modified nodal analysis approach reduces the system equations (1), (2) to the system

$$
\begin{array}{r}
A_{1} \frac{\mathrm{d}}{\mathrm{d} t} q\left(A_{1}^{\top} \boldsymbol{e}, t\right)+A_{1} g\left(A_{1}^{\top} \boldsymbol{e}, t\right)+A_{2} \boldsymbol{i}_{2}=0, \\
\frac{\mathrm{d}}{\mathrm{d} t} \phi\left(\boldsymbol{i}_{2}, t\right)+r\left(\boldsymbol{i}_{2}, t\right)-A_{2}^{\top} \boldsymbol{e}=0,
\end{array}
$$

having as unknown variables only the nodal potentials $e$ and the currents $i_{2}$ of the voltage controlling elements, see e.g. Estévez Schwarz and Tischendorf (2000). Here, the incidence matrix $A=\left(A_{1}, A_{2}\right)$ is split with respect to the current and voltage controlling elements.

\section{WHY REGULARIZATION?}

Why are we interested in regularization approaches? In order to explain it, we consider the following very simple example: A circuit having exactly one loop with one capacitance $C$ and one voltage source $v(t)$. The MNA equations are given by

$$
\frac{\mathrm{d}}{\mathrm{d} t}(C e)+i=0, \quad e=v(t)
$$

with $e$ being the node potential different from the mass node and $i$ being the current through the voltage source. Obviously, the solution of this system reads

$$
e=v(t), \quad i=-C v^{\prime}(t) \text {. }
$$

Comparing this solution $(e, i)$ with the solution $\left(e_{\delta}, i_{\delta}\right)$ of the perturbed system

$$
\frac{\mathrm{d}}{\mathrm{d} t}\left(C e_{\delta}\right)+i_{\delta}=\delta_{1}(t), \quad e_{\delta}=v(t)+\delta_{2}(t)
$$

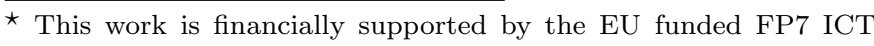
project nanoCOPS GA619166. we find

$$
e_{\delta}-e=\delta_{2}(t), \quad i_{\delta}-i=-C \delta_{2}^{\prime}(t)+\delta_{1}(t) .
$$

It shows that even small perturbations., e.g. of the form $\delta(t)=\varepsilon \sin (\omega t)$, may lead to very high deviations from the exact solution, here e.g. of the size $\omega \varepsilon$.

Such a critical behavior is known for all differential algebraic systems of higher index, see e.g. Lamour et al. (2013), and in particular dangerous for circuits with very high frequency signals. Several investigations of the circuit equations have shown that the MNA equations may lead to DAEs with an arbitrary high index but often not higher than 2, see e.g. Günther and Feldmann (1991), Estévez Schwarz and Tischendorf (2000), Tischendorf (2001).

Notice that each electric engineer would avoid circuit models with loops of capacitances and voltage sources ( $C V$-loops). However, the combination of sophisticated equivalent circuit models for transistor elements may cause circuits containing $C V$-loops.

\section{REGULARIZATION APPROACHES}

Since $C V$-loops and $L I$-cutsets (that means cutsets of inductances and current sources) are known to yield a higher index MNA equation system, the easiest way of a regularization would be the inclusion of the resistances in series to each voltage source belonging to a $C V$-loop and the addition of small capacitances and/or resistances in parallel to $L I$-cutsets. However, this procedure has several drawbacks. First, the dimension of the model equation system increases. Secondly, it is not easy to decide which resistance/capacitance values are appropriate for the added elements. But the most critical fact is the observation that the solution of the regularized system can be much more expensive because of much stronger time step size restrictions, see e.g. Kampowsky et al. (1991).

There have been a series of approaches to bring the MNA equations into a state space description, see e.g. Natarajan (1991). The main goal of these procedures was not a regularization because of stability issues but a faster simulation, e.g. for the application of explicit methods. 
However, the state space equation systems suffer also from higher computational costs mainly caused by the loose of sparsity.

A general index reduction of DAE systems has been introduced by Kunkel and Mehrmann (2004) using a minimal extension of the original DAE system. In principle, this can directly be applied to the MNA equation system but it does not support the automatic generation of the model equation systems from net lists.

Therefore, Bächle and Ebert (2007) adapted this approach in such a way that the regularized systems can be formed from a modified net list. In more detail, they replace one capacitance of each $C V$-loop by a controlled current source reflecting the differentiated Kirchhoff's voltage loop equation. Analogously, they replace one inductance of each $L I$-cutset by a controlled voltage source reflecting the differentiated Kirchhoff's current cutset equation. There are two remaining difficulties with this approach. Since the $C V$-loops usually arise from equivalent circuit descriptions of transistors, the approach requires a modification of (at least some of) the transistor models. But they are in general not easily accessible. Secondly, two new variables are introduced for each independent $C V$-loop. This number can be considerable large.

Estévez Schwarz and Lamour (2001) proposed an index reduction for general index-2 DAEs and explored it especially for MNA network equations. However they used it for the computation of consistent initial values but not for solving the circuit equations. It seems to be too costly for a transient simulation. Recently, Reis and Stykel (2010) presented an efficient procedure for the computation of the spectral projectors for the MNA circuit equations exploiting the topological structure. They used it for a passivity preserving balanced truncation approach but it can also be used for an index reduction. Unfortunately, also here the sparsity can get completely lost.

Chen et al. (2012) introduced an efficient approach combining topological reduction methods with elimination strategies that allows a regularization of large-scaled MNA circuit systems preserving the sparsity structure. So far, the approach has been elaborated for linear systems but it seems to be applicable also for certain nonlinear ones.

Finally, we would like to direct a view onto regularization approaches using hybrid modeling techniques. Iwata et al. (2012) have shown that a hybrid representation of (1), (2) in terms of loop and cutset equations lead to DAEs with at most index 1 if the circuit is passive and the tree voltages of current controlling elements as well as the link currents of voltage controlling elements are eliminated. Riaza (2011), Garcáa de la Vega and Riaza (2013) extended this hybrid reduction models to circuits with nonlinear active elements and in particular also to memristive systems. Unfortunately, practical tests for real large-scaled semiconductor circuits could not be performed so far since the transistor models are only accessible in MNA formulation.

\section{CONCLUSION}

There exist several modeling approaches for electric circuits that result in differential algebraic systems of index 1 at most. However, none of them is used in commercial circuit simulation programs since the typical SPICE model formulation of transistor elements requires the MNA modeling approach.

\section{REFERENCES}

Bächle, S. and Ebert, F. (2007). Index reduction by element-replacement for electrical circuits. In G. Ciuprina and D. Ioan (eds.), Scientific Computing in Electrical Engineering, volume 11 of Mathematics in Industry, 191-197. Springer Berlin Heidelberg.

Chen, Q., Weng, S., and Cheng, C. (2012). A practical regularization technique for modified nodal analysis in large-scale time-domain circuit simulation. ComputerAided Design of Integrated Circuits and Systems, IEEE Transactions on, 31(7), 1031-1040.

Estévez Schwarz, D. and Lamour, R. (2001). The computation of consistent initial values for nonlinear index-2 differentialalgebraic equations. Numerical Algorithms, 26(1), 49-75.

Estévez Schwarz, D. and Tischendorf, C. (2000). Structural analysis of electric circuits and consequences for MNA. Internat. J. Circ. Theor. Appl., 28(2), 131-162.

Garcáa de la Vega, I. and Riaza, R. (2013). Hybrid analysis of nonlinear circuits: Dae models with indices zero and one. Circuits, Systems, and Signal Processing, 32(5), 2065-2095.

Günther, M. and Feldmann, U. (1991). Cad-based electriccircuit modeling in industry. i. Mathematical structure and index of network equations. Surveys on Mathematics for Industry, 8, 97129.

Iwata, S., Takamatsu, M., and Tischendorf, C. (2012). Tractability index of hybrid equations for circuit simulation. Math. Comput., 81, 923939.

Kampowsky, W., Rentrop, P., and Schmidt, W. (1991). Classification and numerical simulation of electric circuits. Surveys on Mathematics for Industry, 2(1), 2365.

Kunkel, P. and Mehrmann, V. (2004). Index reduction for differential-algebraic equations by minimal extension. ZAMM - Journal of Applied Mathematics and Mechanics / Zeitschrift für Angewandte Mathematik und Mechanik, 84(9), 579-597.

Lamour, R., März, R., and Tischendorf, C. (2013). Differential-Algebraic Equations: A Projector Based Analysis: A Projector Based Analysis. Springer, Heidelberg.

Natarajan, S. (1991). A systematic method for obtaining state equations using MNA. Circuits, Devices and Systems, IEE Proceedings G, 138(3), 341-346.

Reis, T. and Stykel, T. (2010). Pabtec: Passivitypreserving balanced truncation for electrical circuits. Computer-Aided Design of Integrated Circuits and Systems, IEEE Transactions on, 29(9), 1354-1367.

Riaza, R. (2011). Explicit ODE reduction of memristive systems. International Journal of Bifurcation and Chaos, 21(03), 917-930.

Tischendorf, C. (2001). Model design criteria for integrated circuits to have a unique solution and good numerical properties. In U. Rienen, M. Günther, and D. Hecht (eds.), Scientific Computing in Electrical Engineering, volume 18 of Lecture Notes in Computational Science and Engineering, 179-198. Springer Berlin Heidelberg. 


\title{
Comparison of Regularisation Methods Referring to a Multi-Pendulum Case Study
}

\author{
I. Hafner ${ }^{*}$ C. Pöll ${ }^{* *}$ \\ * dwh GmbH, Simulation Services, Vienna, Austria (e-mail: \\ irene.hafner@dwh.at). \\ ** Institute for Analysis and Scientific Computing, Vienna University \\ of Technology, Austria (e-mail: carina.poell@tuwien.ac.at).
}

\begin{abstract}
This paper compares several regularisation methods for differential-algebraic equation systems of high differential index by applying them to two model problems. Both problems are mechanical systems which are described by equation systems of index three, a pendulum and a double pendulum, where the latter furthermore shows chaotic behaviour. Some methods turn out to be not suitable at all for high index differential-algebraic equation systems while the quality of many regularisation methods depends on an adequate choice of parameters, implementation and the system itself to be solved with the respective method.
\end{abstract}

Keywords: DAE, Differential Index, Regularisation Methods, Mechanical System, Pendulum

\section{INTRODUCTION}

The description of mechanical systems in general leads to differential algebraic equation systems (DAEs) of high index. To solve these DAEs, different methods for regularisation or index reduction can be applied, see Hairer, and Wanner (2002). In this paper, six of those methods are compared by two case studies. The two considered case studies are mechanical systems of index three. On the one hand the equations of motion of a pendulum on a circular path in Cartesian coordinates are considered. On the other hand, the equations of motion of the double pendulum in Cartesian coordinates, which shows chaotic behaviour, are used. For the comparison of the considered regularisation methods, the obtained numerical solutions and the deviation from the constraint equations are taken into account. The presented methods are differentiation and substitution of the constraint equations ( $\mathrm{DaS} \mathrm{C}$ ), the Baumgarte-Method (BM) (see Eich, and Hanke (1995)), the Pantelides algorithm $(\mathrm{P})$, the orthogonal projection method (OP), the symmetric projection method (SP) (see Hairer (2000)) and transformation of the state space (SST).

\section{CASE STUDIES}

\subsection{Pendulum}

The equations of motion of a pendulum in Cartesian coordinates are given by

$$
\begin{gathered}
\dot{x}=v_{x} \quad \dot{y}=v_{y} \\
\dot{v}_{x}=-F x \quad \dot{v}_{y}=\mathrm{g}-F y \\
x^{2}+y^{2}=1,
\end{gathered}
$$

where $F$ is the force and $\mathbf{g}$ is the gravitational acceleration, see Cellier, and Kofman (2006). The constraint equation of this system is given by $x^{2}+y^{2}-1=0$. In the following the constraint equation and its derivatives with respect to $t$ are considered

$$
\begin{gathered}
x v_{x}+y v_{y}=0 \\
v_{x}^{2}+v_{y}^{2}-F\left(x^{2}+y^{2}\right)+\mathbf{g} y=0,
\end{gathered}
$$

where it can be observed that from the second deriviative the force $F$ can be obtained. This shows that the given DAE has differential index three.

All following simulations are done with MATLAB R2012b. The initial values for the presented scenario are $x=1$, $y=0$ and $v_{x}=v_{y}=F=0$, where the initial value for the force $F$ is not necessary for every method. Fig. 1 shows that the substitution of the constraint by its second derivative (3) is no suitable method for this problem as a clear numerical drift-off can be observed due to the loss of information by differentiation. The Baumgarte-Method

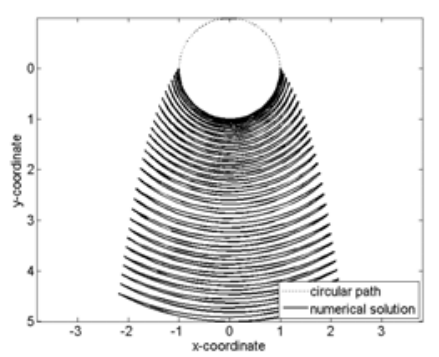

Fig. 1. Drift-off occuring after differentiation and substitution of the constraint, calculated with ode15s (MATLAB)

substitutes the constraint by a linear combination of the constraint and its derivatives, see (4).

$$
\ddot{g}+2 \alpha \dot{g}+\beta^{2} g=0
$$

Due to the consideration of the original constraint in the new system, there is no loss of information. Nevertheless, the choice of suitable values for the parameters $\alpha$ and $\beta$ can be challenging.

For the application of the Pantelides algorithm, four different systems have to be considered for different areas of 
the coordinate system due to the squares in the constraint, which has to be transformed to become an assignment for either $x$ or $y$.

The results of the two projection methods both stay close to the circular path, but the positions themselves differ gravely. This is caused by a seemingly unbounded increase of speed with the orthogonal projection method, see Fig. 2. For this system, a global transformation - the common po-

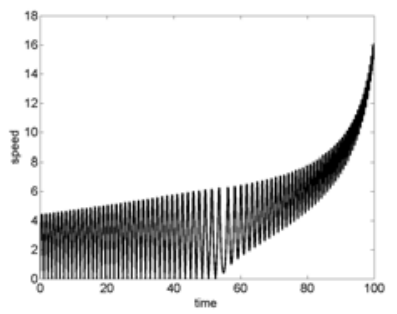

Fig. 2. Increase of speed with the orthogonal projection method (solved with an own implementation of the explicit Euler method)

lar coordinatisation, resulting in (5) - can be found. These equations represent an ordinary differential equation which can easily be solved with common ODE solvers.

$$
\begin{gathered}
\dot{\varphi}=\eta \\
\dot{\eta}=g \cos \varphi .
\end{gathered}
$$

\subsection{Double Pendulum}

The second case study is the double pendulum, where the equations of motion in Cartesian coordinates are given by

$$
\begin{array}{ll}
\dot{x}_{1}=v_{x_{1}} & \dot{v}_{x_{1}}=-F_{1} x_{1}-F_{2}\left(x_{1}-x_{2}\right) \\
\dot{y}_{1}=v_{y_{1}} & \dot{v}_{y_{1}}=\mathbf{g}-F_{1} y_{1}-F_{2}\left(y_{1}-y_{2}\right) \\
\dot{x}_{2}=v_{x_{2}} & \dot{v}_{x_{2}}=-F_{2}\left(x_{2}-x_{1}\right) \\
\dot{y}_{2}=v_{y_{2}} & \dot{v}_{y_{2}}=\mathbf{g}-F_{2}\left(y_{2}-y_{1}\right) \\
x_{1}^{2}+y_{1}^{2}=1 & \left(x_{1}-x_{2}\right)^{2}+\left(y_{1}-y_{2}\right)^{2}=1,
\end{array}
$$

where $F_{1}$ and $F_{2}$ are forces and $\mathbf{g}$ is the gravitational acceleration. The constraint equations are given by $x_{1}^{2}+$ $y_{1}^{2}-1=0$ and $\left(x_{1}-x_{2}\right)^{2}+\left(y_{1}-y_{2}\right)^{2}-1=0$. Deriving the constraint equations two times with respect to the time $t$ shows that the system has differential index three in analogy to section 2.1.

The method of differentiation and substitution of the constraint equations again causes a grave numerical driftoff. Figure 3 shows that the Baumgarte method provides different results for different values of $\alpha$ and $\beta$. The

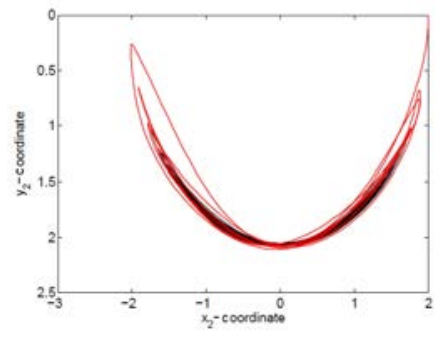

(a) $\alpha=\beta=10$

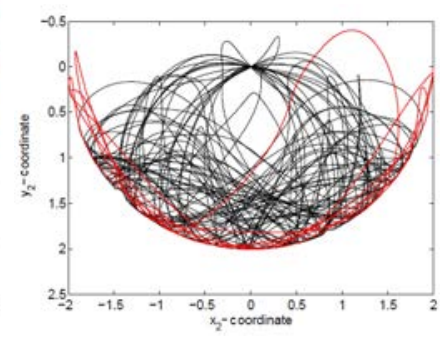

(b) $\alpha=\beta=100$
Fig. 3. Results of the Baumgarte-Method for different values of $\alpha$ and $\beta$, calculated with ode45 (MATLAB)

Pantelides algorithm results in sixteen equation systems of 22 equations each, which represents a very complex description of the given problem. With the orthogonal projection method, an unbounded increase of speed can again be observed, while the symmetric projection method delivers quite reasonable results but takes a long time to simulate due to iteration in each step.

Similar to the pendulum, a global transformation can also be found for the double pendulum, see (7).

$$
\begin{array}{ll}
x_{1}=\cos \varphi_{1} & x_{2}=\cos \varphi_{1}+\cos \varphi_{2} \\
y_{1}=\sin \varphi_{1} & y_{2}=\sin \varphi_{1}+\sin \varphi_{2} \\
v_{x_{1}}=-\eta_{1} \sin \varphi_{1} & v_{x_{2}}=-\eta_{1} \sin \varphi_{1}-\eta_{2} \sin \varphi_{2} \\
v_{y_{1}}=\eta_{1} \cos \varphi_{1} & v_{y_{2}}=\eta_{1} \cos \varphi_{1}+\eta_{2} \cos \varphi_{2}
\end{array}
$$

\subsection{Results}

In Table 1 the maximal error (deviations to the circular paths) and the computing time of all methods until 100 seconds simulation time are shown. It becomes clear that

\begin{tabular}{|c|c|c|c|c|c|}
\hline & \multicolumn{2}{|c|}{ pendulum } & \multicolumn{3}{c|}{ double pendulum } \\
\hline method & max err $e$ & $\mathrm{t}(\mathrm{s})$ & max err $e_{1}$ & max err $e_{2}$ & $\mathrm{t}(\mathrm{s})$ \\
\hline \hline DaS C & 24.731 & 1.1 & 1.008 & 9.495 & 4.9 \\
\hline$\alpha \neq \beta$ & $1.909 \cdot 10^{-5}$ & 1.7 & $1.612 \cdot 10^{-4}$ & $1.605 \cdot 10^{-4}$ & 11.7 \\
\hline$\alpha=\beta$ & $2.464 \cdot 10^{-4}$ & 0.7 & $2.242 \cdot 10^{-5}$ & 0.013 & 3.8 \\
\hline $\mathrm{P}$ & $3.345 \cdot 10^{-4}$ & 8.5 & $1.550 \cdot 10^{-4}$ & $2.677 \cdot 10^{-4}$ & 21.0 \\
\hline OPM & $5.551 \cdot 10^{-16}$ & 76.2 & $4.441 \cdot 10^{-16}$ & $6.662 \cdot 10^{-16}$ & 166.6 \\
\hline SPM & $2.701 \cdot 10^{-8}$ & 71.7 & $2.538 \cdot 10^{-7}$ & $4.764 \cdot 10^{-7}$ & 129.6 \\
\hline SST & $2.221 \cdot 10^{-16}$ & 0.6 & $2.221 \cdot 10^{-16}$ & $2.221 \cdot 10^{-16}$ & 3.7 \\
\hline
\end{tabular}

differentiation and substitution of the constraint and the orthogonal projection method (in spite of staying close to the circular path, compare Fig. 2) lead to unreasonable results while state space transformation proves to be the most suitable for the given problem.

\section{CONCLUSION}

Differentiating and substituting the constraint equations turns out to be not suitable, therefore other approaches for solving the case studies are necessary. The orthogonal projection method has problems with the correct positions due to the increasing speed and hence does not provide reasonable results. The Baumgarte-Method shows good results if the parameters are chosen well. The Pantelides algorithm has the disadvantage of a complex implementation and the used ode-solver has problems to solve the equation systems. In contrast to the orthogonal projection method the symmetric projection method has bounded speed and leads to reasonable results. The last method is the state space transformation which can be done globally for both case studies. All in all, it can be stated that the most suitable method depends highly on the given problem.

\section{REFERENCES}

Hairer, E. and Wanner, G. (2002). Solving ordinary differential equations II. Springer, Germany.

Eich, E. and Hanke, M. (1995). Regularization methods for constrained mechanical multibody systems. ZAMM - Journal of Applied Mathematics and Mechanics.

Hairer, E. (2000). Symmetric projection methods for ordinary differential equations on manifolds. BIT Numerical Mathematics.

Cellier, F.E. and Kofman, E. (2006). Continuous System Simulation. Springer, USA. 
MATHMOD 2015 Abstract Volume 


\section{MATHMOD 2015 ABSTRACT VOLUME}

MATHMOD Poster Contributions 
MATHMOD 2015 Abstract Volume 


\title{
Methods of Inverse Simulation for Nonlinear Ship Steering Systems Investigations
}

\author{
David J. Murray-Smith
}

University of Glasgow, Glasgow, G12 8QQ U.K; (e-mail: David.Murray-Smith@ glasgow.ac.uk).

\begin{abstract}
The paper describes the application of two inverse simulation methods based on continuous system simulation principles to a ship model which incorporates a rudder actuator submodel with saturation and rate limits. One approach depends on feedback principles while the other involves the use of an approximate differentiation method. If the actuator is operating in a linear fashion the two inverse simulation methods give very similar results. However, the differentiation method cannot be used with hard limiting and nonlinear oscillatory phenomena may cause problems with the feedback approach in such cases. An approximate two-stage procedure that avoids these difficulties but provides useful insight about the effects of rudder saturation and rate limiting is presented. Inverse simulation allows the onset of limiting to be detected and can provide insight about vehicle handling and manoeuvrability more directly than conventional simulation methods.
\end{abstract}

Keywords: Simulation, inverse, nonlinear, model, feedback, differentiators, actuators, ship, limit.

\section{INTRODUCTION}

Analytical approaches to model inversion can present problems with nonlinear models in many engineering applications. Simulation techniques are therefore used extensively for finding inverse solutions. Examples include aircraft handling qualities studies (see e.g. Thomson and Bradley (2006)) and applications involving other engineering systems (see, e.g. Murray-Smith (2011) and Tagawa et al. (2012)). In applications involving vehicles of any kind inverse simulation can provide information, in a very direct way, about practical difficulties that may arise in performing different manoeuvres and about available margins of control.

\section{APPLICATION TO A NONLINEAR SHIP MODEL}

A nonlinear ship model, which is commonly-used to represent the manoeuvring characteristics of a course-stable ship in yaw, was developed in the 1960s by Norrbin (van Amerongen (1982)). This nonlinear model has the form:

$$
T \ddot{\psi}+n_{1} \dot{\psi}+n_{3}(\dot{\psi})^{3}=K \delta(t)
$$

where the variable $\psi$ is the yaw angle of the vessel, $\delta$ is the rudder angle and $T$ is an inertia constant. Parameters $n_{1}$ and $n_{3}$ are positive damping constants, known as Norrbin coefficients. For the specific case of the R.O.V. Zeefakkel, which is a $45 \mathrm{~m}$ long training ship belonging to the Royal Netherland Naval College, the parameters $n_{1}$ and $n_{3}$ have been estimated for a number of different forward speeds (van Amerongen (1982)) and are therefore known quantities within the simulation model..
The rudder and its associated actuator are modelled using a first-order lag with saturation and rate limits. In the linear mode of operation the actuator model is characterised by a time constant of $1 \mathrm{~s}$.. The saturation limits for the rudder are $\pm 35 \mathrm{deg}$. and the rate limits are $\pm 7 \mathrm{deg} . . / \mathrm{s}$.

\subsection{Inverse simulation of the ship model using feedback principles.}

The use of feedback principles for inverse simulation is well established. (see, e.g., papers by Buchholz and von Grünhagen (2004), Tagawa et al. (2012) and Murray-Smith (2011)). Application of this approach to the ship model is straight-forward for cases where the actuator operates linearly. However, results found for large heading changes involve saturation and rate limiting and have a very different character, showing oscillatory transients which are a property of the feedback approach and not of the system for which the inverse response is required.

A two-stage procedure allows the feedback methodology to be used with actuator nonlinearities present, but without these unwanted oscillations (Murray-Smith (2014)). In the first stage of the procedure an inverse simulation based on the feedback approach is used to find an input to the actuator model that would achieve a desired heading-rate output response time history if no limits were present. The time history of rudder deflection can then be examined, to determine whether or not the amplitude and rate limits are being exceeded. The effect of including the saturation and rate limits may then be investigated quantitatively in the second stage of the procedure by applying this idealised actuator input, found from the inverse simulation process, to 
a forward simulation of the ship with the full nonlinear actuator sub-model. Figure 1 shows a typical result found using this two-stage approach, indicating clearly the effects of the rate limit over the first $12 \mathrm{~s}$. of the response.

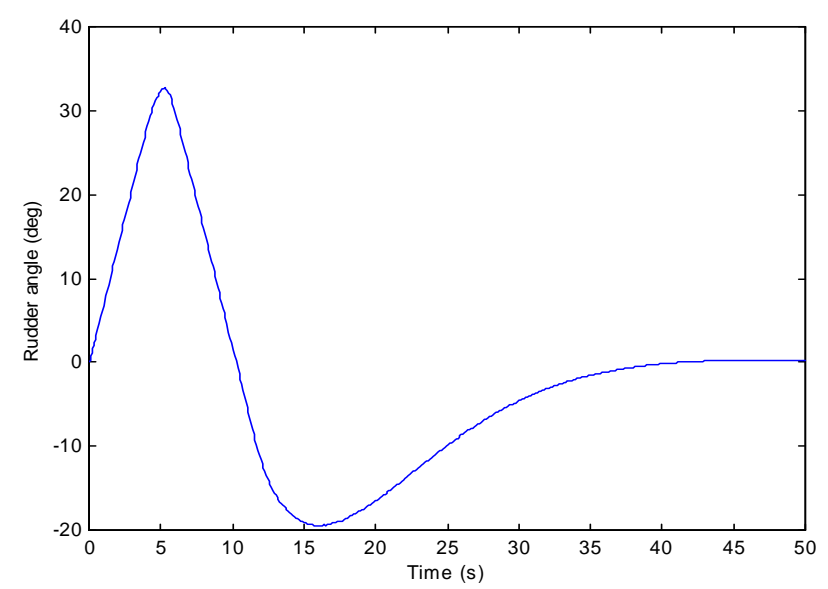

Fig. 1: Plot of rudder angle (deg.) versus time (s.) found by inverse simulation with the two-stage feedback method for forward speed of $2.6 \mathrm{~m} / \mathrm{s}$ and heading change of $30 \mathrm{deg}$. with rudder angle limit of $\pm 35 \mathrm{deg}$. and rate limit of $\pm 7 \mathrm{deg} / \mathrm{s}$.

\subsection{Inverse simulation using a two-stage approximate differentiation approach}

The second approach is based on an approximate differentiation method (Murray-Smith (2013)). However, this cannot be applied directly with amplitude and rate limits and a two-stage approach is again needed (Murray-Smith (2014)). An ideal actuator signal, generated using the approximate differentiation method for the case of the linear actuator model, is passed to a forward simulation model which includes the actuator nonlinearities. Figure 2 shows results for this two-stage procedure involving use of the approximate differentiation method in the first stage.

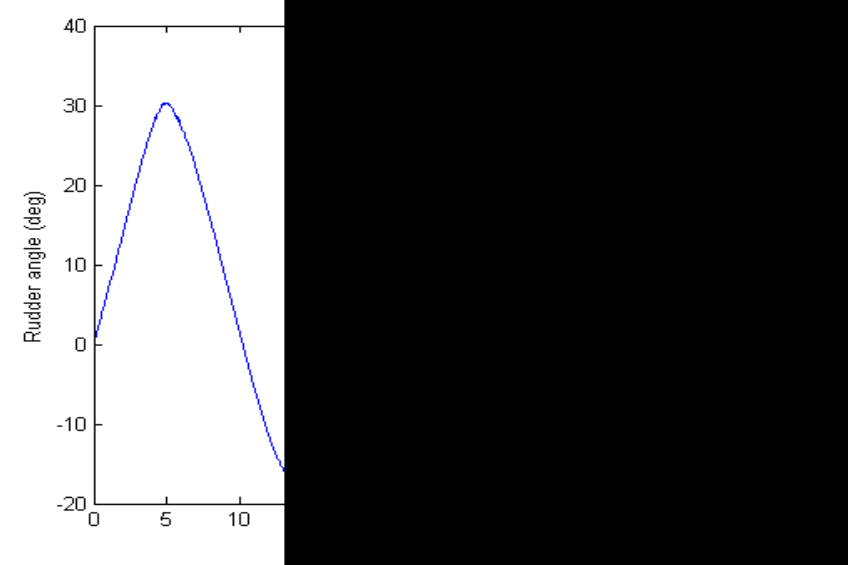

Fig. 2: Plot of rudder angle (deg.) versus time (s.) found by the two-stage differentiation approach for same conditions that applied for the feedback method results of Figure 1.

Comparison of the rudder deflection time histories of Figures 1 and 2 show very similar forms of curve, although the peak value of rudder angle found using the approximate differentiation method is slightly smaller than that obtained by the two-stage feedback method. However, the time history of the error in heading obtained by forward simulation using the rudder deflection shown in Figure 2 as input is found to be very close to that obtained by the two-stage feedback method. Additional comparative results may be found in Murray-Smith (2014).

\section{DISCUSSION AND CONCLUSIONS}

For applications in which actuator amplitude and rate limits are active, a two-stage approach is needed when using either the feedback or approximate differentiation methods. This involves an inverse simulation stage based on a linear actuator sub-model and a second-stage involving a forward simulation model with the actuator nonlinearities included. Tests have shown that these two approaches give very similar.results in all cases considered (Murray-Smith (2014)).

It should also be noted that, for cases that do not involve the actuator nonlinearities, a more conventional single-stage approach is adequate, both for the feedback and differentiation methods. Results found using these two methods are almost identical, for the same sets of conditions.

Although computationally simple, the approximate differentiation method has one major disadvantage. Any changes applied to the forward model of the system require some re-structuring of the inverse simulation. With the feedback approach no alteration of this kind is needed in the inverse simulation if changes are made in the forward model.

\section{REFERENCES}

Buchholz, J.J. and von Grünhagen, W. (2004), Inversion Impossible? Technical Report, University of Applied Sciences Bremen, Germany.

Murray-Smith, D.J. (2011), Feedback methods for inverse simulation of dynamic models for engineering applications, Math. \& Comp. Modelling of Dynamical Syst., 17 (5), 515-541.

Murray-Smith, D.J. (2013), Looking at problems the other way round: engineering applications of inverse simulation based on continuous system simulation methods. In; $8^{\text {th }}$ EUROSIM Congress, Cardiff, Wales, UK, September 1012 2013. Keynote Lecture No.4. Extended abstract and slides. Online: http://eurosim2013.info/. (accessed 12th January 2015).

Murray-Smith, D.J. (2014), Inverse Simulation Applied to Nonlinear Actuation Problems in Ship Steering. Internal Report, DJMS14-01, School of Engineering, University of Glasgow.

Tagawa, Y., Tu, J.Y. and Stoten, D.P. (2012), Inverse dynamics compensation via 'simulation of feedback control systems', Proc. Institution of Mech. Engineers, Part I: J. Systems and Control Eng., 225, 137-153.

Thomson, D. and Bradley, R. (2006), Inverse simulation as a tool for flight dynamics research - Principles and applications, Prog. in Aerosp. Sciences, 42 (3), 174-210. van Amerongen, J. (1982), Adaptive Steering of Ships, $\mathrm{PhD}$ Thesis, Delft University of Technology. 


\title{
Closed form model of manipulators with highly flexible links
}

\author{
Bruno Scaglioni * Gianni Ferretti * \\ * Politecnico di Milano, Dipartimento di Elettronica, Informazione e \\ Bioingegneria, 20133 Milan, Piazza Leonardo da Vinci 32, Italy \\ (e-mail: [bruno.scaglioni,gianni.ferretti]polimi.it).
}

A closed form dynamic model of manipulators with highly flexible links is presented in this abstract. The model is based on a Newton-Euler formulation of motion equations and a substructuring approach is used to account for large deformations. The model, formulated in closed form with respect to joints and elastic coordinates, accounts also for quadratic velocity terms. The formulation of the motion equations starts from a data set which can be either analytically or numerically computed by FE codes. Validation has been carried out by comparing simulation results with two different multibody softwares.

In the classical floating frame of reference (FFR) approach (Shabana (1998)), where superposition of large body motion and small linear deformations expressed in local reference frame is considered, every link $i$ is characterized by a local FFR $\left\{O_{i}, \boldsymbol{x}_{i}, \boldsymbol{y}_{i}, \boldsymbol{z}_{i}\right\}$ as shown in Fig.1, while another local frame $\left\{O_{i}^{\prime}, \boldsymbol{x}_{i}^{\prime}, \boldsymbol{y}_{i}^{\prime}, \boldsymbol{z}_{i}^{\prime}\right\}$ placed at the link tip, is here considered, with the same orientation of the FFR in the undeformed configuration. The angle $\theta_{i}$ is the coordinate of the joint connecting link $i$ to link $i-1$, while $\hat{\boldsymbol{z}}_{i-1}^{\prime}$ is the axis of rotation in the frame $\left\{O_{i-1}^{\prime}, \boldsymbol{x}_{i-1}^{\prime}, \boldsymbol{y}_{i-1}^{\prime}, \boldsymbol{z}_{i-1}^{\prime}\right\}$.

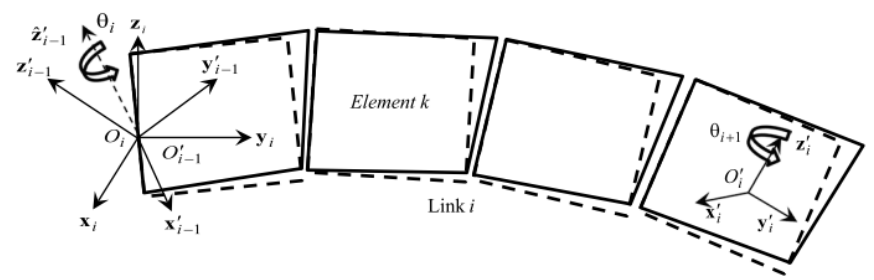

Fig. 1. Reference frames and substructures

The motion equations for link $i$, expressed in the local reference frame, can be then developed using the principle of virtual work (Meirovitch (1967); Shabana (1998))

$$
\begin{aligned}
{\left[\begin{array}{ccc}
m_{i} \boldsymbol{U} & m_{i} \tilde{\overline{\boldsymbol{d}}}_{C, i}^{T} & \overline{\boldsymbol{C}}_{t, i}^{T} \\
m_{i} \tilde{\boldsymbol{d}}_{C, i} & \overline{\boldsymbol{J}}_{i} & \overline{\boldsymbol{C}}_{r, i}^{T} \\
\overline{\boldsymbol{C}}_{t, i} & \overline{\boldsymbol{C}}_{r, i} & \boldsymbol{M}_{e, i}
\end{array}\right]\left[\begin{array}{c}
\dot{\overline{\boldsymbol{v}}}_{i} \\
\dot{\overline{\boldsymbol{\omega}}}_{i} \\
\ddot{\boldsymbol{q}}_{i}
\end{array}\right] } & =\left[\begin{array}{c}
\mathbf{0}_{3} \\
\mathbf{0}_{3} \\
-\boldsymbol{K}_{e, i} \boldsymbol{q}_{i}-\boldsymbol{D}_{e, i} \dot{\boldsymbol{q}}_{i}
\end{array}\right] \\
& +\left[\begin{array}{c}
\boldsymbol{h}_{\omega, i}^{r} \\
\boldsymbol{h}_{\omega, i}^{\theta} \\
\boldsymbol{h}_{\omega, i}^{f}
\end{array}\right]+\left[\begin{array}{c}
\boldsymbol{h}_{e, i}^{r} \\
\boldsymbol{h}_{e, i}^{\theta} \\
\boldsymbol{h}_{e, i}^{f}
\end{array}\right]
\end{aligned}
$$

where $\boldsymbol{M}_{e, i} \in \mathbb{R}^{M_{i} \times M_{i}}, \boldsymbol{D}_{e, i} \in \mathbb{R}^{M_{i} \times M_{i}}, \boldsymbol{K}_{e, i} \in \mathbb{R}^{M_{i} \times M_{i}}$ are the structural mass, damping and stiffness matrix respectively, regarding the flexible dofs, $\boldsymbol{h}_{\omega, i}^{r} \in \mathbb{R}^{3}, \boldsymbol{h}_{\omega, i}^{\theta} \in$
Table 1. Link data

\begin{tabular}{ll}
\hline$M_{i}$ & Number of modal coords. \\
$I_{i}^{1}, \mathbf{I}_{i}^{2}, \mathbf{I}_{j, i}^{3}, \mathbf{I}_{i}^{4}, \mathbf{I}_{j, i}^{5}, \mathbf{I}_{i}^{6}, \mathbf{I}_{i}^{7}, \mathbf{I}_{j, i}^{8}, \mathbf{I}_{j k, i}^{9}$ & Inertia invariants \\
$\mathbf{K}_{e, i}$ & Structural stiffness matrix \\
$\mathbf{S}_{i}, \hat{\mathbf{S}}_{i}$ & Shape functions matrices \\
$\overline{\mathbf{u}}_{0 i}$ & rel. position between FFRs \\
$\hat{\boldsymbol{z}}_{i-1}^{\prime}$ & Joint rotation axis \\
\hline
\end{tabular}

$\mathbb{R}^{3}, \boldsymbol{h}_{\omega, i}^{f} \in \mathbb{R}^{M_{i}}$ are the vectors of gyroscopic and centripetal terms and $\boldsymbol{h}_{e, i}^{r} \in \mathbb{R}^{3}, \boldsymbol{h}_{e, i}^{\theta} \in \mathbb{R}^{3}, \boldsymbol{h}_{e, i}^{f} \in \mathbb{R}^{M_{i}}$ are the vectors of external forces, applied at the body connectors. The terms of the generalized mass matrix, as well as the gyroscopic and centripetal terms, can be computed starting from data of Table 1 as described in Ferretti et al. (2014).

Adopting a spatial vector notation (Fijany and Featherstone (2013)), thus defining:

$$
\boldsymbol{V}_{i}=\left[\begin{array}{c}
\boldsymbol{\omega}_{i} \\
\boldsymbol{v}_{i}
\end{array}\right] \in \mathbb{R}^{6}, \quad \boldsymbol{F}_{i}=\left[\begin{array}{c}
\boldsymbol{n}_{i} \\
\boldsymbol{f}_{i}
\end{array}\right] \in \mathbb{R}^{6}
$$

where $\boldsymbol{\omega}_{i}$ and $\boldsymbol{v}_{i}$ are the angular and linear velocities of each FFR expressed in the global reference frame and $\boldsymbol{n}_{i}$ and $\boldsymbol{f}_{i}$ are the force and torque applied from link $i$ to link $i-1$ at $O_{i}$ respectively, all the kinematic and dynamic equations relative to the each single link can be collected into global equations, defining a closed form model of the flexible manipulator:

$$
\begin{aligned}
\mathcal{M}_{\theta \theta}(\boldsymbol{\theta}, \boldsymbol{q}) \ddot{\boldsymbol{\theta}}+\mathcal{M}_{\theta q}(\boldsymbol{\theta}, \boldsymbol{q}) \ddot{\boldsymbol{q}}+\mathcal{C}_{\theta}(\boldsymbol{\theta}, \boldsymbol{q}, \dot{\boldsymbol{\theta}}, \dot{\boldsymbol{q}})=\boldsymbol{\tau} \\
\mathcal{M}_{\theta q}^{T}(\boldsymbol{\theta}, \boldsymbol{q}) \ddot{\boldsymbol{\theta}}+\mathcal{M}_{q q}(\boldsymbol{\theta}, \boldsymbol{q}) \ddot{\boldsymbol{q}}+\mathcal{D}_{e} \dot{\boldsymbol{q}}+\mathcal{K}_{e} \boldsymbol{q}+ \\
+\mathcal{C}_{\boldsymbol{q}}(\boldsymbol{\theta}, \boldsymbol{q}, \dot{\boldsymbol{\theta}}, \dot{\boldsymbol{q}})=\mathbf{0}
\end{aligned}
$$

Where $\boldsymbol{\theta}=\operatorname{col}\left(\theta_{i}\right), \boldsymbol{q}=\operatorname{col}\left(\boldsymbol{q}_{i}\right), \boldsymbol{q}_{i}$ being the vector of elastic coordinates of link $i$. It must be recalled that equations (3,4), in particular matrices $\mathcal{M}_{\theta \theta}, \mathcal{M}_{\theta q}, \mathcal{M}_{q q}$, $\mathcal{D}_{e}, \mathcal{K}_{e}$ and vectors $\mathcal{C}_{\theta}$ and $\mathcal{C}_{\boldsymbol{q}}$, can be computed in closed form starting from the links data summarized in Table 1.

However, the standard FFR approach is generally not applicable in the case of large deflections, thus when the elastic displacements are not "small". In this case, the absolute nodal coordinates formulation (ANCF) has been proposed (Yakoub and Shabana (1999)), requiring the definition of global shape functions for every element with respect to the absolute reference frame. On the other hand, the ANCF approach is actually only suitable for beams and shells elements, but it is hardly applicable to complex shapes and cannot benefit of the results of FEM analysis. Accuracy of results for large deformation fields can be 


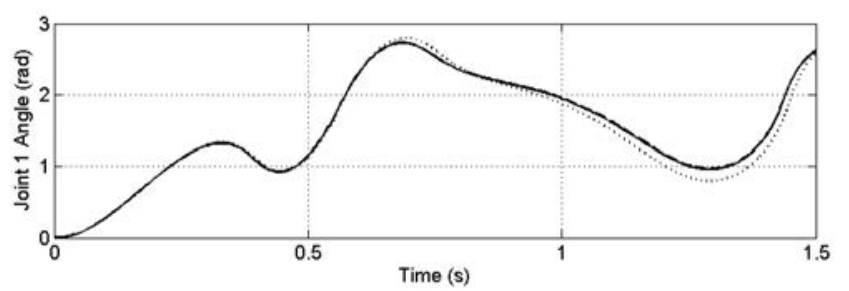

Fig. 2. Angle of the first joint

anyway achieved in the context of the FFR approach by means of the substructuring technique (Shabana (1985)) as demonstrated in the context of this work.

Highly flexible bodies can thus be substructured in several elements, applying on each element the theory of linear elasticity. To this aim, link $i$ can be subdivided into more rigidly connected flexible elements or substructures, as shown in Fig. 1, each one described through a FFR approach introducing $M_{k}$ modal coordinates. Define with $N_{e}$ the total number of elements of the whole manipulator and with $M=\sum_{k=1}^{N_{e}} M_{k}$ the total number of modal coordinates. Let $\theta_{i}=\theta_{\bar{k}}^{e}, i=1, \ldots, N$ denote the "real" joint angle between element $\bar{k}-1$ and $\bar{k}$ and consider a "dummy" joint angle $\theta_{k}^{e}$ if a rigid connection exists between element $k-1$ and $k$. Then, the model remains formally identical by removing the columns corresponding to the "dummy" joint angles in the assembly matrices.

The developed model has been validated by comparison of simulation results obtained with a Matlab/Simulink implementation of the closed form model with multibody simulations, obtained with Modelica/Dymola and with MSC/Adams. The benchmark has been taken from Yakoub and Shabana (1999), where the ANCF is used to describe links compliance in the case of a flexible double pendulum.

The structure consists of two flexible bodies connected together and to the ground by revolute joints, initially in horizontal position and free to fall under the effect of gravity. The physical parameters, summarized in Table 2, have been chosen in order to allow large deformation on the second pendulum. The models, developed in the different environments, implements substructuring by subdividing the first link in two elements with length $0.1 \mathrm{~m}$ and the second link in 12 elements with lentgh $0.075 \mathrm{~m}$.

All figures show comparisons among Matlab/Simulink simulation (solid line), Modelica/Dymola simulations (dashed line) and MSC/Adams simulations (dotted line). It must be pointed out that the Modelica model accounts for damping in exactly the same way as the closed form model, while Adams adopts a different approach, hence, small differences in the simulation results are appreciable. Figures 2 and 3 show the relative angle of the first and second joint, while figure 4 shows the transverse deflection of the tip point of the second link. As it is apparent, results are in good accordance, in particular, the results obtained from Matlab/Simulink and Modelica/Dymola simulations are undistinguishable, while the results provided by MSC/Adams shows some small differences. A sequence of 3D snapshots of the simulation at intermediate instants is shown in Fig. 5.

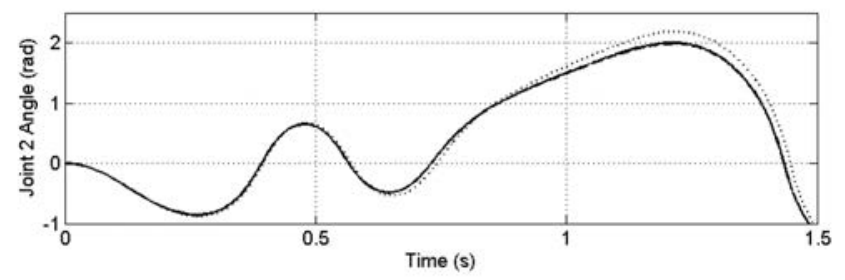

Fig. 3. Angle of the second joint

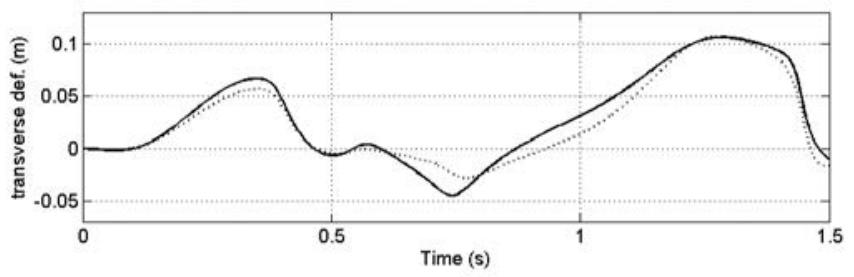

Fig. 4. Transverse deflection of second pendulum tip

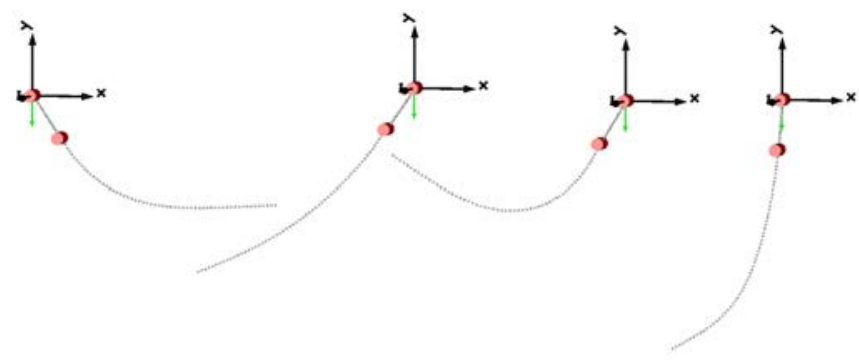

Fig. 5. 3D representation of the simulation

Table 2. Double Pendulum data

\begin{tabular}{lll}
\hline Property & Body 1 & body 2 \\
\hline Mass $(\mathrm{Kg})$ & 0.6810972 & 2.4740052 \\
Length $(\mathrm{m})$ & 0.2 & 0.9 \\
Cross sectional Area $\left(\mathrm{m}^{2}\right)$ & $1.26 E-03$ & $1.26 E-03$ \\
Second moment of area $\left(\mathrm{m}^{4}\right)$ & $1.26 E-07$ & $1.26 E-07$ \\
Mass moment of inertia $\left(\mathrm{Kg} \times \mathrm{m}^{2}\right)$ & $2.27 E-03$ & $1.67 E-01$ \\
Modulus of elasticity $\left(\mathrm{N} / \mathrm{m}^{2}\right)$ & $8.00 E+07$ & $5.00 E+05$ \\
\hline
\end{tabular}

\section{REFERENCES}

Ferretti, G., Leva, A., and Scaglioni, B. (2014). Objectoriented modelling of general flexible multibody systems. Mathematical and Computer Modelling of Dynamical Systems, 20(1), 1-22.

Fijany, A. and Featherstone, R. (2013). A new factorization of the mass matrix for optimal serial and parallel calculation of multibody dynamics. Multibody System Dynamics, 29, 169-187.

Meirovitch, L. (1967). Analytical Methods in Vibration. Macmillan Publishing, New York.

Shabana, A.A. (1998). Dynamics of Multibody Systems. Cambridge University Press, New York.

Shabana, A. (1985). Substructure synthesis methods for dynamic analysis of multi-body systems. Computers and Structures, 20(4), $737-744$.

Yakoub, R. and Shabana, A. (1999). Use of Cholesky coordinates and the absolute nodal coordinate formulation in the computer simulation of flexible multibody systems. Nonlinear Dynamics, 20(3), 267-282. 


\title{
Modeling of Elastic Robotic Arm using a Soft-Computing Algorithm
}

\author{
Hammam Tamimi * Dirk Söffker * \\ * Chair of Dynamics and Control, University of Duisburg-Essen, \\ Duisburg, Germany (e-mail: hammam.tamimi@uni-due.de)
}

\begin{abstract}
This paper proposes the use of the least square support vector machine (LS-SVM) algorithm to model an elastic robotic arm. Dynamic system modeling is important as the first step in obtaining a suitable controller for any system. Acquiring an accurate model of elastic robotic based on input-output measurements using the LS-SVM algorithm requires less knowledge about the physical-laws of the system. The LS-SVM algorithm achieves global, unique solution, and requires less training time compared with other soft computing algorithms. In this paper, a successful use of the LS-SVM algorithm to model the elastic robotic arm as multi-input multi-output system is demonstrated. The simulation results illustrate the efficiency and high performance of the proposed approach.
\end{abstract}

Keywords: System identification, multi-input multi-output system, soft-computing algorithm, support vector machine, multi-step ahead perdition.

\section{INTRODUCTION}

This paper focuses on the modeling of elastic robotic arm. Building light weight manipulators is advantageous since smaller actuators can be used for driving the joints of the manipulator. Consequently, this translates to less energy consumptions. Additionally, damage to the manipulator system due to accidental collisions can be avoided by adding flexibility. Further, light-weight manipulators exhibit higher speed manipulators compared to conventional rigid manipulators.

Due to the flexibility of the elastic robotic arm varies problem rises such as the dynamic model is considered highly nonlinear, structural vibrations, and the accurate positioning of the end effector is reduced.

This paper concerns with the use of least squares support vector machine (LS-SVM) to model an elastic robotic arm. The implemented method has two goals, the result model should be able to make accurate multi-step ahead prediction, and the method should allow the modeling of the elastic robotic arm as multi-input multi-output system.

\section{SYSTEM IDENTIFICATION USING SUPPORT VECTOR MACHINE}

The Support Vector Machine (SVM) algorithm was proposed in Vapnik et al. (1996) . Although the SVM algorithm is widely used in the field of classification application, the use of SVM algorithm in the field system identification is not fully explort . The adaptation of the LS-SVM for the modeling of dynamic system was proposed in Suykens and Vandewalle (1999), where the LSSVM algorithm was successfully implemented to model the double scroll system. The multi-step ahead prediction model of unknown dynamic system with the input vector $u \in \mathbb{R}^{m}$ and output vector $y \in \mathbb{R}^{r}$ is done by estimating the function $f()$ given as

$$
\begin{aligned}
& \hat{y}(k)=f(\hat{y}(k-1), \hat{y}(k-2), \ldots, \hat{y}(k-p), \\
&u(k), u(k-1), \ldots, u(k-p)),
\end{aligned}
$$

where $\hat{y}$ is the estimated output, $k$ is the time step, $p$ the number of previous steps.

According to Suykens and Vandewalle (1999), the dynamic model of the unknown system estimated using LS-SVM is given as

$$
\hat{y}(k)=\sum_{j=1}^{M} \alpha_{j} K\left(q(k), q_{j}\right)+b,
$$

where $q(k)=[\hat{y}(k-1), \hat{y}(k-2), \ldots, \hat{y}(k-p), u(k), u(k-$ $1), \ldots, u(k-p)], \alpha_{j}$ is Lagrange multiplier, $M$ is the number of nonzero Lagrange multipliers, $q_{j}$ is called support vector, kernel function $K($,$) , and b$ is a bias term.

The graphical representation of recurrent LS-SVM model is shown in figure 1. It should be noted that the multi-step ahead prediction is achieved by feeding back the output to the model input vector.

As outlined by Yan et al. (2003), the SVM has less tuning effort in comparison to multilayer perceptron (MLP) network. Also, the computational time required by the SVM algorithm is less than MLP network; due to the fact that the SVM algorithm uses linear equations to obtain the results while MLP network uses backpropagation algorithm. Yan et al. (2003) noted that models obtained using the SVM algorithm has better generalization ability than ones obtained using MLP network. Additionally, for the SVM there is no risk of getting stuck into local minima, thus the SVM always results in a unique and global solution. 


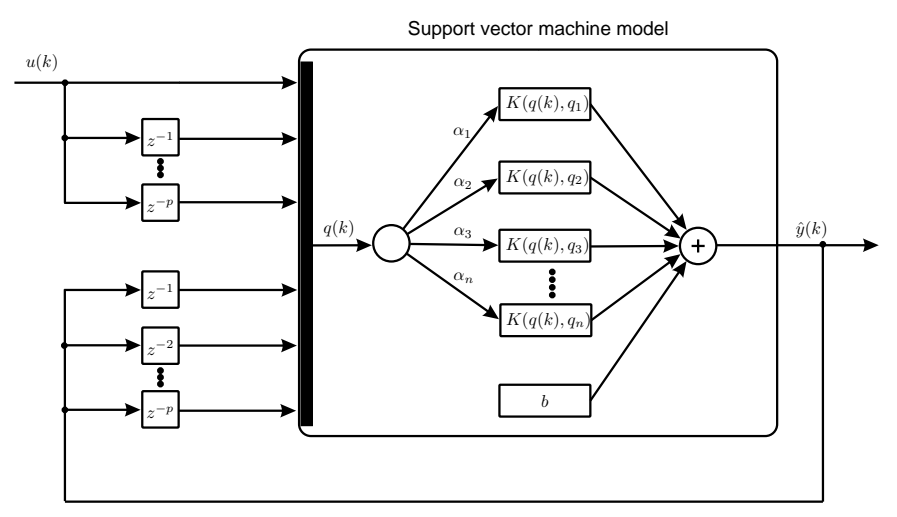

Fig. 1. Recurrent LS-SVM model

\section{PRACTICAL ASPECTS OF USING SOFT-COMPUTING ALGORITHMS FOR MODEL BUILDING}

To ensure accuracy of the dynamic model the following two major practical aspects have to be considered:

- The cross-validation technique is used to overcome the bias-variance dilemma. The most popular method in cross-validation technique is the $k$-fold crossvalidation. In this work the 2 -fold cross-validation, which is the simplest form of $k$-fold cross-validation, is used. In this method the data are divided into two parts; the first part is used for training, second part is used for validation, then the process is reversed.

- For a perfect dynamic model, the input error crosscorrelation should show no correlation and the error autocorrelation should show one nonzero value at zero lag. The dynamic model accuracy can be improved by increasing the number of delay $p$ Beale et al. (2014).

\section{SIMULATION RESULTS}

In this paper, the elastic robotic arm is considered as cantilever beam. The simulation model is obtained using finite element method (FEM) assuming that the cantilever beam consists of five elements with a control input applied at the tip of the beam. The cantilever beam parameters are given in table 1 . The simulation model is excited using two distinctive signals; pseudo random signal and sweep signal. For simplicity, in this paper the displacement signals $\left[x_{1}\right.$ to $\left.x_{5}\right]$ are considered. It is important that the excitation signals have wide range of dynamic properties in order to stimulate all the dynamic of the system. In this work the sweep signal is used to train the LS-SVM model and the pseudo random signal is used for the validation of the LS-SVM model.

Table 1. Beam parameters

\begin{tabular}{|l|l|}
\hline Density $(\rho)$ & $2700 \mathrm{~kg} / \mathrm{m}^{3}$ \\
\hline Length $(l)$ & $1 \mathrm{~m}$ \\
\hline Cross section area $(A)$ & $3.2 \times 10^{-4} \mathrm{~m}^{2}$ \\
\hline Modulus of elasticity $(E)$ & $10 \times 10^{10} \mathrm{~N} / \mathrm{m}^{2}$ \\
\hline
\end{tabular}

The sum of squared error (SSE) function is considered as a performance measure of the LS-SVM model. The process of selecting $p$ is an iterative process, $p$ is increased until the performance is maximized. Once the number of delays is obtained ( $p=53$ ), an accurate LS-SVM model is calculated. In Figure 2 the prediction of the LS-SVM model red and black curves compared to the FEM model curve in blue and green curves while exiting the system with two different pseudo random signals is shown. It can observed that predictions of the LS-SVM model are accurate.

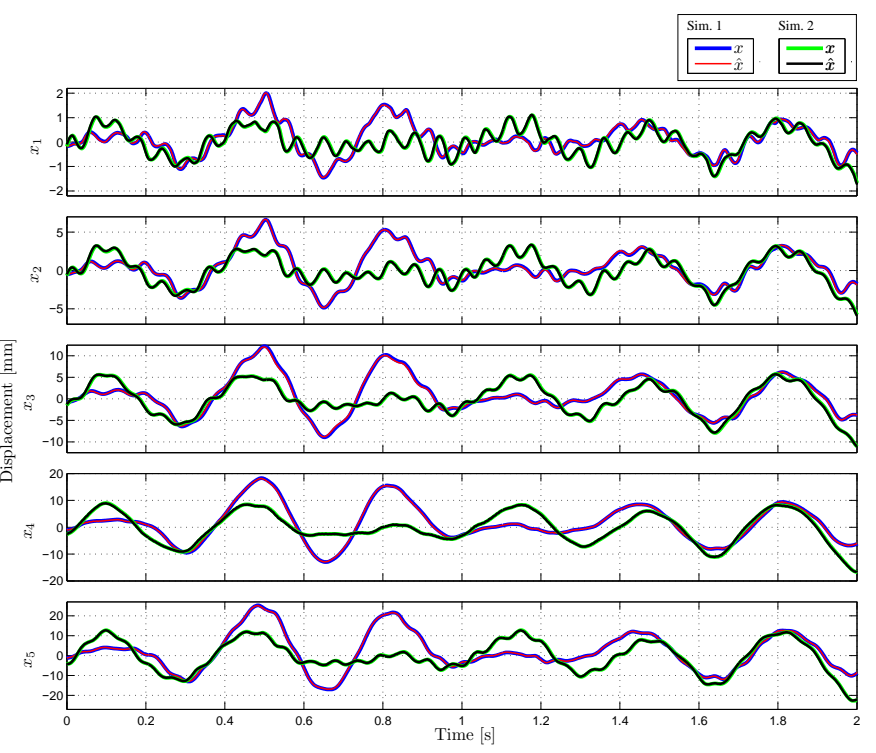

Fig. 2. LS-SVM model prediction vs. simulation model

\section{SUMMARY}

In this paper, modeling of elastic robotic arm as multipleinput multiple-output system using LS-SVM algorithm is presented. The LS-SVM algorithm has less tuning parameters, is faster to train, also it achieves an unique and global solution. The simulation results show the successful multistep-ahead prediction of LS-SVM model of the elastic robotic arm at different positions.

\section{REFERENCES}

Beale, M.H., Hagan, M.T., and Demuth, H.B. (2014). Neural network toolbox: getting started guide. Mathworks.

Suykens, J. and Vandewalle, J. (1999). Least squares support vector machine classifiers. In Neural Processing Letters, volume 9, 293-300.

Vapnik, V., Golowich, S., and Smola, A. (1996). Support vector method for function approximation, regression estimation and signal processing. Advances in Neural Information Processing Systems, 9, 281-287.

Yan, R., Liu, Y., Jin, R., and Hauptmann, A. (2003). On predicting rare classes with SVM ensembles in scene classification. IEEE International Conference on Acoustics, Speech, and Signal Processing, 3, 21-24. 


\title{
Modeling, Control, and Powermanagement Optimization for an Emulated Multi-Source Hybrid Drivetrain
}

\author{
B. Moulik* D. Söffker* \\ * Chair of Dynamics and Control, University of Duisburg-Essen, \\ Duisburg,47057Germany (e-mail: bedatri.moulik@uni-due.de)
}

\begin{abstract}
Hybrid vehicles have gained a lot of importance in the past years. Their ability to save energy and simultaneously satisfy the vehicle power demands makes them particularly significant in conserving resources. Although significant developments have been made, consumption minimization options for guiding the power flows between its different components, could be taken into consideration. In this paper, a three-power-source hybrid powertrain along with online powermanagement control is realized using DC/DC-converters. A new embedded online optimization approach is proposed and its application in two experimental set-ups is discussed.
\end{abstract}

\section{INTRODUCTION}

For the past several years, hybrid vehicles have mostly implied two power sources with various powermanagement techniques. The main aim of this contribution is to determine the flow of power between three main components of the multi-power hybrid namely, fuel cell, battery, and supercapacitor such that the entire process is capable of being carried out online with reduced computational effort. The first step is to formulate control rules for a rule-based controller based on the type of control strategy chosen for each of the components, the mode of operation, etc. This controller takes input from the components (Fig. 1) and sends desired signals to the DC/DC converters. As a next step, with the help of a suitable optimization algorithm, the parameters from PM such as battery/supercapacitor State-of-Charge (SoC), battery current, etc. are analysed for mimimum fuel consumption on a case-specific basis depending on the velocity-time profile/mode of operation of the vehicle. For multiple cases, multiple iterations of offline optimization are required. Therefore, the optimization is decoupled from online PM. The final step is to combine the results from optimization with the PM so as to obtain an online solution. This a achieved by storing the optimal parameter values in a Look-up-Table (LUT). The advantage of such a strategy (Fig. 2) is a reduction in computational effort. Due to its applicability in real-time systems, here, two experimental set-ups can be considered. One is a hybrid electric vehicle (HEV) set-up consisting of components emulating real drivetrain components, and the other a coupled system of HEV and driving simulator where instead of a predefined load cycle, a human driver generates an unknown velocity profile. The disadvantage of the proposed embedded online optimiation is the generation of sub-optimal solutions.

\section{POWERTRAIN SYSTEM MODELING}

The considered powertrain system is a three source hybrid vehicle. Here fuel cell acts as pure power source and the supercapacitor and battery act as both source and stor-

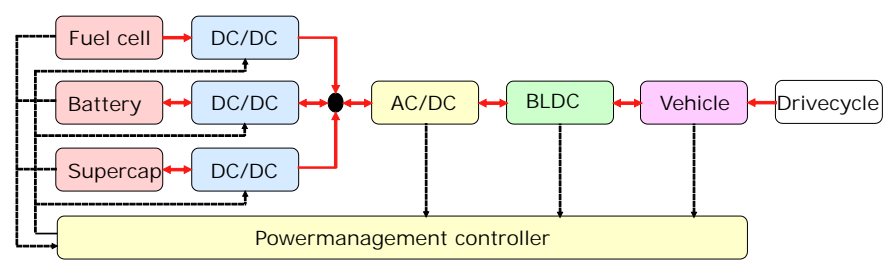

Fig. 1. Dynamic modeling used for PM

\begin{tabular}{|c|c|c|c|c|}
\hline Online & & & Offline & \\
\hline $\begin{array}{l}\text { Accurate \& } \\
\text { dynamic } \\
\text { model }\end{array}$ & $\begin{array}{l}\text { Rule-based } \\
\text { controller }\end{array}$ & LUT & $\begin{array}{l}\text { Parameter } \\
\text { optimization }\end{array}$ & $\begin{array}{l}\text { Simplified } \\
\text { model }\end{array}$ \\
\hline
\end{tabular}

Fig. 2. Embedded online optimization strategy

age devices. Using three DC/DC-converters, the power flow between the components is controlled. Mono- and bi-directional DC/DC-converters are used (Fig. 1). The modeling (Oei et al. (1997), Akella et al. (2001), Marx et al. (2014)) takes into account the dynamics of the components. The accuracy at which the modeled components represent real components is kept as high as possible. However, an integration of the optimization algorithm might result in increased complexity, computational time, and effort. Therefore a simplified model is considered. The objective function is defined in terms of fuel consumption.

\section{CONTROL ALGORITHMS AND PARAMETER OPTIMIZATION}

The control actions considered in this contribution are specific to the component controlled namely fuel cell, battery, and supercapacitor. The methods include a two-position control of the primary source, a current rate limitationbased controller, and a bus voltage-regulation controller Marx et al. (2014). For optimization, a suitable parameter optimization algorithm is chosen which generates optimal solutions in multiple iterations. It is run as a separate offline process and only the results from these iterations are implemented online using a LUT (Fig. 2). 


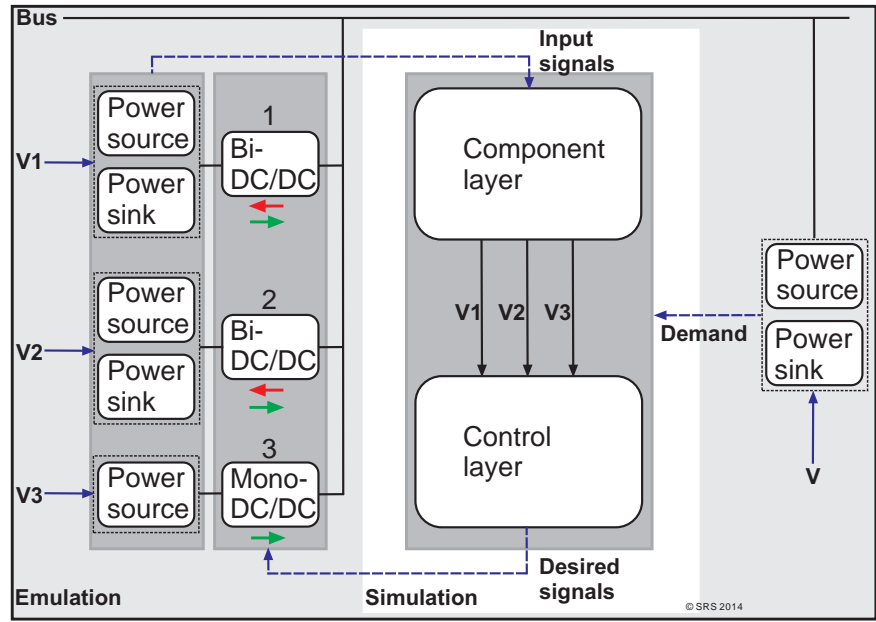

Fig. 3. Theoretical concept for HEV emulation

\section{APPLICATION EXAMPLES}

The first experimental set-up considered is a hybrid electric vehicle. The real vehicle components such as fuel cell, battery, and supercapacitor are replaced by controllable power sources and sinks that are capable of emulating the real components Gao et al. (2011). For example, a fuel cell can be emulated by a source, battery by a sourcesink combination, and the vehicle by a power consumer in acceleration mode and power supplier in deceleration mode. Apart from power sources and sinks, both mono and bi-directional DC/DC converters are used. The advantage of such a set-up is the possiblity to make the hardware cheap and compact. Powermanagement is achieved with the help of rule-based control that takes into account power flow control methods Marx et al. (2014) and executes them online. The dynamic and nearly accurate model of FCHEV is considered in combination with this PM (Fig. 1). The parameters involved in PM relevant for optimization are the SoCs of battery and supercapacitor and the battery current, so an information on the optimal boundaries of these parameter values in terms of minimum fuel consupmtion can be sent to the PM in order to ensure optimal power exchange between components. This is achieved with a suitable optimization algorithm that calculates optimal values for each case of PM and stores them in a LUT, which, containing only the results from multiple iterations of the optimization algorithm, can be integrated with the PM. The powertrain modeling and rule-based PM are implemented in the simulation environment and all the signal flows between the simulation and hardware/emulation environments are realized with the help of a real time interface (Fig. 3). Here the desired outputs from the rule-based PM can be fed to the real DC/DC converters. The LUT contains optimal parameter values for each of the driving modes which act as a reference for the PM controller. In other words, the PM controller can attain optimal parameter information corresponding to each driving mode from the LUT. Thus, the task of the LUT is to store optimal parameter values corresponding to each mode (modes: power from/to one/more source/source-sinks, etc.). The second experimental set-up considered is that of a hybrid electric vehicle and driving simulator coupling. Here, the emulator set-up can be kept the same as the previous set-up but instead

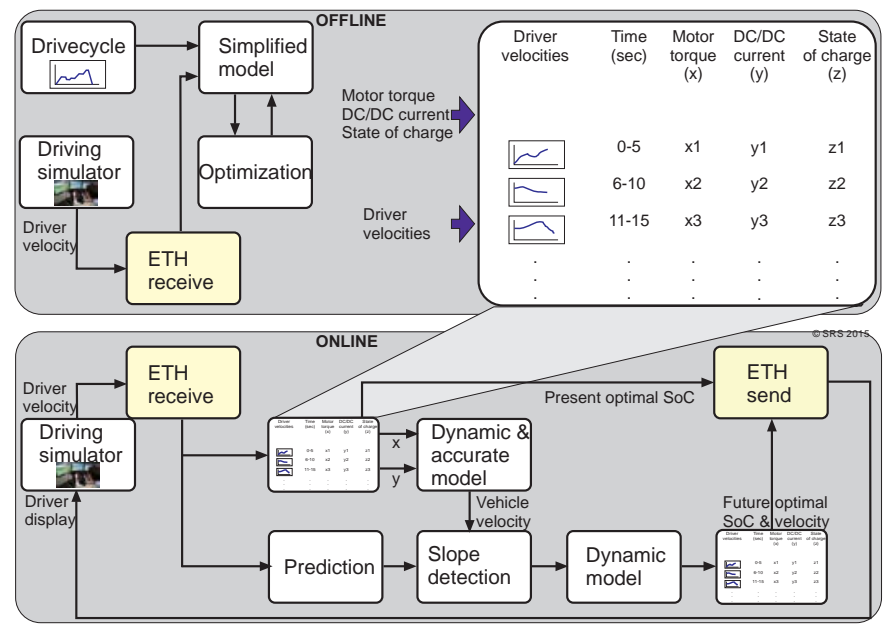

Fig. 4. Theoretical concept for HEV-driving simulator

of a feeding a predetermined drive cycle to the vehicle, the velocity pattern information of a real human driver is used. Unlike the previous set-up, where PM controller was combined with the LUT, the human acts as a controller. Here, the task of the LUT is only to supply velocity pattern-specific optimal values as an information to the driver. The decision on changing the driving pattern is left to the driver. The entire process is shown in Fig. 4. Thus, online realization of embedded results of offline optimization is possible in both application examples.

\section{SUMMARY AND CONCLUSION}

In this contribution, we propose a method to carry out online optimization with the results generated from multiple iterations of offline optimization. The embedded-online optimization can be applied to a HEV emulation set-up in combination with powermanagement or to a HEV-driving simulator set-up with human-in-the loop. The advantages include reduction in computational effort and the suitability of both experimental set-ups for lab purposes.

\section{REFERENCES}

Akella, S., Sivashankar, N., and Gopalswamy, S. (2001). Model-based systems analysis of a hybrid fuel cell vehicle configuration. In Proceedings of the American Control Conference. Arlington.

Gao, F., Blunier, B., Bouquain, D., Miraoui, A., and .ElMoudn, A. (2011). Model based DC power source emulator for electrical and hybrid electrical vehicles drive train tests. In Proceedings of IEEE Vehicle Power and Propulsion Conference. Chicago.

Marx, M., Özbek, M., and Söffker, D. (2014). Power management of a hybrid electric powertrain system design, power flow control, and optimisation targets. International Journal of Powertrains, 3, 221-241.

Oei, D., Adams, J.A., and Kinnelly, A.A. (1997). Directhydrogen-fueled proton-exchange-membrane fuel cell system for transportation applications. National Technical Information Service, Transportation Research Information Services TRIS, USA. 


\title{
Network Based Simulation In Water Construction
}

\author{
Stefan Brunner, Gerhard Höfinger \\ STRABAG AG, Zentrale Technik Wien \\ Donau-City-Straße 9,1220 Vienna, Austria \\ (e-mail: stefan.brunner@strabag.com,gerhard.hoefinger@strabag.com)
}

\section{INTRODUCTION}

Simulation is currently not very widely used in the construction industry. In a research project at Zentrale Technik Wien it could be shown that simulation is a suitable instrument to examine the influence of certain phenomena such as bad weather, technical defects, interruptions of supply, etc. on a dredging project. These phenomena are outside the sphere of influence of the construction contractor, are mostly of a stochastic nature and can often have a significant impact on the cost of the relevant construction activity. Simulation supports in quantifying the influence of these phenomena and can therefore help reduce price risk for the construction contractor and/or its client.

Herein, we present a framework used in the construction industry to dynamically generate simulation models of arbitrary harbor dredging projects with variable equipment fleets.

\section{PROBLEM FORMULATION}

In many harbors it is necessary to remove silt from basins and channels from time to time in order to provide for navigability. Usually these works are carried out with dredging platforms and a fleet of barges that transport the material from the dredgers to spoil areas at some distance from the harbor entrance.

In addition to the dredgers and barges, tugs are needed to manoeuver the barges in the vicinity of the dredgers and to tow the barges of the non-automobile type to the spoil areas and back to the dredgers. Modern tugs with enhanced maneuverability are used for moving the barges in the vicinity of the dredging platforms (platforms that are fixed to the seafloor in the area of operation with a bucked excavator mounted on top). Simpler and more inexpensive tugs are used to tow the barges to the spoil areas and back. Usually there are several dredgers, several dredging and spoil locations and a variety of barges and tugs involved in this kind of construction activity.

The equipment cost is essentially time based. However a merely performance based approach does not lead to reliable results due to presence of phenomena influencing the construction process that are often interlinked and clearly outside the sphere of influence of the construction firm. These phenomena include but are not limited to: Unsuitable weather conditions, damage of barges or other equipment, necessary refuelling of the excavators, the passage of large ships, unsuccessful landing of barges, and the relocation of dredging platforms. All these items lead to a disruption of the construction process, reducing the degree of utilization of the dredgers, hence increasing total construction cost. The key to minimizing costs is maximizing the degree of capacity utilization of the dredgers as the most costly elements which is done by ensuring a continuous servicing of the dredgers with barges.

Finding the right fleet composition for the barges is vital for achieving cost efficiency, especially because changing the fleet is very costly and time consuming. A sound simulation model enables the estimator to work with scenarios in order to find an "optimal" fleet composition before the works start.

Compared to earthworks on land (as in conventional road construction), which can be optimized using linear programs (Bogenberger et al., 2013), the costs are higher and less units (barges, dredgers) are in use.

In order to create a more solid basis for cost calculation a framework for automatic simulation model creation was developed. The resulting models enable the user to carry out Monte-Carlo-Analysis as well to further secure cost calculation results.

\section{THE MODEL}

The nature of the problem described above suggests using discrete event simulation in order to quantify the bandwidth of the influence of the disruptions on the duration of a given construction project described before.

In summer 2013 a subsidiary of STRABAG was carrying out a dredging project in the harbor of Yuzhne, Ukraine. Thus, activity reports of 3 dredging platforms for almost 1 year were available, making it possible to determine the probability distributions for the disrupting phenomena of interest. Data analysis has shown that a lognormal distribution is a suitable model for all these phenomena.

The simulation model was created using the simulation software Anylogic (6.8.1), which had proven to be suitable for modeling construction related processes before (e.g. for tunnel construction, material supply in tunnel construction, material flow in a quarry, influence of construction activities on traffic, etc.).

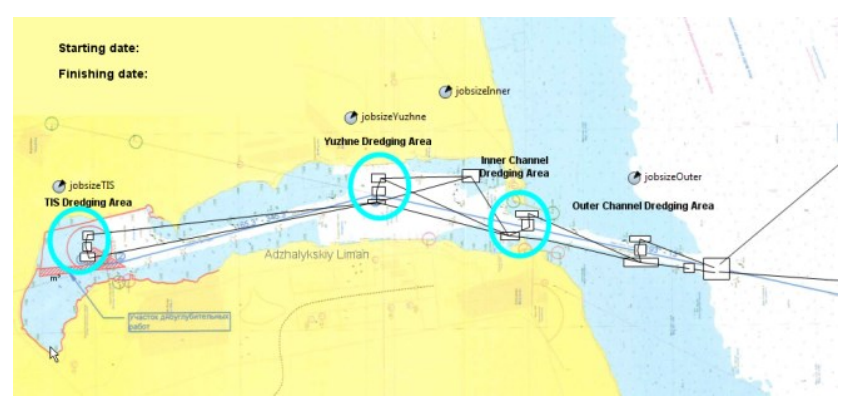

Fig. 1. The network drawn on a sea map

For the model presented in this paper a network based modelling approach was used. Herein the locations, such as 
dredging and spoil sites and anchorage are the nodes and the routes along which the ships move are the vertices.

The network geometry is taken from a map of the relevant area. In the first phase of the research the network was literally drawn on a sea map of Yuzhne as can be seen in Figure 1.

Dredging platforms and barges are entities that populate the network. The tugs are modelled as network resources, which means that the barges request the tugs in accordance with the task that has to be carried out and the network object itself provides the entities with the requested resource as soon as it is available.

A close to optimum disposition of the barges to the dredgers is crucial to minimize idleness of the excavators. Therefore, on site a human dispatcher controls the operation of the barges.

In the model, the dispatcher is represented by the "Disponent" object, which iterates over all operational dredgers once a barge is unloaded at a spoil area and identifies the dredger with the least shipping space on the way and then sends the relevant barge to its dredging area. As in reality, the "Disponent" object is called each time a barge has completed a task and a new destination for the respective barge has to be determined.

The events in the simulation are triggered by a random number generator using the frequency distributions and durations calculated from the reports of the dredgers. Parameters were calibrated using site data.

The validated model was then used to try a variety of different sets of equipment for the given project in order to find an optimal equipment configuration.

\section{VALIDATION}

Similar to the real system the dredging platforms in the simulation model can create activity reports. This data was used to validate the model. The data of 30 days in the real system were compared to 30 simulated days. Simulation results match the real world results satisfactorily (see Table 1). Observing a longer timespan obviously leads to a better agreement with real world results. A denser data basis would have been desirable.

Table 1. Validation results

\begin{tabular}{|l|r|r|r|r|}
\cline { 2 - 5 } \multicolumn{1}{c|}{} & \multicolumn{2}{c|}{ count } & \multicolumn{2}{c|}{ mean duration } \\
\cline { 2 - 5 } \multicolumn{1}{c|}{} & $\begin{array}{l}\text { real } \\
\text { data }\end{array}$ & $\begin{array}{l}\text { sim } \\
\text { data }\end{array}$ & $\begin{array}{l}\text { real } \\
\text { data }\end{array}$ & $\begin{array}{l}\text { sim } \\
\text { data }\end{array}$ \\
\hline loading & 493 & 543 & 79,30 & 77,35 \\
\hline position change & 57 & 38 & 56,43 & 58,32 \\
\hline repair & 56 & 33 & 226,55 & 113,91 \\
\hline ship traffic & 52 & 33 & 87,45 & 115,89 \\
\hline barge shortage & 188 & 212 & 70,05 & 136,11 \\
\hline refuelling & 19 & 13 & 64,44 & 65,12 \\
\hline bad weather & 27 & 39 & 416,35 & 661,14 \\
\hline
\end{tabular}

\section{GENERALISATION}

In order to allow application of the model to other construction sites of the discussed type, dependency of expert model developers had to be reduced. A tool had to be created which allows estimators without knowledge of modeling software to adapt the model to their respective construction project. Acceptance of this tool by estimators and simplicity of usage is crucial for introduction of simulation as a method for cost estimation and risk management.

To achieve this, object replication and connectivity features of Anylogic were used. In a generic model, location of dredging sites, spoil areas, and ship routes are input via kml-files which are produced using GoogleEarth. Additional information on barges and dredgers are input via Microsoft Excel into standardized forms, which are partly created dynamically via VBA. The Anylogic model reads the kmland the Excel-file and sets up the model accordingly.

Training of estimators and application to new construction sites is part of an ongoing development project.

\section{CONCLUSIONS}

Simulation of logistic processes is currently not very widely used in the construction industry (an overview of existing approaches can be found in Günther et al., 2012), despite the fact that many of its processes are predestined for simulation due to the circumstance that construction processes are very often so costly that real experiments cannot reasonably be carried out and external influences, which are hardly quantifiable, have an important impact on construction processes.

Yet it could be shown that simulation can be put to use for cost calculation and risk estimation, generating a significant benefit for the user. This project shows that highly flexible tools can be created in a way that estimators unfamiliar with modelling and simulation can use them.

Through application of simulation models in certain niches the degree of familiarity with and the trust in these instruments can certainly be increased. If we succeed in reproducing the complexity of large scale construction activities with semi- or fully automatized generated models, maybe also in connection with improved data models, it will be possible to facilitate a number of decisions with simulation models that can be generated quickly and inexpensively. Of course, in the future the majority of construction projects will still be satisfactorily planned and executed by experienced project managers. But more and more complex projects are harder and harder fully to perceive by a single human being and therefore, prospects for simulation models in the construction industry are increasing and therewith the prospects for the players in the construction industry using the instrument of simulation. (Höfinger, 2014)

\section{REFERENCES}

Bogenberger, C., Dell'Amico, M., Hoefinger, G., Iori, M., Novellani, S., Panicucci, B. (2013, May). Earthwork Optimization Models for the Construction of a Large Highway System, International Network Optimization Conference.

Günthner, W.A., Borrmann, A (ed) (2011), Digitale Baustelle - innovativer Planen, effizienter Ausführen, SpringerVerlag, Berlin

Höfinger, G. (2014), Chancen für die Simulation in der Bauwirtschaft, WING Business, 47/1, p. 10-12 


\title{
A new tool for visual modeling - Rand Model Designer 7.
}

\author{
Andrey A. Isakov *, Yury B. Kolesov*, \\ Yury B. Senichenkov*

\begin{abstract}
*National Research University St. Petersburg State Polytechnical University, Russia, 195251, St. Petersburg, Polytechnicheskaya,29 (Tel: +7 (812)297-1616; e-mail: senyb@dcn.icc.spbstu.ru).
\end{abstract}

\begin{abstract}
MvStudium research group has announced the new version of visual environment for modeling and simulation of complex dynamical systems Rand Model Designer 7. It has two principal differences in comparison with previous versions: a) now it is possible using dynamic objects of components with «input-output» or «contact-flow» external variables for solving problems of queueing theory («agent-based»approach); b) global system of equations for a local behavior of component model with hybrid behavior is always built, analyzed and transformed on run time.
\end{abstract}

Keywords: modeling and simulation, visual environment, complex dynamical systems, hybrid systems, component models, automatic building and transforming equations, algebraic-differential equations, structural analysis, block triangular form.

MvStudiumGroup research group supports three visual tools for modeling and simulation complex dynamical systems: Rand Model Designer (www.rand-service.com, www.mvstudium.com) for common using, TransasProf (www.transas.com) for designing real time marine training simulators, and open-source tool OpenMVLShell (https://den.ftk.spbstu.ru/) for education (Isakov and Senichenkov $(2010,2011)$ ). All of them use object-oriented modeling language Model Vision Language (Kolesov and Senichenkov (2007)). Model Vision Language (MVL), likewise Modelica language (Fritzson (2011)), oriented on modeling hierarchical event-driven component systems. It is based on Unified Modeling Language's de facto standards, accommodating them for hybrid systems. Hybrid systems are considered as an extension of classical dynamical systems (Senichenkov (2004), Kolesov and Senichenkov (2006), Kolesov and Senichenkov (2012,2014).

The main concept of MVL is «active dynamical object» with global behavior described by Behavior-Chart (modification of UML's State Machine) and local behaviors in the form of algebraic-differential equations (Kolesov and Senichenkov (2007)).

Building global systems of equations for composition of component's automata is the main difficulty for component models with hybrid behavior. When number of components and number component's hybrid automata states increase, the number of possible states for composition became extraordinarily large. Alternative for building all possible systems beforehand is building only realized systems directly on run time. In large scale event-driven systems the number of realized local behaviors is large too. It is very important to know the structure of solving system. Systems with block triangular structure are abundant problems in practice. Using numerical methods taking block triangular structure in account increases speed of computer modeling. The problem of automatic building global equations for local behavior of component model with hybrid behavior and automatic detecting or transforming its structure on run time is considered.

The simplest RMD-7 user's equation form is a form for describing local behavior of isolated hybrid system. It is a system of differential equations with substitutions (1):

$$
\begin{aligned}
& w=\operatorname{Subst}(w, C), F\left(\frac{d s}{d t}, s, w, t, C\right)=0, \operatorname{Out}(y, s, w, C)=0, \\
& w \in \mathfrak{R}^{k 1} ; C \in \mathfrak{R}^{k 2} ; F, \frac{d s}{d t}, s \in \mathfrak{R}^{n} ; \text { Out }, y \in \mathfrak{R}^{m}
\end{aligned}
$$

It contains algebraic-differential equations $F\left(\frac{d s}{d t}, s, w, t, c\right)=0$, respect to state variables $s \in \mathfrak{R}^{n}$, with constancies $\quad C$ and substitutions $w=\operatorname{Subst}(w, C)$, and equations $\operatorname{Out}(y, s, w, C, t)=0$ respect to output variables $y \in \mathfrak{R}^{m}$. Systems of equations in form $F\left(\frac{d s}{d t}, s, w, t\right)=0$ will be automatically transformed with the help of Gear's substitution to

$$
\left\{\frac{d s}{d t}=z ; \quad F(z, s, w, t)=0, s(0)=s_{0}\right.
$$

Special cases (linear and non-linear variants) of form (2) are

$$
\begin{aligned}
& \left\{\begin{array}{l}
\frac{d s}{d t}=F(s, w, t) \\
G(y, s, w, t)=0, s(0)=s_{0}
\end{array}\right. \\
& \left\{\begin{array}{l}
\frac{d s}{d t}=F(s, w, t), s(0)=s_{0}, \\
\{G(s, w, t)=0
\end{array}\right.
\end{aligned}
$$

The linear form is recognized automatically.

There are special Solvers for each type of equations. The special role plays Solvers for Non-linear Algebraic Equations (NAE). To solve NAE is necessary in implicit methods for Ordinary Differential Equations (ODE), in methods for finding consistent initial conditions and solving AlgebraicDifferential Equations (DAE). NAE are solved with the help 
of Newton method, and in case of its failure, Powell method is used. Solving of linear systems of algebraic equations (SLAE) is the main operation for Newton method in turn. Newton method may choose appropriate modification of Gauss method with taking structure of solved system matrix in account: dense, band, sparse, block triangular.

Any techniques decreasing computational costs of numerical solution are very important. Computing matrix block triangular form if possible is well known and commonly used trick (Duff (1977)). In RMD-7 this technique is used for structural matrix of equations. Structural matrix of a system is a matrix with $\{0,1\}$ elements pointing out occurrence of unknowns in equations. RMD computes block triangular form for structural matrix of any allowed types of systems (SLAE, NAE, ODE, DAE) with the help of Tarjan algorithm and solves only subsystems for strongly connected blocks.

Tarjan algorithm in RMD is used for reordering substitutions to computable sequence of formulas and detecting equations («algebraic loops») among them too.

Building and transformation global equations cause maximum difficulties for component models with «contactsflow» external variables («acausal blocks»). It is well known that if even local behaviors of components' automata do not contain high-index DAE, they can appear in their composition. So it is necessary to analyze all states of composition, if we want detect and build all high-index DAE systems beforehand.

RMD's Analyzer detects high-index DAE on run time and builds new additional equation for numerical differentiation.

The structure of RND's Numerical Library and algorithm of interaction between Numerical library and Model Engine have been changed for implementing new approach to building global equations on run time.

If any new event has occurred, the control program estimates necessity of rebuilding of current solved system. New system is built if it is necessary.

If the current global system has full transversal:

Tarjan algorithm is used for building block triangular form of structural matrix.

Strongly connected components (diagonal blocks of reordered structural matrix and associated with them systems of equations) are analyzing for choosing appropriate Solvers. Solving a system of equations corresponding to diagonal block with the help of suitable Solver will be named «subtask».

Next step is building the condensation of the Tarjan's algorithm graph. The condensation is used for construction subtask queue. Subtasks may be executed sequentially or parallel. The information about computer hardware (number of processors, number of kernels for a processor) needed for creation a thread pool for parallel execution is determined automatically. Threads are loaded by subtasks which are ready for execution. The subtask readiness for execution determinates using condensation. Initially all nodes (subtasks) of the condensation are marked as «Unresolved». If an «unresolved» node has no input edges or input edges start from the nodes with solved systems then control program changes its status for «Ready to start», otherwise it will have status «Not ready to start». Solving subtask's system has name «Solving», after ending of solving it becomes «Solved». The calculation comes to an end when all nodes become «Solved».

The new approach with block triangular form, different Solvers for each subtask, and threads was compared with old one. For comparison was used a set of models developed by Transas company (http://www.transas.com/products). The results of numerical experiment for most difficult problem are shown in Table 1. The computer used for calculations had four processors, so it was possible to create maximum four threads, but even if only one thread was used then total time of calculations decreased in two times.

Table 1. Product Tanker, Cargo System, about 2500 equations.

\begin{tabular}{|l|l|l|l}
\hline Thread Count & 1 & 2 & 4 \\
\hline Old Numerical Library & 8,53 & - & - \\
\hline New Numerical Library & 4,72 & 3,80 & 3,67 \\
\hline Time difference & $\mathbf{4 4 , 6 7 \%}$ & $\mathbf{5 5 , 4 5 \%}$ & $\mathbf{5 6 , 9 8 \%}$ \\
\hline $\begin{array}{l}\text { Time on Right Part } \\
\text { Calculation }\end{array}$ & 2,44 & 2,47 & 2,47 \\
\hline Right Part Calculation Count & 28964 & 28964 & 28964 \\
\hline Control Time & 2,28 & 1,33 & 1,20 \\
\hline From one thread & $100,00 \%$ & $\mathbf{5 8 , 3 3 \%}$ & $\mathbf{5 2 , 6 3 \%}$ \\
\hline
\end{tabular}

\section{REFERENCES}

Duff I.S. (1977). MA28-A set of Fortran subroutines for sparse unsymmetric linear equations. Technical Report AERE R8730 (SR-2040 8.0 edition) HMSO, London

Isakov A.A., Senichenkov Yu. B. (2010). OpenMVL is a tool for modelling and simulation. //Computing, measuring, and control systems, pp. 84-89, SPBSPU, St. Petersburg.

Isakov A.A., Senichenkov Yu. B. (2011). Program Complex OpenMVL for Modeling Complex Dynamical Systems // «Differential equations and control processes» Journal, Mathematics and Mechanics Faculty of Saint-Petersburg State University, St. Petersburg.

Kolesov Yu.B., Senichenkov Yu.B. (2006) Modelling of systems. Dynamical and Hybrid systems. 224 pp, BHV, St. Petersburg.

Kolesov Yu.B., Senichenkov Yu.B. (2007) Modelling of systems. Object-oriented approach. 256 pp, BHV, St. Petersburg.

Kolesov Yu.B., Senichenkov Yu.B. (2012) Mathematical Modelling of component systems. 223 pp, SPbSTU, St. Petersburg.

Kolesov Yu.B., Senichenkov Yu.B. (2014) Mathematical Modelling of hybrid systems. 236 pp, SPbSTU, St. Petersburg.

Pissanetsky S. (1984). Sparse matrix technology, Academmic Press, London.

Senichenkov Yu. B. (2004) Numerical modelling of hybrid systems.206 pp, SPbSTU, St. Petersburg.

Fritzson P. (2011) Introduction to Modeling and Simulation of Technical and Physical Systems with Modelica, 232 pp, Wiley-IEEE Press. 


\title{
Efficient Modelling Approaches in Control
}

\author{
Borut Zupančič \\ University of Ljubljana, Faculty of Electrical Engineering, 1000 \\ Ljubljana, Slovenia (e-mail: borut.zupancic@fe.uni-lj.si)
}

\section{INTRODUCTION}

Due to many disadvantages of traditional causal block oriented modelling approaches new modelling techniques were developed in nineties, e.g. Bond graphs and $\mathrm{OO}$ acausal and multi domain modelling which preserve the realisation aspects of the systems being modelled. The result was the Modelica language [Fritzson (2004)] and also the development of several environments (Dymola, Math Modelica, Open Modelica, MapleSim, ...) [Dymola (2014)]. Traditional modelling techniques were used in our long term activities in modelling of thermal and radiation flows in buildings. [Škrjanc et al. (2001), Lah et al. (2005)]. However using Matlab-Simulink it is not possible to build the library of reusable components. Namely when we wanted to use our one room model for a several rooms model, it was simply not possible. Every new configuration demanded the design almost from the scratch. Therefore we switched to the Dymola-Modelica environment and a new model for thermal and radiation flows in buildings was developed.

\section{MODELLING, SIMULATION AND CONTROL OF THERMAL AND RADIATION FLOWS IN BUILDINGS IN MODELICA}

The model of the room was built from the prepared model classes[Sodja and Zupančič (2009)]. Mostly the model classes from the standard Modelica library for one dimensional thermal processes were used (e.g. HeatCapacitor, ThermalConductor, Convection, Body Radiation). The standard connector Heatport was also used with heat flow and temperature interface variables. Some new components were added.

\subsection{Preparation of the Modelica model for Matlab-Simulink}

Dymola-Modelica is an extremely powerful tool for true physical modelling. However for more complex experimentations (e.g. optimization, linearization, steady state calculation, etc.), for results presentation it is far from Matlab possibilities. So we decided to use Dymola-Modelica just for the 'physical' part and Matlab-Simulink for all other needs. We prepared a top level Modelica model which can be used as a Dymola (Modelica) block in the Matlab-Simulink environment. Actually we had to prepare appropriate connectors, which are compatible with other Simulink blocks. We prepared five inputs (outdoor temperature, roller blind position, direct solar radiation, diffuse solar radiation and artificial heating-cooling) and one output (indoor temperature). Then we prepared Simulink environment to accept Dymola block. This block has to be compiled within Simulink before the simulation is started.

\subsection{Contol systems optimisation in Matlab}

Of course there is no need to use the Matlab environment for pure simulation runs as these can be performed efficiently also in Dymola. However Matlab is efficient if we programe more sophisticated experiments using Toolboxes. In the design of control systems we can determine the PID contoller parameters $k_{P}, T_{I}, T_{D}$ using optimisation. Optimization toolbox and unconstrained optimization with the function fminsearch were used.

\subsection{Experiments with $P$ and PI controllers}

Although the basic goal was to harmonise the thermal and also radiation flows which influence temperatures and illuminations, we started with more basic experiments to control the internal temperature with additional heating/cooling. The controller minimises the error between the desired and the actual room temperature. Prior to that, we also performed a number of open loop experiments [Zupančič and Sodja (2013)]. We used a variety of test signals: constants, the step changes as well as signals derived from actual measurements on the test room. Beside usual controller inputs - reference temperature and actual temperature, we added additional input - the signal of direct solar radiation. With this input we intend to improve the control with appropriate feed forward control.

\section{REALISATION-PRESERVING MODEL REDUCTION OF MODELS IN MODELICA}

Beside described examples we used Modelica with Matlab in many other applications. We learned that $\mathrm{OO}$ and multi-domain modelling approach is very efficient especially in model definition phase, but unfortunately not so much in model execution. Namely under the surface of very transparent models very complex structures for execution are obtained. If we use well tested components it does not mean that the model will produce accurate results when many components are put together into a model. If one room model performes accurate results it does not assure that the model with several rooms is also accurate and usable. A simplification and/or model reduction is therefore very important in each modelling application. The emphasise of our activities was given to realisation-preserving model reduction approaches in Modelica environments.

\subsection{Realisation-preserving reduction at object-diagram level}

The simplest procedure for reducing models represented with a scheme (graph) is to remove connections (edges) or 
Table 1. Ranking of the room-model components according to the activity metric

\begin{tabular}{lccc} 
Element & $\begin{array}{c}\text { Activity } \\
{[\mathrm{J}]}\end{array}$ & $\begin{array}{c}\text { Relative } \\
{[\%]}\end{array}$ & $\begin{array}{c}\text { Accumulated } \\
{[\%]}\end{array}$ \\
\hline window & $1.38 \cdot 10^{7}$ & 22.32 & 22.325 \\
OppositeWall & $7.76 \cdot 10^{6}$ & 12.59 & 34.91 \\
WinPort & $7.55 \cdot 10^{6}$ & 12.25 & 47.17 \\
WallOppositePort & $5.95 \cdot 10^{6}$ & 9.65 & 56.82 \\
Ceiling & $3.68 \cdot 10^{6}$ & 5.97 & 62.79 \\
WallOnLeft & $3.63 \cdot 10^{6}$ & 5.88 & 68.67 \\
WallOnRight & $3.63 \cdot 10^{6}$ & 5.88 & 74.56 \\
WinWall & $3.44 \cdot 10^{6}$ & 5.59 & 80.14 \\
CeilingPort & $3.23 \cdot 10^{6}$ & 5.24 & 85.39 \\
Floor & $2.77 \cdot 10^{6}$ & 4.49 & 89.88 \\
WallOnRightPort & $1.40 \cdot 10^{6}$ & 2.28 & 92.15 \\
WallOnLeftPort & $1.40 \cdot 10^{6}$ & 2.28 & 94.43 \\
WinWallPort & $1.39 \cdot 10^{6}$ & 2.26 & 96.69 \\
FloorPort & $1.16 \cdot 10^{6}$ & 1.88 & 98.57 \\
Interior & $8.08 \cdot 10^{5}$ & 1.31 & 99.88 \\
OutsideAir & $7.35 \cdot 10^{4}$ & 0.12 & 100.00 \\
RadiationBox & 0.01 & 0.00 & 100.00 \\
Infiltration & 0.00 & 0.00 & 100.00 \\
\hline
\end{tabular}

components (nodes) estimated to have insignificant effect on salient dynamics of the system. Very intuitive approach to determine these connections or components is to use energy and power related metrics in bond graphs[Louca (1998)]. Energy, which a component exchanges with its environment, is not so explicitly available in Modelica as in bond-graph formalism [Sodja (2012)]. However, it can be obtained by inspecting the connections of the components. There are only few different types of physical interactions and therefore types of connections, so if a connector is defined appropriately, a list of rules for calculating power of each connection-type is generated and power associated with a component is calculated as the sum of powers of its connections. Elimination of low ranked components (or connections) in Modelica is even more difficult, because components usually can't be classified in generalised inductance, capacitance and resistance as in case of bond graphs. After ranking of the component is done, it can be whether left to the user to decide how to reduce the model (which is adequate in some cases) or the rules for proper removal of components are derived by automatic manipulation of underlying equations.

\subsection{Example: Ranking components in the room model}

It was mentioned in in Sodja (2012) that for each connector of Modelica Standard Library it is possible to determine associated energy-flow considering only information provided by connector's definition.

Consider the model presented in section 2 that we developed for the thermal behaviour of the test room. It uses almost exclusively connectors for 1-dimensional heat transfer. In Table 1, components of the room submodel are listed and sorted according to their activities, which were calculated (Eq. 1) for a simulation experiment using measured data for three autumn days. $\dot{e}_{j}(t)$ designates the $j$-th energy flow through the boundary of an element.

$$
\mathrm{A}_{i}=\int_{t_{1}}^{t_{2}}\left|\sum_{j} \dot{e}_{j}(t)\right| \cdot d t \quad \mathrm{AI}_{i}=\frac{A_{i}}{\sum_{j=1}^{n} A_{j}}
$$

The modelled room has a cubic shape with equal walls, so it was expected that activities of the walls are roughly the same. The results at the bottom of the Table 1 where components RadiationBox and Infiltration have allegedly zero activity are more surprising. That is because these two components only transfer heat without storing it. Therefore sum of all energy flows on their borders is zero at any time instant. Choice of connector variables where extensive variable is energy flow thus causes that only energy-storing components are considered while transferonly components are ignored what is by no means acceptable. Sodja (2012) used entropy generation rate in Eq. 1 (in place of $\dot{e}$ ) to evaluate activity metric for a component instead of using heat flow. However the order of components was the same as in Table 1 but with nonzero but still small values of the last two components.

Of course the main question is, what to do with the Table 1 . Of course we can not just eliminate the components with low activity, because some classes can not be directly compared. But nevertheless we can find sometimes a very useful information: e.g. the window is very important, the walls have similar importance - perhaps some walls can be modelled with one unified wall etc. Of course if one component between several similar components has much lower activity, we can think how to eliminate this component from the model.

\section{REFERENCES}

Dymola (2014). Dymola, Dynamic Modeling Laboratory, User's Manual. Dassault Systèmes.

Fritzson, P. (2004). Principles of Object Oriented Modeling and Simulation with Modelica 2.1. IEEE Press, John Wiley \& Sons, Inc., USA. Publication.

Lah, M.T., Zupančič, B., and Krainer, A. (2005). Fuzzy control for the illumination and temperature comfort in a test chamber. Building and environment, 40(12), $1626-1637$.

Louca, L.S. (1998). An Energy-based Model Reduction Methodology for Automated Modeling. Ph.D. thesis, University of Michigan.

Sodja, A. and Zupančič, B. (2009). Modelling thermal processes in buildings using an object-oriented approach and Modelica. Simulation modelling practice and theory, $17(6), 1143-1159$.

Sodja, A. (2012). Object-oriented modelling and simulation analysis of the automatically translated models. Ph.D. thesis, University of Ljubljana, Fac. of El. Eng.

Škrjanc, I., Zupančič, B., Furlan, B., and Krainer, A. (2001). Theoretical and experimental fuzzy modelling of building thermal dynamic response. Building and environment, 36(9), 1023-1038.

Zupančič, B. and Sodja, A. (2013). Analysis and control design of thermal flows in buildings: efficient experimentation with a room model in matlab-modelica environment. In Proceedings of the 8th Eurosim Congress on Modelling and Simulation, 155-160. Cardiff, UK. 


\title{
Numerical Solution of an Optimal Control Problem in Cancer Treatment: Combined Radio and Anti-Angiogenic Therapy
}

\author{
Kurt Chudej* Lisa Wagner ${ }^{* *}$ Hans Josef Pesch ${ }^{* * *}$ \\ * Lehrstuhl für Ingenieurmathematik, Universität Bayreuth, 95440 \\ Bayreuth, Germany (e-mail: kurt.chudej@uni-bayreuth.de). \\ ** Numerische Mathematik und Wissenschaftiches Rechnen, \\ Universität Darmstadt, Germany \\ *** Lehrstuhl für Ingenieurmathematik, Universität Bayreuth, 95440 \\ Bayreuth, Germany (e-mail:hans-josef.pesch@uni-bayreuth.de).
}

\begin{abstract}
Several therapies and also combined therapies for cancer treatment exist mathematical models of which have partly been also optimized by means of optimal control methods. Here we focus on an optimal control problem describing a combined radiotherapy and an antiangiogenic treatment. The underlying model is taken from literature where, however, despite rigorous analytical investigations a complete numerical solution is missing. In order to fill this gap, a direct solution approach has been developed for which both state and control functions of the optimal control problem are discretized. The resulting nonlinear programming problem is solved via the modelling language AMPL and the interior point solution method IPOPT. In an a posteriori step we try to check the main necessary conditions of optimal control theory in order to verify the candidate optimality of the continuous problem via the approximate discrete optimal solution. The most interested feature of this model from the viewpoint of optimal control theory is the fact that singular control subarcs exist for a two-dimensional control vector. However, the arising numerical difficulties caused by chattering controls prevented us so far from a complete candidate optimal solution.
\end{abstract}

Keywords: Tumor treatment, optimal control, radiotherapy, anti-angiogenic treatment, vector-valued singular control.

\section{OPTIMAL CONTROL MODEL}

Starting point is the article of Ledzewicz et al (2012) which describes a 2-compartment cell-population-based optimal control problem for the primary tumor volume $p$ and the carrying capacitiy of the tumor vasculature $q$, as state variables. The tumor is attacked by radiotherapy (control $w$ ) and an anti-angiogenic agent (control $u$ ). Tumor anti-angiogenic treatment is known as an indirect cancer therapy to prevent a tumor's ability to grow. By anti-angiogenic drugs the development of the vasculature is to be inhibited to cut off the tumor from nutrients and oxygen.

For the growth of the tumor volume $p$ we use a logistic growth model based on the Gompertz's growth function $F(x):=-\ln (x)$ with $x=\frac{p}{q}$. The carrying capacity $q$ is modelled by a balance between stimulatory and inhibitory effects, $S=\left(q p^{\frac{2}{3}}\right) /\left(q p^{-\frac{1}{3}}\right)$, resp. $I=q p^{\frac{2}{3}}$, which both are based on certain consumption-diffusion equations according to Hahnfeldt et al (1999). The first two equations of the subsequent model are controlled by the anti-angiogenic so-called $\log$-kill term $\kappa(u, q):=-\gamma u q$ which is based on the assumption that the number of killed cells is proportional to the dosage $u$ and the size $q$ of the cell population. In addition a linear-quadratic term enters the ode for the tumor volume. It describes the influence of the radiation therapy on the tumor volume by radiation ionisation. Its linear part $-\alpha p w$ is a second log-kill term. For the temporal development of the tumor volume under a radiation therapy we apply the so-called LQ-model of Ergun et al (2003),

$$
\dot{p}(t)=-p(t)\left(\alpha+\beta \int_{0}^{t} w(s) \exp (-\rho(t-s)) \mathrm{d} s\right) w(t),
$$

where $w$ is the dosage by which the amount of radiation is controlled.

Hence, the following optimal control problem can be summarized:

$$
\min p\left(t_{f}\right)
$$

s.t. to ode constraints

$$
\begin{aligned}
& \dot{p}=-\xi p \ln \frac{p}{q}-(\alpha+\beta r) p w, \\
& \dot{q}=b p-\left(\mu+d p^{2 / 3}\right) q-\gamma q u-(\eta+\delta r) q w, \\
& \dot{r}=-\rho r+w, \\
& \dot{y}=u, \\
& \dot{z}=(1+\theta s) w,
\end{aligned}
$$




$$
\dot{s}=-\sigma s+w,
$$

with the initial conditions

$$
p(0)=p_{0}, q(0)=q_{0}, r(0)=y(0)=z(0)=s(0)=0,
$$

and subject to the control constraints

$$
\begin{aligned}
& 0 \leq u \leq u_{\max }, \\
& 0 \leq w \leq w_{\max },
\end{aligned}
$$

and the boundary inequalities

$$
\begin{aligned}
& y\left(t_{f}\right) \leq y_{\max } \\
& z\left(t_{f}\right) \leq z_{\max }
\end{aligned}
$$

The final time $t_{f}$, at which we want to minimize the tumor's volume, is assumed to be free.

In summary, we have the following variables:

the state variables:

$p$ primary tumor volume,

$q$ carrying capacity of the tumor vasculature,

$r$ tissue repair,

$y$ accumulated amount of used anti-angiogenic agent,

$z$ accumulated radiation dose in tumor,

$s$ accumulated radiation dose in healty tissue.

the control variables:

$u$ dosage of anti-angiogenic agent,

$w$ radiation dose.

\section{Constants are:}

$\alpha, \eta$ linear damage caused by radiation of the tumor,

$\beta, \delta$ quadratic damage caused by radiation of the tumor, $\rho$ repair rate of the tumor and endothelial cells,

$\sigma$ repair rate for the healty cells,

$\theta$ parameter of the healty tissue.

In the paper of Ledzewicz et al (2012) very elaborated theoretical investigations have been carried through, particularly in deriving complicated optimality conditions, which the optimal solution has to fulfill necessarily. However, no numerical results were presented. This presentation will try to fill this gap at least in a first step.

\section{NUMERICAL SOLUTION}

Using a direct approach we discretize both states and controls over a time grid. This yields a nonlinear programming problem which is solved by the interior point method IPOPT of Wächter (URL) — see also Wächter (2002) and Wächter and Biegler (2006) — via the modelling language AMPL of Fourer et al (2003). An advantage of this combination is the built-in automatic differentiation modus in AMPL which provides the NLP-solver IPOPT with exact derivatives. Moreover the implementation of ODE optimal control problems is very user-friendly by using AMPL.

We will present first numerical results derived in the Master Thesis of Wagner (2014). Additional a posteriori tests of the computed approximations are performed by using the discretized Lagrange multipliers from IPOPT and the optimality conditions of Ledzewicz et al (2012).
The approximations for both controls show a chattering behaviour the analysis of which needs further investigations, theoretically as well as numerically. For example, the switching time optimization approach of Maurer and Pesch (2008) may give more insight, since it allows to make use of the feedback formulas on singular control subarcs in a postprocessing optimization step.

\section{REFERENCES}

Ergun, A., Camphausen, K., and Wein, L.M. (2003) Optimal Scheduling of Radiotherapy and Angiogenic Inhibitors. Bull. Math. Biol. 65, 407-424.

Fourer, R., Gay, D.M., and Kernighan, B.W. (2003) AMPL: A Modeling Language for Mathematical Programming. 2nd Edition, Duxbury, Thomson.

Hahnfeldt, P., Panigrahy, D., Folkman, J., Hlatky, L. (1999) Tumor Development under Angiogenic Signaling: a Dynamical Theory of Tumor Growth, Treatment Response, and Postvascular Dormancy. Cancer Res. 59, 4770-4775.

Ledzewicz, U. and Schättler, H. (2012) Multi-input Optimal control Problems for Combined Tumor Antiangiogenic and Radiotheray Treatments. J Optim Theory Appl 153, pp.195-224.

Maurer, H. and Pesch, H. J. (2008) Direct Optimization Methods for Solving a Complex State-Constrained Optimal Control Problem in Microeconomics. Applied Mathematics and Computation 204 (2), 568-579.

Wagner, L. (2014) Optimale Steuerung in der Krebstherapie. Masterarbeit, University of Bayreuth, Bayreuth, Germany.

Wächter, A.: IPOPT Online Dokumentation. web site http://www.coin-or.org/Ipopt/documentation.

Wächter, A. (2002) An Interior Point Algorithm for LargeScale Nonlinear Optimization with Applications in Process Engineering. Phd Thesis, Carnegie Mellon University, Pittsburgh, PA, USA.

Wächter, A. and Biegler, L. T. (2006) On the Implementation of an Interior-point Filterline-search Algorithm for Large Scale Nonlinear Programing. Mathematical Programming 106, 25-57. 


\title{
Information Management Platform Model for Local Sightseeing and Disaster Prevention Information
}

\author{
Takeshi Tsuchiya*, Hiroo Hirose*, Tadashi Miyosawa*,

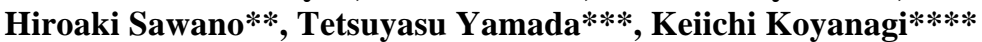 \\ * Tokyo University of Science, Suwa, Toyohira 5000-1, Chino, Nagano 391-0213 \\ JAPAN (Tel: 81-266-73-9674; e-mail: \{tsuchiya.takeshi, hirose, miyosawa\}@ rs.tus.ac.jp). \\ **Aichi Institute of Technology, (e-mail:sawano@aitech.ac.jp) \\ ***NTT Software Corporation, (e-mail: tetsuyasu@gmail.com) \\ ****WASEDA University, (e-mail:keiichi.koyanagi@waseda.jp)
}

\begin{abstract}
We discuss the information management model by crawling/analyzing technology used to retrieve information diffused on the Internet, and propose local information platform that collects information on sightseeing, as a main item, in Suwa Area of Nagano Prefecture. The information includes several types of it such as reputable and interesting events that the users may transmit. This platform is also able to gather and deliver not only the above-mentioned information but also the disaster information, regardless of official/unofficial information, based on fault tolerance of the Internet with distributed nodes in an emergency. This research clarifies our proposed platform and demonstrates operation of a platform integrated Internet model.
\end{abstract}

Keywords: Sightseeing information,

\section{INTRODUCTION}

The value of the Internet that is not only the technical system but also the social infrastructure has been recently regarded as very important from the viewpoint among users. And it enables to realize direct collaboration as community among information suppliers and the consumers in the point of economic activity. Especially, the Internet enable to provide more flexible public relations to cope with production costs and assumed users' taste than providing by mass media such as newspapers and television. Generating community is flexible on logical space based on the parameters of interest and orientation without depending on physical distance. Current communities are created in the absolutely specific Internet services: 4travel regarded as for travel information, and kakaku.com regarded as for price of OA product information. On these service, general consumer expresses individual his/her experience and opinion not only on such communities and but blog and SNS (Social Network Service). But, there are several cases in non-updated old information may be held on a blog and SNSs. These left old information that a person expresses in the SNS and blog would be possible as important piece of information in a small community.

In this paper, we propose the information platform technology that diffusing information on the Internet. Especially, our proposal is to generate some community based on physical locality, which does not exist in logical space provided by the services on the Internet. In detail, we survey the local information platform for "sightseeing information" that is a characteristic Internet community in Suwa Area of Nagano Prefecture. We use this platform to extract information on "Suwa Area of Nagano Prefecture" from "sightseeing information" diffused on the Internet and to produce information to be placed on the local information portal site that will be a community consisting of information on "Suwa Area of Nagano Prefecture".

\section{MODEL}

Our proposal information platform has following functions. Its outline is shown in Fig. 1.

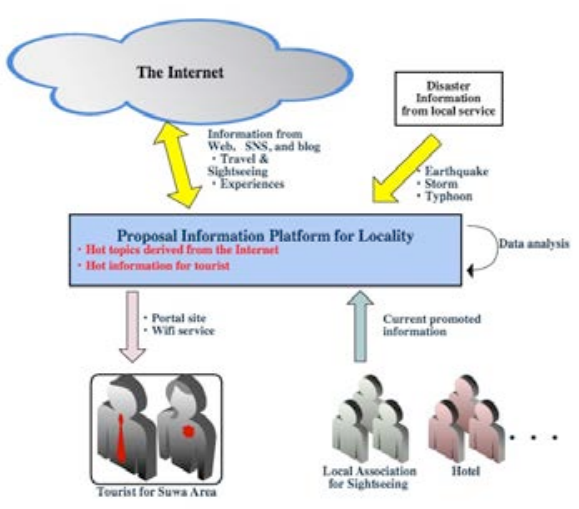

Fig.1 Outline of proposal model

Acquirement and classification of information diffused over the Internet by previous our activities is crawled and collected based on cloud computing technology [1][2]. In this manner, similar information exist close of the information sources. So the collection by sophisticated crawling is 
inefficient for locality from the whole in the specified information sources. Therefore, our manner determined that the range of crawling is limited under 3 hops from crawled source information. This limitation is depended by current hardware resources such as CPU and databases, and more neighbor information linked to sources are crawled and collected with improving such points.

The collected information stored on the proposal platform is executed the morphological analysis by natural language technology and classify the proper nouns for analysis. After that, extracted terms are as candidate of hot topics, and analyze them based on the statistical manners. For analysis, it is impossible to express and judge the terms only with simple co-occurrence in information. For example, "Shirakabako" in Suwa area differs the meaming greatly from "Shirakaba (white birch)" + "ko (lake)", and out of their original meaning. Proposal platform manages registered several important keywords as important knowledge terms and it also learn how to extract proper nouns and technical terms locality by machine learning manner. These extracted terms and topics are ordered by using the simple importance and supervisor's experience and subjective points of view by the effective fuzzy outranking method. In this manner, rank information clearly in the paired comparison method by replacing qualitative data with quantitative data. The ranked topics and terms are outputted XML periodically, and it is used as Web and so on.

Though the information for disaster prevention using on our proposal is required responsibility, it is used as the responsible information provided by government including area one. The information is followings: J-Alerts and Chino city emergent mail service. At the same time, un-responsible information is also used as for indicating current conditions of disasters with analyzing from SNS. This manner enables to collect and indicate row voice from the victims of disaster.

\section{Implementations}

All functions in section 2 are constructed independently shown in fig. 2 Especially, there are two independent servers: one is database server which stores crawled and collected information. Another is analyzer server that extracts topics and terms from stored data. This function holds special terms of locality, and extract terms by TermExtract [3].
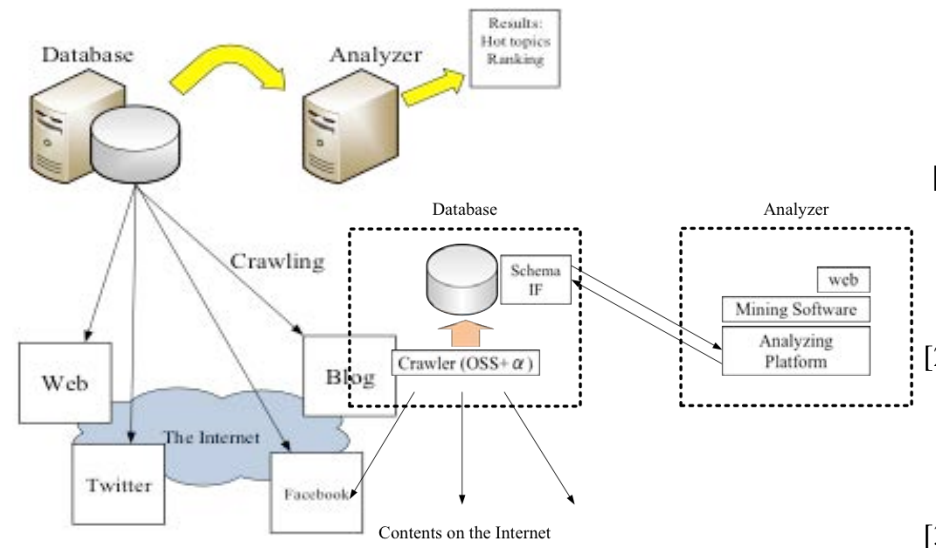

Fig.2 Implementations

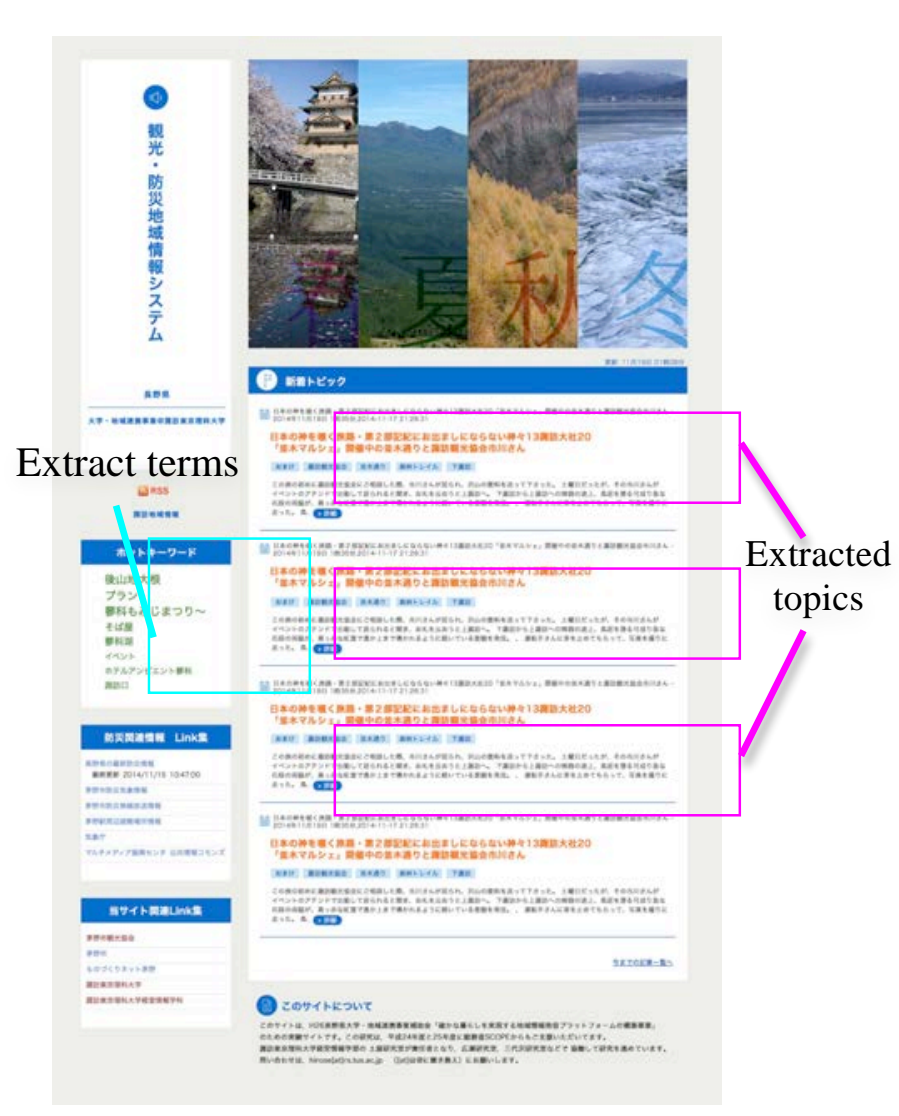

Fig.3 Extract Information

\section{CONCLUSIONS}

This paper propose information management platform for local sightseeing and "disaster "preventive" information in Suwa Area of Nagano Prefecture in Japan from diffusing over the Internet. Our proposal performs autonomously without management by professional engineers. Therefore, anyone enables to construct and manage local information. Realizing actual service, current implementation is required to improve and evaluate service quality including the effect of using services on the sites.

\section{Acknowledgements}

This work was partly supported by MEXT KAKENHI Grant Number 14485676

\section{REFERENCES}

[1] Tsuchiya, T., Yoshinaga, H. and Koyanagi, K.: P2P Distributed Information Retrieval Method by the Independent Indices Management for the Ubiquitous Services Environment, Journal of Japan Society for Fuzzy Theory and Intelligent Information, Vol. 21, No. 1, February 2009

[2] Tsuchiya, T., Yoshinaga, H. and Koyanagi, K.: Research on Distributed Database Service to Integrate Diffusion Data by Peer-to-Peer Cloud Computing Technology, Journal of Japan Society for Fuzzy Theory and Intelligent Information, Vol. 25, No. 1, February 2013

[3] Term Extract: http://gensen.dl.itc.u-tokyo.ac.jp/termextract.html 


\title{
Influence of sample period variation to MPC trajectory tracking of mobile robot
}

\author{
Gregor Klančar* Igor Škrjanc* \\ * Faculty of Electrical Engineering, University of Ljubljana, Tržaška \\ 25, SI-1000Ljubljana, Slovenia (e-mail: gregor.klancar@fe.uni-lj.si, \\ igor.skrjanc@fe.uni-lj.si)
}

\section{INTRODUCTION}

This work applies predictive path tracking approach similarly as done in previous works such as Normey-Rico et al. (1999), Ollero and Amidi (1991), Kühne et al. (2004). The main difference of the proposed model predictive control (MPC) approach Klančar and Škrjanc (2007) is that it obtains optimal solution analytically without numeric optimization.

In case of discrete control the main problem presents uncertainty nature of used algorithms time complexity and control delay. If computational time and/or control delay are long and deterministic then this can be simply solved by extending the sampling time of discrete control law if this is still acceptable with system dynamics. Time slot between samples in discrete control must be deterministic by definition which is in contradiction to information from sensors which is usually obtained at random time (nondeterministic) due to processing algorithms.

The main contribution of this work is the validation of the model predictive trajectory-tracking law proposed in Klančar and Škrjanc (2007) against variable sample period. To improve performance of the control law its continuous representation is suggested.

\section{TRAJECTORY TRACKING CONTROL}

Trajectory tracking control of differential drive is done using feedforward and feedback control law as suggested in Klančar and Škrjanc (2007), Kanayama et al. (1990), Samson (1993), Blažič (2014). The reference trajectory is given as a function of time $\left(x_{r}(t), y_{r}(t)\right)$ and by the reference robot input $u_{r}$ consisting of translational and angular velocity obtained by first and second derivatives of the reference trajectory.

The tracking error of the robot $e(t)=\left[e_{x}(t) e_{y}(t) e_{\theta}(t)\right]^{T}$ is expressed in local robot coordinates

$$
e(t)=\left[\begin{array}{ccc}
\cos \theta & \sin \theta & 0 \\
-\sin \theta & \cos \theta & 0 \\
0 & 0 & 1
\end{array}\right]\left(q_{r}-q\right)
$$

where $q=[x, y, \theta]^{T}$ is the robot pose relative to global frame, $q_{r}=\left[x_{r}, y_{r}, \theta_{r}\right]^{T}$ is the reference pose where $\theta_{r}=$ $\arctan \frac{\dot{y}_{r}(t)}{\dot{x}_{r}(t)}$. The kinematics of the differential drive is

$$
\dot{q}(t)=\left[\begin{array}{cc}
\cos \theta(t) & 0 \\
\sin \theta(t) & 0 \\
0 & 1
\end{array}\right]\left[\begin{array}{l}
v(t) \\
\omega(t)
\end{array}\right]
$$

where robot inputs $v(t)$ and $\omega(t)$ are translational and angular velocity, respectively. Control vector $u=\left[\begin{array}{ll}v & \omega\end{array}\right]^{T}=$ $u_{f}+u_{b}$ consists of feedforward part $u_{f}=\left[\begin{array}{ll}v_{r} \cos e_{\theta} & \omega_{r}(t)\end{array}\right]$ and feedback part $u_{b}=\left[v_{b}(t) \omega_{b}(t)\right]^{T}$ which is calculated by MPC as described in the following.

Nonlinear tracking error dynamics considering (Oriolo et al. (2002)) is

$$
\dot{e}=\left[\begin{array}{rrr}
0 & \omega_{r} & 0 \\
-\omega_{r} & 0 & v_{r} \frac{\sin e_{\theta}}{e_{\theta}} \\
0 & 0 & 0
\end{array}\right] e+\left[\begin{array}{rr}
-1 & e_{y} \\
0 & -e_{x} \\
0 & -1
\end{array}\right] u_{b}
$$

which is in the vicinity of the reference trajectory approximated by a linear model in the form $\dot{e}=A e+B u_{b}$.

\subsection{Discrete $M P C$ trajectory tracking}

Feedback control law derivation is done in Klančar and Škrjanc (2007). The main idea is to find control variable that minimizes quadratic cost function in the recedinghorizon $h$

$$
J\left(u_{b}, k\right)=\sum_{i=1}^{h} \epsilon^{T}(k, i) Q \epsilon(k, i)+u_{b}^{T}(k+i) R u_{b}(k+i)
$$

where $\epsilon(k, i)=e_{r}(k+i)-e(k+i \mid k)$ and $e_{r}(k+i)$ and $e(k+i \mid k)$ stands for the reference robot-following trajectory and the robot-following error, respectively, $h$ is horizon time and $Q$ and $R$ stand for the weighting matrices where $Q \geq 0$ and $R \geq 0 . e_{r}(k+i)$ is the reference error trajectory which the robot must follow to converge to the reference trajectory $\left(x_{r}(t), y_{r}(t)\right)$. At current time instant $k$ it is initialized by $e_{r}(k)=e(k)$ and in prediction horizon it decreases according to the reference error model $e_{r}(k+i)=A_{r}^{i} e(k)(i=1, \cdots, h)$ where $A_{r}$ is the reference-error transition matrix. The control law can be obtained by optimization search or solved analytically. To be solved analytically the nonlinear robot model (3) is linearized around the reference trajectory and expressed in discrete state space form $e(k+1)=A_{d} e(k)+B_{d} u_{b}(k)$ with sampling period $T_{s}$ (for details see Klančar and Škrjanc (2007)). The feedback part of the control signal, $u_{b}(k)$ is the solution of the criteria function (4) optimization.

\subsection{Continuous MPC for trajectory tracking}

The main reason for implementing continuous MPC is to make control performance independent of sampling period 
variation. At the same time also higher robustness of control law parameters such as horizon and control law dynamics to different sample period of the closed loop is achieved.

Similarly as in (4) the control variable $u_{b}$ is determined to minimize the criteria function in the horizon prediction time $T_{h}$. The quadratic cost function is

$$
\mathcal{J}=\int_{o}^{T_{h}}\left[\varepsilon^{T} Q \varepsilon+u_{b}^{T}(t+\tau) R u_{b}(t+\tau)\right] d \tau .
$$

where $\varepsilon=e_{r}(t+\tau)-e(t+\tau)$ and $Q$ and $R$ are diagonal weighting matrices. The control signal is approximated by second order Taylor series expansion $u_{b}(t+\tau) \doteq$ $\left[\begin{array}{lll}I_{2} & \tau I_{2} & \frac{\tau^{2}}{2 !} I_{2}\end{array}\right]\left[u_{b}(t) \dot{u}_{b}(t) \ddot{u}_{b}(t)\right]^{T}$ where $I_{2}$ is $2 \times 2$ identity matrix. The optimum solution of (5), i.e. the control variable $u_{b}(t)$, can again be obtained analytically where linearized tracking error model of (3) is used for prediction. Optimal value of $u_{b}$ is obtained by calculating $\frac{\partial \mathcal{J}}{\partial u_{b}^{*}}$ and finding the solution of expression $\frac{\partial \mathcal{J}}{\partial u_{b}^{*}}=0$. This covers only the most important steps to indicate the derivation idea of the continuous control law. Due to continuous formulation no discretization of the linearized model is required which means that control law performance is less sensitive to variable sampling period (control delay is still present).

\section{VARIABLE SAMPLING SIMULATION SCENARIO}

Simulation study is performed where results obtained on discrete and continuous MPC are evaluated against nonuniform sampling period caused by varying computational time of simulated sensor pre-processing and variable control delay. Both the sensor pre-processing time and control delay are nondeterministic and modelled with normal distribution

From simulation results (Figs. 1 and 2) it is seen that tracking results of continuous MPC (CMPC) contain less control noise than discrete MPC (DMPC). The variable control delay affects both the CMPC and DMPC while the noise in sampling period duration mostly affects DMPC which is not evaluated at regular sampling period and therefore the error due to discretization is in a closed loop propagated to the control noise. Generally the control noise is caused in a closed loop from output noise and noise in sampling period duration and variable delay. The control noise is usually larger if controller dynamics is faster. However from CMPC results a faster tracking is obtained at lower control noise.

\section{REFERENCES}

Blažič, S. (2014). On periodic control laws for mobile robots. IEEE Transactions on industrial electronics, 61(7), 3660-3670.

Kanayama, Y., Kimura, Y., Miyazaki, F., and Noguchi, T. (1990). A stable tracking control method for an autonomous mobile robot. In Proceedings of the 1990 IEEE International Conference on Robotics and Automation, volume 1, 384-389. Cincinnati, $\mathrm{OH}$.

Klančar, G. and Škrjanc, I. (2007). Tracking-error modelbased predictive control for mobile robots in real time. Robotics and Autonomous Systems, 55(6), 460-469.

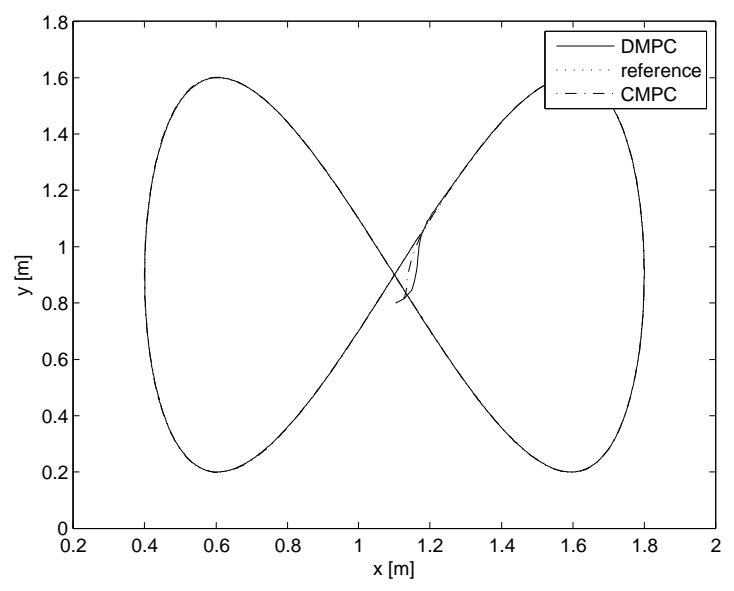

Fig. 1. Trajectory tracking of the simulated vehicle using continuous and discrete MPC with variable simulated sample period and control delay.
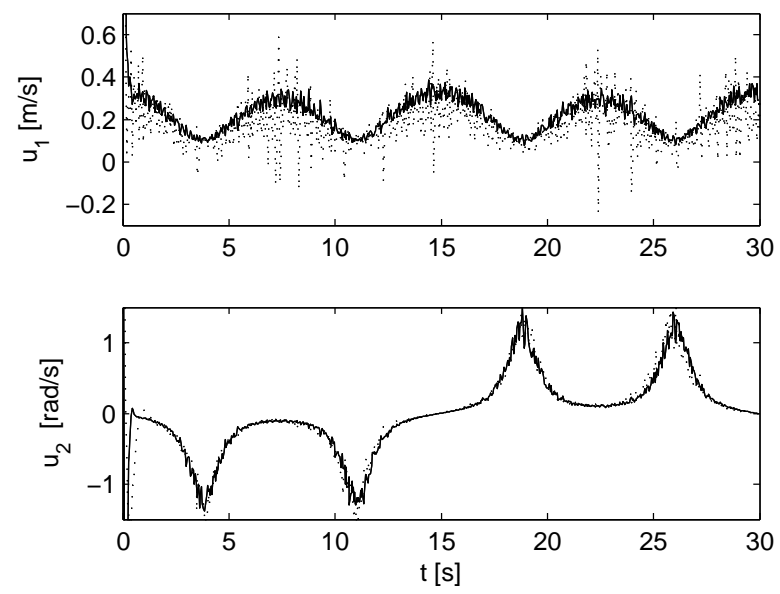

Fig. 2. Simulated vehicle inputs at trajectory tracking where the noise in the period sampling reflects to the process input noise. Tangential velocity $u_{1}$ and angular velocity $u_{2}$ for CMPC (-) and DMPC (..).

Kühne, F., Lages, W., and Gomes da Silva Jr., J. (2004). Model predictive control of a mobile robot using linearization. In Proceedings of Mechatronics and Robotics 2004. Aachen, Germany.

Normey-Rico, J., Gomez-Ortega, J., and Camacho, E. (1999). A smith-predictor-based generalised predictive controller for mobile robot path-tracking. Control Engineering Practice, 7(6), 729-740.

Ollero, A. and Amidi, O. (1991). Predictive path tracking of mobile robots. application to the cmu navlab. In Proceedings of 5th International Conference on Advanced Robotics, Robots in Unstructured Environments (ICAR '91), volume 2, 1081-1086. Pisa, Italy.

Oriolo, G., Luca, A., and Vandittelli, M. (2002). Wmr control via dynamic feedback linearization: Design, implementation, and experimental validation. IEEE Transactions on Control Systems Technology, 10(6), 835-852.

Samson, C. (1993). Time-varying feedback stabilization of car like wheeled mobile robot. International Journal of Robotics Research, 12(1), 55-64. 


\title{
Modeling and Design of high-speed FM-AFM driver electronics using Cadence Virtuoso ${ }^{\circledR}$ and Simulink ${ }^{\circledR}$
}

\author{
Benedikt Schlecker* Anna Eichel* Maurits Ortmanns* \\ Georg Fantner** Jens Anders* \\ * Institute of Microelectronics, University of Ulm, D-89081 Ulm, \\ Germany (e-mail: see http://www.uni-ulm.de/in/mikro/). \\ ** Laboratory for Bio- and Nano-Instrumentation, École Polytechnique \\ Fédérale de Lausanne, CH-1015 Lausanne, Switzerland (e-mail: see \\ http://lbni.epfl.ch/).
}

\begin{abstract}
We present an architecture and modeling approach for cantilever driver electronics required in FM-AFM experiments. To verify the topology, our overall system is modeled and implemented on top level in Simulink with the most important building blocks realized hierarchically on circuit level in Cadence Virtuoso. The resulting heterogeneous model is simulated using Spectre-Simulink cosimulations. The system performance is evaluated and it is shown that it is suitable to produce a stable closed-loop cantilever drive signal, whose frequency can be adjusted by an external reference.
\end{abstract}

Keywords: Atomic force microscopy (AFM), frequency-modulated AFM (FM-AFM), cantilever driver.

\section{INTRODUCTION}

Atomic force microscopy (AFM) as a type of scanning probe microscopy (SPM) is a versatile imaging technique which offers the capability of visualizing arbitrary samples at very high resolutions. Since its invention by Binnig et al. (1986), the technique has been used in many different research disciplines. Due to relaxed requirements imposed by AFM experiments on the sample material and the experimental conditions, compared to other SPM applications (e.g. scanning tunneling microscopy (STM)), nowadays, it is widely used in life science applications, cf. e.g. Fantner et al. (2010) and Ando (2013). In contrast to applications in material science, the frame rate is of prime importance for the real time observation of biological processes where several tens of frames per second are desirable. Therefore, to enable such a high speed image acquistion, all components of the AFM setup need to provide sufficient bandwidth. To meet these stringent speed requirements, in Schlecker et al. (2014), the authors have presented a singlecycle demodulation architecture for high-speed FM-AFM experiments. However, while the demodulator electronics used in these experiments were technically mature, the driver electronics still required cumbersome manual operator adjustments and lacked the possibility of working at an externally definable reference frequency. The latter feature is desirable since it allows to operate the cantilever in a region with a close-to-linear phase response. In this paper, we address these two shortcomings and present an improved architecture together with a model used for mixed system-circuit level simulations of the resulting system.

\section{FM-AFM PRINCIPLE OF OPERATION}

A common FM-AFM setup is shown schematically in Fig. 1. To obtain an image in an FM-AFM experiment, the

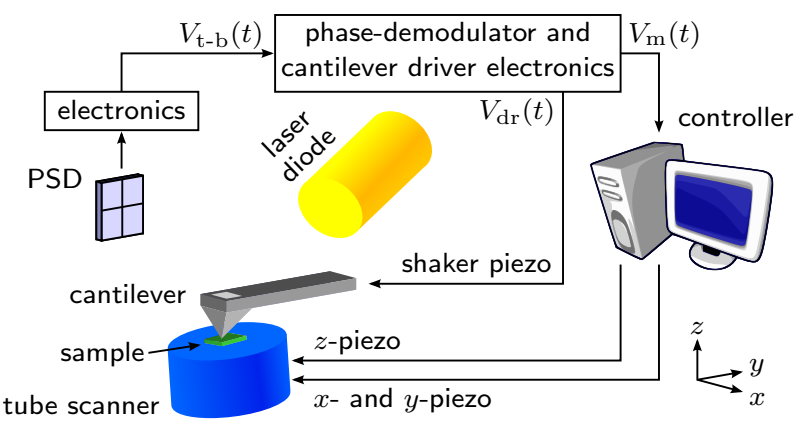

Fig. 1. Schematic AFM measurement setup.

cantilever is brought into self-oscillation near its resonance frequency $f_{\text {res }}$ by embedding it into an oscillator loop. The actual image is then acquired by laterally scanning the sample surface and measuring the cantilever deflection at each location using the reflected light of a laser diode on a position sensitive detector (PSD) generating a so-called top-minus-bottom signal $V_{\mathrm{t}-\mathrm{b}}$. In FM-AFM, the topography information is contained in the cantilever resonance frequency shifts, which are induced by the tipsample interaction and which produce corresponding shifts in the phase of the closed-loop cantilever oscillation. These information are then extracted by a phase-demodulator generating the demodulated signal $V_{\mathrm{m}}$ which is then fed back to the $z$-control of the scanner to actuate a piezo crystal to ensure a constant distance between the cantilever's tip and the sample under investigation.

The phase-demodulator is accompanied by cantilever driver electronics which generate the signal $V_{\mathrm{dr}}$ for the shaker piezo to close the oscillator loop. The driver ensures that the Barkhausen oscillation criterion remains satisfied under all operating conditions. The remainder of this pa- 


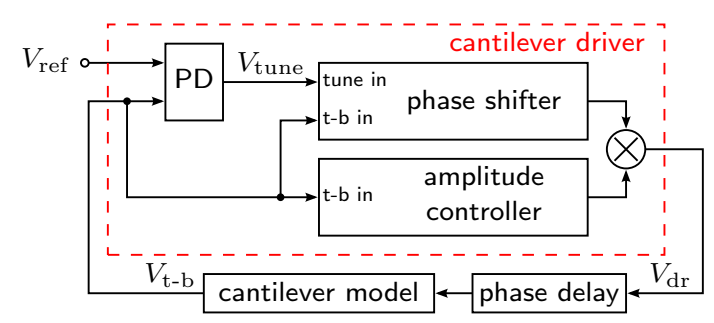

Fig. 2. Proposed system architecture of the cantilever driver in closed-loop configuration

per addresses the design and modeling of this cantilever driver.

\section{DESCRIPTION AND SIMULATION OF THE PROPOSED CANTILEVER DRIVER}

The proposed system architecture is shown in Fig. 2 and consists of three building blocks: a phase detector (PD), a phase shifter and an amplitude controller. In addition to the driver electronics, the figure contains a block modeling the cantilever and a phase delay block, accounting for any additional phase delay generated in the loop. Moreover, it accounts for the $-90^{\circ}$ phase shift between a mechanical resonator and the electrical resonator $\left(2^{\text {nd }}\right.$ order LRCband pass filter) we used to model the cantilever in Cadence Virtuoso.

The closed-loop system can be seen as an electromechanical oscillator for which the Barkhausen stability criterion needs to be fulfilled. To achieve this, the driver consists of two separate signal paths. The first path contains the variable phase shifter to ensure that the Barkhausen oscillation criterion is satisfied. The second path implements the amplitude control mechanism.

Phase Shifter The purpose of the phase shifter in the proposed driver is to compensate for phase losses occurring in the overall loop, thereby ensuring a stable oscillation at the desired frequency. The phase shifter is realized as an active all-pass filter. To render the circuit electronically controllable the capacitor inside the all-pass is implemented by means of a varicap. In all simulations, the varicap is modeled using a Verilog-A model fitted to the $C$ - $V$-curve of the varicap BB202 from NXP.

Amplitude Controller To form an electromechanical oscillator, an amplitude control circuit is required which ensures the existence of a stable limit cycle of known and adjustable amplitude. The circuit is responsible for demodulating the current amplitude and adjusting the excitation signal to match a defined set point. Here, a PI controller is used with the values $K_{\mathrm{p}}$ and $T_{\mathrm{n}}$ determined by the Ziegler-Nichols method, cf. Ziegler and Nichols (1942).

Phase Detector Using a PD, it is possible to define an external reference for the cantilever's oscillation frequency by comparing the phases of the two signals according to Fig. 2. The PD is realized by an XOR-gate with subsequent level conversion and low-pass filter to provide an appropriate tuning signal $V_{\text {tune }}$ feeding the all-pass.

Closed-loop Simulation The results of an exemplary closed-loop simulation are shown in Fig. 3. Here, the driver's response to a sudden step of the oscillation fre-
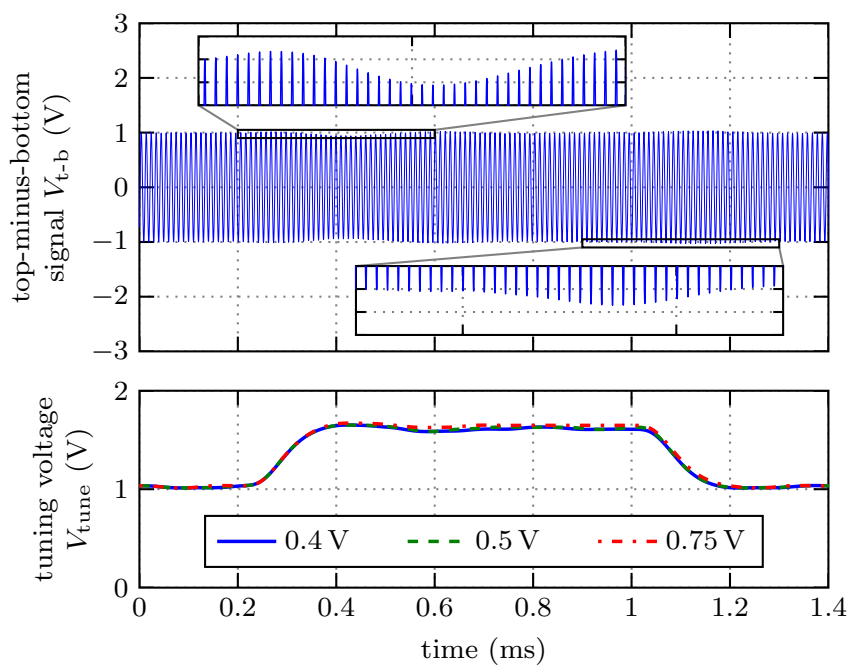

Fig. 3. Closed-loop response on a frequency step input.

quency is simulated by changing the properties of the cantilever model to generate a step in $f_{\text {res }}=100 \mathrm{kHz}$ of $\Delta f \approx 1.9 \mathrm{kHz}$. In the results one can see an automatic adjustment of $V_{\text {tune }}$ and a stable oscillation despite the change in $f_{\text {res }}$.

\section{CONCLUSION AND OUTLOOK}

In this paper we have presented a novel system architecture for a cantilever driver for the use in AFM experiments. It consists of an auto-adjusting amplitude controller and a phase shifter to ensure a stable oscillation even in the presence of unknown phase shifts and long term drifts in the system. The functionality of the proposed architecture was verified in closed-loop Spectre-Simulink cosimulations. The simulations clearly indicate that the system is capable of producing stable oscillations at a predefined frequency and amplitude. Our next step will be the fabrication of a hardware prototype of the proposed architecture in CMOS technology and to use it in real AFM experiments. Here, the proposed mixed Simulink Spectre model allows for a one by one replacement of Simulink blocks by transistor level implementations and thereby greatly facilitates the design process.

\section{REFERENCES}

Ando, T. (2013). High-speed atomic force microscopy. Microscopy, 62(1), 81-93. doi:10.1093/jmicro/dfs093.

Binnig, G., Quate, C.F., and Gerber, C. (1986). Atomic force and microscope. Physical Review Letters, 56, 930934.

Fantner, G.E., Barbero, R.J., Gray, D.S., and Belcher, A.M. (2010). Kinetics of antimicrobial peptide activity measured on individual bacterial cells using high-speed atomic force microscopy. Nat. Nanotechnol., 5(4), 280285.

Schlecker, B., Dukic, M., Erickson, B., Ortmanns, M., Fantner, G., and Anders, J. (2014). Single-cycle-pll detection for real-time fm-afm applications. Biomedical Circuits and Systems, IEEE Transactions on, 8(2), 206215. doi:10.1109/TBCAS.2014.2307696.

Ziegler, J.G. and Nichols, N.B. (1942). Optimum settings for automatic controllers. Transactions of the ASME, 64, 759-768. 


\title{
Is driving assisted behaviour infectious? \\ Using spectral analysis to understand car following behaviour of naïve drivers \\ Eric Stemmler*, Katharina Oeltze*, Caroline Schieß1*
}

\author{
*German Aerospace Center, Braunschweig, 38108 \\ Germany (Tel: +49 531 295-3411; e-mail: eric.stemmler@dlr.de).
}

\begin{abstract}
The development of future driver assistance systems has a strong tendency towards both automation and cooperative intelligent systems (ITS). Two types of ITS can be defined: Car-to-car (C2C) and car-to-infrastructure (C2I). Either way, the driver is equipped with technology to communicate with other equipped drivers and thus ease cooperation. In regard to nowadays relatively low penetration rate of those systems, the question arises of how non-equipped drivers might react to equipped drivers' behavior. In daily traffic, a driver anticipates the behavior of a vehicle in front, e.g. at a traffic light. It may be anticipated that the drivers maintain their speed when approaching a green traffic light. However, the equipped driver, may act differently, e.g. decelerating when the cooperative system communicates that there is no time left to pass the traffic light in time. The goal of this work is evaluating and modelling the effect of those systems on the interaction between differently equipped drivers by using a new data analysis approach. So the impact of cooperative systems e.g. on safety and user acceptance aspects can be estimated reliably.
\end{abstract}

The data was collected with a multi-driver-simulator that can be used to let multiple drivers interact freely with each other in the same simulation scenario. The scenario was designed with two drivers following a computer controlled leading vehicle. This platoon was driving on a ring-shaped road passing several signalized intersections. The leading vehicle was programmed simulating the behavior of a driver equipped with traffic light assistance. Two different use cases were defined and implemented across the intersections: Firstly, stopping at a red traffic light, start during red phase and crossing the stop line simultaneously with the signal switching to green and secondly, approaching a red traffic light that switches $t$ green before the leading vehicle stops. Test runs were executed to collect both experimental and baseline data, where for the latter the computer controlled vehicle simulated non-equipped driving behavior. 64 participants contributed to the dataset within 32 separate trials. For each trial a participant was either in the first (Nr. 2) or the second (Nr. 3) following car. Both baseline and experimental run where completed respectively.

Car-following situations are prominent in today's urban traffic. Thus, especially reacting to changes in speed of a leading vehicle is a fundamental task in driving. In 2013, 13.9\% of all crashes with physical injuries were caused by unadapted velocities (Statistisches Bundesamt, 2014). So on the other hand for the sake of safety, it is important to estimate a system's impact on car following behavior. On the other hand, an adaption of non-equipped drivers on equipped ones would enhance the efficiency of the whole traffic flow and thus the benefit of the ITS.

Car-following analysis inherently requires taking into account the course of the speed trajectory. Most descriptive statistics do not come up to this challenge. Thus more complex measures are needed to quantify this aspect of behavior. A potential adaptation process of the non-assisted driving behavior (NADB) to the assisted driving behavior (ADB) of a leading vehicle is analyzed. Car-following is considered from a signal-processing point of view, where the driver of a following vehicle perceives the behavior of the leading vehicle (input signal) and responds to these stimuli with his own driving (output signal). The speed trajectories of both vehicles are measured and transformed into the frequency domain. In this way, possible co-occurring rhythmic changes in speed are expressed in terms of coherence, gain and delay within its dominant frequency band. There coherence and gain measures are evaluated by the weighted average in terms of the power spectrum. Time delay was estimated by iteratively lagging one of the speed trajectories until coherence reached its maximum. The lag-time with maximum coherence was taken as an estimate for the delay time.

To the best of our knowledge, there is no publication where driving behavior adaption in the context of assisted driving has been investigated with spectral analysis of coherence. As the quantitative results may show it might be interesting to see that changes in coherence between different use cases are possible to detect even when analyzing the starting vehicles alone. 
It is shown that frequency analysis of the cross-correlation spectrum of each of the pairs is a narrow banded one located around $0.003 \mathrm{~Hz}$. Coherence and delay show plausible differences between the combinations of vehicles $1 \& 2$ and $1 \& 3$. A first analysis showed a lower coherence and a higher delay for the driving behavior of the non-equipped drivers when the leading vehicle was equipped in the first use case. However coherence is generally high and mostly above 0.9 for the first use case and significantly lower in the second one. Time delay estimation is mostly consistent with expectations of human reaction times of around $1 \mathrm{~s}$.

Spectral-Analysis seems to be a suitable tool for evaluating car following behavior as the results meet with common-sense expectations. Especially the time delay estimation as a rather new approach to describe the reaction time of non-equipped drivers shows consistent normal human reaction times of around $1.5 \mathrm{~s}$. Thus, a first step towards parameters quantifying the interaction between drivers is made and will be validated in further research to evaluate cooperation and behavioral adaptation of drivers.

Keywords: Driving behavior, spectral analysis, time delay estimation, car following, assisted driving 


\title{
Modelling of Low Power Electronic Loads in Harmonic Analysis
}

\author{
Amir Tokić*, Admir Jukan** \\ Vedran Suljkanović*** \\ *Faculty of Electrical Engineering, University of Tuzla, Tuzla, Bosnia and Herzegovina \\ (e-mail:amir.tokic@untz.ba) \\ **Faculty of Electrical Engineering, University of Tuzla, Tuzla, Bosnia and Herzegovina \\ (e-mail:ajukan@muptk.ba) \\ ***Department EAAE - ABB AG Wien, Austria \\ (e-mail:vedran.suljkanovic@at.abb.com)\}
}

\begin{abstract}
Low power electronic loads are one of the main sources of harmonic currents in distributive electrical networks. In this paper a model of typical low power electronic load is presented and its applicability to calculations of current harmonics is demonstrated. The real power diode is modelled by using corresponding nonlinear resistor. The eigenvalue analysis of differential equation systems, that describe load behaviour, has revealed a very stiff nature. The obtained equation systems are solved by using an A- and L-stable BDF numerical method. The simulation results are verified by comparison with the available experimental results. The suggested electronic load model can be efficiently used in harmonic studies and its implementation in the EMTP or SIMULINK programs are recommended.
\end{abstract}

Keywords: electronic load, power diode model, stiff system, harmonics.

\section{INTRODUCTION}

The widely spread single-phase low power electronic loads such as personal computers, televisions, light-dimmers and battery chargers, generate harmonic currents in the electrical network and generally can cause power quality degradation. Individually, harmonic currents injected by these loads are relatively small to cause a significant distortion in distributive networks. However, the cumulative effect of these loads has the capability of causing serious harmonic distortion levels. Regarding this, harmonic analyses are currently receiving more attention due to the increasing number of these loads. In general, these loads use single-phase diode bridge rectifiers with dc-smoothing capacitors, shown in Figure 1.

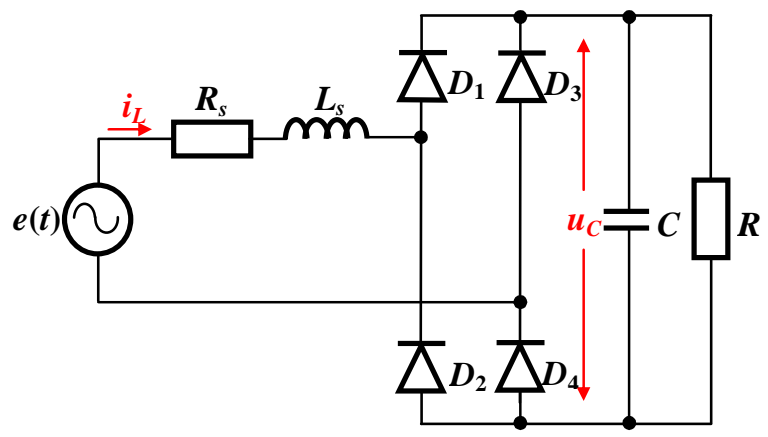

Fig. 1. Typical low power electronic load model.

System voltage $e(t)$ is represented by the sum of harmonic voltage sources. The parameter $R_{s}$ represent equivalent total source and filter resistance, and $L_{s}$ inductance, whereas $C$ represent smoothing capacitor and $R$ model equivalent load resistance. The parameters $R$ and $C$ define acceptable ripple in the rectifier's output voltage (dc voltage ripple). The power diodes $D_{i}, i=1,2,3,4$ are commonly modelled as ideal switching elements, Johnson et al. (2005). However, in this paper diodes are modelled considering their real nonlinear current-voltage characteristics i.e. diodes are modelled as nonlinear resistors.

\section{LOW POWER ELECTRONIC LOADS MODEL}

In general, the nonlinear resistor can be described using the curve that describes the dependence of the resistor voltage $u_{R}$ from the corresponding resistor current $i_{R}$. This curve is usually defined as a set of points:

$\left(i_{r_{k}}, u_{r_{k}}\right), k=1,2, \ldots, M$

where $M$ is the total number of core resistor curve segments. The resistor current within the $k$-th linear segment of the resistor curve, is calculated by using the following relation:

$i_{R}=\frac{1}{R_{k}} u_{R}+\operatorname{sign}\left(u_{R}\right) I_{r_{k}}=\frac{1}{R_{k}} u_{R}+S_{k}$

where: $R_{k}=\left(u_{r_{k+1}}-u_{r_{k}}\right) /\left(i_{r_{k+1}}-i_{r_{k}}\right), I_{r_{k}}=i_{r_{k}}-u_{r_{k}} / R_{k}$.

Based on the equation (1) it is possible to create the nonlinear resistor equivalent model using a linear resistor $R_{k}$ and the corresponding current source $S_{k}$, as presented in Figure 2.

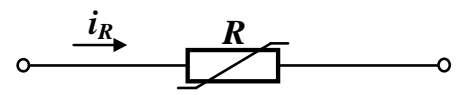

(a)

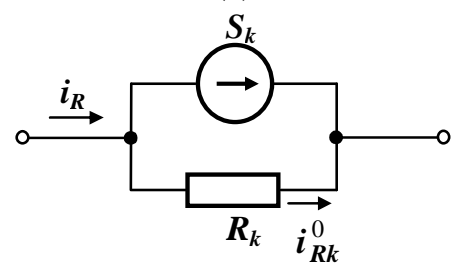

(b)

Fig. 2. (a) Nonlinear power diode, (b) equivalent model 
Accordingly, the following state-space equation describing low power electronic loads behaviour is developed:

$$
\frac{d X(t)}{d t}=A_{k_{1}, k_{2}, k_{3}, k_{4}} X(t)+B_{k_{1}, k_{2}, k_{3}, k_{4}} U(t)=F(X, t)
$$

where:

$$
X(t)=\left[\begin{array}{ll}
u_{C} & i_{L}
\end{array}\right]^{\mathrm{T}}, U(t)=\left[\begin{array}{lllll}
e(t) & S_{k_{1}} & S_{k_{2}} & S_{k_{3}} & S_{k_{4}}
\end{array}\right]^{\mathrm{T}} .
$$

It is very important to evaluate if the obtained system of differential equations (2) is a stiff- or a very stiff system. In general stiff systems are categorized as those whose different parts have a much dispersed time constants (real parts of eigenvalues).

\section{LOW POWER ELECTRONIC LOADS MEASUREMENTS AND SIMULATIONS}

The suggested model is implemented and tested on the laboratory low power electronic load with these parameters: $R_{s}=17.5 \Omega, L_{s}=91.9 \mathrm{mH}, R=750 \Omega, C=100 \mu \mathrm{F}$

The nonlinear characteristic of real power diode (nonlinear resistor) is shown in Figure 3.

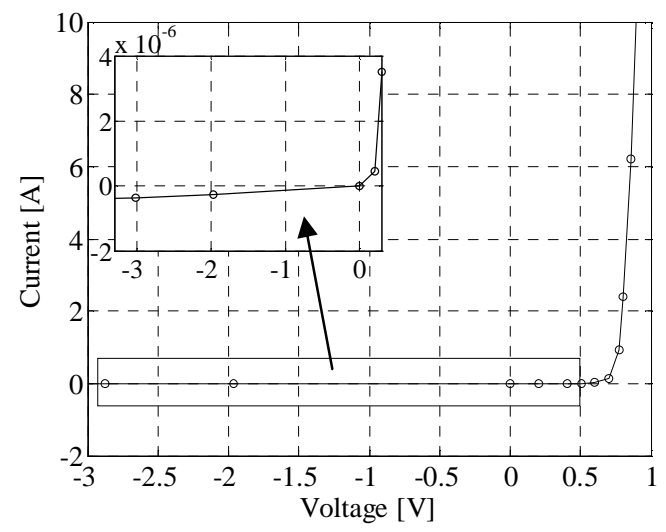

Fig. 3. Real nonlinear characteristics of diode.

In order to examine the character of equation (2), in every integration step of the simulation time the maximal and the minimal eigenvalues of system matrix are computed as shown in Figure 4.
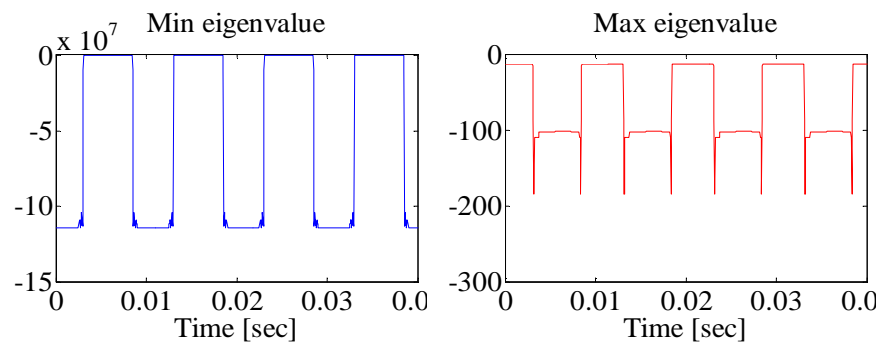

Fig. 4. Propagation of real part of eigenvalues during the simulation time.

It is clear that in this case very stiff matrixes appear. For this reason, for simulating the low power electronic loads the $A$ and $L$ stable BDF2 numerical methods were used, Tokić et al. (2013). The comparison between the measured and the simulated input ac current $i_{L}$, their harmonic content, as well as dc rectifier voltage $u_{C}$, are shown in Figures 5-7. The simulations were performed by using the BDF2 numerical method with the integration step of $\Delta t=50 \mu \mathrm{sec}$.

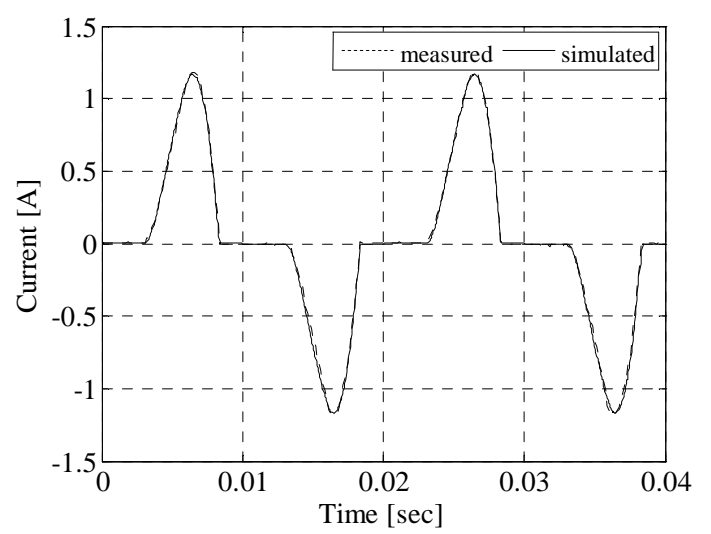

Fig. 5. Measured and simulated input ac current.

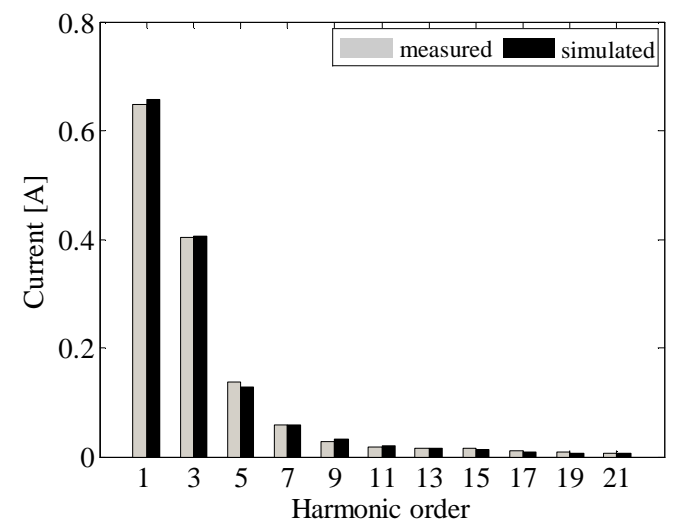

Fig. 6. Harmonic content of measured and simulated current.

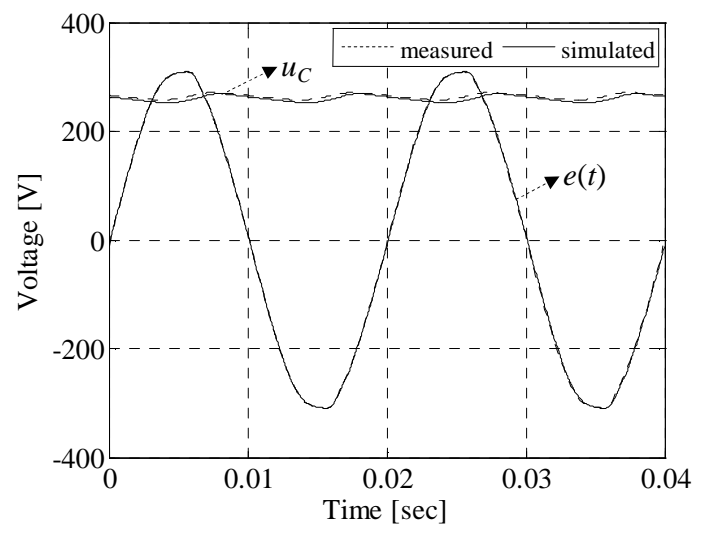

Fig. 7. Measured and simulated ac source voltage and dc rectifier voltage.

\section{REFERENCES}

Johnson, B., Hess, H., and Martinez J.A. (2005). Parameter Determination for Modeling System Transients-Part VII: Semiconductors, 2086-2092, IEEE Transaction on Power Delivery, Vol. 20., No. 3.

Tokić, A., Uglešić, I., Štumberger, G. (2013). Simulations of transformer inrush current by using BDF-based numerical methods, 1-10, Mathematical Problems in Engineering, Vol. 2013. 


\title{
p(t)MOR: Time-Varying Parametric Model Order Reduction and Applications for Moving Loads
}

\author{
Maria Cruz Varona, Matthias Geuss, Boris Lohmann \\ Institute of Automatic Control, Technische Universität München \\ Boltzmannstr. 15, D-85748 Garching, Germany \\ e-mail: \{maria.cruz, matthias.geuss, lohmann\}@tum.de
}

Keywords: time-varying; parameter-dependent systems; model reduction; moving loads.

\section{INTRODUCTION}

In this contribution we investigate Model Order Reduction (MOR) for linear systems with time-varying parameters $\mathbf{p}(t)$. Such systems arise, for instance, in structural dynamics and multibody simulations when the position of an acting force on the physical structure varies with time. This behaviour is found in many industrial applications, e.g. in meshing gears, milling processes, bridge cranes or any system with moving load components. The varying load location can generally be considered as a parameter dependency of the system model. Thus, a spatial discretization of such systems leads to large-scale linear parameter-varying (LPV) models, and model order reduction becomes necessary to enable a fast simulation keeping the computational expense and the storage requirements low. Our aim is to develop time-varying parametric model order reduction, which we call $\mathrm{p}(\mathrm{t}) \mathrm{MOR}$, to obtain a reduced order model of a large-scale LPV system.

After giving a review of standard pMOR by matrix interpolation to generate a reduced order model of a parametric LTI dynamical system, linear parameter-varying systems are examined in Section 3, where two different cases are presented: First, quasi-static parameter variations are considered, where the parameters are assumed to vary slowly with time. For this case, standard pMOR from Section 2 can be adapted and applied. Secondly, we consider the dynamic case where the parameters may change very quickly and present the challenges arising from this concern.

\section{PARAMETRIC MODEL ORDER REDUCTION}

In the following, a high-dimensional parametric first order dynamical system is considered:

$$
\begin{aligned}
\mathbf{E}(\mathbf{p}) \dot{\mathbf{x}}(t) & =\mathbf{A}(\mathbf{p}) \mathbf{x}(t)+\mathbf{B}(\mathbf{p}) \mathbf{u}(t), \\
\mathbf{y}(t) & =\mathbf{C}(\mathbf{p}) \mathbf{x}(t),
\end{aligned}
$$

where $\mathbf{E}(\mathbf{p}), \mathbf{A}(\mathbf{p}) \in \mathbb{R}^{N \times N}, \mathbf{B}(\mathbf{p}) \in \mathbb{R}^{N \times m}$ and $\mathbf{C}(\mathbf{p}) \in$ $\mathbb{R}^{p \times N}$ are the system matrices which depend on the parameter vector $\mathbf{p} \in \mathcal{D}$ with parameter domain $\mathcal{D} \subset \mathbb{R}^{d}$. As a standard, projection-based pMOR is carried out to approximate the original large-scale parametric system (1) by a low-dimensional system.

\subsection{Projection-based pMOR}

In projection-based parametric model order reduction we aim to approximate the state-vector $\mathbf{x}(t) \in \mathbb{R}^{N}$ by $\mathbf{x}(t) \approx$ $\mathbf{V}(\mathbf{p}) \mathbf{x}_{r}(t)$, where $\mathbf{x}_{r}(t) \in \mathbb{R}^{n}$ and $n \ll N$. To this end, appropriate projection matrices $\mathbf{V}(\mathbf{p}), \mathbf{W}(\mathbf{p}) \in \mathbb{R}^{N \times n}$ which span respective $n$-dimensional subspaces have to be found. Applying suitable projection matrices on (1) that impose the Petrov-Galerkin condition leads to the reduced order model

$$
\begin{aligned}
\mathbf{E}_{r}(\mathbf{p}) \dot{\mathbf{x}}_{r}(t) & =\mathbf{A}_{r}(\mathbf{p}) \mathbf{x}_{r}(t)+\mathbf{B}_{r}(\mathbf{p}) \mathbf{u}(t) \\
\mathbf{y}_{r}(t) & =\mathbf{C}_{r}(\mathbf{p}) \mathbf{x}_{r}(t)
\end{aligned}
$$

with

$$
\begin{aligned}
\left\{\mathbf{E}_{r}, \mathbf{A}_{r}\right\}(\mathbf{p}) & =\mathbf{W}(\mathbf{p})^{T}\{\mathbf{E}, \mathbf{A}\}(\mathbf{p}) \mathbf{V}(\mathbf{p}), \\
\mathbf{B}_{r}(\mathbf{p}) & =\mathbf{W}(\mathbf{p})^{T} \mathbf{B}(\mathbf{p}), \\
\mathbf{C}_{r}(\mathbf{p}) & =\mathbf{C}(\mathbf{p}) \mathbf{V}(\mathbf{p}) .
\end{aligned}
$$

For calculating $\mathbf{V}$ and $\mathbf{W}$ with fixed $\mathbf{p}$, different methods are available, like e.g. the Modal Reduction approach, Balanced Truncation, Proper Orthogonal Decomposition (POD) or Krylov Subspace Methods (see Antoulas (2005)). In order to find a parameter-dependent reduced model, there exist several parametric model order reduction techniques, which can be generally categorised in either global or local approaches (Benner et al. (2013)). In the following, local pMOR by matrix interpolation is considered as an example and applied for the reduction.

\section{2 pMOR by matrix interpolation}

The main idea of parametric model order reduction by matrix interpolation is to compute the original system (1) at certain parameter values $\mathbf{p}_{i}$ with $i=1, \ldots, k$, then reduce each of the $k$ local models separately to the same order $n$ using different projection matrices $\mathbf{V}_{i}:=\mathbf{V}\left(\mathbf{p}_{i}\right)$ and $\mathbf{W}_{i}:=\mathbf{W}\left(\mathbf{p}_{i}\right)$ calculated according to one specific order reduction method mentioned above and finally interpolate between the local reduced system matrices. Since the projection matrices $\mathbf{V}_{i}$ are not equal, the individually reduced models

$$
\begin{aligned}
\mathbf{E}_{r, i} \dot{\mathbf{x}}_{r, i}(t) & =\mathbf{A}_{r, i} \mathbf{x}_{r, i}(t)+\mathbf{B}_{r, i} \mathbf{u}(t), \\
\mathbf{y}_{r, i}(t) & =\mathbf{C}_{r, i} \mathbf{x}_{r, i}(t),
\end{aligned}
$$

are first modified applying state transformations of the form $\mathbf{x}_{r, i}(t)=\mathbf{T}\left(\mathbf{p}_{i}\right) \hat{\mathbf{x}}_{r, i}(t)$ with regular matrices $\mathbf{T}_{i}:=\mathbf{T}\left(\mathbf{p}_{i}\right)$ 
and multiplying (4) from the left by $\mathbf{M}_{i}^{T}$ to ensure that the local reduced models are described in the same set of coordinates $\hat{\mathbf{x}}_{r}(t)$ (see Panzer et al. (2010); Amsallem and Farhat (2011)). The resulting transformed reduced models are thus given by

$$
\begin{aligned}
\overbrace{\mathbf{M}_{i}^{T} \mathbf{E}_{r, i} \mathbf{T}_{i}}^{\hat{\mathbf{E}}_{r, i}} \dot{\mathbf{x}}_{r, i}(t) & =\overbrace{\mathbf{M}_{i}^{T} \mathbf{A}_{r, i} \mathbf{T}_{i}}^{\hat{\mathbf{A}}_{r, i}} \hat{\mathbf{x}}_{r, i}(t)+\overbrace{\mathbf{M}_{i}^{T} \mathbf{B}_{r, i}}^{\hat{\mathbf{B}}_{r, i}} \mathbf{u}(t), \\
\mathbf{y}_{r, i}(t) & =\underbrace{\mathbf{C}_{r, i} \mathbf{T}_{i}}_{\hat{\mathbf{C}}_{r, i}} \hat{\mathbf{x}}_{r, i}(t) .
\end{aligned}
$$

After this transformation, an interpolation between the reduced system matrices can be performed as

$$
\begin{aligned}
& \hat{\mathbf{E}}_{r}(\mathbf{p})=\sum_{i=1}^{k} \omega_{i}(\mathbf{p}) \hat{\mathbf{E}}_{r, i}, \quad \hat{\mathbf{A}}_{r}(\mathbf{p})=\sum_{i=1}^{k} \omega_{i}(\mathbf{p}) \hat{\mathbf{A}}_{r, i}, \\
& \hat{\mathbf{B}}_{r}(\mathbf{p})=\sum_{i=1}^{k} \omega_{i}(\mathbf{p}) \hat{\mathbf{B}}_{r, i}, \quad \hat{\mathbf{C}}_{r}(\mathbf{p})=\sum_{i=1}^{k} \omega_{i}(\mathbf{p}) \hat{\mathbf{C}}_{r, i}
\end{aligned}
$$

with weighting functions $\omega_{i}(\mathbf{p})$ with $\sum_{i=1}^{k} \omega_{i}(\mathbf{p})=1$ in order to obtain a reduced model for any new parameter value $\mathbf{p}$.

Choice of the matrices $\mathbf{T}_{i}$ and $\mathbf{M}_{i}$ : Panzer et al. (2010) proposed to make the state vectors $\hat{\mathbf{x}}_{r, i}(t)$ compatible with respect to a reference subspace spanned by the columns of the orthogonal matrix $\mathbf{R}$. To this end, $\mathbf{T}_{i}$ and $\mathbf{M}_{i}$ are chosen as $\mathbf{T}_{i}:=\left(\mathbf{R}^{T} \mathbf{V}_{i}\right)^{-1}$ and $\mathbf{M}_{i}:=\left(\mathbf{R}^{T} \mathbf{W}_{i}\right)^{-1}$, where the columns of $\mathbf{R}$ are determined by the $n$ most important directions of the matrix

$$
\mathbf{V}_{\text {all }}=\left[\begin{array}{llll}
\mathbf{V}_{1} & \mathbf{V}_{2} & \ldots & \mathbf{V}_{k}
\end{array}\right]
$$

which can be calculated by Singular Value Decomposition (SVD) of $\mathbf{V}_{\text {all }}=\mathbf{U} \boldsymbol{\Sigma} \mathbf{N}^{T}$. This approach for choosing the matrices $\mathbf{T}_{i}$ and $\mathbf{M}_{i}$ is indeed a special case of the more general framework for pMOR by matrix interpolation proposed by Geuss et al. (2013), which can also be applied to select the remaining degrees of freedom.

\section{TIME-DEPENDENT PARAMETRIC MODEL ORDER REDUCTION}

After considering parametric LTI dynamical systems, we now examine high-dimensional linear parameter-varying first order systems of the form

$$
\begin{aligned}
\mathbf{E}(\mathbf{p}(t)) \dot{\mathbf{x}}(t) & =\mathbf{A}(\mathbf{p}(t)) \mathbf{x}(t)+\mathbf{B}(\mathbf{p}(t)) \mathbf{u}(t), \\
\mathbf{y}(t) & =\mathbf{C}(\mathbf{p}(t)) \mathbf{x}(t),
\end{aligned}
$$

where the parameter vector $\mathbf{p}(t)$ now explicitly depends on time. In a similar manner like before, we apply projectionbased $\mathrm{p}(\mathrm{t}) \mathrm{MOR}$ in the following to reduce the original LPV system to one of smaller dimension.

\subsection{Projection-based $p(t) M O R$}

In the case of projection-based time-varying parametric model order reduction we aim to approximate (8) using $\mathbf{x} \approx \mathbf{V}(\mathbf{p}(t)) \mathbf{x}_{r}$,

$\dot{\mathbf{x}} \approx \dot{\mathbf{V}}(\mathbf{p}(t)) \mathbf{x}_{r}+\mathbf{V}(\mathbf{p}(t)) \dot{\mathbf{x}}_{r}=\frac{\partial \mathbf{V}}{\partial \mathbf{p}} \dot{\mathbf{p}} \mathbf{x}_{r}+\mathbf{V}(\mathbf{p}(t)) \dot{\mathbf{x}}_{r}$ and a properly chosen projection matrix $\mathbf{W}(\mathbf{p}(t))$ that imposes the Petrov-Galerkin condition. As a result we obtain the reduced order model

$$
\begin{aligned}
\mathbf{E}_{r}(\mathbf{p}(t)) \dot{\mathbf{x}}_{r}= & \left(\mathbf{A}_{r}(\mathbf{p}(t))-\mathbf{W}(\mathbf{p}(t))^{T} \mathbf{E}(\mathbf{p}(t)) .\right. \\
& \left.\frac{\partial \mathbf{V}}{\partial \mathbf{p}} \dot{\mathbf{p}}\right) \mathbf{x}_{r}+\mathbf{B}_{r}(\mathbf{p}(t)) \mathbf{u} \\
\mathbf{y}_{r}= & \mathbf{C}_{r}(\mathbf{p}(t)) \mathbf{x}_{r}
\end{aligned}
$$

with

$$
\begin{aligned}
\left\{\mathbf{E}_{r}, \mathbf{A}_{r}\right\}(\mathbf{p}(t)) & =\mathbf{W}(\mathbf{p}(t))^{T}\{\mathbf{E}, \mathbf{A}\}(\mathbf{p}(t)) \mathbf{V}(\mathbf{p}(t)), \\
\mathbf{B}_{r}(\mathbf{p}(t)) & =\mathbf{W}(\mathbf{p}(t))^{T} \mathbf{B}(\mathbf{p}(t)) \\
\mathbf{C}_{r}(\mathbf{p}(t)) & =\mathbf{C}(\mathbf{p}(t)) \mathbf{V}(\mathbf{p}(t)) .
\end{aligned}
$$

Depending on whether the parameters vary slowly or significantly change with time, we distinguish between quasi-static and dynamic $\mathrm{p}(\mathrm{t}) \mathrm{MOR}$.

\subsection{Quasi-static p(t)MOR}

Here, it is assumed that the parameters change slowly over time and are therefore called quasi-static. Since in this case $\dot{\mathbf{p}} \approx \mathbf{0}$ holds for small time intervals, equation (9) simplifies to (2) with $\mathbf{p}$ substituted by $\mathbf{p}(t)$. Thus, standard methods of pMOR (e.g. pMOR by matrix interpolation according to (6)) can be carried out for the reduction: in each time step of a numerical simulation, a corresponding interpolated reduced order model is recalculated using (6) and evaluated (see also Fischer and Eberhard (2014)), without need to perform any large-scale calculations. Hence we obtain different interpolated reduced order models, one for each simulation step.

\subsection{Dynamic $p(t) M O R$}

For the case of fast-changing parameters the timederivative of the parameter vector in (9) should no longer be ommited, and extended reduction steps are needed. First results and a discussion comparing the quasi-static and the dynamic approach will be presented at the conference.

\section{REFERENCES}

Amsallem, D. and Farhat, C. (2011). An online method for interpolating linear parametric reduced-order models. SIAM Journal on Scientific Computing, 33(5), 21692198.

Antoulas, A.C. (2005). Approximation of Large-Scale Dynamical Systems. SIAM.

Benner, P., Gugercin, S., and Willcox, K. (2013). A survey of model reduction methods for parametric systems. Preprint MPIMD/13-14, Max Planck Institute Magdeburg.

Fischer, M. and Eberhard, P. (2014). Simulation of moving loads in elastic multibody systems with parametric model reduction techniques. Archive of Mechanical Engineering, 61(2), 209-226.

Geuss, M., Panzer, H., and Lohmann, B. (2013). On parametric model order reduction by matrix interpolation. In Proceedings of the European Control Conference, 3433-3438.

Panzer, H., Mohring, J., Eid, R., and Lohmann, B. (2010). Parametric model order reduction by matrix interpolation. at-Automatisierungstechnik, 58(8), 475-484. 


\title{
Advances on the Adaptive Selection of Both Shifts and Reduced Order in $\mathcal{H}_{2}$-Pseudo-Optimal Model Reduction *
}

\author{
Alessandro Castagnotto, Heiko K. F. Panzer, Thomas Wolf, \\ Boris Lohmann \\ Institute of Automatic Control, Technische Universität München, \\ Boltzmannstr. 15, D-85748 Garching, Germany \\ (e-mail: \{a.castagnotto, panzer, lohmann\}@tum.de).
}

\begin{abstract}
Keywords: Model reduction; large-scale systems; Krylov subspace methods; strictly dissipative realization
\end{abstract}

\section{INTRODUCTION}

The accurate description of dynamical systems in the fields of engineering and applied sciences can result in highfidelity models (HFM) of prohibitively large dimensions. This is the case, for instance, in the very-large-scale integration of electrical circuits or for models resulting from the spatial discretization of partial differential equations over a fine grid. Simulations, optimizations, and control design algorithms based on these large models are computationally expensive, if at all feasible.

To resolve this issue, reduced order models (ROM) are required that capture the dominant behavior of the HFM and possibly preserve fundamental characteristics such as stability, passivity, or second-order structure. Model order reduction (MOR) based on Krylov subspace methods stands out due to its generality ${ }^{1}$ and low computational cost, making it a predestined candidate for the reduction of so-called truly large-scale systems. Even so, the inherent flexibility of the methods can lead to quite unsatisfying results as well, attaching even more importance to the careful selection of free design parameters. In this contribution, we demonstrate how both the size of the reduced model and the complex frequencies (shifts) at which the ROM and HFM share the same transfer function values can be chosen adaptively in a cumulative reduction framework. Current advances based on recent work are addressed and an overview on some open challenges in this field is given.

\section{PRELIMINARIES}

Many systems that arise in practice can be modeled, at least locally, by a linear time-invariant state space model of the form

$$
\left\{\begin{aligned}
E \dot{x} & =A x+B u \\
y & =C x+D u
\end{aligned}\right.
$$

where $x \in \mathbb{R}^{N}$ is the state, $E, A \in \mathbb{R}^{N \times N}$ represent the dynamics of the unforced system, and $u \in \mathbb{R}^{m}$ and

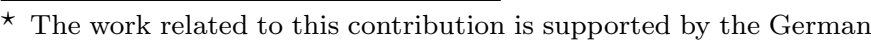
Research Foundation (DFG), Grant LO408/19-1.

1 It has been shown by Gallivan et al. (2002) that every reduced model, at least in the SISO case, can be obtained through Krylov subspace methods.
}

$y \in \mathbb{R}^{p}$ represent the inputs and outputs of the system respectively $(p, m \ll N)$. A unifying framework to describe reduction techniques based on representation (1) is given by projection methods, in which the ROM is obtained by applying a Petrov-Galerkin projection of the differential equation in (1) with respect to the subspaces defined by the matrices $V, W \in \mathbb{R}^{N \times n}$. The resulting $\mathrm{ROM}$ is subsequently given as

$$
\left\{\begin{aligned}
E_{r} \dot{x}_{r} & =A_{r} x_{r}+B_{r} u \\
y_{r} & =C_{r} x_{r}+D u
\end{aligned}\right.
$$

where $x_{r} \in \mathbb{R}^{n}, n \ll N, E_{r}:=W^{\top} E V, A_{r}:=W^{\top} A V$, $B_{r}:=W^{\top} B$, and $C_{r}:=C V$.

The matrices $V, W$ defining the ROM can be computed in several ways, for example through modal reduction or balanced truncation. However, due to their generality and low computational cost, Krylov subspace methods have been studied in the past few decades as tools to reduce large-scale dynamical systems. If $V, W$ are generated using input and output Krylov subspaces respectively, then the ROM implicitly matches some coefficients $m_{i}$ (moments) of the transfer function Taylor series

$$
G(s)=-\sum_{i=0}^{\infty} m_{i}\left(s-s_{0}\right)^{i}
$$

at given complex frequencies $s_{0}$, called shifts. Since the quality and properties of the ROM are determined by the reduced order $n$ and the complex shifts $s_{0}$, past developments have focused on the appropriate selection of these design parameters. Trivial choices for the shifts are implemented in Padé approximations $\left(s_{0}=0\right)$ and partial realizations $\left(s_{0} \rightarrow \infty\right)$, whereas the more general case with multiple shifts in the range $0 \leq\left|s_{0}\right|<\infty$ is known under the name of multipoint Padé or rational interpolation. In this latter case, a convenient selection strategy is required. The Iterative Rational Krylov Algorithm (IRKA) introduced by Gugercin et al. (2008), for instance, conducts a fixed-point iteration of the shifts that yields a local $\mathcal{H}_{2^{-}}$ optimum - provided convergence is reached. The desired reduced order $n$ must be specified in advance, so that an improvement in accuracy of the resulting ROM requires a reiteration of the whole reduction process. 


\section{AN ADAPTIVE FRAMEWORK}

In this contribution, we present the current developments on the adaptive selection of a) the reduced order $n$ and b) the position of the shifts $s_{0}$, both introduced by Panzer et al. (2013a). The cornerstone of the discussion is given by an unconventional representation of the error system $G_{e}(s)$. In fact, if the matrix $V$ is the basis of an input Krylov subspace, then the error system can be factorized as

$$
G_{e}(s)=G(s)-G_{r}(s)=G_{\perp}(s) \cdot G_{f}(s),
$$

where $G_{\perp}(s)$ represents the HFM (with modified input matrix) and $G_{f}(s)$ represents the ROM (with modified output and unity feedthrough). Starting from (3), we make use of the concept of $\mathcal{H}_{2}$-pseudo-optimality introduced by Wolf et al. (2013). If the ROM is $\mathcal{H}_{2}$-pseudo-optimal, which in the SISO case is equivalent to requiring

$$
G\left(-\lambda_{r, j}\right)=G_{r}\left(-\lambda_{r, j}\right)
$$

for all reduced eigenvalues $\lambda_{r, j}(j=1, \ldots, n)$, then the factor $G_{f}(s)$ is a unity all-pass. These results motivate a cumulative reduction framework (CURE), in which at each step only the $G_{\perp}(s)$ factor of the remaining error systems is reduced. In addition to this, $\mathcal{H}_{2}$-pseudo-optimal reduction at every step guarantees monotonic decrease of the $\mathcal{H}_{2}$-norm of the error system. CURE allows for an adaptive selection of the reduced order $n$ and yields an $\mathcal{H}_{2}$-pseudo-optimal ROM.

An additional advantage introduced by CURE is the free choice of reduced order $n_{k}$ at each reduction step $k$. Therefore, it is possible to restrict this choice to reduced models of the order $n_{k}=2$. Assuming asymptotic stability of the HFM, the choice of shifts can be parametrized by two positive real numbers and a greedy, trust-region based algorithm for the detection of locally $\mathcal{H}_{2}$-optimal shifts can be implemented, bypassing the convergence issues related to IRKA. This algorithm was first introduced by Panzer et al. (2013a) under the name of Stability Preserving Adaptive Rational Krylov (SPARK). It can be shown that in $\mathcal{H}_{2}$-pseudo-optimal reduction, the minimization of the $\mathcal{H}_{2}$-norm of the error system (3) corresponds to the maximization of the $\mathcal{H}_{2}$-norm of the ROM, yielding a cheap cost functional for SPARK.

That said, cumulative reduction requires a stopping criterion that detects when the desired approximation accuracy has been reached. A typical choice is usually given by some upper bound on the error norm, since the evaluation of the actual, high dimensional error system is exceedingly expensive. For state-space systems (1) in strictly dissipative form, meaning

$$
E=E^{\top} \succ 0, \quad A+A^{\top} \prec 0
$$

rigorous global error bounds introduced by Panzer et al (2013c) were derived that yield an a posteriori stopping criterion for CURE. In addition to this, these error bounds can be also included in the greedy search for optimal shifts. In fact, we propose a change of paradigm within the optimization in SPARK and show how the minimization of the error bound - as opposed to the intuitive approach of minimizing the actual error - can deliver a better suited stopping condition for the cumulative framework. This course of action is motivated by the fact that, in a large scale setting, the error bound is the only error measure we are actually able to compute.

Finally, it was shown how the conditions in (4) are rarely restrictive if the original HFM is given in a second order structure

$$
\left\{\begin{aligned}
M \ddot{z}+D \dot{z}+K z & =F \\
y & =S z
\end{aligned}\right.
$$

with positive definite matrices $M, D, K$, as it is often the case in structural mechanics and electrical circuits. For this case, a transformation from the second order to a strictly dissipative first oder structure (1) was derived by Panzer et al. (2013b).

\section{ADVANCES AND OPEN CHALLENGES}

The adaptive framework introduced in the last section builds the basis for the current research endeavors. The recent work within this framework is aimed at expanding its validity to other classes of systems not included so far. Current results, for instance, show how the concept of strictly dissipative realizations can be enlarged to comprise descriptor systems with positive semi-definite descriptor matrix $E$, allowing an adaptive reduction with rigorous, global error bounds also in this case. The improvement of the greedy algorithm in SPARK, as well as the expansion of this framework to both multiple-input multiple-output systems and reduction preserving the second order structure in (5) is topic of future research.

\section{ACKNOWLEDGEMENTS}

The authors express their sincere appreciation to Dr. Klaus-Dieter Reinsch for his fundamental support on the extension of strict dissipativity to descriptor systems.

\section{REFERENCES}

Gallivan, K.A., Vandendorpe, A., and Van Dooren, P. (2002). On the generality of multipoint padé approximations. In 15th IFAC World Congress on Automatic Control.

Gugercin, S., Antoulas, A.C., and Beattie, C.A. (2008). $\mathcal{H}_{2}$ model reduction for large-scale linear dynamical systems. SIAM Journal on Matrix Analysis and Applications, 30(2), 609-638.

Panzer, H.K.F., Jaensch, S., Wolf, T., and Lohmann, B. (2013a). A greedy rational Krylov method for $\mathcal{H}_{2^{-}}$ pseudooptimal model order reduction with preservation of stability. In American Control Conference, 55325537.

Panzer, H.K.F., Kleinherne, B., and Lohmann, B. (2013b). Analysis, interpretation and generalization of a strictly dissipative state space formulation of second order systems. In Methoden und Anwendungen der Regelungstechnik - Erlangen-Münchener Workshops 2011 und 2012. Boris Lohmann and Günter Roppenecker (Hrsg.). URL http://mediatum.ub.tum.de/doc/1175736.

Panzer, H.K.F., Wolf, T., and Lohmann, B. (2013c). $\mathcal{H}_{2}$ and $\mathcal{H}_{\infty}$ error bounds for model order reduction of second order systems by Krylov subspace methods. In European Control Conference, 4484-4489.

Wolf, T., Panzer, H.K.F., and Lohmann, B. (2013). $\mathcal{H}_{2}$ pseudo-optimality in model order reduction by Krylov subspace methods. In European Control Conference. 


\title{
Equations of motion with redundant coordinates for mechanical systems on manifolds
}

\author{
M. Konz ${ }^{*}$ J. Rudolph* \\ * Chair of Systems Theory and Control Engineering, \\ Saarland University, 66123 Saarbrücken, Germany, \\ (e-mail: \{m.konz, j.rudolph\}@lsr.uni-saarland.de)
}

\begin{abstract}
The Lagrange formalism for deriving equations of motion for lumped mechanical systems is reviewed. When dealing with a configuration space that is not isomorphic to $\mathbb{R}^{n}$, the choice of generalized coordinates is local and may result in awkward expressions. It is shown how redundant coordinates can lead to more convenient global expressions.
\end{abstract}

Keywords: energy methods, Lagrange equations, redundant coordinates, embedding

\section{INTRODUCTION}

Lagrange's equations are widely used in engineering for tasks such as modeling, control design, and optimization. To model a holonomic rigid body system one has to choose generalized coordinates $q \in \mathbb{R}^{n}$ and formulate the Lagrangian $L$ (kinetic minus potential energy) in terms of the chosen coordinates $q$ and their time derivatives $\dot{q}$ (generalized forces are neglected for the sake of brevity). The equations of motion can then be derived from

$$
\frac{\mathrm{d}}{\mathrm{d} t} \frac{\partial L}{\partial \dot{q}_{i}}-\frac{\partial L}{\partial q_{i}}=0, \quad i=1, \ldots, n .
$$

When dealing with a system whose configuration space is a smooth manifold that is not isomorphic to $\mathbb{R}^{n}$, the choice of generalized coordinates is the choice of a necessarily local chart. In these cases the Lagrangian approach might lead to awkward expressions as shown in the following example.

\section{MOTIVATING EXAMPLE: RIGID BODY}

Consider a rigid body in three dimensional space that is fixed at one point and subject to no external forces. A popular choice of generalized coordinates are Euler angles $q=(\alpha, \beta, \varphi)$. The Lagrangian $L$ (which here is just kinetic energy) expressed in terms of $q$ has the form

$$
L=\frac{1}{2} \dot{q}^{\top} M(q) \dot{q} .
$$

Evaluating (1) yields equations of the form

$$
M(q) \ddot{q}+c(q, \dot{q})=0 .
$$

The entries of $M(q)$ and $c(q, \dot{q})$ are quite cumbersome and dependent on $q$. Also problematic is that the inertia matrix $M(q)$ is singular where the Euler angles are.

On the other hand, for this particular example, there are Euler's equations

$$
\dot{R}=R \text { Skw } \omega, \quad \Theta \dot{\omega}+\operatorname{Skw} \omega \Theta \omega=0,
$$

with rotation matrix $R \in \mathrm{SO}(3)=\left\{R \in \mathbb{R}^{3 \times 3} \mid R^{\top} R=\right.$ $I_{3}$, det $\left.R=1\right\}$, angular velocity $\omega \in \mathbb{R}^{3}$, and moment of inertia $\Theta \in \mathbb{R}^{3 \times 3}$. The skew-symmetric matrix Skw $\omega \in$ $\mathbb{R}^{3 \times 3}$ is defined such that $(\operatorname{Skw} \omega) v=\omega \times v, \omega, v \in \mathbb{R}^{3}$.
The Lagrangian expressed in terms of the angular velocity $\omega$ is

$$
\tilde{L}=\frac{1}{2} \omega^{\top} \Theta \omega
$$

Of course, Euler's equations (4) and Lagrange's equations (3) describe the same motion. Also (5) and (2) are different expressions for the same quantity, the kinetic energy. But it is not possible to derive Euler's equations directly by using (1) as the angular velocities $\omega$ are not derivatives of some coordinates. Another aspect of (4) is that the attitude (3 degrees of freedom) of the rigid body is expressed by 9 quantities, the entries of the rotation matrix $R$.

This example should motivate the question: Is it possible to modify Lagrange's equations to derive (4) and can this be generalized?

\section{DERIVATION OF EQUATIONS OF MOTION FROM HAMILTON'S PRINCIPLE}

The derivation of Lagrange's equations (1) from Hamilton's principle of least action can be found in most textbooks on analytical mechanics, e.g. Arnold [1989]. The following is very similar but differs in allowing redundant coordinates that are used constructively in sec. 3.2.

\subsection{Problem statement}

Assume the configuration of a mechanical system can be described by the coordinates $x \in \mathbb{R}^{m}$ which are (possibly) redundant due to $m-n$ geometric constraints

$$
\phi(x)=0, \quad \operatorname{rank} \frac{\partial \phi}{\partial x}=m-n \geq 0,
$$

where $n \leq m$ is the degree of freedom of the system.

If there is an expression for the Lagrangian $\hat{L}(x, \dot{x})$, the system has to obey Hamilton's principle

$$
\delta \int_{t_{0}}^{t_{1}} \hat{L}(x(t), \dot{x}(t)) \mathrm{d} t=0
$$


with fixed endpoints $x\left(t_{0}\right)$ and $x\left(t_{1}\right)$. Bringing the variation inside the integral and integrating by parts yields

$\int_{t_{0}}^{t_{1}}\left(\frac{\partial \hat{L}}{\partial x} \delta x+\frac{\partial \hat{L}}{\partial \dot{x}} \delta \dot{x}\right) \mathrm{d} t=\int_{t_{0}}^{t_{1}}\left(\frac{\mathrm{d}}{\mathrm{d} t} \frac{\partial \hat{L}}{\partial \dot{x}}-\frac{\partial \hat{L}}{\partial x}\right) \delta x \mathrm{~d} t=0$.

If the coordinates $x$ are independent, i.e. $m=n$, so are the variations $\delta x$. As the integral has to vanish for arbitrary variations $\delta x$, the quantity in the parentheses must vanish, yielding (1).

In the case of redundant $m>n$ coordinates, this argument is not applicable as the variations $\delta x$ are constrained by

$$
\delta \phi=\frac{\partial \phi}{\partial x} \delta x=: \Phi \delta x=0,
$$

i.e. they are not independent.

\subsection{Ansatz: Construct a basis}

Augment $\Phi(x) \in \mathbb{R}^{(m-n) \times m}$ by $Y(x) \in \mathbb{R}^{n \times m}$ to an invertible matrix and get $A(x) \in \mathbb{R}^{m \times n}$

$$
\left[\begin{array}{l}
\Phi \\
Y
\end{array}\right] \delta x=\left[\begin{array}{l}
0 \\
h
\end{array}\right], \quad \operatorname{det}\left[\begin{array}{c}
\Phi \\
Y
\end{array}\right] \neq 0 \Rightarrow \delta x=A h .
$$

The $n$ quantities $h=Y \delta x$ cover the domain of allowed variations and are independent. So they can be regarded as a basis of the variations.

In the same way, define the basis of the velocity

$$
\frac{\mathrm{d}}{\mathrm{d} t} \phi=\Phi \dot{x}=0, Y \dot{x}:=\xi \quad \Rightarrow \quad \dot{x}=A \xi .
$$

Interpretation: If the initial conditions $x\left(t_{0}\right)=x_{0}$ obey the constraint $\phi\left(x_{0}\right)=0$, the kinematic relation (10) ensures that $x(t)$ evolves only within its constraints, as $\frac{\partial \phi}{\partial x} A=0$.

For the following, the so-called Boltzmann-symbols $\gamma_{i j s}$, defined as

$$
\delta \xi_{i}-\dot{h}_{i}=\gamma_{i j s} h_{j} \xi_{s}, \quad \gamma_{i j s}=\left(\frac{\partial Y_{i l}}{\partial x_{k}}-\frac{\partial Y_{i k}}{\partial x_{l}}\right) A_{l j} A_{k s},
$$

will be useful. Here, and in the following, the summation convention over double indices $i, j, s=1, \ldots, n$ and $k, l=$ $1, \ldots, m$ is understood.

\subsection{Equations of motion}

Based on the previous discussion, it is convenient to express the Lagrangian in terms of the redundant coordinates $x \in \mathbb{R}^{m}$ and the velocity coordinates $\xi \in \mathbb{R}^{n}$ as $\tilde{L}(x, \xi)$. Hamilton's principle now yields

$$
\begin{aligned}
& \delta \int_{t_{0}}^{t_{1}} \tilde{L}(x, \xi) \mathrm{d} t=\int_{t_{0}}^{t_{1}}\left(\frac{\partial \tilde{L}}{\partial \xi_{i}} \delta \xi_{i}+\frac{\partial \tilde{L}}{\partial x_{k}} \delta x_{k}\right) \mathrm{d} t \\
& =\int_{t_{0}}^{t_{1}}\left(\frac{\mathrm{d}}{\mathrm{d} t} \frac{\partial \tilde{L}}{\partial \xi_{i}}-\gamma_{j i s} \xi_{s} \frac{\partial \tilde{L}}{\partial \xi_{j}}-\frac{\partial \tilde{L}}{\partial x_{k}} A_{k i}\right) h_{i} \mathrm{~d} t=0 .
\end{aligned}
$$

The quantities $h$ are independent and arbitary which implies that the terms in the brackets have to vanish.

Summing up, the equations of motion consist of the kinematic part from (10) and the kinetic part from (11)

$$
\begin{aligned}
\dot{x}_{k}=A_{k i} \xi_{i}, & k=1, \ldots, m, \\
\frac{\mathrm{d}}{\mathrm{d} t} \frac{\partial L}{\partial \xi_{i}}-\gamma_{j i s} \xi_{s} \frac{\partial L}{\partial \xi_{j}}-\frac{\partial L}{\partial x_{k}} A_{k i}=0, & i=1, \ldots, n,
\end{aligned}
$$

and the constraints on the initial condition $\phi\left(x\left(t_{0}\right)\right)=0$. These equations have a similar structure as the formulation proposed by Hamel [1904] or Poincaré [1901] (see also
Bloch et al. [2009] for a derivation in a more geometric fashion) but do not require generalized coordinates. Note that, when generalized coordinates are chosen $(m=n)$, the constraint vanishes and $A=Y^{-1}$. Then (12) is identical to the ones proposed by Hamel [1904]. Furthermore, if $Y=I_{n}$ is chosen, then $\xi=\dot{x} \Rightarrow \gamma_{s i j}=0$ and (12) is identical to Lagrange's equations (1).

The formulation by Hamel [1904] also covers nonholonomic systems. By using the same arguments, it should be straightforward to extend (12) for this case, too.

\subsection{Application to the motivation example}

The redundant coordinates $x \in \mathbb{R}^{9}$ are the coefficients of the rotation matrix $R \in \mathrm{SO}(3) \subset \mathbb{R}^{3 \times 3}$. The geometric constraints $\phi(x)=0$ are the $m-n=6$ independent equations formed by $R^{\top} R=I_{3}$. The angular velocities $\xi:=\omega=\operatorname{skw}\left(R^{\top} \dot{R}\right)=: Y \dot{x}$ are a convenient choice for the basis as $\operatorname{det}\left[\left(\frac{\partial \phi}{\partial x}\right)^{\top}, Y^{\top}\right]^{\top}=\operatorname{det} R=1$. Inverting the augmented matrix $\left[\left(\frac{\partial \phi}{\partial x}\right)^{\top}, Y^{\top}\right]^{\top}$ gives $\dot{x}=A \xi$ which is identical to $\dot{R}=R$ Skw $\omega$ up to some reordering. The Boltzmann-symbols computed from $Y$ and $A$ turn out to be identical to the Levi-Civita symbol in this case, so $\gamma_{j i s} \xi_{s} \frac{\partial L}{\partial \xi_{j}}=\left(\xi \times \frac{\partial L}{\partial \xi}\right)_{i}$. Finally, evaluating $(12 \mathrm{~b})$ with the Lagrangian $L=\frac{1}{2} \omega^{\top} \Theta \omega$ and $\xi=\omega$ yields Euler's equations.

\subsection{Geometric interpretation and conclusions}

Assume a mechanical system whose configuration space $\mathrm{X}$ is a smooth manifold with dimension $n$. Choosing generalized coordinates is the choice of a map $X \rightarrow \mathbb{R}^{n}$ which is necessarily local if the manifold is not isomorphic to $\mathbb{R}^{n}$. Choosing appropriate redundant coordinates $x$ is an embedding $\mathrm{X} \rightarrow\left\{x \in \mathbb{R}^{m} \mid \phi(x)=0\right\}$ which is global for sufficiently large $m$. Rather than minimal coordinates, the derivation requires a minimal set of $n$ variations. As they are defined on the tangent space $\mathrm{T}_{x} \mathrm{X}$ (i.e. a vector space), this parameterization is trivial.

While this interpretation and the original idea for this method come from differential geometry, the derivation of (12) and its application requires only elementary calculus.

\section{DISCUSSION}

Is the proposed formulation (12) known in the literature? It turns out to be useful for modeling, simulation, and control, especially when interested in global solutions.

\section{REFERENCES}

V. I. Arnold. Mathematical Methods of Classical Mechanics, volume 60 of Graduate Texts in Mathematics. Springer, New York, 1989.

A. M. Bloch, J. E. Marsden, and D. V. Zenkov. Quasivelocities and Stabilization of Relative Equilibria of Underactuated Nonholonomic Systems. In Proc. 48th IEEE Conf. on Decision and Control, 2009.

G. Hamel. Die Lagrange-Euler'schen Gleichungen der Mechanik. Zeitschrift für Mathematik und Physik, 50, 1904.

H. Poincaré. Sur une forme nouvelle des équations de la mécanique. C.R. Acad. Sci., 132:369-371, 1901. 


\title{
Combining Discrete Optimization and Simulation to Understand Stochastic Hub Location Problems *
}

\author{
Peiman Dabidian* J. Fabian Meier* Ina Goedicke \\ Uwe Clausen* \\ * Institute of Transport Logistics, TU Dortmund University, \\ Leonhard-Euler-Strasse 2, 44227 Dortmund, Germany \\ (e-mail: \{dabidian,meier,clausen\}@itl.tu-dortmund.de, \\ goedicke@tu-dortmund.de)
}

\begin{abstract}
Hub location problems model the strategic design of transport networks for depots which mutually exchange shipments: A number of hubs is chosen from possible locations and every depot is connected to every other depot using one or two hubs in between. The aim is to minimize the sum of hub and transport costs. Most of the hub location literature consider models with simplified cost structure and complete knowledge of the shipments. In reality the shipping volumes can only be approximated by probability distributions. Hence the planning of vehicles and hub capacities should take this into account. Because of the algorithmic complexity of stochastic hub location models, we opt to combine the optimization of a simplified model with a detailed event-driven simulation. This allows us to adapt our simplified model in an iterative circuit and, therefore, to construct models which are realistic and quickly solvable.
\end{abstract}

Keywords: Heuristics; Event-driven Simulation; Hub Location; Transportation

\section{INTRODUCTION}

Hub location problems model economies of scale in the transportation business: Bundling shipments decreases unit transport costs. Instead of using direct transport from every depot to every other depot, we choose to built hubs and use them for transhipment. Usual hub location problems adopt the modelling scheme of O'Kelly (1987) and use unit transport costs that are reduced by a fixed factor on hub-hub-connections because these connections usually carry more weight. Vehicle sizes are ignored. Furthermore, exact knowledge about the shipping volumes is assumed.

Little research was done on stochastic hub location problems: The most important contributions Contreras et al. (2011) and Alumur et al. (2012) consider simplified models without vehicle or hub capacities. We know that the simultaneous consideration of vehicle capacities and stochastic effects can lead to very difficult hub location models. Hence we opt to extend a model from Meier (2014) with a simplified version of stochastic effects and measure the accuracy of this integration by an event-driven simulation.

\section{THE MATHEMATICAL MODEL}

We start by writing down a deterministic model which is a slightly simplified version of M2 from Meier (2014). Let $G=(N, P)$ be a graph, where $N$ is the disjoint union of the source-sink-set $S$ and the possible hub set $H$. Furthermore, we have a set of commodities $C$ which have a source $c^{\text {so }}$ and

\footnotetext{
* We thank the Stiftung Mercator for financing the project Lösung von Hub-Location-Problemen in der Stückgutlogistik mittels simulationskalibrierter mathematischer Optimierung
}

a sink $c^{\text {si }}$ in $S$ and a transport volume $c^{\mu}$. An admissible path for a commodity $c \in C$ is given by $c^{\text {so }} \rightarrow l \rightarrow m \rightarrow c^{\mathrm{si}}$ where $l \neq m \in H$ or $c^{\text {so }} \rightarrow l \rightarrow c^{\text {si }}$ with $l \in H$. The choice of path is represented by the binary variable $x_{c l m}$, $l \neq m \in H$ or $x_{c l}, l \in H$.

To every element $l$ of $H$ we can assign a hub level $q \in$ $Q_{l}=\left\{1, \ldots, q_{l}\right\}$ of size $\xi_{l q}$ and of cost $k_{l q}$, restricting the incoming flow from sources. The choice of hub level is encoded in the binary variable $z_{l q}$. We consider costs $t_{i j}$ per truck, and define the truck size to be $r_{i j}(i, j \in N)$. Furthermore, we introduce the integer variable $v_{i j}$ for the number of vehicles on each connection $i \rightarrow j, i, j \in N$, where $v_{i j}=0$ for $i, j \in S$ as direct transport is not allowed.

$$
\begin{gathered}
\operatorname{Min} \sum_{l \in H} \sum_{q \in Q_{l}} k_{l q} z_{l q}+\sum_{i, j \in N}^{i \neq j} t_{i j} v_{i j} \\
\sum_{l \in H} x_{c l}+\sum_{l, m \in H}^{l \neq m} x_{c l m}=1 \quad c \in C \\
x_{c l}+\sum_{m \in H}^{m \neq l} x_{c l m}+x_{c m l} \leq \sum_{q \in Q_{l}} z_{l q} \quad l \in H, c \in C \\
\sum_{c \in C} c^{\mu}\left(x_{c l}+\sum_{m \in H} x_{c l m}\right) \leq \sum_{q \in Q_{l}} \xi_{l q} z_{l q} \quad l \in H \\
\sum_{q \in Q_{l}} z_{l q} \leq 1 \quad l \in H
\end{gathered}
$$




$$
\begin{gathered}
\sum_{c \in C}^{c^{\mathrm{so}}=i} c^{\mu}\left(x_{c l}+\sum_{m \in H} x_{c l m}\right) \leq r_{i l} \cdot v_{i l} \quad i \in S, l \in H \\
\sum_{c \in C} c^{\mu} x_{c l m} \leq r_{l m} \cdot v_{l m} \quad l \neq m \in H \\
\sum_{c \in C}^{c^{\mathrm{si}}=j} c^{\mu}\left(x_{c m}+\sum_{l \in H} x_{c l m}\right) \leq r_{m j} \cdot v_{m j} \quad m \in H, j \in S
\end{gathered}
$$

Equation (1) is the sum of hub cost and costs per vehicle. Equation (2) ensures that every shipment is routed, (3) states that intermediate nodes have to be hubs, (4) derives the hub capacity level and (5) restricts the number of hub levels per hub to at most one. In $(6),(7),(8)$ we compute the total transport volume on the arc $i \rightarrow j$ and calculate the number of vehicles that is necessary for that volume.

\section{STOCHASTICS}

Now we assume that the shipping volumes $c^{\mu}$ for $c \in C$ are normally distributed with variance $c^{\sigma^{2}}$. If a transport arc or a hub is traversed by a subset $\hat{C} \subset C$, then we know that the total volume is again normally distributed with average $\sum_{c \in \hat{C}} c^{\mu}$ and variance $\sum_{c \in \hat{C}} c^{\sigma^{2}}$.

We assume that the vehicles can be planned according to realised transport volume (on an operational level) while the hub capacities cannot be adjusted. Hence the transport cost function can be approximated by averaging over different scenarios while the hub capacity has to be chosen on a security level (depending on the standard deviation of the transhipment volume distribution).

Both procedures cause problems: A large number of scenarios gives a better approximation but makes the model hard to solve, while a small number might lead to an inadequate result. Furthermore, the security level for the hub capacity cannot be chosen large enough to care for any event; the hub capacity costs have to be balanced with the prospective costs of capacity exceedance. These capacity exceedance costs are hard to include properly into an optimization model because overrunning hubs postpone transport volume to the future.

Thus we built different models for the same situation with different choices for the stochastic aspects, i.e. a different number of scenarios for the transport cost function and different security levels for the hub capacities. These models are then fed into the Simulated Annealing algorithm described in Meier (2014). As such algorithms rely on checking huge numbers of neighbouring solutions for improvement, we know that a complicated objective function (with a lot of stochastics) is a significant burden. The solutions created by the Simulated Annealing approach can then be evaluated by an event-driven simulation.

\section{SIMULATION}

Solving the mathematical model (with included simplified stochastic) gives us a hub size for each chosen hub and a route for each shipment. To evaluate the objective function in a more detailed way, we use event-driven simulation. A simulation model is developed based on the simulation software Enterprise Dynamics by Incontrol Simula- tion solutions. Within the simulation suite modelling of the moved objects and resources takes place on a macroscopical level. Furthermore, all handling and controlling processes of each hub in the main network can be modelled and parametrized (Clausen et al. (2012)).

We classify three types of modelling data as explained in Clausen et al. (2013): Firstly, the technical data for describing the topology and structure of the system (e.g., number of hubs in the network, the connections between hubs, capacities and the shipping times), then the organizational data to describe the process structure and other aspects (e.g., routing rules and restrictions of handling operations) and the system load data which describes the weight of the shipments and the hubs to be used between source and destination for each shipment. A large number of periods (e.g., 52 weeks) is considered. In each period, new shipments are created using the normal distributions; then they are routed through the network. This leads to different transport and transhipment volumes in each period. The handling of the varying transport volumes is quite easy, as we pick the appropriate number of vehicles in each period separately. The transhipment volumes are more complex: If the chosen hub capacity is exceeded, the remaining shipments are passed over to the next period.

Due to a large number of simulation periods we can get a good approximation of the average total costs, which form a far more accurate version of the objective function of the hub location problem. In this way we can decide which of our models gives the best account of stochastic behaviour and choose a proper stochastic modelling scheme.

\section{CONCLUSION}

We have shown that it is possible to solve a hub location problem with stochastics in reasonable time by proper metaheuristics and see that the stochastic integration is close to the results of the simulation if we choose the scenarios and security level properly.

\section{REFERENCES}

Alumur, S.A., Nickel, S., and Saldanha-da Gama, F. (2012). Hub location under uncertainty. Transportation Research Part B: Methodological, 46(4), 529-543.

Clausen, U., Dabidian, P., Diekmann, D., Goedicke, I., and Pöting, M. (2013). Analysis of assignment rules in a manually operated distribution warehouse. In Proceedings of the 2013 Winter Simulation Conference: Simulation: Making Decisions in a Complex World, WSC '13, 3430-3439. IEEE Press, Piscataway, NJ, USA.

Clausen, U., Goedicke, I., Mest, L., and Wohlgemuth, S. (2012). Combining simulation and optimization to improve LTL traffic. Procedia - Social and Behavioral Sciences, 48(0), 1993 - 2002. Transport Research Arena 2012.

Contreras, I., Cordeau, J.F., and Laporte, G. (2011). Stochastic uncapacitated hub location. European Journal of Operational Research, 212(3), 518-528.

Meier, J.F. (2014). A versatile heuristic approach for generalized hub location problems. Preprint.

O'Kelly, M.E. (1987). A quadratic integer program for the location of interacting hub facilities. European Journal of Operational Research, 32(3), 393-404. 


\title{
System Entity Structure Ontology Toolbox for MATLAB/Simulink: Used for Variant Modelling
}

\author{
T. Pawletta, D. Pascheka \\ A. Schmidt \\ Hochschule Wismar - University of Applied Sciences, RG CEA, Wismar, PF 1210, D-23952 \\ Germany (Tel: ++4938417537406; e-mail: thorsten.pawletta@hs-wismar.de, \\ danielpascheka@yahoo.com, artur.schmidt@hs-wismar.de).
}

\begin{abstract}
In computer science ontology is often understood as a formal specifi cation of a shared conceptualization in form of a model. The considered doma in of conceptualiza tion is mod eling and simulation of modular, hierarchical systems. In this context, the System Entity Structure (SES) Ontology has been introduced for specifying a set of various system structure $\mathrm{s}$ or parameter settings. In combination with configurable dynamic models, organized in a model base (MB), the SES ontology has been advanced to a modeling and simulation framework. Based on the original SES elements and axioms as well as the introduction of some new features, the impleme ntation of an SES toolbox for MATLAB/Simulink is discussed. Subsequently, the usage of the toolbox is illustrated by a well known example of variant modeling.
\end{abstract}

Keywords: Modeling, Simulation, Ontology, Variant Modeling, System Entity Structure .

\section{INTRODUCTION}

Modelling and experimentation with different system designs or variants is a requir ement that becomes more and more important in engineering . Some engineering software environments, such as MATLAB/Simulink according to The MathWorks Inc. (2014), recently support variant modelling . Here, different system variant $\mathrm{s}$ are directly mapped on the level of dynamic system models . The activation of a certain variant is controlled by specific variables . However, the approach becomes quickly unc lear when faced with complexity.

In computer sci ence ontologies are often employed for conceptual or meta-modelling, as stated by Henderson-Sellers (2012). For the domain of modelling and simulation of modular, hierarchical systems , Zeigler et al. (2007) defined the System Entity Structure Ontology (SES ) for specifying a set of various system structures and parameter settings. In combination with configurable basic models, which describe a dynamic behaviour and are organized in a model base (MB), the SES ontology has been advanced to a model ling and simu lation framework (SES/MB). Following, essential parts of the SES ontology and some new introduced features are briefly summarized. Particular focus is thereby placed on the implementation of an SES toolbox for

MATLAB/Simulink and its usage in context with variant modelling.

\section{SES/MB FRAMEWORK AND SES TOOLBOX}

The SES/MB framework introduced by Zeigler is pictured in Fig.1. Configurable basic models, describing a dynamic behavior, are organized in a MB. The set of various system structures and parameter sett ings are specified in an SES. Thereby, an SES represents a kind of "variable system construction plan" coded as a tree structure. The selection of a system structure including parameter settings, which represents a certain system variant, is derived by a $p$ runing operation. The result is called Pruned Entity Structure (PES). In case of combination with a MB, a SES can specify references to basic models, describing a dynamic behavior Then, an executable simulation model can be generated based on the PES, if an appropriate translator is available.

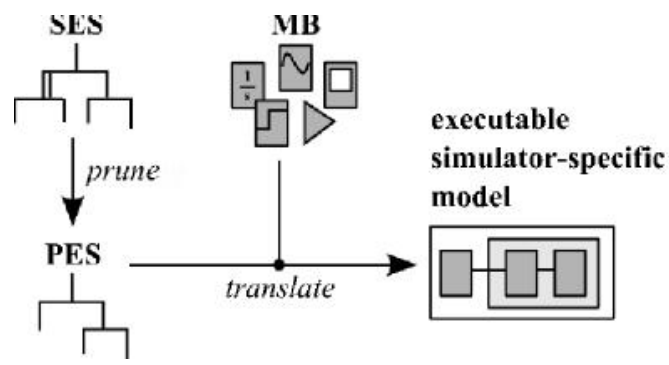

Fig. 1. SES/MB framework according to Zeigler et al. (2000).

The SES ontology published in Zeigler et al. (2007) is a basis ontology that provides a set of descriptive elements and defining a set of axioms. The developed SES toolbox implements nearly the complete ontology within MATLAB/Simulink. Addition ally, it introduces some advanced features, such as SES functions that are fully compatible with the MATLAB $p$ hilosophy. The toolbox is implemented in MATLAB with an own GUI. It provides a graphical SES editor with an integrated parser for error checking, different pruning methods, a merging method for composing SESs and a translation script for generating executable Simulink models. 


\section{EXAMPLE FOR VARIANT MODELING}

Fig. 2 shows an example of variant modelling in Simulink published in The MathWorks Inc. (2014). It comprises two alternative controller designs mapped as variant subsystems: (i) LinearController and (ii) NonLinearController.

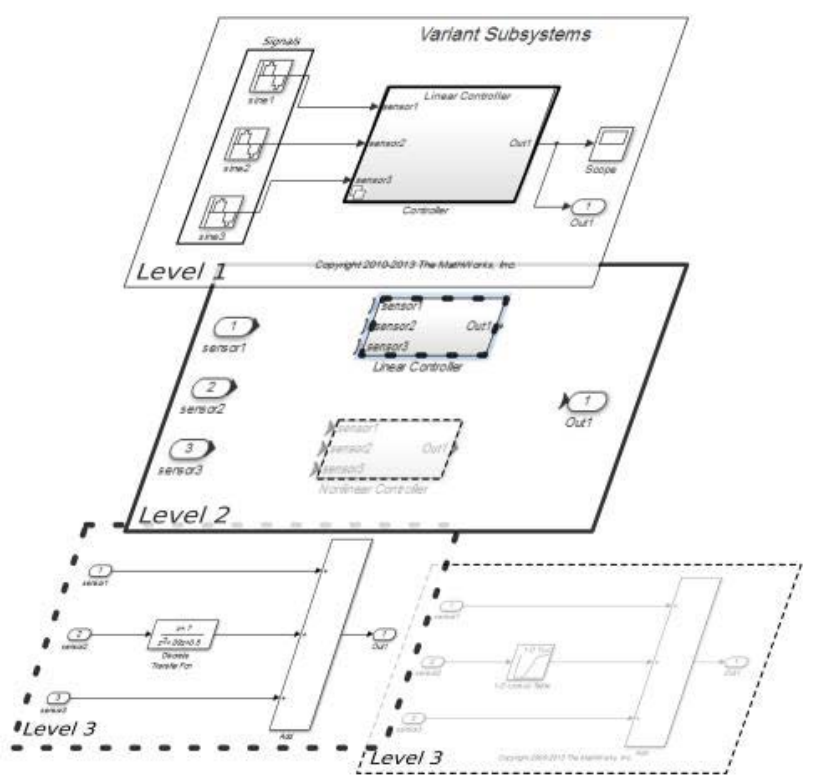

VariantSubsystems $=\{$ Signals, Controller, Scope $\}$;

Signals $\quad=\{$ Sine1, Sine2, Sine 3$\}$;

Controller $\quad=\{$ LinearController $\mid$ NonLinearController $\}$;

LinearController $=\{$ DiscreteTransferFcn, Add $\}$;

NonLinearController $=\{$ LookUp-Table, Add $\}$

Fig. 2. Simulink example with Variant Subsystems.

The same problem specified using the SES toolbox is partly illustrated in Fig. 3. Nodes with the Symbol "e" represent entity nodes and the others SES specific nodes. Leaf nodes are always refe rring to basic systems in a MB, in this c ase blocks in Simulink libraries (see attributes of node Sine). The root and inner entity nodes represent composed systems or specialized systems for mapping variants. Their characteristics are described by its subsequent SES specific node. For example: (i) ModelDec specifies in its attribute coupling the composition of predecessor VariantSubsystems and (ii) ControllerSpec describes by its attribute specrule the possible variants of predecessor Controller. The identifier VSS_MODE in the attribute of specrule is an SES variable. By setting the SES variables the user can affect all kinds of decisions during a pruning operation and thereby the subsequent translation process (Fig. 1). In the same manner like ModelDec the node SignalMAsp specifies an attribute how the type-identical entities Sine have to be composed. Additionally it has to define the number of replications (numRep) of the entity, in this case with numRep $=3$.

In contrast to the built -in variant modeling in Simulink the SES toolbox allows the specific ation of a variant number of signal generators. This extension causes different couplings and different numbers of input/output ports of systems, depending on the number of signal gen erators. Using the advanced concept of SES functions such variability can be easily specified by means of the same function pattern. In this case, SignalMAsp defines the attribute numRep using an SES variable, such as NumSignals, and affected nodes, like ModelDec define their attribute coupling using an SES function call, like [coupling]=couplingFcn1(Children, NumSignals). SES function $\mathrm{s}$ are defined like regular MATLAB functions and they can use internal variables, such as Children or Parent, for specifying couplings in the manner \{Children $\{1\}, ' 1$ ', Children $\{2\}, ' 2\}$ ' .

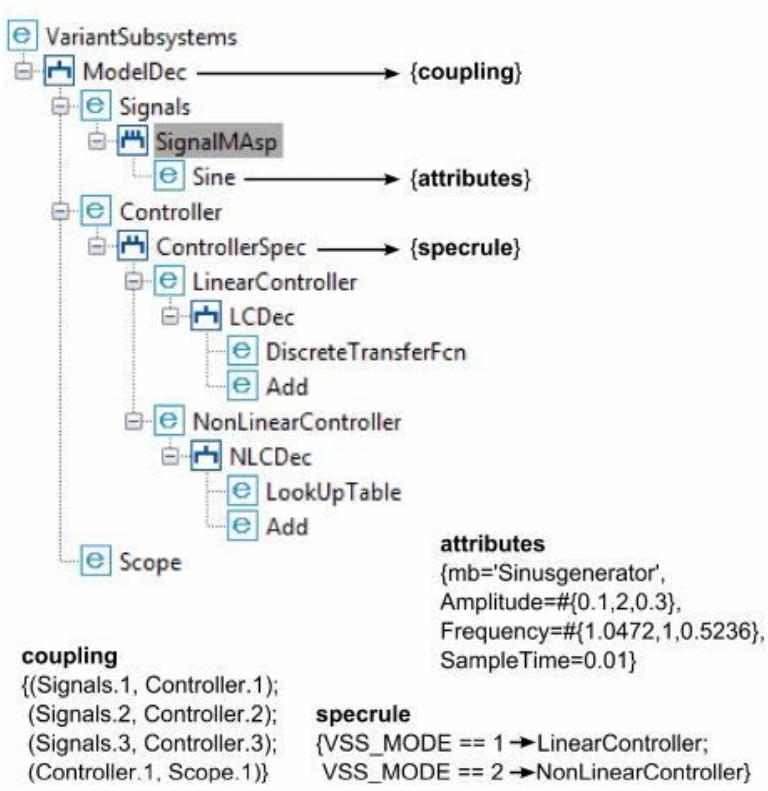

Fig. 3. SES tree mapping the problem pictured in Fig. 2.

\section{CONCLUSIONS}

Beside the original SES ontology, th e toolbox provides advanced features compatible with the MATLAB/Simulink philosophy. They are particularly useful in combination with an automated generation of executable MATLAB/Simulink models. A more detailed description of the toolbox can be found in Pawletta et al. (2013).

\section{REFERENCES}

Henderson-Sellers, B. (2012). On the Mathematics of Modelling, Metamodelling, Ontologies and Modelling Languages, Springer, Heidelberg.

Pawletta, T., Pascheka, D., Sc hmidt, A. and Pawletta, S. (2013). Ontology-Assisted System Modeling and Simulation within MATLAB/Simulink. SNE Simulation Notes Europe, 23(2), 10 pages (accepted publication).

The MathWorks Inc. (2014). Variant $\mathrm{Su}$ bsystems, http://www.mathworks.de/de/help/simulink/examples/va riant-subsystems.html, (date of reading 2014/08/21).

Zeigler, B.P., Praehofer, H. and Kim, T.G. (2000). Theory of Modeling and Simulation, chapter 18. Academic Press, San Diego.

Zeigler, B.P. an d Hammonds, P.E. (2007). Modeling and Simulation-Based Data Engineering, Elsevier Academic Press, San Diego. 


\title{
Robotic Control \& Visualization Toolbox for MATLAB
}

\author{
T. Pawletta, B. Freymann \\ C. Deatcu, A. Schmidt

\begin{abstract}
Hochschule Wismar - University of Applied Sciences, Research Group CEA, PF1210, D-23952 Wismar, Germany (Tel: +49 3841753 7406; e-mail: thorsten.pawletta@hs-wismar.de, birger.freymann@hs-wismar.de,christina.deatcu@hs-wismar.de,artur.schmidt@hs-wismar.de).
\end{abstract}

\begin{abstract}
Industrial robots are used in various application fields and many robot manufacturers are active on the market. In most cases, their software solutions are proprietary and thereby they cannot be used for third party robots. Moreover, the integration of external hard- or software is highly restricted. Long term standardization efforts for robot programming languages, such as the Industrial Robot Language (IRL) and its successor, the Programming Language for Robots (PLR), have been mostly ignored by robot manufacturers. This fact leads to a restriction on the combined usage of robots. Multirobot applications where robots have to interact are usually limited to software solutions and robots of one manufacturer. On the other hand, control design in engineering is often carried out by the usage of Scientific and Technical Computing Environments (SCEs) like MATLAB. The Robotic Control \& Visualization (RCV) toolbox for MATLAB tries to close the gap between robot manufacturer-specific software solutions and SCEs. The current version of the RCV toolbox supports a uniform and integrated control development for KUKA and KAWASAKI robots in the MATLAB/Simulink environment. An extension to other robot types is straight forward. Thus, the implementation of heterogeneous multi-robot applications is considerably simplified.
\end{abstract}

Keywords: robot control, rapid programming, simulation, visualization, state machine, hardware integration, MATLAB, Stateflow

\section{INTRODUCTION}

Research in robotics is proceeding rapidly and new fields of application for robots are made up continually. However, the requirements concerning robot control development are increasing, too. Fast and easy control programming, integration of external hardware or software components and multi-robot operation are of particular importance. In this context it is often desirable to use a Scientific and Technical Computing Environment (SCE), such as MATLAB, for Rapid Control Prototyping (RCP). RCP is understood as an integrated, continuous control development from early design to operating phase in a homogenous environment.

Today, various robot manufacturers are established on the market. They offer proprietary software environments with special robot programming languages as e.g. KRL (KUKA Robotics), AS (Kawasaki Robotics), or RAPID (ABB Robotics). From software engineering point of view, all those robot languages are pretty similar. Nevertheless, long-term standardization efforts like the IRL (Industrial Robot Language) and its successor, the PLR (Programming Language for Robots) still are unsuccessful. In addition almost all robot manufacturers almost all offer a Computer Aided Robotic (CAR) system, which is also referred to as 3D robot simulation software. CAR systems provide physicbased robot models of its manufacturer as well as interfaces to $3 \mathrm{D}$ CAD systems. Thus, a simulation and $3 \mathrm{D}$ visualization of complete robot cells is supported. Robot controls can be developed within these virtual environments using the proprietary robot languages. Such CAR systems simplify the development and start-up of robot applications. However, the proprietary software limits applications to products of its manufacturer. There are some third-party CAR systems available, such as 3DRealize-R by Visual Components Corporation (2014). They offer a comprehensive solution for simulation and 3D visualization of heterogeneous robot types. However, the control programming is still based on the different proprietary robot languages. Hence, the development of interacting multi-robot controls is complicated for robots of different manufacturers.

Inspired by the idea of Rapid Control Prototyping (RCP) in control theory the Research Group CEA started in 2004 with the development of a MATLAB KRL toolbox. The toolbox supports control programming of KUKA robots within MATLAB including the usage of all available MATLAB features. Moreover, it provides a first MATLAB based CAR system (Maletzki et al. (2006)). Users benefit from the powerful methods as well as the various interfaces provided by MATLAB and its toolboxes. Almost all of the proprietary robot languages are imperative languages containing similar programming elements. Thus, the approach of the MATLAB KRL toolbox was generalized. A uniform robot control language for robot types of different manufacturers has been developed with the Robotic Control and Visualization (RCV) toolbox for MATLAB (Research Group CEA (2011)). 
Moreover, the simulation and 3D visualization tools have been enhanced.

\section{COMPONENTS AND USAGE OF RCV TOOLBOX FOR MATLAB}

Fig. 1 illustrates basic concepts and major components of the toolbox. In this example a control PC with one instance of MATLAB controls a cyber-physical system with different types of real and virtual robots.

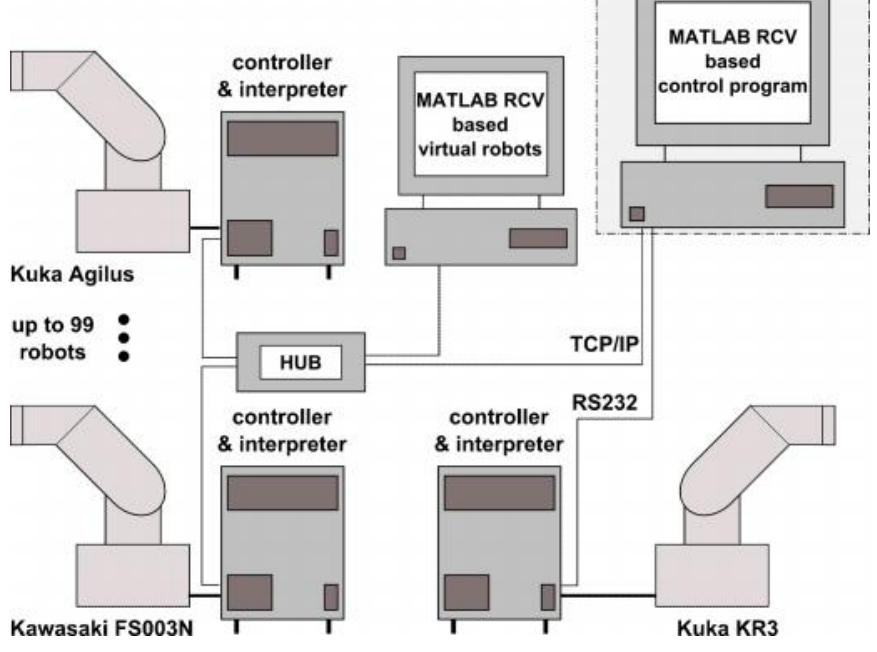

Fig. 1. Installation of a multi-robot control with heterogeneous robot types using the RCV toolbox

Actually, the RCV toolbox unites two toolboxes which act in concert. The Robotic Control toolbox provides an abstract set of robot-oriented programming features within MATLAB. The language elements are closely based on the widely used robot languages: (i) KRL from KUKA and (ii) AS from Kawasaki. A small hardware dependent command interpreter is necessary to control each robot type. The interpreter has to be installed on the robot controller. Analogous to the former MATLAB KRL toolbox, the proper control program is completely developed within MATLAB and all available MATLAB features can be used. The path planning algorithms, security audits, etc. remain tasks of the specific robot controllers. The data exchange between the MATLAB based control program on a PC and the command interpreters on the robot controllers takes place via data links (serial or TCP/IP). Currently, the RCV toolbox contains preimplemented interpreters for KUKA and KAWASAKI controllers. However, the implementation of interpreters for other robot controllers is straight forward.

The second part, the Visualization toolbox supports physicsbased simulation and $3 \mathrm{D}$ visualization of robots, called virtual robots, and other objects. The core elements are kinematic models and simulated command interpreters. Each virtual robot needs such an interpreter as interface to the MATLAB based control program. The concept is analogous to command interpreters on real robot controllers. Hence, from control program's perspective there is no difference between a virtual and real robot.
The Visualization toolbox is designed object-oriented and can, as the Robotic Control toolbox, easily be extended to support new types of robots. At present, kinematic models for KUKA KR 3, Kawasaki FSN003 and soon for KUKA Agilus as well as simulated command interpreters for virtual robots of type KUKA and Kawasaki are implemented. In addition, the toolbox provides an STL file format interface. CAD files can be imported, which simplifies the integration of new types of robots and the mapping of any other objects.

An example for the combination with other MATLAB tools is pictured in Fig. 2. Control commands of RCV toolbox are embedded in Stateflow to implement a state based control. A much more complex integration with MATLAB tools is described in Schwatinski et al. (2012).

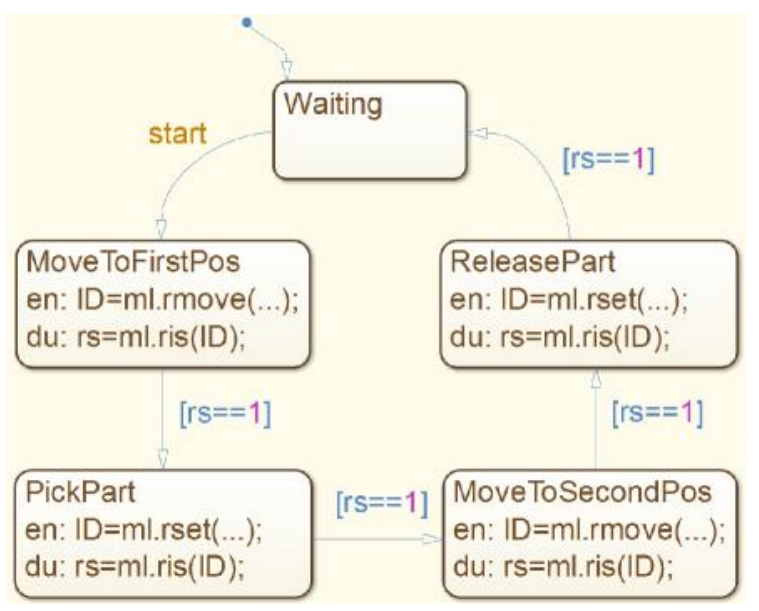

Fig. 2. State based control using Stateflow \& RCV toolbox

\section{CONCLUSION}

The RCV toolbox provides a uniform approach for nonproprietary robot programming, including usage of 3D CAR system functionality. Due to its seamless integration with MATLAB/Simulink, it supports robot control development according to the powerful concept of RCP and using all available MATLAB tools. The number of users of RCV toolbox from universities and research institutes indicates its broad acceptance in the field of training and research.

\section{REFERENCES}

Maletzki, G., Pawletta, T., Pawletta, S. and Lampe, B.P. (2006). A model-based robot programming approach in the MATLAB/Simulink environment. In Advances in Manufacturing Technology $-X X$, $4^{\text {th }}$ Int. Conf. on Manufacturing Research (ICMR06), Liverpool, UK, Sept. 05-07, 2006, 377-382.

Research Group CEA. (2011). Robotic Control \& Visualization (RCV) Toolbox for MATLAB, http://www.mb.hs-wismar.de/cea/sw_projects.html, (date of reading 2014/10/24)

Schwatinski, T., Pawletta, T. and Pawletta, S. (2012). Flexible Task Oriented Robot Controls Using the System Entity Structure and Model Base Approach. SNE Simulation Notes Europe, 22(2), 107-114.

Visual Components Corporation (2014). 3DRealize-R, http://www.visualcomponents.com/products/3drealizer-r, (date of reading 2014/10/24). 


\title{
Two Dimensional Mathematical Model of Heat-transmission Using MAPLE
}

\author{
Ildikó Perjési-Hámori* \\ *University of Pécs, Pécs, 7624 \\ Hungary (Tel: 3672 503650; e-mail: perjesi@pmmik.pte.hu).
}

\begin{abstract}
In our poster we show the two dimensional steady-state temperature distribution on rectangular shape surfaces under different boundary conditions. We compare the results of the analytical (Fourier series solution) and that of the numerical (finite-difference method) methods using Maple. We demonstrate the possibility to automatize the numerical algorithm with different boundary conditions.
\end{abstract}

Keywords: computer algebra, numerical methods.

\section{INTRODUCTION}

Heat-transmission by conduction is the flow of thermal energy within solids and non-flowing fluids (Incropera, Bejan). Our goal was to continue our previous works (Vajda, Perjési-Hámori) in the field of determination of temperature distribution inside a two-dimensional plane region, when the temperature distribution is known on the boundary of the region (Dirichlet boundary conditions.). The mathematical model of the problem is to solve elliptical type partial differential equations:

$$
\frac{\partial^{2} T}{\partial x^{2}}+\frac{\partial^{2} T}{\partial y^{2}}=0
$$

The result of the solution of such elliptical type secondordered partial-differential equations is the temperature distribution. The solution depends on the physical conditions at the boundaries of the medium.

The following conditions are taking into consideration:

- homogeneous material;

- $T(x, y, z)$ is expressed in Cartesian coordinates;

- $\mathrm{d} x \mathrm{~d} y \mathrm{~d} z$ infinitesimally small (differential) control volume;

- no internal energy source;

- steady-state condition

- the surface is rectangular- and corner-shaped.

In the present contribution we compare the results of the analytical (Fourier series solution) and that of the numerical (finite-difference method) methods using Maple, and show the Maple procedure to automatize the calculation in a general case, where the PDE is

$$
\frac{\partial^{2} u}{\partial x^{2}}+\frac{\partial^{2} u}{\partial y^{2}}=f(x, y)
$$

where $u(x, y)$ is the linear transform of $T(x, y)$.

\section{ANALITICAL SOLUTIONS}

We determined the analytical solution in different boundary conditions for rectangular shape surfaces. It can be used in case of special surfaces and boundary conditions. The solution is given with the help of Fourier series.

\section{1. Rectangular shape surface, two different temperatures}

Case study 1: As shown on Fig. 1., the simplest case, the temperatures of three sides of the rectangular are similar $\left(T_{1}\right)$ and the temperature of the fourth side is $T_{2}$, and $f(x, y)=0$.

$$
u(x, y)=\frac{T(x, y)-T_{1}}{T_{2}-T_{1}}
$$

The boundary conditions are:

$$
\begin{aligned}
& R=\{(x, y) \mid 0<x<a, 0<y<b\} \\
& \begin{aligned}
u(x, 0) & =f_{1}(x)=0, \\
u(x, b) & =f_{2}(x)=1 \\
T_{1} T_{T(x, y) T_{T_{1}}} & \\
& u(0, y)=g_{1}(y)=0, \\
u(a, y) & =g_{2}(y)=0
\end{aligned}
\end{aligned}
$$

Fig. 1. Case study 1.

The coefficients of the Fourier series are:

$$
\begin{aligned}
& A_{n}:=\frac{2\left(\int_{0}^{a} f 1(x) \sin \left(\frac{n \pi x}{a}\right) \mathrm{d} x\right)}{a \sinh \left(\frac{n \pi b}{a}\right)} ; B_{n}:=\frac{2\left(\int_{0}^{a} f 2(x) \sin \left(\frac{n \pi x}{a}\right) \mathrm{d} x\right)}{a \sinh \left(\frac{n \pi b}{a}\right)} ; \\
& C_{n}:=\frac{2\left(\int_{0}^{b} g 1(y) \sin \left(\frac{n \pi y}{b}\right) \mathrm{d} y\right)}{b \sinh \left(\frac{n \pi a}{b}\right)} ; E_{n}:=\frac{2\left(\int_{0}^{b} g 2(y) \sin \left(\frac{n \pi y}{b}\right) \mathrm{d} y\right)}{b \sinh \left(\frac{n \pi a}{b}\right)}
\end{aligned}
$$

The solution, the 20. partial sum, the isotherms and gradients can be seen on Figure 2 . 
$u(x, y)=\frac{2}{\pi} \sum_{n=1}^{\infty} \frac{(-1)^{n+1}+1}{n} \cdot \frac{1}{\sinh \frac{n \pi b}{a}} \sin \frac{n \pi x}{a} \sinh \frac{n \pi y}{a}$
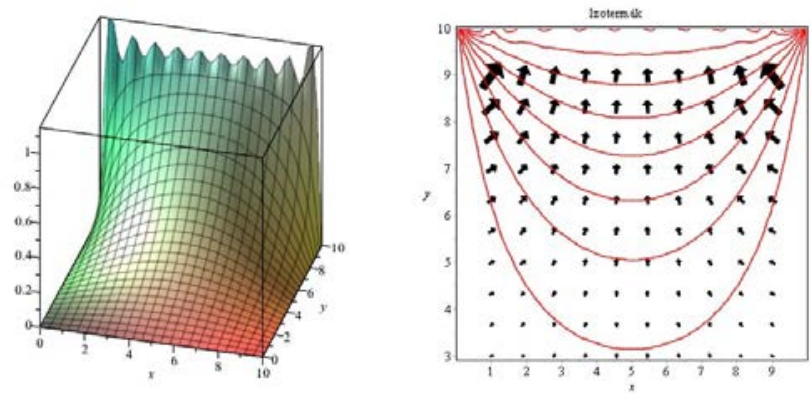

Fig. 2. Isotherms and gradients for Case study 1.

Case study 2: The boundary conditions are:

$u(x, 0)=f_{1}(x)=0, \quad u(x, b)=f_{2}(x)=\frac{x^{2}}{a^{2}}$

$u(0, y)=g_{1}(y)=0, \quad u(a, y)=g_{2}(y)=\frac{y}{b}$

The first three parts of the solution are:

$\frac{2\left(-4+\pi^{2}\right) \sinh \left(\frac{1}{10} \pi y\right) \sin \left(\frac{1}{10} \pi x\right)}{\pi^{3} \sinh (\pi)}+\frac{2 \sinh \left(\frac{1}{10} \pi x\right) \sin \left(\frac{1}{10} \pi y\right)}{\pi \sinh (\pi)}-\frac{\sinh \left(\frac{1}{5} \pi y\right) \sin \left(\frac{1}{5} \pi x\right)}{\pi \sinh (2 \pi)}-$.

$$
+\frac{2}{27} \frac{\left(-4+9 \pi^{2}\right) \sinh \left(\frac{3}{10} \pi y\right) \sin \left(\frac{3}{10} \pi x\right)}{\pi^{3} \sinh (3 \pi)}+\frac{2}{3} \frac{\sinh \left(\frac{3}{10} \pi x\right) \sin \left(\frac{3}{10} \pi y\right)}{\pi \sinh (3 \pi)}
$$

The 3-d contour plot and the contours are shown on Figure 3.
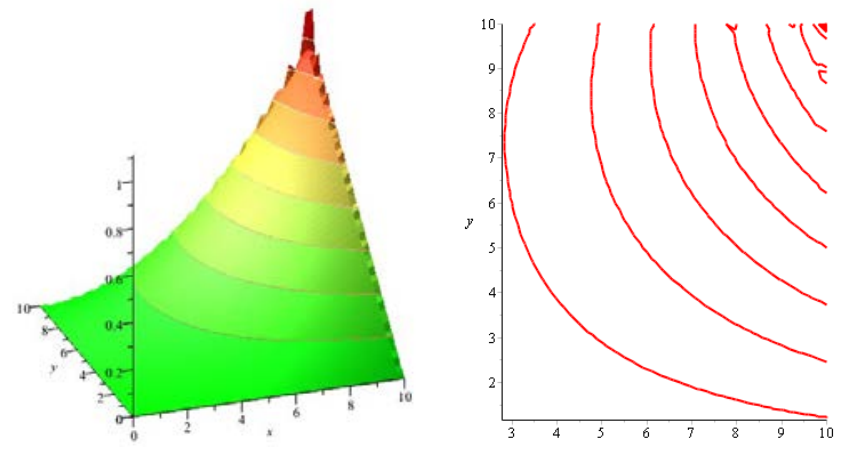

Fig. 3. Three and two dimensional contour plots for Case study 2 .

\section{NUMERICAL SOLUTIONS}

We generalized the problem to solve equation (2), where $u(x, y)=g(x, y)$ on the boundary. Figure 3 shows the procedure which transforms the coordinates of the inner mesh points into one label and creates a linear system, which solution gives the inner mesh points coordinates.

The analytical and numerical results for the Case study 1 are shown on Figure 5.

The difference between the two solutions was smaller than $2.5 \%$ on the average.

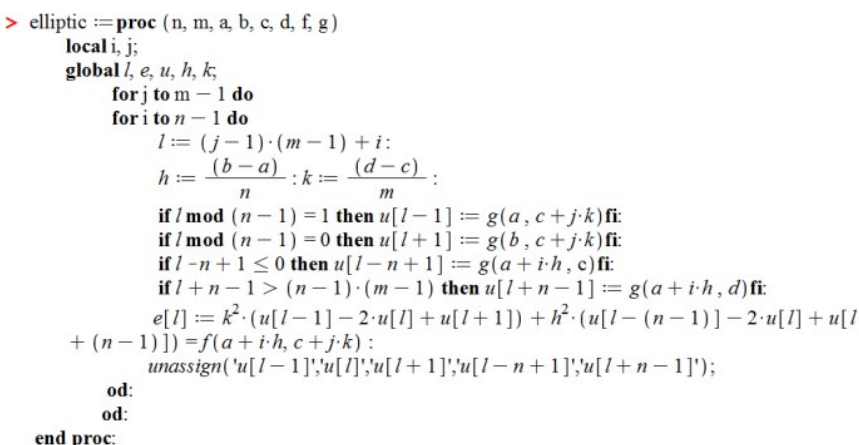

Fig. 4. The procedure which creates the linear system for temperature of the inner mesh points.

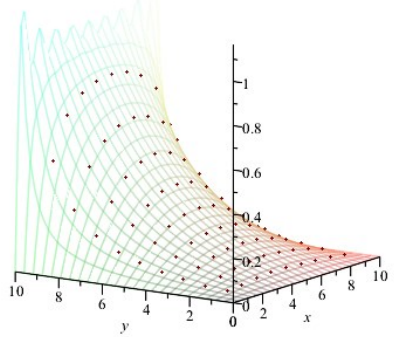

Fig. 5. The analytical and numerical results for the Case study 1 .

The poster will present other two dimensional temperature distribution, including not only rectangular but corner shape surface as well.

\section{CONCLUSION}

Heat conduction problems can generally be expressed by hardly solvable partial differential equations or it is not solvable analitically. The analytical solution of these equations assumes deep knowledge of mathematical analysis; the numerical solution issue in great linear equation systems. With help of computer algebra systems the solutions of these problems are simplified. The modified problems are quickly solvable with the help of visualization of changes. The solutions are easily understandable even for those who have not got enough routine in solution of differential equations.

\section{REFERENCES}

Incropera F.P., DeWitt, D.P. (1996) Introduction to heat transfer, John Wiley and Sons, New York.

Bejan, A. (1996) Heat transfer, John Wiley and Sons, New York.

Vajda J., Perjési-Hámori I. (2007) Two dimensional mathematical model of heat-transmission of one- and double-layer building Pollack Periodica Vol. 2, No.3, pp. 25-34.

Perjési-Hámori I. (2012) Simulation of Heat Radiation Asymmetry with Maple 7th Vienna Conference on Mathematics Modelling Feb. 15-17. 


\title{
Distributed parameter model of the buck converter with constant inductive load
}

\author{
Chenzi Huang* Frank Woittennek* Klaus Röbenack* \\ * Institute of Control Theory, Faculty of Electrical and Computer \\ Engineering, Technische Universität Dresden, 01062 Dresden, Germany
}

\begin{abstract}
In this contribution we present a new variant for the well-known buck converter. We substitute the lumped parameter components of the circuit by the distributed parameter model of a transmission line. For discussion, methods for modeling and simulation will be explained followed by some simulation results.
\end{abstract}

Keywords: Buck converter, distributed parameter model, transmission line

\section{INTRODUCTION}

The step down DC to DC converter, also known as buck converter, is a common electrical device used for transforming constant input voltage into lower output voltage, see Erickson and Maksimovic (2001). The basic circuit diagram is shown in Fig. 1 with an ideal switch which may be implemented with a transistor and a diode in practice. The ratio of switched-on time to the switching period which is called the duty cycle defines the ratio of average output voltage to input voltage. The set-up of inductance and capacitance assures the low-pass character of this circuit. The dimensioning of both elements is an important design parameter which e.g. determines the eigenfrequency of the device.

In a previous paper we introduced a new model of the buck converter with constant resistive load using the distributed parameter model of a transmission line, cf. Huang et al. (2013). More precisely we took the wire between switch and load as the converter itself and compared it with the lumped parameter model in terms of modeling and system behavior. With this new topology we wanted to omit the reactive elements of the classical buck converter in order to achieve a further reduction of the costs of the converter.

In this contribution the same distributed model of converter is investigated but with respect to a constant inductive load. In terms of practical applications, this load could represent an electrical drive. With it, the state space of this model possesses one more dimension than the system with pure resistive load. Furthermore the distributed parameter model is connected with an ordinary differential equation.

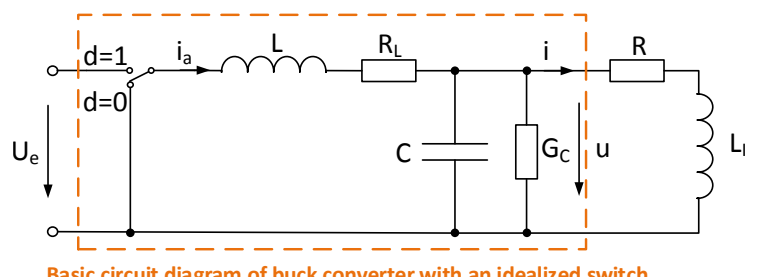

Fig. 1. Circuit diagram of the lumped parameter model
In this abstract, the models of both lumped and distributed parameter buck converter are presented. For both models state-space representations can be found, although both state spaces are fundamentally different. For further discussion, we will carry out a steady state analysis and compare the two converter topologies by numerical simulations.

\section{MODELING}

\subsection{Lumped parameter model}

The lumped parameter model of the buck converter is based on the circuit diagram shown in Fig. 1. The parameters $C$ and $L$ denote the capacitance and inductance of the circuit whereas $R_{L}$ and $G_{C}$ take into account the ohmic loss of both components. The corresponding mathematical model can be derived:

$$
\begin{aligned}
& L \frac{d i_{a}}{d t}+R_{L} i_{a}+u=d U_{e} \\
& C \frac{d u}{d t}+G_{C} u+i=i_{a} \\
& L_{I} \frac{d i}{d t}+R i+u=0
\end{aligned}
$$

The constant input voltage $U_{e}$ is assumed to be known as well as the load $L_{I}$ and $R$. Hence the system input is the switch signal $d$ with $d \in\{0,1\}$.

\subsection{Distributed parameter model}

For the distributed parameter model of the buck converter we use the model of transmission line described by Telegrapher's Equations to substitute all lumped components between switch and load, resulting into the equivalent circuit diagram shown in Fig. 2. It is equivalent to a series connection of infinite number of lumped parameter buck converters. The Telegrapher's Equations are:

$$
\begin{array}{ll}
\frac{\partial}{\partial z} u(z, t)+L^{\prime} \frac{\partial}{\partial t} i(z, t)+R^{\prime} i(z, t) & =0 \\
\frac{\partial}{\partial z} i(z, t)+C^{\prime} \frac{\partial}{\partial t} u(z, t)+G^{\prime} u(z, t) & =0
\end{array}
$$

It is a system of partial differential equations with current $i(z, t)$ and voltage $u(z, t)$ not only depending on time $t$ 


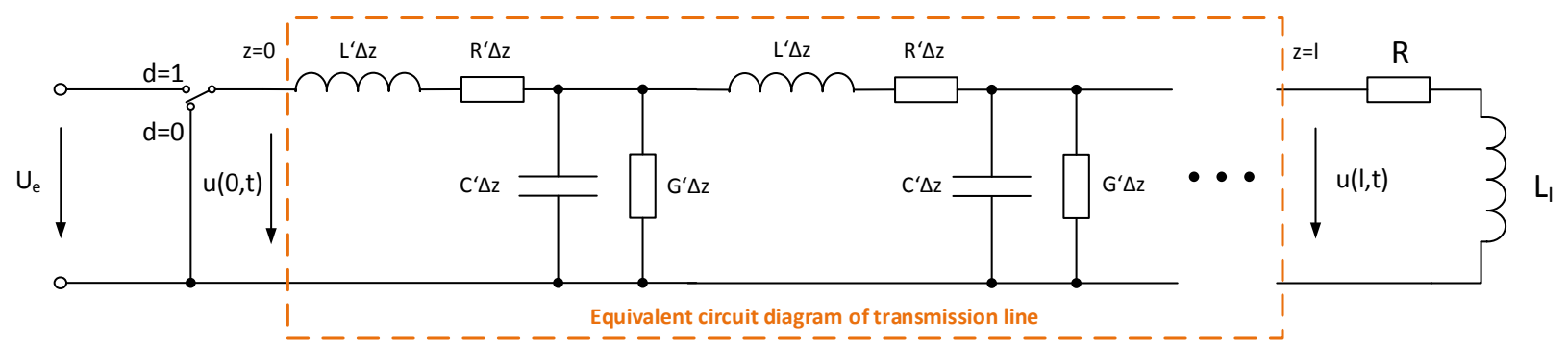

Fig. 2. Equivalent circuit diagram of the distributed parameter buck converter

but also on position $z \in[0, \ell]$ within the transmission line of length $\ell$. Other distributed parameters of the system are inductance $L^{\prime}$, capacitance $C^{\prime}$, resistance $R^{\prime}$ and conductance $G^{\prime}$, whereas $R^{\prime}$ and $G^{\prime}$ determine the loss of the transmission line.

To fully describe the circuit diagram of Fig. 2 boundary conditions need to be specified. At the beginning of the wire the voltage $u(0, t)$ is defined by input voltage $U_{e}$ as well as switch position $d$. At the end of the wire $z=l$ the voltage and current is defined via an ordinary differential equation. These lead to two boundary conditions:

$$
\begin{aligned}
u(0, t) & =U_{e} d(t) \\
i(\ell, t) & =y(t)
\end{aligned}
$$

whereas $y$ satisfies the following differential equation:

$$
\dot{y}(t)=-\frac{1}{L_{I}}(R y(t)-u(\ell, t))
$$

With equations (2) as well as conditions (3) and (4) the model of distributed parameter buck converter is obtained.

\section{SYSTEMS ANALYSIS}

The lumped parameter model (1) is essentially already in state space representation with the state vector $\boldsymbol{x}=$ $\left(i_{a}, u, i\right)^{T}$. The state space is the finite dimensional vector space $\mathbb{R}^{3}$.

In case of the distributed parameter model (2) with (3) and (4) the corresponding state vector can be defined as $\boldsymbol{x}=\left(x_{1}, x_{2}, x_{3}\right)^{T}=(i, u, y)^{T}$ and an abstract state space description can be introduced:

$$
\dot{\boldsymbol{x}}=A \boldsymbol{x}+\boldsymbol{b} d
$$

with $\boldsymbol{x} \in X=L^{2}\left(\Omega, \mathbb{R}^{2}\right) \times \mathbb{R}$ where $\Omega=[0, \ell]$ and $L^{2}\left(\Omega, \mathbb{R}^{2}\right)$ denotes the space of square integrable functions on $\Omega$ with values in $\mathbb{R}^{2}$. The unbounded linear operator $A: X \supset D(A) \rightarrow X$ can be directly read off from (2) and (4):

$$
A \boldsymbol{x}=\left(\begin{array}{ccc}
-\frac{R^{\prime}}{L^{\prime}} x_{1}(\cdot, t) & -\frac{1}{L^{\prime}} \frac{\partial}{\partial z} x_{2}(\cdot, t) \\
-\frac{1}{C^{\prime}} \frac{\partial}{\partial z} x_{1}(\cdot, t) & - & \frac{G^{\prime}}{C^{\prime}} x_{2}(\cdot, t) \\
\frac{1}{L_{I}} x_{2}(\ell, t) & - & \frac{R}{L_{I}} x_{3}(t)
\end{array}\right) .
$$

Its domain $D(A)$ results from the boundary conditions:

$$
D(A)=\left\{\boldsymbol{x} \in H^{1}\left(\Omega, \mathbb{R}^{2}\right) \times \mathbb{R}: x_{2}(0)=0, x_{3}=x_{1}(\ell)\right\} .
$$

Moreover, since the control input enters at the boundary the unbounded input operator

$$
\boldsymbol{b}=U_{e}(0, \delta, 0)^{T}
$$

stems from the dual space of $D\left(A^{*}\right)$, where $A^{*}$ is the adjoint operator of $A$ and $\delta$ is the dirac distribution.
For steady state analysis of periodically switched finite dimensional systems, switched or averaged models can be used, cf. Gensior et al. (2006). In particular the averaged model (see e.g. Sanders et al. (1991) or Middlebrook and Cuk (1977) for its derivation) is a further approximation of the switched model. Its input is a continuous function with respect to time and is therefore also suited for control design purposes. For steady state analysis of the finite dimensional buck converter, both models are derived and analyzed. In case of the infinite dimensional system we use a switched and an input-averaged model.

In order to compare the distributed with the lumped parameter model of the buck converter qualitatively, the distributed transmission line parameters are set appropriately. E.g. $L^{\prime}$ is chosen in a way such that the overall inductance of the line with length $\ell$ corresponds to the inductance $L$ of the lumped parameter converter: $L^{\prime} \ell=L$.

For numerical simulation of the infinite dimensional system we pursue two approaches. First a modal transformation can be conducted so that the solutions of (2) with (3) and (4) are approximated by the solutions of a system of ordinary differential equations. Therewith common ODEsolvers can be used. Second if we consider a lossless or distortionless line, exact solutions of the system in terms of difference equations can be obtained using e. g. the method of characteristics (see e. g. Woittennek (2011)).

\section{REFERENCES}

Erickson, R.W. and Maksimovic, D. (2001). Fundamentals of Power Electronics. Kluwer Academic Publishers.

Gensior, A., Woywode, O., Rudolph, J., and Güldner, H. (2006). On differential flatness, trajectory planning, observers, and stabilization for DC-DC converts. IEEE Trans. on Circuits and Systems I, 53(9), 2000-2010.

Huang, C., Woittennek, F., and Röbenack, K. (2013). Steady-state analysis of a distributed model of the buck converter. In European Conference on Circuit Theory and Design (ECCTD), 1-4.

Middlebrook, R.D. and Cuk, S. (1977). A general unified approach to modelling switching-converter power stages. International Journal of Electronics, 42(6), 521-550.

Sanders, S., Noworolski, J., Liu, X., and Verghese, G. (1991). Generalized averaging method for power conversion circuits. Power Electronics, IEEE Transactions on, 6(2), $251-259$. doi:10.1109/63.76811.

Woittennek, F. (2011). On flatness and controllability of simple hyperbolic distributed parameter systems. In Proc. 18th IFAC World Congress, 14452-14457. Milano, Italy. 


\title{
Taylor Series Based Solution of Linear ODE Systems and MATLAB Solvers Comparison
}

\author{
V. Śátek ${ }^{*, * *}$ F. Kocina ${ }^{* *}$ J. Kunovský ${ }^{* *}$ A. Schirrer Su* $^{* * *}$ \\ * IT4Innovations, Vঙ̆B Technical University of Ostrava, \\ 17. listopadu 15/2172, 70833 Ostrava-Poruba, Czech Republic, \\ (e-mail: vaclav.satek@vsb.cz) \\ ** Brno University of Technology, Faculty of Information Technology, \\ Božetěchova 2, 612 66 Brno, Czech Republic \\ *** Institute of Mechanics and Mechatronics, Vienna University of \\ Technology, Getreidemarkt 9, 1060 Vienna, Austria
}

\begin{abstract}
The Modern Taylor Series Method (MTSM) is employed here to solve initial value problems of linear ordinary differential equations. An automatic computation of higher Taylor series terms and an efficient, vectorized coding of explicit and implicit schemes enables a very fast computation of the solution to specified accuracy. For a set of benchmark problems from literature, the MTSM significantly outperforms standard solvers. Finally, ideas of parallelizing the MTSM computations are discussed.
\end{abstract}

Keywords: Ordinary differential equations, Initial value problems, Taylor series, MATLAB

\section{INTRODUCTION}

The "Modern Taylor Series Method" (MTSM) is used for numerical solution of differential equations. The MTSM is based on a recurrent calculation of the Taylor series terms for each time interval. An important part of the MTSM is an automatic integration order setting, i.e. using as many Taylor series terms as the defined accuracy requires. Thus it is usual that the computation uses different numbers of Taylor series terms for different steps of constant length. The MTSM has been implemented in the TKSL software package (Kunovský, 1994).

Several papers focus on computer implementations of the Taylor series method in a variable-order and variable-step context (see, for instance, Barrio et al. (2005), the TIDES software implemented in Wolfram (2014), or in Jorba and Zou (2005)). The reduction of rounding errors (Rodríguez and Barrio, 2012) and utilization of multiple arithmetics (Barrio et al., 2011) improve the applicability of Taylor series based algorithms.

This paper demonstrates that the MTSM, specialized to directly solving linear ODE systems, solves non-stiff and stiff systems very fast (in explicit and implicit formulations, respectively) and outperforms standard solvers in the considered benchmark problems.

\section{EXPLICIT SCHEME OF TAYLOR SERIES}

In this article, we have focused on effective solution of linear systems of ODEs using Taylor series scheme. The bestknown and most accurate method of calculating a new value of a numerical solution of ordinary differential equation $y^{\prime}=f(t, y), \quad y(0)=y_{0}$ is to construct the Taylor series (Hairer et al., 1987).
The $n$-th order method uses $n$ Taylor series terms in the explicit form

$$
\begin{aligned}
y_{i+1}= & y_{i}+h f\left(t_{i}, y_{i}\right)+\frac{h^{2}}{2 !} f^{[1]}\left(t_{i}, y_{i}\right)+\cdots \\
& +\frac{h^{n}}{n !} f^{[n-1]}\left(t_{i}, y_{i}\right) .
\end{aligned}
$$

Equation (1) for linear systems of ODEs in the form $\boldsymbol{y}^{\prime}=\boldsymbol{A} \boldsymbol{y}+\boldsymbol{b}$ could be rewritten

$$
\begin{aligned}
\boldsymbol{y}_{i+1}= & \boldsymbol{y}_{i}+h\left(\boldsymbol{A} \cdot \boldsymbol{y}_{i}+\boldsymbol{b}\right)+\frac{h^{2}}{2 !} \boldsymbol{A}\left(\boldsymbol{A} \boldsymbol{y}_{i}+\boldsymbol{b}\right)+ \\
& \ldots+\frac{h^{n}}{n !} \boldsymbol{A}^{(n-1)}\left(\boldsymbol{A} \boldsymbol{y}_{i}+\boldsymbol{b}\right)
\end{aligned}
$$

where $\boldsymbol{A}$ is the constant Jacobian matrix and $\boldsymbol{b}$ is the constant right-hand side.

Vectorized MATLAB code of explicit Taylor series expTay with a variable order and variable step size scheme for linear systems of ODEs (2) has been implemented. This algorithm was compared on a set of "non-stiff" linear systems (see Enright and Pryce (1987)) with vectorized MATLAB explicit odeNN solvers. Benchmarking results are shown in Table 1 (each reported runtime is the median value of 100 computations). Ratios of computation times ratio $_{e}=$ ode23/expTay $>1$ indicate faster computation of the MTSM in all test cases. Exact solutions were obtained by the Maple software package (Maplesoft, 2014). All solvers' tolerances were set to obtain relative and absolute tolerances of $10^{-4}$ with respect to the exact solutions.

\section{IMPLICIT SCHEME OF TAYLOR SERIES}

The implicit Taylor series scheme for linear systems of ODEs are constructed as follows: 
Table 1. Median computation time: explicit Taylor expTay and MATLAB explicit odeNN solver comparison

\begin{tabular}{cccccc} 
& $\begin{array}{c}\text { ode23 } \\
{[\mathrm{s}]}\end{array}$ & $\begin{array}{c}\text { ode45 } \\
{[\mathrm{s}]}\end{array}$ & $\begin{array}{c}\text { ode113 } \\
{[\mathrm{s}]}\end{array}$ & $\begin{array}{c}\text { expTay } \\
{[\mathrm{s}]}\end{array}$ & ratio $_{e}$ \\
\hline $\mathrm{A} 1$ & 0.00497 & 0.00537 & 0.00751 & 0.000831 & 5.98 \\
$\mathrm{~B} 2$ & 0.00633 & 0.00758 & 0.0128 & 0.00218 & 2.9 \\
$\mathrm{C} 1$ & 0.00653 & 0.00574 & 0.0111 & 0.00114 & 5.72 \\
$\mathrm{C} 2$ & 0.01 & 0.0147 & 0.0277 & 0.00651 & 1.54 \\
$\mathrm{C} 3$ & 0.00636 & 0.00805 & 0.0156 & 0.003 & 2.11 \\
$\mathrm{C} 4$ & 0.00679 & 0.00836 & 0.0166 & 0.00359 & 1.89 \\
\hline
\end{tabular}

$$
\begin{aligned}
\boldsymbol{y}_{i+1}= & \boldsymbol{y}_{i}+h\left(\boldsymbol{A} \boldsymbol{y}_{i+1}+\boldsymbol{b}\right)-\frac{h^{2}}{2 !} \boldsymbol{A}\left(\boldsymbol{A} \boldsymbol{y}_{i+1}+\boldsymbol{b}\right)- \\
& \cdots-\frac{(-h)^{n}}{n !} \boldsymbol{A}^{(n-1)}\left(\boldsymbol{A} \boldsymbol{y}_{i+1}+\boldsymbol{b}\right)
\end{aligned}
$$

Implicit Taylor series method with recurrent calculation of Taylor series terms and Newton method (impTay) based on (3) was implemented in MATLAB using vectorization. The Jacobian matrix is computed using Broyden's method.

A benchmark problem set of "stiff" linear ODEs from Enright and Pryce (1987) was used for tests. Comparisons of the problems A1, A3, A4, and B1-B5 whose analytic solutions are known (from the Maple software package (Maplesoft, 2014)) have been completed. The simulated intervals were adopted from Enright and Pryce (1987), and the integration time step was set to the entire time interval (just 1 integration step was needed). Relative and absolute tolerances for the computations were again set to $10^{-4}$. Comparisons of MATLAB "stiff" odeNNs solvers with impTay are shown in Table 2 . High ratios of computation times ratio $_{i}=$ ode15s/impTay show that the MTSM method significantly outperforms the standard solvers.

Table 2. Time of solutions: implicit Taylor impTay and MATLAB implicit odeNNs solvers comparisons

\begin{tabular}{cccccc} 
& $\begin{array}{c}\text { ode15s } \\
{[\mathrm{s}]}\end{array}$ & $\begin{array}{c}\text { ode23s } \\
{[\mathrm{s}]}\end{array}$ & $\begin{array}{c}\text { ode23tb } \\
{[\mathrm{s}]}\end{array}$ & $\begin{array}{c}\text { impTay } \\
{[\mathrm{s}]}\end{array}$ & ratio $_{i}$ \\
\hline A1 & 0.0605 & 0.169 & 0.101 & 0.0003 & 194.6 \\
A3 & 0.085 & 0.243 & 0.144 & 0.00001 & 263 \\
A4 & 0.111 & 0.478 & 0.192 & 0.0003 & 294.8 \\
B1 & 0.268 & 1.473 & 0.8 & 0.0003 & 244.4 \\
B2 & 0.069 & 0.285 & 0.134 & 0.00003 & 172.4 \\
B3 & 0.073 & 0.308 & 0.146 & 0.00003 & 211.4 \\
B4 & 0.117 & 0.549 & 0.242 & 0.00003 & 348.4 \\
B5 & 1.155 & 1.529 & 0.664 & 0.00003 & 3306.7 \\
\hline
\end{tabular}

\section{PARALLEL IMPLEMENTATION}

As can be seen from (2), each term of Taylor Series for a linear system can be computed independently. So their computation can be distributed into multiple computation units (utilizing a distributed memory architecture). Hence thread $j \in\{1 \ldots m\}$ evaluates

$$
\boldsymbol{A}_{j}=\sum_{k=0}^{\frac{n}{m}-1} \frac{h^{m k+j}}{(m k+j) !} \boldsymbol{A}^{m k+j-1}
$$

and the final sum is computed afterwards. Therefore expression (2) can be transformed to

$$
\boldsymbol{y}_{i+1}=\left(\left(\sum_{j=1}^{m} \boldsymbol{A}_{j}\right) \boldsymbol{A}+\mathbf{I}\right) \boldsymbol{y}_{i}+\left(\sum_{j=1}^{m} \boldsymbol{A}_{j}\right) \boldsymbol{b}
$$

where $\mathbf{I}$ is the identity matrix.

\section{CONCLUSION}

The Taylor series scheme is highly efficient in solving linear ODEs. It significantly outperforms standard solvers on the considered benchmark problems. Results for double precision arithmetics and a maximum Taylor series order of 90 have been shown. Multiple arithmetics is needed for higher orders. Future studies will address the efficiency and scalability of MTSM ODE solvers in different parallelization architectures.

\section{ACKNOWLEDGEMENTS}

This paper has been elaborated in the framework of the project New creative teams in priorities of scientific research, reg. no. CZ.1.07/2.3.00/30.0055 (as well as the the IT4Innovations Centre of Excellence CZ.1.05/1.1.00/ 02.0070), supported by Operational Programme Education for Competitiveness and co-financed by the European Social Fund and the state budget of the Czech Republic. The paper includes the solution results of the international AKTION research project Number 69p22 and the internal BUT projects FIT-S-12-1 and FIT-S-14-2486.

\section{REFERENCES}

Barrio, R., Blesa, F., and Lara, M. (2005). VSVO Formulation of the Taylor Method for the Numerical Solution of ODEs. In Computers and Mathematics with Applications, volume 50, 93-111.

Barrio, R., Rodríguez, M., Abad, A., and Blesa, F. (2011). Breaking the limits: The taylor series method. Applied Mathematics and Computation, 217, 7940-7954.

Enright, W.H. and Pryce, J.D. (1987). Two fortran packages for assessing initial value methods. In $A C M$ Trans. Math. Softw., volume 13, 1-27. ACM.

Hairer, E., Nørsett, S.P., and Wanner, G. (1987). Solving Ordinary Differential Equations I. vol. Nonstiff Problems. Springer-Verlag Berlin Heidelberg. ISBN 3-54056670-8.

Jorba, A. and Zou, M. (2005). A software package for the numerical integration of ODE by means of high-order Taylor methods. In Exp. Math., volume 14, 99-117.

Kunovský, J. (1994). Modern Taylor Series Method. FEIVUT Brno. Habilitation work.

Maplesoft (2014). MAPLE software. URL http://www.maplesoft.com [online].

MathWorks, T. (2014). MATLAB and Simulink software. URL http://www. mathworks.com [online] .

Rodríguez, M. and Barrio, R. (2012). Reducing rounding errors and achieving brouwers law with taylor series method. Applied Numerical Mathematics, 62, 10141024.

Wolfram (2014). MATHEMATICA software. URL http://www.wolfram.com/mathematica/ [online]. 


\title{
SVD-AORA method for Dynamic Linear Time Invariant Model Order Reduction
}

\author{
K. Mohamed* A. Mehdi* M. Abdelkader* \\ * Université de Tunis El Manar,Ecole Nationale d'Ingenieurs de Tunis, \\ Laboratoire d'Analyse et de Commande des Systemes, BP 37, LE \\ BELVEDERE 1002, Tunis, Tunisie (e-mails: \\ koukimohammed@hotmail.com,mehdiabbes@yahoo.fr, \\ mami.abdelkader@planet.tn).
}

\section{INTRODUCTION}

In this paper we present a combined model order reduction for dynamic linear time invariant (LTI) system, named SVD-AORA (Singular Value Decomposition-Adaptive Order Rational Arnoldi). The SVD-AORA method is an extension of the SVD-Krylov based method. It is based on linear projection using two projection matrices $(V$ and $Z$ ). The first matrix $V$ depends on the Krylov technique through the AORA method, the second matrix $Z$ depends on the SVD technique by the resolution of the Lyaponuv equation, the last is used in determining the observability Gramian matrix $g_{o}$, which is used in calculating the $Z$ matrix. The use of the Krylov technique through the AORA method is attached to the numerical efficiency and a relative lower computation complexity and the use of the SVD technique preserves the stability of the reduced system. The proposed method gives a reduced order model asymptotically stable, capture the essential dynamics of the original model and minimize the absolute error between the original and the reduced one. To evaluate our method, we take one model of high order and we give a comparative study between the proposed method SVDAORA and the SVD-Krylov method.

\section{PRELIMINARY}

Technological world, physical and artificial processes are mainly written by mathematical models which can be used for simulation or control. Among these models there exist the LTI of high order. However, these high order models are difficult to manipulate and analyze, the resolution of these models is indeed very demanding in computational resources, storage space, and mainely in CPU time. The use of model order reduction technique presents a good solution. This technique is based on the detailed description of original model. However, in the literature there exist different reduction methods of linear time invariant system (Arnoldi, Lanczos, Rational Arnoldi, AORA, AOGRA, AORL)A.C.Antoulas (2005); Lee et al. (2006), but they differ in the performances level. Among these performances we can mention:

- A significantly reduced number of variables or states (required for description of a given model) compared to the original model,

- The simulation should be quick and not requiring large memory space,
- The computational complexity associated with the evaluation of the reduced model should be significantly lower than the original model,

- Stability of reduced model must be guaranteed,

- Minimization of error between the original model and reduced one.

To ring this performances, we depict in this paper the SVD-AORA method. This paper in organized as follow, in section 3, a description of SVD-AORA method is given with application in theoretical models, section 4 , a comparative study is presented.

\section{SVD-AORA METHOD}

In this section we give a mathematical formulation of our problem, we present the main steps of the proposed method and the results obtained by the theoretical model.

\subsection{Mathematical problem formulation}

Our mathematical problem consists on determining the state space parameters (order $k<<n$ ) of the reduced model (2) from the state space parameters (order $n$ ) of the original model (1):

$$
\frac{d x(t)}{d t}=A x(t)+B u(t), y(t)=C x(t)+D u(t)
$$

In which $A \in \mathbb{R}^{n \times n}, B \in \mathbb{R}^{n \times p}, C \in \mathbb{R}^{p \times n}$ and for simplicity we take $D=0$.

$$
\frac{d \hat{x}(t)}{d t}=\hat{A} \hat{x}(t)+\hat{B} u(t), \hat{y}(t)=\hat{C} x(t)+\hat{D} u(t)
$$

With, $\hat{A} \in \mathbb{R}^{k \times k}, \hat{B} \in \mathbb{R}^{k \times p}, \hat{C} \in \mathbb{R}^{p \times k}$.

The details of the SVD-AORA algorithm can be found in table 1 :

\subsection{Application}

To test the algorithm we take one SISO model of order 58 (Eady) Y.Chahlaoui and P.V.Dooren (2002), it presents the frequency response of the original model and the reduced one, gives the absolute error between original model and reduced one and the poles distribution of the reduced model. 
Table 1. SVD-AORA algorithm

SVD-AORA algorithm:(Inputs: $A ; B ; C ; D ; S ; k$; Outputs: $V ; Z)$

(1): Define a frequency range $S$

(2): Compute the first projection matrix $\mathrm{V}$ using the AORA algorithm

(3): Compute the Gramian observability matrix by solving the following Lyaponuv equation: $A^{T} g_{o}+g_{o} A+C^{T} C=0$

(4): Compute the second projection matrix $\mathrm{Z}$ through the following relation: $Z=g_{0} V\left(V^{T} g_{0} V\right)^{-1}$

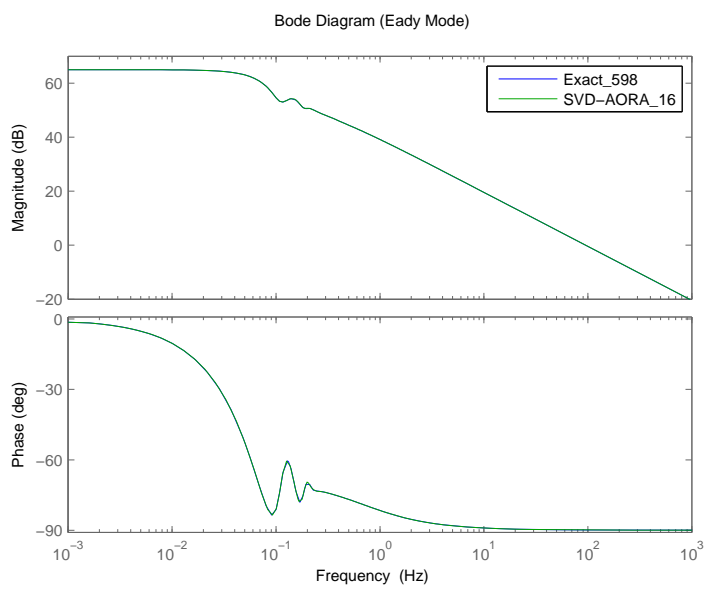

Fig. 1. Frequency response of original system (Exact-5 8) and reduced one (SVD-AORA-16)

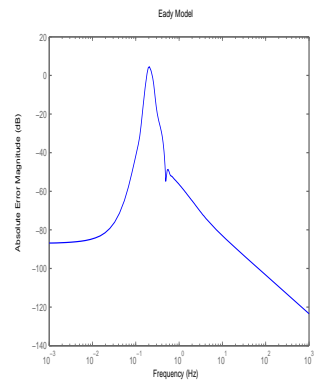

(a)

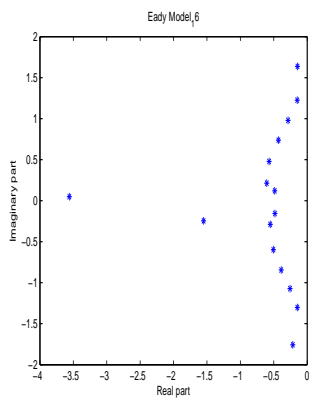

(b)
Fig. 2. (a) Absolute error between original model (5 8) and reduced one (16), (b) Poles Distribution of Eady reduced model with SVD-AORA method

We see from figures 1 and 2(a) a good correlation between the original model and reduced one. We also note from the figure $2(\mathrm{~b})$ that all poles are negative real part, which explain the preservation of stability.

\section{COMPARATIVE STUDY}

In this part we present a comparative study between the SVD-AORA method and the SVD-Krylov one.

Noting from the figure 4 (b) the existence of positive real part poles, which explain the instability of reduced model. We note from the figures 3, 4(a) and from the table 2 that the best performance is obtained by the proposed method SVD-AORA.

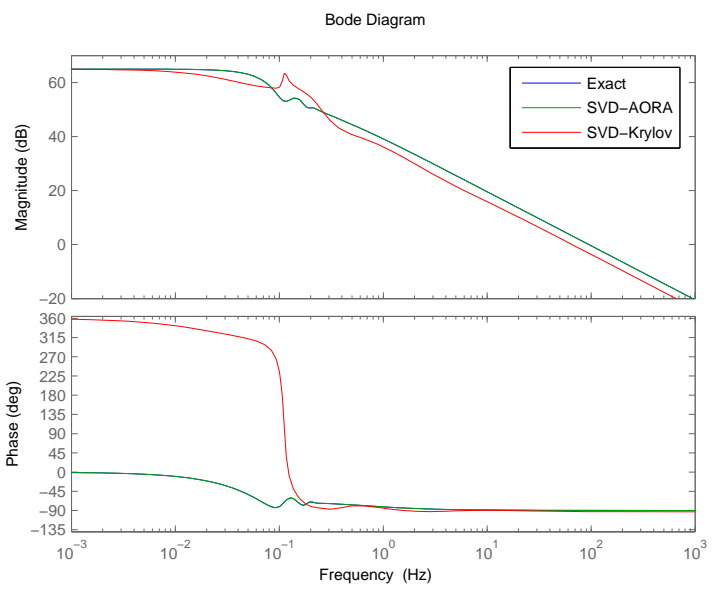

Fig. 3. Frequency response of original system (Exact-5 8) and reduced one (16) with two methods

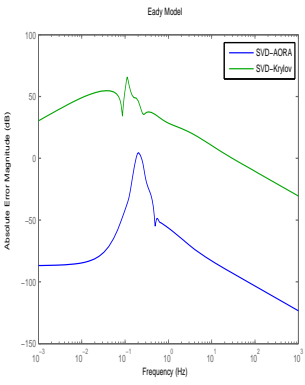

(a)

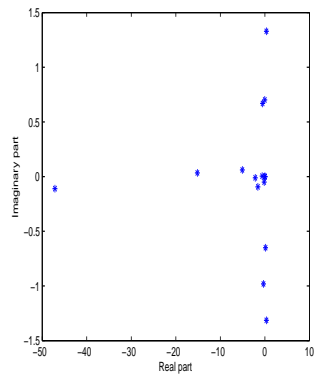

(b)
Fig. 4. (a) Absolute error between original model (5 8) and reduced one (16), (b) Poles Distribution of Eady reduced model with SVD-AORA method

Table 2. $H_{\infty}$ norms and CPU-Time for each method

\begin{tabular}{|l|l|l|l|l|}
\hline Methods & $\begin{array}{l}\text { SISO LTI } \\
\text { System }\end{array}$ & $\min H_{\infty}$ & $\max H_{\infty}$ & $\begin{array}{l}\text { CPU- } \\
\text { Time }\end{array}$ \\
\hline $\begin{array}{l}\text { SVD- } \\
\text { AORA }\end{array}$ & Eady598 & $1: 51110^{-7}$ & $2: 76010^{-5}$ & $525.308 \mathrm{~s}$ \\
\hline $\begin{array}{l}\text { SVD- } \\
\text { Krylov }\end{array}$ & Eady598 & 0.044 & 6.6928 & $721.755 \mathrm{~s}$ \\
\hline
\end{tabular}

\section{CONCLUSION}

A combined method SVD-AORA for dynamic linear time invariant model order reduction have been presented. We seed that this method is efficient in reduction of high order model.

\section{REFERENCES}

A.C.Antoulas (2005). Approximation of large-scale Dynamical systems. Advances in Design and Control.

Lee, H. ., Chu, C., and Feng, W. (2006). An adaptiveorder rational arnoldi method for model-order reductions of linear time-invariant systems. Linear Algebra an its Applications, 415, 235-261.

Y.Chahlaoui and P.V.Dooren (2002). A collection of benchmark examples for model reduction of linear time invariant dynamical systems. URL http:niconet. NIC2 . benchmarks . html. 


\title{
Modelling "Breaking Bad": An economic model of drugs and population dynamics to predict how the series itself feeds back into the drug market
}

\author{
Christiane Rössler*Magdalena Witzmann*Thomas Schmickl* \\ *Department for Zoology, University of Graz, 8010 Graz, Austria \\ (e-mail: \{christiane.roessler, magdalena.witzmann\}@edu.uni-graz.at; thomas.schmickl@uni-graz.at)
}

\begin{abstract}
This stock\&flow-model predicts population dynamics of crystal meth addicts related to the price development of drugs, inspired by the TV series "Breaking Bad". The potential impact of the broadcasting of the TV series on the system is tested by using sudden (pulsed) changes of selected flows and rates to reveal the sensitivity of selected variables: Addicts, price relationship, dealers' saturation and Stock of Crystal Meth. While consumption, purchase and production show strong responses to those changes, other variables like getting_addicted and weaning_off show weaker responses. These flows' reactions to pulsed changes of model parameters are analyzed and their significance is discussed.
\end{abstract}

Keywords: economic systems, modelling, intensity changes, population dynamics, drugs, breaking bad

\section{INTRODUCTION}

The TV show "Breaking Bad" (2008-2012) shows a school chemistry teacher who starts producing and selling crystal meth. "I'll continue to wonder about the long-term effects of mainstreaming such a dangerous drug into popular culture", Blake Ewing said (Ewing 2013). The model (Fig. 1) shows addicts' and drug dynamics in correlation to price development. It is used to test the system's sensitivity to outside influences such as the influence of the TV series "Breaking Bad" on the modelled drug market. These influences can cause an increase in meth users, for example, in reaction to the TV series.

\section{METHODS}

The stock\&flow-model is built in Vensim 5.11A. It is based on the given conditions in "Breaking Bad" and parameterized with data from the world drug report (United Nations Office on Drugs and Crime 2014). The model runs for 120 months, integration type is Euler and time step is defined with $\Delta \mathbf{t}=1$ month. Units for crystal meth are [g], Addicts $\left(\mathbf{N}^{\mathbf{a}}\right)$ and Non_Addicted $\left(\mathbf{N}^{\text {na }}\right)$ are [persons]. The models' main structure is shown in Fig. 1. The total Stock of Crystal Meth $\left(\mathbf{C}^{\text {stock }}\right)$ is specified by

$$
\frac{\Delta C^{\text {stock }}}{\Delta t}=+\alpha \cdot C^{\text {stock }}+g-\beta \cdot C^{\text {stock }} \cdot\left(1-\frac{C^{\text {circ }}}{k}\right)
$$

where $\boldsymbol{\alpha}$ is the intrinsic production rate, $\boldsymbol{g}$ represents the smuggled goods, $\boldsymbol{\beta}$ is the actual purchasing rate and $\mathbf{C}^{\text {circ }}$ is the amount of Crystal Meth in Circulation, which is further defined by (2) that follows from the constant actual purchasing rate $\boldsymbol{\beta}$, consumption rate $\boldsymbol{\gamma}$ and distribution rate $\boldsymbol{d}$. The dynamics of $\mathbf{N}^{\mathbf{n a}}$ are modelled by (3), where the actual growth rate is $\boldsymbol{\delta}$, normal death rate is $\boldsymbol{\varepsilon}$, weaning off rate is $\boldsymbol{\mu}$, addiction rate is $\boldsymbol{\varphi}$ and the dealers' saturation of crystal meth is $\boldsymbol{s}$. Equation 4 determines the dynamics of $\mathbf{N}^{\mathrm{a}}$ that grow in number with $\boldsymbol{\varphi}$ and $\boldsymbol{s}$ and drop with $\boldsymbol{\mu}$ and drug related death rate $\omega$. The purchasing price relationship $\boldsymbol{p}$ is a regulator for the drug flow; it is defined by supply - $\mathbf{C}^{\text {stock }}$ and demand $-\mathbf{N}^{\mathbf{a}}, \boldsymbol{s}, \boldsymbol{\gamma}$ and $\boldsymbol{r}$ - the daily requirement. The price elasticity $\boldsymbol{a}$ is the measurement of how responsive the price relationship is to a change in the proportion of demand to supply. These relationships are described in (5).

$$
\begin{aligned}
& \frac{\Delta C^{\text {circ }}}{\Delta t}=+\beta \cdot C^{\text {stock }} \cdot\left(1-\frac{C^{\text {circ }}}{k}\right)-M I N\left\{\begin{array}{l}
C^{\text {circ }} \cdot d \\
\gamma \cdot N^{a} \cdot s
\end{array}\right\} \\
& \frac{\Delta N^{n a}}{\Delta t}=+\delta \cdot N^{n a}-\varepsilon \cdot N^{n a}+\mu \cdot N^{a} \cdot(1-s)-\varphi \cdot N^{n a} \cdot s \\
& \frac{\Delta N^{a}}{\Delta t}=+\varphi \cdot N^{n a} \cdot s-\mu \cdot N^{a} \cdot(1-s)-\omega \cdot N^{a} \\
& p=\frac{a \cdot \frac{N^{a} \cdot(1-s) \cdot \gamma \cdot r}{C^{s t o c k}+1}}{\left.\sqrt{1+\left(a \cdot \frac{N^{a} \cdot(1-s) \cdot \gamma \cdot r}{C^{s t o c k}}+1\right.}\right)^{2}}
\end{aligned}
$$

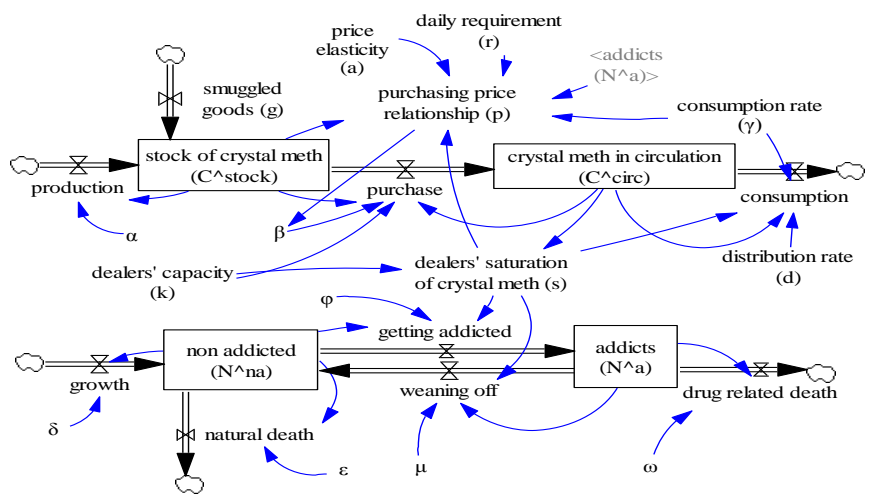

Fig. 1: Excerpt of the stock\&flow-model of "Breaking Bad", showing the main structure of its system components.

The dealers' saturation $s$ has influence on consumption and on $\boldsymbol{p}$, as apparent from (6). The dealers' saturation also 
regulates the getting_addicted and the weaning_off flow. A $100 \%$ dealers' saturation implies a maximum addiction rate. Thus forming the link between drugs and addicts. Equation 6 is given by

$$
s=\frac{C^{\text {circ }}}{k}
$$

where $\mathbf{C}^{\text {circ }}$ is given in (2) and $\boldsymbol{k}$ is the dealers' maximum capacity. A PULSE function was used to test the drug system's sensitivity to the series' influence. This PULSE changes a chosen flow in a defined time interval from 1 to $\mathrm{X}$ by multiplication. The series ran for 5 years, thus we applied the PULSE for 5 years to perturb the system with different strengths $(0 \%$ to $+50 \%)$. To quantify the influence of the PULSE, the data at $\mathrm{t}=73$ are observed.

\section{RESULTS}

Fig. 2 shows the reactions of Addicts, purchasing price relationship, dealers' saturation and Stock of Crystal Meth after the PULSE influenced the affected flows. The abscissa shows the increase and the decrease of the PULSE effect relative to the starting conditions. The ordinate shows the relative change of the observed variables. In general, responses are almost linear.

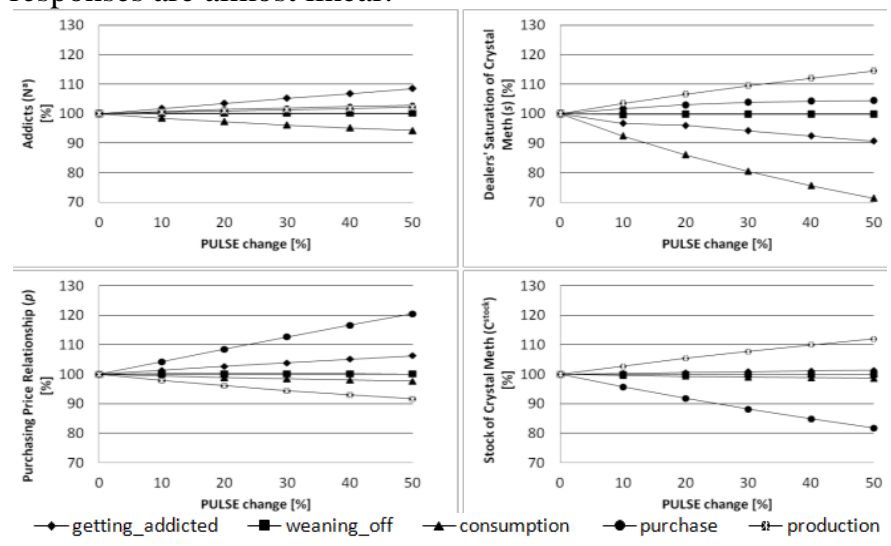

Fig. 2: Results of sensitivity tests by PULSE change

$\mathbf{N}^{\mathbf{a}}$ is most reactive to positive changes of the flow getting_addicted and negative changes of consumption. Purchase and production show less reaction and similar linear scaling of reaction. The price relationship increases with a positive change in purchase and decreases with a rise in production. Getting_addicted shows a slight increase, production is reacting least to a change. Dealers' saturation grows strongest with a rise in production and decreases with an increase in consumption; with changes in getting_addicted and purchase it shows little decrease. $\mathbf{C}^{\text {stock }}$ is most influenced by increased production volumes and by decreased purchase. The other observed flows show similar linear responses, but on a low level. Weaning_off universally remains constant throughout all perturbation experiments.

\section{DISCUSSION}

On the one hand, we showed that the modelled drug market is intrinsically stable in response to extrinsic perturbations. On the other hand, significant medium-term effects of perturbations are predicted, as they can be caused by the TV series itself. These responses, which are shifts of equilibria during the perturbed period, have been analyzed systematically in a quantitative way generating testable hypotheses. Results show different sensitivities of analyzed observed variables to the changes in parameters governing important flows in the system: The more consumption increases, the more dealers' saturation decreases. Consequently the price relationship and $\mathbf{N}^{\mathrm{a}}$ decrease. This seems implausible at first sight but it is accurate according to the model's hypothesis of market regulation. Since demand is determined by the consumption rate and not by the consumption flow, the price relationship does not react as expected. The saturation reacts with a time delay, which is a result of the multi-stock structure of the modelled system. With regard to production, the model's behaviour is governed by the feedback loop "C $\mathbf{C}^{\text {stock }}$ to purchase", which prevents oversaturation. The growing purchase flow reacts with an enhancement of $\mathbf{N}^{\mathbf{a}}$, price relationship and dealers' saturation while $\mathbf{C}^{\text {stock }}$ decreases. This is consistent to the underlying hypothesis of market regulation. A positive change in getting_addicted leads to an increase in $\mathbf{N}^{\mathbf{a}}$. As price relationship is not very responsive to small changes, it grows subtly, due to a remote growth in supply $\left(\mathbf{C}^{\text {stock }}\right)$. Dealers' saturation decreases with higher consumption caused by more $\mathbf{N}^{\mathbf{a}}$. Weaning_off conspicuously is insensitive in consequence of a low weaning_off rate. Since there are no over-proportional changes the system is considered to be stable. This stability is caused by the negative feedback loop established by the interaction of demand and price relationship. The higher the price elasticity is, the more stable the system becomes due to higher flexibility in the price relationship, which induces an enhancement of the negative feedback loop. One disturbance of the drug market that was simulated by the PULSE experiment (on all flows) was found to be the effect of the broadcast of the series itself (Ewing 2013). In agreement to our model predictions, the UNODC reports an increase in $\mathbf{C}^{\text {stock }}, \mathbf{N}^{\mathbf{a}}$ and $\mathbf{C}^{\text {circ }}$ between 2008-2012 (United Nations Office on Drugs and Crime 2014, Fig. 49.). Based on various stories it can be assumed that "Breaking Bad" still has an impact on the rise in meth-use. State-Time plots (not shown here) indicate long-term effects, concerning the ratio of $\mathbf{N}^{\text {na }}$ to $\mathbf{N}^{\mathbf{a}}$ without a decrease of total population. $\mathbf{N}^{\mathbf{a}}$ get more while $\mathbf{N}^{\text {na }}$ decline in amount. It is remarkable that the price relationship always reaches its equilibrium after the PULSE ceases to act on an altered flow. This is a consequence of (5) which varies the terminal point by alternating $\boldsymbol{p}$ in a selfstabilizing way.

\section{REFERENCES}

Blake Ewing: Breaking Bad Normalizes Meth, Argues Prosecutor. Edited by Time Inc. 2013 [cited 2014 October]. Available online from http://ideas.time.com/2013/09/20/breaking-bad-promotesmeth-use-argues-prosecutor/

United Nations Office on Drugs and Crime. 2014. World Drug Report 2014: UNODC, United Nations publication, Sales No. E.14.XI.7 


\title{
Physiolibrary 2.3 - An Intuitive Tool for Integrative Physiology
}

\author{
Marek Mateják*, Filip Ježek**, Martin Tribula*, Jiří Kofránek**** \\ *First Faculty of Medicine, Charles University in Prague, U Nemocnice 5, Prague 2, 128 53, Czech Republic \\ **Faculty of Electrical Engineering, Czech Technical University, Prague, Czech Republic
}

\author{
marek@matfyz.cz
}

Physiolibrary (www.physiolibrary.org) is an open-source Modelica library (Mateják et al., 2014) that can be used for mathematical modelling of cardiovascular circulation (Kulhánek, Kofránek, \& Mateják, 2014), metabolic processes, nutrient distribution, thermoregulation, gas transport, electrolyte regulation, water distribution, hormonal regulation and pharmacological regulation.

Modelica language allows drag-and-drop drawing of electrical circuit diagrams, setting of parameters by dialogs, automatic algebraic manipulation and numerical simulation (Mattson, Elmqvist, \& Broenink, 1997). Graphical diagrams are an analogy for the textual mathematical description behind them, where a set of equations can be generated from exactly defined diagram blocks (Mattsson, Elmqvist, \& Otter, 1998). These sets of equations can be translated and numerically solved with sophisticated tools and solvers inside Modelica environments.

Taking analogies from electrical circuits, other physical, physiological and chemical domains can be expressed. The main principle of these diagrams is connections, which obey Kirchhoff's circuit laws for connectors with two types of variable: flow and non-flow. The electrical domain includes the electric current flow, while non-flow is electric potential, and the laws are the "zero sum of connected flows" and "the same connected non-flow values". The connectors of Physiolibrary listed in Table 1 also obey the same laws as the electric connector in an electric circuit. With these connectors, there is only a small step needed to define analogies of the diagram elements. For example, the analogies of electrical resistance and the electrical capacitor connected to ground are expressed in Table 2.

However, it seems to be more useful to have some specific components because of the different properties of domains. For example, the chemical reaction, the main component of the chemical domain, is implemented by a specific equation of the form:

reactionRate $=k_{f}$ volume $\left(\prod_{i}\left[S_{i}\right]^{s_{i}}-\right.$ $\left.\mathbf{1} / K \prod_{j}\left[P_{j}\right]^{p_{j}}\right)$. This could describe

Table 1

\begin{tabular}{|c|c|c|}
\hline Connector: & Flow & Non-Flow Variable \\
\hline$\square \square$ chemical & molar & concentration \\
\hline$\checkmark$ hydraulic & volumetric & pressure \\
\hline$\square \square$ thermal & heat & temperature \\
\hline$\square$ osmotic & volumetric & osmolarity \\
\hline$\square$ population & change & number of members \\
\hline
\end{tabular}

any amount of substrates $\boldsymbol{S}$ and products $\boldsymbol{P}$ with any fixed stoichiometric coefficients $\boldsymbol{s}$ and $\boldsymbol{p}$. The main parameter here is the dissociation constant $\boldsymbol{K}$, which is equal to the ratio between the forward reaction rate $\boldsymbol{k}_{\boldsymbol{f}}$ and the backward reaction rate, and can also be dependent on temperature (Van't Hoff's law). Specific properties are also components for gas solubility (Henry's law), for hydrostatic column (Pascal's law) or for the ideal radiator, where the heat transfer from medium to environment is calculated to reach the same temperature as the outflowing medium and the environment, etc.

Table 2

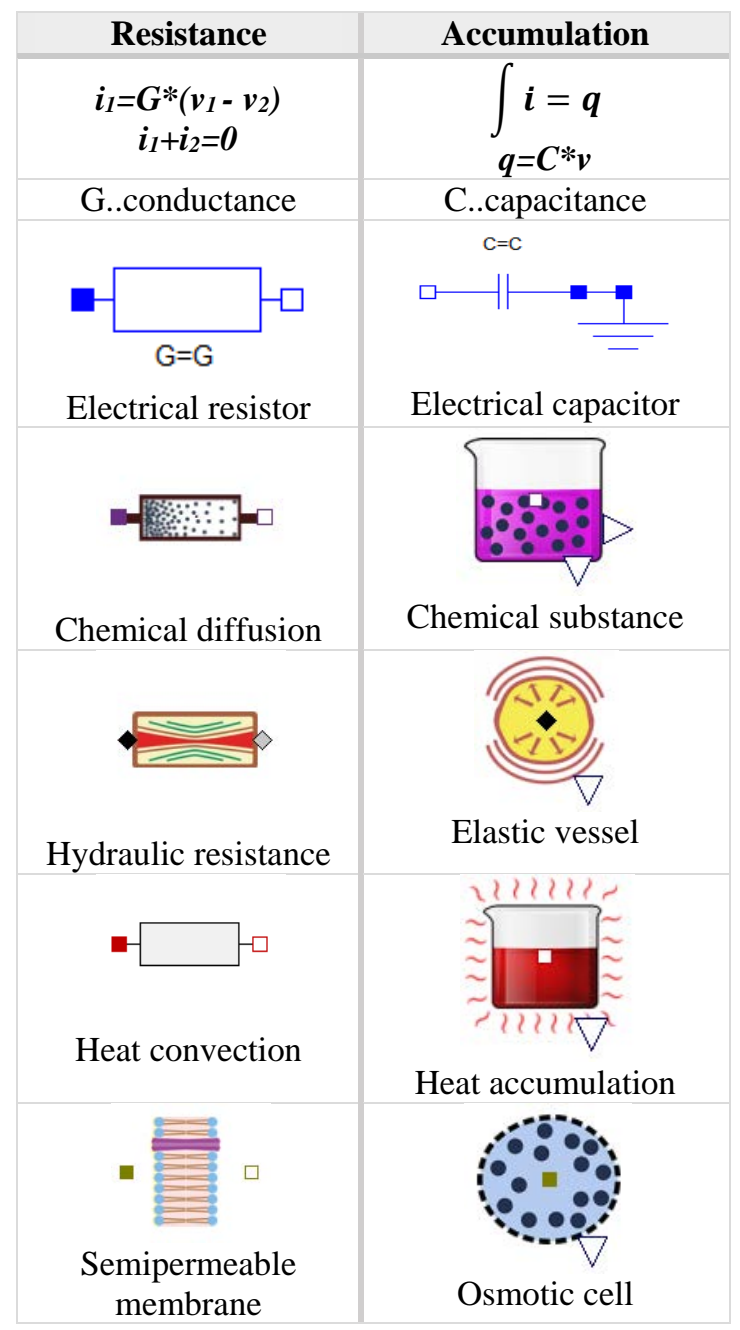


Creating a Modelica model using Physiolibrary is very intuitive (Mateják, 2014). Moreover, the models implemented by graphical diagrams are self-describing and easily expandable. We can demonstrate this, for example, in creating enzymatic reactions. First it is necessary to drag and drop the instances of library components to our new model. Then we set the parameter values using parameter dialogs. A possible setting is the substrate (solute_start=10 mmol), freeEnzyme (solute_start $=\mathrm{tE})$, chemicalReaction1 $\left(\mathrm{nS}=2, \mathrm{~K}=2 / \mathrm{K}_{\mathrm{m}}\right.$, $\left.\mathrm{kf}=2 * \mathrm{k}_{\mathrm{cat}} / \mathrm{K}_{\mathrm{m}}\right), \quad$ chemicalReaction2 $\quad(\mathrm{nP}=2$, $\mathrm{K}=$ Modelica.Constants.inf, $\mathrm{kf}=\mathrm{k}_{\mathrm{cat}}$ ), where $\mathrm{tE}$ is total concentration of enzyme, $\mathrm{k}_{\mathrm{cat}}$ is the maximum rate and $\mathrm{K}_{\mathrm{m}}$ is the Michaelis constant as usual. All other parameters can be set at default values. Because we define two substrates of chemicalReaction1 (parameter nS) and two products of chemicalReaction2 (parameter $\mathrm{nP}$ ), we can connect the different indexes for the chemical connector of the reactions to different substances. All connections are done by drawing a line from connector to connector, resulting in the final model in Figure 1.

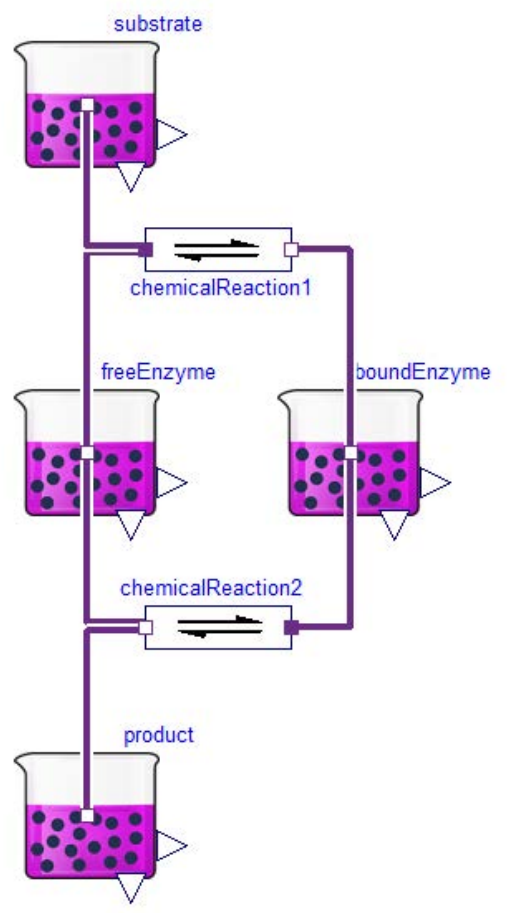

Figure 1

This model of enzymatic reaction dynamically changes substrate into product, as can be seen from the simulation results. But usually the reactions are quick enough to be calculated in equilibrium, which in Physiolibrary is called steady state, and is also supported. To examine these principles, please see the examples in the Physiolibrary.SteadyStates package.

The biggest model today based on Physiolibrary is called Physiomodel (www.physiomodel.org). Physiomodel was developed from the model HumMod Golem Edition (Mateják \& Kofránek, 2010, 2011), which extends the model HumMod (www.hummod.org) with more a complex acid-base and gas transports. These models integrate more than 600 original research studies listed in reference lists www.zotero.org/groups/physiomodel and www.zotero.org/groups/hummod_references. The model has 10 subsystems: cardiovascular, gases, water, extracellular proteins, electrolytes, hormones, nerves, status, nutrients and metabolism. Each subsystem is hierarchically composed with specific parts. The cardiovascular subsystem includes heart, pulmonary and systemic circulation. The subsystem of gases includes oxygen and carbon dioxide. The water and extracellular protein subsystem includes gastrointestinal lumen, plasma, torso, kidney and bladder. The electrolyte subsystem includes acid-base, sodium, chloride, potassium, phosphates, sulfate and ammonium. The hormonal subsystem has catecholamine, RAAS hormones, atriopeptin, erythropoietin, insulin, glucagon, leptin, thyrotropin and thyroxin. The neural subsystem has baroreceptors, metaboreflexes, sympathetic and parasympathetic pathways, kidney ganglia, hypothalamic thermoreflexes and the heart sinoatrial node. The metabolism subsystem features liver, tissues, glucose, fat, keto acids, amino acids, urea and lactate. Each of these parts is built upon Physiolibrary and can also be simulated separately.

Physiolibrary reduces implementation errors and significantly simplifies the subsequent extensions and integration of models, because components are based on elementary physical laws, the acausal connector interfaces with physical quantities, and SI units are compatible with other Modelica models and other Modelica libraries. This gives the user a very comfortable platform on which are automatically solved all the algebraic equations, the numeric solutions and the non-SI unit recalculation.

\section{REFERENCES}

Kulánek, T., Kofránek, J., \& Mateják, M. (2014). Modeling of short-term mechanism of arterial pressure control in the cardiovascular system: Object-oriented and acausal approach. Computers in Biology and Medicine, 54(0), 137-144.

Mateják, M. (2014). Physiology in Modelica. MEFANET Journal, 2(1), 10-14.

Mateják, M., \& Kofránek, J. (2010). Rozsáhlý model fyziologických regulací v Modelice. [Large scale model of physiological regulations in Modelica]. Medsoft, 126-146. In Czech.

Mateják, M., \& Kofránek, J. (2011). HumMod-Golem Edition-Rozsáhlý model fyziologických systémů. [Golem Edition - large scale model of physiological systems]. Medsoft, 182-196. In Czech.

Mateják, M., Kulhánek, T., Šilar, J., Privitzer, P., Ježek, F., \& Kofránek, J. (2014, March 10-12). Physiolibrary Modelica library for physiology. Paper presented at the 10th International Modelica Conference, Lund, Sweden.

Mattson, S. E., Elmqvist, H., \& Broenink, J. F. (1997). Modelica: An international effort to design the next generation modelling language. Journal A, 38(3), 1619.

Mattsson, S. E., Elmqvist, H., \& Otter, M. (1998). Physical system modeling with Modelica. Control Engineering Practice, 6(4), 501-510. 


\title{
Model predictive control in small family house: extravagance or future of energy consumption in households \\ Ante Marušić*, Dražen Lončar*
}

\author{
*Faculty of Mechanical Engineering and Naval Architecture, University of Zagreb, Ivana Lučića 5 \\ CROATIA (Tel: +38516168 172; e-mail: ante.marusic@fsb.hr).
}

Keywords: model predictive control, heating

\section{INTRODUCTION}

As one of fastest spreading control technologies since 1980s, model predictive control (MPC) has gained significance in process engineering and other similar industrial applications with fast responses and small time constants. Furthermore, public and academic pressure on energy savings during last few years has opened the doors for MPC in heating, ventilation and air conditioning (HVAC) systems, especially in research papers. Most of the energy management applications in buildings using MPC are devoted to complex office and residential buildings with advance HVAC systems, where detailed models can help reduce energy consumption and increase heat comfort at the same time. Additionally MPC is often presented as most cost effective solution when considering improvements of heating systems. MPC predicts impact of measured or estimated disturbance to the system on the controlled variables and sequently adjusts the control signals to achieve the desired behaviour of the process (Gruber et al. 2014). MPC potential for zero energy houses with heat pumps, passive and active energy storages, solar collectors etc. has been shown in number of examples. However, as complexity of the system (HVAC system and building thermal properties) decreases, control possibilities for MPC decrease as well. Therefore, benefits of MPC for small, conventional family house heating system are examined in this paper.

\section{SIMULATION}

Detailed model of a simple, one floor, family house was created with only simple heating system, detailed hourly weather and insolation profiles were acquired and used as boundary conditions. Model predictive controller was based on the same house model. Behaviour of the system with controller was simulated in the MATLAB Simulink. Weather forecasting with it's error was replicated with disturbance curve (error) that was superposed to temperature and insolation (because of clouds) vectors.

Results of simulation were compared with results of simulation with simple on/off relay regulation. Different relay thresholds were used to compare energy consumption and inhouse temperature behaviour. Two different thermal masses of heating device were used to affect response time of heating device. Significant energy consumptions were not observed, while temperature oscillations using simple regulation are much more prominent.

\section{HOUSE MODEL}

House model is based on a very simple, one floor house with a flat roof located in Zagreb, Croatia. House is divided in two rooms, $35 \mathrm{~m} 2$ each divided with a $35 \mathrm{~cm}$ thick wall. Outside walls are also $35 \mathrm{~cm}$ thick with $10 \mathrm{~cm}$ of XPS heat insulation on the outer side. House is positioned so each wall is perpendicular to one of four cardinal directions. The wall separating two rooms is positioned east-west. One window is placed on the south and north wall respectively.

Outside temperatures, insolation profiles for horizontal surfaces and vertical surfaces were downloaded from Photovoltaic Geographical Information System with resolution of $15 \mathrm{~min}$. Since simulation is performed only for first two months of the year, ground temperature is assumed constant and around $7{ }^{\circ} \mathrm{C}$.

Heating system is simulated as simple radiator heating device with control of inlet temperature and on-off control of constant inlet temperature water flow for MPC and relay control respectively. As it will be shown, thermal capacity of radiator (response speed) is one of the most influential parameters to discern results of two control techniques in such simple examples.

\section{MODEL PREDICTIVE CONTROLOR}

Since primary object of this article is detailed theoretical comparison of MPC and simple control techniques, different MPC controller configurations were used. Most important controller variables (number of prediction steps $(\mathrm{P})$, number of control steps (C) and weight coefficients) were varied in order to achieve best responses. Larger prediction horizons require more computer resources, and consequently more time to calculate optimal response. This is not of particular importance in heating applications because of very slow thermal behaviour of the building. Two different variables were used to determine control quality: Heat comfort (temperature variations in room) and overall heat consumption.

MPC controllers usually have prediction horizon in days, ie. from 2 days ( $\mathrm{Li}$ et al. 2015) to 7 days (Oldewurtel at al. 2012.).

Time step for MPC was set to 5 minutes. Control interval was varied in range from 0.4 hours ( 5 steps) to 6 hours ( 72 steps); prediction horizon was between 17 hours (200 steps) and 2 days (576 steps). Only constraint was set for inlet water temperature so it corresponds to the real values. Same method 
was used for insolation forecast. Thus obtained temperature and insolation vectors were used as measured disturbance for feed-forward control of the model.

\section{RESULTS}

Benefits of MPC on thermal comfort can be observed on temperature profiles for 6 days between January and February for both MPC and relay control presented in Fig. 1. While indoor air temperature varies over range of $1{ }^{\circ} \mathrm{C}$ for relay threshold of $0.4{ }^{\circ} \mathrm{C} \quad\left(19.6-20.4{ }^{\circ} \mathrm{C}\right)$, MPC controlled temperature varies in range smaller than $0.1^{\circ} \mathrm{C}$. Using smaller threshold range does not improve heat comfort proportionally $\left( \pm 0.1^{\circ} \mathrm{C}\right.$ results in room temperature behaviour $\left.\pm 0.4^{\circ} \mathrm{C}\right)$, but has harmful influence on valve, and higher energy consumption.

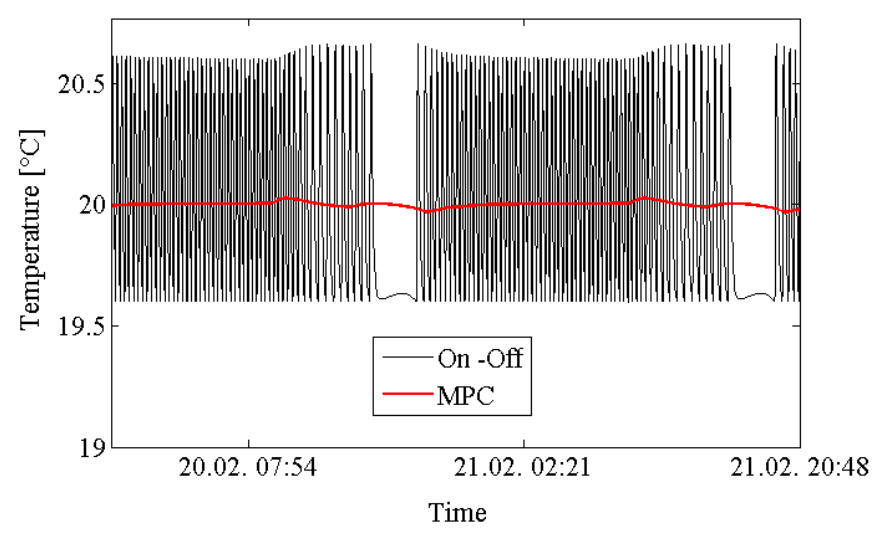

Fig. 1. Temperature profile in the south room (blue On/Off, red MPC)

Advantage of MPC is obvious and could deceptively lead to conclusion that usage of MPC also results in significant energy savings which, as can be seen from Fig. 2, is not the case. Also, small weather forecast error was simulated, therefore simulation results would be even worse if weather forecast error was more realistic.

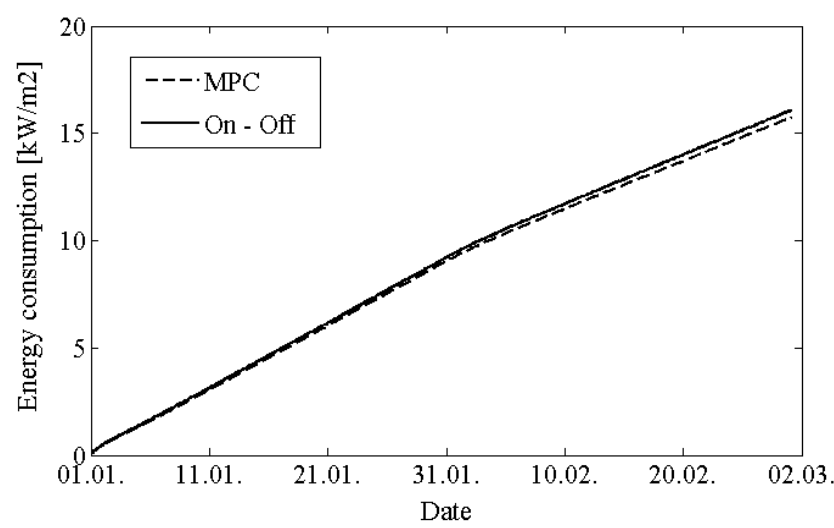

Fig. 2. Integral of heat consumption per square meter during simulated period

Prediction and control horizon length effect on energy savings can be seen in Fig 3. Simplicity of the system results in very small heat profiles deviation. Nevertheless longer prediction horizons show better energy consumption with insignificant effect on temperature deviations in house.

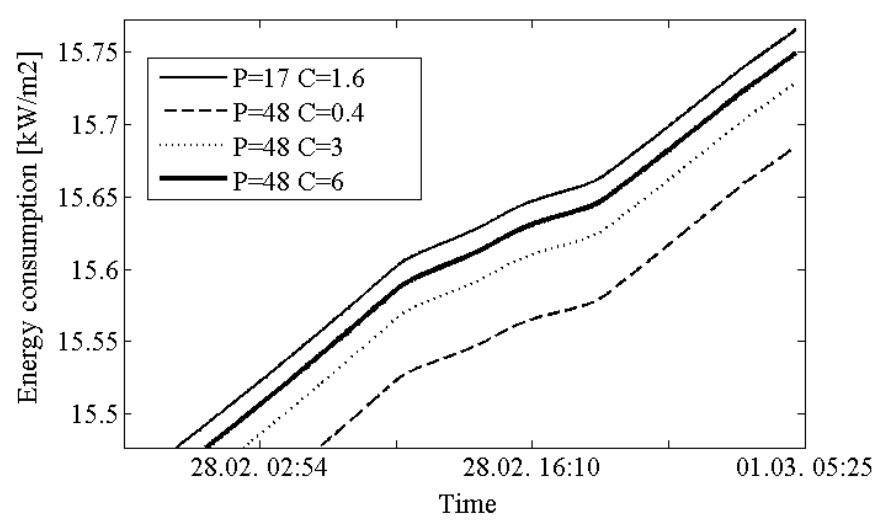

Fig. 3. Heat consumption at the end of simulation for different controller setups

During analysis it was also observed that variations of radiator thermal capacity have significant influence on benefits from MPC. Larger radiator heat capacity results in bigger savings on the behalf of MPC, this corresponds with expected MPC behaviour where predictive control has bigger influence. Adding electricity price to analysis could result in bigger benefits of MPC usage as whole house is acting like energy storage and therefore providing opportunity to shift electricity demand in time. However, in houses as simple as presented one, these benefits would still be far from justifying investments in MPC systems.

\section{REFERENCES}

Mattias Gruber, Anders Trüschel, Jan-Olof Dalenbäck (2014) Model-based controllers for indoor climate control in office buildings - Complexity and performance evaluation, Energy and Buildings, (68) 213-222

Mesut Avci, Murat Erkoc, Amir Rahmani, Shihab Asfour (2013) Model predictive HVAC load control in buildings using real-time electricity pricing, Energy and Buildings, (60) 199-209

Siwei Li, Jaewan Joe, Jianjun Hua, Panagiota Karava (2015) System identification and model-predictive control of office buildings with integrated photovoltaic-thermal collectors, radiant floor heating and active thermal storage, Solar energy, (113) 139-157

Frauke Oldewurtel, Alessandra Parisio, Colin N. Jones, Dimitrios Gyalistras, Markus Gwerder, Vanessa Stauch, Beat Lehmann, Manfred Morari (2012) Use of model predictive control and weather forecasts for energy efficient building climate control, Energy and Buildings, (45) 15-27 


\title{
Comparison between a non-linear and linearized three-compartment model of a bioreactor for hepatocyte culturing
}

\author{
Seyed Danial Naghib a, Alberto Di Renzo a, Efrem Curcio a,b, Loredana De Bartolo b, \\ Francesco Paolo Di Maio ${ }^{\text {a }}$ \\ ${ }^{a}$ Department of Environmental and Chemical Engineering, University of Calabria, \\ Via P. Bucci, cubo 44A, 87030 Rende (CS), Italy \\ b Institute on Membrane Technology, National Research Council of Italy, ITM-CNR, \\ via P. Bucci, cubo 17C, 87030 Rende (CS), Italy \\ danial.naghib@unical.it
}

Bioreactor solutions are being increasingly explored as alternatives to surgery treatments of severe hepatic failures. However, complications related to hepatocyte culturing requirements in vitro renders design, operation and optimization of proposed bioreactor solution extremely hard, with stable and durable cultivation of viable and functional cells hardly exceeding ten days. Several factors may limit the survival of hepatocyte cultures in artificial environment including nutrient transport, oxygen diffusion as well as removal of catabolites, but also provision for anchorage support and very limited flow shear stress [1, 2, 3]. In the present work, two versions of a simplified dynamic model of a crossed-hollow-fiber membrane bioreactor are discussed and compared. A linear and non-linear, inputoutput process models were developed fundamentally, i.e. starting from the equations governing flow, mass transfer, permeation, (bio)chemical reactions, in dynamic conditions.

With respect to the actual bioreactor configuration, in this preliminary modelling effort, various simplifying assumptions are considered in order to simplify the derivation. The reactor system is modelled as composed of three distinct zones, which are assumed as lumped parameter compartments (Fig 1):

1. A feed zone composed of a dead-end hollow-fiber polyetheretherketone (PEEK) membrane bundle (FB) providing oxygen and medium/serum feeds;

2. An intermediate space occupying the reactor shell and surrounding the two membrane bundles, similar to the extra-capillary space (named ECS), which contains the cell culture;

3. A second bundle of dead-end hollow-fiber polyethersulphone (PES) membranes in crossed configuration for the effluent stream (EB).

Seven-state model equations have been developed. The non-linear model, from which a linearized version has been derived, is detailed below. Input variables are inlet concentrations $C_{A 0}, C_{B 0}$ (concentration of oxygen (A) and urea (B)), and ECS inlet and outlet flow-rates $\left(F_{1}, F_{2}\right)$. State variables are all other (6) concentrations and the variable liquid volume in the ECS $V_{2}$. The overflow stream rate from compartment $2, F_{o f}$, is set to zero.

The non-linear, original set of model equations is composed of:

$$
\begin{array}{ll}
\text { 1- } \frac{d C_{A 1}}{d t}=\frac{F_{1}}{V_{1}}\left(C_{A 0}-C_{A 1}\right) & \text { 2- } \frac{d C_{B 1}}{d t}=\frac{F_{1}}{V_{1}}\left(C_{B 0}-C_{B 1}\right) \\
\text { 3- } \frac{d C_{B 2}}{d t}=\frac{F_{1}}{V_{2}}\left(C_{B 1}-C_{B 2}\right)+\frac{V_{\max } C_{A 2}}{K_{m}+C_{A 2}} & \text { 4- } \frac{d C_{A 2}}{d t}=\frac{F_{1}}{V_{2}}\left(C_{A 1}-C_{A 2}\right)-\frac{V_{\max } C_{A 2}}{K_{m}+C_{A 2}} \\
\text { 5- } \frac{d C_{A 3}}{d t}=\frac{F_{2}}{V_{3}}\left(C_{A 2}-C_{A 3}\right) & \text { 6- } \frac{d C_{B 3}}{d t}=\frac{F_{2}}{V_{3}}\left(C_{B 2}-C_{B 3}\right)
\end{array}
$$

7- $\frac{d V_{2}}{d t}=F_{1}-F_{2}$

The linearized model differs in that it contains the first-order Taylor series truncation of the non-linear terms expanded around the steady-state values of the variables.

The biochemical reaction rate is described using the Michaelis-Menten enzymatic model. Here, $V_{\max }$ represents the maximum rate achieved by the system at maximum (saturating) substrate concentrations. The 


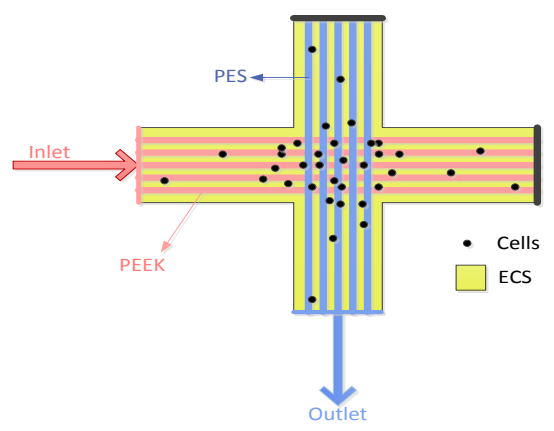

a)

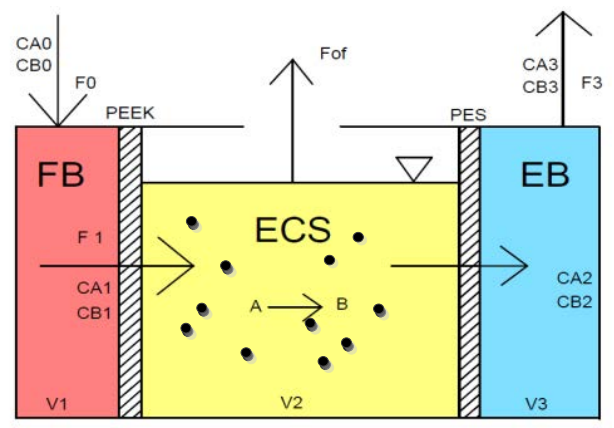

b)

Figure 1. a) Hollow fiber membrane bioreactor scheme, b) Three-compartment schematic model of the membrane bioreactor. FB = feed membrane bundle, ECS = extra capillary space, EB = effluent membrane bundle.
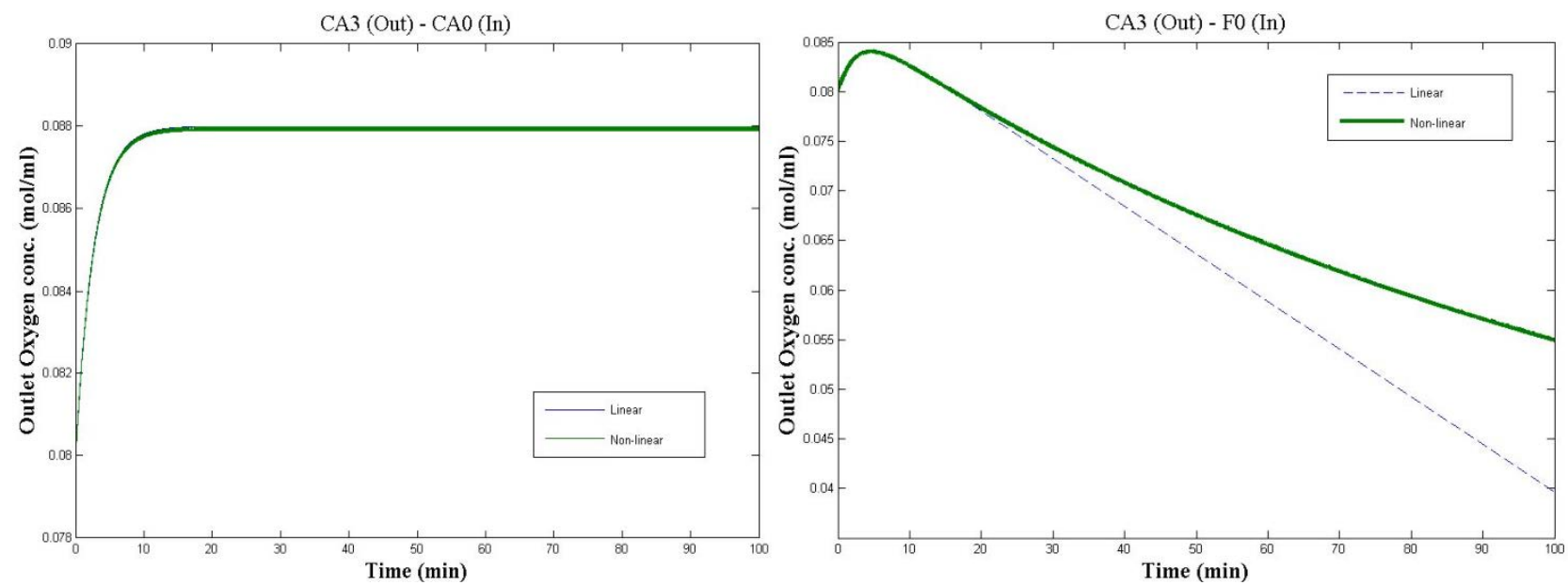

Figure 2. Non-linear vs. linear model: oxygen output concentration $C_{A 3}$ for a $10 \%$ step change in the input concentration $C_{A 0}$ (left) and the inlet flow-rate $F_{0}\left(=F_{1}\right)$ (right).

Michaelis constant $K_{m}$ is the substrate concentration at which the reaction rate is half of $V_{\max }$. Since progenitor hepatocytes are considered, the number of cells in the system is constant.

Predictions of the responses produced by the linearized model (more flexible for control design) and the nonlinear model (more complex but realistic) are compared for a step change in selected inputs. The analysis reveals how changes in the inlet compositions produce negligible differences between the two versions, while a change in the inlet flow-rate (e.g. as a result of membrane fouling effects) affects significantly the evolution of the outlet concentrations. Fig. 2 shows a comparison between non-linear and linear model responses of the outlet oxygen concentrations $C_{B 3}$ for a $10 \%$ step change in inlet oxygen concentration $C_{A 0}$ and in inlet flowrate $F_{1}$. Differences, when present, are evident after about 20 min of operation. Notably, as it can be inferred from Fig. 2, negative concentrations can be obtained (after $180 \mathrm{~min}$ ) using the linearized model, which is clearly unacceptable.

In the future, the present work will serve to improve the understanding of the behaviour of the reactor in transient conditions and as a basis for the development of an instrumentation and control system aimed at enhancing the bioreactor operation under optimal conditions.

\section{Acknowledgment}

The financial support of the European Union through the Project FP7-PEOPLE-2012-ITN "Training network for developing innovative bioartificial devices for treatment of kidney and liver disease" (G.A. no. 316690) is gratefully acknowledged. 


\title{
Taylor Series Based Differential Formulas
}

\author{
Gabriela Nečasová ${ }^{*}$ Jiří Kunovský* Václav Šátek ${ }^{*, * *}$ \\ Jan Chaloupka* Petr Veigend* \\ * University of Technology, Faculty of Information Technology, \\ Božetěchova 2, 612 66 Brno, Czech Republic, (e-mail: \\ inecasova@fit.vutbr.cz) \\ ** IT4Innovations, VŠB Technical University of Ostrava, \\ 17. listopadu 15/2172, 70833 Ostrava-Poruba, Czech Republic
}

\begin{abstract}
The paper is a part of student cooperation in AKTION project (Austria-Czech) and concentrates on numerical computations using high-order forward, backward and symmetrical formulas. As an example, the hyperbolic PDE is analyzed, together with multiple integral computations. A multiple integral of a continuous function of $n$ variables can be computed by $n$-ary integration of the function fixing the remaining variables.
\end{abstract}

Keywords: Differential equations, Multiple integrals, Taylor series method, Partial Differential Equations

\section{INTRODUCTION}

The aim of the paper is to show an easy way of very high order difference formulas construction using Taylor series terms. For the point $u_{1}$ using forward formula (with respect to Fig. 1) we have following equations.

$$
\begin{aligned}
& u_{2}=u_{1}+h u_{1}^{\prime}+\frac{h^{2}}{2 !} u_{1}^{\prime \prime}+\frac{h^{3}}{3 !} u_{1}^{\prime \prime \prime}+\frac{h^{4}}{4 !} u_{1}^{\prime \prime \prime \prime} \\
& u_{3}=u_{1}+2 h u_{1}^{\prime}+\frac{(2 h)^{2}}{2 !} u_{1}^{\prime \prime}+\frac{(2 h)^{3}}{3 !} u_{1}^{\prime \prime \prime}+\frac{(2 h)^{4}}{4 !} u_{1}^{\prime \prime \prime \prime} \\
& u_{4}=u_{1}+3 h u_{1}^{\prime}+\frac{(3 h)^{2}}{2 !} u_{1}^{\prime \prime}+\frac{(3 h)^{3}}{3 !} u_{1}^{\prime \prime \prime}+\frac{(3 h)^{4}}{4 !} u_{1}^{\prime \prime \prime \prime} \\
& u_{5}=u_{1}+4 h u_{1}^{\prime}+\frac{(4 h)^{2}}{2 !} u_{1}^{\prime \prime}+\frac{(4 h)^{3}}{3 !} u_{1}^{\prime \prime \prime}+\frac{(4 h)^{4}}{4 !} u_{1}^{\prime \prime \prime \prime}
\end{aligned}
$$

Above mentioned forward formula considers only five points.

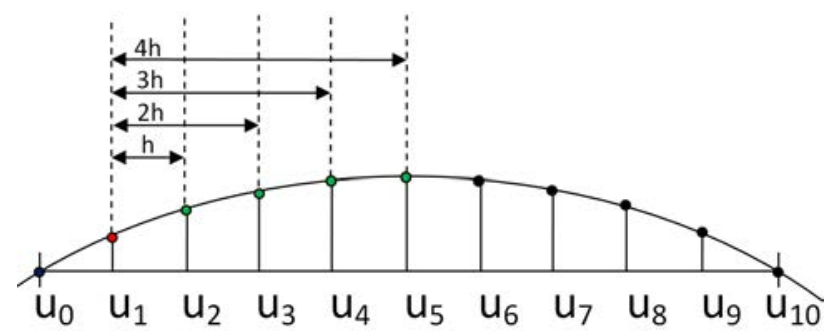

Fig. 1. Forward method

After expressing

$$
\begin{aligned}
& u_{2}-u_{1}=D U 1+D U 2+D U 3+D U 4 \\
& u_{3}-u_{1}=2 D U 1+2^{2} D U 2+2^{3} D U 3+2^{4} D U 4 \\
& u_{4}-u_{1}=3 D U 1+3^{2} D U 2+3^{3} D U 3+3^{4} D U 4 \\
& u_{5}-u_{1}=4 D U 1+4^{2} D U 2+4^{3} D U 3+4^{4} D U 4
\end{aligned}
$$

the Taylor series terms $D U 1, D U 2, D U 3, D U 4$ can be calculated, where $D U i=\frac{u_{1}{ }^{(i)}}{i !} h^{i}$.

Similarly, the Taylor series terms can be calculated for the backward and symmetrical differential formulas.

\section{HYPERBOLIC PDE}

One of the most common hyperbolic PDE is the wave equation Burden and Faires (2010); Collins (2006); Grasselli and Pelinovsky (2008); Cheney and Kincaid (2013); Kunovský (1995).

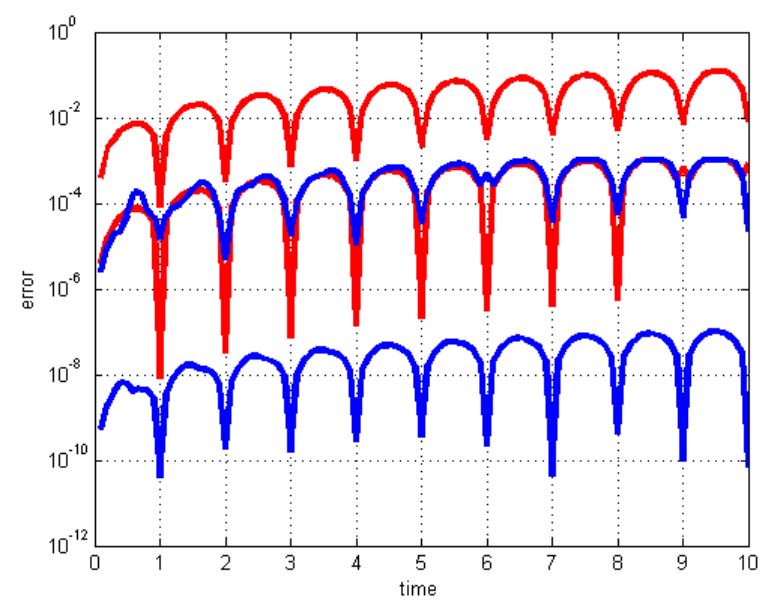

Fig. 2. "Error" functions

The wave equation may describe the oscillations of an ideal string of unit length. The "error" function of hyperbolic PDE solution using a three-point approximation is plotted in Fig. 2- the upper red function for 10 segments and the lower red function for 100 segments. The "error" can be more effectively decreased by an increase in the order of the difference formula. The upper blue function of Fig. 2 
plots the "error" function for 12 segments and also for 100 segments (shown in the blue function down) supposing that a five-point approximation has been used.

\section{MULTIPLE INTEGRAL COMPUTATION}

Here we transform the solution of integral

$$
F(x)=\int_{a}^{b} f(x) \mathrm{d} x
$$

into the ordinary differential equation

$$
F^{\prime}(x)=f(x)
$$

with initial condition $F(a)=0$, see Hirayama (2008).

Taylor series method can be used in solution of (9) in form

$$
\begin{aligned}
F\left(x_{1}\right) & =F\left(x_{0}\right)+\frac{h}{1 !} F^{(1)}\left(x_{0}\right)+\frac{h^{2}}{2 !} F^{(2)}\left(x_{0}\right)+\cdots \\
F\left(x_{2}\right) & =F\left(x_{1}\right)+\frac{h}{1 !} F^{(1)}\left(x_{1}\right)+\frac{h^{2}}{2 !} F^{(2)}\left(x_{1}\right)+\cdots \\
\vdots & \\
F\left(x_{n}\right) & =F\left(x_{n-1}\right)+\frac{h}{1 !} F^{(1)}\left(x_{n-1}\right)+\frac{h^{2}}{2 !} F^{(2)}\left(x_{n-1}\right)+\cdots
\end{aligned}
$$

where the sequence $x_{0}, x_{1}, \ldots, x_{n}$ represents a discretization of the interval $\langle a, b\rangle$.
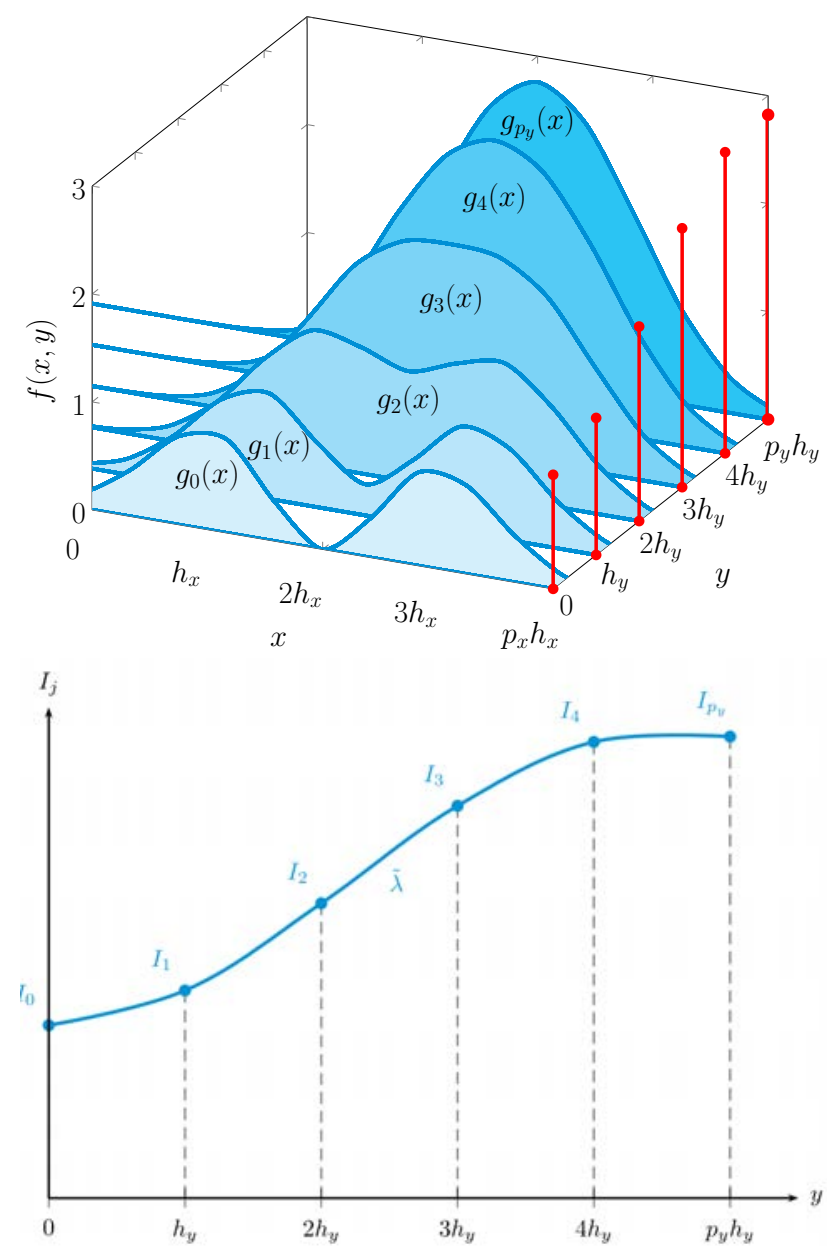

Fig. 3. Sampled integrals as a new function $I_{j}=\Psi\left(j h_{y}\right)$

\subsection{Double integral}

Without any loss of generality we can suppose all double integrals are in form 11.

$$
\int_{0}^{b_{2}} \int_{0}^{b_{1}} f(x, y) \mathrm{d} x \mathrm{~d} y
$$

Numerical solution starts by sampling both the $x$ and $y$ axis see Fig. 3.

After sampling we get a new function $\psi$ with values defined at multiples of $h_{y}$ (see Fig. 3). Using $\psi$, we can approximate the double integral by the following formula.

$$
\int_{0}^{b_{2}} \int_{0}^{b_{1}} f(x, y) \mathrm{d} x \mathrm{~d} y \approx \int_{0}^{b_{2}} \psi(y) \mathrm{d} y
$$

Using differential formulas, Taylor series terms can be calculated and Taylor series method presented in (10) can be used for double integral computation.

\section{CONCLUSIONS}

The process of multiple integral can be generalized for $n$ ary integrals by repeating the process above for all subintegrals. Triple integral is computed from a sampled double integral as a function of one variable. The number of integrals grows exponentially with $n$. Integrated function is sampled, continuously integrated by above mentioned process, integrals are composed into a function. The functions then decrease the multiplicity of integration. Once we get a double integral, the last iteration of the process is executed.

\section{ACKNOWLEDGEMENTS}

This paper has been elaborated in the framework of the project New creative teams in priorities of scientific research, reg. no. CZ.1.07/2.3.00/30.0055 (as well as the IT4Innovations Centre of Excellence CZ.1.05/1.1.00/ 02.0070), supported by Operational Programme Education for Competitiveness and co-financed by the European Social Fund and the state budget of the Czech Republic. The paper includes the solution results of the international AKTION research project Number 69p22 and the internal BUT projects FIT-S-12-1 and FIT-S-14-2486.

\section{REFERENCES}

Burden, R.L. and Faires, J.D. (2010). Numerical Analysis. Cengage Learning.

Cheney, E.W. and Kincaid, D.R. (2013). Numerical Mathematics and Computing. Cengage Learning, 7th edition.

Collins, P.J. (2006). Differential and Integral Equations. Oxford University Press.

Grasselli, M. and Pelinovsky, D. (2008). Numerical Mathematics. Jones \& Bartlett Learning.

Hirayama, H. (2008). Fast numerical integration method using Taylor series. In C. Constanda and S. Potapenko (eds.), Integral Methods in Science and Engineering, 135-140. Birkhuser Boston.

Kunovský, J. (1995). Modern Taylor Series Method. Habilitation work, Brno University of Technology, Brno. 


\section{MATHMOD 2015 ABSTRACT VOLUME}

Index of Authors 
MATHMOD 2015 Abstract Volume 
Alessandro, Alla

Antoulas, Athanasios C.

Araujo, Pablo

Araujo Pimentel, Guilherme

Aschauer, Guilherme

Aschemann, Harald

Atanasijevic-Kunc, Maja

Atitallah, Asma

Bachler, Martin

Bader, Eduard

Bakhshande, Fateme

Banagaaya, Nicodemus

Bascetta, Luca

Bastin, Georges

Becker, Felix

Belkhir, Fethi

Benavides, Micaela

Bianca, Carlo

Bicher, Martin

Bodi, Michael

Bolotnik, Nikolay

Bück, Andreas

Buhr, Andreas

Butt, Saif Siddique

Buwaya, Julia

Cafferkey, Neil

Caló, Antonio

Caponetto, Riccardo

Carla, Pinto

Caron, Simon

Carvalho, Ana

Casella, Francesco

Castagnotto, Alessandro

Chernousko, Felix L.

Chernyshov, Kirill

Chudej, Kurt

Costeseque, Guillaume

Couenne, Francoise

Cruz Varona, Maria

Dabidian, Peiman

Dahmen, Thorsten

Delgado Londoño, Sergio

DeSimone, Antonio

Detz, Hermann

Dickinson, Jillian L

Dirk, Peschka

Dullinger, Christian

Duque Guerra, David José

Dürr, Robert

Dyczij-Edlinger, Romanus

Dziwnik, Marion

Edelmann, Johannes

Endel, Florian

Faustner, David
137 Fehr, Joerg 131

117 Fernandes, Sofia 216

223 Figurina, Tatiana 200

$222 \quad$ Forstinger, Martin 57

231 Fries, Clemens 266

207 Gahleitner, Reinhard 85

79 Garofalo, Gianluca 54

73 Geiser, Juergen 163, 167

258 Gentile, Basilio 275

145 George, K.M. 100

80 Geuss, Matthias 127

289 Gilson, Marion 11

157 Glas, Silke 147

214 Goodwine, Bill 175

203 Gotthans, Tomas 291

23 Goupil, Philippe 9

220 Graube, Markus 282

168 Grimaud, Ghjuvan Micaelu 49

36 Grüner, Sten 280

89 Gugercin, Serkan 119

202 Haase, Joachim 68

41 Hafner, Irene 330

150 Harald, Piringer 256

86 Helmers, Michael 240

99 Herrmann, Lukas $\quad 84$

44 Himpe, Christian 148

315 Höfinger, Gerhard 343

178 Höger, Christoph 33

107 Holm, Sverre 173

66 Holzwarth, Philip 140

109, 111, $113 \quad$ Huang, Chenzi 375

155 Iapichino, Laura 143

363 Jadachowski, Lukas 186

199 Jansen, Lennart 141

$75 \quad$ Kampert, David 273

$87 \quad$ Kerler, Johanna 135

$35 \quad$ Khairudin, Nurulhuda 219

$183 \quad$ Khlopov, Dmytro 125

$361 \quad$ Kimmerle, Sven-Joachim 244

$367 \quad$ Klancar, Gregor 353

$301 \quad$ Klodmann, Julian 53

$55 \quad$ Konz, Matthias $\quad 365$

195, 197 Kostin, Georgy 96

67 Kouki, Mohamed 379

$47 \quad$ Kramer, Bernhard 18

237 Kuo, Chung-Hsien 205

$232 \quad$ Kuznetsov, Nikolay $\quad 69,70$

287 Lakehal, Abdelaziz 65

19 Lames, Martin 309

129, 138 Lang, Norman 121

238 Lebacque, Jean-Patrick 95

229 Lee, Alpha Albert 241

251 Lefevre, Laurent 184

63 Lein, Claudius 133 
Lemarchand, Annie

Leser, Roland

Lindhorst, Henning

Liukkonen, Mika

Lopez-Quiroga, Estefania

Lorenz, Wolfgang E.

Louca, Loucas

Louis, Jean-Nicolas

Macchelli, Alessandro

Maione, Guido

Manhartsgruber, Bernhard

Marušić, Ante

Maschke, Bernhard

Mateják, Marek

Mayr, Simon

McKenzie, Ross

Mercorelli, Paolo

Michael, Johannes

Mikota, Gudrun

Montorsi, Luca

Moriwaki, Katsumi

Moulik, Bedatri

Muffato, Leonardo Angelo

Murray-Smith, David.

Music, Gasper

Muthig, Olga

Naghib, Seyed Danial

Nazari, Shaghayegh

Necasova, Gabriela

Njiri, Jackson Githu

Novikov, Anton

Orlov, Igor

Ortner, Thomas

Özdemir, Denis

Paltrinieri, Fabrizio

Parragh, Stephanie

Pawletta, Thorsten

Pecha, Petr

Penasso, Harald

Perjesi-Hamori, Ildiko

Pesch, Hans Josef

Peters, Bernhard

Pöll, Carina

Proell, Stefan

Pumhössel, Thomas

Puntel Schmidt, Philipp

Putek, Piotr Adam

Rabenstein, Rudolf

Rauh, Andreas

Richelle, Anne

Rieck, Christian

Ritzberger, Daniel

Rooman, Marianne

Sakrutina, Ekaterina
Samani, Nozar

Sano, Hideki

50,76

303

22

316,319

224

102

56

314

185

177

269

385

189

383

159

324

21,64

247

268

263

88

341

82

335

101

26

387

277

389

83

42

208

255

156

264

17

369,371

48

299

373

349

169

327

253

234

279

293

20

210

221

28

230

215

74

Sarhan, Ahmed A.D. $\quad 27$

Sarkheyli, Arezoo 25

Satek, Vaclav 377

Scaglioni, Bruno 337

Schitter, Georg $\quad 12$

Schlacher, Kurt 187

Schlecker, Benedikt 355

Schleicher, Stephan 245

Schmidt, Andreas 144

Schneckenreither, Günter 34

Schneider, Eugenia 213

Schöberl, Markus 192

Scholz, Lena 323

Schöps, Sebastian 10

Schörgenhumer, Markus $\quad 165$

Senichenkov, Yury B. 345

Skvaril, Jan $\quad 317$

Soltan, Ibrahem 29

Specker, Thomas 59

Steinboeck, Andreas $\quad 190$

Steinbrecher, Andreas $\quad 326$

Steindl, Alois 227

Stemmler, Eric $\quad 357$

Stöckl, Michael 307

Strohm, Christian 295

Sueur, Christophe 81

Tamimi, Hammam 339

Tilp, Markus $\quad 305$

Tischendorf, Caren $\quad 328$

Tokic, Amir 359

Tsuchiya, Takeshi 351

Tulzer, Gerhard 94

Van der Linden, Franciscus L.J 158

Van Impe, Jan F.M. 217

Vetyukov, Yury 191

von Lieres, Eric 218

Wagnerova, Renata $\quad 60$

Winkler, Stefanie $\quad 37$

Witzmann, Magdalena $\quad 381$

Wurzer, Gabriel 103

Yebra, Luis J. $\quad 313$

Zaal, Martijn 243

Zauner, Günther $\quad 259$

Zeman, Paul 58

Zhang, Yongjin 123

Zupancic, Borut 347 


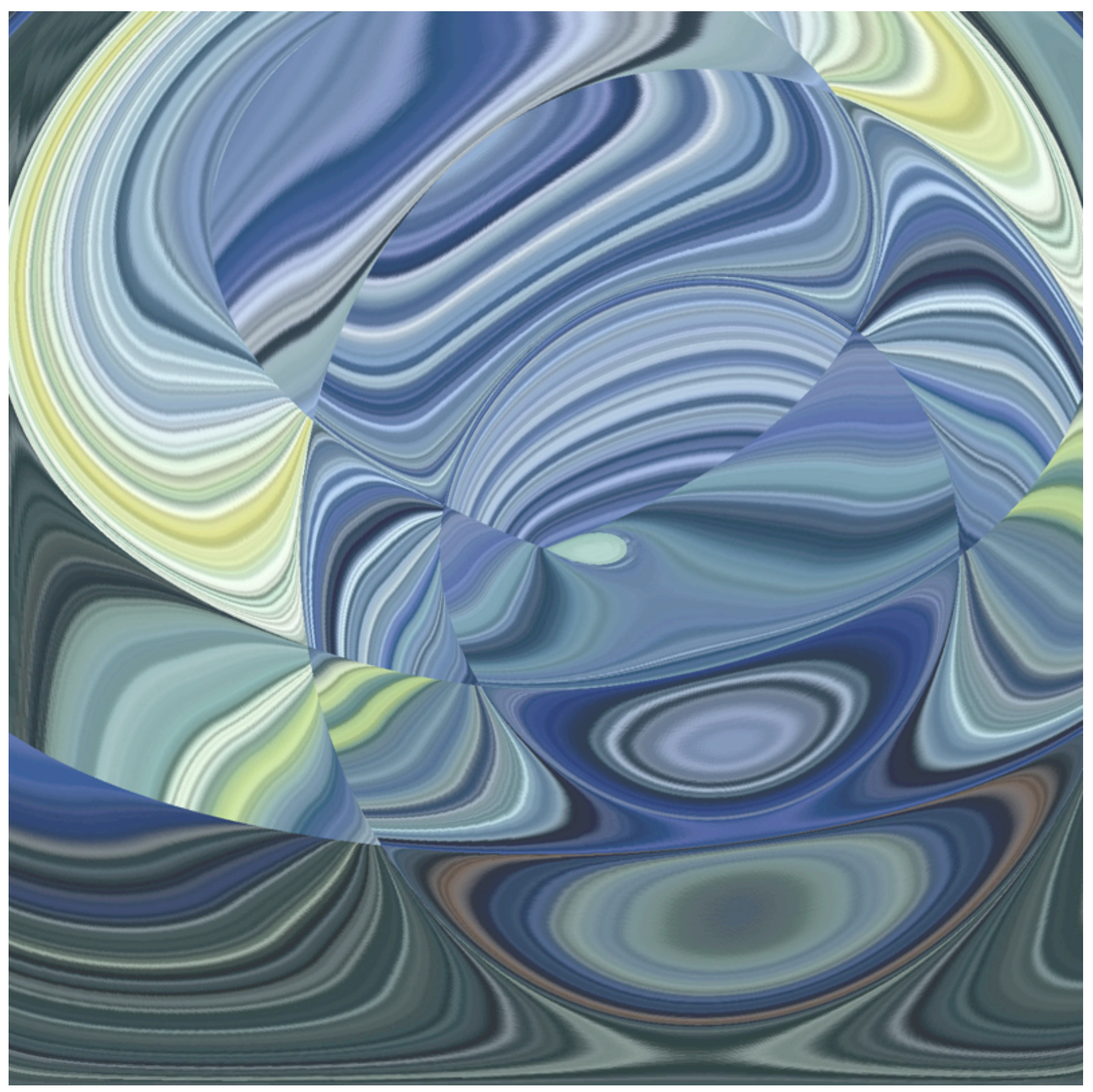

ARGESIM REPORT No. 44 